\title{
Towards an in vitro diagnosis for neurodegeneration
}

Citation for published version (APA):

Teunissen, C. E. (2001). Towards an in vitro diagnosis for neurodegeneration. [Doctoral Thesis, Maastricht University]. Datawyse / Universitaire Pers Maastricht. https://doi.org/10.26481/dis.20011130ct

Document status and date:

Published: 01/01/2001

DOI:

$10.26481 /$ dis.20011130ct

Document Version:

Publisher's PDF, also known as Version of record

\section{Please check the document version of this publication:}

- A submitted manuscript is the version of the article upon submission and before peer-review. There can be important differences between the submitted version and the official published version of record.

People interested in the research are advised to contact the author for the final version of the publication, or visit the DOI to the publisher's website.

- The final author version and the galley proof are versions of the publication after peer review.

- The final published version features the final layout of the paper including the volume, issue and page numbers.

Link to publication

\footnotetext{
General rights rights.

- You may freely distribute the URL identifying the publication in the public portal. please follow below link for the End User Agreement:

www.umlib.nl/taverne-license

Take down policy

If you believe that this document breaches copyright please contact us at:

repository@maastrichtuniversity.nl

providing details and we will investigate your claim.
}

Copyright and moral rights for the publications made accessible in the public portal are retained by the authors and/or other copyright owners and it is a condition of accessing publications that users recognise and abide by the legal requirements associated with these

- Users may download and print one copy of any publication from the public portal for the purpose of private study or research.

- You may not further distribute the material or use it for any profit-making activity or commercial gain

If the publication is distributed under the terms of Article $25 \mathrm{fa}$ of the Dutch Copyright Act, indicated by the "Taverne" license above, 
Towards an in vitro diagnosis for neurodegeneration 
C.E. Teunissen, Maastricht 2001

ISBN $905278325 X$

Omslagontwerp: Geertjan van Zonneveld

Druk: Datawyse I Universitaire Pers Maastricht 


\section{Towards an in vitro diagnosis for neurodegeneration}

\section{PROEFSCHRIFT}

Ter verkrijging van de graad van doctor aan

de Universiteit Maastricht, op gezag van de Rector Magnificus,

Prof.dr. A.C. Nieuwenhuijzen Kruseman, volgens het besluit van het College van Decanen, in het openbaar te verdedigen

op vrijdag 30 november 2001 om 16.00 uur

door

Charlotte Elisabeth Teunissen

Geboren te Deventer op 15 augustus 1971

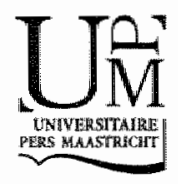




\section{Promotores:}

Prof.dr. H.W.M. Steinbusch

Prof.dr. C. de Bruijn

\section{Co-promotor:}

Dr. J. de Vente

\section{Beoordelingscommissie:}

Prof.di. J. Troost (voorzitter)

Profidr. A. Bast

Prof.dr. J. Delanghe (Universitair Ziekenhuis Gent, België)

Prof.dr. C. Dijkstra (Vrije Universiteit Amsterdam)

Prof.dr. M. Maes

Financial support for the publication of this thesis was given by:

Internationale Stichting Alzheimer Onderzoek, Lilly Nederland, Novartis Pharma Nederland BV, Paes Nederland BV, Servier Nederland BV, Stichting Alzheimer Nederland and UCB Pharma BV. 
Ritannädle 



\section{Contents}

\section{Chapter 1}

Introduction: biochemical markers ralated to Alzheimer's dementia in serum and

cerebrospinal filuid

\section{Chapter 2}

Whole brain spheroid cultures as a model to study the development of nimic oxide synthase - guanylate cyclase signal transduction

\section{Chapter 3}

Presence of soluble and particulate guanylyl cyclase in the same hippocampal

astrocytes

\section{Chapter 4}

Evaluation of 3-nitrotyrosine as a marker fior 3-nitropropionic acid induced oxidative stress in Lewis and Wistar rats and straim-specific whole brain spheroid cultures

\section{Chapter 5}

Behavioural correlates of striatal glial fibrillary acidic protein in the 3-nitropropionic acid rat model: disturbed walking pattem and spatial orientation

\section{Chapter 6}

Concentrations of different sterols in the striatum and serum of 3-nitropropionic acid treated Wistar and Lewis rats

\section{Chapter 7}

Stability of Interleukin 6 , Interleukin 6 Receptor, Interleukin 10 and C 16 in lnuman serum

\section{Chapter 8}

Inflammation markers are related 10 cognition in a healthy ageing population

\section{Chapter 9}

Serum cholesterol precursors and metabolites in relation to cognitive performance in an ageing population. A longitudinall follow-up study

\section{Chapter 10}

Homocysteine: a marker for cognitive performance? A longitudinal follow-up and patient study

\section{Chapter 11}

Evaluation study of 29 markers in serum of patients with Alzheimer's and Parkinson's 
Chapter 12

Ceneral discussion

Summary

Samenvatting 223

Publications

Curriculum vitae

Dankwoord 


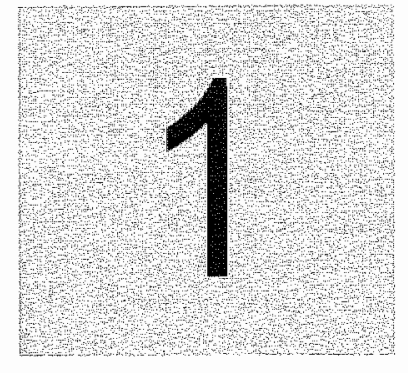

\section{Biochemical markers related to Alzheimer's disease in serum and cerebrospinal fluid}

Charlotte Teunissen, Jan de Vente, Harry Steinbusch and Chris De Bruijn

European Graduate School of Neuroscience (EURON), Maastricht University, Department of Psychiary and Neuropsychology, The Nethertands

Neurobiology of Aging, in press 


\section{Introduction}

Agerelated neurodegenerative diseases including Alzheimer's (AD) and Parkinson's disease (PD) are posing an increasing impact on our society mainly due to an increasing life expectancy (Table 1). Not only will the emotional buten increase for more and more patients and their social environment (family or caretakers) $[222,265]$, but also the economic costs for society in general will increase. For example, in the Netherlands the annual costs for an $A D$ patient living at home is estimated at 5,000 - 6,000 Euro. The annual costs for admission to a residential home is estimated at 22,000 Euro, white the costs for admission to nursing homes is estimated at 4547,000 Euro (prices of 1996$)[302]$.

To date, the diagnosis of probable or possible AD is based on neuropsychological examination using criteria such as insidious onset and progressive impairment of memory as well as loss of other cognitive functions [197]. Differentiation between Alzheimer and other types of dementia is based on exclusion, for example: reversible causes, tumours or vascular dementia. The feversible causes of dementia, e.g. metabolic disorders vitamin B12 deficiency, thyroid disorders, or drug intoxication, can be excluded via additional blood tests. Vascular dementia is suspected based on the medical history (e.g. cerebrovascular disease), and is confimed by

\begin{tabular}{|c|c|}
\hline Prevalence of demendia in the European population oven gen yil. & $n=25.8 .10): 6.4 \%[168]$ \\
\hline 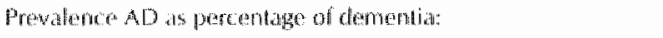 & $53.7(38.5-78 \%) \%[168]$ \\
\hline Odds Ratio womary compared to man ager $>80$ wears: & $1.2-1.3 \llbracket 87 \rrbracket$ \\
\hline linc idence $\mathrm{AD}$ people aged 65.59 years ": & $1.240 .6-2.3)[87]$ \\
\hline Incidance AD puople uged $70-74$ yeass : & $3.3(2.2-4.3)[97]$ \\
\hline Un idence AD proople aget $75-79$ years"; & $9.1[7.1-10.7)[197]$ \\
\hline Incichofe $A D$ people aged b0-BA years & $21.8(17.6-24.0) 978$ \\
\hline Inc inlences AD people anged $85-89$ years " & $35.3(25.5-36.3)[87]$ \\
\hline Iroidente AD people anged $>90$ years ": & $53.5(36.5 \times 55.8) 1877$ \\
\hline 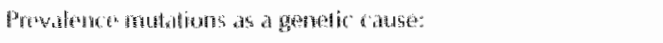 & $-2 \% 11611$ \\
\hline 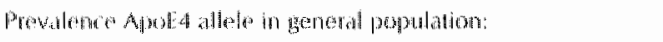 & $16 \%[2811$ \\
\hline 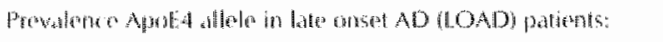 & $50 \%[281]$ \\
\hline 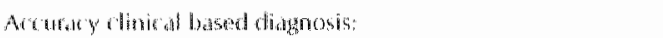 & $0.620 .92 \mid 1411$ \\
\hline
\end{tabular}

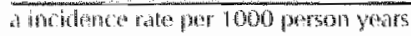

magnetic resonance imaging (MRD) or single photon emission computed tomography (SPECT) [2611. Conclusive diagnosis of AD may only be made after post mortem examination of brain lissue for the presence of large numbers of plaques and tangles. However, it has to be realised that the mere presence of plaques and tangles in the brain is not sufficient evidence for a conclusive diagnosis of $\mathrm{AD}$ as these have also been reported in cognitive healthy elderly $[61,126]$.

Several genetic risk factors for familial AD are known, including mutations in the amyloid precursor genes on chromosome 21 or in the genes coding for presenitin on chromosomes and 14 [161]. The Apolipoprotein 4 (ApoE4) allele is a major risk factor for getting sporadic AD at an early age. This alfele is found in only a small part of the general population and in only part of the AD patients 281 ]. Since these mutations and the ApoE4 allele are found in only a 
Subpopulation of all AD patients, identification of these mutations can also not be used as a conclusive diagnostic test.

Nomally, the degenerative process has already progressed to an advanced stage with massive loss of cell mass before diagnosis can be made. For example, substantial enlargement of ventricles and of corticat sulci as well as hippocampal atrophy is observed at the time of diagnosis of $A D$ [39]. The transition from nomal cognitive perfomance to the AD phenotype is probably gradual. Patients with so-called mild cognitive impairment are prone to develop AD [123]. Considering the limited capacity of the central nervous system (CNS) tissue to repair, early intervention in the degenerative processes, thus in palients with mild cognitive impairment, will be crucial to spare as much tissue as possible. Early diagnosis may then help to increase the possibilities for developing and testing new preventive strategies, such as antiinflammatory therapy [196], antioxidant supplementation $[257]$ or oestrogen replacement therapy [230].

In the ideal situation, early diagnosis should be possible with an easy and inexpensive blood test. Many studies have investigated if dementia may be reflected in altered body fluid composition, including proteins, micronutrients, sterols and DNA as will be discussed in this review. However, so far no single peripheral biological marker with specificity for AD has been found $[30,218,310]$.

AD is a heterogeneous disease with respect to several possible pathophysiological causes and mechanisms contributing to a common stage of cell death. Oxidative stress is an important condition preceding cell dleath $[106,178]$.

Oxidative stress is defined as a imbalance between the increased production of oxygen raclicals, e.g. superoxide or nitric oxide (NO), and the anti-oxidative delences and repair mechanisms against oxygen radical-induced protein-, lipid-, RNA- or DNA-damage [104]. The production of reactive oxygen species is a nomal phenomenon in tissue, for example during mitochondrial energy production, the transformation of xanthine into hypoxanthine, during NO synthesis or in the immune defence [105]. The organism has a number of defence mechanisms against these aggressive chemical species and a permanently increasing oxidative slress may result from the stow decline in efficiency of these diefience mechanisms during normal ageing [118]. Increased oxidative stress can be induced during conditions of excitoxicity 222$]$, during inflammatory processes [77] and during vascular abnormalities [271]. These conditions maly in tum be influenced by a large number of environmental and life-style influences such as dietary intake of vitamins [230], smoking [284], physical exercise [67,241] and exposure to pesticides [245],

It is not known what the role of each of these influences is. They can be causalive or involved in the propagation of the destructive process or both. In addition, the relative contribution of each of the different mechanisms may be different for each individual. In our opinion, this may explain why changes in allmost every biomarker investigated are found only in subgroxps of patients and as will be shown for several markers in this review, why a large overlap in the concentration of cerebrospinal fluid (CSF) or serum markers exists between patients and controls. Another implication of the involwement of several processes in neurodegenerative diseases is that a combination of matkers rom the different disease mechanisms may have increased diagnostic value $[30]$.

\section{Aim of the present review}

The purpose of this review is to evaluate the current literature on possible marker molecules for oxidative stress, inflammation reactions, cholesterol homeostasis, plaque formation and vitamin status. These processes are likely involved in the neurodegenentive processes occurring in dementia and in this respect should not be viewed as completely independent entities. The 
focus of this review is on altered marker concentrations in biological fluids, i.e. blood (serum/plasma) and CSF, in relation to dementia. The makers will be evaluated on consistency of reported changes in relation with AD, specincity of the marker for bran-related processes and the presence of alterations in early stages of dementia. Each chapter will start with an introduction on the relation of the disease mechanism with dementia and the current knowledge about the relation of the markers with dementia processes. Subsequently, the litealure on different markers in blood (serum/plasma) and CSF is discussed. A table summarising the data is included in each chapter. Finally, we will evaluate which markers may be interesting for further research that aims to combine markers for the different pathophysiological mechanisms.

\section{Markers of inflammatory processes}

\section{Inflammation and $A D$}

In the late 80 's, the first reports appeared showing that microglia in senile plaques contained a protein nomally associated with inflammatory processes, i.e. HLA-DR, a protein of the class II major histrocompatibility complex (MHC) [194,195]. Other known mediators of inflammation have also been found in plaques, such as interleukin-1 $\beta$ (11.-1 $\beta$ ), interleukin-6 (1L-6) and tumour necrosis factor (TNF-a] $[96,99]$. Additional evidence for the involvement of inflammation in AD is provided by epidemiological data and retrospective clinical data showing positive effects of non-steroid-anti-inflammatory drugs $[41,196]$, though a protective effect of these drugs in clinical trials has not been unequivocally shown so far $[3,255]$. A classic immune response as defined by the involvement of T-cells or immunoglobulins has not been identified in the senile plaques of AD brains.

The inflammation related proteins in the brain were shown to originate primarily from aclivated microglia and astroglia (reviewed in [190]). It was shown that microglia, astroglia and even neurones produce interleukins constitutively at low level [319]. However, when microglia are activated, for example atter bran trauma or events leading to oxidative stress, they produce high amounts of $11-1 \beta$ and TNF $\alpha$. This could be via an oxidant sensitive nuclear factor $b$ (nfkb) partway that is activated by interleukins such as TNF-a 11911. Next, IL-1B and TNF-a induce the expression of several proteins in astrocyles, e.g. gliallibrillary acidic protein (GFAP), $S$ 100p, Apot, inducible NO synthase and cytokines as a-antichymotrypsin $(\alpha-A C T), 1 L-6,1 L-1 \beta$ and TNF- $\alpha[60,237]$. This pathway can thus induce a self-propagating process via $\| \mathrm{L}-1 \beta$ and TNF- $\alpha$. In addition, II-78 can stimulate the APP promotors 73,90 , which are widely expressed in both neurones and glial cells, with some forms preferentially expressed in particular cell rypes (e.g. APP695 in neurones) [190]. As a consequence anyloid beta (AB) production is increased. $A B$ can in tum increase the production of both $A B$ and interleukins by microglia $[26,78]$.

The involvement of inflammation reactions in $A D$ is also evidenced by change in concentrations of inflammation related proteins in CSF and serum, as will be shown below and in Table 2. As the inflammation related proteins $11-1 / \mathrm{p}$ and $1 \mathrm{~L}-6$ are not able to cross the bloodbrain-barrier (BBB), altered serum concentrations may not be due to increased flow of inflammation related proteins from the brain when the $B B B$ is intact. It might be possible that a whole-body change in concentrations of interleukins occurs, which affects the brain tissue specifically. Alfernatively, damage to brain lissue may be one of the various effects of an increased peripheral concentration of interleukins. 


\section{Interleukin-6 (lL-6) in CSF or serum (plasma) (Table 2)}

Serum 1 L-6 levels have been shown to increase during ageing $[8,72,134]$, being undetectable in juveniles and gradually increasing to detectable levels in older mice, monkeys, and humans [83].

The majority of studies report no difference in $11-6$ levels in CSF of AD patients compared lo controls $152,81,107,111,134,155,181,290]$. Two studies reporled increased levels of $1 \mathrm{~L}-6$ in $\mathrm{CSF}$ $[34,179]$, and one study reported decreased levels only in early-onset AD patients (i.e. disease onset $<65$ yrs) [312].

A major problem with evaluating the role of $1 \mathrm{~L}-6$ in CSF is that normally no CSF is avalable from healthy control individuals. Most studies compare AD patients to pattients with other neurological diseases as control groups, so the results can have diagnostic value only for discrimination of $\mathrm{AD}$ patients from other CNS patients. In one study [181] even depressed patients are included in the control groups whilst depression may also be accompanied by a systemic acute phase response (i.e. elevated $1 L-1 \beta, I L-6)$ [174].

Results obtained from studies investigating serum levels of $I L-6$ in $A D$ patients have been controversial. Several studies observed increased levels of IL-6 in serum or plasma of AD patients compared to controls $[36,134,165,175,290]$, while others found no difference $[13,52,155,303]$.

A further problem in the analysis of $1 \mathrm{~L}-6$ is the low level of $1 \mathrm{~L}-6$ present in serum or plasma in combination with the IOW sensitivity of the ELSA systems used. For example, in one study, plasma IL.6 could be detected only in $53 \%$ of the AD patients compared to $27 \%$ of the controls [165]. Similarly, in another study (detection limit IL-6 ELISA $0.7 \mathrm{pg} / \mathrm{mL}$ ) $1 \mathrm{~L} \cdot 6$ concentration in the majority of samples was below the detection limit [13]. Another problem in stuclies on interleukins could arise from the use of bioassays, i.e. stimulation of cultured cells by the interleukins. In one of the studies, it was reported that the sera were theat-inactivated before measurement of 1L-6 using a bioassay [52]. However, we have observed that heat-inactivation reduced the $1 \mathrm{~L}-6$ concentration between 50-80\%, and there was large variation in the percentage reduction between samples, as measured at least with our ELISA (unpublished data). IL-6 exerts its biological actions by complex interactions with specilic soluble and membrane bound receptors, forming the IL-6 receptor complex. Two different receptors are included in this complex: $1 \mathrm{~L} 46$ receptor $(80 \mathrm{kD})$ and glycoprotein 130 (gp/30) (130 kD). Binding of 11.6 to the IL-6 receptor leads to binding of the thus formed complex $10 \mathrm{gp} 130$, and the IL-6 receplor complex then formed can generate a neuronal differentiation signal 1140,1821. So far, four studies have investigated the soluble $\mathrm{HL}-6$ receptor or gp 130 concentrations in CSF or serum of patients with AD. One study found no difference in ILw6 receptor or gp 130 concentrations in CSF [181]. However, two studies reported a decrease in 1L-6 receptor or gp 130 concentrations in CSF $[108,109]$. Serum IL-6 receptor concentrations were decreased in AD patients compared to controls \$131. Further studies on $\$ L-6$ receptor concentration in serum must confirm these results.

\section{Interleukin-1 (IL-1B) in CSF or serum (plasma) (Table 2)}

Most of the studies were unable 10 find any difference in mean II-1/P concentration in the CSF of AD patients compared to controls with other neurological diseases $193,111,155,180,240,290]$. Howewer, one author $[93]$ did report an increase in the ll-1 \{ receptor (IL-1 $\beta$ receptor II) concentrations in AD patients compared to other neurolongical controls. Only two studies have reported increased 11 - 1 \% levels in CSF of AD patients compared to contrals with other neurological diseases (such as multiple sclerosis, headache, 
hydrocephalus, discrete polyneuropathy [34,49]. As stated already in the paragraph addressing IL-6 concentrations, the interpretation of these studies needs caution as the control groups consisted of patients with other neurologicall diseases. Nevertheless, given the fairly consistent lack of changes in $14-18$ concentration after comparing CSF from AD pattents with controls, we may conclude that this marker may not be interesting as a diagnostic CSF marker in dementia studies.

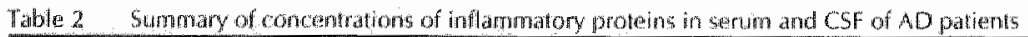

\begin{tabular}{|c|c|c|c|}
\hline & makar & |aven & references \\
\hline \multirow[t]{17}{*}{ CSt: } & $\| L-6$ & \& & $194,179]$ \\
\hline & & $=$ & $152,81,107,111,134,155,121,2901$ \\
\hline & & - & {$[312$} \\
\hline & HLw recepton & $=-$ & $\| 181$ \\
\hline & & - & $1108,109]$ \\
\hline & Ge130 & 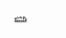 & {$[181]$} \\
\hline & & - & {$[109]$} \\
\hline & HL $1 B$ & + & {$[34,49]$} \\
\hline & & $\Rightarrow$ & {$[93,111,155,180,240,290]$} \\
\hline & [L-1] recentom il & $\dot{+}$ & 1931 \\
\hline & $\operatorname{TWF} \cdot \mathrm{a}$ & + & 2901 \\
\hline & & $=$ & {$[93,1 \| 1,155]$} \\
\hline & TNHa receptors & $=$ & {$[93,1 \| 1,155]$} \\
\hline & A-ACT & + & {$[110,184,186]$} \\
\hline & & $=$ & {$[155,240]$} \\
\hline & Hip & $=$ & 1801 \\
\hline & Hip fragments & + & $\llbracket 130,139]$ \\
\hline \multirow[t]{13}{*}{ Seram/plasma } & $11 \cdot 6$ & + & $136,134,165,175,290]$ \\
\hline & & $=$ & $\llbracket 13,52,155,301]$ \\
\hline & 11. 6 receptor & - & {$[109]$} \\
\hline & $\| 1-1 \beta$ & + & $\{16.5\}$ \\
\hline & & $\Leftrightarrow$ & $134,249,155,240,290$ \\
\hline & 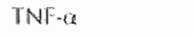 & f & $\| 8,4,84 \pi$ \\
\hline & & - & {$[52,155,175,200]$} \\
\hline & & -- & 448 \\
\hline & $(x+A C)$ & + & $194,163-165,2331$ \\
\hline & & $\Rightarrow$ & $\mid 155,2401$ \\
\hline & $\mathrm{Hp}$ & $=$ & 180,941 \\
\hline & $H p 2-1$ & + & 1306 \\
\hline & & - & {$[35,79,88,197]$} \\
\hline
\end{tabular}

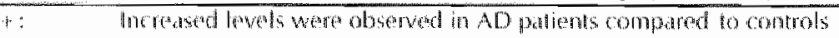

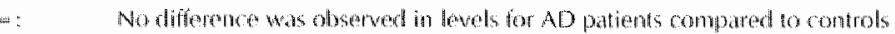

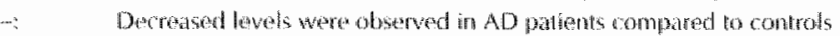

Similar to the findings in CSF, most studies that investigated serum show no difference in serum or plasma $1 \mathrm{~L}-13$ levels between $\mathrm{AD}$ patients and controls $34,49,155,240,290]$. However, one recent large sludy, including $145 \mathrm{AD}$ patients and 51 controls, showed higher concentrations of serum IL-1B in AD patients compared to controls [165]. 
Similar difficulties as with the data on IL-6 are present for IL-1B. i.e. the low lavel of IL-1p present in serum of nomal controls in combination with the low sensitivity of the ELISA systems used. For example, in the large study mentioned above, plasma ll-18 could be detected in only $13 \%$ of the patients $(n=145)$ and $2 \%$ of the controls $(n-51)$ [165]. Likewise, in another study the majority of samples had interleukin levels below the detection level of the ELSA assays used (detection limit IL-1B ELISA $10 \mathrm{pg} / \mathrm{mL}$ ) [240]. So in the majority of AD patients it is not possible to measure $11 \mathrm{~L}-1 \beta$ in serum and this may explain why differences in mean concentrations can only be measured when a large group of AD patients is included. Consequently, an increase in IL-1 3 concentration in serum may have very limited diagnostic value for single patients.

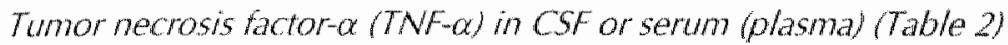

The studies on TNF-a in CSF of patients with $A D$ are not unequivocal. One study has reported an increase in TNF- $\alpha$ concentrations [290], while three studies reported no difference in TNF- $\alpha$ levels or the levels of the TNF- $\alpha$ receptors TNF receptor I and TNF receptor II in CSF of AD patients compared to controls $[93,111,155]$.

There is consensus that TNF- $\alpha$ and the soluble TNF-a receptor concentrations in serum increase with age $[44,111]$. The results on TNF- $\alpha$ in serum of patients with AD are not consistent. One study reported that the serum TNF $\alpha$ level was decreased in serum of demented patients (multinfard dementia and ADI compared to healthy controls $[481$. In contrast, increased TNF-a concentration was observed in serum of AD patients in three other studies $[8,44,84]$, while several studies reported no significant differences $[52,155,175,290]$.

Given the contradictory results on concentrations of TNF $\alpha$ and its receptors in both serum and CSF, we conclude that these markers are not suitable for our investigations in reation to dementia.

\section{ar-antichymotrypsin ( $\alpha$-ACT) in CSF or serum (plasma) (Table 2)}

Several reports showed increased concentrations of $\alpha-A C T$ in CSF $[110,184,186]$ or serum $[94,163,164,233]$ of $\mathrm{AD}$ patients. In a more recent study that used a large pallient sample [165], increased a-ACT levels in sera of $A D$ patients $(n-145)$ compared to non-demented controls $(n=51)$ were observed (mean concentration $A D:-125 \%$ of control concentration). A negative corration was observed with the scones on the minimental state exam, (MMSE: a measure of severity of dementia [85]), which indicates that higher a-ACT concentrations are observed in more severely affected patients. Several authors [155,240] did not observe a difference in the mean a-ACT concentrations in serum nor in CSF. However, the number of patients in each group of these latter studies was smaller than in the study of Licastro [165] observing incrased $\alpha$-ACT concentration in AD patents (AD: $n=8$; other CNS disease: $n=9$ [155]; $A D: n=40$, controls: $n=42[240]$ ). Thus, the differences may become significant only when large populations are compared.

Again, the variation in results seems to limit the sulability of serum a-ACT measurements for discriminating single $A D$ patients. However, the correlation with severily of $A D$ as recently reported [165] may point to the use of this marker in monitoring the progression of the severity of dementia symptoms, rather than in diagnosing dementia. 


\section{Haptoglobin (Ho) in CSF or serum (plasma) (Table 2)}

In 1973 an article was published which reported an increased presence of the Hp 2-1 phenolype in AD patients [306]. Several groups tried to replicate the finding, though without success. The results of those studies did not show a significantly increased frequency of any of Hp phemotypes or alleles in AD patients compared to controls $[35,79,88,187]$. This discrepancy may be explained by variations in sample size (varying from 20 10 65 individuals in control groups, and from 18 to 2297 in $\mathrm{AD}$ groups), as the numbers in some sample may have been too small for performing genetic analysis. In addition, there are some differences in the diagnostic criteria used. The studies performed in the seventies did not use DSM-IV and NINCDS-ARDA criteria, which originate from 1984 [197]. Or there may simply be no reproducible relation between AD prevalence and Hp phenotype and, in that case the findings of the first study on this subject [306] may have been biased by other factors.

Hp is an iron transporting protein as weil as an acute phase protein. Hp binds haemoglobin released after haemolysis and the thus formed complex has a high affinity for the CD163 receptor on macrophages [143]. Binding leads to internalisation of the complex into macrophages, which transport the haemoglobin to the bone marrow [1]. In this way, oxidative damage is prevented which can occur via the Fenton reaction, a hydroxyl radical generating reaction that is calalysed by iron $[308$ ].

The Hp gene is expressed in hepatocytes and hepatic synthesis of $\mathrm{Hp}$ is induced by cytokines, such as IL-6, IL-1 13 and TNF- $\alpha[38,247]$. Hp is predominantly present in blood and so far there is no proof of a brain origin [295]. The phenotype of the Hp protein is an expression of the combination of wo genelic loci (i.e. $H_{p}$ and $\left.H p_{p}\right)$. The expression of different combinations of the $H p_{a}$ alleles leads to a polymorphism (phenotypes 1-1, 2-1,2-2) [151]. The proteins of the 22 phenolype tend to form large polymerised Hp proteins of $170-800 \mathrm{kD}$ size, compared to a 86 KD protein for the $1-1$ phenotype and a $86-300 \mathrm{kD}$ protein size for the 2-1 phenotype [151]. Combining the fact that subjects with a $2-2$ phenotype have lower blood $H$ p protein concentration, expressed in $\mathrm{g} / \mathrm{L}$, with the relatively high molecular weight, consequently even less moleculles are present in subjects with this phenotype [304]. The large polymeric proteins of the 2-2 phenotype have probably less access to interstitial fluids, such as intracerebral fluids [151]. This, in combination with the low protein concentration, might cause subjects with the 2* 2 phenotype to have a lower antiroxidative defence in body fluids due to the lower iron binding capacity $[151]$. It was shown that the $2-2$ phenotypes have lower serum vitamin $C$ values, while levels of other anthoxidanls (uric acid, biliburin, albumin, ceruloplasmin and tolal antioxidative staltus were comparable among the phenotypes [151]. These findings are in agreement with in vitro experiments that showed a lower stability of L-ascorbic acid in blood of subjects with the HP $2-2$ type, and an inverse contation between Lascorbic acid depletion and Hp concentration [152]. Finally, human immunodeficiency virus (HIV) patients expressing the Hp 2-2 phenolype had a higher 5 -years mortality (median survival time was 7.3 years compared to 11 years for palients with the $2-1$ or $1-1$ phenotype) $[65]$.

In view of the probable decreased anti-oxidative defence in people with lower Hp concentrations, it might be interesting to know if AD patients also have lower serum or CSF Ha concentration. Some authors investigated the mean serum and/or CSF concentrations of the HP protein and failed to find any differences between $A D$ patients and healthy controis $[80,94]$. In view of the dependence of the serum concentration on phenotype, it would be interesting to repeat concentration measurements while controlling for $H p$ phenotype, which was not detemined in the studies mentioned. 
Other studies showed increased presence of some lower weight isotoms of the Hp protein in CSF of AD patients using a two-dimensional electrophoresis technique $[130,189]$. In these studies only a small number of patients was investigated, i.e. 10 AD patients vs. 11 controls [130]. In conclusion, these results showing differences in CSF Hp concentrations between AD patients and controls as determined in small patient samples are suggestwe for a promising discriminative role of CSF Hp between individual AD patients and controls. However, there is still overlap between patients and controls. Furthermore, the laborious iwo-dimensional gel electrophoresis method for identification of small protein fragments is a disadvantage for using Hp protein isoform determination for diagnostic practice.

\section{Other makkers related to infiammation}

Several siludies have appeared on other inflammation markers. The concentrations of the immune suppressors TGF- $\alpha$ and TGF $\beta$ have also been investigated in CSF, but no differences have been reported between dementia patients and controls [305]. The few studies on $C$ reactive protein performed showed contradictory results [44,94, 163]. Increased concentrations in AD patients compared to controls have been obseved for lithostathine in CSF [74], the proteinase kallikrein in both serum and CSF [68], histamine in serum [8], and anti-GFAP, antineve growth factor, anti-histone and anti-double stranded DNA antibodies in serum $\llbracket 69,199 \rrbracket$. Decreased concentrations were observed in AD patients compared to controls for the markers mannan-binding lectin in CSF (while the serum lectin levels were not changed $[154 \rrbracket$, and cleavage products of the high molecular weight kininogen in CSF [24]. These other inflammation markers have in common that the main focus was on CSF. Inflammation markers are not specific for brain diseases, though the presence in CSF may increase the significance for brain-related processes.

\section{Additional comments on the inflammatory pioteins}

It is possible that the severity of dementia influenced the differences in results from studies investigating inflammation markers. For example, one study observed an increase in IL-6 serum levels only in the most severely affected patients compared to controls [134]. Untortunately not all studies measured the duration or the severity of dementia (MMSF). In addition, elevated serum $1 L-1 B$ values were observed only when early-onset $A D$ patients were compared to healthy controls [8]. Such results have little implication for the late onset patients, who form the majority. The low number of patients included in several studies may also limit the possibilites to establish a relation with severity. For example in one study [34] measuring IL-1/3 and IL -6 in serum and CSF, only 11 AD patients and 12 controls were included. In another study $\| 155]$ on IL-1B, IL-6 and TNF-o in serum and CSF, only 8 AD patients and 9 patients with other CNS diseases were included.

In summary, the definite statements concerning inflammation marker differences between control and $\mathrm{AD}$ patients require the use of sensitive assays, large patient groups, in combination with the measurement of MMSE. This is imperative not only for measuring very low concentrations of interleukins but for all possible AD markers. 


\section{Markers for oxidative stress}

\section{Markers for oxidative stress and AD}

It appears to be widely accepted in the literature that oxiclative stress plays an important role in the $\mathrm{AD}$ disease process $[4,22,178,270,275]$. The brain has a thigh oxygen consumption and is relatively rich in polyunsaturated fatty acids that are easily oxidised. Decreased cerebral perfusion results in decreased oxygen and gucose delivery to the brain parenchyma. This then leads to energy deprivation, an impontant causal factor of oxidative stress in the brain [64].

Evidence of oxidative stress in $A D$ is obtained by increased protein carbonyl content and lipid, proten and DNA oxidation products and by the inactivation of oxidation-sensitive enzymes, such as glutamine synthase or creatine kinase $[112,169,276]$. A recent study showed increased concentration of the DNA oxidation product B-hydroxy-2'-deoxyguanosine in post-mortem ventricular fluid of $A D$ patients compared to controls, with no overlap between the groups [170]. These results are therefore very interesting with respect to the search for a CSF marker that discriminates between AD patients and controls.

Other manifestations of oxidative stress include increased brain tissue concentrations of F2isoprostanes, Isoprostanes are free radical-catalysed peroxidation products of aracthidonic acid and structural isomers of prostaglandins (see $[242,254]$ for reviews). The peroxidation of arachidonic acid occurs independently of cycloxygenase $A$ enzyme activity, the enzyme responsible for the melabolism of arachidonic acid to prostaglandins. Isoprostanes can originate from arachidonic acid esterified to phospholipids and subsequent hydrolysis of esterified isoprostames, yielding free F2-isoprostanes $[213,215]$. When isoprostane formation occurs on membrane phospholipids, such as phosphatidylserine, the conformation of these molecules will be distorted and in this way affact membrane fluidity [253]. Quantification of F2-isoprostanes has emerged as an accurate approach to assess oxidative damage in vivo [212].

Increased levels of $\mathrm{F} 2$-isoprostanes were shown in frontal and temporal cortical areas compared to the cerebellum in AD patients and these levels were also higher compared to levels in the same cortical areas of patients with schizophrenia, PD or non-neuropsychiatric disorders [244]. Ventricular fluid (post mortem) isoprostane levels were shown to correlate positively with the severity of neurodegeneration based on histological examination of plaques and tangle load (Braak shage) 139], as well as with the reduction in brain weight. However, no correlation with amoun of placues, langles or Apot phenowpe was observed [208]. Other isoprostane-like compounds; D4-, E4- 1214,249] and F4- isoprostanes or neuroprostanes [231,253], may be more interesing for identifying CNS damage, as these isoforms are derived from dacosahexaenoic acid. Docosahexaenoic acid is highly enriched in the brain and more easily oxidised than athohidonic acid [216]. Levels of F4-isoprostanes were elevated in temporal and occipilal lobe, but not in the parietal lobe of ab patients 231$]$.

3 Nitrotyrosine is thought to be a promising early marker for oxidative stress in various neurodegenerative processes $123,40,277]$. Since the identification of 3 -nitrotyrosine in Cu/Zn superoxide dismulase as a marker for protein modification by peroxynitrite [120], evidence of 3 nitrotyrosine occurrence in various neurodegenerabe diseases in humans is accumulating. In AD brains, 3-nitrotyrosine and 3,3'dityrosine were found in neocortical and hippocampal regions, compared to unchanged $3,3^{*}$-dityrosine levels and a decrease in 3-nitrotyrosine in the cerebellum of these patients 1113]. 3-Nitrotyrosme was found in neurones with DNA damage in the visual contex of $\mathrm{AD}$ patients before signs of tangle formation were observed [282]. The involvement of 3-nitrotyrosine residues in neurodegenerative processes and ageing has been confimed in other disedses such as multiple sclerosis [57], amyotrophic tateral sclerosis (ALS) 
[43] and Huntingion's disease 192] and by in vivo $[58,238,272]$, and in vitro models $[191,311,315]$.

Although the mechanism is not universally accepted, 3-nithoiyrosine formation is thought to occur by reaction of peroxynutrite with brosine residues in proteins. Peroxynitrife is a strong oxidising agent formed from superoxide and NO. Under physiologic circumstances in the brain, NO functions as an intercellular messenger molecule and it is produced after activation of the constiturively expressed neuronal and endothelial isoforms of NO synthase. Peroxynitrite formation may occur under conditions of oxidative stress in combination with the ongoing synthesis of $\mathrm{NO}$ by the inducible form of NO synthase in microglial and astroglial cells [40]. However, as protective effects of NO have been reported too and the physiological function of $\mathrm{NO}$ is still insufficiently understood. The exact role of $\mathrm{NO}$ during neurodegenerative processes is also still under discussion $[53,62,298]$.

\section{Isoprostanes and 3-nitrotyrosine in CSF or serum (plasma) (Table 3)}

Increased levels of F2-isoprostanes could be detected in both ventricular and lumbar CSF of AD $[205,207,210]$ or Huntington's disease [206] patients, while in amyotrophic lateral sclerosis (ALS) patients the levels remained similar to controls $[205]$. Ventricular fluid F2-isoprostane levels were significantly negatively conelated with brain weight $\left(-0.3 \mathrm{pg} / \mathrm{mL} / \mathrm{g}, \|^{2}=0.32\right)[207]$. F4-isoprostane levels in CSF of patients with AD (110 $112 \mathrm{pg} / \mathrm{mL})$ were significantly higher than age-matched controls $(64 \pm 8 \mathrm{pg} / \mathrm{mL})(p<0.05)[253]$.

So far, one study observed no differences in serum F2-isoprostane level between AD patients and controls [209], while another recent study observed increased 8,12-isoprostane F2a-VI concentrations in CSF, blood and urine of AD patients 1243$]$. The 8,12-isoprostane F2q-VI levels correlated with cognitive and functional impairment as well as with number of ApoE alleles [243].

An important difficulty in detemining isoprostanes in body liquids is that they can be autooxidised easily. In serum, this would cause a problem even when samples are immediately centrifuged and stored at $-20^{\circ} \mathrm{C}$. Immediate centrifugation and storage at $-80^{\circ} \mathrm{C}$ may circumvent this problem [20]. The isoprostanes can be measured in various biologic matrices, e.g. tissues, serum, CSF and urine. The levels are not affected by lipid content of the diet 1250$]$ but the formation is modulated by antioxidant status $117 \%, 254]$. The method for determining isoprostanes requires expensive equipment (CCMS). In this respect it looks promising that eftorts are being undertaken to develop ELISA methods for determining isoprostanes.

The specificity of changes in F4-isoprostane (meuroprostane) levels for brain lissue related processes has to be clarified further. However, isoprostane-like compounds are sill likely candidates as oxidative stress markers that are specific for certain neurodegenenative processes.

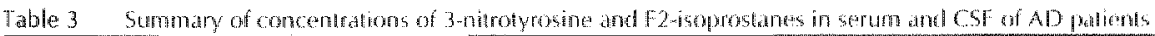

\begin{tabular}{|c|c|c|c|}
\hline & trourker & level & 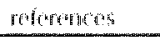 \\
\hline \multirow[t]{4}{*}{$\mathrm{cs}$} & 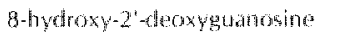 & 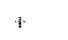 & $\| 1701$ \\
\hline & F2-isoprositantas & i. & $1205,207,2101$ \\
\hline & 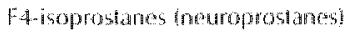 & 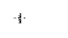 & {$[25]$} \\
\hline & 3u-bitrotyosine & s. & $114,113,29 \%$ \\
\hline \multirow[t]{2}{*}{ SEन } & $8,12-i 50-\frac{i}{2}|P| 2 a-4 /$ & . & $|2+4|$ \\
\hline & F2-isoprosiandres & $\infty$ & 2001 \\
\hline
\end{tabular}

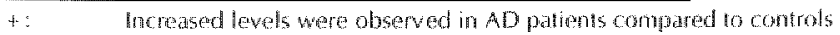

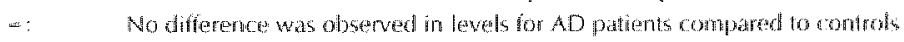

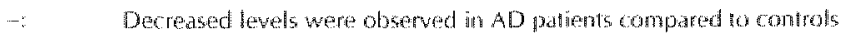


Increased vyrosine nitration of Mn-superoxide dismutase has recently been shown in CSF of AD, $\mathrm{PD}$ and ALS patients [14]. A six-fold increased concentration of 3-nitrotyrosine in lumbar CSF of AD patients was shown by one study and litle overlap with controis was seen [297]. This 3nitrotyrosine concentration correlated negatively with MMSE, i.e. the lower the MMSE score the ligher the 3-nitrotyrosine concentration. A positive correlation of 3-nitrotyrosine in CSF with age was observed within the group of neurological nomal patients [297]. Likewise, a slight, but not significant increase in 3-nitrotyrosine in post mortem wentricular fluid was abserved in AD patients [113]. Nitrotyrosinylation of specific brain proteins, e.g. GFA. protein tau or S-100 , would increase the specificity of this marker for brain related processes.

No studies on 3-nitrotyrosine in serum of patients with $A D$ are known yet. This may be expected due to several reasons. First, 3-nitrolyrosine is not stable in serum, caused by breakdown of the nitrated protein or metabolism of the 3-nitro-group [136]. The presence of enzymes with denitrase activity in homogenates and crude extracts of rat brain and heart have been demonstrated [146]. Another study also showed the occurrence of 3-nitrolyrosine breakdown via an inducible factor with denitrase activity in supernatants from spleen homogenates, while no such achivity was found in homogenates from rat liver or kichey [136]. The characteristics of denitrating enzymes in brain tissue remain to be demonstrated. Another problem may be that increased proten nitration occurs at, as yet undetectable levels during. chronic diseases and is deteclable only during acute phases. Thus, increased levels were observed only during the acute phases in monocytes/macrophages of patients with multipte sclerosis [234] or celiac disease [291]. Similar relation with the severity of disease was found for patients with systemic lupus erythematosis, where subjects with adtive lupus nephritis had higher levels of serum 3-nitrotyrosine than those without renal disease and where serum 3nitrotyrosine correlated positively with disease activity [232].

\section{Vitamins}

\section{Vilamins and $A D$}

The vitamins $A, B, C$, and $E$ are important antioxidants, whose levels can be influenced by dieary habits. Vitamins have been advocated as modulators of cognitive performance [1.50,230].

Vitamin $E$ is known as a major chain breaking antioxidant in biological membranes. It is especially important for the brain, considering the high lipid content and a relatively high proportion of polyunsaturated fatty acids of the bran [102]. Intake of 2000 IU vitamin E every daty for up to two years slowed the progression of AD in moderately severe patients on outcomes as time to death, institutionalisation or loss in the ability to perform simple daily activities. Unfortunately, no positive effect of intake of Vilamin E on cognition was observed in this study [257]. One reason for the lack of cognitive improvement nay be that the treatment was started in already cognitive deteriorated patients. It might be that intake of vitamin $E$ before the onset of clinical symptoms improves the positive effects on cognitive outcomes.

Oher vitamins possibly related to cognitive deterioration are vitamin B6, B12, and in this context, the related compounds folic acid and homocysteine. Homocysteine is a sulfur containing amino acid formed from methionine as a product of S-adenosylmethoninedependent transmethylation (Fig. 1). Homocysteine can be reconverted into methionine by methylation, callysed by the enzyme methionine synthase, a process requiring vitamin 812 and folic acid as cofactors. Altematively, it can be transtormed to cystathionine catalysed by orstathionine b-synthetase, a vilamin B6-dependent enzyme. In the liver, remethylation can also 
be catalysed by the enzyme betaine-homocysteine transmethylase. Cellular expont represents an additional mechanism of homocysteine removal. Excess intracellular homocystene leads to export of homocysteine and may thus lead to increased plasma homocysteine levels in the circulation $[248,299]$.

Elevated levels of homocysteine are known as a risk factor for vascular heart diseases and impaiments of both the peripheral and cerebral vasculature [54,248]. An increase in homocysteine of 1 pmoll $L$ is associated with an $-10 \%$ increased risk for cardiovascular disease. Table 4 summarises several determinants of elevated homocysteine levels.

Up to now, there has been limited insight in the mechanism of interaction between cognitive impairment and elevated homocysteine levels or decreased vitamin B6, B12 or folic acid levels in blood. Data of the few studies addressing this subject suggest that excitotoxicity may be involved. It has been reported that homocysteine induced epileptic seizures in rats [145]. In another sludy glutamate uptake in synaptic vesicles was inhibited by homocysteic acid, al

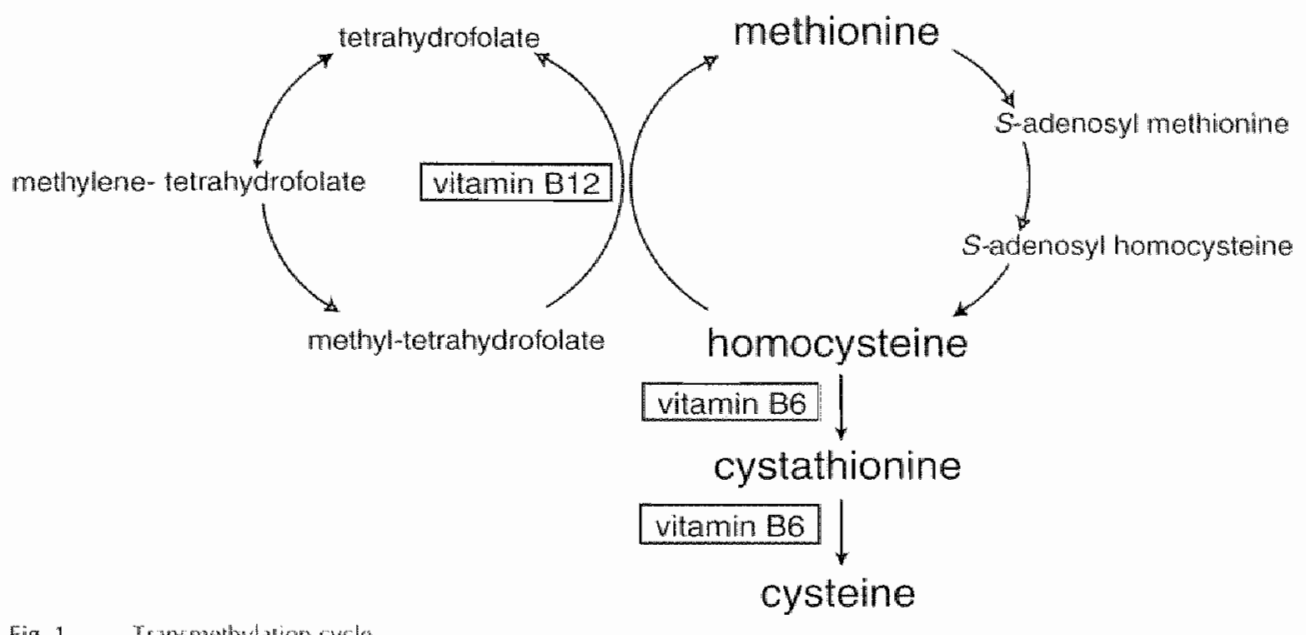

Fig. 1 Thingmethytation able.

Cof actors are roprenened in haxes.

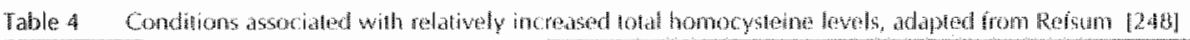

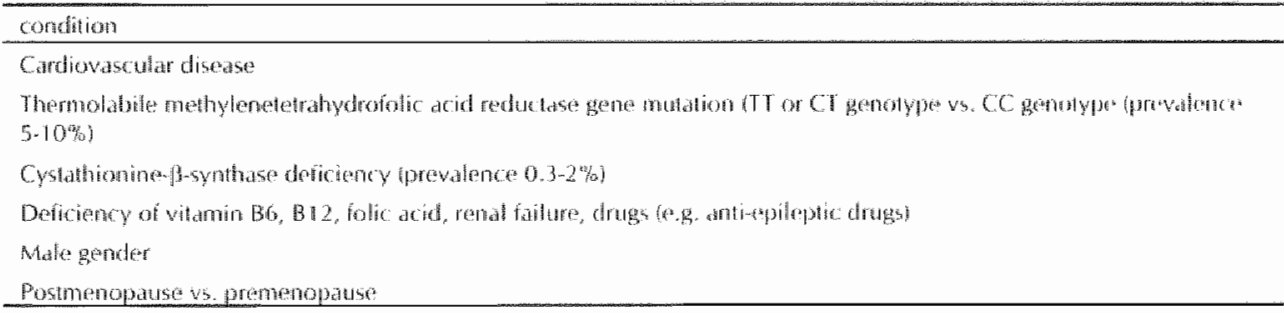

metabolite of homocysteine [89]. Homocysteine rapidly reacts with NO fo form Snitrosohomocysteine. The neurotoxicity in cultured cortical neurones induced by homocysteine was lower after exposure to S-nitrosohomocysteine $[66,139]$. If was recently reported that homocysteine can induce neuronal apoptosis and can increase neuronal vulnerability to excitotoxicity by a rrechanism involving DNA damage and poly-ADP-ribose polymerase 
activation [144]. Cerebral oxygen supply may be at risk when homocysteme levels are increased since the NO-mediated cerebrovascular response may be inhibited by homocysteine [318]. Decreased methionine may lead to decreased methyl transfer via s-adenosylmethionine, one of the mechamisms involved in the degradation and synthesis of neurotransmitters [21] and methylation of membrane phospholipids [115].

\section{Vitamin A, B6, B 72, C, E, folic acid and homocysteine in serum (plasma) or CSF}

Decreased concentrations of vitamin $A, C$, and $E$ in serum of pattents with dementia have been reported Table 5] $[86,125,129,252,270,316]$. This difference was found in AD as well as in vascular dementia patients, under nomal dietary circumstances, i.e. without any supplementation, whille total antioxidant capacity was comparable between the groups. Decreased plasma vitamin E level has been observed also in Down's syndrome patients with AD compared to non-AD Down's symdrome palients [124]. However, some studies observed no difference in plasma vitamin C levels in CSF or plasma [236] or vitamin E in plasma [2]. Decreased vitamin $E$ levels in CSF have also been reported and the vitamin $E$ concentration in serum correlated positively with the CSF concentration [129]. However, no correlation was found between CSF vitamin $\mathbb{E}$ levels and disease severity, age, or disease duration [129]. Similarly, decreased CSF vitamin E levels have been observed in AD patients [296].

Epidemiological data have suggested a relation between lower vitamin A, C, E or B12 levels and poor memory performance in the elderly $[25,97,150,239]$. The studies did not report consistent results on every vitamin and it is to be established if increased oxidative status is reflected by an individual pattern of decreased vitamin concentration.

Increased llevels of homocysteine in serum in combination with decreased vitamin B12 or folic acid values are considered as a possible risk factor for $A D$, wascular dementia or cognitive deterioration (see: [266] for a recent review). The results of one study showed a significant increase in total homocysteine levels, and decreased folic acid and vitamin B12 levels. This was a case control study including 164 patients with $A D$ and 108 controls. Surprisingly, the total

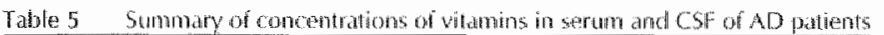

\begin{tabular}{|c|c|c|c|}
\hline Mediliz & matking & dawe! s & Radprences \\
\hline \multirow[t]{3}{*}{$\operatorname{cst}$} & witafnin $C$ & $=$ & 2368 \\
\hline & vitimin $\mathbb{L}^{2}$ & -- & $\llbracket 1129$ \\
\hline & & $=$ & {$[296]$} \\
\hline \multirow[t]{11}{*}{ Simom/plaghal } & homocysteine: & + & $\{55,75,172,159,193,225\}$ \\
\hline & & se & $|246|$ \\
\hline & vithatene $\mathrm{BO}_{3}$ & - & 246 \\
\hline & Vilanaia 312 & $\ldots$ & {$[55]$} \\
\hline & & $\ldots$ & {$[225]$} \\
\hline & lotic acid & $\ldots$ & $\mid 55.225]$ \\
\hline & vilamin $A$ & - & $186,125,3161$ \\
\hline & vitamin $C$ & - & $186,125,252,2701$ \\
\hline & & $=$ & $|230|$ \\
\hline & vitamin : & - & {$[86,123,129,252,270,316]$} \\
\hline & & 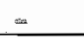 & 21 \\
\hline
\end{tabular}

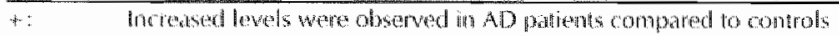

- : No difference Wos observed in levels for AD palionts compared to controls

-: Dexpeased lawals were obsarwed in AD patients compured to controls 
homocysteine levels turned out to be stable throughout 3 years of follow-ups and the homocysteine levels did not correlate with duation of symptoms 155]. The increased homocysteine concentration in demented elderly has been confirmed by several but not all studies (See Table 5) $[75,132,159,193,246]$.

Similarly, in epidemiological population-based studies a relation between plasma homocysteine and vitamin B6, B12 or folic acid concentrations and scores on cognitive tests was observed $[46,97,132,150,166,225,226,251]$. For example, in 70 male neurologically healthy subjects aged $54-81$ years, the performance on spatial copying lests was correlated significantly with homocysteine concentrations [251]. Homocysteine appears to be stronger related to cognition (significant and related to several cognitive functions) than folic acid or vitamin 312 in both epidemiological and patient studies $[55,132,193,225,226,251]$. Although there appears to be a rather broad consensus on the relation between high homocysteine and low performance on cognitive tests, there are important exceptions. In a large study on 702 individuals, no relation between increased homocysteine concentrations and cognitive decline was observed [135]. In addition, the correlation between high homocysteine levels and poorer cognitive performance was not found in every test. Thus, one study reported no significant correlation between homocysteine concentration and outcomes on several other fests, including word memory rest, pattern comparison test, language tests and spatial reasoning tests [251].

Studies comparing all three parameters homocysteine, vtamin $B^{1} 12$ and folic acid in relation to cognitive performance could give more insight in this relation. Since homocysteine levels were found to be increased and stable during three years follow-up in $\mathrm{AD}$, it would be interesting to know whether homocysteine is a mere risk factor for $A D$, as it is for cardiovascular disease [248], or if it may be of use as an early marker molecule. In support of this risk factor-hypothesis are the results of a study that observed increased homocysteine levels in individuals with only subjective symptoms [159]. However, it must be considered that vitamin B 12 and folic acid deficiencies are fairly common among the elderly [6,259]. Evidence against elewated homocysteine levels as a risk factor for AD is the fact that there was no increased prevalence of cognitive impaiment in aged people (>85 years) with chronically moderately increased homocysteine concentrations, as was found in people with a thermolabile methylenetetrahydrofolic acid reductase geve mutation (Table 4) [103].

\section{General comments on vitamins as markers for $A D$}

Considering the variable results obtained on vittamin $A, C$ and $E$ tevels in the healthy elderly, if may be hypothesised that decreased levels of already one vitamin may reflect a change in oxidative balance. Biasing factors, such as age, education or sex, should be carrefully talken into account while evaluating such markers, which are strongly influenced by life-style. for example, after adjustment of the data to those three biasing factors the effects of vilamins on cognition disappeared for vilamin A, C and $E$ in one population-based study [202]. However. the relation between vilamin $\mathbb{E}$ and cognitive performance remained significant in another population-based study after adjustment to those confounders [264].

A low vitamin status may give an indication of the susceptibility of an individual to oxidarive damage. Since the results were so bar obtained from correlation studies, the causality in the relation between cognitive impairment and vilamin status is not known. A deficiency in these vitamins is common in older individuals, due to an insufficient dietary intake or a reduced intestinal absorption [51]. Thus, dietary intake or supplementation can influence the levels of these markers. Studies showing improvement of cognive functions atter supplementation of folic acid or $\mathrm{B} 12$ are awaited with interest. 


\section{Markers related to characteristic pathological alterations in AD}

\section{Proteins of plaques, tangles and $A D$}

The characteristic post-montem pathological lesions in AD are neurofibrillary tangles and neuritic plaques accompanied by astroghiosis. The main proteins in these plaques are the amyloid $\beta$ (AB) proteins, with several other proteins associated, such as ApoE, 3w nilrotyrosinylated proteins and interleukins. The $A B$ proteins are derived after proteolytic processing of a larger precursor protein, amyloid precursor protein (APP) (fig. 2), vielding the protein fragments $A B(1-40)$ and $A \beta(1-42)$ [95]. Although $A \beta(1-40)$ is present in higher concentrations in the brain than $A(\beta(1-42)$, the latter is highly amyloudogenic and an initial component of senile plaque amyloidosis [122]. The main protein in the neurofibrillary tangles is the hyperphosphorylated form of the cytoskeletal protein tau [100,101].

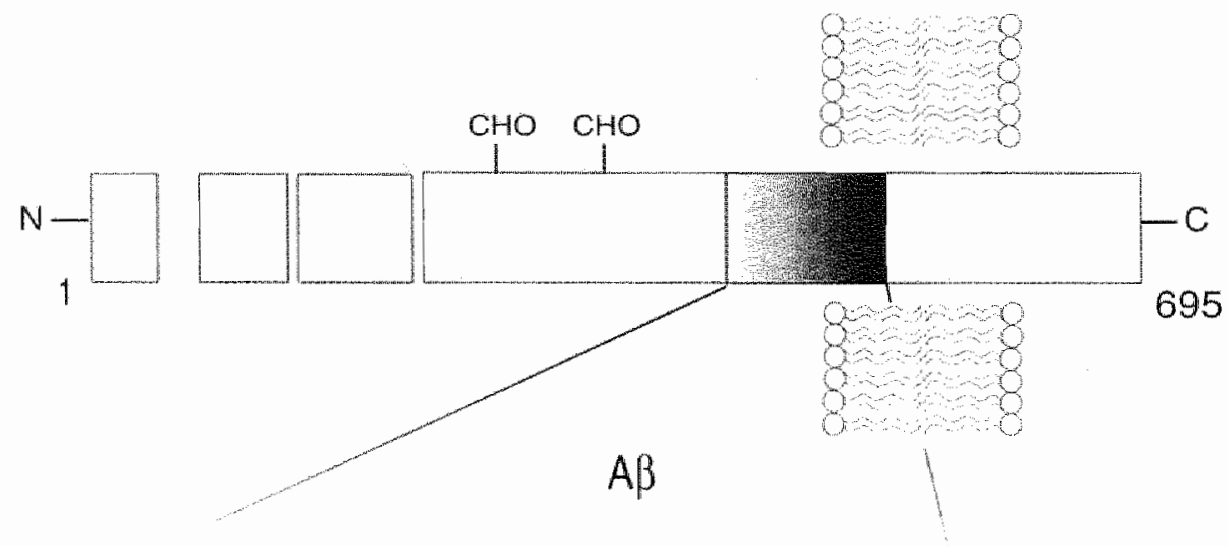

DAEFRHDSGYEVHHQKLVFFAEDVGSNKGAIIGLMVGGVVIAT

1

$\uparrow$

B-SECRETASE

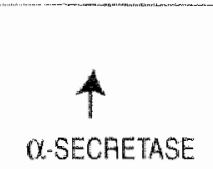

43

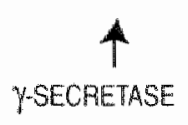

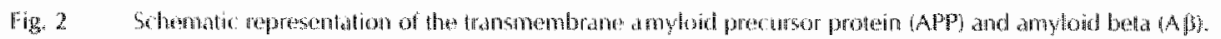

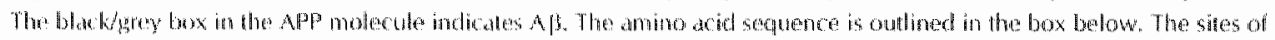

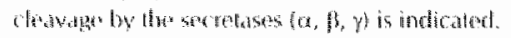

Another protein associated with plaques is GFAP. GFAP is the major intermediate filament cytoskeletat protein expressed primarily by astrocyles (MW $50 \mathrm{kD}$ ). Increased GFAP is the hallmark of astrogliosis, the vigorous response of astrocytes to brain injury [76]. Increased tissue concentrations of GFAP are found in many neurodegenerative diseases, such as ischemia, acquired immunodeficiency syndrome (AIDS) dementia, prion-associated spongiform encephatopathies, ALS or AD. In AD, GFAP forms all important component of the plaques, in close association with the microvasculature [59]. 


\section{$A \beta$ and tau in CSF or serum (plasma) (Table 6)}

Promising results have been obtained from studies on measurements of CSF concentration of $A \beta(1-40), A B(1-42)$ and tau or the combination of $A, 3(1-40)$ to $A(\beta(1-42)$ ratio with tat concentration in $\mathrm{AD}$ patients.

The concentration of $A B(1-42)$ in CSF has repeatedly been reported to be decreased in $A D$ patients compared to controls, while the total $A \beta$ protein or $A \beta(1-40)$ concentration remained unchanged $[9,12,119,137,217,267]$. This decreased CSF $A \beta(1-42)$ concentration in relalion to the increased $A B$ concentration in the plaques is unexplained, but may be due to increased deposition of the protein, which has also been shown in peripheral muscles of AD patients [148]. The decreased CSF A $\beta(1 \% 42)$ concentrations are al ready observed at moderate severity of the disease (MMSE $>25$ ), indicating a possible use of this marker in early diagnosis [12]. However, the use of $A B(1-42)$-concentration measurements in differential diagnosis is limited, as levels of $A B(1-42)$ were similar in $A D$ and in patients with major depression [116]. The correllation of $A 3$ peptides with MMSE score has yielded controversial results. For example, in several studies lower total $A \beta[116,128,228], A B(1-42)$ or $A \beta(1-40)[256]$ concentrations were found in more severely affected patients or after thee-year follow-up of AD patients [289], though in other studies no correlation of $A \beta(1-40 / 1-42)$ on follow-up or with disease severity or duration was found $[12,137]$. In this respect it is interesting to note that plaque and tangle density in at least certain cortical or isocortical areas does correlate with severity of cognitive deterioration $[50]$.

For patients with the Swedish APP mutation similarly no correlation between CSF AB level and cognitive performance was observed, though the level of a-secretase cleaved APP in CSF correlated positively with cognitive performance [7]. The mean level of CSF AB was decreased in Swedisch APP mutation carriers compared to non-carriers [153]. In cases of familial AD with the APP, presenilin 1 or presenilin 2 mutation (See Table 1 increased plasma and decreased CSF $A \beta(1-42)$ concentrations have been reported [262]. So, increased plasma $A \beta$ concentrations might be expected in sporadic $A D$ patients.

Plasma $A B$ concentrations have also been examined in relation to sporadic $A D$. Some studies found no difference between plasma $A / 3(1-42) 43$ and $A B(1-40)$ in sporadic cases of $A D$ compared to controlls $[119,286]$. In contrast, an increased $A B(1-42)$ concentration in plasma of [some) AD patients thas been found in the some studies [147,185] of which one also showed rapid disappearance of $A \beta$ epitopes, possibly by proteolytic degradation or sequestration. Another study investigated plasma $A B(1-42)$ and $A B(1-40)$ in 96 nomal healthy people [192]. The data showed that people with $A B(1-42)$ concentrations in the upper wo quartiles had a three to four fold increased risk of developing AD compared to the people in the lowest quartile [192].

Recently, it was reported that: CSF protein tau concentrations increase with age [32]. II has repeatedly been shown that protein tau was elevated in CSF of AD patients. A distinction could be made based on the mean tau concentration between $A D$ and vascular dementia, frontal lobe or alcoholic dementia, depression or PD $[10,15,17,45,127,198,211,221,227]$. Changes in tau concentration have been observed already at mild cognitive impairment (MMSE 25-28) [149]. Correlation with neurofibrillary tangle load was found [288], while no correlation with alge, disease duration, severity (MMSE, or Apot4 genotype was observed $[98,149,198,221,228,283]$. These data indicate that protein tau could be a marker for early diagnostic purposes rather than for clinical follow-up. This has also been confirmed by the increased concentration in patients with mild-cognitive impairment [16]. 
Interestingly, the ratio of $A g(1-42)$ to $A B(1-40)$ in combination with the increased taw concentration vielded an increased specificity and sensitwity in several studies, including large multicenter studies $[11,91,117,137,267]$. For example in one study [117] a sensitivity of $85 \%$ and specificity of $86 \%$ was shown for the combined analysis of $A B(1-42)$ and tau concentrations in CSF for discrimination between AD patients and other neurological patients and controls. The specificity of the single markers was $55 \%$ for $A B(1-42)$ and $65 \%$ for tau at the reported sensitivity of $85 \%$. Others obtamed similar results. One study [267] showed that the combined analysis of tau and $A \beta$ ratio yelded a lest, which could discriminate $A D$ patients with increased sensitivity and specificity. Furthermore, in a study of 16 subjects with mild cognitive impaiment, decreased baseline CSF AB(1-42) and increased baseline CSF tau turned out to be a good predictor for $A D$ at follow-up [12], confirming the value of these markers for early diagnosis.

The measurement of protein tau in CSF will need some slandardisation on possible genetic and geographic differences, as absolute tau levels of AD patients reported by different groups are variable. For example, one research group $[16,18]$ reported a level of around $20 \mathrm{pg} / \mathrm{mL}$ of protein tau in AD CSF in Japanese controls as measured by the ELISA from Innotest. Un contrast, others [137] reported a much higher level of $217 \mathrm{pg} / \mathrm{mL}$ in Japanese controls while again others [98] reported a level $612 \mathrm{pg} / \mathrm{mL}$. CSF in British controls, bolh studies using an ELISA from Innogenetics. Similarly, groups that inwestigated populations on different geographical sites each using a different ELISA reported different concentrations for $A \beta(1-42)$. The AB(1-42) concentrattions for controls reported by one study in Munich were $15.1 \mathrm{ng} / \mathrm{mL}$ [1 16]; another study in the USA reported a concentration of $1.49 \mathrm{ng} / \mathrm{mL}$ [91]; and a study in Japan [137] reported 1.36 innol/mL for controls. Finally, better discrimination between AD patients and controls or other neurological diseases may be obtained when hyperphosphorylated tau is used instead of tau $[18,121]$.

Although the sensitivity and specificity of the combined assay is satisfactory and may increase the preciston of the diagnosis in non-acadenic clinics, the use of CSF limits the possibility to develop this assay for routine diagnostic purposes or as a population screening method.

\begin{tabular}{|c|c|c|c|}
\hline mastitix & mataker & lanve! & feferences \\
\hline \multirow[t]{7}{*}{ CSF } & Nis & - & {$[9,10,119,137,217,228,256,267,289]$} \\
\hline & tetu & זे & {$[15,17,45,99,127,149,199,211,221,227,228,293,2891$} \\
\hline & 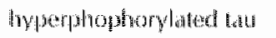 & t. & $\llbracket 121 \rrbracket$ \\
\hline & nut combined willi & If. with - & $\|11,91,117\| 37,267)$, \\
\hline & AfH $(1-42) A=(1-40)$ ratio & & $|12|$ : increased risk for $A D$ \\
\hline & Grip-protein & + & {$[56,229,276,307]$} \\
\hline & 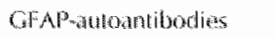 & + & {$[287,29,3]$} \\
\hline \multirow[t]{3}{*}{ Serum/phasmes } & $A$ & 4 & {$[147.185][192]^{\prime \prime}$} \\
\hline & & $\Rightarrow$ & 119,286 \\
\hline & GFAP-anlibodiess & + & {$[200,20 \pi, 287,293\rfloor$} \\
\hline
\end{tabular}

\begin{tabular}{|c|c|}
\hline i: & Increased lovel so were observed in ab patients compared to congrols \\
\hline- : & No dibenence wits observed in tevels for AD patients compared wo controls \\
\hline- : & Decraind levels were obsenved in AD patients compared to aontrols \\
\hline a & Inereated risk for AD associated whth wher two quartiles \\
\hline
\end{tabular}




\section{Glial fibrillary acidic protein (GFAP) in CSF or serum (plasma) (Table 6)}

Disorders associated with an increased CSF concentration of CFAP include dementia $[56,229,278,307]$ and brain tumours [5]. A transient increase in GFAP and $\$-100 p$ concentration during the first week after stroke was obseved in CSF [19]. In studying GFAP as a possible marker molecule, it has to be realised that during normal ageing, GFAP MRNA levels increase in human and rat brain [223] and in human CSF [307].

In serum, both GFAP and $5-100 \beta$ concentrations were increased in stroke patients, as measured with novel sensitive ELISA. GFAP was found to be the most sensitive marker of the two for smaller lacunar lesions or minor stroke [114]. Using western blot analysis, another study [224] also showed that the serum GFAP protein content was increased during five weeks follow-up after stroke.

The brain has long been regarded as an immunological privileged area. However, intrathecal production of immunoglobulins has been reported in some $A D$ patients $(n=56)$, compared to none of 33 healthy control persons [31]. And indeed, antibody titres against GFAP were higher in both sera and CSF of patients with AD compared to controls [287,293]. This latter study investigated the presence of autoantibodies in various patient groups including patients with AD, vascular dementia, PD, ALS, multiple sclerosis, Guillain-Barré syndrome, chronic inflammatory demyelinating polyradiculoneuropathy, patients with other neurological diseases, and controls. [293]. These authors showed that anti-GFAP autoantibodies were more frequently present in the sera of the dementia groups (AD: $33 \%$; vascular dementia: $44 \%$ ) compared to only $19 \%$ in serum of ALS patients and less then $6 \%$ in any of the other groups cincluding normal healthy controls: $4.5 \%$. Anti-GFAP autoantibodies were also present in the CSF of $23 \%$ of AD patients, compared to $13 \%$ of $\mathrm{PD}$ patients and none of 30 non-neurological controls [293]. In other studies, higher anti-GFAP titres could also be detected in serum of pattients with vascular dementia and senile AD, but lower titres were observed in earlyonsel AD $[200,201]$. In conclusion, anti-GFAP autoantibodies may be markers with high specificily for brain damage or dementia. However, the fact that no anti-GFAP autoantibodies were observed in presenile dementia suggests limited applicability as an early diagnostic marker. The characteristics of the subgroups with presence of autoantibodies need further investigation. Finally, the recent development of a sensitive ELISA for measuring GFAP protein [114] may help to address the concentration of this protein in serum or plasma in rellation to dementia.

\section{Markers related to cholesterol homeostasis}

\section{Cholesterol homeostasis and $A D$}

Cholesterol is the main lipid constituent of neuronal membranes and myelin. It is known that cholesterol is synthesised in the brain in situ [279] and that extracerebral cholesterol does nol contribute significantly to brain cholesterol content [133]. Excess of brain cholesterol has to be removed into the periphery (Fig. 3). The mechanism of transport is unclear, but may be mediated by ApoE and by facilitated transport of oxidised products like 24S-hydroxycholesterol 127,172]. However an additional, as yet unknown, transport mechanism may be involved (reviewed by [71]). It has been hypothesised that during neurodegenerative processes an increased removal of cholesterol from the brain occurs [29]. Aberration of cholesterol homeostasis may indeed be involved in AD. For example, a decreased level of brain cholesterol led to a reversible decreased formation of $A \beta$ in cultured hippocampal neuromes $[269]$. In addition, the unesterified cholesterol to phospholipid ratio was decreased in the temporal gyrus 
of AD patients, while the total cholesterol concentration was unchanged [183]. Recenlly, it was shown that use of cholesterol lowering drugs was associated with a decreased prevalence of $A D$ [309]. Research on serum cholesterol levels in relation to $\mathrm{AD}$, did not yield consistent results. In some studies $[203,268]$, decreased serum high density lipoprotein (HDL)-cholesterol concentration in patients with $A D$ were observed compared to controls, whereas others observed increased total and LDL-cholesterol in AD patients [37, 162].

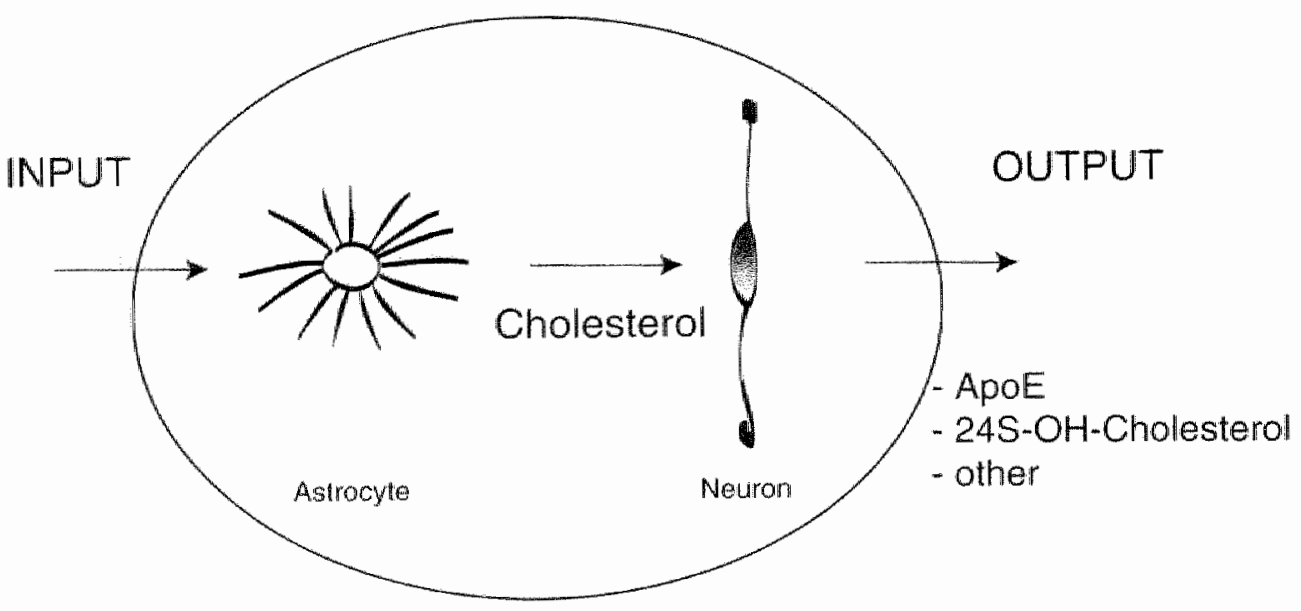

\section{CNS}

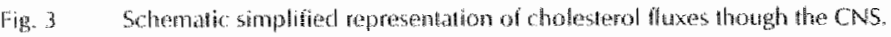

ApoE is involved in lipid transpont and cholesterol homeostasis. Circulating ApoE is associated with very low density lipoprotein (VLDL and involved in reverse cholesterol transport mediated by high density lipoprotein (HDL from cells to the liver [176,220]. There are three different alleles of the ApoE gene in humans coding for the ApoE isotorms: 82,83 and 84 occurring with trequencies of $10 \%, 74 \%$ and $16 \%$ respectively (Fig. 4) [317]. The phenotypes formed from these solforms are thus $2 / 2,2 / 3,2 / 4,3 / 3,3 / 4,4 / 4[300]$.

Apot is attracting a lot of attention in the context of $\mathrm{AD}$ as the ApoE4 alles is one of the major risk factors known at present for getting AD at a relatively early age, although the mechanism underlying this risk is far from understood $[160,281]$. The risk is significant in early-onset as well as the lateonset type of $\mathrm{AD}$; ApoE $4 / 4$ phenotypes have a risk to get $\mathrm{AD} 16$ years earlier than non- 4 cariers. The ApoE 4 allele is more frequently found among late-onset $A D$ patients when compared with age-matched controls $(50 \% \mathrm{vs}$. $16 \%$, respectively) $[281]$. In contrast, the ApoE4 isolom is thought to offer some protection against AD. The ApoE 4 allelle likely also correlates with impaired cognition in the healthy elderly. In a large epidemiological study a correlation of the 4 allele with cognitive dysfunction was demonstrated in the healthy elderly (e.g. worse performance on tests for reaction time, free recall and vocabulary in $3 / 4$ and $4 / 4$ subjects compared $102 / 2$ and $3 / 3$ homozygotes] [280]. In contrast, in a population based sudy in the Netherlands [274], no effect of ApoE allele type on MMSE decline over average 2.9 years (SD 0.4 of follow-up was observed. In agreement with this, a lack of correlation between the amount of ApoE4 alleles and global cognitive performance was observed in AD patients [157] 


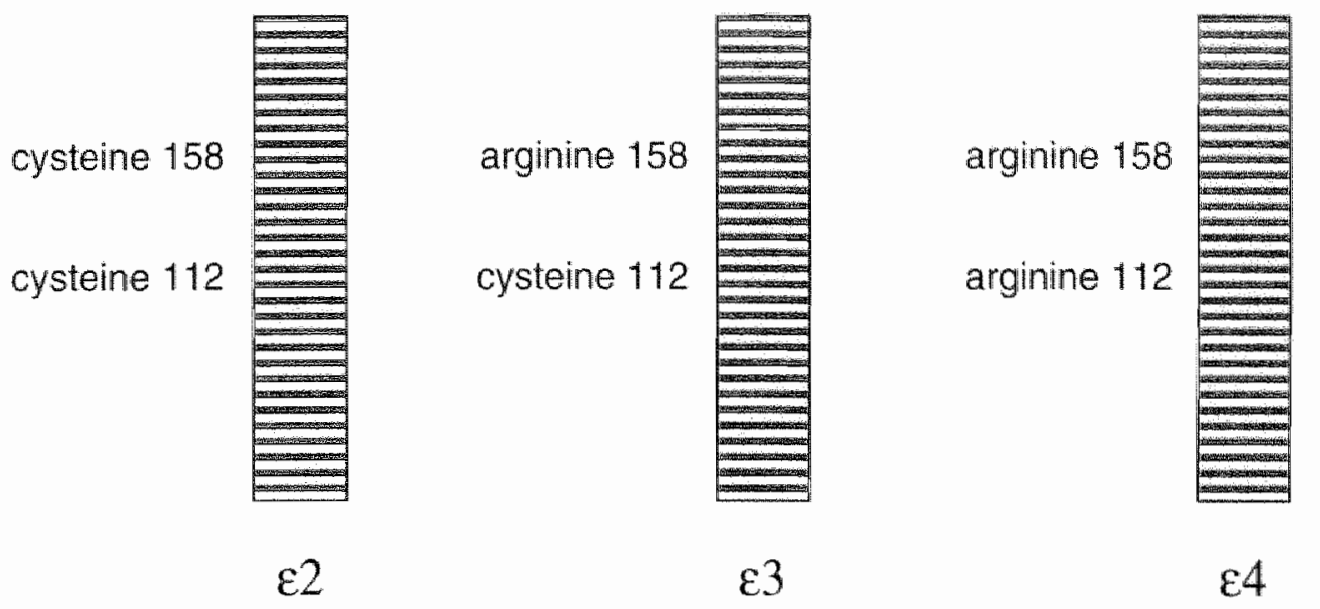

Fig. 4 Difterent MpoE allleles.

The amino acid substitutions ane indicated.

Recent views hypothesise that not the ApoE polymorphism but other defects in or close to the ApoE gene could be responsible for the increased susceptibility for AD [63]. For example, a 491AA polymorphism in the ApoE gene was associated with AD [47,158]. The ApoC1*2 gene, which is located close to the ApoE gene on chromosome 19, increased the risk for AD independently from the ApoF genotype [260].

ApoE is predominantly produced by and located in astrocytes in the CNS [70]. However, in the case of a neurodegenerative atlack (e.g. ischemic insult, status epilepticus, hypoglycaemia) it is found in neurites as well [156]. Increased ApoE mRNA and protein was observed in astrocytes in the frontal and temporal cortex of AD patients [70,313].

The function of ApoE in the brain is not yet clear, let alone that its role in the pathogenesis of $A D$ is elucidated. Similar to its role in the periphery, the rolle of ApoE is probably to mediate cholesterol transport and thus repair and the removal of excess cholesterol from dying cells. Recently, it was shown that ApoE knockout mice on high fat diel present hopaired BBB functioning [219]. ApoE may have a protective role against oxidative stress as ApoE deficient mice had higher levels of 3-nitrotyrosine [188]. In order to understand the mechanism behind the relation of $A$ poE with $A D$, the relation of the Apof isoform distribution on concentrations of several biomarkers in CSF, plasma and serum have been investigated. So far, no dear differences between ApoE isoforms and concentrations of these markers have been found, though a correlation befween ApoE concentration or number of 4 alleles and a du concentration may exist [157]. During a mean follow-up of 20 months, CSF tau was increased in AD patients with the ApoE4 allele [138]. Similarly, in another study tau concentrations were increased in AD patients with the ApoE4 allele during 14 months follow-up of [33]. Likewise, the concentration of ApoE in CSF correlated positively with tau levels in another study [167]. The mechanisms behind the relation between the two markers and the significance of those findings are not yet clear.

Recently, another mechanism of cholesterol removal from the brain has been determined: viat enzymatic oxidation into 245-hydroxycholesterol. It has long been known that this bydroxylated product of cholesterol is present in relatively high concentrations in the brain $[29,82,172]$. 24S-hydroxycholesterol is more polar than cholesterol and it is formed anter enzymatic oxidation of cholesterol catalysed by 245-hydroxylase [27] (See Fig. 1 in Chapter 9]. 
245-hydroxylase is an enzyme of the $P 450$ enzyme family and is almost exclusively located to the brain, except some small amounts in the adrenals [171]. Within the brain it is predominantly located in neurones [171]. However, like other oxysterols, 245hydroxycholesterol may have toxic properties for cells. Using neuroblastoma cells, loss of cell viability was shown at physiological concentrations of 245 -hydroxycholesterol, as measured by lactate dehydrogenase release and increased intracellular calcium concentrations [142]. In addition, 24S-hydroxycholesteral is known to be a potent inhibitor of cholesterol synthesis [258]. Experiments in rats suggested that 245-hydroxycholesterol is impontant for elimination of cholesterol from the rat brain by lacilitating transfer over the BBB [28].

\section{Apoliprotein E (ApoE) in CSF or serumplasma (Table 7)}

It has been thypothesised that ApoE is important for cholesterol transport from the brain into the circulation [71]. In case of increased levels of brain ApoE, as in neurodegeneration, increased ApoE levels in CSF might be expected. At least in aged individuals higher CSF ApoE concentrations were observed than in younger individuats [167].

Reports on concentrations of ApoE in CSF were conflicting. One group found increased ApoE levels in CSF of patients with disease duration up to 5 years compared to patients that were affected for longer lime [314]. Other's reported no differences in CSF ApDE concentrations of AD patients, or a decrease $[160,204]$. No correlation between isoforms and concentrations of ApoE in CSF have been observed $[160,292]$.

From literature in connection with atherosclerosis it is known that serum ApoE levels are dependent on the isoform of $A p o E, \varepsilon 2$ carriers having the highest concentration and 84 carriers the lowest ApoE serum concentration [263]. As there is an overrepresentation of the ApoE4 allele in $A D$ patients, it might be expected that ApoE levels in serum of AD patients are decreased too. Some reports indeed confirm the relation between the ApoE4 isotorm and ApoE concentrations in serum and indicate that ApoE serum concentrations are decreased in AD patients $[268,273]$. However, others were not able to confirm this observation $[160,260,285]$. The results of a recent study are very enlightening in this respect [268]. In this multi-centre study a significant decrease was observed in only some of the nine participaling European centres. The average serum concentration in the whole group, counting 489 AD patients and 429 controls, was significantly decreased with considerable ovenlap between the groups $142.95 \mathrm{D}$ $11.7 \mathrm{mg} / \mathrm{l}$. compared $1048.3 \mathrm{SD} 14.9 \mathrm{mg} / \mathrm{L}$ respectively) [268]. In other studies only $72-129$ patients were included and compared to various amounts of controls (n $=44-890$ ) and no unequivocal results were reportted $1260,273,285]$. Thus, the differences in ApoE serum or plasma levels between AD patients and controls may be of a magnitude to be detected only in large panels, and thus have limited value for diagnosis of individual patients.

\section{5-hydroxycholesterol in serum or plasma (Table 7)}

Infants and children have higher circulating levels of 245 -hydroxycholesterol than adults, while brain concentrations increase with age [172]. In mice, serum 245-hydroxycholesterol concentrations are low in mewly bom mice, peaking between postnatal day 12 and 15 and declining thereafter to baseline levels. Thus, lower serum levels of 245 -hydroxycholesterol at increasing age is observed in both humans and mice. In contrast, a continuous developmental increase in level was shown for the 245-hydroxycholesterol forming enzyme 245-hydroxylase
in the brains of mice and humans [171].

In view of the increased serum concentrations during development, it was hypothesised that altered levels of 245 -hydroxycholesteral can occur in the circulation of patients with 
neurodegenerative diseases [29]. In one study, serum 245-bydroxycholesterol concentration was increased in mildly affected $A D$ patients compared to controls, though there was considerable overlap in 24S-hydroxycholesterol concentrations [173]. The 245hydroxycholesterol concentrations correlated negatively with severity, i.e. the highest levels were observed in the less affected patients. Other recent studies showed decreased 245 hydroxycholesterol levels in plasma of severely affected AD patients [42,235]. Thus, increased levels of this oxysterol may be observed during early stages of $A D$ and decreased levels in more advanced states.

These findings may suggest that 245-hydroxycholesterol may be a state marker of neurodegenerative processes. Increased concentrations at early stages of the disease night be indicative of increased cellular degeneration and increased removal of cholesterol from the CNS. Decreased concentrations would then be indicative for a lower cholesterol level due to the lower tissue mass in later stages. In addition, since 245 hydroxycholesterol is present almost exclusively in the brain, it might constitute a biological marker with high specificity for alterations in brain cholesterol homeostasis. Though, considering the overlap between patients and controls, its use as a single marker diagnosing individual patients will be limited.

Table 7 Summary of concentratons of Ahot and 24S-OH-Chol in serum and CSF of AD pations

\begin{tabular}{|c|c|c|c|}
\hline maltrix & marker & |ravel & ruferences \\
\hline \multirow[t]{3}{*}{$\mathrm{CSF}$} & ApoE & 4 & {$[3[4]$} \\
\hline & & $\therefore$ & {$[204]$} \\
\hline & & $\ldots$ & {$[160]$} \\
\hline \multirow[t]{4}{*}{ Serumplasma } & ApoE & $=$ & {$[160,260.285]$} \\
\hline & & - & {$[268,273 \|$} \\
\hline & 2.45 modroxycholesterol & + & $|173|$ \\
\hline & & - & {$[42,235]$} \\
\hline
\end{tabular}

\begin{tabular}{|c|c|}
\hline$+:$ & Inereased hewels ware observad on AD patiants compared to controls \\
\hline$=:$ & 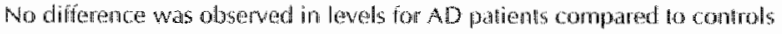 \\
\hline$\ldots$ & ed levels were observed an $\mathrm{AD}$ pathents compared to com rols \\
\hline
\end{tabular}

\section{Concluding remarks}

This paper intended to provide an overview of the current knowledge of biological markers in blood or CSF that are related to AD. In line with the heterogeneous pathology of AD, changes in markers related to the severall pathophysiological mechanisms addressed in this overview have been observed, i.e. inflammation, oxidative stress, vitamin status, brain specific structural alterations and cholesterol homeostasis. Furthemore, the aim of this overview was to identify markers that may be of interest for our studies on how to combine markers from these different mechanisms in order to arrive ultimately at an in vitro diagnostic test for AD.

CSF is not a matrix that can be easily used for diagnostic purposes, let alone for screening a population on risk factors. Nevertheless, we summarise the most promising results of the markers described in this rewiew. Concerning the brain-specific proteins, the characteristic plaque marker lau is a relativelly specific and sensitive marker for AD. Decreased AB concentrations are found in early stages of AD, but this decrease is not very specific for AD (vs. vascular dementia). The combination of CSF tau and AP vields a probable accurate diagnostic tool. GFAP protein and CFAP antibodies in CSF are brain-specific markers, for more severe stages. The CSF levels of markers for inflammation present a variable picture. So far, only consistent changes have been reported for the increase in haptoglobin fragments. The studies 
on the oxidative stress markers 3-nitrotyrosine, the DNA oxidation product 8-hydroxy-2:deoxyguanosine, and isoprostanes have reponted increased concentrations of these markers in CSF. Most of the data of these (not brain-specific markers are based on post-montem CSF examination and the relevance of these markers for early stages of $A D$ remains to be established. Conceming the vitamins, a decreased CSF vitamin $E$ concentration has low specificity for $\mathrm{AD}$, but may still be interesting for further studies since these levels can be changed by dietary supplementation.

Serum (plasma) investigations may be more promising for a population-screening test, but alterations observed in the periphery may be less specific for CNS disorders. In this respect, 245-OH-Chol and GFAP antibody concentrations may present the currently most specific and promising markers. $245-\mathrm{OH}$-cholesterol may be an early marker, while the GFAP autoantibodies may be late markers. The inflammation markers $14-6$ and TNF a have shown varlable results and the relation of these markers with dementia is still not clear. $\alpha-A C T$ concentration in serum may be the most convincing, much investigated, inflammation marker to date, while other markers such as haptoglobin, and IL-6 receptor need further replication. The vilamins $A_{3} C$, and $E$ as serum markers for oxidative stress may be too much influenced by diet but may give an indication of the oxidative status in generat. Homocysteine must be considered for inclusion in a set of markers, due to the relative consistently reported increased mean concentrations in both AD and normal cognitive ageing. However, increases in this marker are not specific to brain related processes and thus, this marker may have diagnostic value only in combination with other markers.

\section{Outline of the thesis}

The pathophysiology of neurodegenerative diseases is very complex. For the most frequently occurring neurodegenerative clinical pictures, $A D$ and $P D$, the initial pathology is still unknown. Processes like excitotoxicity, inflammation, changes in energy or lipid metabolism, insufficient DNA repair, and mRNA misreading, have all been described in relation to AD or other forms of neurodegeneration. These processes may be influenced by a large number of envirommental factors andior life events. Although not of necessity, evaluation of serum concentrations of a number of relevant substances related to the above mentoned processes, commonly named biomakers or markers, was rationalised to be presently the best approach for an in vitro test with prognostic value for neurodegeneration.

For quite a number of possible or putative biomarkers the relevance of reflecting one of the abovementioned processes in relation to neurodegeneration is not known. Thus, inflammation is a process, which can be found in any tissue, similar to oxidative stress. Therefore the relation of a number of these markers with neurodegeneration, which was controversial or unclear in the Herature, had to be investigated. Betore starting our endeavour to evaluate a large number of serum markers, we decided to study a number of these markers in established models for neurodegeneration. To this aim we chose the 3-nitropropionic acid treated cell cultures and the 3-mitropropionic acid treated rat model.

In the CNS, oxidative stress is imposed by highly reactive, oxygen containing species, like $\mathrm{H}_{2} \mathrm{O}_{2}, \mathrm{O}_{2}{ }^{*}, \mathrm{NO}$, and $\mathrm{OH}^{\circ}$. As $\mathrm{NO}$ is an essential intermediate in excitotoxicity, we needed for our studies a cell culture system in which the presence of a functional NO synthase was highly likely. This was round in a three-dimensional culturing system, known as spheroids. Spheroids are cultured from foetal whole brain single cell suspensions [294]. In essence, shaking the cell 
suspension will make the cells reaggregate into spheres, which can be cultured as such for prolonged periods of time.

The first part of this thesis addresses the characterisation of these spheroids and the validation of the presence of a glutamate - NOS - CGMP signalling pathway (Chapter 2). In addition, we wanted to study the similarity of the cellular localisation of this pathway between the spheroids and a hippocampal slice preparation (Chapter 3 (as a spinmoff of Chapter 2)).

Next we wished to investigate the effects of an oxidative stress on the presence of a number of biomarkers known to be vulnerable to oxidative modification. Therefore, we treated the spheroid cultures with the toxin 3-nitropropionic acid for different periods of time. Our special attention was focussed on the oxidative stress marker 3-nitrotyrosine (Chapter 4).

Having established the 3-nitropropionic acid treatment of spheroids as a model for severe oxidative stress, we wished to explore the effects of 3-nitropropionic acid treatment in an in vivo rat model. Therefore we had to evaluate the effects of this treatment on several behaviounal parameters and on markers for neurodegeneration and inflammation in Lewis and Wistar rats (Chapter 4 and 5). The effects of 3-nitropropionic acid treatment were also assessed on parameters which give information about the integrity of the blood brain barrier which is known to be affected in this model) and lipid metabolism in the brain (Chapter 6 ).

Biomarkers for inflammation had to be measured in sera from a group of nomally ageing humans (MAastricht Ageing Study (MAAS). MAAS is a longitudinal population based follow-up study imvestigating determinants of nomal ageing [131,301]. As some of these serum samples were stored for more than six years, we had to establish that storage conditions had no adverse effect on concentrations measured after such a long period of time. Indeed, it proved not possible to perform all our intended measurements, as some possible biomarkers deteriorated under prevailing conditions (Chapter 7 ).

Thereafter, the relation befween cognitive performance during nomal ageing and biomarkers for inflammation (Chapter 8), markers for cholesterol homeostasis (Chapter 9) or homocysteine metabolism (Chapter 10) was to be investigated in serum of the MAAS.

Finally, in order to construct an in vitro diagnostic tool, we wished to evaluate all serum marker patterns in patient groups diagnosed for AD (Chapter 11). The sensitivity and specificity of a model based on serum markers was investigated in discriminating AD patients from patients with PD or other cognitive disorders and a healthy control group. As a test for our ullimale aim to establish an in vitro test with prognostic value for neurodegenerative cliseases, we wanted to apply the best model for discriminating between AD and healthy controls in the six year followup study (MAAS). Thus, the suitability of this model as a prognostic test for cognitive performance was evaluated.

\section{Acknowledgement}

The assistance of A. Scheepens is gratefully acknowleciged for critically evaluating the English spelling of the mainuscript.

\section{References}

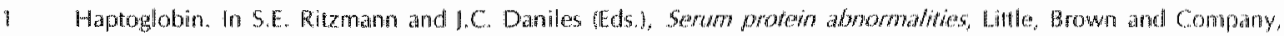
1975.

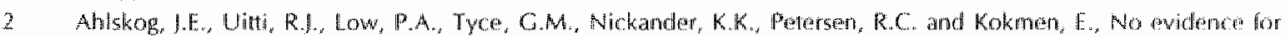
systemic oxidant stress in Parkinson's or Alzhenter's disedse, Mov Disord 1001995556673.

3 Assen, P.S. Anti-inflanmatory therapy for Alzheimer's distase: implications of the predn isone drial, Arat Neurol 


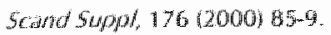

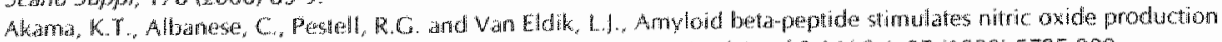

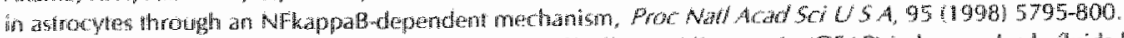

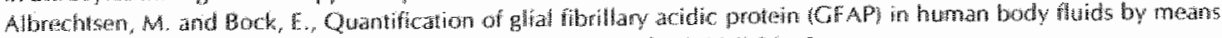

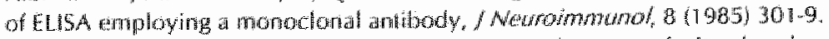

Alen, R.H. Stabler, S.P", and Lindenbaum, I., Retrevance of witamins, homocysteine and other metabolites in

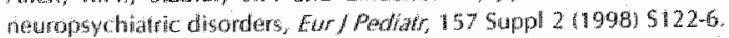

Almkwist, O, Busun, H., Wagner, 5.1., Rowe. B.A., Wahtund, L.O. and Lanntelt, L., Cerebrospinal thid levels of alphatsecretaseclaved saluble anwotd precursor protein mirror cognition in a Swedish family with Alpheimer diserate and a gene mulation, Arch Newol, $54(1997) 641-4$

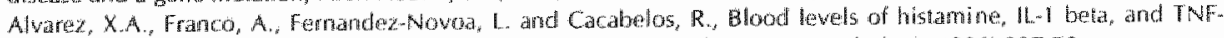

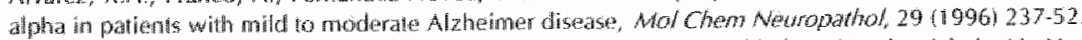

Andrasen, N, Hesse, C., Davidsson, P., Minthon, L, Wallin, A., Winblad, B., Vanderstichele, H., Vanmechelen, E.

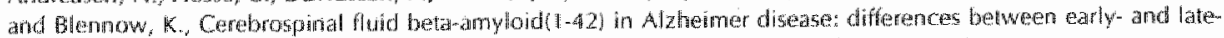
onset Altheimer disease and stabliv during the course of disease, Ard Neuro/, 56 (1999) 67380.

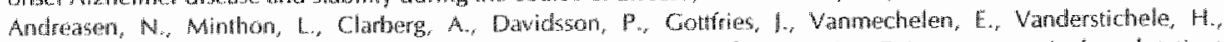

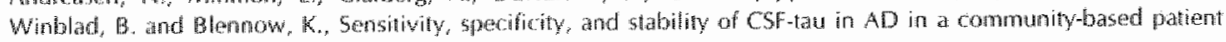
sample, Nexology, 5391999$) 1488-94$.

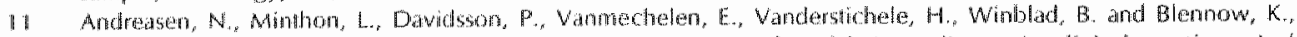
Evatuation of CSF tau and CSF-Abeda42 as diagnostic mankes for Alzheimer disease in clinical praction. Arch Newo/s 58 2001) 373.9.

12 Andrasen, N., Minthon, L., Vammechelen, F., Vanderstichelle, H., Davidsson, P., Winblan, B. and Blennow, K.

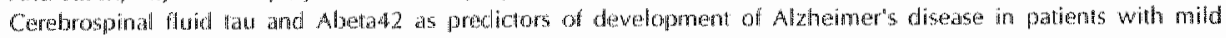

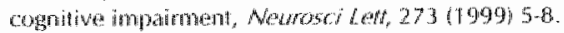

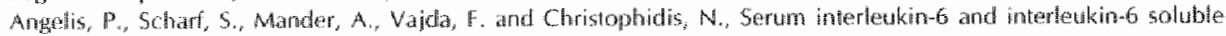

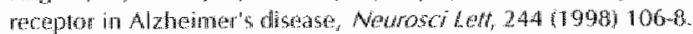

14 Aoyana, K, Malsibara, K., Fujikawa, Y., Nagahiro, Y., Shimizu, K., Umegae, N., Hayase, N., Shiono, H. and Kobatyashi, 5. Nifration of manganese superoxide dismutase in cerebrospinat fluids is a marker for perowentritemediated oxidative siress in neurodegenerative diseases, Anm Newrol, 4702000$] 5247$.

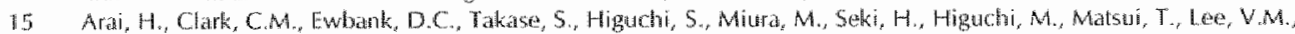
Trojanowski, 1.Q. and Sasaki, H. Cerebrospinal thid tau protein as a potental diagnostic marker in Alzheirner"s disease, Newobiol Aging $19(1998) 125$-6.

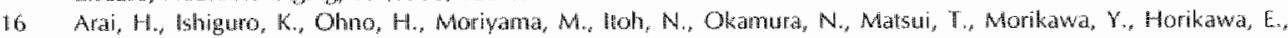
Kohno, H. Sasak, H. and mahori, K., CSF phosphorylated tau protein and nild cognilive impairment: a prospoctivestudy, Exp Newrol, $1606(2000) 201-3$.

17 Arai, H., Satoh-lNakagawa, T., Higuthi, M., Morikawa, Y., Mitad, M, Kawakami, H., Seki, H., Takase, 5, and Sasaki, H. No increasa in cerebrospinall fluid tau protetn lewels in patuents with wascular dementia, Newosci Let, 256 (1998) $\| 746.6$

18 Arai, H., Teraima, M. Mätra, M., Higuchi, S., Muramatsu, T., Machida, N., Seiki, H., Takase, S., Clark, C.M., Lee,

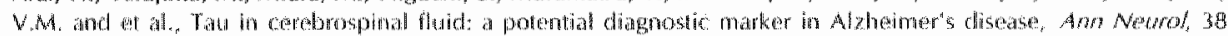
$(1995) 649 \times 52$

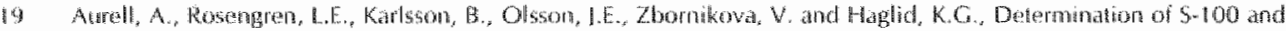

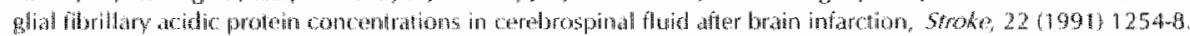

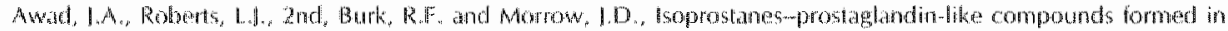

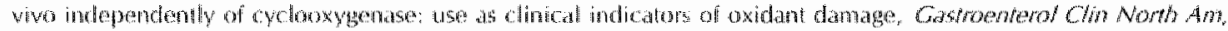
$25(1096) 409-27$.

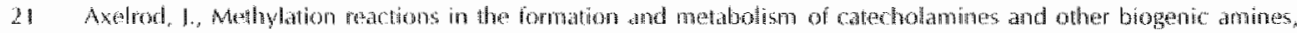

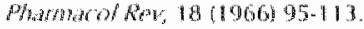

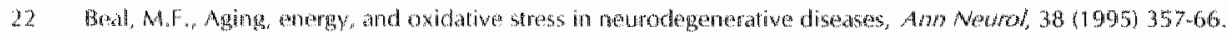

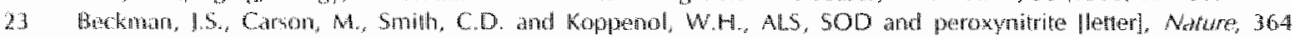
[1993) 584 .

24 Bergamaschini, L., Parmetti, L., Patwhor, D., Canziati, S., Cugho, M, and Agostoni, A., Actiwation of the conlact

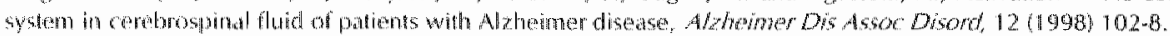

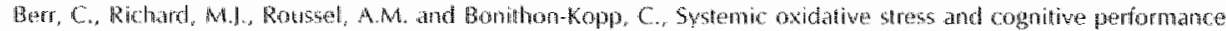

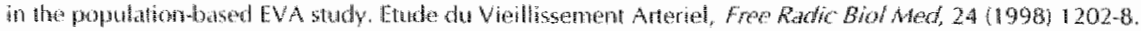

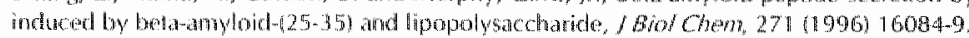

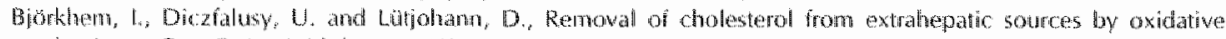

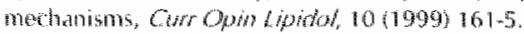

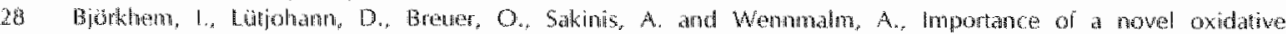

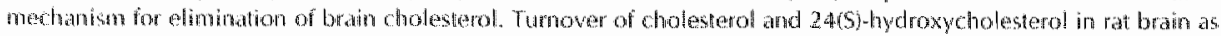

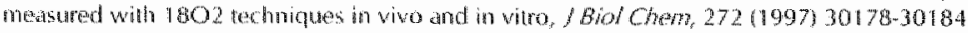




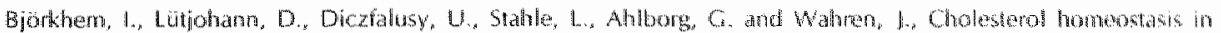

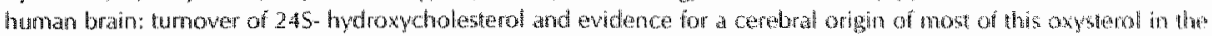
circulation, /Lipro Res, 39 (1990) 1594-1600.

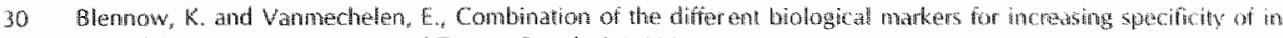

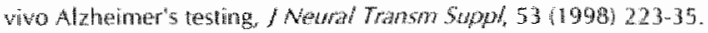

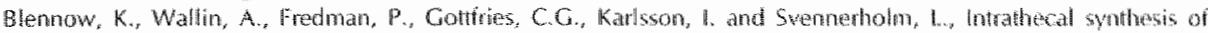

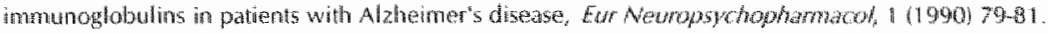

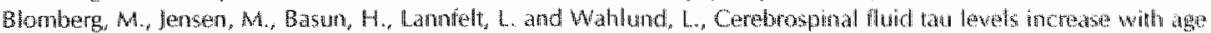

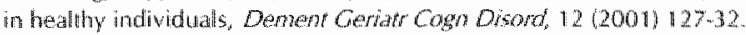

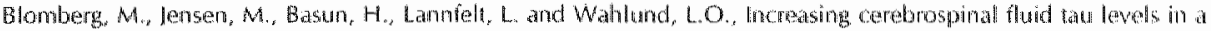

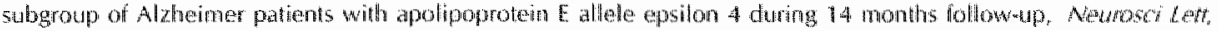
$21419969 \div \div 3: 6$

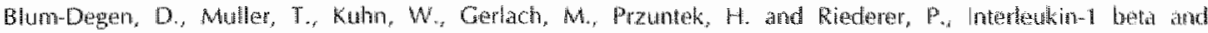

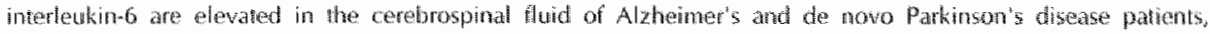
Neurosci Lest, $202(199591720$.

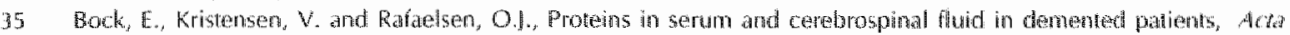
Neurol Scand, 50 11974 91-102.

36 Bonaccorso, S., Lin, A. Song, C. Verkerk, R., Kenis, G. Bosmans, E., Scharpe, S., Vandewowde, M., Dosshe, A and Maes, M., Serofonin-inmune interactions in elderly volumeers and in patients with Alzheimar"s disease (DAT): tower plasma tryptophan awatability to the brain in the plderly and increased serum interteuting in DAT, Algho (Ahlanol, 10 (1998) $316-23$.

37 Bonarek, M., Baberger-Gateat, $P$., Letenneur, L, Deschmps, V., tron, A., Dubroca, B. and Dartigues, ff.

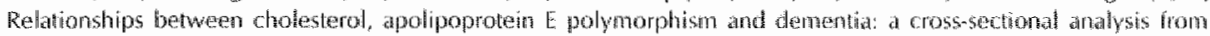
the PAQUID study, Newrogidemiology, $19(2000) 141.8$.

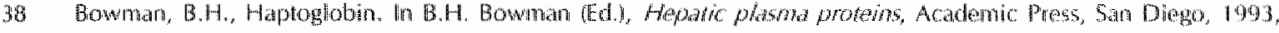
pp. 159-167.

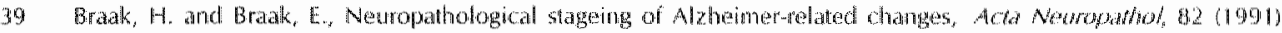
239.59.

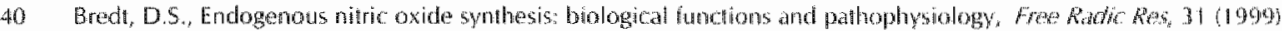
$577-96$.

41 Breimer, J.C., Wethi, K.A. Helens, M. G. Gaskell, P.C., Gat, B.A., Roses, A. D. Pericak-Vance, M.A. and 5 aumders, A.M., Delayed onset of Alzheimer's disease with nonsteroidal antianflanmatory and hisfamine H2 blocking drugs, Newobriot Aging, 1611995$) 528.30$

42 Bretillon, l., Lütjohann, D., Stahle, L., Widhe, T., Bindl, L., Eggersen, G., Diczlalus, U. and Björkhem, I., Plasma levels of 245 -hydroxydnolesterol reflect the ballance between centabl production and hepatic metatbolsin and are inversely related to body surface, /lipid Res, 41:2000i840-845.

43 Bruijn, L.1., Beal, M.E., Beqher, M.W., Schulz, I.B., Wong, P.C., Price, D.L and Cleveland, O.W., Elewated tree nitrotyrosine levels, but not protein-bound nitrotyrosine or hydroxyl radicals, throughout amyotrophic lateral

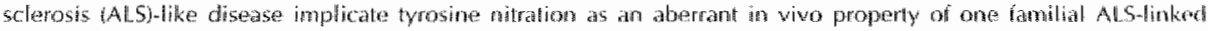

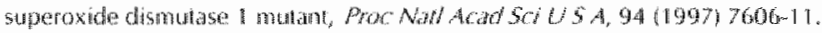

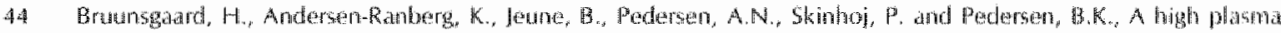

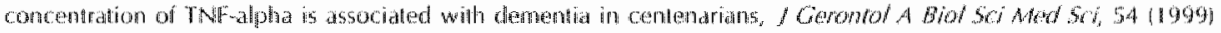
M357-64.

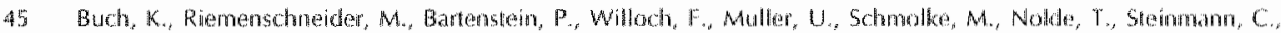

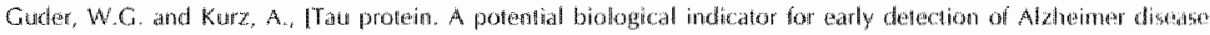
[see commentsi]. Nenveriot, 65 (1998) 379-85

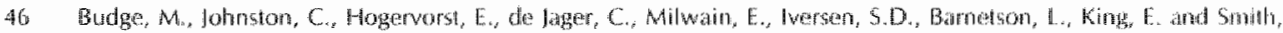

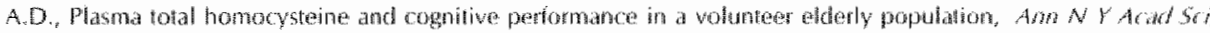
$903020001407-10$.

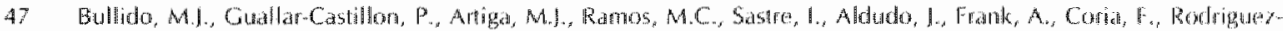

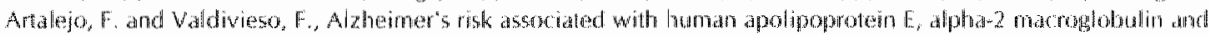
lipoproten receptor related protein polwoworphisms: absence of genetic intoractions, and modution by gendes,

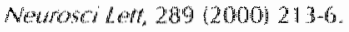

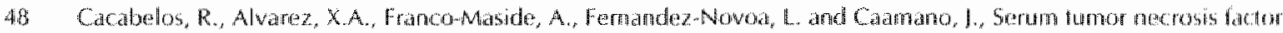

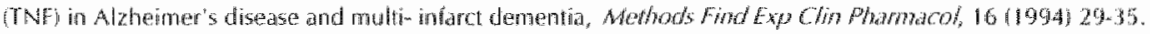

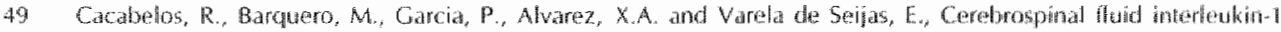

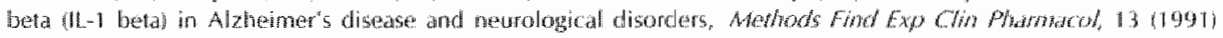
455.8.

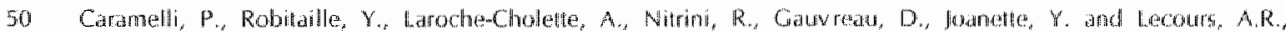

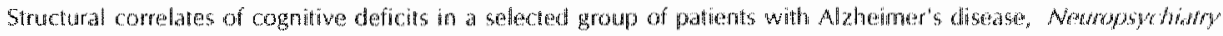
Newropsy hol Behar Netwol, 11 (1998i) $184-90$.

51 Carmel, Ka, Got, P.S., Waters, C.H. Cains, K., Green, R., Bonderef, W., DeGiongio, C.M. Cummings, 1.L, 


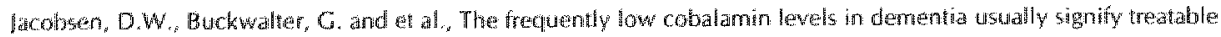

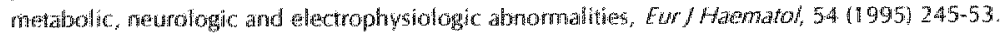

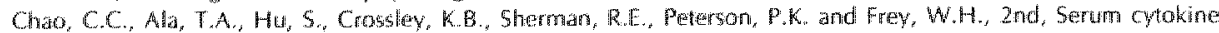

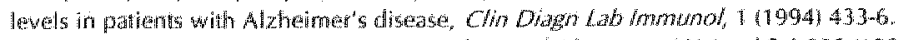

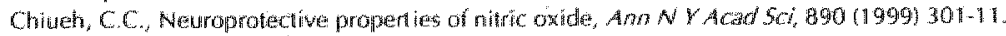

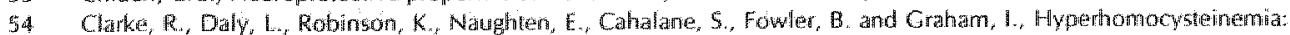

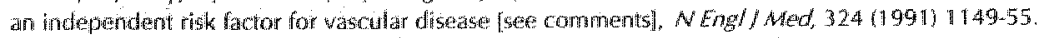

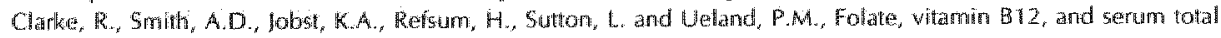

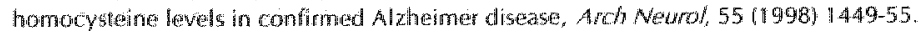

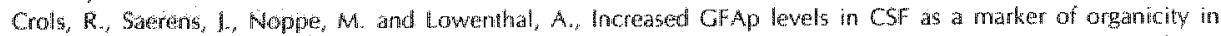

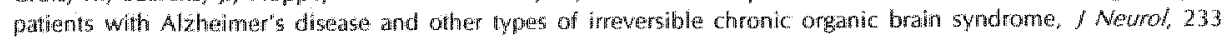
(1986) $15 \% 60$.

57 Cross, A.H. Mannings, P.T., Keeling, R.M., Sehridt, R.E. and Misko, T.P., Peroxynitrite formation within the central

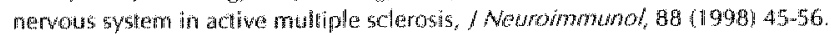

58 Cross, A.t. Marning, P.T., Stern, M.K. and Misko, T.P., Evidence for the production of peroxymitrite in

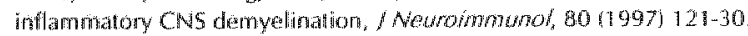

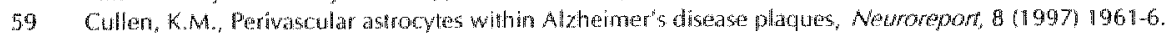

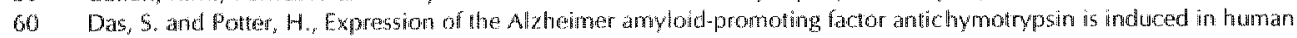
asilocyles by 11,1, Neetrom, 1411995$) 447.56$

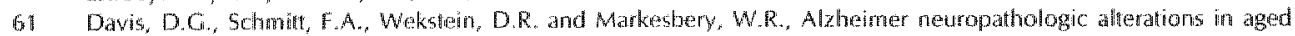

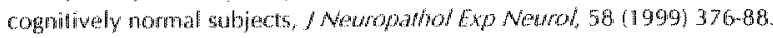

62 Dawsor, V.L. and Dawson. T.M. Nitric oxide in neurodegeneration, Prog Brañ Res, 1190 (19984 $215-29$.

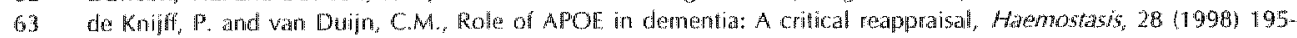
201.

64 de ta Torr. I.C, Cerobral hy popurfusion, capillary degeneration, and development of Alsheimer disease, Alzheimer Dis, Asoc Disom, $1412000 \mathrm{~S} 572 \times 81$.

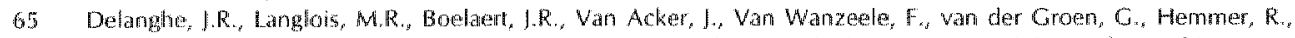
Verholstede, C., De Buyzere, M. De Bacquer, D., Arendi, W. and Plum, I., Haptoglobin potymorphism, iron metabolism and morlality in HIV infection. Aids, $12(1998) 102 \%-32$.

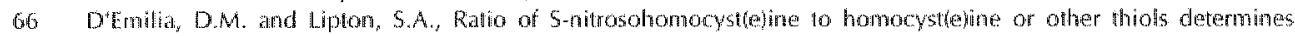
neurotoxicily in tat cerebrocortical cultures, Newosci Lent, $265(1999) 103.6$.

67 Di Meo, S. and Venditt, P., Mitochondra in exercisemduced oxidatiwe stress, Biol Signals Recept, 10 (2001) $125 \mathrm{n}$ 40.

68 Diamandis, E.F., Youse, G.M. Petraki, C. and Soosaipillä, A.R., Human kallikrein 6 as a biomarker of alzheimer"s disease, Clin Blochern, 33 (2000) $663-7$.

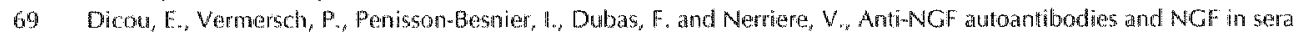
of Alzhemer patients and in normal subjects in relatom to age, Awoimnumity, 26 11997) 189494.

70 Diedrich, J.F., Mimigan, H., Carp, R.I. Whitaker, I. W., Race, R., Frey, W. d. and Haase, A. T. Neuropathological changes in scrapia and Alzheimer's disease are associated with increased expression of apolipoprotein $E$ and

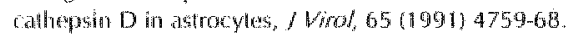

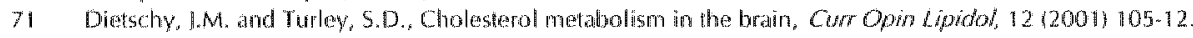

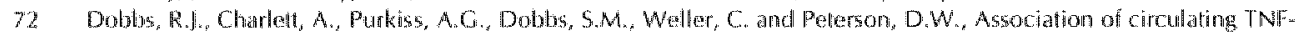

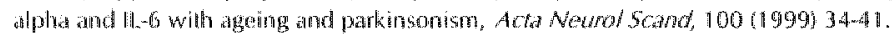

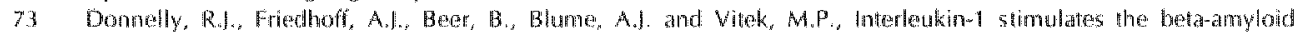
precursor protein promoter, Ce// Alol Neurobio/ 10 (1990) 495-95.

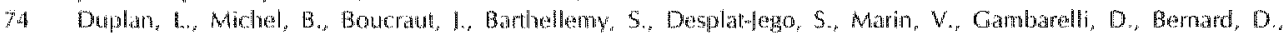

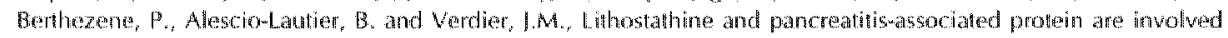
in the very early stages of Alameiner's disease, Newobol Aging, 22 (2001) $79-80$.

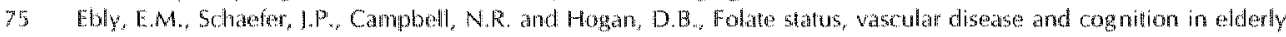

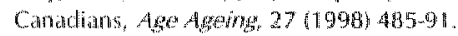

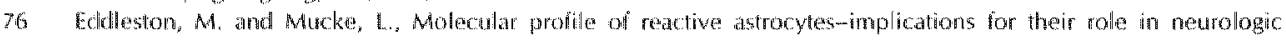

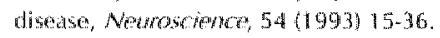

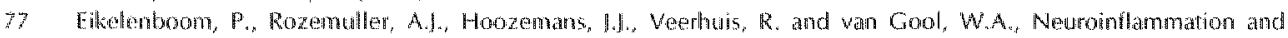

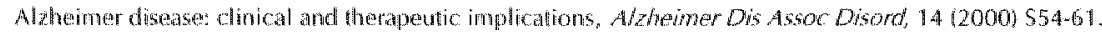

70 Eikelenboon, P. and Weethuis, R., The importance of inflammatory mechanisms for the devellopment of Alzheimer"s Wisease, Exp Geronol 3411999$) 45361$.

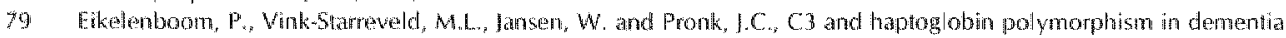

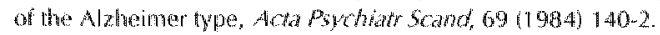

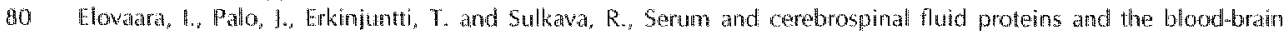
Warrier in Alzheimer"s disease and multinfanct dementia, Eur Netrof, 26 (1987) 229-34.

91 Ergetborghs, S., De Brabander, M., De Cree, J., DHooge, R., Geens, H., Verhaegen, H. and De Dewn, P.P., Unchanged lavals of intergukins, neopterin, interferon-gamma and tumar necrosis factoralpha in cerebrospinal 


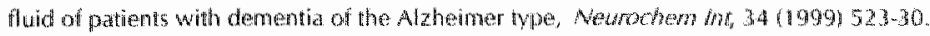

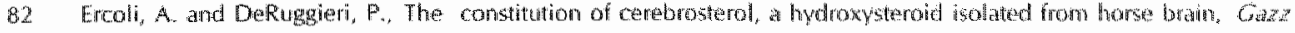
Chinn /hat $83(1953973-86$.

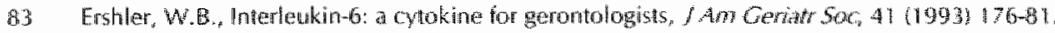

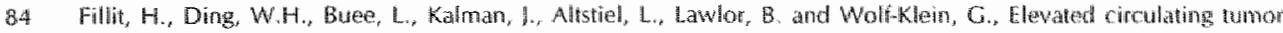

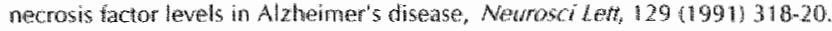

85 Folstein, M.F., Folstein, S.E. and MCHugh, P.R., "Minimental state'. A practical method for grading the cognitive state of putients for the clinician, / Pspctials Res, 1211975$) 180-98$.

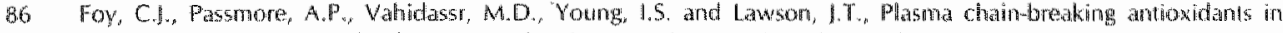
Alshentimer's disease, wascular dementa and Parkinson's disease, Qm 92 (1999) 3945.

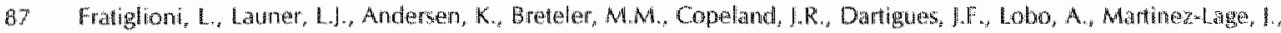
Soininen, Fit. and Hofman, A. Incidence of dementia and major subtypes in Europe: A collaborative shuty of population-based cohorts. Newnologic Diseases in the Elderly Research Group, Neurology, 54 (2000) $510-5$.

88 Frohlander, $N$. and Adolfson, R. Haptoglobin groups in dementia of Alzheimer type and multi-infarct dementia, Hum thered 35 (17985) $25 \% 9$

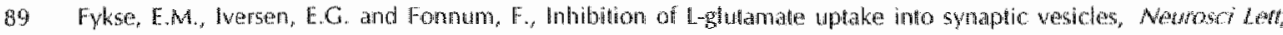
$135(1992) \div 25-88$

90 Gahan, E. and Owermier, IB., Inflammatory pathogenesis in Alzheimer's disease: biological mechanisms and cognilive sequeli, Neurosi Biodehaw Rew $23(1999) 615-33$.

91 Galasko, D. Chang, L., Motter, R., Clank, C.M., Kaye, I., Knopman, D., Thomas, R, Kholodenko, D., Schenk, D. Lieberburg, I., Miller, B., Green, R., Basherad, R., Kentles, L. Boss, M.A. and Seubert, P. High cercubrospanal fluid tan and low amyloid beta42 lewels in the dirical diagnosis of Alzheimer disease and relation to apolipopourun genotype, Arch Neurol, 55 (1998) $937-45$.

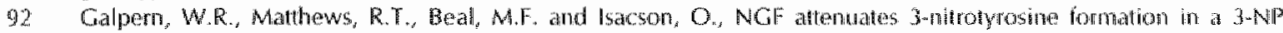
model of Huntington's disease, Neurorepont, 7 (1996) 2639-42.

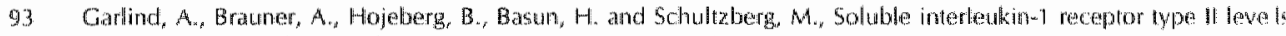

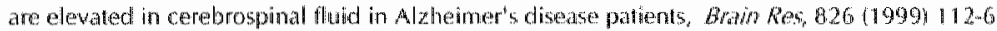

94 Giometto, B. Argentiero, $V$., Sanson, F. Ongaro, $G$. and Tavolato, B. Actiophase proterins in Alzheimer"s disedese, Eur Newrol $28(1988) 30-3$.

95 Gienwer, G.C. and Wong, C.W., Azheimer's disease: initial report of the purifation and othataterization of novel cerebrovascular amyloid protein, Biochem Biophys Res Commun, $120(1984) 83.5-90$.

96. Gonzalez-Scarano, F. and Batuch, G. Microglia as mediators of intlammatory and degenerative diseases, Anwh hel Neurasci, $22(1999) 219 m 40$.

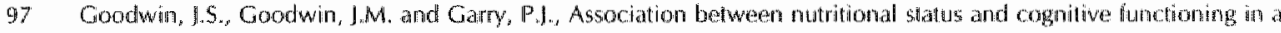
healthy elderly poputation, darma, 249 (1983) $2917-21$.

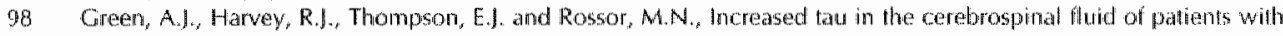
from lotemporal dementia and Alzheimer's disease, Neurosci Ledt, 259 (1999) 133-5.

99 Girffin, W.S., Sheng, 1.G., Robents, G.W. and Mrak, R.E, lnterleukin 1 expression in different placue types in

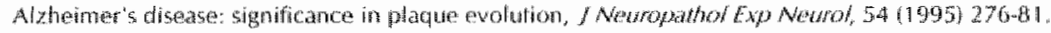

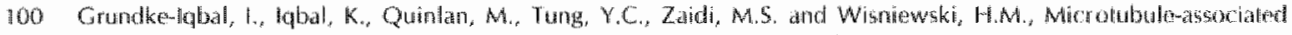

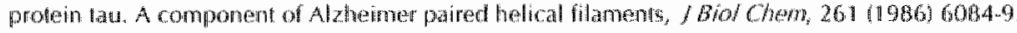

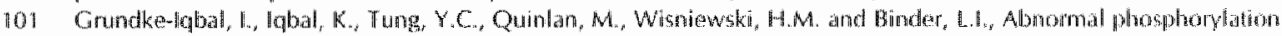

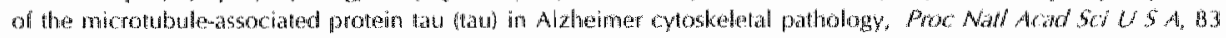
(11986) $4913-7$.

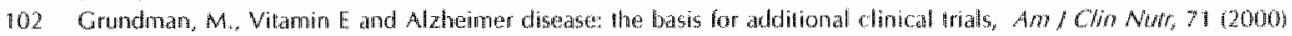
$6305-6365$.

10 G Gussekloo, 1. Hehmans, B.T., Sloghoom, P.E, Lagay, A.M., Knook, D.L. and Westendorp, R.C., Thermolabile

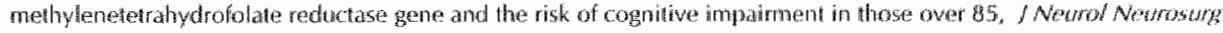
Psychianth, 67 (1999) 53,5-8.

104 Hallwesl, B. Reactive oxygen species and the centrat newots spatem, / Neurocharn, 59 (1902) $1609-23$.

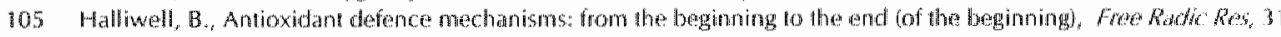
(1999) $261-72$.

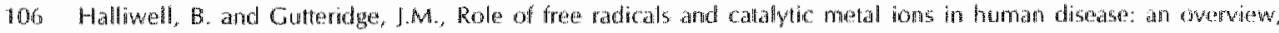
Wethods EnZWhod 18641990$) 1-85$.

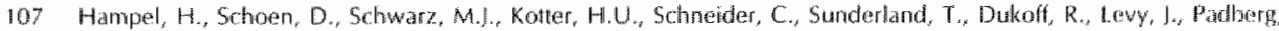

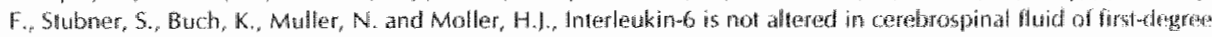
relatives and patients with Alzheimer"s disease, Netrowci Lat, 228 (1997) 143-6.

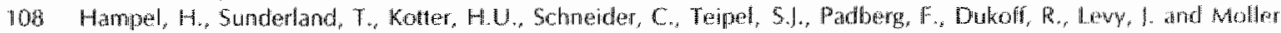

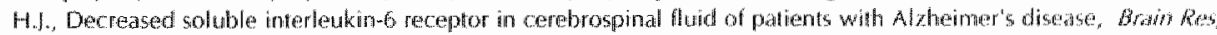
$780(1998) 3569$.

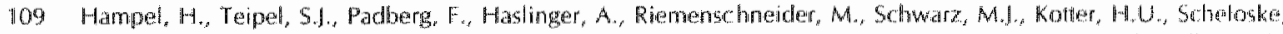

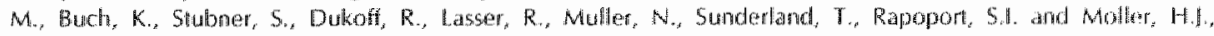




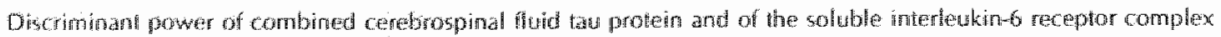

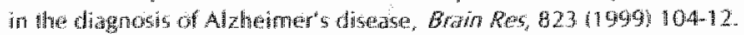

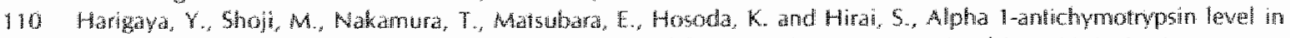

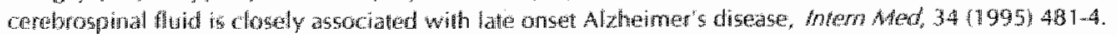

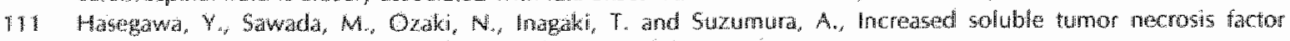
receptor lews in the serum of elderly people, Gerontology, 46 2000) $185-8$.

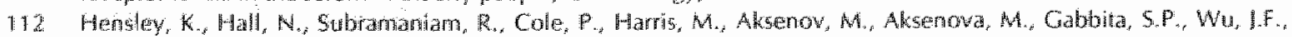
Carney, M. and al, Brint regiond correspondence batween Azheimer's disease histopathology and hiomarkers

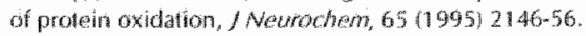

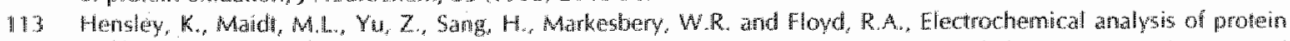

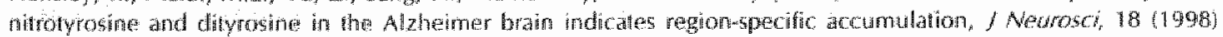
E1 2632

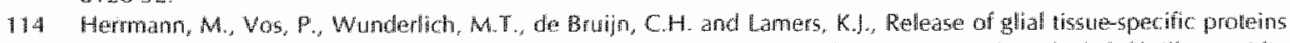
affer acute stroke: A comparative aralysis of serum concentrations of protein $5-100 \mathrm{~B}$ and glal fibrilary acidic protein, Stroke, 31 12000 $2670-7$.

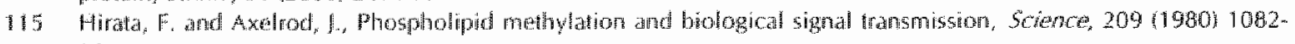
90.

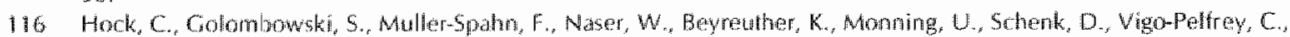

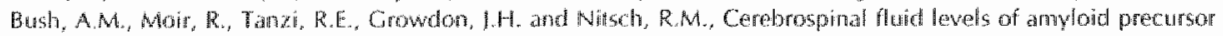
profen and amylotd betapeptide in Alzheimer's disedse and major dropession * inverse corretation with dementia seweriay, Eur Newhol, 39 (1998) 11118.

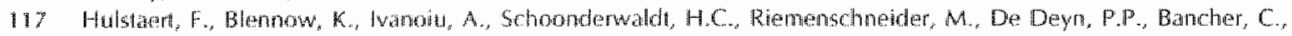

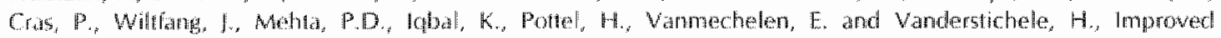

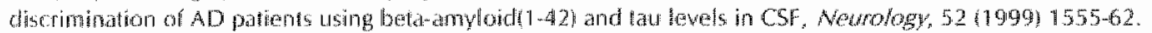

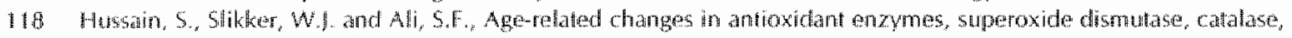
glatalhione peroxidase and glutathone in diferent regions of mouse twains, fot. J. Dew. Nevroscience, 13 (1995) 81) 1817

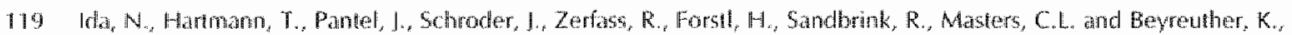

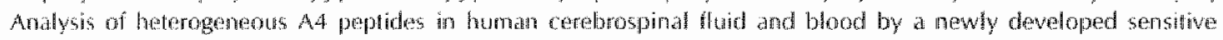
Westerm blot assay, J Biol Chom, 271 19996) 22908-14.

120 Ischiropoulos, H., Zhu, L., Chen, I., Tsai, M. Marrin, 1.C., Smith, C.D. and Berkman, I.S. Peroxymitrite-medialled fyrosine nitration calalyzed by superoxide dismulase, Awh Biochem Biophy, 298 (1992) $431-7$.

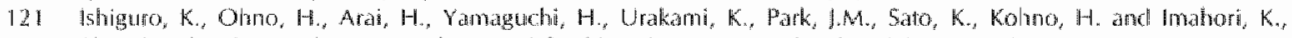

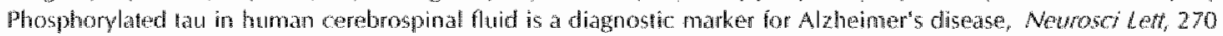
(1909) 91-4.

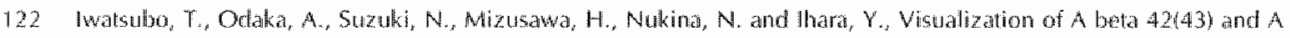
beta 40 in sente plaques with end specific A beta monoclonats: evidence that an initially deposited species is $A$ beta $42(43)$, Newon, $13(1994) 45.53$.

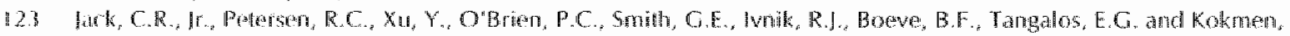

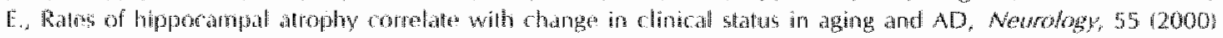
48499

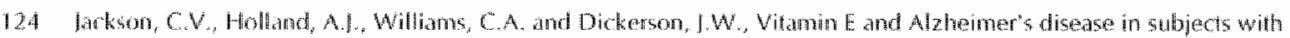

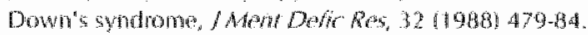

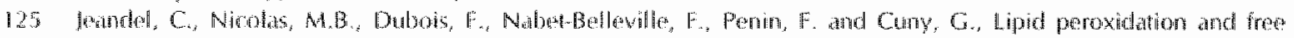

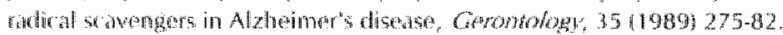

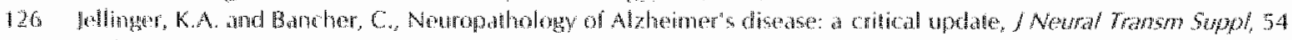
(11098. 79.95

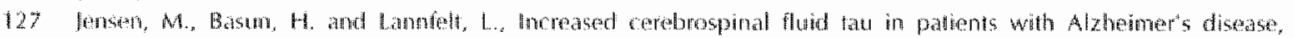

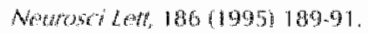

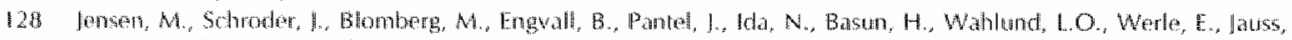

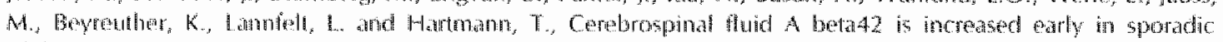

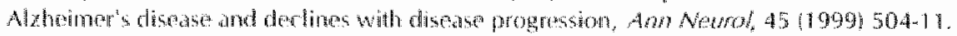

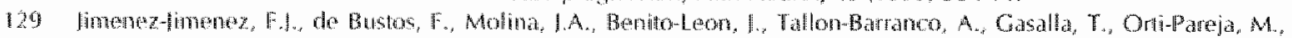

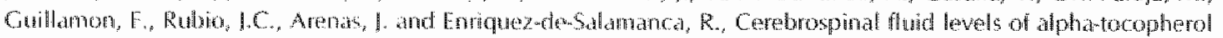

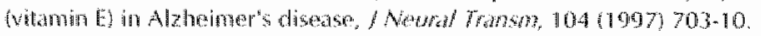

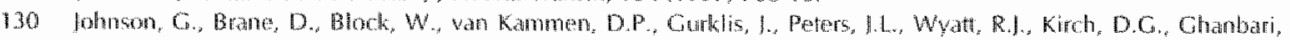
H.A. and Merrit, C.R. Ceretrospinal huid protein variations in common to Azheimer's disease and schizophrenia. Ary theor therowhor, $3(1992) 47-53$.

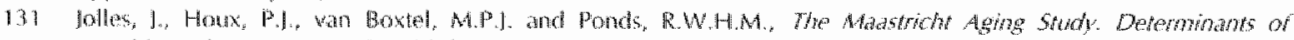

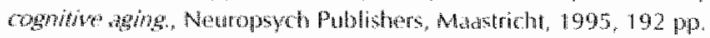

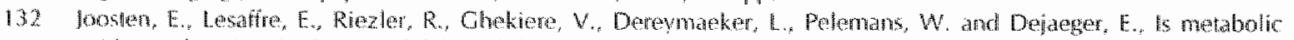

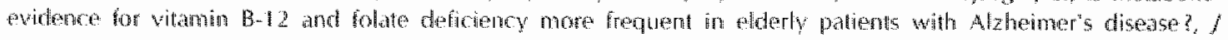




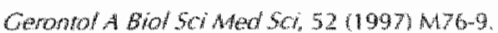

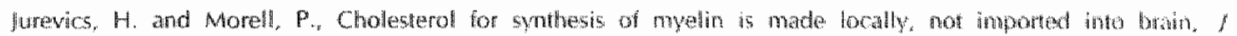
Newrochem, 64 (1995) 895.901 .

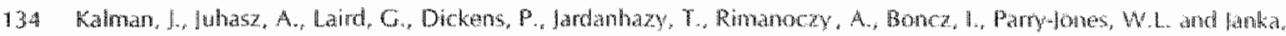

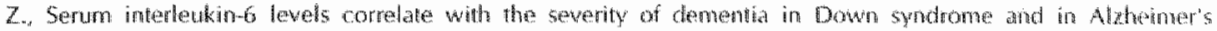
disease, Acha Newol Scand $9611997236-40$

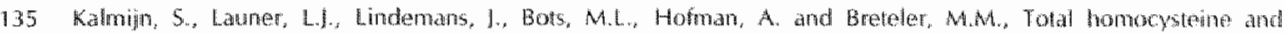

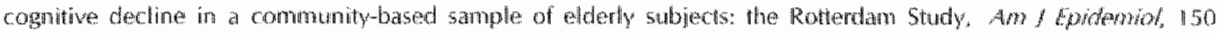
(1959) $283-9$

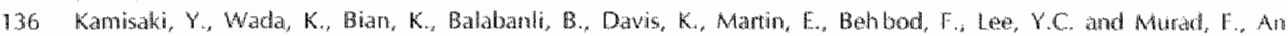

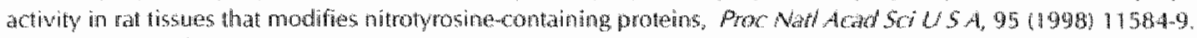

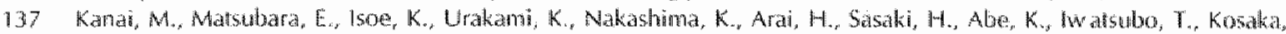

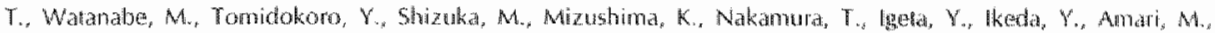

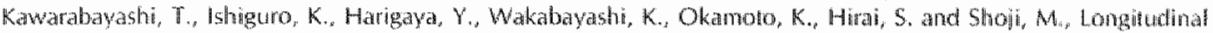

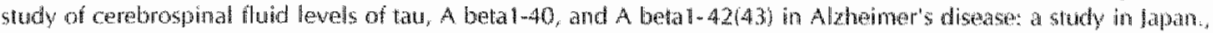
Anm Neurol, $4411998 ; 17-26$.

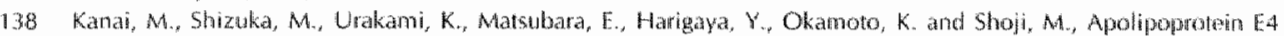

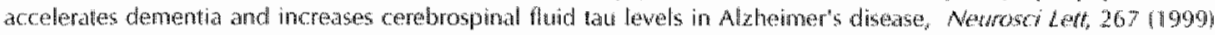
658.

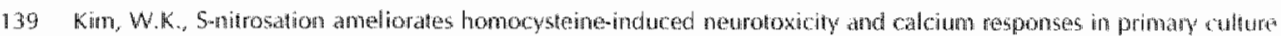
of rat contical neurons, Newosen Lent, $265(1929) 99 \% 102$

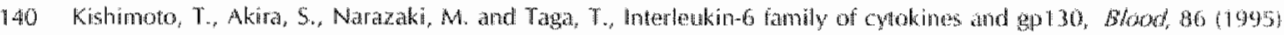
$1243-54$

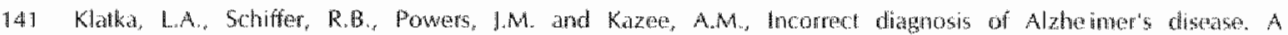
Clinicopathologic study, Ard Newat, $53:$ (1996) 35-42.

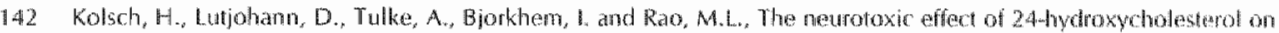
SH-SYSY human neurobtastoma cells, Braif Res, 018 (1999) $17 \pi-5$.

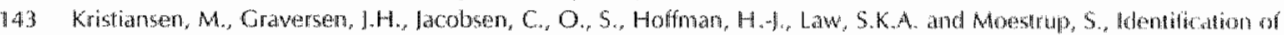
the haemoglobin scavenger receptor, Matwe, 409 (2001) 198-201.

144 Kruman, II. Culmser, C., Chan, S,L, Kruman, Y., Guo, Z., Penix, L. and Matison, M.P., Homorwsteme elicits a DNA

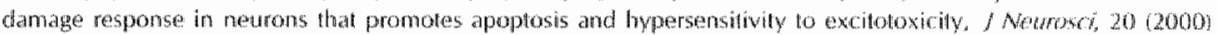
6920-6.

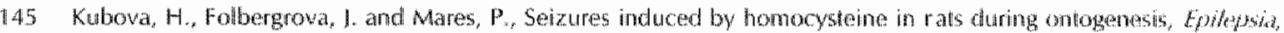
$36(1995)>50-6$.

146 Kko, W.N. Kanadia, R.N, shanbhag, V.P. and Toro, R., Denitration of peroxynititetreated prote ins by 'prokein nitratases." from rat brain and heart, sol Cel Bioctem, 201 (1999) 11-6.

147 Kub Y.M. Ementing, M.R., Lampert, H.C, Hempelman, S.R., Kokjohn, T.A., Woods, A.S., Colter, R.J. and Roher, A.E. High tevells of circulating abetid2 are sequestered by plasma proteins in Alzheimer's disenso, Biochom

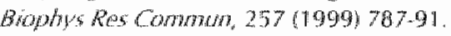

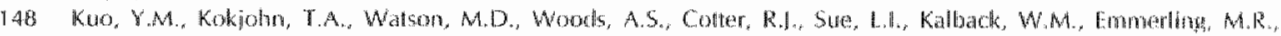

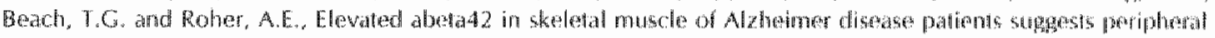

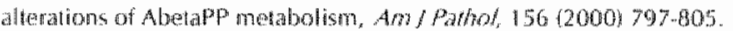

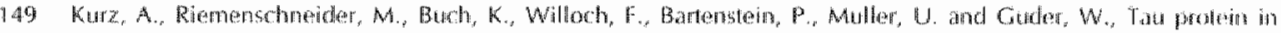

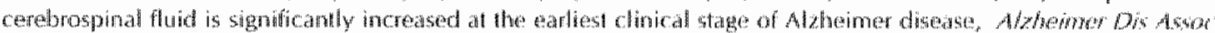
Disord, 12119903727.7

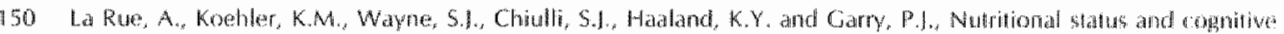

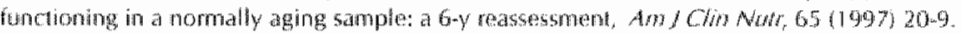

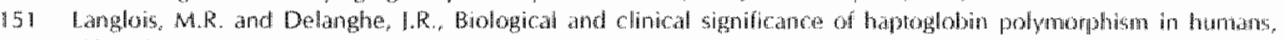
Ch Chom, 4211996$) 1589-600$.

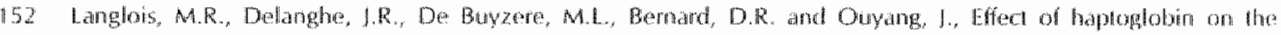

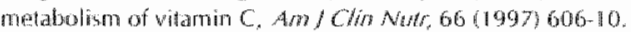

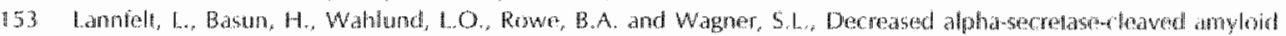

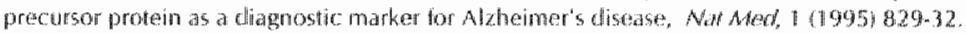

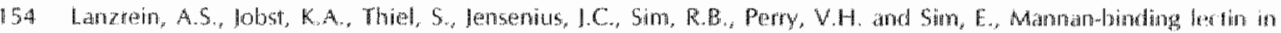

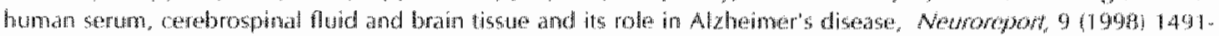
5.

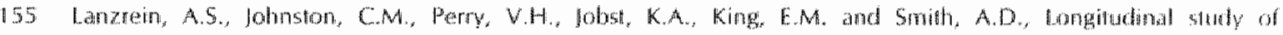

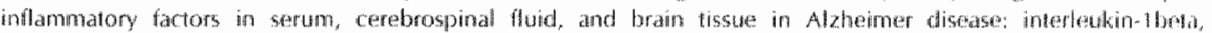

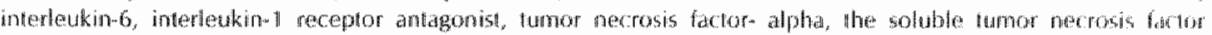

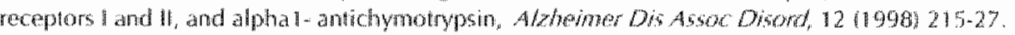

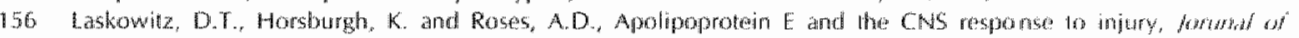
cerebral blood tow and metabolsim, 1809999$) 465-4 \%$. 
157 Lasser, R.A., Dukef, R, Lewy, L, Lewin, R, Lethimaki, T., Geuben, P, and Sunderlard, T., Apolipoprotein E epsilon 4

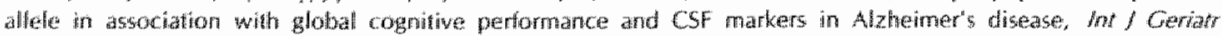
Psphollow, $13(1998) 767-74$

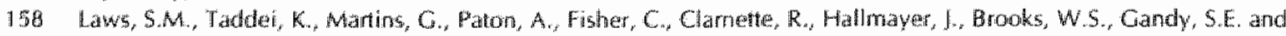
Martins, R.N. The 491 AA polymorphism in the APOE gene is associathed with increased plasma apoE levels in Alzhemer's disesse, Neuromoport, 1010999187982

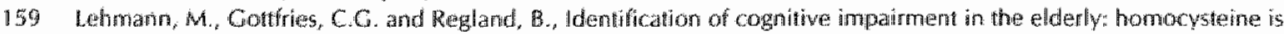

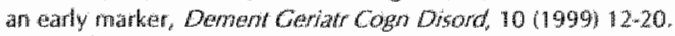

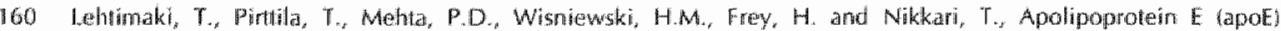
polymonphism and its influence on ApoE concentrations in the cerebrospunal fluid in Finnish patients with Alzheimer's disease, Hom Gentit, 95 (1995) 39.42

161 Lendon, C.L., Ashal!, F. and Goate, A.M., Exploring the ethology of Alzheimer disease using molecular gendetics, fame, $277(1997) 825-31$.

162 Lesser, G., Kandiah, K., Libow, L.S., Likourazos, A., Breuer, B., Mallin, D. Mohs, R., Haroutunian, V. and Neufeld, R. Elevated senum total and LDL cholesterol in very old patients with Alzheimer's disease. Dement Geriatr Cogn Disort, $12(2001) 138-45$.

16. Licasiro, F., Masliah, E, Pedrini, 5. and Thal, L., Blood levels of alpha-l-antichymotrypin and risk tactors for Alzhemer"s disease: effects of gender and apolipoprotenin $\mathbb{E}$ genowpe, Dement Cierratr Cogn Disord, 11 (2000) 25 off.

164i Licastro, F., Mormi, M.C. Polazzi, E, and Davis, L.., Increased serum alpha lwantichymotrypsin in patients with probable Alzheimer's disease: an acute phase reactant without the peripheral acute phase response, / Neuroismminol 57 (1995) $71-5$.

165 Licastro, F, Pedrini, 5, Capulo, L., Ammoni, O., Davis, L., Ferri, C., Casadei, V. and Grimaldi, LM, Increased plasma levels of interleukin-1, interlatkin-6s and alpha-1m antichynotrypsin in patients with Alzheimer's disease: peripheral inflammation or signalls from the bran?, J Neurommunot, $103000097-102$.

166 Lindeman, R.D., Ronero, L.J, Koebler, K.M., Liang, H.C., LaRue, A., Baumgartner, R. N. and Garry, P.J, Serum whamin B12, C and tolate concentrations in the New Mexico elder health survey: correthat bons with cogritive and affective functions, / Am Coll wer, 19 (2000) 68-76.

167 Lindh, M., Blomberg. M., Iersen, M., Basun, H., Lamielt, L., Engwall, B., Schamage, H., Marz, W., Wathlund, L.O. and Cowburn, R.F. Cerebrospinal fuid apolipoprotein $\mathbb{E}$ lapole) levels in Alzhemer's disease patients are increased at follow up and show a correlation whth levels of tau protein, Neurosci Lett, $22911997 \mathrm{y} 85 \%$.

16 Lobo, A., Launer, L..., Fratiglioni, L., Andersen, K., Di Cario, A., Breteler, M.M. Copeland, J.R., Dartigues, IF, lagger, C., Martinez-Lage, J., Sotninen, H, and Hofman, A. Prewalence of dementia and major subtypes in Europe: A collaborative study of population-based cohorts. Neurologic Diseases in the Eldery Reseanch Group, Neurofogy, 54 (2000) $\$ 49$

169 Lovel, M.A., Gabbita, S.P. and Markesbery, W.R., Increased DNA oxidation and decreased levels of repair products in Azheimer's disease ventrictal ar CSF, / Neurochem, 72 (1999) $7711-6$.

170 Lovell, MA and Markesbery. W.R., Ratio of 8 Hydroxyguanine in Intact DNA to Free BuHydroxyguanine Is Increased in Alzheimer Disedse Ventricular Cerebrospinal Fluid, Arch Neyro/ $58001201392-396$.

171 Lund, E.,., Guileyardo, J.M. and Russell, D.W., CDNA cloning of cholesteral 24tyydroxylase, a mediator of cholegterol homeostasis in the brain, Proc Nat, Acad sci U S A, 96 (1999) 7238-7243.

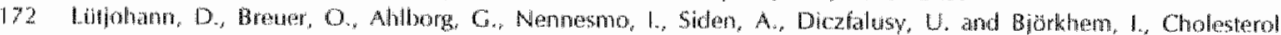

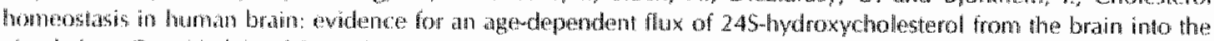

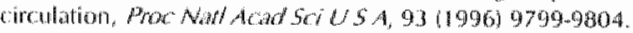

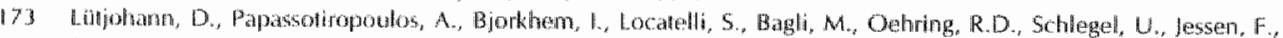

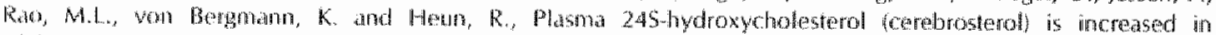

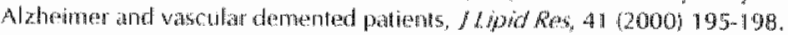

174 Mans, M. A rewirw on the acule phase responsa in major tepression, Rev Neurosi, 4 (1993) 407-16.

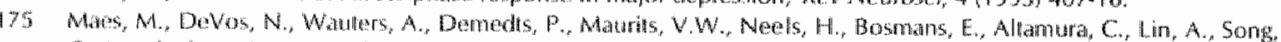
C., Vathdenbroutke, M. and Scharpa. S. Inflammatory markers in younger vs elderly nomal volunteens and in

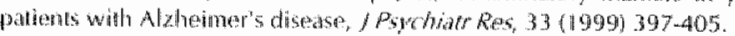

176 Mahley, R.W. and Wuang, Y., Apolizpoprotein E: from atherosclerosis to Alzheimer's disease and beyond, Curr Opin Lipkiklol, $10(1999) 207-1)$

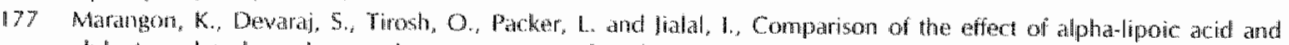
alpha-tocopherol stiplementation on measures of oxidative stress, Free Radic Biol Med, 27 (1999) 11 14-21.

178. Makesbery, WR, The role of oxidative styess hin Alzeimer disease, Arch Newrol 56 (1999) 1449.52.

179 Martinez, $M$, Fernandez-Vivancos, E. Frank, A., De la Fuente, M. and Hernanz, A., Irecreased cerebrospinal fluid fas (Apo-1) levels in Alzheimer"s disease. Relitionshap with IL-6 concentrations, Bran Res, 869 (2000) $216-9$.

100 Martinez, M. Frank, A and Herranz, A, Relationship of interleukinm beta and beta z-microglobulin with meuropeptides in cerebrospinal fuscl of patients with dementia of the Alzheimer lype, INeturommunof, 48 (1993) 235.40

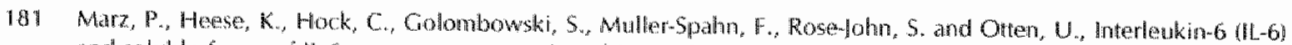
and soluble torms of tLa receptors ane not altered in cercbrospinal thid of Alahemer's disease patients, Neuroso 


\section{Lext, 29911997$) 29-32$}

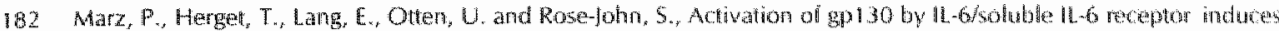

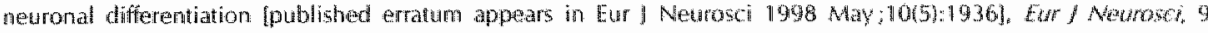
$109972765 \mathrm{~m} 73$.

183 Mason, R.P., Shoemaker. W.l., Shajenko, L, Chambers, T.E and Hebente, L. G. Fvidence for changes in the Alzheimer"s disease brain contcal membrane structure mediated by cholesterol, Newnbrol Agring: $13(1992) 413$. 419.

184 Matsubara, E., Amari, M. Shoï, M., Harigaya, Y., Yamaguchi, H., Okanuolo, Kand Hirai, S, Serum concentration

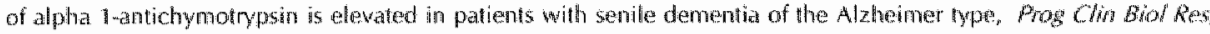
$317119891707-14$.

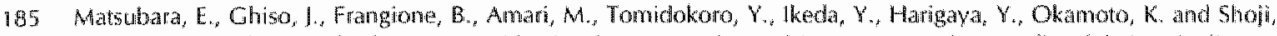
A. Lipoprotein-free amyloidogenic peptides in plasma are elewated in patients with sporadic Alzhemeras disedise and Down's syndrome, Anm Neurd, 45 (1999) $537-41$.

186 Matsubara, E, Hirei, S., Amari, M., Shoii, M., Yamaguchi, H., OKamoto, K., Ishiguro, K., Hanigaya, Y. and

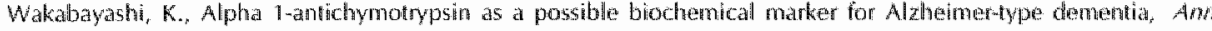
Newrol, 2801990$) 561-7$.

187 Matsurama, S.S., Cripe, A.T. and joseph, f., Haptoglobin phenotypes in dementia of the Alaheimer type, Horn Hered 36 (19986) $93-6$.

188 Mathews, R.T. and Beal, M.F, Increased 3-nitrotyrosine in brains of Apo E-deficient mice, Bran Res, 718 (1996) 1011 . औ.

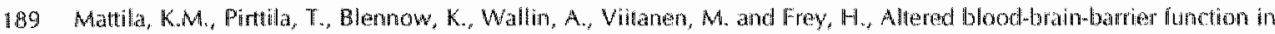
Alzheimer's disease?. Acta Neurol Scand, 399 (1994) 192-8.

190 Mattson, M.P., Barger, 5.W. Furukawa, K, Bruce, A.l. Wyss-Conay, T. Mark, Ru, and Mucke, L. Cellular signaling

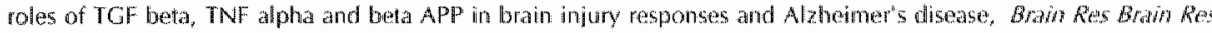
Rew, $23(1997) 47-61$.

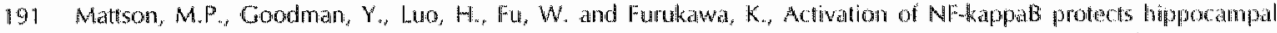
neurons against oxidative stress-induced apoptosis evidence for induction of manganese superoxide dismutase and suppression of peroxynitrife production and protein tyrosine nithation, $/$ Newroso Res, 49 (1997) 601.97.

192 Mayeux, R., Tang, M.X., Jacobs, D.M., Manly, I., Bell, K. Merchant, C., Smatl, S.A., Stern, Y., Whisniewsiki, H.M. and Mehta, P.D., Plasma amyloid beta-peptide 1-42 and incipient Alzheimer's disease, 4ho Nound, 46019991412 6.

193 McGaddon, A., Davies, G., Hudson, P., Tandy, 5. and Cattell, H., Total serum homoryseine in semile dementia of Alzheinen type, tht / Geriatr Pswchatry, 13 (1998) $235-9$.

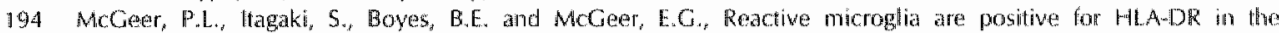
substamtia nigra of Parkinson's and Alzheimers disease brans, Nenology, 38 (1988) $1285-91$.

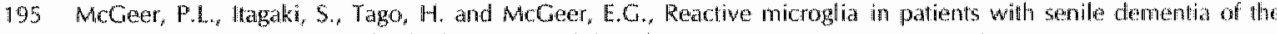
Alzheimer type are positive for the histocompatibilly glycoproten HLA-OK, Neturosci Lett, 79 (1987) 195-200.

196 McGeer, P.L., Schulzet, M. and McGeer, E.G. Arthitis and anti-inflammatory agents as possible protectwe factors

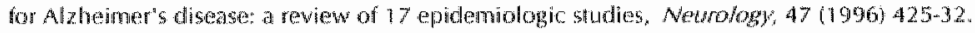

197 Mckhann, G., Drachman, D. Folstein, M., Katzman, R., Prica, D. and Stadlan, IE.M., Climical diagnosis of

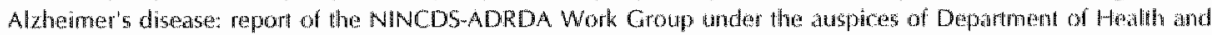
Human Serwices Task Force on Alzheiner's Disease, Newrology, 34 1984) 939-44.

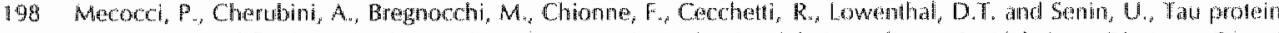

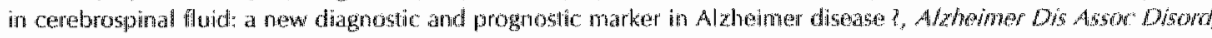
\#2 (1998) 21)-4.

199 Mecoci, P., Ekman, R., Parnetii, L. and Senim, U., Antihistone and anti-d sDNA, autoantibodies in Alaheimeat"s disease and vascular dementia, Bial Psychiday, 34 (1993) 360.5.

200. Mecoci, P., Parnesti, L., Donato, R., Santucci, C., Santucci, A, Cadlini, D., Foa, E, Cecheoti, Re and Senin, U.,

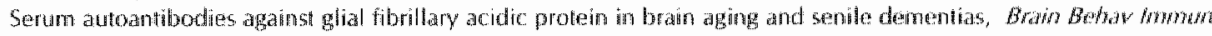
$6(10992) 286-92$

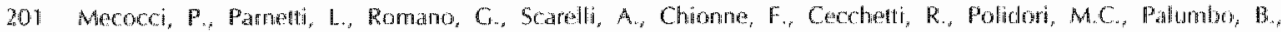

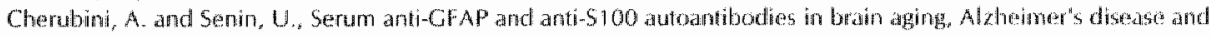
vascular denuentia, / Neuroimmunol 57 (1995) $165-70$.

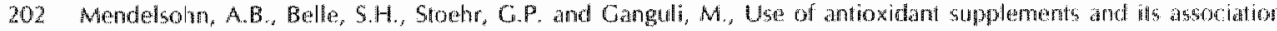

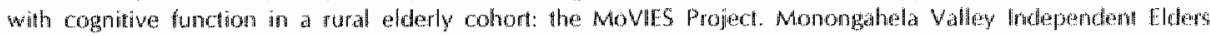
Survey, Am/ Epidemiod, 1486 (1998) 3044.

209 Merched, A, Xia, Y., Visvikis, S., Serot, IM. and Siest, G. Decreased high-density lipoproteais cholesterol and serum apolipoprotein Al concentrations are highly corretated with the sewerity af Aldeiners disedse. Nawotsol Aging, 21 (2000) 27-30.

20.4 Montine, K.S., Bassett, C.N., Ou, I.I., Markesbery, W.R., Swift, L.L. and Montine, T.J., Apolipoprotein E alletia inftuence on human cerebrospinal fluid apolipoproteins, J Lipjof Res, 39 (1999) 244751 .

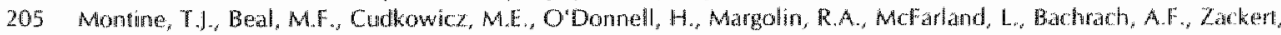




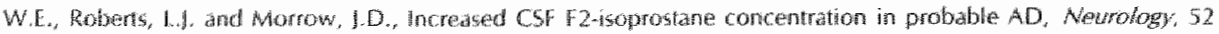
(1995) $562-3$

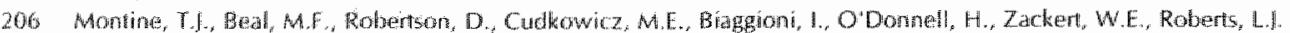

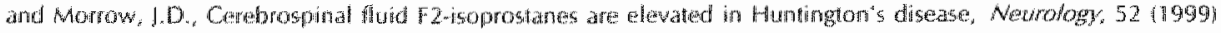
$1104-5$.

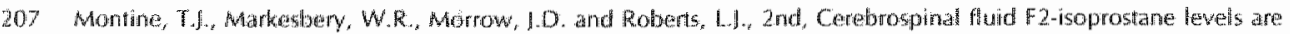

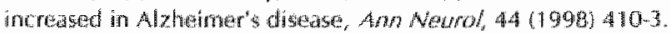

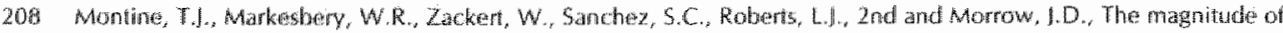

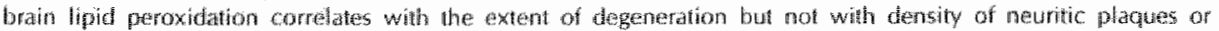

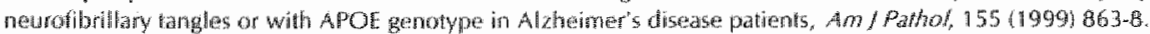

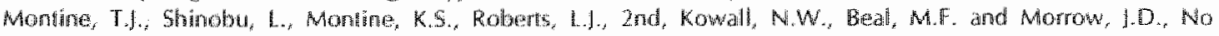

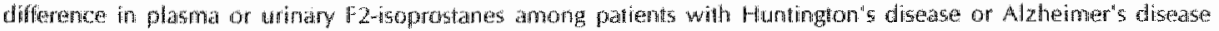

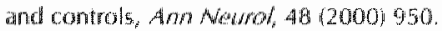

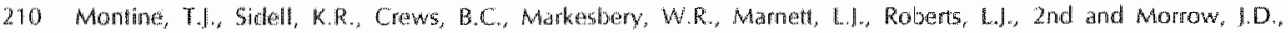
Elewated CSF prostaghandin E2 levels in patarents with probathe AD, Neurotog\%, 53 (1999) 1495-8.

211 Morikawa, Y, Arai, H. Matsushita, S., Kato, M., Higuchi, S, Miura, M. Kawakami, H., Higuchi, M., Okamura, M.

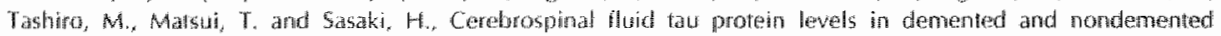

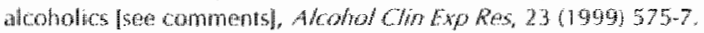

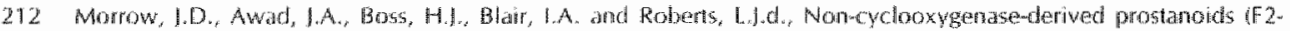

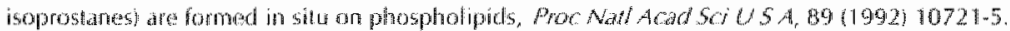

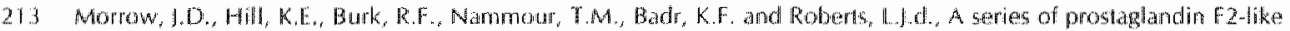

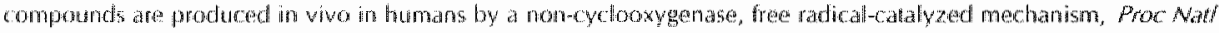

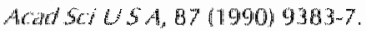

214 Morraw, I.D. Minton, T.A., Mukundan, C.R., Campleall, M.D., Zacken, W.E., Darniel, V.C., Badr, K.F., Blair, I.A.

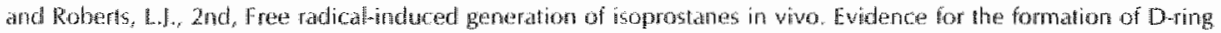
and E-ring isoprostianes, / Bio/ Chem, 269) (1994) 4317-26.

215 Morrow, 1.D. and Roberts, L., 2nd, The isoprostanes, Current knowledge and directions for future research, Brochen phandacot 51 (1996) 1-9.

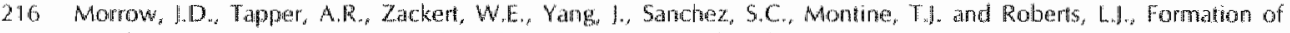
novel isoprostane-like conpounds from docosahexaenoic acid, Adv Emp Mtod Brol, 469 (1999) 3437.

217 Motter, R., Vigo-Pelfey, C., Kholodenka, D. Barbour, R., Johnson-Wood, K., Gatasko, D., Chang. L., Miller, B.,

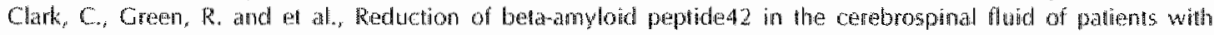
Alzheimer's disease, Ann Newdol, 38, (1995) 643-8.

218 Mulder, C, Schellens, P., Visser, J.J, wan Kamp, G.ll, and Schutgens, R.B., Genetic and biochemical markers ton Alaheimer's disease: recent developmens, Am Clin Biochem, 37 (2000 $593-60 \%$.

219 Mulder, M., Blokland, A., van clen Berg, D. ., Schutten, H., Bakker, A.H., Terwel, D., Honig, W., de Kloet, E.R. Havekes, L.M. steinbusch, H.W. and de Lange, E.C., Apolipoprotein E protects against meuropathology induced by

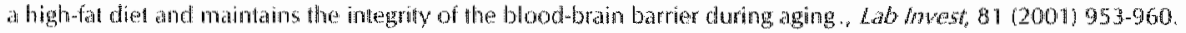

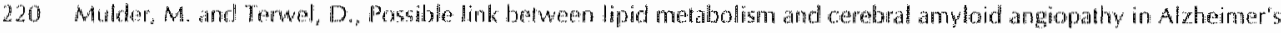

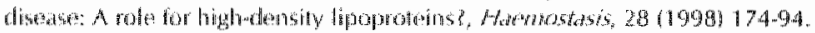

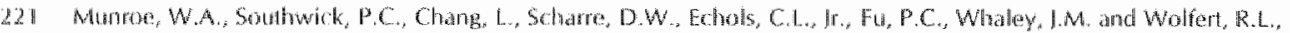

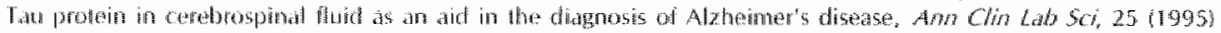
$20 \% 17$.

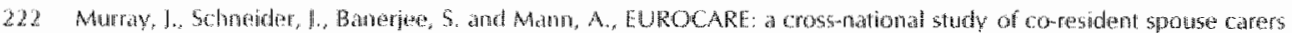

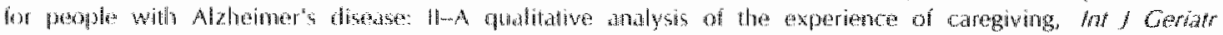
Pstichion, 140199906027.

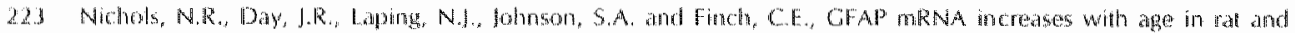

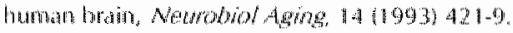

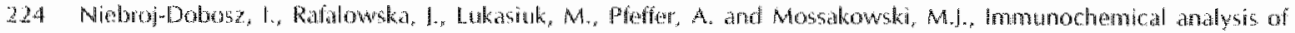

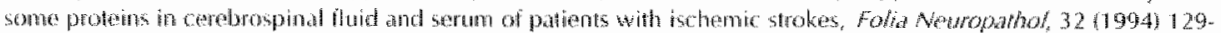
37.

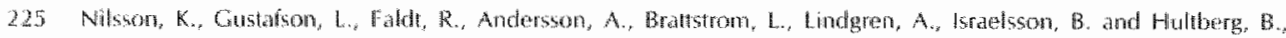

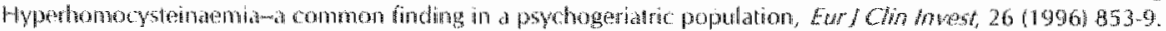

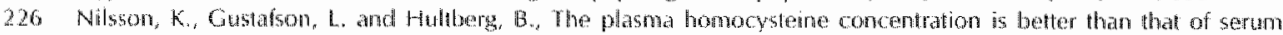
methymalonic acid as a matker for sochopsychological perfomance in a psychogerianic population, Chin Chen, 46 $20008991-6$

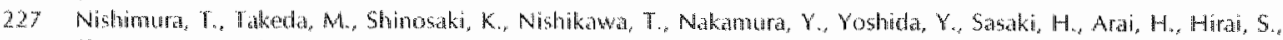

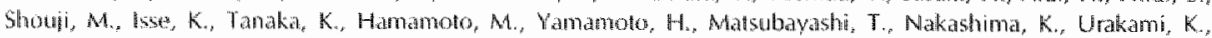
Adath, Y, Nakamura, S., Toj. H, and Yoshida, H., Basic and cinical studies on Apot gene typing by line probe

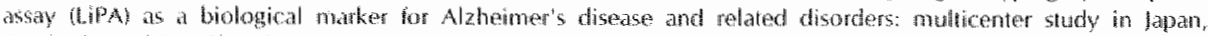

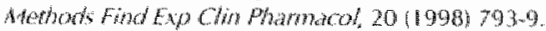

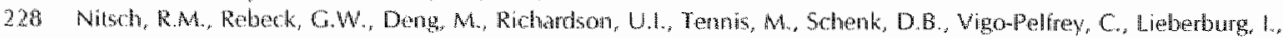




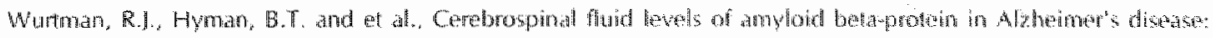

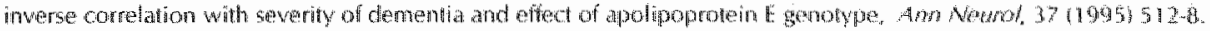

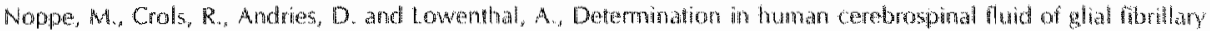

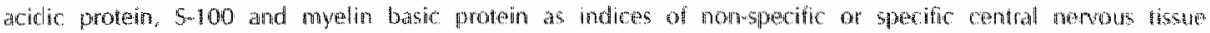
pathology, Chin Chro Atta. $15501986143-50$.

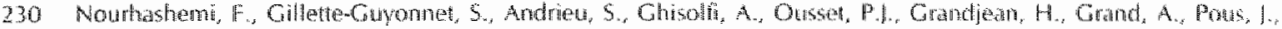

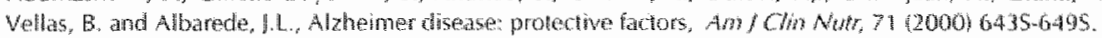

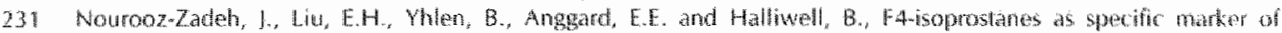

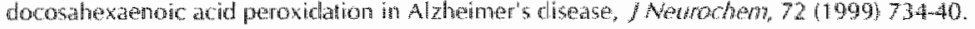

232 Oate, J.C. Chistensen, E.F. Reilly, C.M, Sel, 5.E. and Gilkescon, G.5. Prospewtive measure of serum 3.

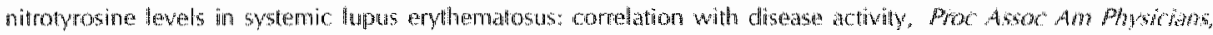
$111(1999) 611 \cdot 2 \%$

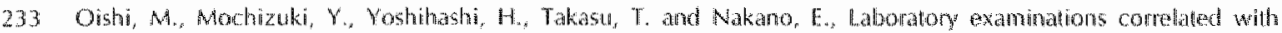
Severity of dementia, Am Clim Lab $5,26(1996) 340-5$.

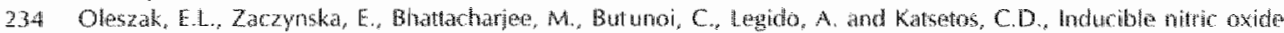
symthase and nizrolyrosine are found in monocytes/macrophages and/or astooves in acule, but inot in thronic. multiple scierosis, Cho Diagr Lab Immund, 5 (1998) 43845.

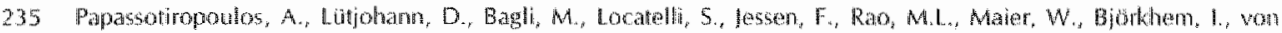

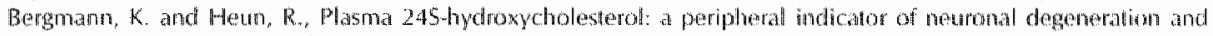

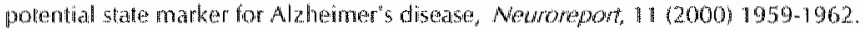

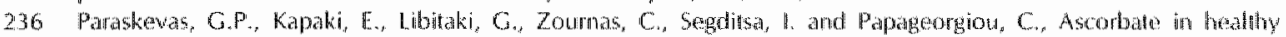

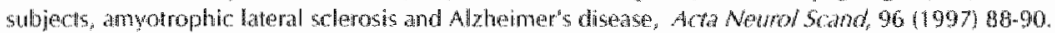

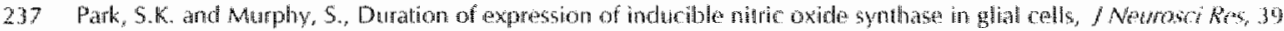
(1994) 405-1\%.

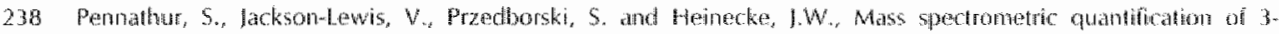

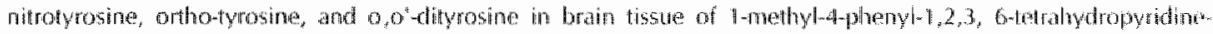
treated mice, a model of oxidative stress in Parkinson's disease, IBho/ Chem $27411999 \%, 3621-8$.

239 Perkins, A.J. Hendrie, H.C., Callahan, C.M., Gao, 5., Unverzagt, F.W., Xu, Y., Hall, K.S. and Hui, S,L., Association of antioxidants with memory in a multiethnic elderly sample using the Third Nallonal Health and Nutrition Examination Survey, Am/ Epideniol, 15041999 , 37-44.

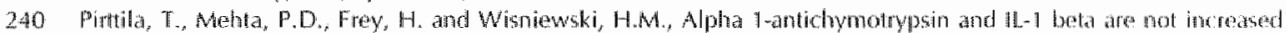
in CSF or serm in Alzheimer's disedse, Neurobrot Aging. 15 (1994) 31137.

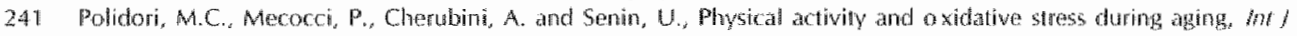
Sports Mat; 21 (2000) 1547 .

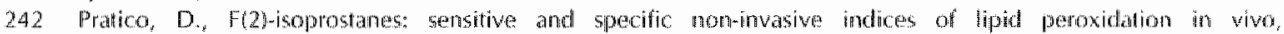
Atheroscterosis, 14711999$) 1-10$.

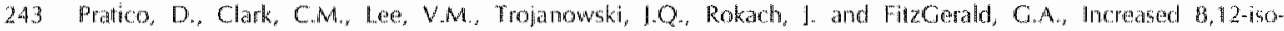

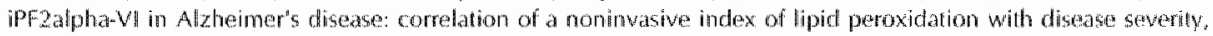
Ann Neurol, 48 i2000) $809-12$.

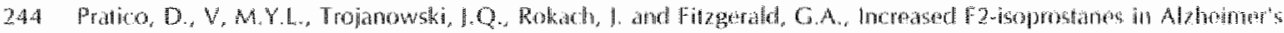

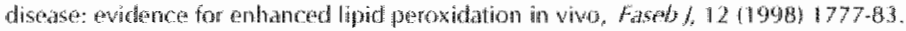

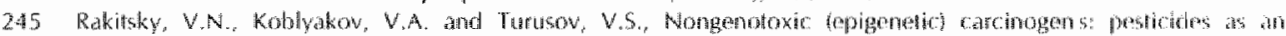

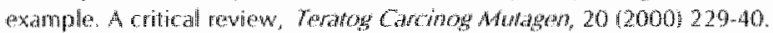

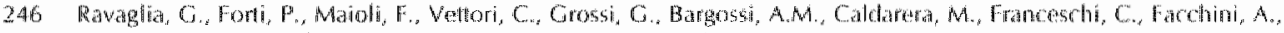

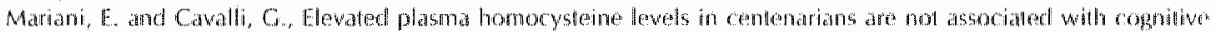

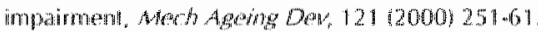

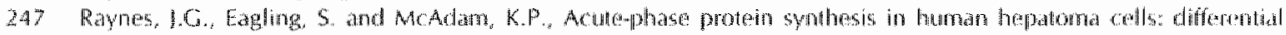

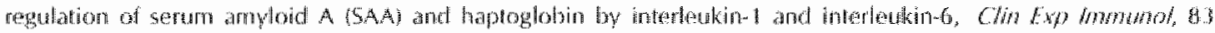
$(1991) 488-9 \%$.

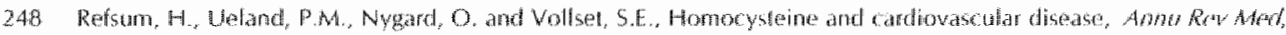
$49(1999) 31-62$.

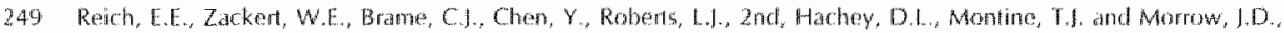

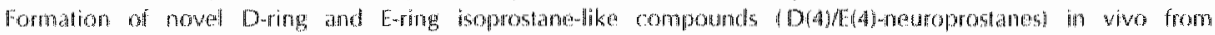

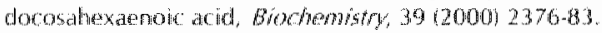

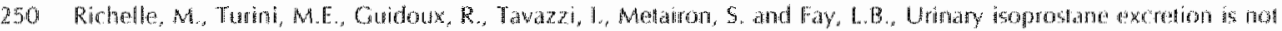
contounded by the lipid conten of the diet, FEBS Let $45901999,259,62$.

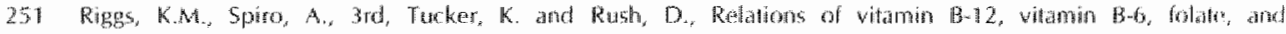

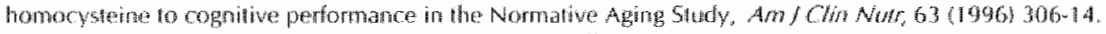

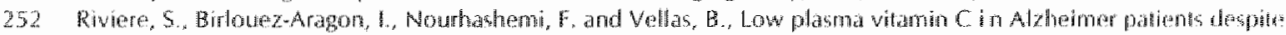

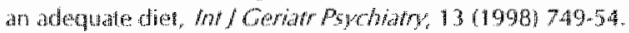

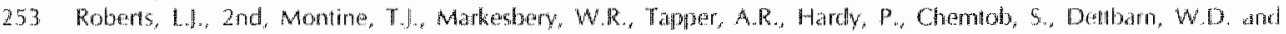

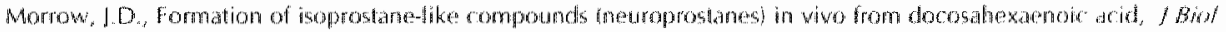


Chern, $2730199941605-32$.

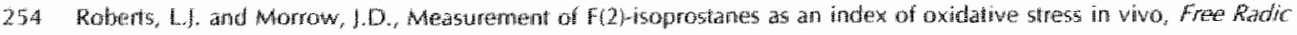
Brol Ated 28 (2000) 505513 .

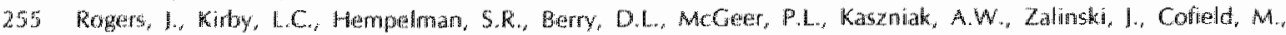
Mansukhari, I. Wilson, P. and at. Clinical trial of indomethacin in Alzheimer's disease, Newrology, 43 11993) $1609-11$.

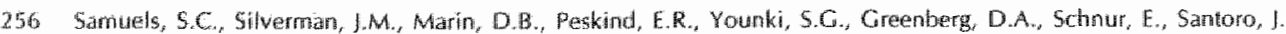
and Davis, K.L. CSf betamanyloid; cognition, and APOE genotype in Ahtheimut's disease, Nezrology, 52 (1999) $547-51$.

257 Sano, M. Enesio, C., Thomas, Ru, Klaber, M.R., Schater, K., Grundman, M., Woodbury, P., Crowdon, 1. Cotman, C.W., Preiffer, E., Schneider, L.S. and Thal. L.H. A controlled trial of selegitine, alpha-tocopherol, or both as

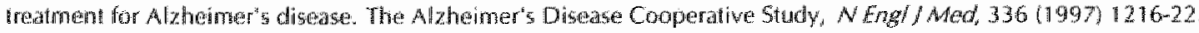

258 Saturier, S.E., Kandutsch, A.A., Caven, A.K. Simahn, D.K. and Spencer, T.A., Oxysterol qegulatlors of 3-hydroxy-3-

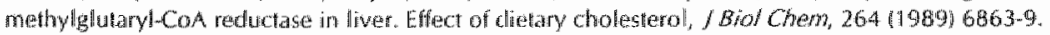

259 Savage, D.C., Lindentatum, 1. Stabter, S.P. and Allen, R.H., Sersitivity of serum methylmalonic acid and total homocysteine deletminations for diagnosing cobalamim and folate deficiencies, Am / Ated, 96 (1994) 23946.

260 Scachi, R., Gambina, G, Ruggeri, M, Martin, H.C., Fretrari, G., Sillvestri, M. Schiawon, R. and Corbo, R.M., Plasma lewels of apotipoprotein E and genctic markers in etderly patients with Alchemer's disease, Neurosci Lett, 259 (11999) 33-6.

261 Scheltens, P. and Hijdra, A.M., Diagnostic criteria for vascular dementia, Hoenow\%sis, 28 (1998) $151-7$.

262 Schetuner, D., Eckman, C., Jensen, M., Song, X., Citron, M., Suzuki, N., Bird, T, D., Hard\%, J., Huttori, M., Kakul,

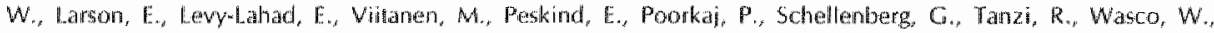
Laniolt, L., Sekoe, D. and Younkin, S., Secretad amyloid betaprotein similar to that in the senite plaques of Alzheimer"s disease is increased in wo by the presenilin 1 and 2 and APp mutations linked to familal Alzheamer's discase [see comments]. Named, 2 (1996) 86470.

263 Schiele F., De Bacquer, D., Vincentwiry, M., Beisiegel, U., Ehnholm, C., Ewans, A., Kahatos, A., Martins, M.C.,

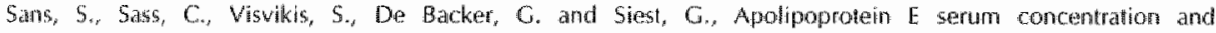
polymorphism in six european countries; the Apo Europe project, Atheroscterosis, 1522000$) 475-88$

264 Schmidt, R., Hayn, M., Rronhart, B., Roob, G., Schmidt, H., Schumacher, M., Watzinger, N. and Launer, I.A., Plama antoxidants and cognitive performance in middle-aged and older adults: results of the Austrian Stroke Prevention Study, /Am Geriate Sor, $46(1998) 1407-10$.

265 Schneider, 1. Murray, 1., Banerjee, S. and Mann, A., EuROCARr a crossmational study of co-resident spouse caners

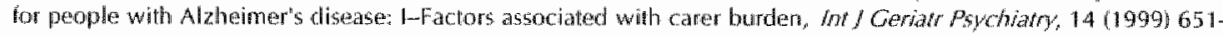
61.

266 Sehub, J., Baglew, L.C. Miller, I and Rosenbeng, I.H., B vitumins, homorysteine, and neurocognitive function in the elderly, Am / C/m Notr, 71 (2000) 6145-6205.

267 Shoji, M., Malsubara, E., Kanai, M. Walanabe, M, Nakambra, T., Tomidokoro, Y., Shizuka, M., Wakabayashi, K.,

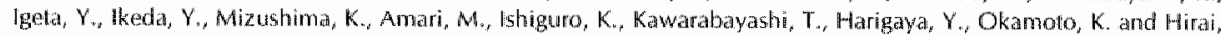

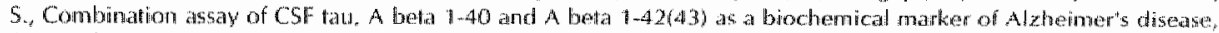
/ Newol $5 \mathrm{C}, 158(1998) 134-40$.

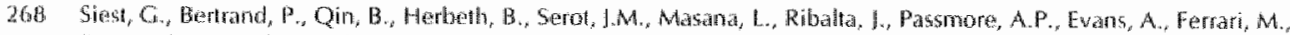
Franceschi, M., Shepherd, 1., Cuchel, M., Beisiegel, U, Zuchowsky, K., Rukavina, A.S., Sertic, 1., Stojanow, M.

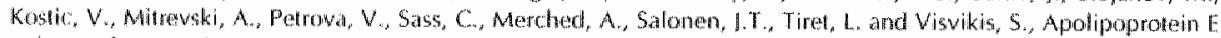
polymorphish and serm concentration in Alzheimer's disease in rine European centres: the Apoturope study. Apoteurope group, Chin Chern Lab Med, 38 (2000) 721-30.

269 Simons, M. Kaller, P., De Strooper, B., Beyreuther, K., Dotii, C.C. and Simons, K., Cholesterol deptetion inhibits

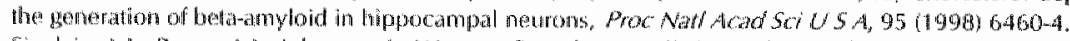

270 Sinclair, A.l., Bayer, A.J., Johnston, I. Warner, C. and Maxwell, S.R., Altered plasma antoxidant status in subiects

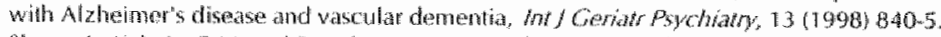

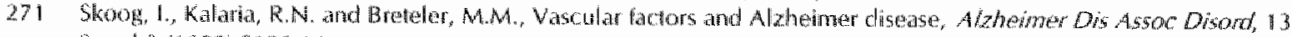
Suppl $3(1999) 5106 \times 14$.

272 Shane. I.A. Hollandes, W. Mhos, M.B., Rosene, D.L, and Abraham, CR., lncreased microghal activation and protein nitration in white matter of the aging monkey, Nowobiol Aging; 20 (1999) 395-405.

27.3 Shooter, A.l, dru Knipt, P.. Howan, A., Cruts, M. Breteler, M.M., Van Broedhowen, C., Havekes, L.M. and wan

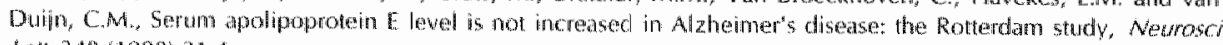
Ledf $248(1998) 21-4$.

274 Slocter, A.J., van Duin, C.M., Bots, M.L., Ot, A., Breteler, M.B., De Voecht, I. Wemert, A., de Knijit, P., Havekes, L.M., Crobbee, D.E., Van Broedhowen, C. and Hofman, A., Apolipoprotein E genolype, atherosclerosis, and cognilive decline: the Rotterdam Study. / Neurad Transm Supp/, 33 (1998, 17-29.

275 Smith, C.D. Camey, I.M. Starke-Reed, PE. Oliver, C.N. Stedtman, E.R., Floyd, R.A. and Markesbery, M.R., Excess brain protein oxidalion and enzyme dystunction in nomal aging and in Alzheimer disease, Proc Nath Acad Sor U S A. 8641991$) 10540-3$. 


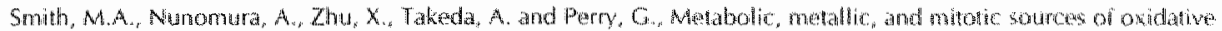
stress in Alzheimer disedse, Antoxid Redox Sigral, 212000141320.

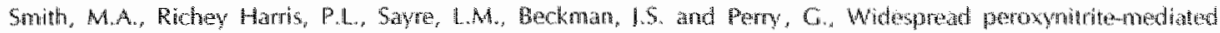
darmage in Alahemer's disease, N Newrasc, 17 (199\%) 26537.

Soler Federspoiel, B.S, Karcher, D. and Lowenthat, A. Blood and cerebrospinal thud anomaties in brain ageing and Azheimen's disease Gerontology, $33(198 \%) 193-6$.

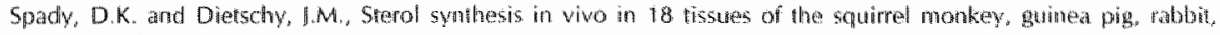
hamster, and rat, /Lipid Res, 24 (1983) 303-315.

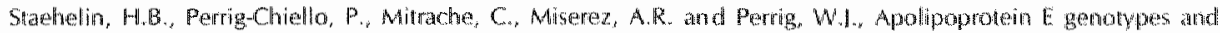

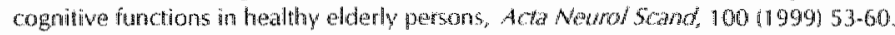

Strittmatter, W.]. Salunders, A.M., Schmechel, D., Pericak-Kance, M., Enghild, 1. Salwesen, G. S. and Roses, A.D., Apolipoprotein $\mathrm{E}$ : high-avidty binding to beta-armwoid and ine reased frequency of type 4 allele in lateonself familal Alzhemer disease, Proc Nall Acad Soi US,4,901993) 1977-81.

Su, 1.H. Deng, G. and Coman, C.W., Neuronat DNA datnage precedes fangle formation and is assoratide with upregutation of nitrotyrosime in Alzhemer's disease brain, Bran Res, 774 (1997) 193-9.

Sturderland, T., Wolozin, B., Galasko, D. Lewy, J., Dukoff, R., Bahro, M., Lasser, R., Motter, R., Lehtimatki. T, and

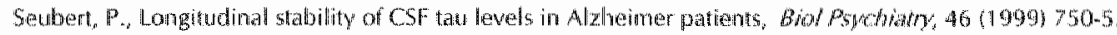

Suzukt. I., Inouse, Y, and Suzuki, S., Changes in the urinary excretion level of 8-hydroxyguanine by exposurea tio

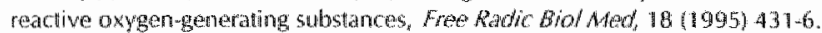

Tadcei, K., Clarnette, R., Gandy, S.E. and Martins, R.N., lincreased plasma apolpoprotein E tapoE, lexwels in Alzhener's discase, Neyrosci Lett, $223(1997) 29-32$

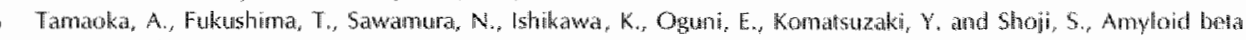
protein in plasma from patients with spondfic. Alzheimer's disease, / Neurof Sci, 141 (1996) 65-8.

Tanaka, J., Nakamura, K., Takeda, M., Tada, K., Suzuki, H., Morita, H., Okado, T. Hariguchi, S. and Mighimufa, 1., Enzyme-linked immunosorbent assay for humark autoantibady to glial fibrillary acidic profain: highes thiter of the

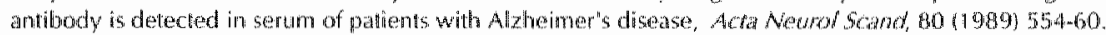

Tapiola, T., Overnyer, M., Lehtovirta, M., Helsalmi, S., Ramberg, I. Alafuzofi, I., Riekkinen, P., Sr, and Soininen,

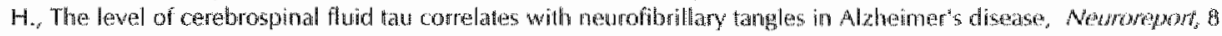
(1997) $3961-3$.

Tapiola, T., Pirtita, T., Mikkonen, M., Mehwa, P.D., Alafuzor, 1., Koiwisto, K. and Soininen, H., Three-year follow -up

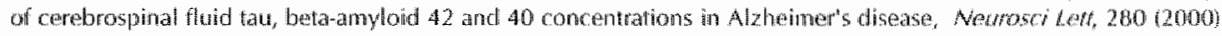
11922.

Tarkowski, E, Blennow, K., Wallin, A. and Tarkowski, A, Infracerebral production of tumor nectosis factor-ipha, a

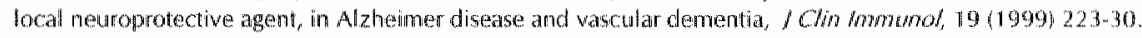

ter Steege, I.C. Kosterkamphus, L, van Straten, E.A. Forget, P.P. and Butuman, W.A. Nitrotyrosine in plasma of celiac disease patients as detected by a now sandwion ELISA, Froe Radic Biol Ated 25 (1998) $953-63$.

Tenrisse, L. Poirier, I. Bertrand, P., Merched, A, Visvikis, S., Siest, G., Milne, R. and Rassant, E. Increased levels of

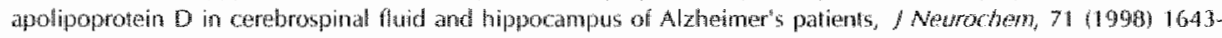
50 .

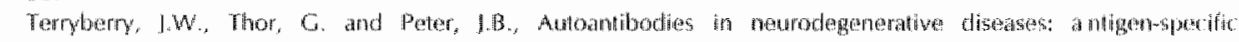

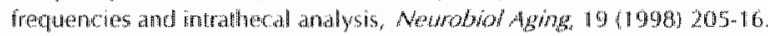

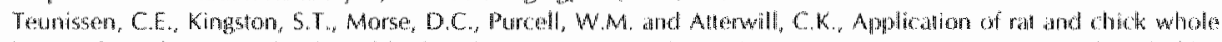

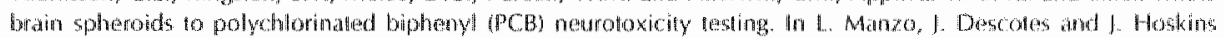

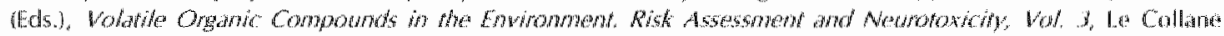
della Fondazione Salvatore Matugeri, Pl-AE Press, Pavia, 1998, pp. 265-272

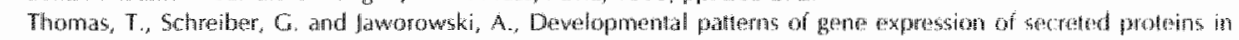

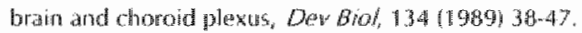

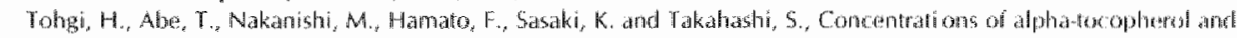

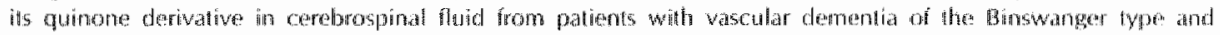

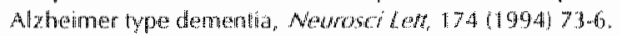

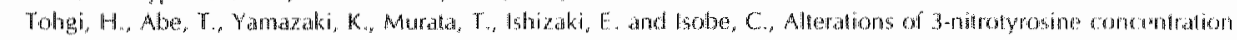

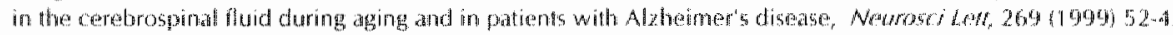

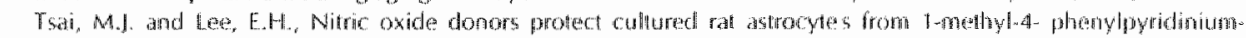
induced toxicity. Free Radic Brol Hed, 2461996$) 705-13$.

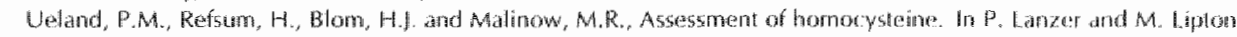

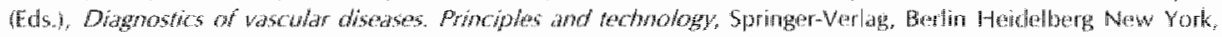
$1997, p p .218-225$.

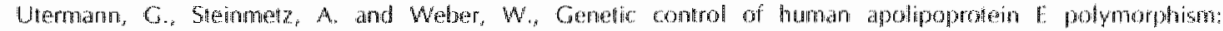

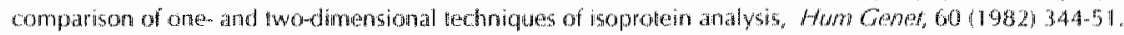

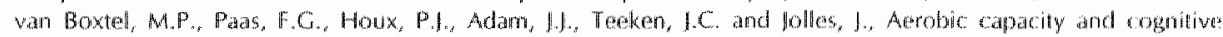
performance in a cross-sectional aging sludy. Mod Sc Sports Exer, $29(1997) 135765$.

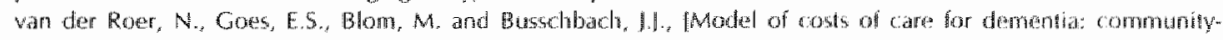




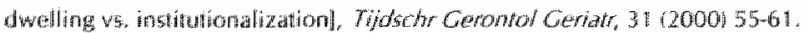

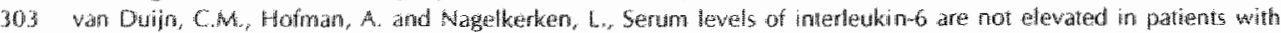
Alzheimer's diseast, Novosed tert, 108 (1990) 350 m.

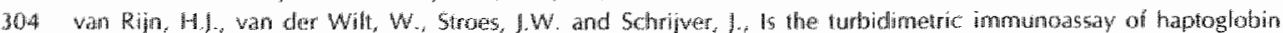

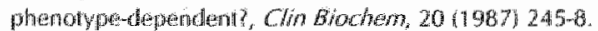

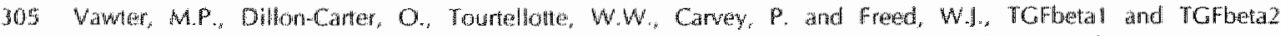

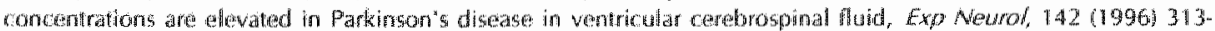
22 .

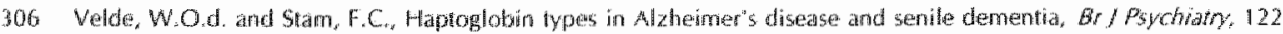
$(1979\} 331-6$.

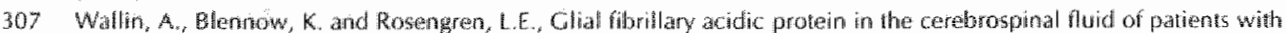

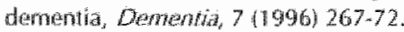

308 Wardman, P. and Candeas, L.P. Fenton chumistry: an introduction, Radiat Res, 145(1996) 523-31.

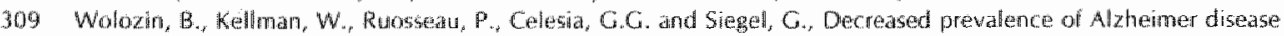

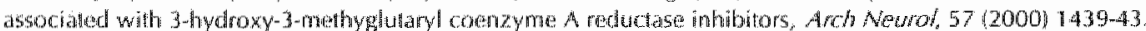

310 Working Group, Ma.B.Mo.A., D. Consensus Rejort of the Working Group on: "Molecular and Biochemical

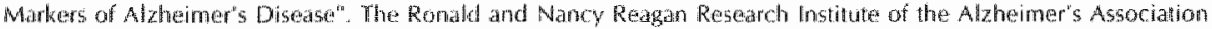

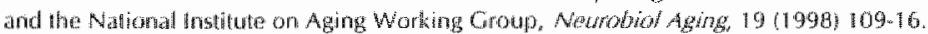

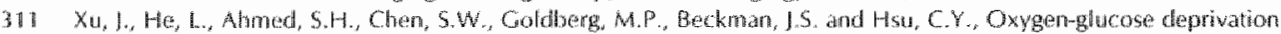

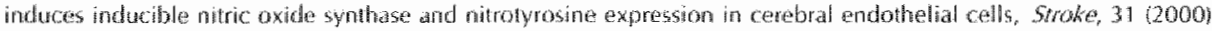
1744.51 .

$\$ 12$ Yamada, K., Kono, K. Unegaki, H. Iguchi, M., Fukatsu, T, Nakalshima, N., Nishimaki, H., Shimada, Y., Sugita, Y,

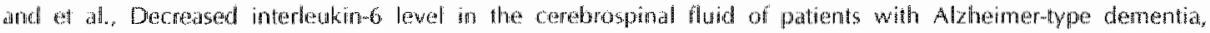

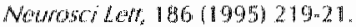

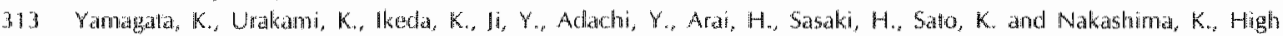
Expression of Apolipoprotwin E mRNA in the Brains with Sporadio Alzheimer's Disease, Dentrent Geriatr Cogs Disord, $12(2001) 5762$

31 H. Yamauchi, K., Tozuka, M., Nakabayashi, T., Sugano, M. Hidaka, H., Kondo, Y. and Katstiyama, T., Apolipoprotan E in cerebospinal fuid: relation to phenotype and plasma apolpoprotein E concentrations, Chn Chemp, 45 (1999) $407-504$.

315 Yang, L., Mathews, R.T., Schule, J.B., Klockgether, T., Liao, A, W., Matinou, J..., Penmey, J.B., Ir., Hyman, B.T. and

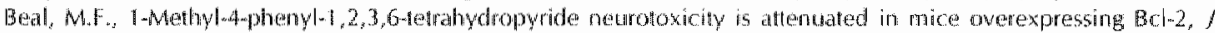
Newrosci, $18(1998) 8145-52$.

316 Zaman, Z. Ruche, S., Fiedden, P., Frost, P.G., Nirialla, D.C. and Cayley, A.C., Plasifa concentraxions of vitamins A and If and carotenoids in Alzhemerts diseaste, Age Ageing, 21 (1992) 91-4.

317 Zannis, V.1. Genetic polymorghism in human apolipoprotein E. Methods Enzymor, 28 (1986) 823-51

113 Zhang, F. Stungaad, A., Vercelouti, G.M. and ladecola, C., Superoxidedependent cerebrowascular effects of homocysteine, Am/ Physial, 274 \& 1998 ) R1704-11.

319 Zhao, B. and Schwartz, J.P., hwolvement of cytokines in nomal CNS development and neurological diseases:

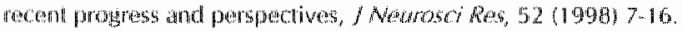




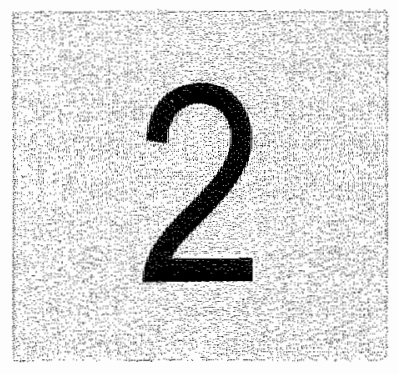

\section{Whole brain spheroid cultures as a model to study the development of nitric oxide synthase - guanylate cyclase signal transduction}

Charlotte Teunissen, Harry Steinbusch, Marjanne Markerink-van Ittersum, Chris De Bruijn, Hubertus Axer and Jan de Vente

European Graduate School of Neuroscience (Euron), Universiteit Maastricht, Department of Psychiatry and Neuropsychology, The Netherlands;

@ Department of Anatomy I, Rheinisch Westfählische Technische Hochschule (RWTH), Aachen, Germany

Developmental Brain Research, 125 (2000) 99-115. 


\section{Abstract}

Whole brain spheroids provide a suitable model to study neurodevelopment. In the literature a role for the nitric oxide (NO)-cyclic guanosine 3;, 5 - monophosphate (cGMP) signalling pathway during development has frequently been suggested. In this study we investigated whether functional CCMP pathways were present in differentiated spheroids. In three-week-old spheroids soluble guanylate cyclase was stimulated with $\mathrm{N}$-methyl D-aspartic acid or sodium nitroprusside (NO donor). The results showed that the NO synthase-CGMP pathway is present in the cullture system. Soluble guanylate cyclase-dependent CGMP formation was found in NO symthase containing neurons, in neurons of the GABAergic, glutamatergic and cholinergic system, and in astroglia and oligodendroglia. Activation of particulate guanylate cyclase by atrial natriuretic peptide also iriggered an increase in CGMP production. Particulate guanylate cyclase was found in astroglia and in microglia as well as in glutamic acid decarboxylase and calbindin containing structures and neuronal $\mathbb{N O}$ synthase containing meurons. Chronic inhibition of NO synthase during cullure development had no effect on soluble or particulate guanylate cyclase functioning. Similarly, inhibition of soluble guanylate cyclase during culture development did not have any effect on NO synthase and particulate guanylate cyclase functioning.

If is concluded that NO synthase and both soluble and particulate guanylate cyclase are present in whole brain spheroid cultures and that their activity can be influenced by several stimuli. The spheroid culture system constitutes a suitable model to study the NO-CGMP pathway during brain development in mammals.

\section{Introduction}

Whole brain spheroids have been and are being used as a cell culture model to study neurodevelopment [44]. The presence of a mixure of cell types (neurons, astrocytes and oligodendroglia) enables the establishment of a three-dimensional cell culture system that supports neuronal differentiation and a considerable life span [3]. The model reflects many aspects of the in vivo situation, including migration, formation of synapses [42] and myelin sheaths [49], synthesis, storage and release of neurotransmitters [68], and spontaneous electrical activity [57]. As spheroid cultures are frequently used as a model to investigate developmental aspects of the rat brain, we were interested in the possibilities to study functional interactions between cells at the level of a second messenger pathway.

It is well established that nitric oxide (NO) has an intercellular messenger function in all parts of the central nervous system. Under physiological conditions in the brain it is produced by the constitutive and calcium-dependent isoforms of nitric oxide synthases (NOS): the neuronal isoform (nNOS) and the enclothelial isoform (eNOS). Under pathological conditions, such as excitotoxicity or oxidative stress, a calcium-independent isoform of NOS (NOS) might be induced in astrocytes and microglia, leading to synthesis of large amounts of $N O[10,16,20]$.

nNOS can be activated as a result of glutamate-triggered calcium influx $[7,19,35,54]$. The $\mathrm{NO}$ thus formed has a high affinity for the hame moiety of soluble guanylate cyclase (GC) and binding to this prosthetic group leads to the formation of cyclic guanosine 3',5'-monophosphate (cGMP) $[32,45]$.

Altematively, cGMP can be produced via activation of the receptor donain of the particulate GC [66]. The activity of this particulate GC is not mediated by NO, but by binding of peptides such as atriall natriuretic peptide (ANP) to the membrane-bound receptor. CGMP is present in 
almost every region of the rat brain [17] and the functions of $6 \mathrm{GMP}$ in the central newous system are presently under intense investigation. Known effects include interactions with protein kinases, cyclic nucleotideregulated ion channels and phosphodiesterases [20]. Cyclic GMP could influence the CAMP signal transduction pathway by the latter effec [33].

The NO-CCMP pathway has been implicated in several forms of synaptic plasticity, for example in long-tem potentiation in the hippocampal CAl region and in long-tem depression in cerebellar climbing and parallel fibres (see [20] for a review). Furthermore, NO-COMP retrograde signalling is thought to play a role in synaptic dewelopment, as shown by increased neuronal sensitivity towards $\mathrm{NO}$ during synaptogenesis in the grashopper [63] and the involvement of the NO-CGMP pathway in activity-dependent sylnaptic suppression at developing neuromuscular symapses in cultures from Xenopus embryos [65]. In the rat brain, NO has been shown to mediate the formation of synaptic connections in developing oltactory receptor neurons $[47,52]$. Recently, it was shown that CGMP could play an important role in determining the orientation of neurites [56]. Previous work by our group on the developing rat brain has shown a high NO-mediated increase in CGMP concentrations immediately atter birth, which declined during the first three postnatal weeks [15]. In another study, we have shown that ANP-induced CCMP formation is also dependent on the developmental stage of the rat brain [41] and a role for natriuretic peptides during development has been proposed by Brown and Zuo $[8]$

In this study we wanted to investigate whether whole brain spheroid cultures contain nNOS, particulate GC or soluble GC and whether it is possible to stimulate all these enzymes to increase cCMP production, using immunohistochemical and biochemical methods. In addition, we investigated the influence of chronically inhibiting elements of the NO-CGMP pathway in a model for foetal development on functioning of the NO-CGMP pathway and on particulate GC activity.

\section{Materials and Methods}

\section{Animals}

Time pregnant Lewis rats were obtained from the local breeding centre for experimental animals. Experiments were performed according to the guidelines of the University Committee on Animal Welfare, which follow the European Communities Council Directive $(86 / 609 / 5 \mathrm{E})$.

\section{Chemicals}

Dulbecco's modification of Eagle's medium (DMEM) was purchased from ICN Biomedical Inc. Aurora, USA and L-glutamine from Serva, Heidelberg, Cemany. Foelal calf serum was oblaired from Gibco BRL, Paisley, Scotland. Isobutylmetthy xanthine (IBMX) was from Aldrich, Steinheim, Gemany, N-methyl D-aspartic acid (NMDA) from Reseanch Biochemicals International, Natick, USA, ANP from Sigma, St. Louis, MO, USA. Sodium nitroprusside dihydrate (SNP) and Nanitro-l-arginine methyl ester hydrochloride (LNAME) were purchased from Fluka, Buchs, Switzerland. $1 \mathrm{H}-[1,2,4]$ oxadiazolo[4,3-a]quinaloxin-1-one (ODQ] was obtained from Tocris Cookson, Bristol, UK, and dimethylsulfoxide (DMSOY was from Janssen Phamaceutica, Beerse, Belgim. The polyclonal antibodies against NNOS and calbindin were kindly provided by P.C. Emson, The Brabaham Institute Cambridge, UK. The polyclonal antibody against the choline acetyltransferase (ChAT) was a gift from the late Dr. Ichikawa. The polyclonal antibody against the presynaptic glutamatetransporter was a giff from Dr. Rothstein, NIH, Bethesa, USA. The antibody against glutamic acid decarboxylase (GAD) was obtained from Bio Trend (Köln, Germany). Mouse monodonal anti-glial fibrillary acidic protein (GFAP). 
anti-2, 3'cyclic nucleotide 3'-phosphohydrolase (CNPase and biotin labeled iso-tectin B4 were obtained from Sigma, St. Louis, MO, USA. The Cy3-labelled donkey anti-rabbit secondary antibody, the biotin-labeled donkey anti-mouse antibody, the streptavidin-Cy 3 complex and the FITC-labeled goat anti-mouse secondary antibody were from Jackson Immunoresearch Laboratories, Inc., Pennsylvania, USA. The Alexa 488-labelled donkey anti-sheep secondary antibody was purchased from Molecular Probes, Leiden, the Netherlands. All further chemicals were purchased from Merck Darmstadt, Germany.

\section{Spheroid cultures}

Whole brain spheroids were prepared as follows: Brains of 12-20 foetuses (gestation day 15) from 2 rats were transferred into a nylon gauze bag (Nytal@200 $\mu \mathrm{m}$, Merck) in ice-cold Hanks' D2 solution $(5.5 \mathrm{mM}$ D-Glucose, $41.32 \mathrm{mM}$ sucrose, $138 \mathrm{mM} \mathrm{NaCl}, 5.4 \mathrm{mM} \mathrm{KCl}, 0.17 \mathrm{mM}$ Na2t $\mathrm{PO}_{4}, 0.22 \mathrm{mM} \mathrm{KH} 2 \mathrm{PO}_{4}, 14 \mu \mathrm{M}$ phenol red, $1.8 \mathrm{mM} \mathrm{CaCl}, 0.8 \mathrm{mM} \mathrm{MgCl}$. After washing three times, the brains were gently extruded into ice-cold Hanks' D1 solution $15.5 \mathrm{mM} \mathrm{D}$ Gilucose, $46.52 \mathrm{mM}$ sucrose, $138 \mathrm{mM} \mathrm{NaCl} 5.4 \mathrm{mM} \mathrm{KCl}, 0.17 \mathrm{mM} \mathrm{NaHPO}$; $0.22 \mathrm{mM}$ $\mathrm{KH} \mathrm{PO}_{4}, 14 \mu \mathrm{M}$ phenol red). The suspension was filtered through a finer nylon mesh $(150 \mu \mathrm{m}$, washed three times by centrifugation $(170 \mathrm{~g}, 5 \mathrm{~min})$ and suspended, and finally plated out in aliquots of $3.5 \mathrm{ml}$ at a density of $1 \times 10^{7}$ cells/m/ DMEM supplemented with $1 \%$ L-glutamine and $10 \%$ foetal calf serum in 25-ml DeLong flasks (Bellco Gilass) and placed on a gyratory shaker $(85 \mathrm{rpm})$ (Innova 2000, New Brunswick, USA) in an incubator $\left(37^{\circ} \mathrm{C}, 9^{\circ} \mathrm{CO}\right.$, humidified air). After two clays, the cultures were transferred into $50 \mathrm{~m} /$ Delong flasks. Medium was changed three times a week. After one week of culture D, L- $\alpha$-tocopherol $(0.1 \mathrm{mM}$, ICN Biomedical Inc., Aurora, USAI was supplied to the medium.

\section{Exposures}

Three-week-old spheroids were plated out into a six-well plate and washed in Krebs-Ringer bicarbonate buffer $(\mathrm{pH} \mathrm{7.4)} \mathrm{shortly} \mathrm{before} \mathrm{exposure.} \mathrm{Subsequently,} \mathrm{all} \mathrm{wells} \mathrm{except} \mathrm{for} \mathrm{the}$ controls were preincubated with the phosphodiesterase inhibitor IBMX (1 mM) in Krebs buffer. The control wells were incubated during the whole experiment in Krebs buffer only. Exposures to NMDA $(0.5 \mathrm{mM}, 2 \mathrm{~min})$, ANP $(0.1 \mu \mathrm{M}, 10 \mathrm{~min})$, or SNP $(0.1 \mathrm{mM}, 10 \mathrm{~min}$ ) were in Krebs butfer with IBMX. For coincubation with the NOS inhibitor L.NAME $(0.1 \mathrm{mM})$, this compound was present from the stant of the preincubation. After exposure, spheroids were fixed for immunohistochemical analysis or tharvested in Krebs buffer with $3 \%$ perchloric acid for adioirmunoassay. The data determined with the radioimmunoassay were obtained from thee experiments in triplicate. One well containing $50-100$ spheroids was exposed to each separate condition in the experiments analysed by immunohistochemistry and these experiments were repeated at least six times. For testing the influence of inhibition of mNOS or soluble $G \mathrm{C}$ on spheroid development, L-NAME $(0.1 \mathrm{mM})$, D-NAME $(0.1 \mathrm{mM})$, DMSO $10.05 \%)$ or ODQ 10.01 MM, dissolved in $0.05 \%$ DMSO) were added to the medium during the whole culture period (thus not during the incubation with NMDA, SNP or ANP) and treated with IBMX, NMDA, SNP and ANP as described above. One culture tlask containing $300-500$ spheroids was used for each condition and these experiments were repeated three times.

\section{Immunohistochemistry}

Spheroids were fixed in $4 \%$ freshly depolymerised paraformaldehyde in $0.1 \mathrm{M}$ phosphate buffer $(\mathrm{pH} 7.4)$ for 1.5 hours. After subsequent overnight immersion in 10\% sucrose solution the spheroids were frozen in Tissue- Tek ${ }^{*}$ and $10 \mathrm{~mm}$ thick cryostat sections were prepared. 
Sections were air-dried at room temperature for $20 \mathrm{~min}$. After washing in Tris-buffered saline containing $0.3 \%(\mathrm{~V} / \mathrm{V})$ Triton X-100 (TBS-T, PH 7.6), sections were incubated ovemight at $4{ }^{\circ} \mathrm{C}$ with the primary antibodies. The specificity's of the primary rabbit anti-cCMP (1:300 dilution) and the sheep anti-cGMP antibodies (1:4,000 dilution) have been described previously [14,61]. The antibody against nNOS was raised in sheep (1:750 dilution) [26]. The antibody against ChAT $(1: 1,000$ dilution) was raised in rabbit [31]. The antibody against calbindin, a protein found in GABAergic structures (dilution 1:1,000) was raised in rabbil [34]. The antibody against GAD, a protein also specific for GABAergic structures (dilution 1:400) was raised in rabbit and its specificity was as described by the supplier. The antibody against a $73 \mathrm{kDa}$ protein of a neuronal glutamate transporter subtype (EAAC1, C-terminal) was raised in rabbit (dilution 1:100) [18]. The specificity's of the mouse monoclonal anti-GFAP (1:1,000 dilution), the mouse monoclonal anti-CNPase (1): 1,000 dilution) and the biotin-labelled iso-lectin B4 antibodies (1:200) were as described in the data sheets of the supplier. The antibody against the $\beta 1$-subunit of the soluble GC was raised in rabbit (dilution 1:1000) and its specificity was described previously $[36]$.

Cyclic GMP immunoreactivity was visualised by means of an Alexa 488-labelled donkey antisheep secondary antibody (1:100 dilution) or a Cy3-labelled donkey anti-rabbit secondary antibody (1:800 dilution). NNOS, B1, EAAC1, ChAT and calbindin immunoreactivity were visualised by the Cy3-labelled donkey anti-rabbit secondary antibody. CNPase and GFAP immunoreactivity were visualised by a FITC-labelled anti-mouse secondary antibody raised in goat (1:50 dilution) and bound iso-lectin B4 was visualised by streptavidine Cy 3 fluorescence (1:1,000 dilution). The secondary and tertiary antibodies were diluted in TBS-T and incubated at room temperature for 1 hour.

Photographs of the immunostaining (IS) were made on an Olympus microscope, model $A \times 70$ TRF, equipped with a narrow band MNIBA-type FITC filter, a MNG filter for Cy3 fluorescence, permitting to photograph $\mathbb{F I T C}$ or Cy3 fluorescence in the complete absence of bleedingthrough of the other fluorescent marker. Double labelling of structures was defined as positive fluorescence observed through both the MNIBA filter and the MNG filter.

\section{Confocal microscopy}

The confocal laser scanning microscopy was performed with a Leica TCS NT (Leica Microsystems, Heidelberg, Germany). The Argon-Krypton laser was used to excite fluorophores at $488 \mathrm{~nm}$ (FITC) and $568 \mathrm{~nm}$ (Cy3). First, the fluorescence emission was separated with a 580 $\mathrm{nm}$ dichroic beam splitter. Then, the FTC fluorescence was selected by a $5300 / 30 \mathrm{n} \mathrm{nm}$ band pass filter and the Cy3 fluorescence was selected by a $590 \mathrm{~mm}$ long pass filter. In this way crossover fluorescence was negligible. Each optical section was averaged four times. FITC fluorescence was visualised as green and Cy3 fluorescence as red pixels and colocalisation of both markers was visualised as yellow in the overlay picture.

\section{Quantitation of COMP immunofluorescence}

Immunofluorescence (IF) was recorded with a Sony (Power HAD) CCD-camera. Images were imported into a computer equipped with an image analysis program (Soff Imaging System, Analysis 3.0, Münster, Germany). Fluorescence intensity of the spheroids in an image was converted to grey values (255 classes) and expressed as mean intensity. In addition, the highest grey value class of the pixels of the spheroid surface was determined and expressed as maximum intensity. All whole spheroid sections on a microscopic slide were included and the total amount of spheroids is indicated in de legends of the figures and in the text. Sections to be processed for image analysis from the different experiments were immunostained 
simultaneously, to minimise experimental wariation. Mean and maximum intensity classes of sections from the same experiment stained with the secondary antibody alone were subtracted.

\section{Electron microscopy}

Spheroids were fixed in $2.5 \%$ glutaraldehyde in $0.1 \mathrm{M}$ phosphate buffer, $\mathrm{pH} 7.4$, overnight at $4{ }^{\circ} \mathrm{C}$. This was followed by one hour wash in buffer and post-fixation in $1 \%$ osmiumternoxide in the same phosphate buffer for one hour. Next, the spheroids were dehydrated and embedded in Epon. Thin-sections $(70 \mathrm{~nm})$ were stained with uranyl acetate and lead citrate and viewed in a Philips CM 10 electron microscope. Scanning electron microscopy was performed as follows. The spheroids were fixed in $2.5 \%$ glutaraldehyde in $0.1 \mathrm{M}$ phosphate buffer, $\mathrm{pH} 7.4$. dehydrated in alcohol and air dried, sputtered with gold $(10 \mathrm{~nm})$ and viewed in a Philips XL30 scanning electron microscope.

\section{Biochemical assays}

Spheroids were homogenised and centrifuged $\left(18,000 \mathrm{~g} ; 15 \mathrm{~min} ; 4^{\circ} \mathrm{C}\right.$. The pellets were dissolved in $1 \mathrm{M} \mathrm{NaOH}\left(65^{\circ} \mathrm{C}, 30 \mathrm{~min}\right)$ for protein determination. The dissolved pellets and the supernatants were stored at $-80^{\circ} \mathrm{C}$ untill determination. Cyclic GMP levels were measured with a radiommunoassay procedure as described by Steiner et al. [59]. Protein content was detemined according to the method of Lowry [39].

\section{Statistics}

Values obtained with the biochemical assays and image analysis are expressed as means with standard error of mean. Statistics were performed with the non-parametric Mann-Whitmey U test or ANOVA with Scherfe's test for posthoc analysis with the help of the SPSS statistics program. The level of significance was taken at $P<0.05$.

\section{Results}

Rat whole brain spheroids were cultured tor 3 weeks. After ane week of oultwe the followsing appearance was observed in the spheroids (Fig. 1): they seemed loosely packed and contained predominantly undifferentiated cells and many processes (Fig. 1E). At the border of the spheroids round mononuclear cells were observed, as is typically for spheroids [62]. The cells seemed viable as avidenced by the presence of many mitochondria, Golgi apparatus and rough endoplasmatic reticulae (Fig. 1A). Synapse and myelin fomation was observed, as well as the presence of other types of cell-cell contacts resembling tight junctions, which are typically for spheroids (Fig. IA-CI [55]. Some dividing cells were also seen (Fig. 1D).

Alter three weeks of culture electron microscopy studies revealed nomally developed spheroids, showing differentiated cells with elaborated processes (Fig. 1F). There were larger cells with bright nuclei, which were presumably neurons (black arrow). Other cells had an irregular appearance and also irregularly shaped, electron-dense nuclei in which the chromatin was distributed evenly. These cells were presumably astrocytes (black arrowhead). Other cells had round nuclei with a dark nucleolus and electron-dense chromatin at the periphery of the nuclei, suggesting that these cells were microglia (white arrow) $[2,58]$. Similar to the one-weekold cultures round mononuclear cells were observed at the spheroid border. Typical appearance of the spheroids on scanning electron microscopy is shown in Fig. 2 . 

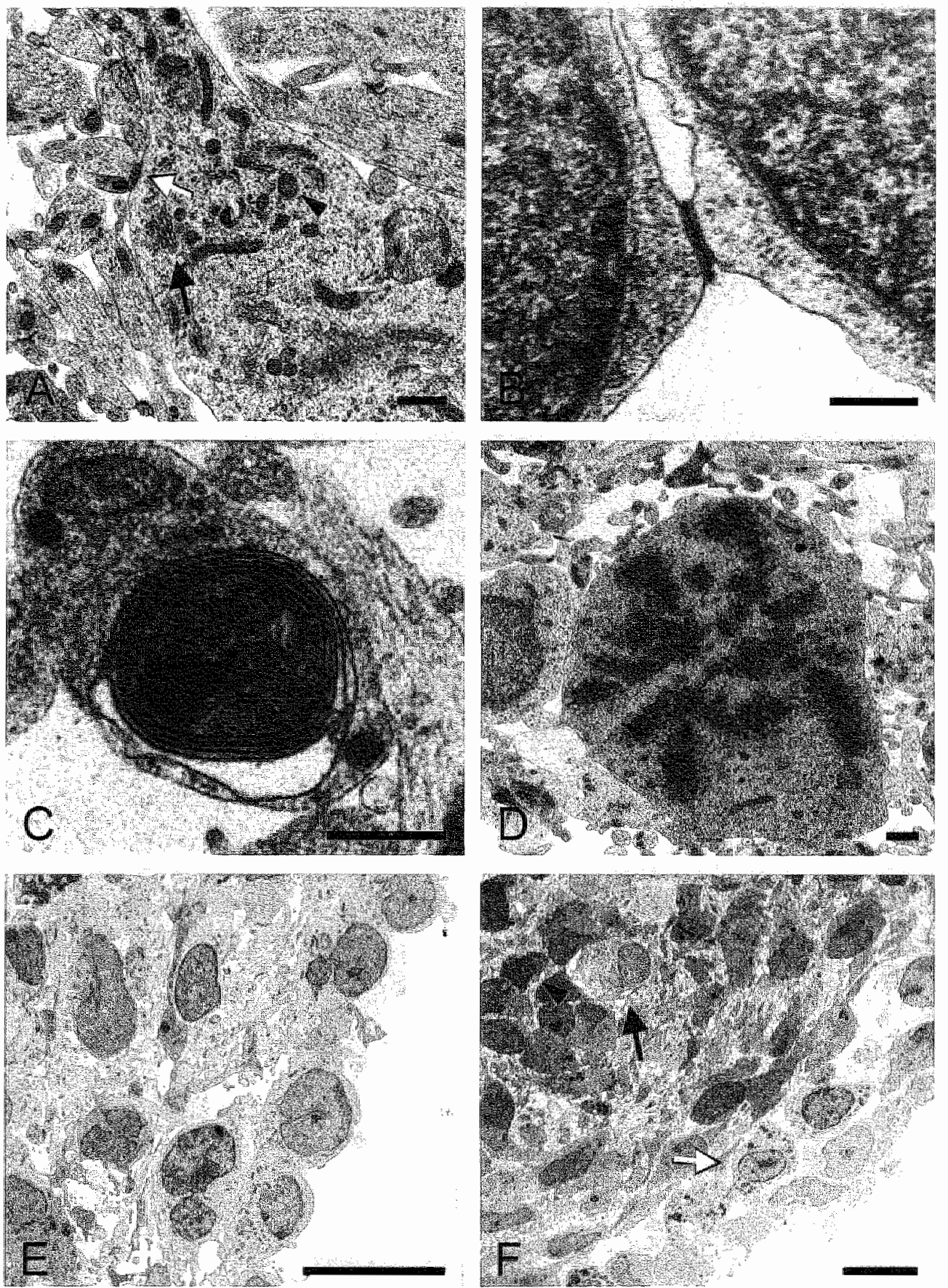

Fig. 1 Electon miroscopic view of one and three-wack old spheroids.

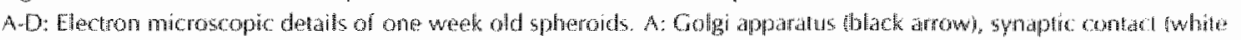

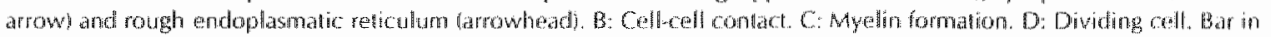

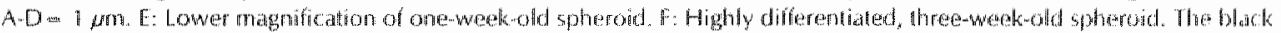

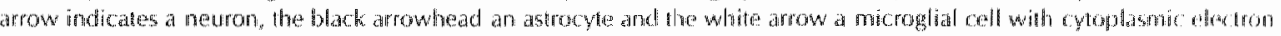
cletse material. Bars in $E$ and F represent 10 urm. 

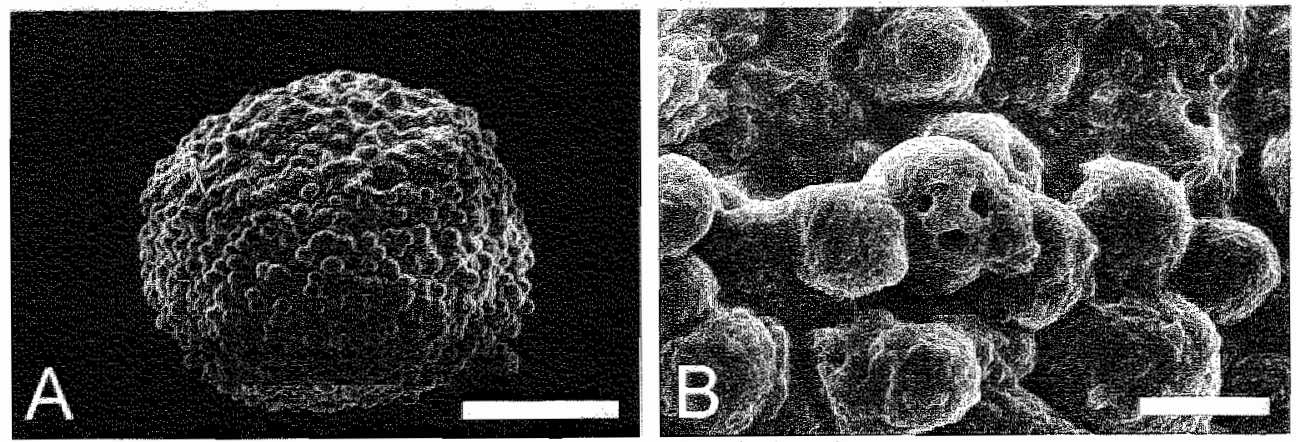

Fig. 2

Scanning electron microscopy photograph of a cullured spheroid.

A: Example of theowed old whole brain spheroids thowing a typical three-dimensional appearance. Bar represents 50

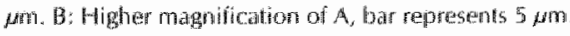

Three-week-old spheroids were stimulated with SNP to increase CGMP concentrations (Table 1). An almost 7 -fold increase in CGMP concentrations $(68.7 \pm 23.7 \mathrm{pmol} / \mathrm{mg}$ protein, $P<0.05$ ) as compared to basal levels (10.7 \pm 4.5$)$ was found as measured with the RIA. In addition, the spheroids were exposed to several conditions and CGMP-IF was measured using immunohistochemistry and an image analysis system, the results of which are shown in Table 1 and Fig. 3 and Fig. 4Fig. When the incubation was performed without IBMX CGMP-IF was not different from background (Fig. 3A). Affer incubation with $1 \mathrm{mM}$ of the phosphodiesterase inhibitor IBMX mean intensity of CGMP-IF rose to $25.2 \pm 1.3$ and maximum intensity was 67.1 土 5.4. Occasionally, CGMP positive cell bodies and fibres appeared in spheroids under these basal conditions (Fig. 3B). Incubation of the spheroids with $0.1 \mathrm{mM}$ of the NOS inhibitor LNAME led to the disappearance of this IF (Fig. 3C). Similar result was obtained after incubation

Table 1 Cyclic GMP content in 3 weel old spherotd cultures. All incubations were done in the presence of 1 mad IBMX.

\begin{tabular}{|c|c|c|c|c|}
\hline & \multicolumn{3}{|c|}{$\begin{array}{c}\text { Image analysis } \\
\text { lgrayvalue intersity) }\end{array}$} & \multirow{2}{*}{$\begin{array}{c}\text { Radioimmunoassay } \\
\text { ipmoling protein } \\
\text { Mean d sem }\end{array}$} \\
\hline & Mean sem & Maximuma + sem & $n$ & \\
\hline IBMX abads & $25.2+1.3^{3}$ & $67.1 \pm 5.4$ & 107 & $0.7+4.5$ \\
\hline $1 . N A M E$ & $0.0 \pm 0.0$ & $0.0 \pm 0.0$ & 22 & n.d. \\
\hline NMDA & $36.2 \pm 0.7^{1}$ & $94.3 \pm 4.5$ & 202 & n.d. \\
\hline NMDA + LWAME & $1.4 \pm 1.4$ & $1.7+1.7$ & 23 & n.d. \\
\hline $5 \mathrm{NP}^{3}$ & $38.1+0.6^{13}$ & $129.9 \pm 4.9^{5}$ & 176 & $68.7 \pm 23.7^{\prime \prime}$ \\
\hline $\mathrm{ODQ}$ & $2.5 \pm 1.7^{d . r}$ & $2.9 \pm 2.0^{\mathrm{los}, \mathrm{c}}$ & 23 & ned. \\
\hline$A N P$ & $269 \pm 1.2$ & $126.4+8.5^{16.7}$ & 130 & $n, d$ \\
\hline$O D Q$ + ANP & $29.2 \pm 1.5^{\circ}$ & $1529+11.9^{\circ}$ & 62 & n.d. \\
\hline
\end{tabular}

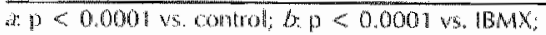

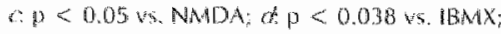

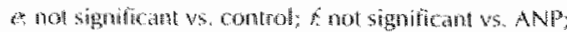

g. $P<0.02$ vi. $18 \mathrm{Br}$ :

and a not dome? 
of the spheroids with $0.01 \mathrm{mM}$ of the soluble GC inhibitor ODQ (Fig. 30 . Treatment of the spheroids with $0.5 \mathrm{mM}$ NMDA during 2 minutes led to an increase of the mean and maximum CGMPAF as compared to basal levels and the appearance of several cell bodies and fibres (Fig. 3E). To further confirm the role of $\mathrm{NO}$ in the activation of soluble $\mathrm{GC}$ in the culture system after stimulation with NMDA, spheroids were exposed to NMDA in the presence of $0.1 \mathrm{mM} \mathrm{L}$ NAME. Under these conditions CGMP levels were reduced to very low levels (Fig. $3 F$ ).
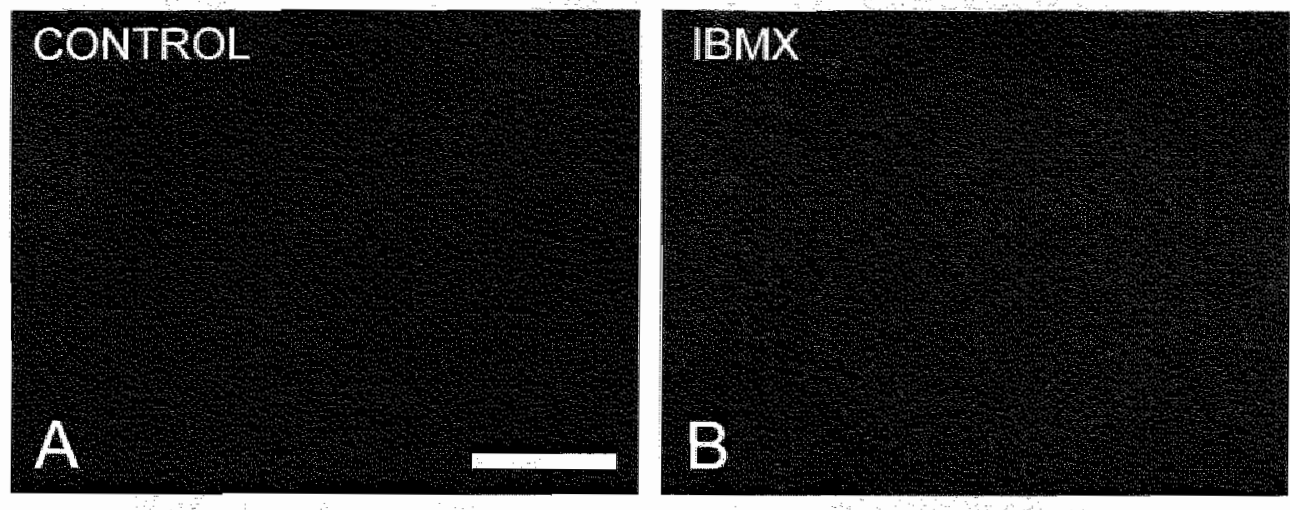

\section{L-NAME}

\section{ODQ}

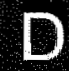

\section{NMDA}

\section{NMDA + L-NAME}

\section{E}

Fig, 3

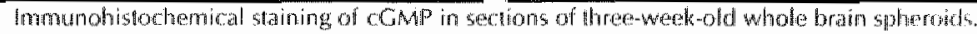

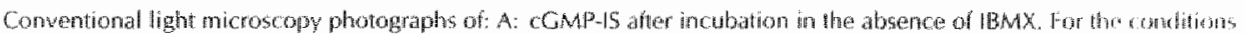

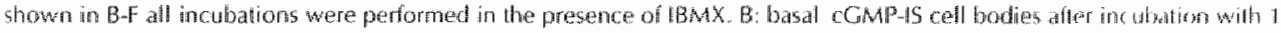

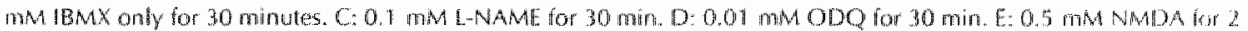

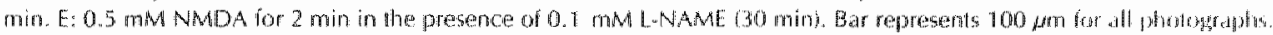



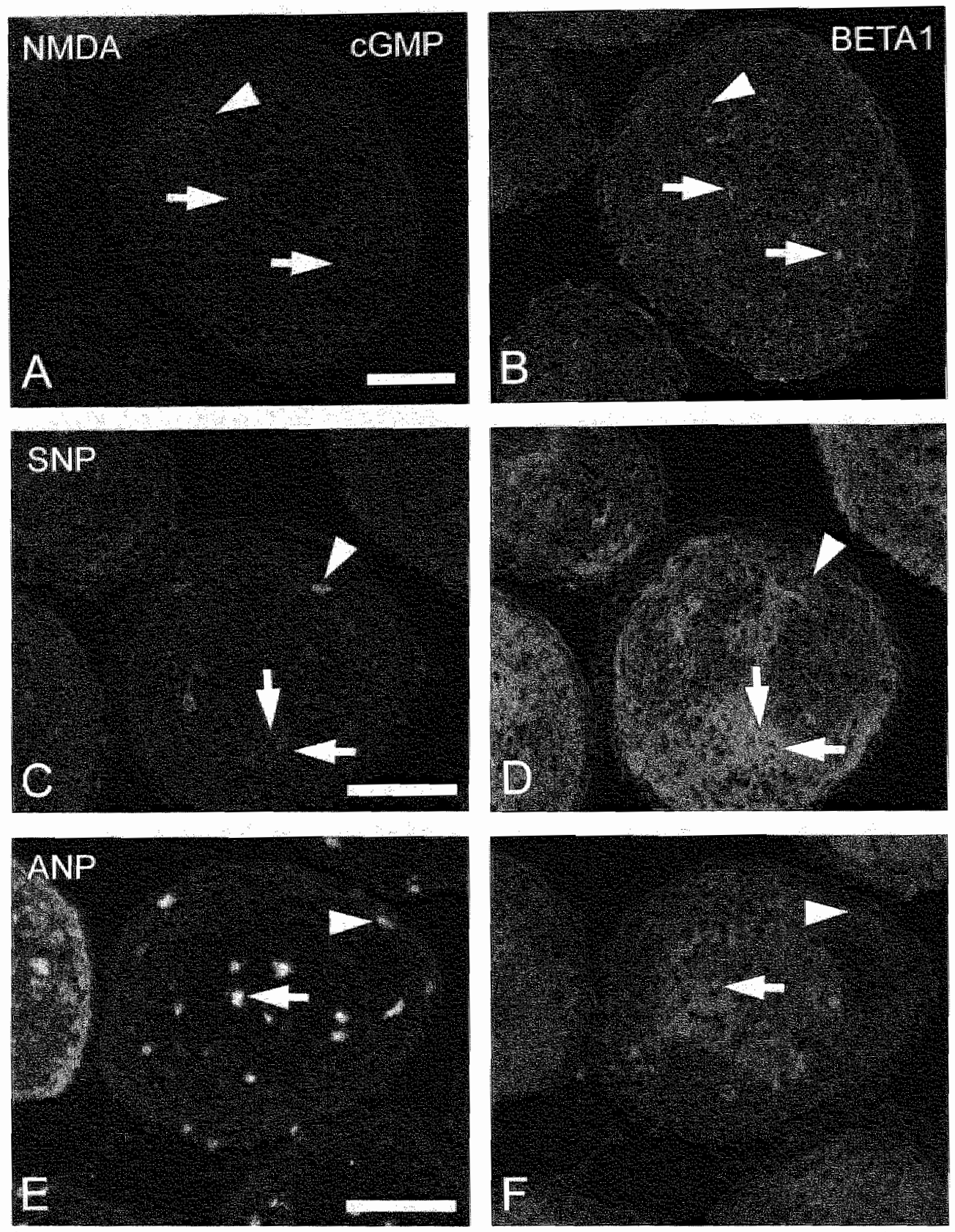

Fig 4

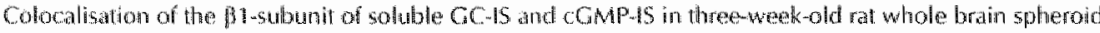
sectoms

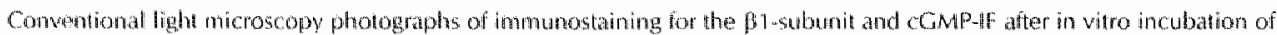

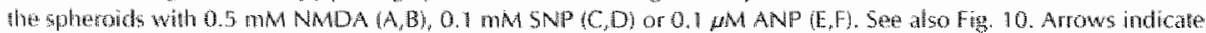
coluchising structures, arrowhedds indicate struchtes without colocalisation. Bars represent 100 pm. 


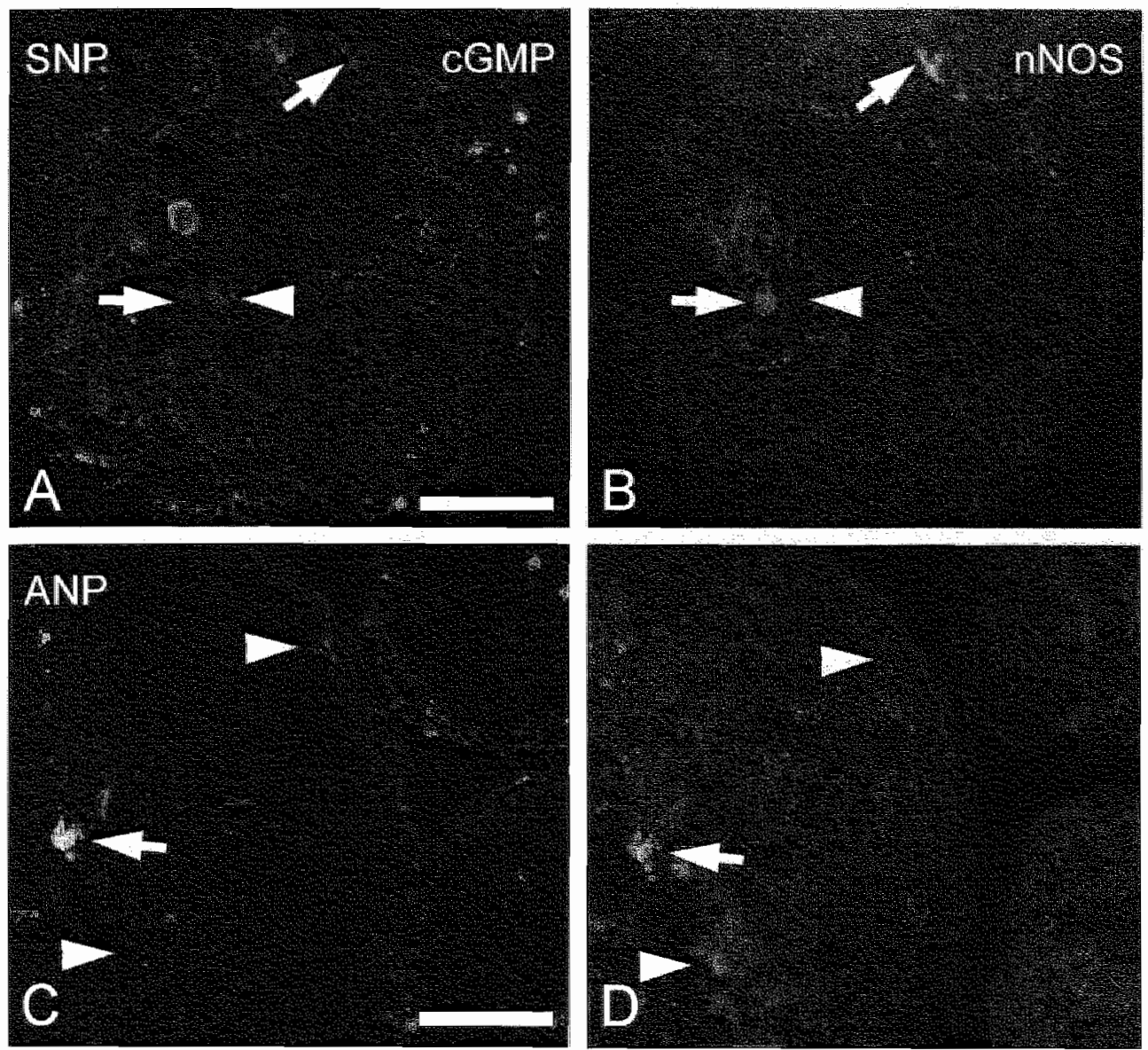

Fig. 5

Colocalisation of mNOS and CGMP in three-week-old rat whole bran spheroid sertions.

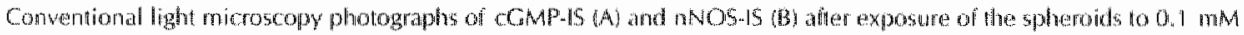

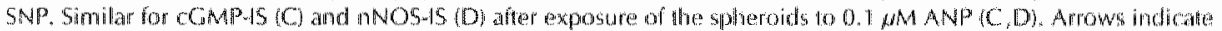
colocalising stuctures, arrowheads indicate struchues willout colocalisalion. Bars rapresent $100 \mathrm{fm}$

To stimulate the activity of soluble GC directly, SNP was used as a NO-donor. Exposure of the spheroids to SNP lead to CGMP-IF cell bodies and fibres (Fig. 4C) and an increase in the mean intensity of the COMP-IF $(38.1 \pm 0.6)$, while the maximum intensity was increased up to two times the basal levels (128.9 \pm 4.8$)$. To activate the particulate isoform of GC, the cullures were exposed to ANP $(0.1 \mu \mathrm{M}, 10 \mathrm{~min})$. This resulted in some intensely staning cell bodies with short processes, with a maximum CGMP.FF of $126.4 \pm 8.5$ (Fig. $4 \mathrm{E}$ ). Inhibition of basal soluble GC activity with ODQ during exposure to ANP resulted in a similar mean and maximum CGMP-IF as after incubation with ANP alone (29.2 \pm 1.5 and $152.9 \pm 11.9$, respectively).

Characterisation of the CGMP-1S structures was addressed by performing double 15 of CGMP with a number of cellular markers. For a colour photomicrograph obtained with contocal microscopy of the majority of colocalisations, see Fig. 10. Colocalisation of CGMP with the B1subunit of the soluble GC was pertormed after activation of $G C$ with different stimuli. As expected, cGMP formation by soluble GC after exposure to $0.5 \mathrm{mMNMDA}$ or $0.1 \mathrm{MM}$ SNP was colocalising with $\$ 1$-containing structures (Fig. 4A-D). However, not all CCMP-Containing structures were positive for the $\beta 1$-subunit. This may indicate that another form of the NO- 
responding soluble GC may be involved. Similarly, not all $\beta 1$-subunit containing cells were responding with CCMP formation after exposure to NO. We found that the B1-subunit of soluble GC was also present in ANP-responding, CGMP synthesising cells (Fig. 4E, Fig. 10A. We found NOS-mediated CGMP symthesis in some structures which were nNOS-positive, as shown by light microscopy (Fig. 5A, B). Similarly, a subpopulation of ANP responsive cells showed CCMP- and nNOS-1S (Fig. 5C, D).

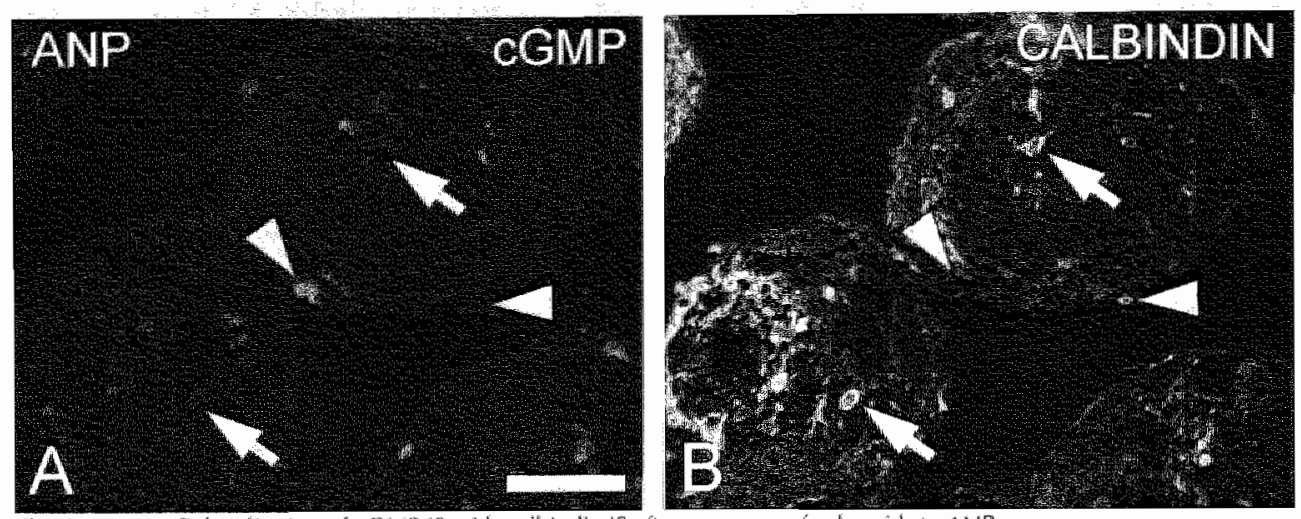

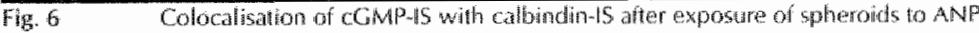

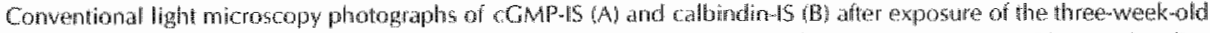

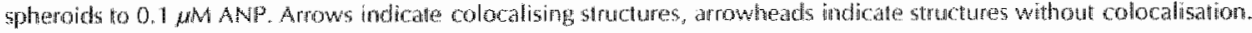
Bestrepresents $100 \mathrm{um}$.

CGMP-IS was combined with staining for EAAC1, ChAT, calbindin, GAD, GFAP (astrocyles), CNPase (oligodendrocytes) or iso-lectin B4 (microglia). The presence of soluble GC in some neurons positive for EAACI was shown after stimulation with SNP (Fig. 10B). Stimulation of CGMP production with NMDA or SNP showed the presence of calbindin in neurons positive for soluble GC (Fig. 10C, D). In addition, extensive colocalisation of calbindin with CGMP was found after exposure to ANP (Fig.A, B). These structures might be astrocytes as colocalisation of GFAP with calbindin was found as well (Fig.10E). Under the same stimulating conditions colocalisation of GAD with CGMP was found (Fig. 10F-H). Sporadically, it appeared that CGMP containing cells also contained ChAT after stimulation with NMDA, indicating the possibility to activate soluble GC via NOS in neurons of the cholinergic system (Fig. 7A, B). Simillar results were obtained aiter stimulation with $\$$ INP (Fig. 7C, D). Double staining of markers for astrocytes or microglia with CGMP revealed that, after exposure of the spheroids to all three slimuli, NMDA, SNP or ANP, the CGMP-IS was found in GFAP positive structures (Fig. 101-K). None of the CGMP positive cells showed iso-lectin B4 co-staining after stimulation of soluble GC with SNP or via NMDA. However, some cells exhibiting double staining for CGMP and iso-lectin were observed after stimulation of the particulate GC with ANP (Fig. 8A, B). When the possible colocalisation of cCMP in oligodendrocytes was tested, it appeared that following exposure to NMDA or SNP, CGMP-IS was found in CNPase positive cell bodies (Fig. 9A, B, Fig. 10L, M). Colocalisation was present in subsets of cells as some cells containing CGMP were positive for CNPase, and some CNPase positive cells were observed without CGMP-IS.

A summary of the double labelling studies is given in Table 2. 


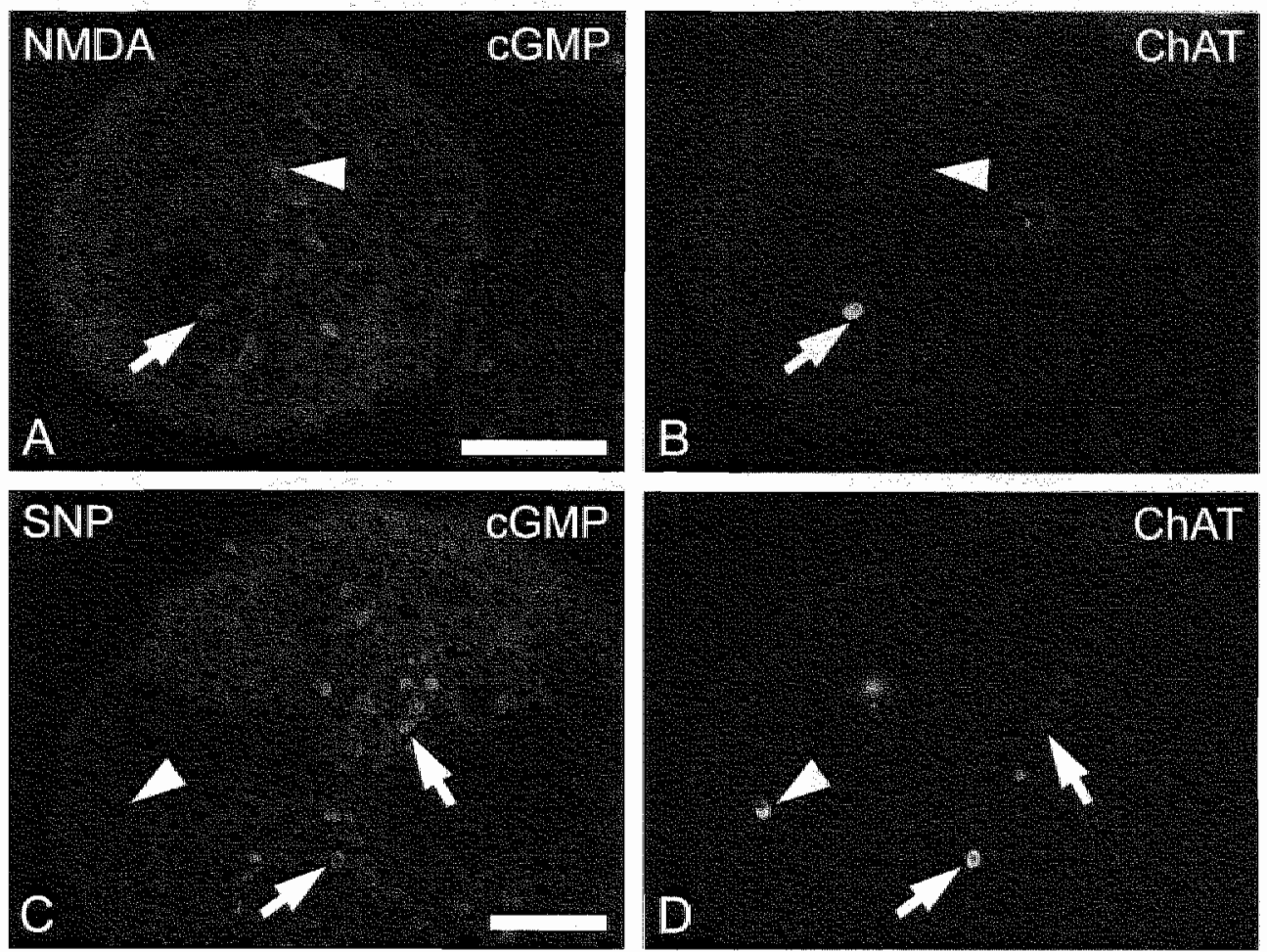

Fis 7

Colocalisation of ChAT and CGMP.IS in theeweek-old rat whole brain spheroid sections.

Comentional light microscopy photographs of CCIMP-IS (A) and ChAT-IS (B) after exposure of the splneroids to $0.5 \mathrm{mth}$ NMDA. C-D: CGMP-IS (C) and CI7.ATHS (D) atter exposure lo $0.1 \mathrm{mM} 5 \mathrm{NP}$. Arrows indicate colocalising structures. arrowheads indicate structures without colocallsation. Bars represent 100 Hm.

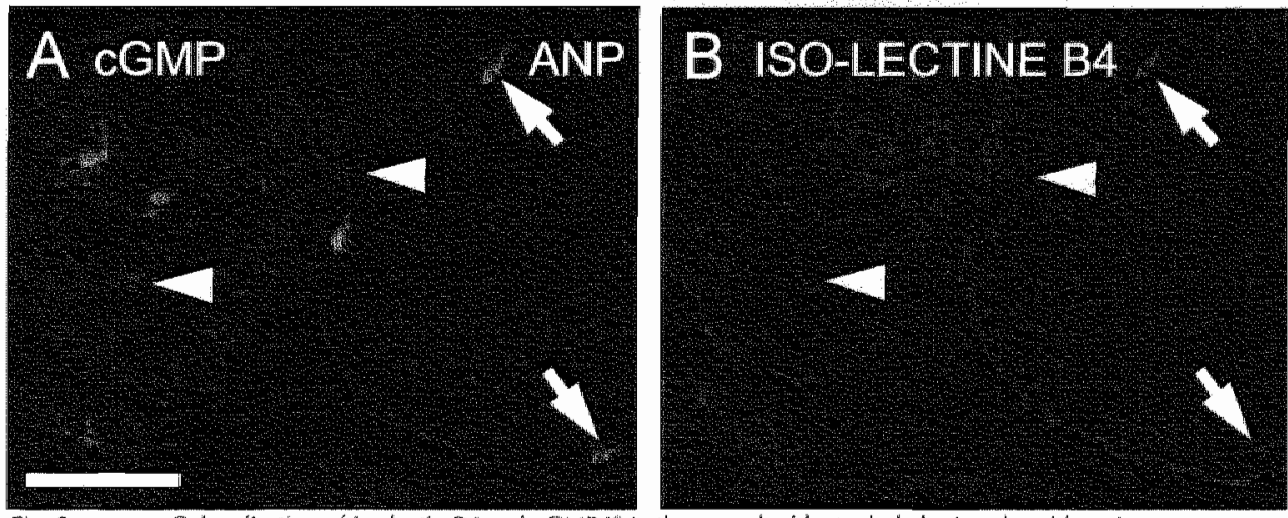

Fig. 8

Colocalisation of iso-lectin $\mathrm{B} 4$ and CGMP-15 in thres-week-old rat whole brain spheroid sections.

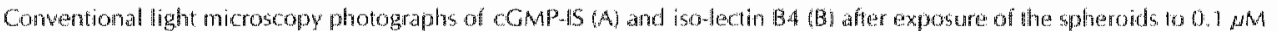
ANP. Anrows indicate colocalising structures, arrowheads indicate structures without colocalisation. Bar reprements 100 pm. 


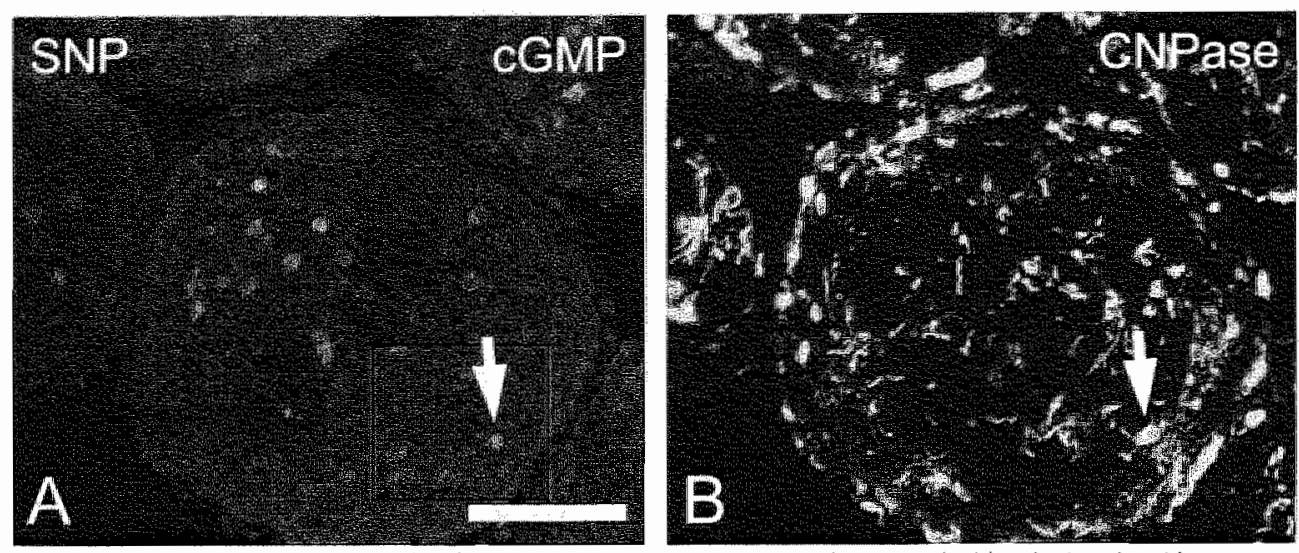

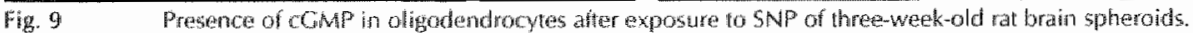

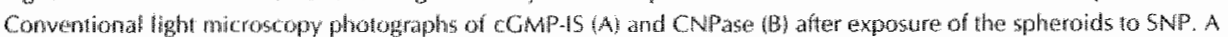

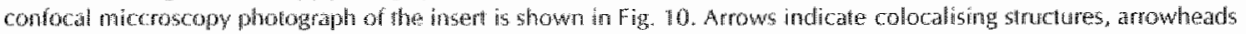
indicate structures withoul colocalisation, Bars repesent 100 wan.

As the experiments showed that an intact NO-CGMP signal transduction pathway was present in three-week-old spheroids, we investigated the influence of prolonged inhibition of the two key enzymes of this pathway, NOS and soluble GC, on culture development and CGMP production in spheroids. Mean intensities of basal CGMP values were found to be decreased in the L-NAME-treated cultures as compared with controls and D-NAME treated cultures (control: $47.6 \pm 0.84, n=113 ;$ L-NAME: $9.81 \pm 2.90, n=38$; D-NAME: $37.1 \pm 0.97, n=99)$ (Fig. 1 1.A). Similarly, NMDA induced CGMP formation was decreased in L-NAME treated cultures (control: $71.26 \pm 1.76, n=105 ;$ L-NAME: $11.2 \pm 3.07, n=37 ;$ D-NAME: $38.6 \pm 0.80, n=76) . \quad$ In contrast, the CGMP production could be stimulated by SNP in the L-NAME treated cultures similar to control cultures (control: $68.4 \pm 1.22, n=140 ; \mathrm{L}-\mathrm{NAME}: 63.1 \pm 1.34, \mathrm{n}=87: \mathrm{D}-$ NAME; $61.0 \pm 1.22, n=611$.

Maximum fluorescence intensity values of basal and NMDA induced CGMP-15 were also lower in L-NAME treated cultures than in the control and D-NAME treated cultures (Fig. 11B). Treatment with D-NAME during development decreased basal and NMDA-induced CGMP formation, although not as much as in L-NAME treated cultures.

The results in Fig. 11 A show that DMSO treatment during culture development had a positive effect on soluble GC stimulation by NMDA or SNP. NMDA induced CGMP fluorescence levells in spheroids exposed to ODQ during development were similar as in DM5O control spheroids (mean intensity DMSO: $87.0 \pm 3.5, n=50 ; \mathrm{ODQ}: 76.6 \pm 3.4, \mathrm{n}=30 ;$ maximal intensity DMSO: $230.0 \pm 6.1, n=50 ; \mathrm{ODQ}+\mathrm{DMSO}: 226.8 \pm 9.8, n=30)$. Mean intensity of COMP-1S after stimulation of soluble GC with SNP was decreased in ODQ treated cultures (DMSO: $111.3 \pm$ 5.2. $n=24 ; \mathrm{ODQ}+\mathrm{DMSO}: 72.2 \pm 2.4, n-37$ ). Maximum fluorescence intensily after stimulation with the NO-donor SNP was similar in DMSO or ODQ + DMSO treated spheroids (DMSO: $253.3 \pm 1.7, n=24 ; \mathrm{ODQ}: 245.9 \pm 5.3, n=37$ ). Mean and maximal intensity of ANP stimulated CGMP.IF was similar in all treatments. 


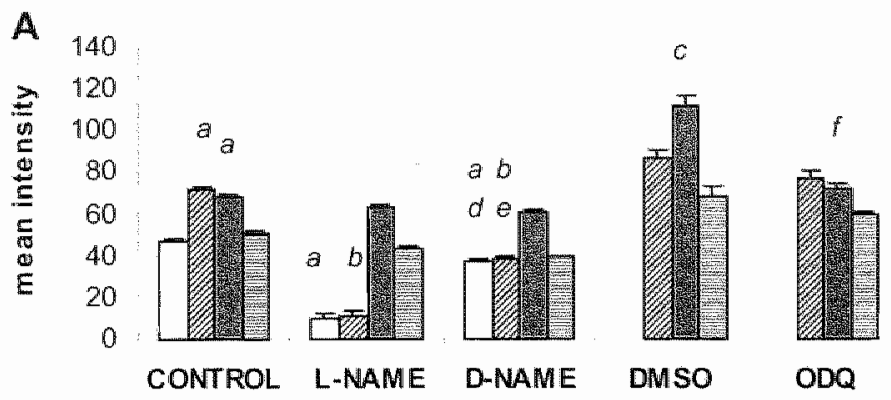

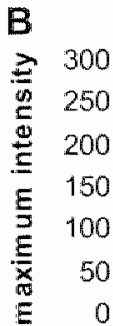
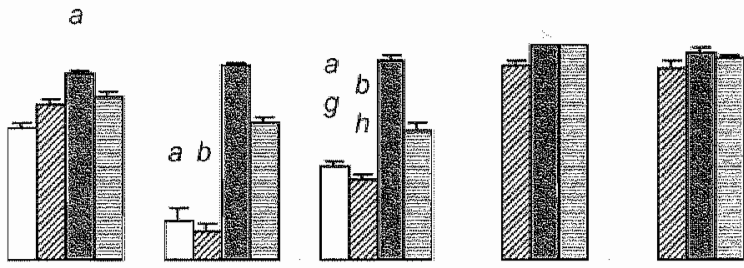

CONTROL

L-NAME

D-NAME

DMSO

ODQ

Fig. 11 Mean and maximum immunofluorescence intensily of COMP-lS after inhibition of WOS using 1 . NAME: 10.1 mMth or soluble $\mathrm{GC}$ asing ODO $10.01 \mathrm{mM}$ ) during three weeks of the spheroid development

White bar indicates level aftar incubation with BBMX; diagonals inclicates levels after stimulation with WMDA whring 2 minutes; black bar indicates cCMP leves after stimulation with 5 NP during 10 minutes; horizonal lines indicales cCMP

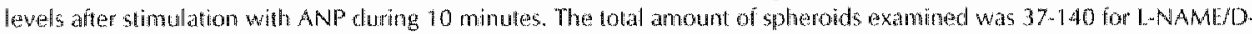

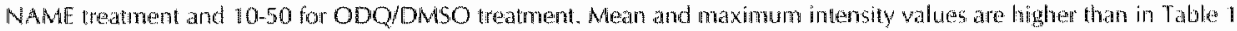
due to culure and staining wariation and differences in thresholds during importing the images.

A: Mean immunofluorescence intensity. B: Maximum immunofluorescence intensity.

a. significanty $(P<0.0001)$ different compared to treatment with IBMX only in control cultures. $b$ signticanty $(P<0.0001$ different compared to treatment with NMDA in conthol collures. $c$ significantly $(P<0.0001)$ different compared to

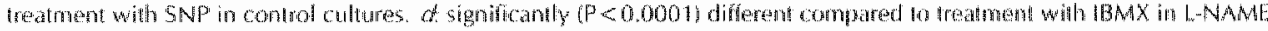

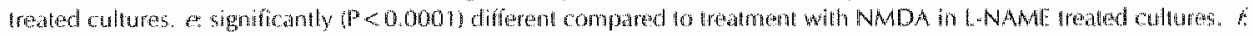

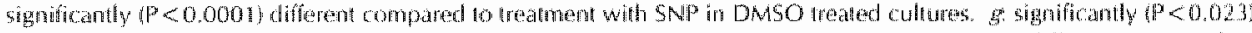

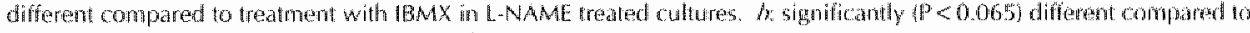

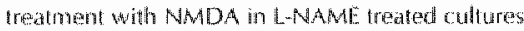

\section{Discussion}

The spheroid culture system has been introduced as a model for brain development. The model has been shown to display several aspects of in wivo brain development, such as migration and extensive differentiation $[25,51,53]$. Our experiments are in agreement with these previous Findings as the morphological results showed that specialised contacts and myelin formation existed in these three-dimensional cell cultures (Fig. 1A,B). In our experiments it was shown that already after one week spheroid cultures are maturing, forming myelin sheets and synapses, processes that peak after four weeks in culture [55].

The aims of our investigation were two-fold: first we wanted to investigate whether functional systems using CGMP as a second messenger were present in spheroids and secondly, if present, whether inhibition of wo key enzymes of this pathway had implications for the development of the spheroids and for the functioning of NO-CGMP signal transduction. We demonstrated 
CCMP production in three-week-old spheroids after exposure of the spheroids to NMDA, SNP or ANP, indicating the presence of both soluble and particulate GCs in this system. Further evidence of the presence of soluble GC was obtained by demonstrating the presence of the $\beta 1$ subunit of soluble GC. In accordance with the literature, CGMP formation in our cultures after NMDA exposure was NOS-mediated, as shown by the absence of CCMP increase when LNAME was present during the exposure to NMDA. Although it has been shown before that spheroids can synthesise CCMP after stimulation with kainic acid [29], the present study demonstrates for the first time the presence of the NO-CGMP signal transduction pathway as well as particulate GC-mediated CGMP production in this culture system.

Current wiews hold that NO produced by NOS will diffuse to target cells to exert its effect on soluble $\mathrm{GC}$ in glutamatergic terminals $[19,28,46]$. Our results confirm this possibility, as cGMP was found in glutamatergic neurons. (Fig. 10B). Howewer, the question arises to what extent soluble $G C$ in the glutamatergic neurons is the only target for $\mathrm{NO}$ as only al small population of the CCMP-IS neurons were glutamatergic. In addition, identification of CGMP positive structures showed that activated soluble GC collocalised with cholinergic and GABAergic neurons. The presence of soluble GC in cholinergic neurons is in agreement with our recent observations in adult rat brain [13]. Another consequence of the original hypothesis was that NO functions as an intercellular messenger. It would be interesting to know if $\mathrm{NO}$ could also function as an intracellular messenger, i.e. if soluble GC can be activated by $N O$ synthesised in the same cell. We found the presence of soluble GC in nNOS containing structures, as was demonstrated recently in brain slices [12]. Cultured astrocytes have been shown to be able to express both NOS and GC $[1,5,40]$. At least in culture, these cells can contain both enzymes, though it is not yet proven whether soluble GC is activated by NO produced in the same call.

Stimulation of particulate GC in our spheroids led to CGMP formation in microglia. This is the first study showing particulate GC in microglia. Microglia are of the same geneity as. macrophages, a cell type known to contain particulate $\mathrm{GC}[30,43]$. Both cell types originate from monocytes and invade the brain during embyonic and early postnatal life $[6,67]$. Furthermore, after injury microglia can transform into cells with characteristics of macrophages $[6,21]$. Although the presence of macrophages in spheroid cultures has mot been reported before, cell culture conditions and procedures may lead to an activated, macrophage-like phenotype of the microglia. Altematively, the cells positive for iso-lectin and CGMP after ANP exposure could be macrophages, as macrophages are reported to be present in the brain and with the marker for microglia iso-lectin $B 4$ no distinction can be made between macrophages and microglia $[67]$.

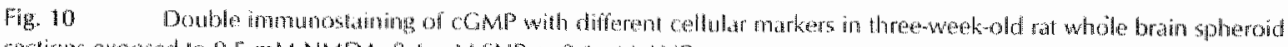

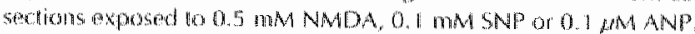

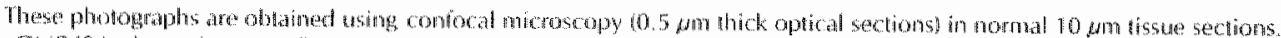

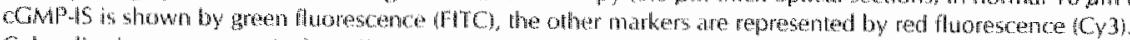

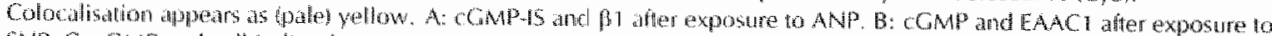
SNP. C. OCMP and calbindin after exposura 10 NMDA, D: CGMP and calbindin afrer exposure to SNP. E: GFAP and

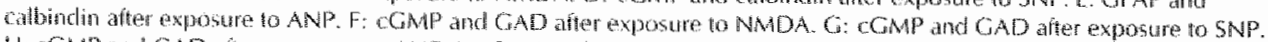

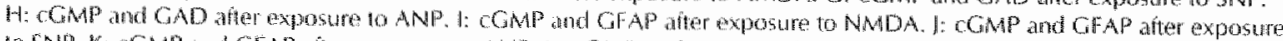
to SNP. K: CCMP and CTAP after exposure to ANP. 1. CGMP and CNPage anter exposune to NMDA. AM: COMP and CNPase after expostare to SNP. Bar in A repregents $100 \mathrm{pm}$ for A H. Bar in M also represents $100 \mathrm{~cm}$ 

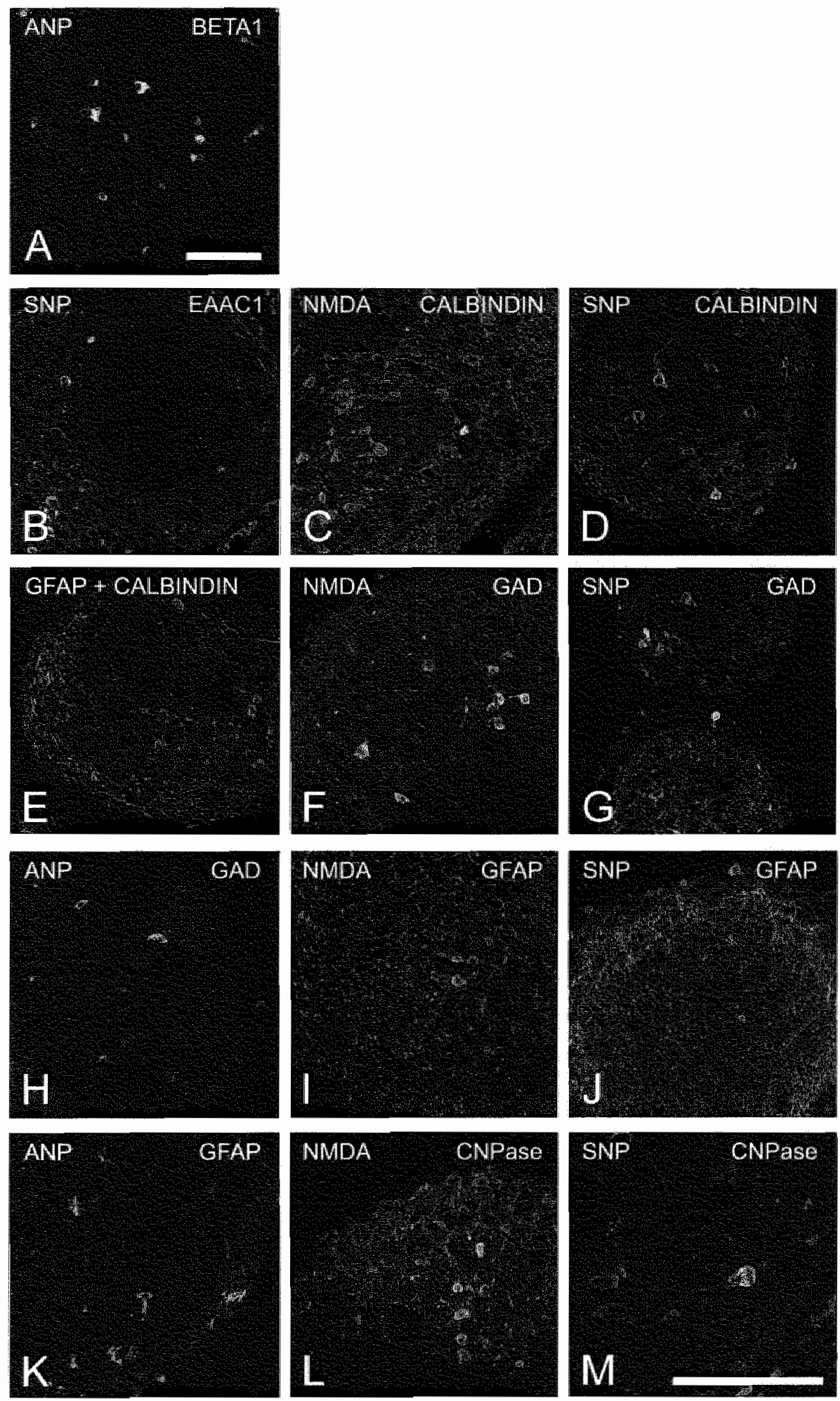


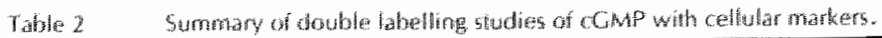

\begin{tabular}{|c|c|c|}
\hline Colomaisdion CMP with & Sormulation & Colocalisation \\
\hline \multirow[t]{3}{*}{ Pl-subunit of soluble $\mathrm{GC}$} & MMDA & + \\
\hline & $5 w^{2}$ & + \\
\hline & $A M P$ & $\stackrel{5}{9}$ \\
\hline \multirow[t]{3}{*}{$\mathrm{nNOS}$} & NMOA & 4 \\
\hline & $S \mathbb{A}_{\mathrm{S}} \mathrm{P}$ & + \\
\hline & $A N P$ & + \\
\hline EAAC: & NMDA & ned. \\
\hline lnetronal gotomate & SNP & + \\
\hline Mangpoter & ANP & - \\
\hline \multirow[t]{3}{*}{ Calbindin } & NMDA & + \\
\hline & SNP & + \\
\hline & $A N P$ & + \\
\hline \multirow[t]{3}{*}{ GAD } & NMDA & + \\
\hline & $5 \mathrm{NP}$ & + \\
\hline & ANP & + \\
\hline \multirow[t]{2}{*}{ CHAT } & MMDA & + \\
\hline & $S N P$ & 4 \\
\hline Iso-kection 34 & NWDA & - \\
\hline \multirow[t]{2}{*}{ (microglia) } & SNP & - \\
\hline & $A N P$ & + \\
\hline CNPase & NMOA & + \\
\hline \multirow[t]{2}{*}{ toligorlendrogstian } & SNP & + \\
\hline & AAP & - \\
\hline GFAP & NMDA & + \\
\hline \multirow[t]{2}{*}{ (astrorytes) } & SANP & + \\
\hline & ANP & + \\
\hline
\end{tabular}

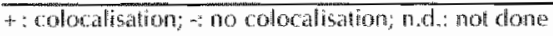

We found evidence of CCMP formation in astrocytes after incubation with NMDA or SNP. These results are in agreement with previous results $[15,61]$. In addition, we found cGMP fomation in astrocytes after incubation with ANP, which is in agreement with previous studies in adut and in developing rat brain $[11,41,61]$. The presence of calbindin in particulate cyclase containing structures suggests that astrocyles in cultured spheroids express calbindin.

We found CGMP-1S after SNP stimulation in oligodendrocyles in owr cultures, suggesting the presence of soluble GC in this cell type. This is in accordance with a previous study showing the presence of soluble GC in oligodendrocytes in the developing rat brain (2 weeks) [61]. We found no CGMP-IS in oligodendrocytes after exposure of the spheroids to ANP, suggesting that these olligodendrocytes did not contain particulate GC. Previous studies demonstrated particulate GC in purified rat brain myelin [22] and in myelinated fibres of the developing frontal rat brain [41]. The differences between our results and results obtained in the rat brain regarding the presence of soluble GC in oligodendroglia might find their explanation in the differences in developmental stage between spheroids and the rat brain in vivo. In addition, our group demonstrated that the development of ANP-responsive myelinated fibres coligodendroglia) was very site specific [41].

As stated in the introduction, evidence is accumulating that the NO-CGMP pathway plays a role in certain mechanisms of brain development, such as neurite orientation $[48,56,60]$, synaps formation [63], growh arrest and proliferation [47]. NO was shown to regulate the formation of 
synapses in Xenopus embryos [65], and NO was able to enhance neurotrophin-induced neurte ougrowth wia a CCMP dependent mechanism in culured PC12 cells [27]. We investigated the functional activity of the NOsoluble GC pathway in the spheroids atter chronic inhibition of NOS or soluble GC during foetal development of the spheroid cultures. We supplied treshly prepared L-NAME to the cultures three fimes a week, and it can be argued that L-nitro-L. arginine would probably have been present during the whole culture period. It was shown that L.NAME is metabolised into the NOS inthibiting compound L-mitro-L-arginine rapidly, whereas L-nitro-L-arginine concentration declines slowly after infusion of L-NAME in humans. The half live was 23 hours and after 72 hours about $10 \%$ of the maximum L-nitro-L-arginine level were still present [4]. Similarly, in canine plasma and blood Luitro-Larginine remained stable for 4 hours after incubation in vitro with L-NAME B B].

In an attempt to sudy the role of NO and CGMP during foetal development, we inhibited NOS or soluble $\mathrm{GC}$ during culturing of the spheroids the development of the typical appearance. such as shown in Fig. 2, of the spheroids was not affected by the chronic presence of L-NAME or ODQ. As expected, analysis of both mean and maximum CGMP immunofluorescence intensity after inhibition of NOS revealed suppressed basal and NMDA stimulated COMP production caused by residual LNAME in the cultures. Soluble GC activity remained preserved, as SMP induced mean and maximum If remained similar as in control conditions. These results suggest that either NOS is not playing a crucial rote for functioning of soluble GC of the residual amount of NOS activity is sufficient to sustain the role of NO. Inhibition of soluble GC during development with ODQ decreased the mean CGMP production by the NO donor SNP. without a similar effect on maximun cGMP-IF. We assume that the observed dectease in mean CGMP-IF is caused by the residual ODQ still present from the pre-reament. These results are in agreement with previous results inhibiting NOS during postnatal development in the in vivo rat brain $[50,64]$.

Occasionally, we found both particulate and soluble GC in the same cell. This is in agreement with our observations of the presence of particulate and soluble GC in the same astrocyle in hippocampus of the in vivo rat brain (Chapter 3). It is not clear what the function is of this phenomenon. It has been demonstrated that particulate $G C$ and soluble GC can coexist in the same cell in myocyte cullures [24]. In another study it was found that the llanscription of particulate GC could be suppressed by ANP possibly via a CGMP-responsive element [9]. In other systems, translocation of CMP-dependent proten kinase to the nuclear comparment has been demonstrated [23] and the nuclear localisation of CGMP has been described [63]. In this way, NO or soluble GC mediated CCMP formation might influence particulate GC expression. Indeed, another study showed that inhbition of NO-mediated soluble GC activity leat fo increased particulate GC acivily in proximal tubular cells $\$ 381$. Athough both cyclases ware present in the same cell, no alteration in particulate GC activity after inhibition of NOS or soluble GC during development was seen in our experiment. The effects of inthibition of particulate $\mathrm{GC}$ on soluble $\mathrm{GC}$ activity is difficult to test at present, as no selective inhibitor of particulate GC is available. However, such influence is not likely to occur via NOS as we saw no attered soluble GC activity after chronic inhibition of NOS activity with LNAME during development (Fig. :1).

In conclusion, we have shown that nNOS and both soluble GC and particulate GC are present in whole brain spheroid cullures showing functionall activily. Furthermore, a celltype dependent pattern in the colocalisation of CMP synthesising systems was found. I1 was shown that soluble GC can be present in glutamatergic, cholinergic and GABAergic neurons as well as in cells containing particulate GC. Chronic inhibition of NO synthase during cullure: development had no effect on soluble or particulate guanylate cyclase functioning. Similarly. inhibition of soluble guanylate cyclase during culture development did not have any effect on NO synthase and panticulate guanylate cyclase functioning. However, this inhibition did not 
lead to clear alterations in the reaggregating process and funther development of the splteroids. Further experiments on $\mathrm{GC}$ (isolforms will be necessary to elucidate the exact role of the NO and CCMP during brain development.

\section{Acknowledgements}

The late Dr. Ichikawa is gratefully acknowledged for providing us the antibody against ChAT. R. van Gool is gratefully acknowledged for the electron microscopic photonicrographs. P. Emson is gratefully acknowledged for providing us the antibody against nNOS. D. Koesling is gratefully acknowledged for providing us the antibody against the $\beta \%$-subunit of GC. I. Rothstein is gratefully acknowledged for providing us the antibody against EAAC1.

\section{References}

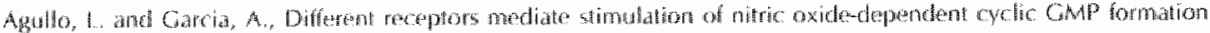

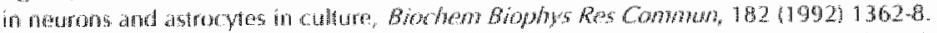

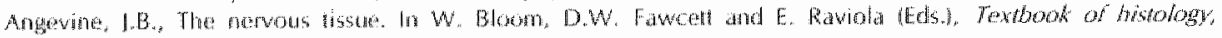
Chapman \& Hathl, Now Yark, 1994, pp. 304-367.

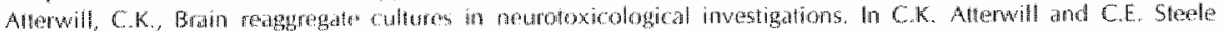

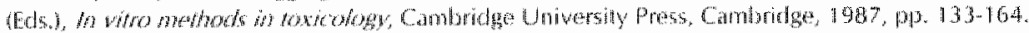

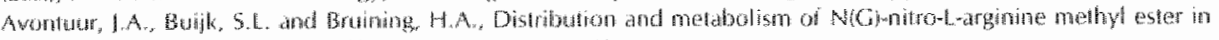

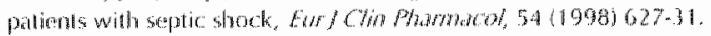

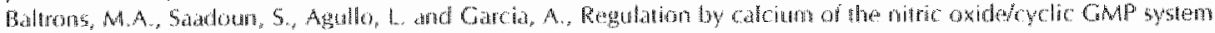

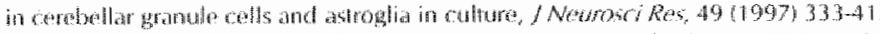

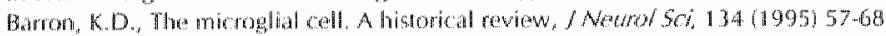

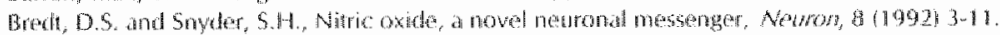

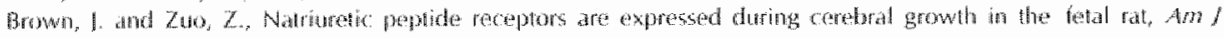

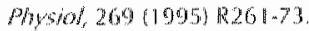

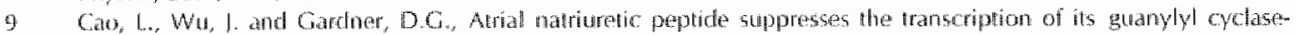

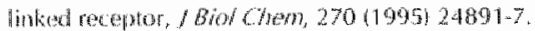

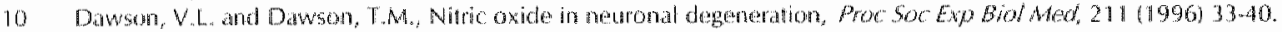

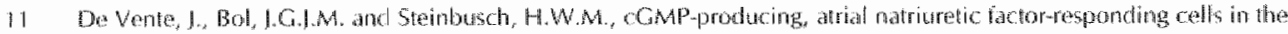
at brain: an immunocytochemical stedy. Eur/ Neurosci, $1(1989) 436-460$.

12 De Wente, H. Hopkins, D.A., Markerink-Van litesum, M. Emson, P.C., Schmidt, H.H.H.W. and Steinbusch,

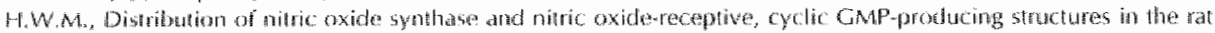

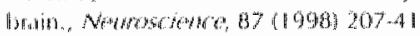

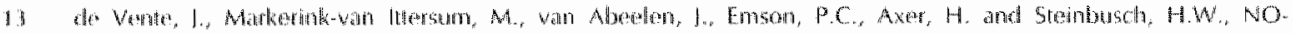

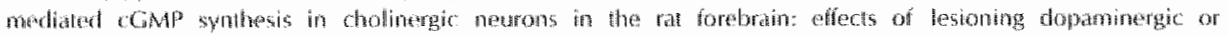

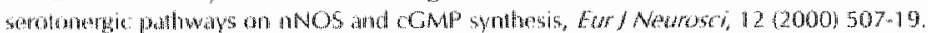

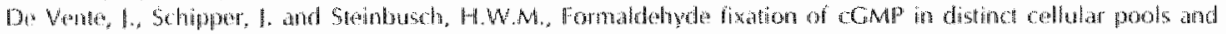

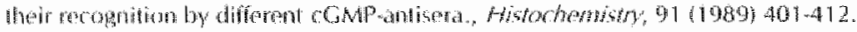

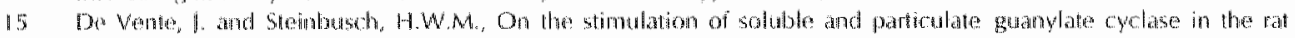

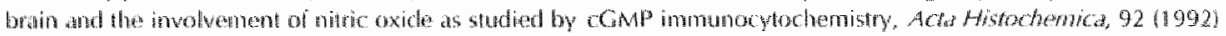
$13-38$

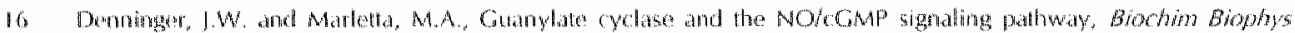

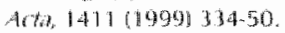

17 Ferrendell, 1.A. Distribution and reguldion of cyclic GMP in the central nervous system, Adw Cyche Nucleotide

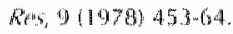

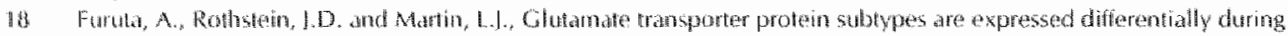
rat CWS development, / Newnari, $17(1997) 8363-75$.

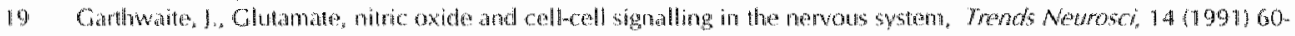
7.

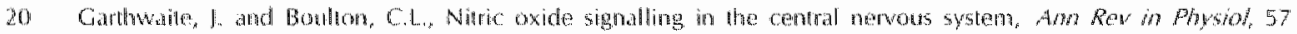
109051683-706.

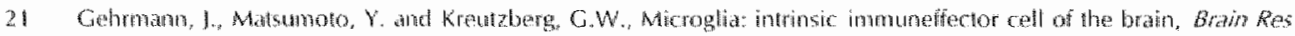
Bran Kes Ker, $20\{1995,269-87$.

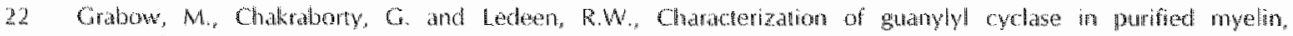
Niturethen Res, $21(1996) 457-62$. 


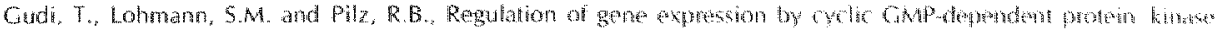

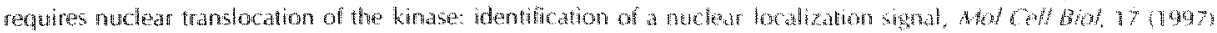
524454.

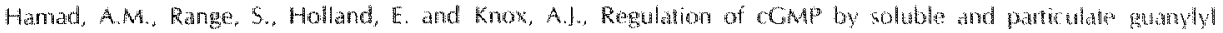

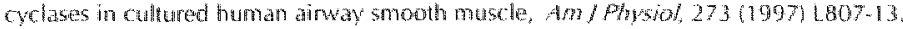

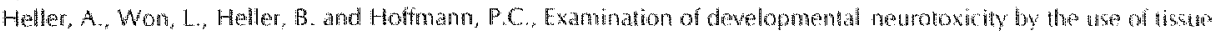

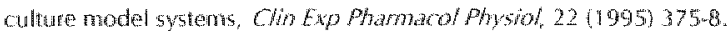

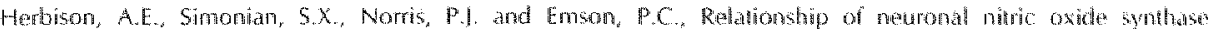

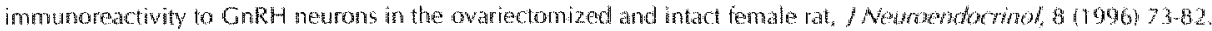

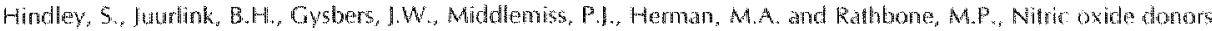

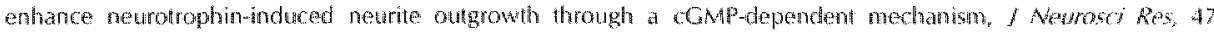
$(1997) 427 \times 39$

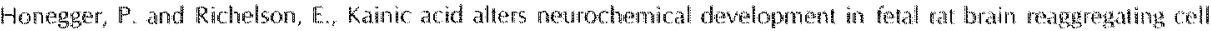
cullutes, Brin Res, 38 (1977) 580 -584.

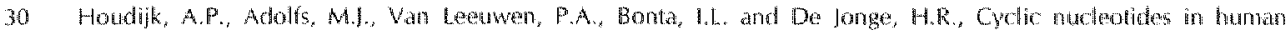

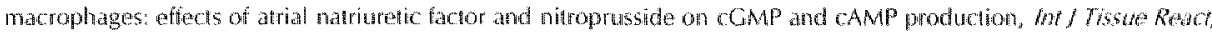
$131199 \div 287-93$.

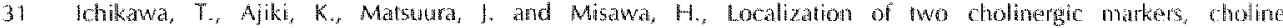

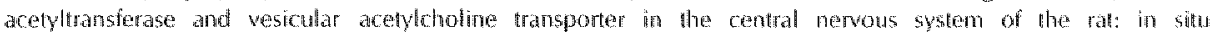

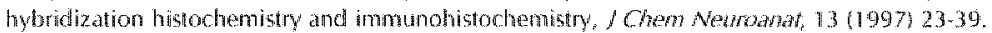

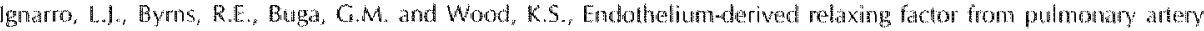

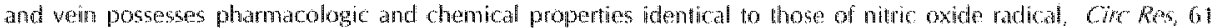
$(1967) 866-79$

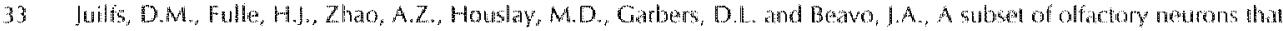

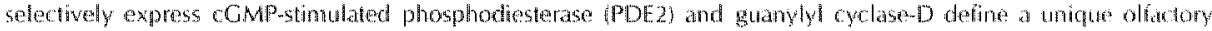

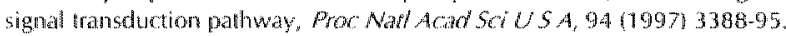

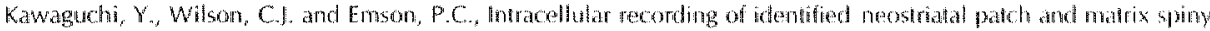

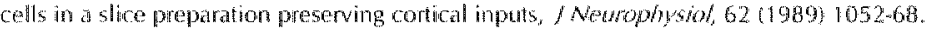

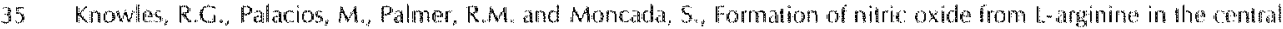

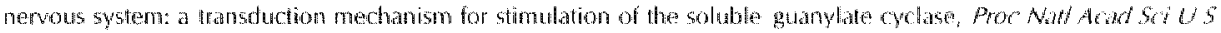
4. $8601989 \div 515962$.

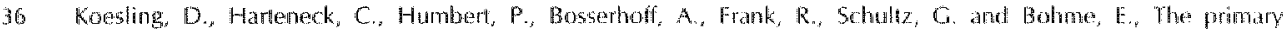

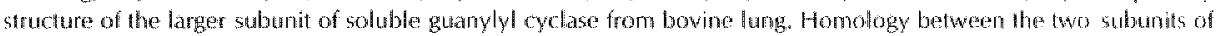
the enzyma, FEBS Lot, 266 (1990) $128-32$

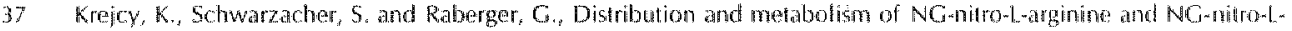

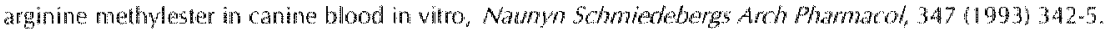

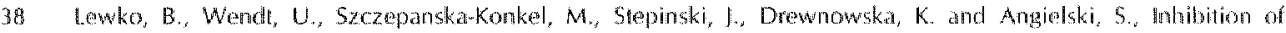

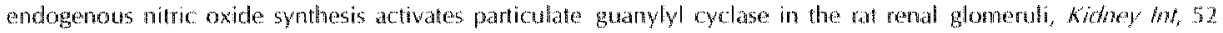
(1997) $654 \times 9$

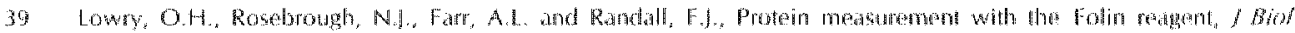
(1) $19 \%, 1991951) 265-275$

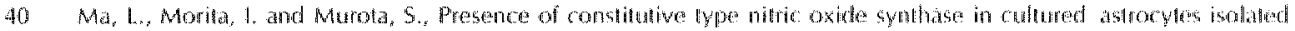
fron rat cerebra, Neurosci Lent, 17411994$) 123-6$.

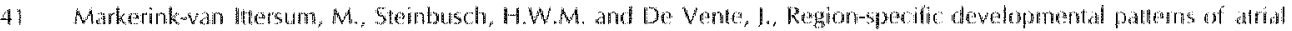

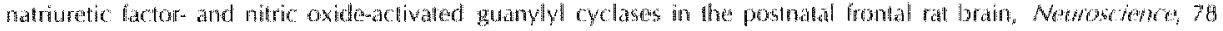
$(1997) 571.587$

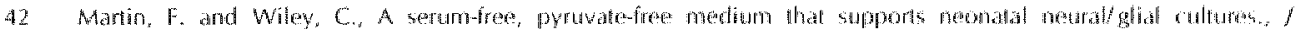
Nownosi Res, $41(1995) 246-258$.

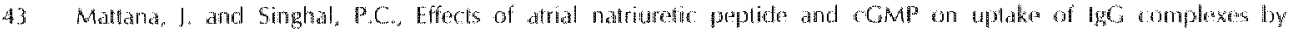
macroph

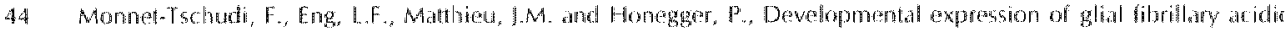

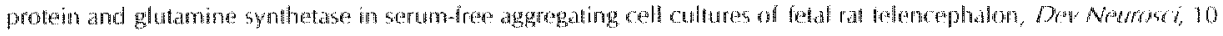
1098169.172

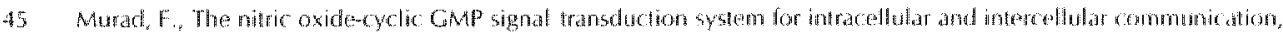
Racrof Prog Horny Ras, 191199423940.

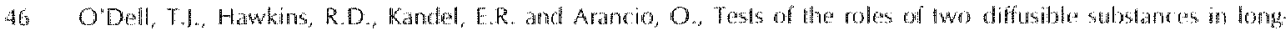

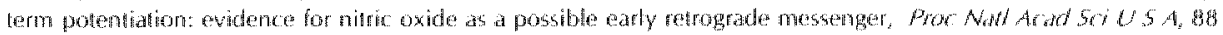
(1991) 112859.

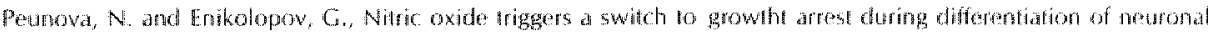

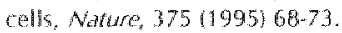

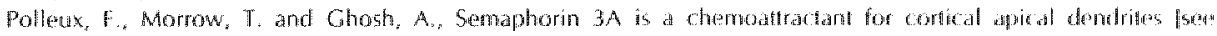
comments!. Ninfire, $404(2000) 567-73$

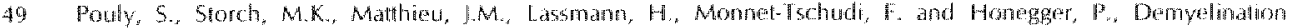




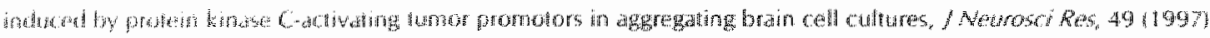
$21-132$.

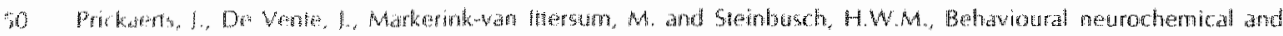

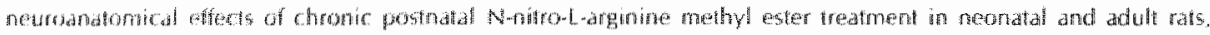

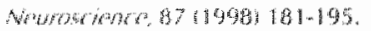

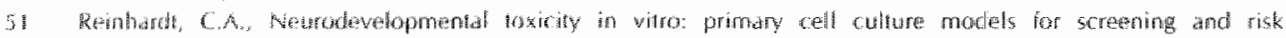

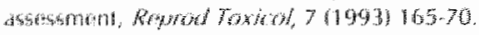

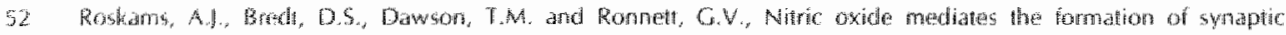

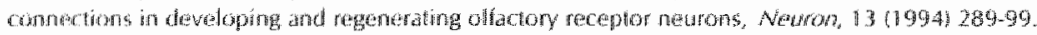

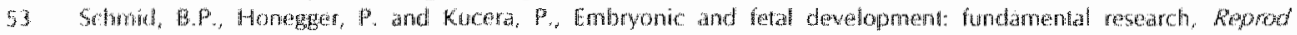
Towrot, 7 (1593) $155-64$.

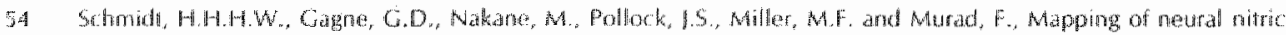

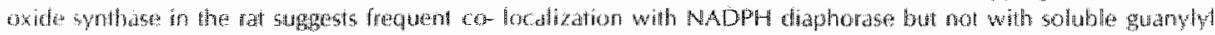

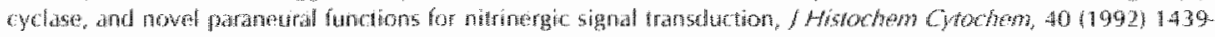
56.

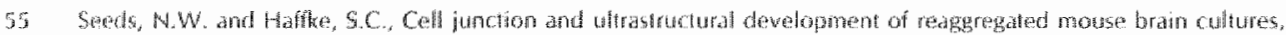

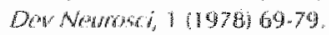

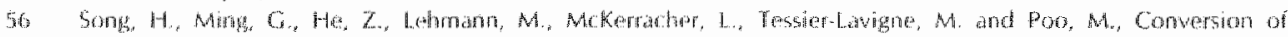

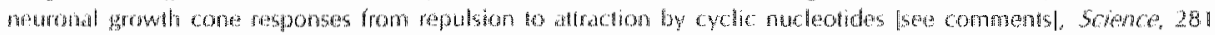
(1906) $1515-8$

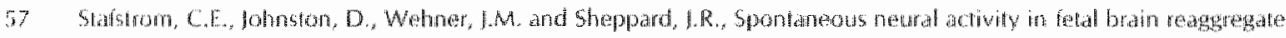

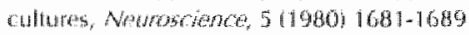

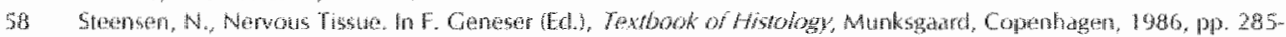
332.

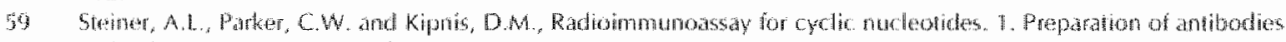

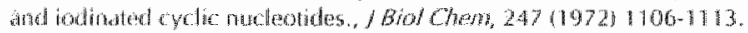

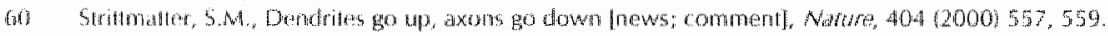

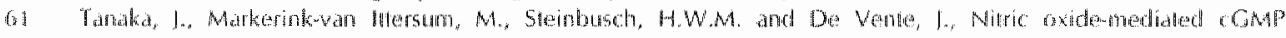

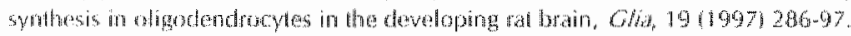

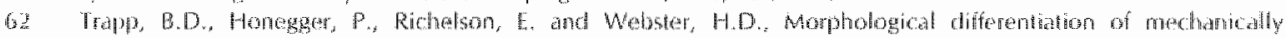

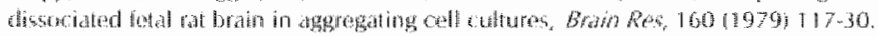

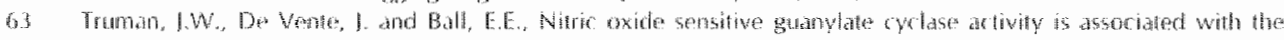

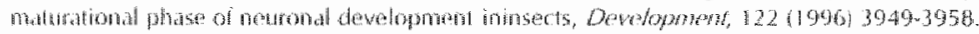

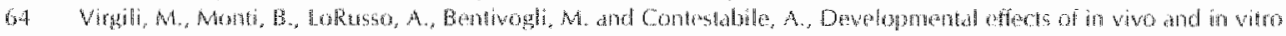

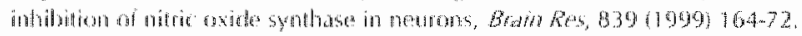

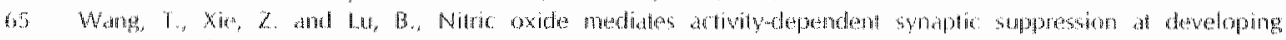

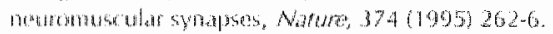

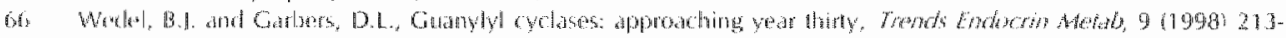
219.

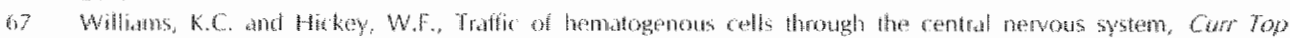

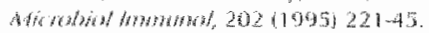

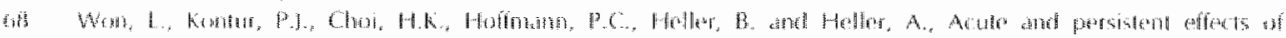

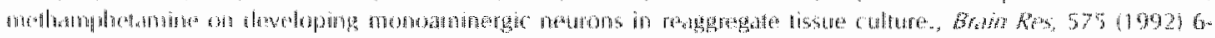
12 


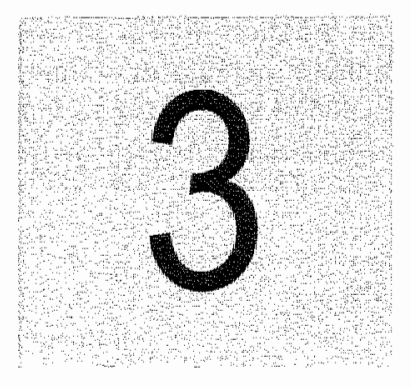

\section{Presence of soluble and particulate guanylyl cyclase in the same hippocampal astrocytes}

Charlote Teunissen, Harry Steinbusch, Marjanne Markerink-van Ittersum, Doris Koesling and Jan de Vente

European Graduate School of Neuroscience (EURON), Madstricht University, Department of Psychiatry and Neuropsychology, The Netherlands a Institut für Pharmakologie, Freie Universitat Berlin, Germany

Brain Research, 891/1-2 (2001) 206-212. 


\section{Abstract}

The localisation of particulate and soluble guanylyl cyclase was studied in hippocampal astrocytes. Counting the colocalisation of CGMP immunoreactivity with the astrocytic marker glial fibrillary acidic protein after stimulation of brain slices with sodium nitroprusside $(0.1 \mathrm{mM})$ or atrial natriuretic peptide $(100 \mathrm{nM})$, we were able to show that at least 67 \% of the hippocampal astrocytes contained both guanylyl cyclase isoforms. In addition, it was shown that a large number of atrial natriuretic peptide, brain-derived natriuretic peptide or sodium nitroprusside responsive cells contain the $\beta 1$-subunit of the soluble guanylyl cyclase. The results show that, in at least a subset of hippocampal astrocytes, soluble and particulate guanyly! cyclases are simultaneously present in the same cells.

\section{Introduction}

The second messenger malecule cyclic guanosine $3^{\prime}, 5^{\prime \prime}$-monophosphate (cGMP) is synthesised by the enzyme guanylyl cyclase. Two different forms of this enzyme are known, conventionally called particulate and soluble guanylyl cyclases (GO) (for reviews see: [58,59]). Particulate cyclase is a homodimeric membrane spanning molecule, with an extracellular domain containing the ligand binding site, a kinase homology domain forming the membraneanchoring region, and an intracellular part responsible for guanylyl cyclase activity. Up to now seven isoforms of the particulate GC receptors are known: GC-A to GC-F $[11,61]$. The ligands of particulate GCS are the matriuretic peptices: atrial natriuretic peptide (ANP), brain derived natriuretic peptide (BNP) and CNP. ANP and BNP have a preference for the ANP-A (GC-A) receptor isoform, and CNP for the ANP-B (GC-B) receptor isoform [61]. the soluble GC is a helerodimer consisting of an $\alpha$ and a $\beta$ subunit [24]. Presently, two isoforms of each subunit are known $(\alpha 1-2, \beta 1-2)[67]$. Both subunits and a haeme-group have to be present in order for the enzyme to be activated. The most important ligand of the soluble GC is nitric oxide, while another ligand, $\mathrm{CO}$, binds with less affinity $[11,33]$.

Guanylyl cyclase activity is found in every brain region studied, although there are large differences between activities in different areas [15]. In the rat brain, there is a close complementary relationship between the localisation of soluble $\mathrm{GC}$ and the enzyme nitric axide synthase (NOS) $[8,50,54]$. The soluble $\mathrm{GC}$ is abundantly present in neurones, however, it: is also found in many regions in astrocytes [10]. The particulate $\mathrm{GC}$ isoform has been described in astrocytes in discrete regions of the rat brain, such as the cingulate cortex, septum, corpus mamillare and hippocampus [7]. Thus, the suggestion has been made that both particulate and soluble GC might occur in the same astrocyte [7,9]. In the present studly we report that particuline GC and soluble GC are Co-localised in the great majority of the astrocytes in the hippocampus.

\section{Material and Methods}

Male Lewis rats were used (8-10 weeks of age). Animals were bred in the local animal facility. All experiments were approved by the local committee on ethical guidelines for animal experimentation according to $\mathrm{EC}$ regulations.

Animals were decapitated without anaesthesia and the brains were removed as quickly as possible and transferred into ice-cold oxygenated Krebs-bicarbonate buffer containing $1 \mathrm{~mm}$ 
IBMX to inhibit phosphodiesterase actiwity, as described previously 181 . Bain slces were prepared using a Vibroslicers, while submerged in the same icecold axygenated Krebs bicarbonate bufter. Experiments with brain slices $1300 \mathrm{~km}$, incubated in vitro, were perfomed as described previously [8]. Slices were slowly warmed to $35^{\circ} \mathrm{C}$ and after $45 \mathrm{~min}$ panticulate GC. was stimulated with 100 nM ANP (atriopeptin 111 , fragment $5-28$, Sigma) or 100 NM BNP (BNP. 45. Sigmaj and soluble GC was stimulated with $0.1 \mathrm{mM}$ sodium nitroprusside (SNP) as NOdonor for 10 minutes. Cryostat sections $(10 \mu \mathrm{m})$ were incubated ovemight at $4 . \mathrm{C}$ with sheep anti-formaldehyde fixed CGMP (1:4,000) [58], mouse anti-GFAP (1: 10, Innogenetics), rabbit anti- $\beta)$ subunit of soluble GC $\{1: 1,000[34])$ or with combinations of these antibodies. The specificity of the antibody against the $\beta \|$ subunit of soluble GC has been described previously [25]. It was demonstrated that preabsorption of the antiserum with the native $\beta-1$ peptide abolished immunostaining [36]. The sheep antibody was visualised using Alexa-488 conjugated donkey anti-sheep immunoglobulins (Molecular Probes) diluted 1:100, whereas the mouse and rabbit antibodies were visualised using Cy3 conjugated donkey anti-mouse antiserum diluted $1: 800$ and donkey anti-rabbit antiserum (1:800, Jackson, respectively. The experiments were repeated 4 times.

Sections were examined with an Olympus AX-70 TRF microscope, equipped with a narrow band MNIBA-lype FITC filter, or a MNG filter for Cy3 fluorescence (Chroma Technology Corporation). These filters made it possible to photograph FITC or Cy3 fluorescence without any bleeding of the other fluorescent marker through the filter [B]. Double labelling of structures was defined as positive fluorescence observed through both the MNIBA filter and the MNG filter in the same structure.

The number of cells in which cGMP immunostaining co-tocalised with the astrocytic marker GFAP was counted in the hippocampal CAUCA3 area of sllices incubated in the presence of $100 \mathrm{nM}$ ANP or $0.1 \mathrm{mM}$ SNP. Therefore 30 fields from the CA1/CA3 area from four experiments were photographed for both CGMP and GFAP (see Fig. 1C.F for representative samples). Counting was performed by two different observers and the average number was calculated. The inter-observer variation was $12 \%$. The procedure was also repeated for randomly chosen pictures, to callculate an intra-observer variation, which was $9 \%$.

\section{Results}

In the absence of a phosphodiesterase inhibitor, CGMP-immunoreactivity (CGMP-IR) was observed only very weakly in blood wessel walls (Fig. 1A). In the presence of $1 \mathrm{mM}$ isobutylmethylXanthine (IBMX), a non-specific phosphodiesterase inhibitor, weatk cGMP-IR was found in blood vessel walls and some varicosity's could be observed Ifig. IB. All further experiments were performed in the presence of $1 \mathrm{mM} I B M X$.

As has been reported before, ANP stimulation of particulate GC in hippocampal stices resulted in CGMP-IR in every area of the hippocampus in cells with the characteristics of actrocytes (Fig. 1Dy. Double immunostaining with GFAP indeed showed that the ANPresponsive cells were astrocytes (Fig. 1O). Similarly, we were able to show that SNP stimulation of soluble GC also resulted in CGMP-IR in astrocyles in the hippocampus (Fig. 1E-F). The effect of SNP in the astrocytes was generally lless pronounced than that caused by ANP stimulation. As expected, the $\beta 1$-subunit of soluble GC was tound in cellular structures, astrocyles or neurones, which are immunoreactive for CGMP after stimulation with $0.1 \mathrm{mM}$ SNP /Fig. 2A-B. Colocalisation of the BI subunit with CGMP-IR in slices stimulated with $100 \mathrm{nM}$ ANP or BNP was seen in cellular structures with the characteristic morphology of astrocytes (Fig. 2C-F). 

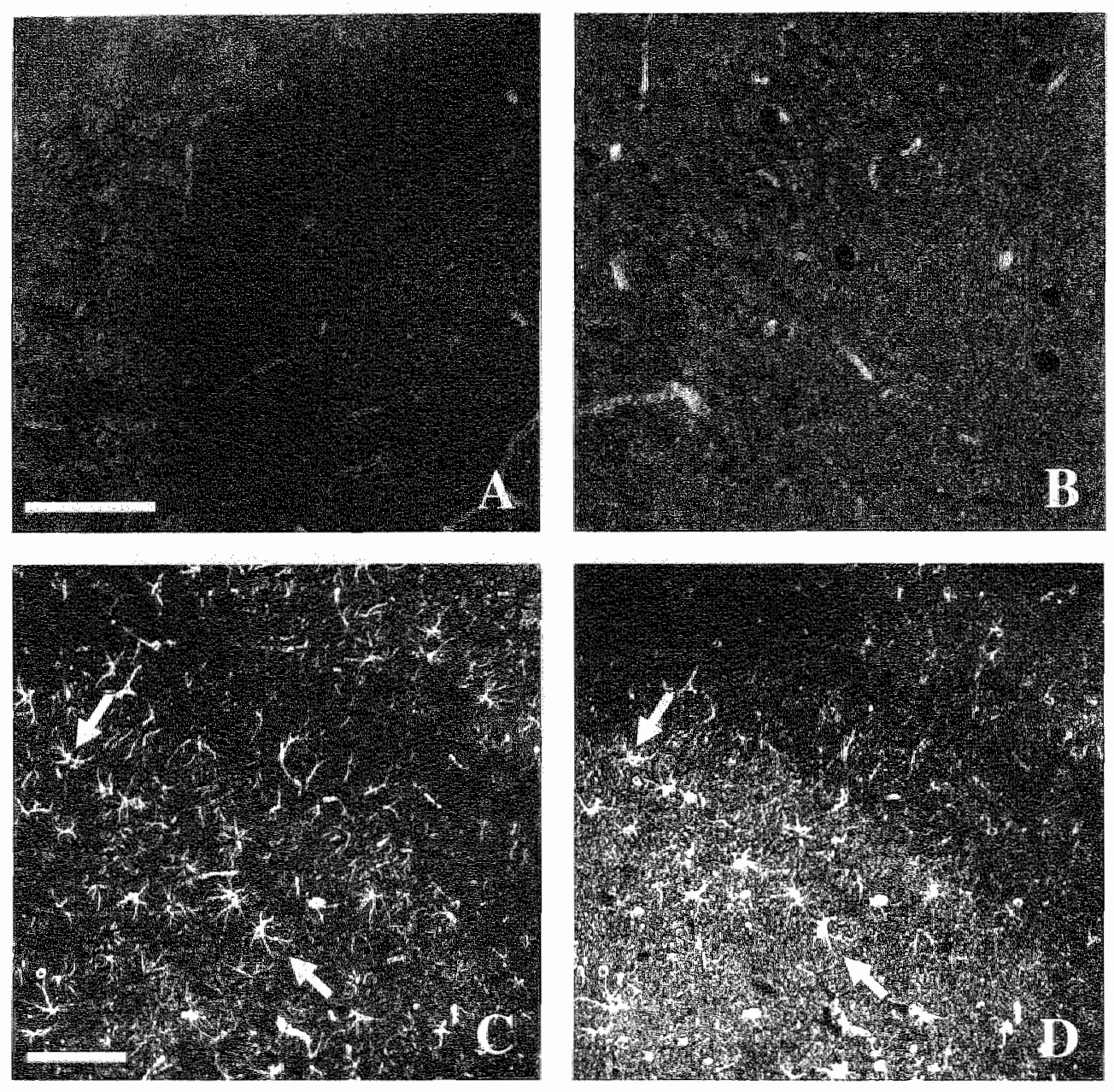

W.7. M. W 1 m,

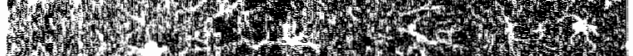
1.

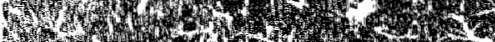
H.

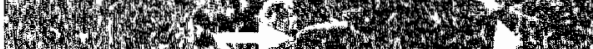

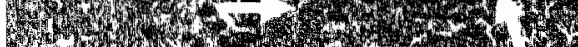
Wry T.7. 17.

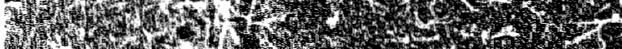

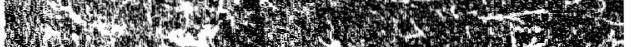
1. W.

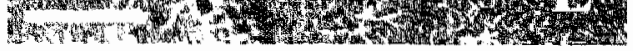

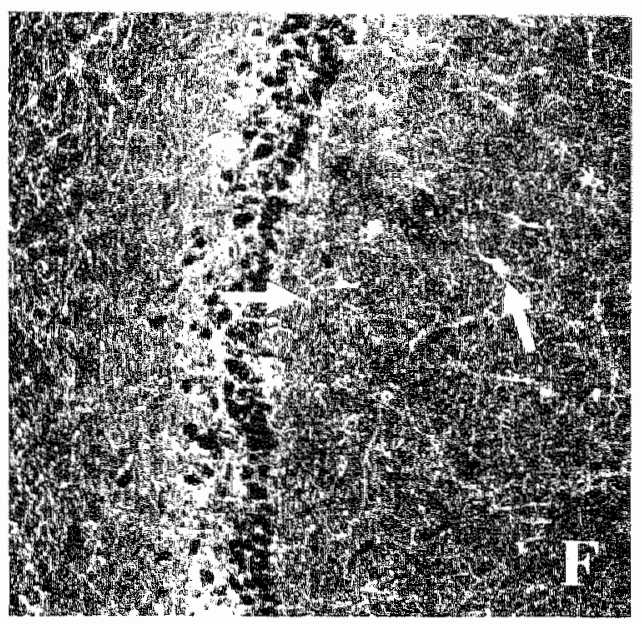


Fig. 1 Localisation of CGMP inmunoreachive in astrocytes,

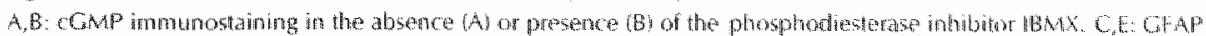

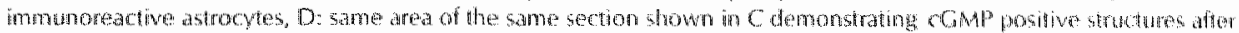

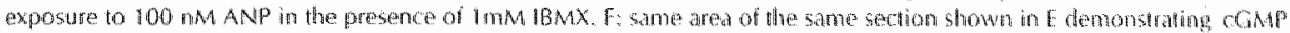
positive structures after exposure to 0 .

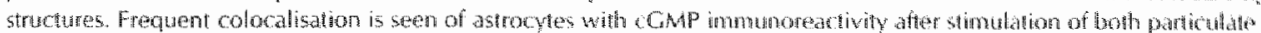
and soluble GC thar wa $100 \mathrm{pmi}$.

We assessed the number of ANP-responding, CGMP-producing astrocytes in the hippocampus. A total number of 870 GFAP-positive cells was counted in 12 sections from 4 experiments. Of these GFAP positive cells $90 \%$ (sem.: $1 \%$ were found to be ANP-responding. Similtarly, in another series of 18 sections we counted a lotal number of 1074 GFAP positive cells, of which $77 \%$ (5.em: $4 \%$ were found to be SNP-responding. Taking into account the $10 \%$ of the ANP responsive cells which were not identified by CFAP as being astrocytes, these figures indicate that at least $67 \%$ of the astrocytes in the hippocampal CA1/CA3 area contain both particulate $\mathrm{GC}$ and soluble $\mathrm{GC}$.

\section{Discussion}

In this investigation we present evidence that particulate and soluble GC can be present in the same astrocyte in the hippocampus. There are several studies using a biochemical assay of cGMP on either particulate or soluble GC activity in astrocyles, which indicate the presence of both enzymes in rat astroglial cell cultures $[12,55,64]$. Similarly, the presence of both GCS has been described in cultured cardiac myocytes and fibroblasts [4], vascular smooth muscle cells (e.g. $[43,45]$, human airway smooth muscle cells [23], human articular chondrocytes [17] and brain endotheliall cells [55]). However, the coexistence of the two forms of GC in the same cell has never been demonstrated. In addition, expression of GC genes in astroglial cultures may be different from the in vivo stuation. This is demonstrated by in vitro slice-studies demonstrating dramatic changes in functional activity of soluble and particulate GC during development $[10,42]$. Thus, using two independent immunocytochemical approaches, double immunostaining for CGMP-IR and the $\beta 1$ subunit for guanylyl cyclase as well as cell counts based on the number of CGMP-IR cells doublestaining for the astrocytic marker CFAP, we demonstrated the presence of both GCs in the same astrocytes.

Several reports described the presence of $\alpha 1$ and $\beta 1$ subunits of $\mathrm{sCC}$ in the hippocampal pyramidal and graritule cell layer $[16,19,41]$. In addition, the presence of sGC was demonstrated in these cells layers using a monoclonal antibody against the a subunit of SGC $[50 \mathrm{~A}$. Although the $B 1$ subunit was found to be present in the pyramidal cells (Fig. 2B,D,F, CCMP-IR was absent in these cells. The reason for this discrepancy is unknown. It might be that the sGC in the pyramidal (and granule cells in the dentate gyrus) is still in a form which cannof be activated by $\mathrm{NO}$.

The role of the CGMP in the various cellular brain compantments is not yet known. Several potential target molecules for CGMP are known. CGMP may be involved in gating cyclic nucleotide regulated ion channels which have a widespread distribution throughout the brain (e.g. $[32,56]$ ). In addition, CGMP might be irvolved in controlling phosphodiesterase activity $[2,27]$. In the past tew years it has become dear that cCMP-dependent protein kinases also have a wide-spread distribution throughout the central nervous system $\llbracket 13,14,40 \rrbracket$. The presence of COMP-dependent protein kinase-ll has been demonstrated in astrocyles and may be involved in gene expressiom [20,39]. Recently, is was shown in cuttured astrocytes that NO-CGMP signalling was involved in regulating the intracellular calcium concentration and intercellular calcium wawes through an interaction with the ryanodine receptor [62]. Although it has been 

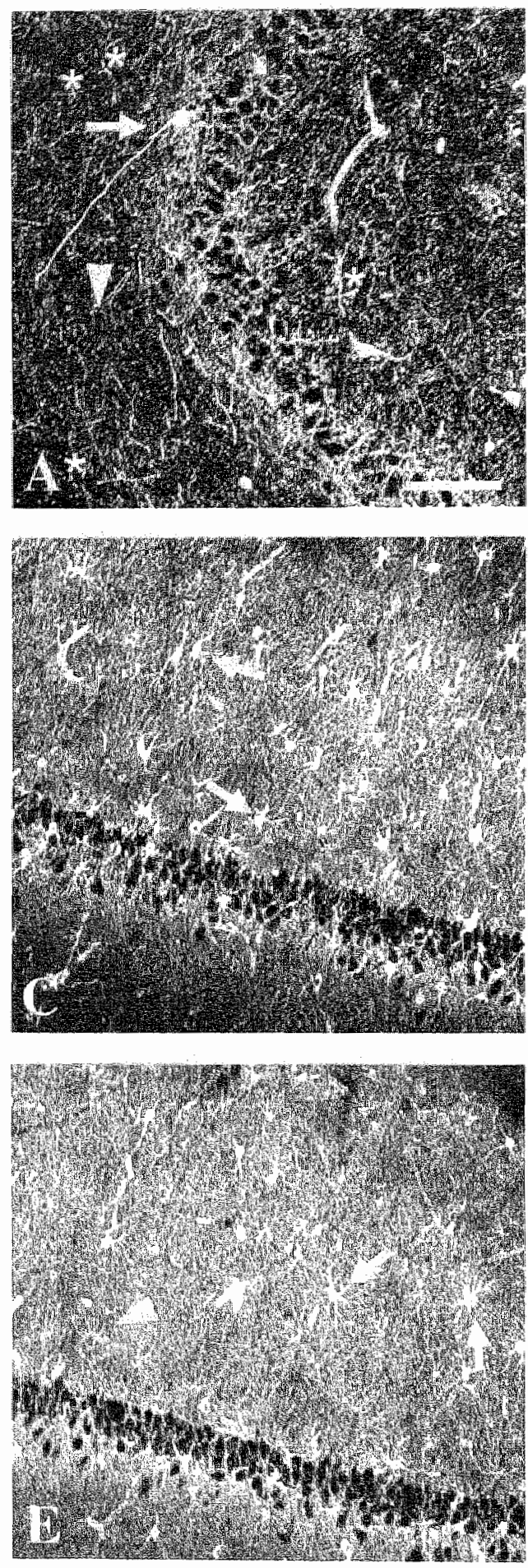
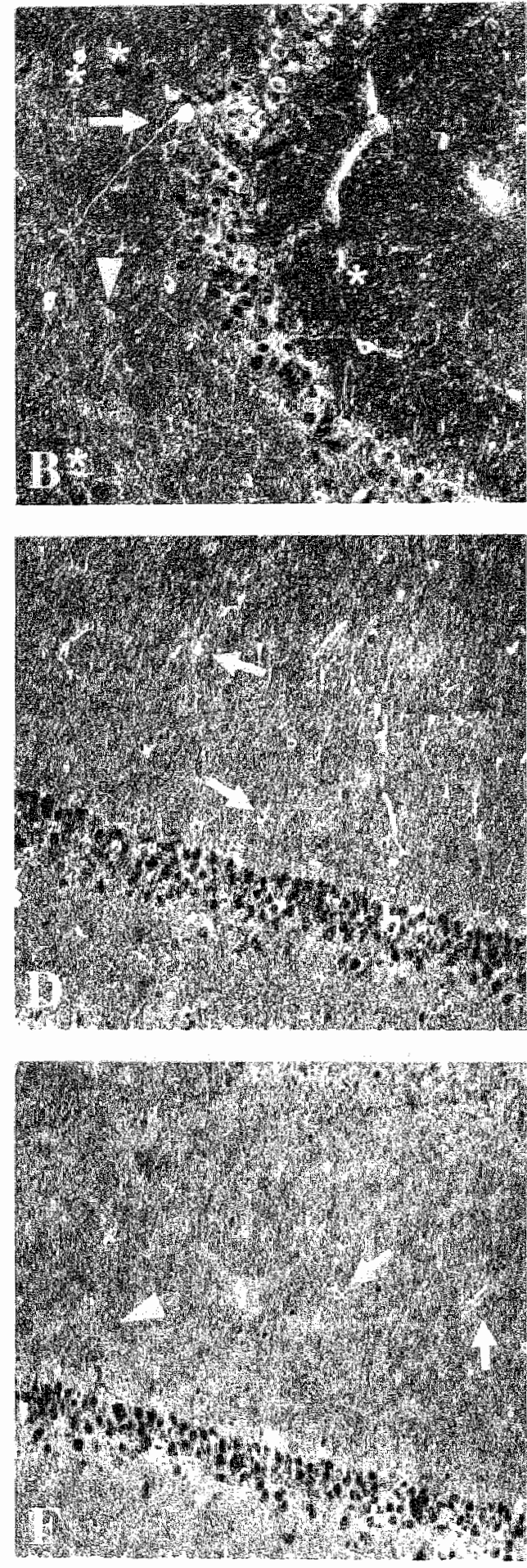


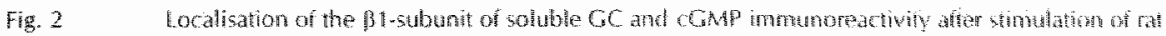

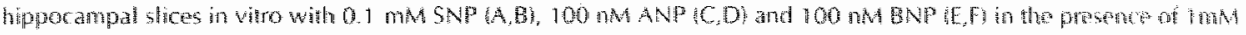
IBMX.

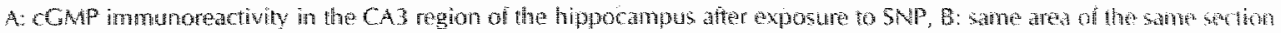

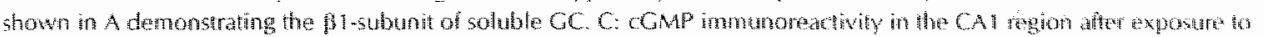

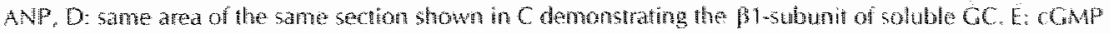

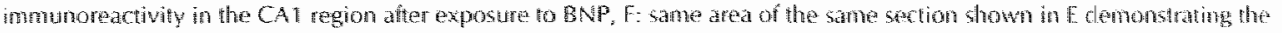

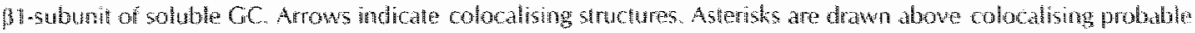
astrocytes. Arrowheads indicate struntures without apparent colocalisation ibar = 100 ynal.

described several times that in neuronal cells cyclic nucleotides can phosphorylate the IP3 receptor, which resulls in a diminished IP3 sensitive callium release $[44,57,63]$, Wilmott et al. 162] found that the IP3 sensitive calcium release in astrocytes was not affected by the NO-CGMP pathway. Our results demonstrate that cGMP concentrations can be increased in astrocytes by wwo different ways, i.e activation of particulate GC or activation of soluble GC. In contrast to the situation in cultured astrocytes [62], neuronal NOS is not expressed constitutively in astrocytes in vivo or in short term incubated slices and this implies that both triggers for the $\mathrm{GC}$ cGMP pathway in astrocytes are extracellular signals. It might be possible that both pathways exert a similar effect as has been described for neurite outgrowth in neuronal PC12 cells [26] and vasorelaxation $[11,30]$.

The presence of ANP in the rat hippocampus has been demonstrated $[49,53]$ and in addition, the ANP binding sites have been reported in low concentration in the same region $[18,37,41,48]$. One major problem is that the function of the matriuretic peptides in the hippocampus is unknowm. Anti-proliferative and/or anti-mitogenic effects of ANP has been reported in astrocytes $[28,38,47]$, mesangial cells $[1,5,31,51]$, airway smooth muscle cells: $[22,29]$, myolibroblastic hepatic stellate cells $[59]$, chondroprogenitor cells $[46]$, cardiac myocytes and fibroblasts [4] and thymocytes [60]. On the other hand, stimulatory or accelerating effects of ANP have been reported on cardiomyocytes $[35,52]$ and chondrocytes $[21,65]$. In addition, it has been demonstrated that natriuretic peptides can regulate gene expression, e.g. tissue factor and plasminogen activator inhibitor- 1 in endothelial cells $[6,66]$. The early presence of matriuretic peptide receptors in the foetal rat brain led Brown and Zuo [3] to suggest that natriuretic peptides may have growh regulationy properties in the embryonic cerebral contex. In addition, we showed that ANP strongly stimulated GMP synthesis in developing astrocytes in the rat forebrain, which effect declined during maluation 1421. Nevertheless, there is presently no evidence that natriuretic peptides have an antiproliferative function in the in vivo rat brain.

In conclusion, we have shown that particulate and soluble GC can occur in situ in the same hippocampal astrocyte. The functional relevance of this observation remains to be clarified.

\section{References}

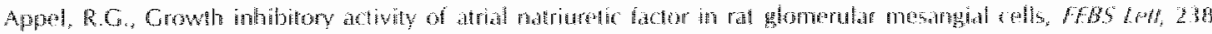
(1) 198$) 135.8$

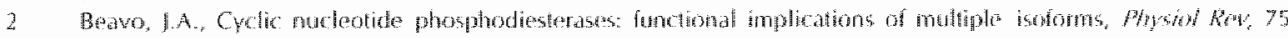
$(1995) 725-48$

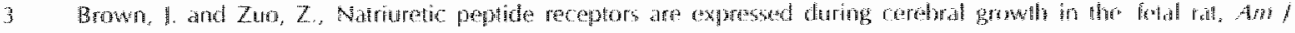

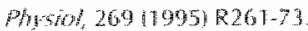

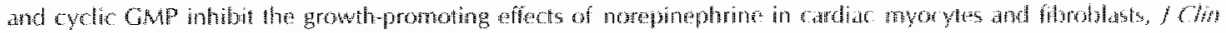

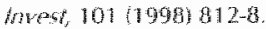

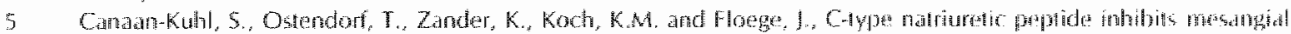

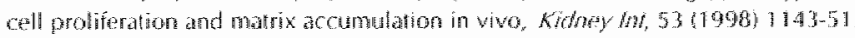

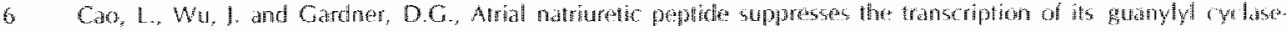

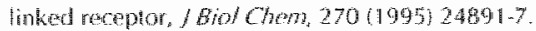




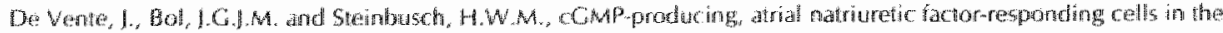

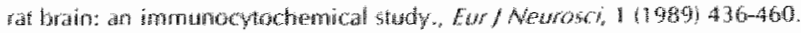

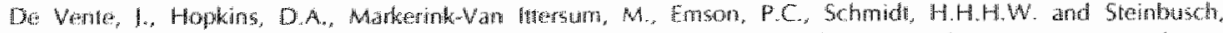

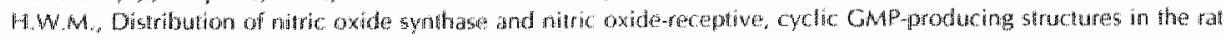

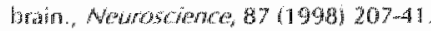

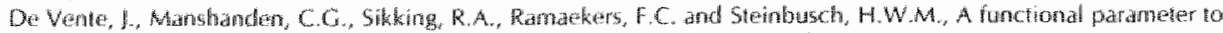

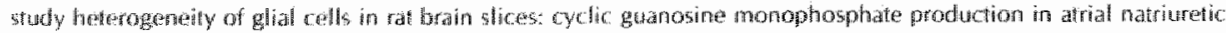

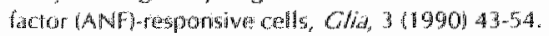

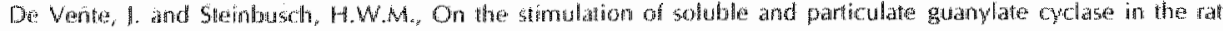

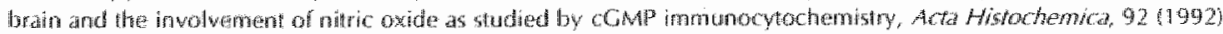
13.38 .

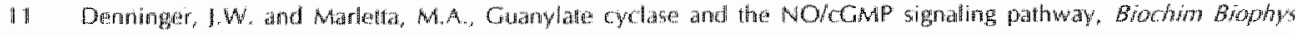
Acta, 44111999133450 .

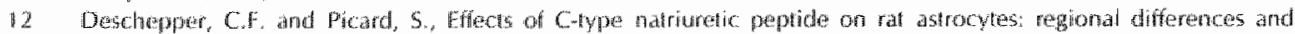

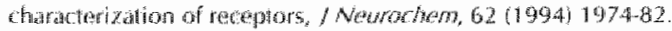

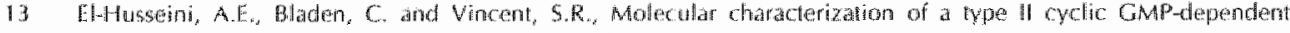

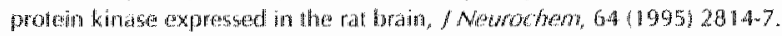

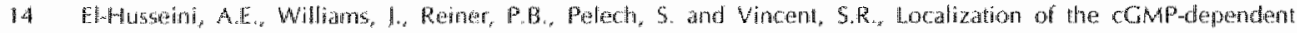

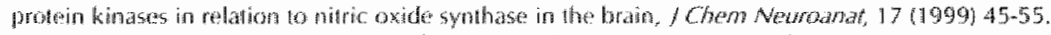

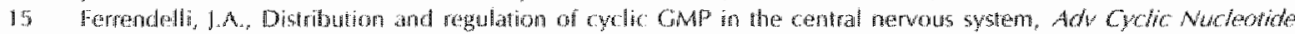
kess, $9(19789453-64$

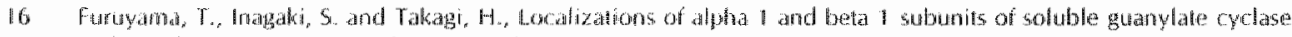
in the ral lyain, Brom Res Alol Brain Ress $20119991335-44$.

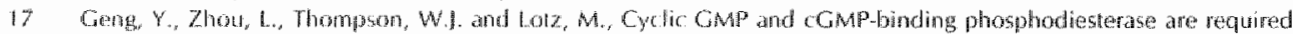

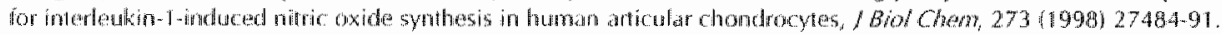
Cibson, T.R., Witdey, G.M. Manaker, S. and Glembotski, C.C., Autoradiographic localization and characterization

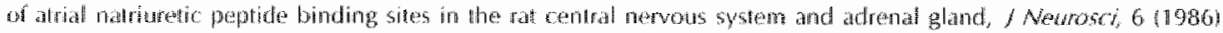
$2004-11$.

19 Giuili, G., Luzi, A. Poyard, M. and Guelawn, G., Expression of mouse bram soluble guanyly cyclase and No synthase during ontlogeny, Bran Res Dew Bran Res, 81 (1994) 269-83.

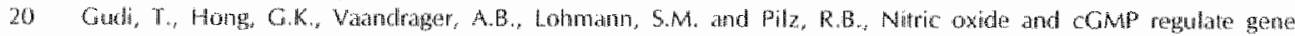

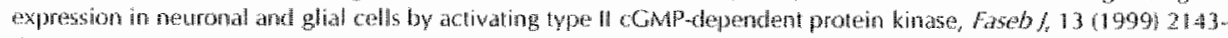
52

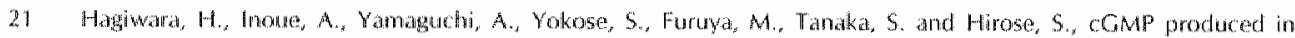

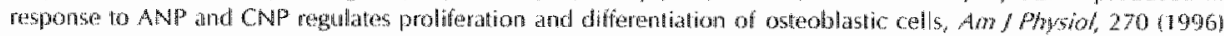
C1311-8.

22 Hamad, A.M., Johnson, S.R. and Knox, A.s. Antiproliferative effects of NO and ANP in cultured humart airway smooth mascle, Am / P/y,sitol, 277 (11999) L910-A.

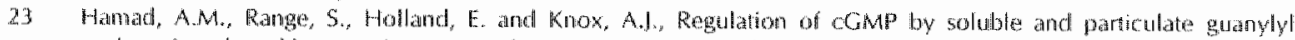
Cyclases in cultured human dirwaly smodh muscle, Aw/ Phy sol, 273 [1997) L807-13

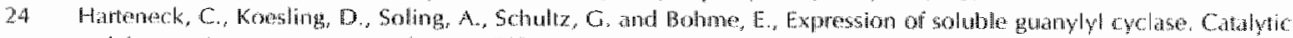

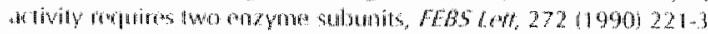

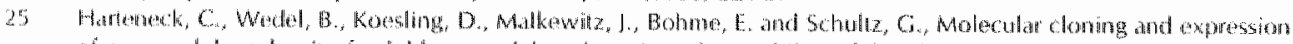

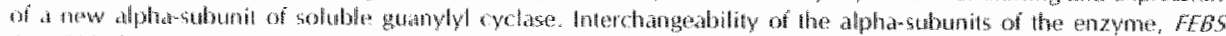

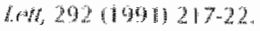

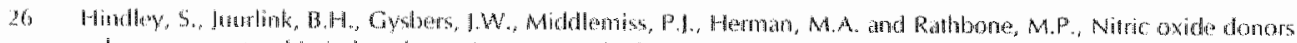

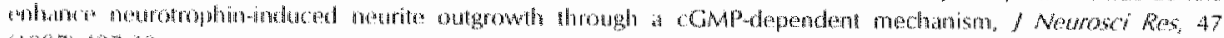
$(1967)+27-39$

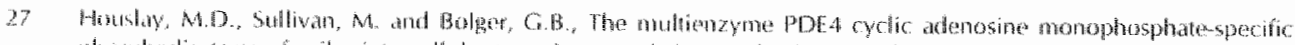

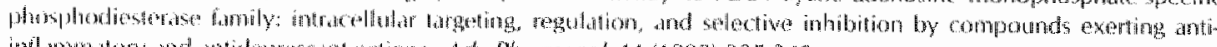

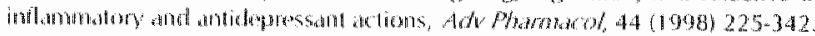

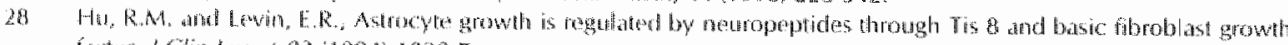

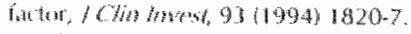

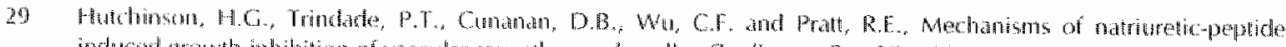

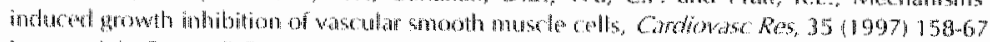

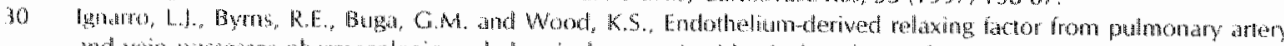

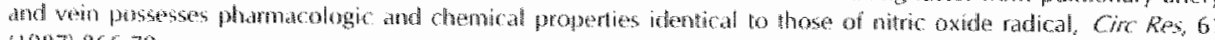
$(1987) 860.79$

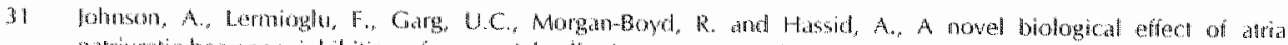

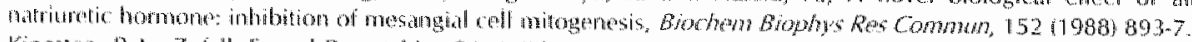

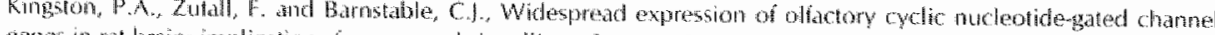

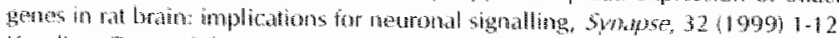

31 Kow

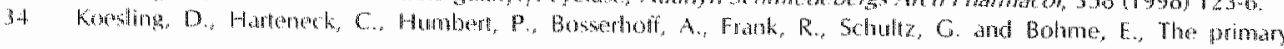




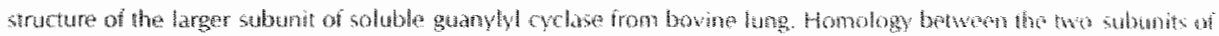

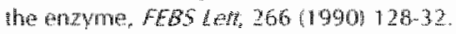

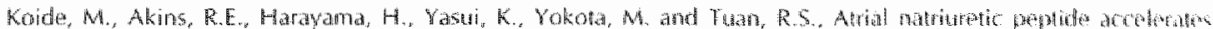

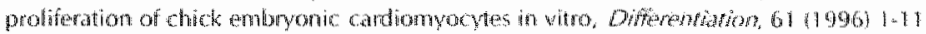

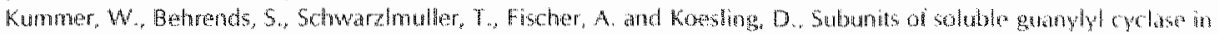

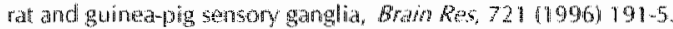

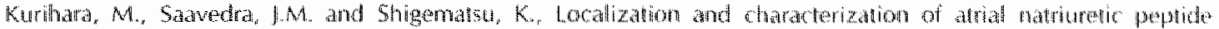

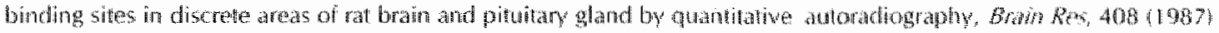
31.9.

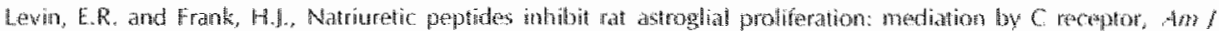
Physil, 261 (1991) R453-7.

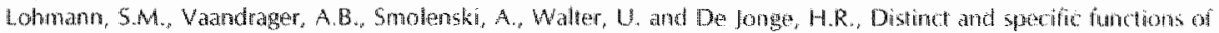
cGMPdependent protein kinases, Trends Biochen, 56,22 (1997) $307-2$.

Lohmann, S.M., Walter, U., Miler, P.E., Greengard, P, and De Camili, P., mamunohisochemeal focalizaton of

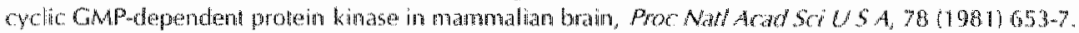

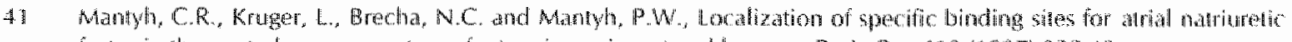
factor in the cental nerwous system of rat, guinea pig, at and thuman, Brom Res, 412 (1987) 32942 .

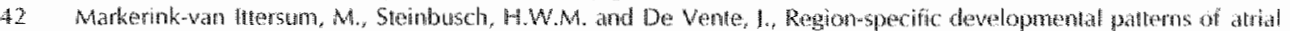

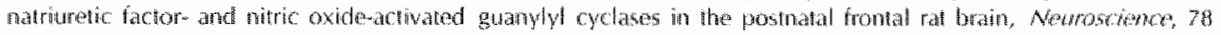
(1997) $571-587$.

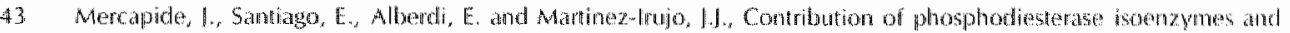

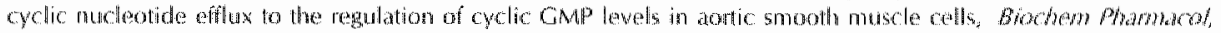
$5811999) 1675-183$.

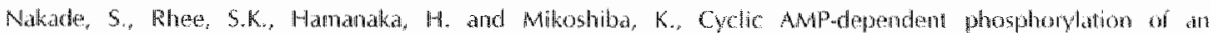

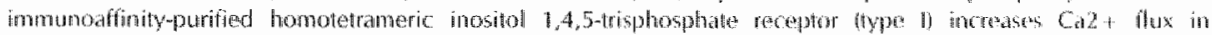
reconstituded lipid wesiches, f Bio/ Chan, $269(1994) 6735 \times 42$.

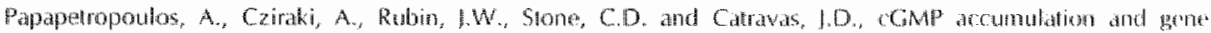

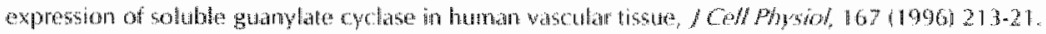

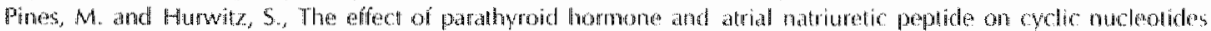

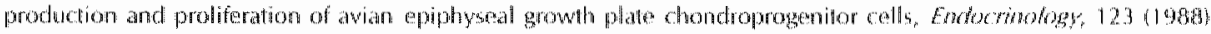
$360-5$.

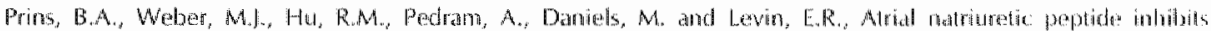

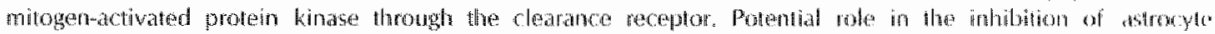
protiferation, / Brö Chern, 271 (1996) 14156062.

48 Quition, R., Dalpe, M. and Dam, T.W., Characterization and distribution of recoptors for the atriat natrunetic peptides ir mentamalian brain, Proc Not Acad Sci L 5 A, 83 (1986) 174-8.

49 Saper, C.B., Wufley, K.M. Moga, M.M., Hotmes, H.R., Adans, S.A. Leahy, K.M. and Needleman, P., Brain

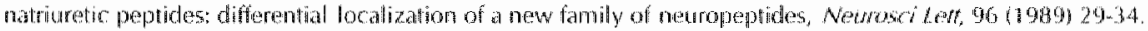

50 Sohmidt, H.H.H.W., Gagne, G.D. Wakane, M., Pollock, J.S., Miller, M.F, and Murid, K, Mapping of puen ail nitric oxide synthase in the rat stggests frequent co localization with MADPH diaphorase but not will soluble getarylyl

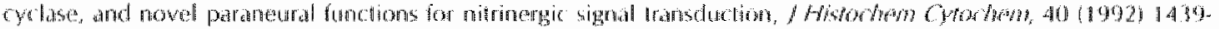
56 .

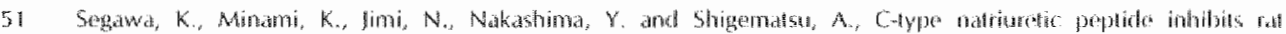

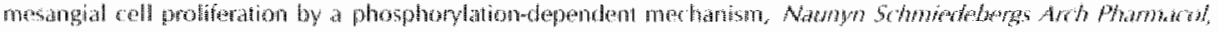
$35701998770-6$.

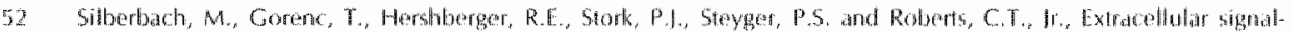

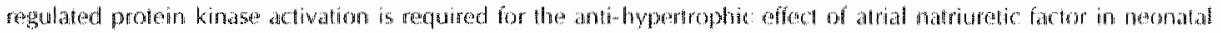
rat wentricular myocytes, 1 bin/ Chent, 274 (1999) $24850-64$.

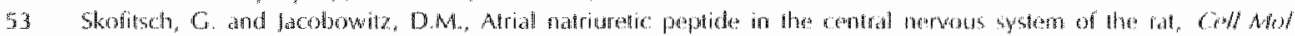

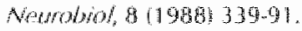

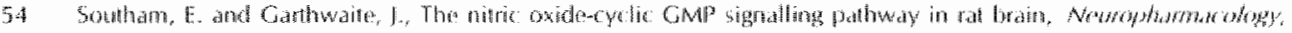
$32(199) \sqrt[1267-77]{ }$

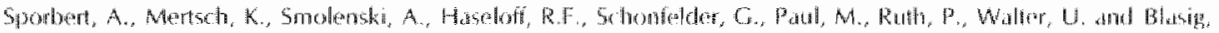

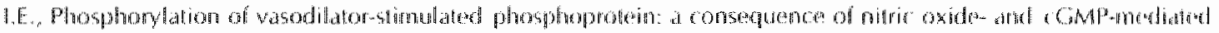

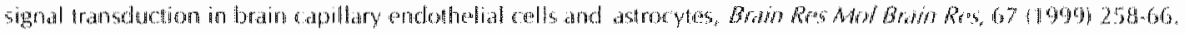

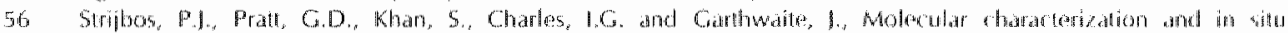

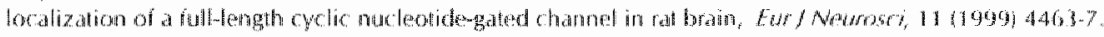

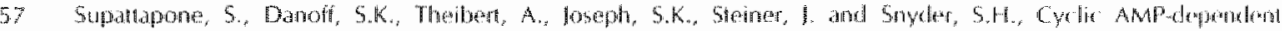

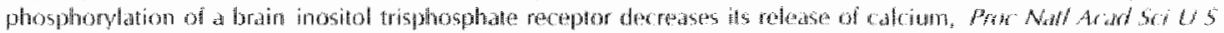
4. 85,198937874750 .

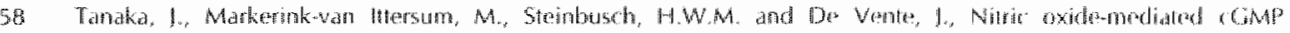

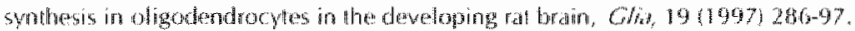

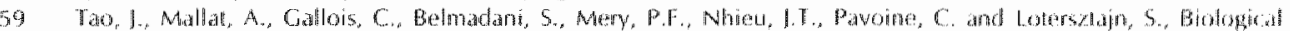

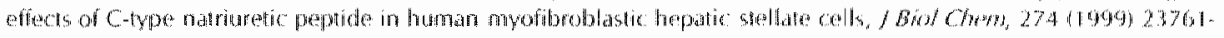


9.

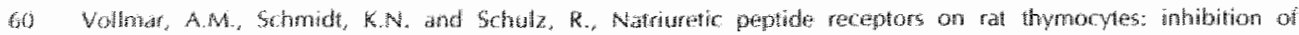

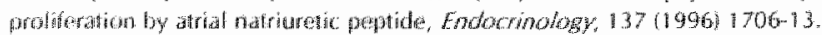

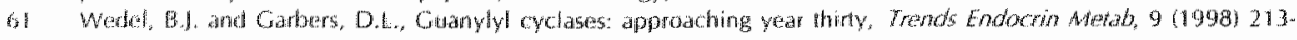
219.

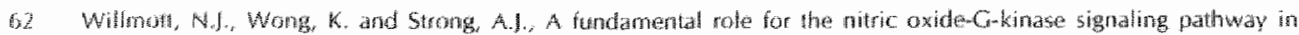

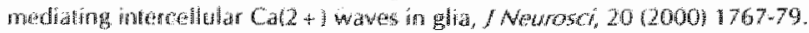

63 Wojcikevicz, R., and Lug, S.C., Phosphoryation of inoshol 1,4,5-trisphosphate receptors by cAm-dependent

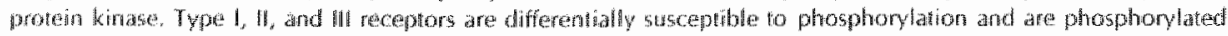

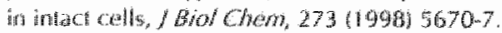

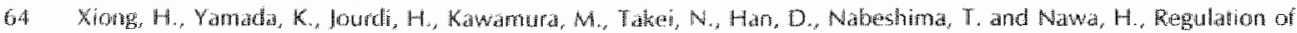
nerwe growh factor release by nitric oxide through cyctic GMP pathwey in cortical glial cells, Mol Pharmacol, 56 19999133947.

65 Yasoda, A, Ogawa, Y, Suda, A., Tanura, N, Mon, K., Sakuma, Y., Chusho, W, Shota, K., Tanaka, K. and Nakao,

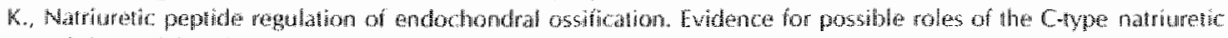

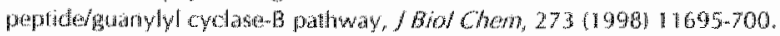

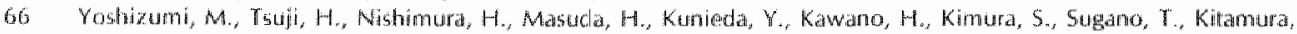

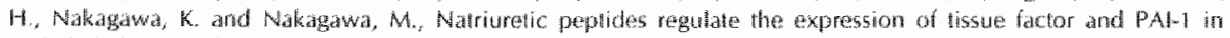

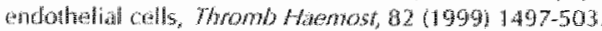

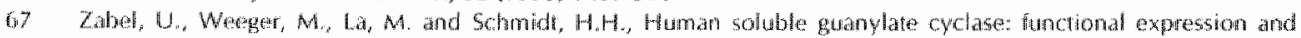

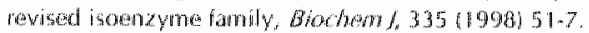




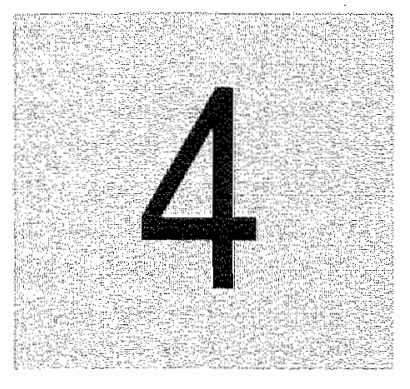

\section{Evaluation of 3-nitrotyrosine as a marker} for 3-nitropropionic acid induced oxidative stress in Lewis and Wistar rats and strainspecific whole brain spheroid cultures

Charlotte Teunissen, Marjanne Markerink-van Ittersum, Chris De Bruijn, Harry Steinbusch and Jan De Vente

European Graduate School of Neuroscience (EURON), Maastricht University, Department of Pychiatry and Neuropsychology, The Netherlands 


\begin{abstract}
3- Witroryrosine has been proposed as a marker for neurodegenerative processes related to oxidative stress or excitotoxicity in the central nervous system. The aim of the present study was to inwestigate whether 3-nitrotyrosine can be used as an early marker for neurodegenerative processes. We investigated the sensitivity of 3-nitrotyrosine formation in a whole brain spheroid culture model as well as in a rat model after 3-nitropiopionic acid exposure, using Lewis and Wistar rats. Increased 3-nitrotyrosine concentration in spheroid cultures from Lewis rats was observed at lower dose of and shorter exposure time to 3-NP as compared to alterations in glial fibrillary acidic protein concentration, decrease in glutamine synthetase activity or cell loss. Exposure to 3-ritropropionic acid resulted in decreased slaining of GABAergic neurons, whilo neuronal nitric oxide synthase staining was preseved. Dithiothreitol and vitamin $\mathbb{E}$, but not glutatione, inhibited the formation of 3-nitrotyrosine. Interestingly, $\mathrm{N}^{C_{-}}$-nitro-L-arginine methyl ester increased the 3-nitrotyrosine fomation. Spheroid cultures obtained from Wistar rats did not show increased 3-nitrotyrosine concentration after exposure to 3-nitropropionic acid during five days. In the striatum of 3-nitropropionic acid exposed Lewis and Wistar rats, no change in 3-nitrotyrosine concentration was observed, whereas only in Wistar rats the glial fibrillary acidic protein concentration was increased in addition to activation of microglial cells.

It is concluded that 3-nitrotyrosine was a more sensitive marker for oxidative stress than glial fibrillary acidic protein and glutamine synthase in spheroid cell cultures of Lewis rats. The strain-clependent formation of 3-nitrotyrosine residues indicates an influence of genetic background on the sensitivity of biological markers for neunodegeneration. Finally, the similarities between the 3-nitropropionic acid spheroid model and the vivo model indicate that the spheroid cultures provide a good alternative for chronic exposure of animals to neurotoxins.
\end{abstract}

\title{
Introduction
}

Neurodegenerative diseases including Alzheimer's disease (AD), Parkinson's disease and Huntington's disease, inwolve processes like excitotoxicity and oxidative stress [4,61]. Altered concentrations of several cellular markers for oxidative stress have been observed in brain parenchyma as well as in cerebrospinal fluid of patients with neurodegenerative diseases. For example, the presence has been shown of increased concentrations of oxidised proteins, such as carbonyl formation, and loss of creatine kinase or glutamine synthetase (GS) activity in brain lissue of AD patients [60]. In addition, increased concentration of oxidised DNA products, such as 8-hydroxydeoxyguanosine, as well as the presence of increased lipid peroxidation products, such as isoprostanes, have been observed in brain tissue and in cerebrospinal fluid of Alzheimer's disease and Huntington's disease patients [12,39,45,53].

Another marker of protein oxidation may be the presence of 3-mitrotyrosine residues in proteins $[7,25]$. 3-Nitrotyrosine is the product of nitration of tyrosine in proteins, presumably via peroxyntrite, which is a strong oxidising agent [32]. Peroxynitrite is formed during the reaction of mitric oxide (NO) and superoxide anion and occurs especially under conditions of oxidative stress when the production of $\mathrm{NO}$ and oxygen radicals is increased. The reaction between $\mathrm{NO}$ and superoxide anion is extremely fast as it is diffusion limited [30]. Since 3-nitrotyrosine formation will impair the conformational changes in proteins, an impairment of functioning of 3-nitrotyrosylatted proteins might be expected. Indeed. Mn-superoxide dismutase activity was decreased after ty tosine nitration [32]. Increased tyrosine nitration of Mn-superoxide dismutase has recently been shown in cerebrospinal fluid of patients with Alzheimer's disease, Parkinson's 
disease or amyotrophic lateral sclerosis [2]. This suggests that 3-nitrotyosme concentration might be a marker for neurodegenerative disonders. In agreement with this hypothesis. increased 3-nitrotyrosine fomation has also been observed in several animal models, including rats treated with 3-nitropropionic acid (3-NP), i.e. an animal model for Huntington's disease, and mice treated with 1-methyl-4-phenyl-1,2,3,6-tetrahydropyride, an animal model for Parkinson "s disease $[35,58,74]$.

In brains of patients with Parkinson's disease, protein 3-nitrotyrosine formation was observed in association with degenerating and intact neurons [23]. In addition, the majority of Lewy bodies in Lewy body dementia, multi-system atrophy and Parkinson's disease contained 3-nitrotyrosine [18]. 3-Nitrotyrosine formation was also associated with the characteristic langles in Alzheimer"s disease [24]. The finding of protein nitration in otherwise intact neurons with DNA damage, as well as in tangle bearing neurons in the visual cortex of patients with Alzheimer's disease led to the suggestion that protein nitration precedes tangle formation [63]. Considering the above findings of the presence of 3-nitrotyrosine in relatively intact cells in Alzheimer"s disease on Parkinson's disease, we hypothesised that 3-nitrotyrosine inight be an early marker common for neurodegenerative processes associated with oxidative stress. So far, experimental evidence for 3-nitrotyrosine as an early marker for neurodegeneration in biological systems is lacking.

Spheroid cell cultures consist of a mixture of neuronal cell types (neurons, astrocytes and oligodendroglia) establishing a three-dimensional cell culture system which supports neuronal differentiation and has a considerable life span [3]. In Chapter 2, we showed that the spheroid cell culture model contains a functional mitric oxide (NO)-cyclic guanosine 3*,5: monophosphate (CGMP) signalling system, which could be stimulated by N-methyl D-aspartic acid [66]. Thus, the spheroid cultures may be a suitable model to study 3-nitrotyrosine formation under conditions of oxidative stress.

$3-N P$ is an inhibitor of succinate dehydrogenase, a mitochondrial enzyme $[11,26]$. There is evidence that chronic systemic treatment of experimental aninals with 3 -NP results in a general oxidative stress syndrome $[1,58]$. This has been characterised at both systemic and molecular levels $[20,41,56]$. As the cultured spheroid is made up of all cell types present in the central nervous system [66], we investigated whether exposure of spheroids to 3-NP resulls in similar. cellular and molecular characteristics as found in the intact animal. As molecular markeris for oxidative stress we studied 3-nitrotyrosine formation in protein residues and loss of glutamine synthase activity, as this latter enzyme has been reported to be especially sensitive to conditions. of oxidative stress [37,60]. Astrogliosis and cell loss were studied as signs of neurodegeneration.

\section{Materials and methods}

\section{Materials}

Ninitro-L-arginine methyl ester hydrochloride (L-NAME) and aprotinin were purchased from Fluka, Buchs, Switzerland. Dulbecco's modification of Eagle's medium (DMEM) was purchased from ICN Biomedical Inc. Aurora, USA and L-glutamine from Serva, Heidelberg, Germany. Foetal calf serum was obtained from Gibco BRL, Paisley, Scotland. D, L- $\alpha$-tocopherol was from ICN Biomedical Inc., Aurora, USA. Dithiothreitol (DTT), L(土 ) Mactate, 2-p-iodophenyl-3nitrophenyl tetrazolium chloride, phenazine methosulfate, soybean trypsin, leupeptide and phenylmethy/sulfonyl fluoride were all from Sigma, St. Louis, MO, USA.

The Delong flasks were from Bellco Glass Inc. (Vineland, NJ, U.S.A.) and the gyratory shaker was from Innova (Innova 2000), New Brunswick, UK. The nylon gauze (Nytal@) was obtained from Merck, Darmstadt, Germany. 
The mouse anticGFP monoclonal antibody was obtained from Sigma (Si. Louis, USA, clone G* A-5). The rabbit anti-CFAP polyclonal antbody was a gift from F.R.C. Ramaekers (Department of Molecular Cell Biology and Genelics, Universitein Maastricht). Purified bovine GFAP protein was obtained form Progen Biotechnik CmbH, Heidelberg, Genmany. The anti-rabbit $\operatorname{lgC}$ labelled with an alkaline phosphatase conjugate was from Vector. Burlinghame, USA, and $p$ nitrophenylphosphate in diehanolamine solution was purchased from Bio-Rad Laboratories, Veenendaal, The Nethertands.

The monoclonal antibodies against 3-nitrotyrosine in proteins used in the 3-nitrotyrosine-ELISA were a gift from W. Buurman, Deparment of General Health, Universiteit Maastricht. The polyclonal antibodies against nNOS and calbindin were kindly provided by P.C. Emson. The Brabaham institute, Cambridge, UK. The polyclonal antibody against the presynaptic glutamate transporter (EAACI) was a gift from Dr. Rothstein. National Institutes of Health, Bethesda, USA. Anti-2; 3'-cyclic nucleotide 3'phosphohydrolase (CNPase) and biotin labelled iso-lectin B4 were obtained from Sigma, SE. Louis, MO, USA. The antibody against activated microglia (ED1) was a gift from ). Damoiseaux (Department of Immunology, Universiteit Maastricht). The Cy3labelled donkey anti-rabbit and antimmouse secondary antibody and the streptavidin-Cy 3 complex were obtained from Jackson Immunoresearch Laboratories, Inc., Pennsylvania (USA). The Alexa 488-labelled donkey anti-sheep secondary antibody was purchased from Molecular Probes, Leiden, The Netherlands. All further chemicals were purchased from Merck, Darmstadt, Germany.

\section{Animals}

Time pregnant Lewis rats and twelve six-months-old male Lewis rats were obtained from the local breeding centre for experimental animals. Time pregnant Wistar rats and welve male sixmonths-old Wistar rats were obtained from lanvier, Le Cienest-Saint-Isle, France. All animals had free access to regular chow and water.

Experiments were performed according to the guidelines of the University Committee on Animal Welfare, which follow the European Communities Council Directive (86/609/EECh. All efforts were made to minimise animal suffering and to use only the number of animals necessary to produce reliable scientific data, and to utilise altematives to in vivo techniques, if available.

\section{Spheroid cuftures}

Whole brain spheroids were prepared as follows: Brains of 12-20 toetuses (gestation day 15) from two nats were transterred into a nylon gauze bag (Nytal@ 200 $\mu \mathrm{m}$ ) in ice-cold Hanks' D2. solution $(55 \mathrm{mM}$ D-Clucose, $413.2 \mathrm{mM}$ sucrose, $138 \mathrm{mM} \mathrm{NaCl}, 5.4 \mathrm{mM} \mathrm{KCl}, 0.17 \mathrm{mM}$ $\mathrm{Na}$.HPOa, $0.22 \mathrm{mM} \mathrm{KH}, \mathrm{PO}$, $14 \mu \mathrm{M}$ phenol red, $1.8 \mathrm{mM} \mathrm{CaCl}, 0.8 \mathrm{mM} \mathrm{MgCl}$ ). After washing three times, the brains were gently extruded into icecold Hanks" D1 solution $(55 \mathrm{mM}$ D. Glucose, $413.2 \mathrm{mM}$ sucrose, $138 \mathrm{mM} \mathrm{NaCl}, 5.4 \mathrm{mM} \mathrm{KCl}, 0.17 \mathrm{mM} \mathrm{NaHPO}, 0.22 \mathrm{mM}$ $\mathrm{KH} \mathrm{PO}_{1}, 14 \mathrm{\mu M}$ phenol red). The suspension was filtered through a finer nylon mesh (150 $\left.\mu \mathrm{m}\right)$, washed three times by centrifugation $(170 \mathrm{~g}, 5 \mathrm{~min})$, resuspended and finally plated out in aliquots of $3.5 \mathrm{~mL}$ at a density of $1.10^{\circ} \mathrm{ce} / \mathrm{s} / \mathrm{mL}$ medium, which consisted of DMIEM supplemented with $1 \%$ L-gltumine and $10 \%$ foetal calf serum, in 25-mL DeLong flasks and placed on a gyatory shaker $\left(85 \mathrm{rpm}\right.$ in an incubator $\left(37^{\circ} \mathrm{C} ; 9 \% \mathrm{CO}\right.$. humidified air). After two days, the cultures were transferred into $50 \mathrm{~mL}$ Delong flasks. Medium was changed three times a week. After one week of culture vitamin $E(D, L-\alpha$-tocopherol, $0.1 \mathrm{mM})$ was supplied to the medium. 


\section{Exposures}

Two-week-old spheroids were plated out into a six-well plate one day before exposure $2 \mathrm{~mL}$ medium/well). Shortly before exposure $1 \mathrm{~mL}$ of medium was replenished by $1 \mathrm{~mL}$ fresh medium. The spheroids were exposed to $3-\mathrm{NP}(0.5$ and $5 \mathrm{mM}$, pH adjusted to $7-8)$ or vitamin $\mathbb{E}$ (0.1 $\mathrm{mM})$, glutathione $(1 \mathrm{mM})$, DTT $(0.25 \mathrm{mM})$, LNAME $(0.1 \mathrm{mM})$, or combinations of $3-\mathrm{NP}$ with these substances during five days. After 2 days, freshly prepared 3-NP and protectors were added to the medium. After finishing the exposures, the spheroids were washed three times with ice-cold PBS, supplemented with $0.5 \mathrm{~mL}$ ice-cold phosphate buffer $12 \mathrm{mM} \mathrm{NaH} \mathrm{PO}_{\text {- }}, 0.5$ mM EDTA, $145 \mathrm{mM} \mathrm{NaCl}, \mathrm{pH} 7.0$ ) and stored at $-80^{\circ} \mathrm{C}$ for biochemical assays. The spheroids were thawed and homogenised by sonification on the day of the GS auctivity assay. Altematively, after washing steps with PBS, the spheroids were fixated in freshly depolymerised $4 \%$ paraformaldehyde during 1.5 hours, quickly frozen in Tissue-Tek and cut into sections 110 $\mu \mathrm{m})$ on a cryostat.

\section{In wivo experiments}

Of each rat strain seven animals were assigned to the 3-NP treatment group and five animals to the control group. The excess number of animals in the treatment groups was based on studies reporting large variation, including death, in treatment effects [9]. All rats were housed individually and were kept at normal light/diark cycle (lights on from 6.00 to $18.00 \mathrm{hr}$ ). Rats were treated with $3-\mathrm{NP}$ as follows: Six months old male Wistar $(518 \pm 11 \mathrm{~g}$ ) and Lewis rats (382 $\pm 8 \mathrm{~g})$ were treated with $3-\mathrm{NP}(20 \mathrm{mg} / \mathrm{kg})$ or saline $(0.9 \% \mathrm{NaCl}, 1 \mathrm{~mL} / \mathrm{kg})$ by intraperitoneal injections every other day during eleven days. One animal of the 3-NP Wistar rats was severely paralysed after the 3 rd injection and was therefore sacrificed and excluded from further analysis. Amother 3-NP treated Wistar rat had lost $35 \%$ of its initial body weight after the sixth injection and showed signs of severe hypoactivity. This animal was injected with saline i.p. (5.5 $\mathrm{mL} / \mathrm{day}$ ) and fed glucose solution (p.0. $5 \mathrm{~mL}$ ) during one week to awoid starvation and dehydration.

During the three weeks after completing the injections, behavioural testing was performed, which has been described in Chapter 5. After finishing these tests, the rats were decapitated, blood samples were taken and the right half of the striatum was dissected, homogenised in phosphate buffer (2 $\mathrm{mM} \mathrm{NaH} \mathrm{PO}_{4}, 0.5 \mathrm{mM}$ EDTA, $145 \mathrm{mM} \mathrm{NaCl}, \mathrm{pH} 7.4,0.1 \mathrm{~g}$ wet tissue/mL) supplemented with the protease inhibitors soybean trypsin $(10 \mu \mathrm{g} / \mathrm{mL}$ ). Veupeptide (1 $\mu \mathrm{M})$. phenylmethylsulfonyl fluoride $(1 \mathrm{mM})$ and aprotinine $(8.1 \mathrm{IU}-1 \mu \mathrm{g} / \mathrm{mL})$ and stored at $-80^{\circ} \mathrm{C}$. The left half of the brain was used to prepare slices of $3000 \mu \mathrm{m}$ thickness and these were fixated in $4 \%$ freshly depolymerised paraformaldehyde during 3 hours. After overnight immersion with $10 \%$ sucrose solution, the slices were quickly frozen in Tissue-Tek and stored at $-20^{\circ} \mathrm{C}$ until preparation of cryostat sections ( $20 \mu \mathrm{m}$ thick).

\section{Biochemical assays}

The 3-nitrotyrosine ELISA was performed following the method described [64] using nillated bovine serum albumin as a standard. The specificity for nitrated tyrosine in proteins has been shown by westem blotting and preincubation with nitrated amino acids and proteins [64]. The concentrations are thus expressed as equivalents of the nitrated bovine serum albumin standard. $100 \mu$ of homogenate (final concentration $0.1 \mathrm{mg} / \mathrm{mL}$ ) was incubated.

Glutamine symthase activity in the homogenates was determined as described previously [67]. GFAP concentration in striatal homogenates (concentration homogenates $0.2 \mathrm{mg}$ protein/ $\mathrm{mL}$ ) was determined using an ELISA as described by O'Callaghan [48]. The primary antibody ffinal concentration $10 \mu \mathrm{g} / \mathrm{mL}$ in PBS) was a mouse anti-CFA.P monoclonal antibody and its specificity 
has been described by the supplier. The secondary rabbit anti-GFAP polyclonal antibody was used in a dilution of 1:500. Purified bowine GFAP protein was used as a standard. Samples were incubaled in the final protein concentration of $0.04 \mathrm{mg} / \mathrm{mL}$. The detection system used was antirabbit $\operatorname{lgC}$ labelled with an alkaline phoshatase conjugate (dilution $1: 3,333$ ) and $\mathrm{p}$ nitrophenylphosphate in diethanolamine solution. Extinction was measured at $405 \mathrm{~nm}$ on a Benchmark microplate reader (Bio-Rad Laboratories, Veenendaal, The Netherlands).

Cell death was determined measuring the total protein concentration of the spheroids per well according to the method of Lowry [40].

\section{Immunohistochemistry}

Cryostat sections were air-dried at room temperature for $20 \mathrm{~min}$ followed by three washing cycles in Tris-buffered saline (TB5) containing $0.3 \%$ (w/V) Triton X-100 (TBS-T, pH 7.6). Sections were incubated overnight at $4^{\circ} \mathrm{C}$ with the primary antibody (diluted in TBS-T). After three washing cycles, the sections were incubated with a secondary antibody labelled with a fluorescent label during one hour. After three additional washes the sections were immersed with glycerol in TBS $(3: 1)$ and coverslipped.

The same mouse GFAP monoclonal antibody as for the GFAP ELISA was Lised for the GFAP immomunohistochemistry $(1: 1,600$ dilution). The antibody against nNOS was raised in sheep (1:750 dilution) [29]. The antibody against calbindin, a protein found in GABAergic structures (dilution 1:1,000) was raised in rabbit [34]. The antibody against a $73 \mathrm{kDa}$ protein of a neuronal glutamate transporter sublype (EAAC1, C-terminal) was raised in rabbit dilution 1:100) [21]. The specificities of the mouse monoclonal anti-GFAP, the mouse monoclonal anti-CNPase (1): 1,000 dilution) and the biotin-labelled iso-lectin B4 antibodies (1:200) were as described in the data sheets of the supplier. GFAP, EAAC1, ED1, CNPase and calbindin immunoreactivity were visualised by a Cy3 fluorescent secondary antibody (dilution 1:800) and bound iso-lectin B4 was visualised by streptavidine $\mathrm{Cy} 3$ fluorescence $(1: 1,000$ dilution). nNOS immunoreactivity was visualised by means of an Alexa 488-labelled donkey anti-sheep secondary antibody (1:100 dilution).

Photomicrographs were made on a Olympus microscope, model AX70 TRF, equipped with a narrow band MNIBA-type FITC filter and a MNG filter for Cy3 fluorescence, permitting to visualise FITC or Cy3 fluorescence in the complete absence of bleeding through of the other fluorescent marker. Images for Figures $2-6$ were imported with a SONY (Power HAD) CCD camera and arranged using Adobe ${ }^{(3)}$ Photoshop ${ }^{(19}$ without further processing.

\section{Quantification of immunot worescence}

Immunofluorescence was recorded with a Sony (Power HAD) CCD-camera. Images were imported into a computer equipped with an image analysis program (Soft Imaging System, AnalySIS 3.0, Muinster, Germany). Fluorescence intensity of the pixels present in spheroids was converted to grey values (255 classes) and expressed as mean staining intensity level. All spheroids present on a microscopic slide were included and the total amount of spheroids is indicated in table. Sections to be processed for image analysis from the different experiments ware immunostained simultaneously to minimise experimental variation. Mean fluorescence intensity of sections from the same experiment stained with the secondary antibody alone were subtracted.

\section{Statistics}

Data from the biochemical determinations in the spheroids are expressed per mg protein and standardised (mean 1 standard error of mean (s.e.m.) to the control values after five days of 
exposure. Data were obtained from at least two experments in triplicate. Diferences in biochemical parameters between control and exposed spheroids were calculated using a Mann-Whitney $U$ test. Differences in grey value intensity between controt and exposed spheroids were evaluated with the Students" "test. Differences in biochemical parameters between treated and control rats were also evaluated with the twest. All statistics were performed using an SPSS statistical program.
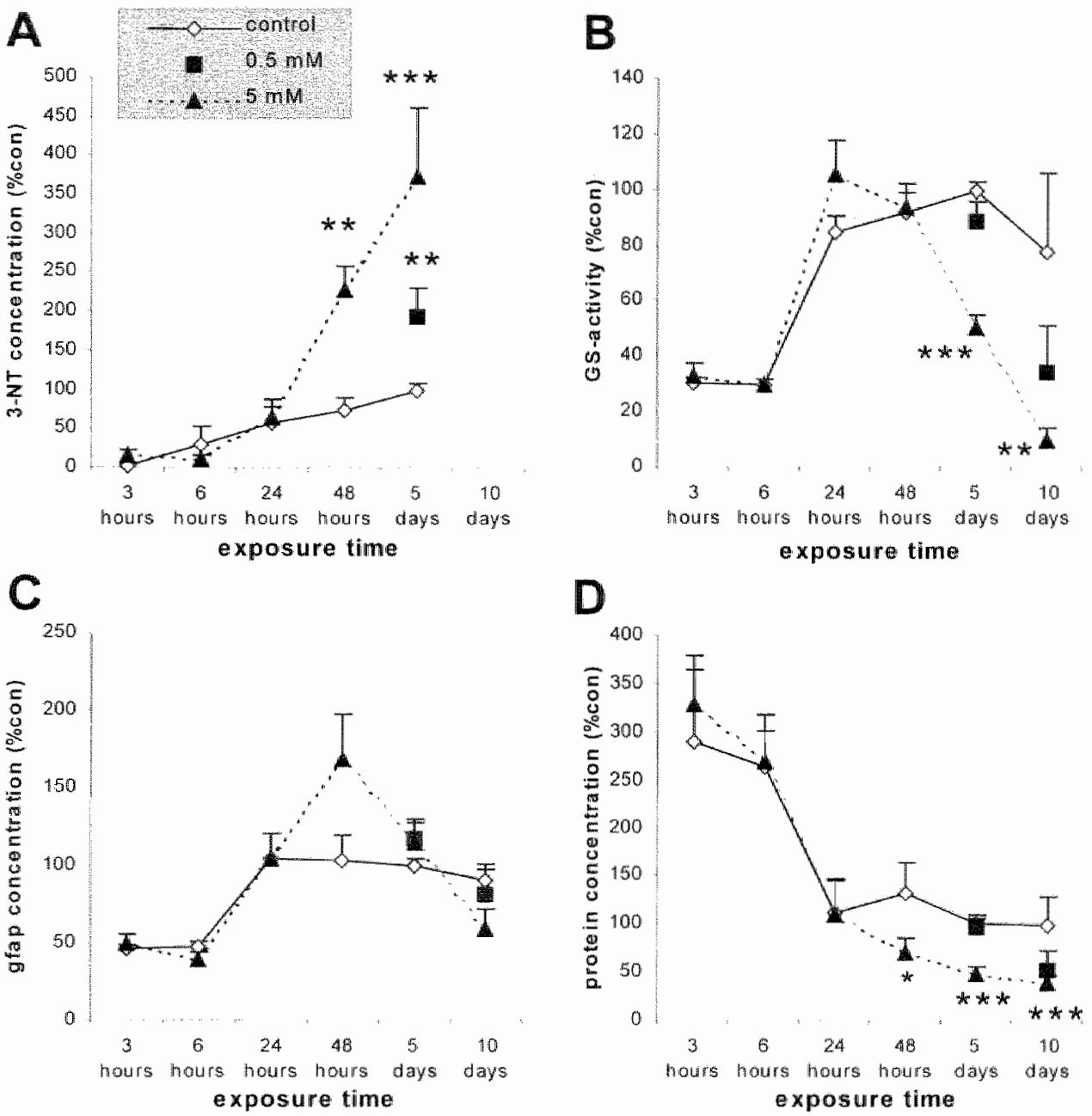

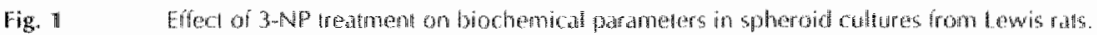

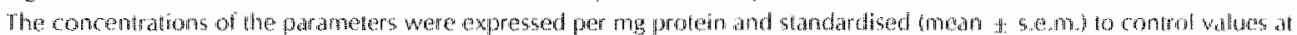

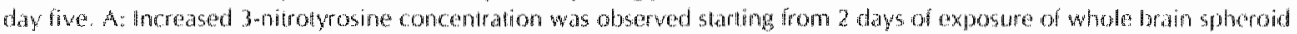

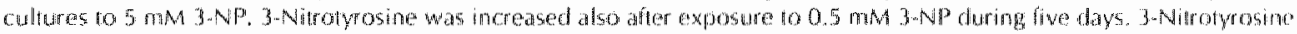
concentration an control culures increased gadually during the expermental lime. B: Gl citamine synhase at livity was

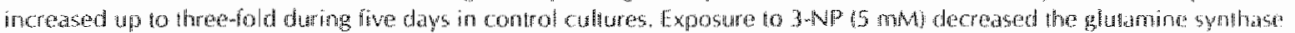

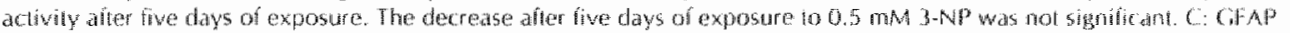

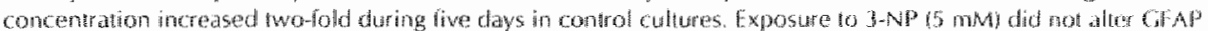

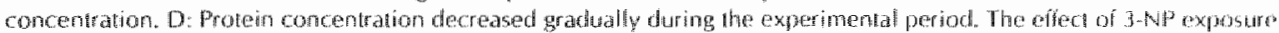
became apparent after two days. $* 00.05 ; * P<0.01 ; * * P<0.001$ 


\section{Results}

\section{Dose-response relation between 3-NP and cellular markers}

Treatment of spheroid cultures from Lewis strain rats with $5 \mathrm{mM}$ 3-NP for various time periods resulted in a three-fold increase in 3-nitrotyrosine levels after 48 hours (Fig. 1A). After five days of exposure to $5 \mathrm{mM} 3-\mathrm{NP}$ an almost four-fold increase in 3-nitrotyrosine levels was observed, although within each of the 14 experiments large variation of 3-nitrotyrosine concentration existed annong the wells. At a reduced concentration of $0.5 \mathrm{mM} 3-\mathrm{NP}, 3-$ nitrotyrosine levels were increased two fold after five days of exposure (Fig. 1A).

Decreased GS activity $150 \%$ of controll after exposure to 3-NP was first observed after five days (Fig. 1B). The reduced concentration of $0.5 \mathrm{mM} 3-\mathrm{NP}$ did not have an effect on GS activity after five days of exposure. After ten days of exposure, GS activity was decreased to $12 \%$ of control values at day five. A ten-fold lower concentration of 3 -NP had an inhibitory effect on GS activity $(50 \%$ of control) after ten days of exposure, although not significantly.

In contrast to the reported astrogliosis induced in vivo by 3NP [71], no effect on GFAP concentration was observed in the spheroids (Fig. 1E). Although protein loss was a general feature of the spheroid cultures, there was a significant decrease in protein concentration after 48 hours of exposure to $3-N$ P (Fig. 1D).

These results indicate that $3 \times N P$ has a toxic effect on cultured spheroids as evidenced by increased 3-nitrotyrosine formation, loss of GS activity and loss of total protein. In addition, changes in 3-nitrotyrosine concentration occurred at a lower dose of 3-NP and exposure time compared to changes in the other markers examined.

\section{Effect of possible inhibitors of free radical formation}

The reactive oxygen species $\mathrm{NO}$ or $\mathrm{O}_{2}$ are presumably involved in 3-nitrolyrosine formation. We investigated if free radicals were involved in 3-NP induced effects in our model. Therefore, spheroids from Lewis rats were exposed to the NOS-inhibitor L-NAME (0.1 MM [66]), the antioxidants DTT $(0.25 \mathrm{mM}$ [65]), glutathione (1 $\mathrm{mM}$ [36]) and vitamin $\mathrm{E}(0.1 \mathrm{mM}$ [38]) during: five days of incubation with 3-NP. The concentration of 3-NP used in these further experiments was $5 \mathrm{mM}$, the dose resulting in effects on GS activity and 3-nitrotyrosine concentration (Table i).

Suprisingly, a large increase in 3-nitrotyrosine concentration was still observed after incubation of the spheroids with $0.1 \mathrm{mM}$ L-NAME during five days: the 3-nitrotyrosine concentration was increased to $650 \%$ of the levels after incubation with L-NAME alone. This level was two-fold higher compared to $3-\mathrm{NP}$ alone $(P<0.05)$. The presence of DTT alone resulted in a slight increase in 3-nitrotyrosine formation ( $P<0.05$ ), the 3-NP induced 3-nitrotyrosine formation was almost completely prevented by the simultaneous presence of DTT. No protective effect of gltathione was observed. Incubation of 3-NP in the presence of vitamin E inhibited the 3-NP induced significant increase in 3-nitrotyrosine concentration. However, this effect varied among the experiments and no significant difference in 3-nitrotyrosine concentration between vitamin E + 3-NP and 3-NP alone was observed.

The decrease in glutamine synthase activity caused by 3-NP could only be abolished by DTT, although the effects were variable and the $n$ was low for this compound.

The GFAP concentration remained comparable to control values after incubation with DTT,

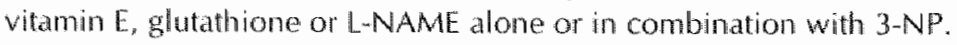

A partial inhibition of total protein loss, as a measure of cell loss, after 3-NP treatment was observed after incubation of the spheroid cultures with vitamin E or DTT. 
Thus, a protective effect of DT was obseved against 3-NP induced changes in 3-nitrotyrosine concentration, total protein concentration, and GS activily. The antioxidant vitamin $E$ also inhibited the changes in total protein concentration and 3mitrotyrosine fomation.

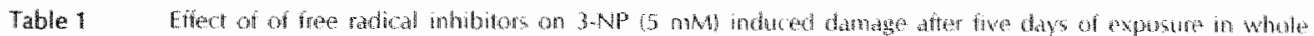
brith spheroids obidinat from Lewis rats.

\begin{tabular}{|c|c|c|c|c|c|}
\hline exposure & $\begin{array}{l}\text { 3nimotyrosine } \\
\text { 4hgingen protein }\end{array}$ & $\begin{array}{l}\text { CS achivity } \\
\text { U/mig proteing }\end{array}$ & $\begin{array}{l}\text { GFAP } \\
\text { inghg protein) }\end{array}$ & $\begin{array}{l}\text { Prottein } \\
\text { (monglons }\end{array}$ & $n$ \\
\hline Control & $100 \pm 9$ & $100+3$ & $100+5$ & $100 \pm 5$ & $43 \times 49$ \\
\hline$\div 3-\mathrm{NP}$ & $373=1899^{3}=$ & $50=5+5$ & $114 \pm 13$ & $49 \pm 0^{4}$ & $34+43$ \\
\hline 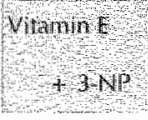 & $\begin{array}{l}38+54 \\
291+40\end{array}$ & 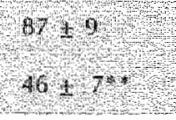 & 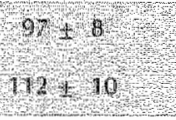 & 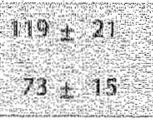 & (6) \\
\hline DTT & $167+60^{\circ}$ & 106 是 4 & $148 \pm 28$ & $139+13^{*}$ & $3-5$ \\
\hline \# $3 \mathrm{NP}$ & $8 \pm 9$ & $50 \div 25$ & $139 \pm 26$ & $105 \pm 18$ & 3.5 \\
\hline Mruththon & 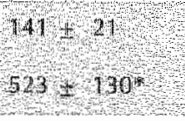 & 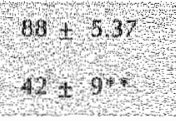 & $\begin{array}{l}46+12 \\
1,1+17\end{array}$ & 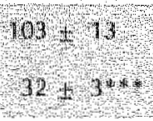 & $\begin{array}{l}19 \\
49\end{array}$ \\
\hline L-NAME & $124 t 7$ & $90 \pm 4$ & $125 \pm 22$ & $B 2+9$ & 6 \\
\hline$+3 N \mathrm{NP}$ & $720 \pm 140 \%$ & $55 \pm 7^{*}$ & $161 \pm 23$ & $25 \pm 5 * 4$ & $4-6$ \\
\hline
\end{tabular}

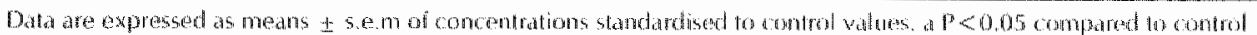

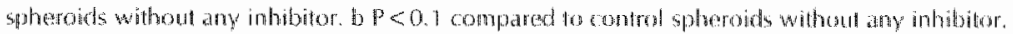

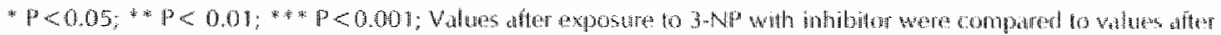

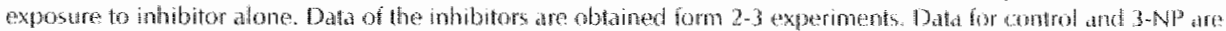
obrained from th 14014 experiments.

\section{Immunostaining of cultures}

To further characterise the effect of 3-NP (5 mM) on spheroids from Lewis rats, we studied the localisation and expression of a number of cellular marker molecules, some of them related to an inflammatory response (Fig. 2-4).

Exposure to 3-NP did not affect nNOS (Fig. 2A,B) and GFAP (Fig. 2C, D) immunostaining in spheroid cullures. The cabindin immunostaining was affected in 3-NP exposed spheroids compared to controls, shown by loss of cell processes concomilant with increased staining intensity of the cell bodies (Fig. $3 \mathrm{~A}$ and B). Quantification of the immunostaining with the help of an Image Analysis system did not reveal decreased mean immunostaining of the spheroids Table 2). The staining for the glutamiate transporter in the spheroid cultures was almost completely absent after exposure to 3-MP (Fig 3C and D). Similarly, the staining for the oligodendroglial marker CNPase was almost completely absent after exposure of the spheroids to 3-NP for five days (Fig. $3 E$ and F).

After immunostaining for ED1, a marker for activated microglia, the control spheroid cultures showed staining of irregular round structures (Fig. 4A). This staining was also almost completely absent after exposure of the cultures to 3-NP for five days (Fig. 4B). In contrast, immunofluorescence intensity of the common marker for microglia, isolectin-B4, was increased atter exposure of the spheroids to $3-N P$ for the same time period (Fig. $4 C$ and D). The increased mean level of immunostaining of isolectin-B4 was also confirmed by quantification of the immunofluorescence intensity (Table 2 ).

Thus, exposure of whole brain spheroid cultures to 3-Np resulted in decreased staining of markers for GABAergic and glutamaterigic neurones, and oligodendrocytes. In contrast, 
isolectir B4. immunostaining pointed to activation of microglial cells in these cultures and nNOS immunostaining was not affected. These results are in agreement with a selective neurotoxic effect of 3.NP on GABAergic and glutamatergic neurons [5, 69].
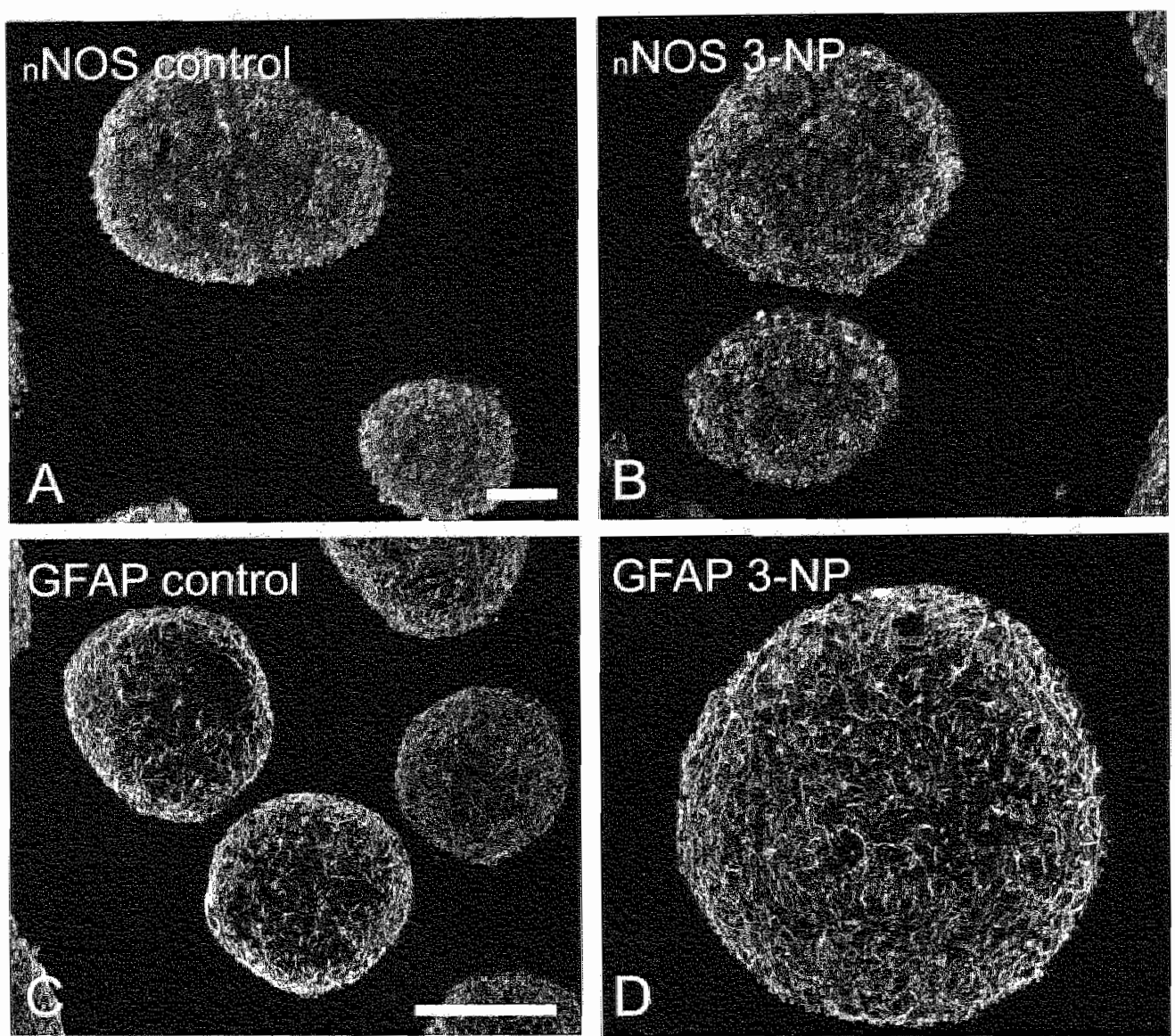

Fig. 2

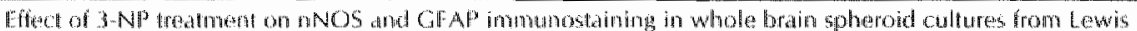

rith.

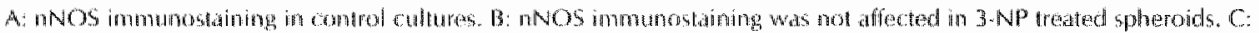

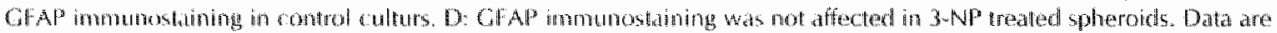

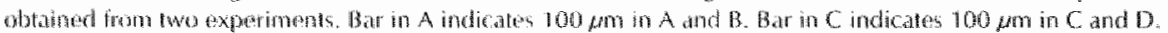

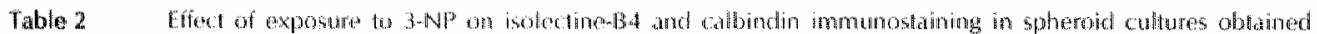

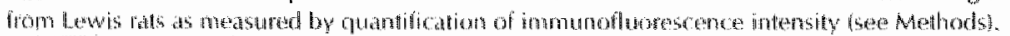

\begin{tabular}{|c|c|c|c|}
\hline & & me an intensidy & ofrom two dxperments) \\
\hline \multirow[t]{2}{*}{ abindin } & omerol & $61.3 \pm 1.3$ & 171 \\
\hline & $3-N l^{b}$ & $57.8+1.8$ & 131 \\
\hline \multirow[t]{2}{*}{ isoletontine $B 4$} & control & $46.7 \pm 0.7$ & 134 \\
\hline & $3-N_{P}$ & $64.1 \pm 1.4 *$ & 110 \\
\hline
\end{tabular}

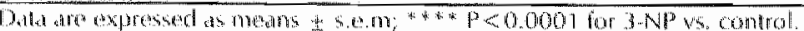



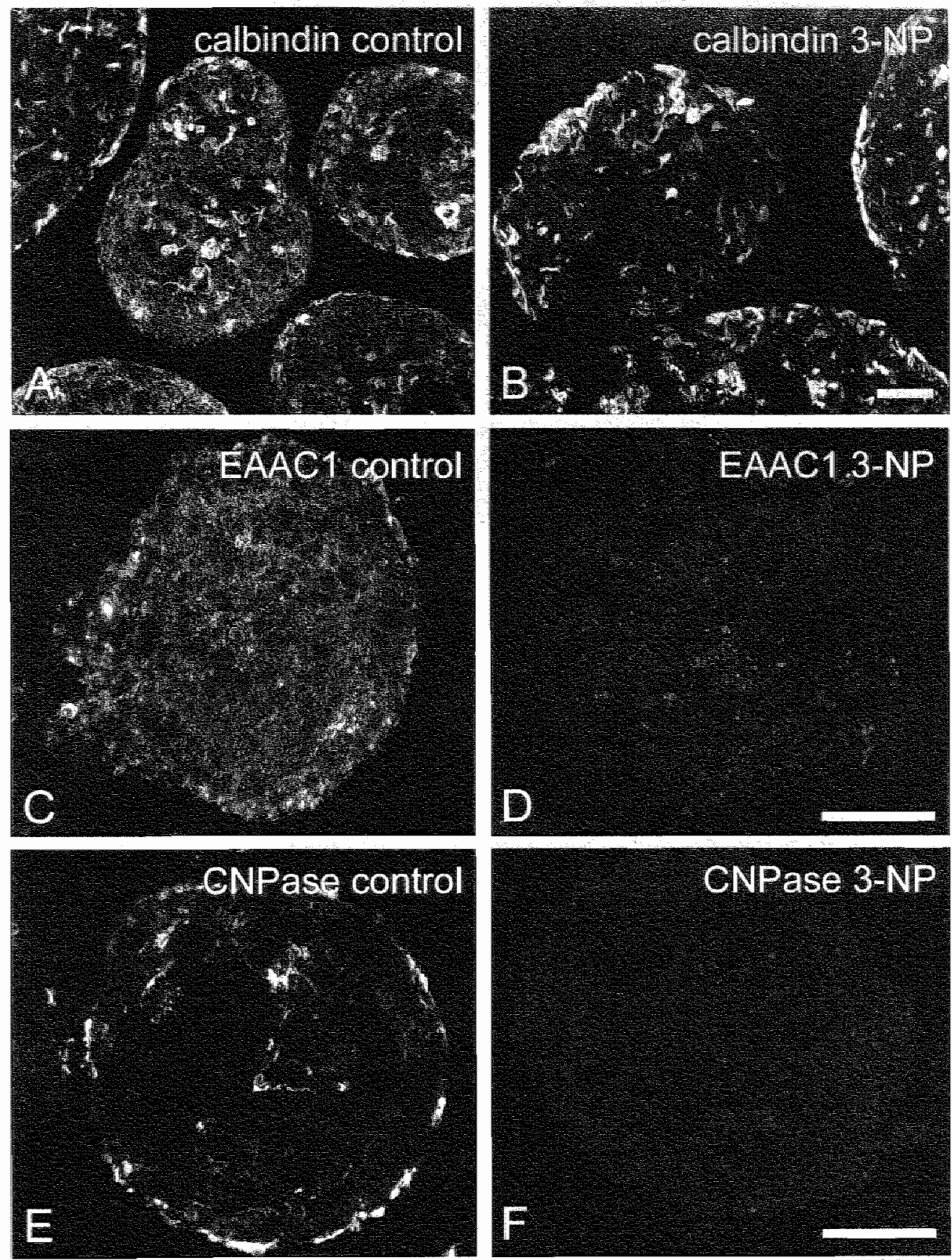

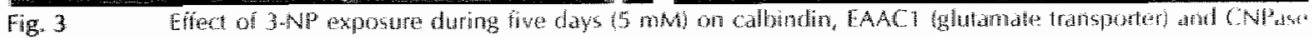

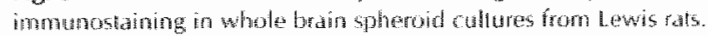

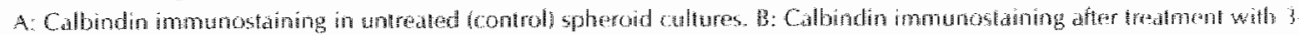

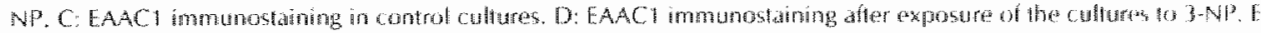

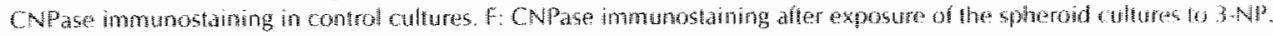
Dita are obtained from two esperiments. Bair indicates $100 \mu m$. 


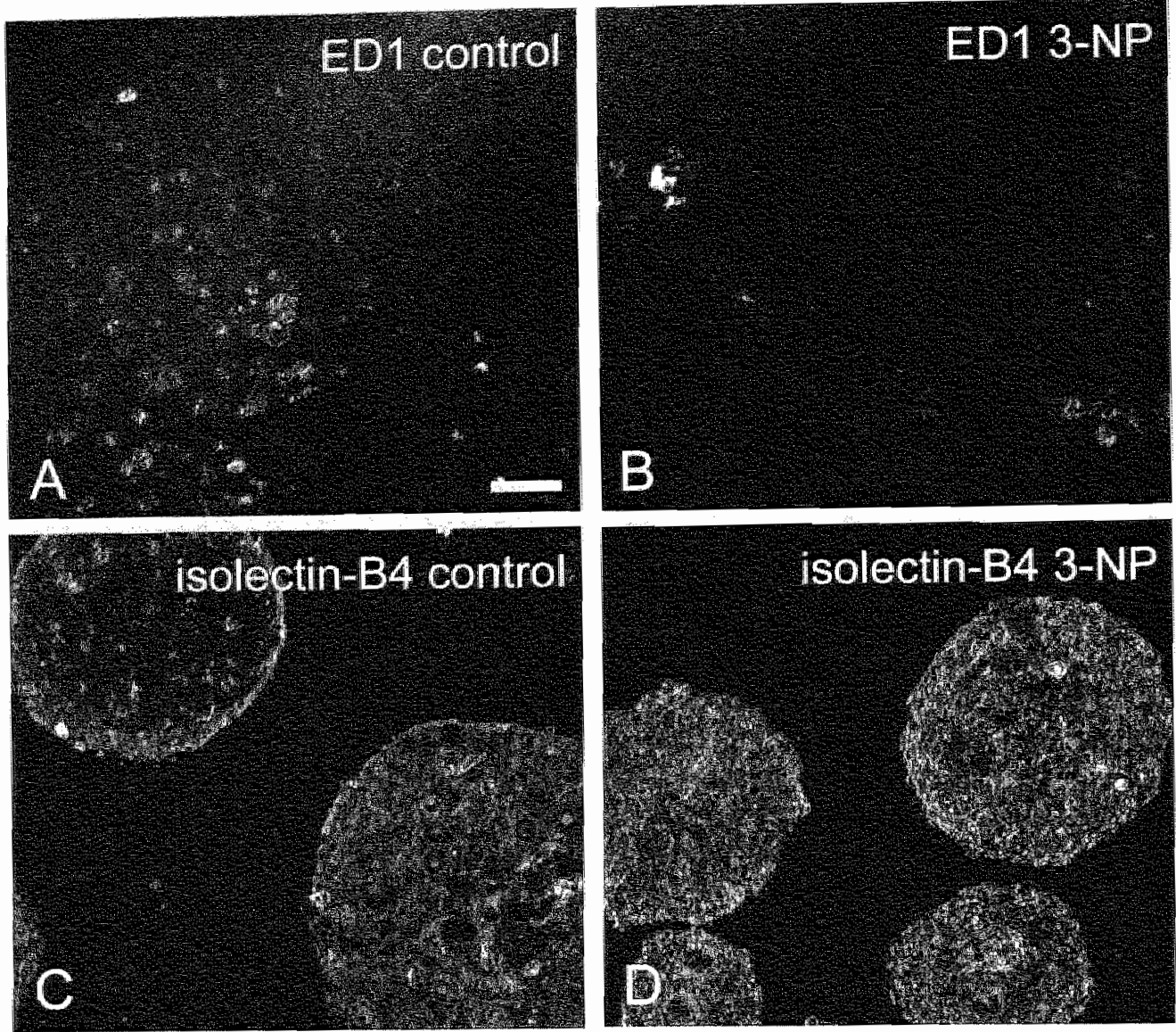

Fïgr 4

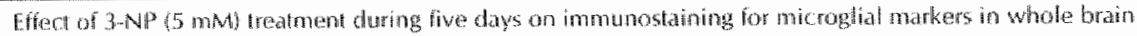
spheroid cullures.

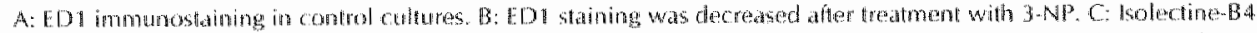

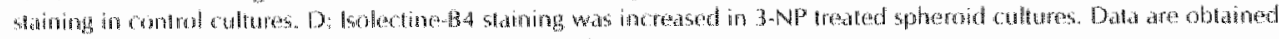

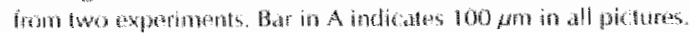

\section{Interstrain differences in spheroid cultures}

We and others thave previously shown that large inter-strain susceptibility exists towards treatment with 3-NP in vivo [50](Chapter 5). For example, we observed weight loss and memory impairments in Wistar rats, while no such effects were observed in the Lewis rats at the same dose. We compared the susceptibility of spheroid cultures from Lewis rats with cultures obtained from Wistar rats towards treatment with $5 \mathrm{mM}$ 3-NP during five days (Table 3). The 3nitrotyrosine concentration was not increased in the spheroids obtained from Wistar rats after exposure to 3-NP (5 mM) during five days. This is in contrast with the three fold increased 3 nitrotyrosine concentration in the spheroids from the Lewis rats, as shown above. A $50 \%$ decrease in glutamine synthase activity was observed in the spheroids obtained from Wistar rats after exposure to $3-\mathrm{NP}$, which was similar to the observations in the spheroids obtained from Lewis als.

The GFAP concentration was slightly, though not significantly, increased in the Wistar spheroids after exposure $103 \times$ NP. Similarly, a non-significant increase in GFAP concentration to $175 \%$ was observed after incubation of the Wistar spheroids with 3-NP in the presence of 
vitamin $E$. These resuls are in contrast to the lack of ffret on GFAP concentration after exposure of the spheroids to 3-NP in the presence vitamin E in Lewis spheroids as shown already in Table 1.

The decrease in proteim concentration induced by 3 -NP could not be prevented by vitamin $E$ in the spheroids obtained from the Wistar rats: this in contrast to the protective effect of vitamin $E$ on protein concentration in spheroids obtained from the Lewis rats.

Table 3 Strain dependen exflects of exposure of whole bran spheroid cultures to 3 -NP.

\begin{tabular}{|c|c|c|c|c|c|}
\hline & & control & $3+\mathrm{NP}$ & vitumin $\mathrm{E}$ & $\begin{array}{l}\text { whambin } \\
+3+N P\end{array}$ \\
\hline 3-hitrotyrosine & Lewis & $100 \pm 9$ & $371 \pm 88^{2}=4$ & $133+54$ & $291 \pm 107$ \\
\hline 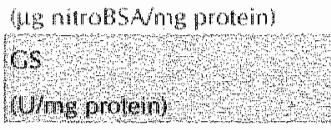 & $\begin{array}{l}\text { Wistat } \\
\text { lowtert } \\
\text { Nistar }\end{array}$ & $\begin{array}{l}100 \pm 7 \\
100+3 \\
100+5\end{array}$ & $\frac{137+59}{450+517}$ & 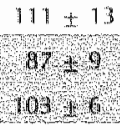 & $129 \pm 29$ \\
\hline GHAP & Lewis & $100 \pm 5$ & $114=13$ & $97+9$ & $112 \pm 10$ \\
\hline (mémg protein) & Wistar & $100 \pm 22$ & $133 \pm 26$ & $90 \pm 12$ & $179+28$ \\
\hline provein & Heress & $100 \div 5$ & $490+8 \%$ & 110,47 & 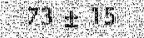 \\
\hline $\mathrm{HWnL}$ & Nistar & $400+8$ & $36+4 \%$ & $80+15$ & $37+1,4$ \\
\hline number of walls oblamed & Lewnis & $38-43$ & $32-41$ & 6 & $9+12$ \\
\hline from at least wwo experiments & Wistar & 9 & 7.9 & $8-9$ & 7.9 \\
\hline
\end{tabular}

Datä äre expressed as means it s.er.m.

* P< 0.01; * P $<0.001$ compared to control spheroids

Walues after exposure to 3 -NP with inhbitor were compared to watues alter exposure to inhibitor atone.

\section{Effect of 3-NP exposure on cellular markers in Lewis and Wistar rats}

In the in vivo experiments, rats were exposed to 3-NP during eleven days and were left to recover for three weeks thereafter. Measurement of striatal concentrations of 3-nitrolyrosine did not reveal any changes due to treatment in both rat strains at that time-point (Table 4). Striatal GS activity was not significantly lower in the 3-NP treated rats compared to control ratts. Striatal GFAP concentration was increased in the 3-NP treated Wistar rats only.

GS activity in serum was decreased in the 3-NP exposed Wistar rats compared to controls, while no effect of treatment was observed in the Lewis rats.

Table 4

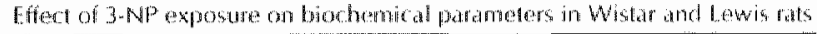

\begin{tabular}{|c|c|c|c|c|c|}
\hline & Matrix & $\begin{array}{l}\text { Whist contral } \\
(n=5)=5\end{array}$ & 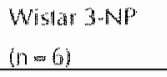 & $\begin{array}{l}\text { Lenwis confroll } \\
(n=5)\end{array}$ & 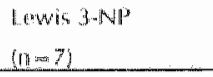 \\
\hline \multirow[t]{2}{*}{ 3-nitrotyrosine } & $\begin{array}{l}\text { striatum " } \\
\text { fug/mg proteing }\end{array}$ & $44.2 \pm 3.4$ & $44.3 \pm 3$ & 38.51 .2 & $52.8+4$ \\
\hline & $\begin{array}{l}\text { serum } \\
\text { He/mol }\end{array}$ & mot detertabses & 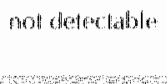 & not detectatble? & not retechalos \\
\hline $\mathrm{CAdP}$ & stratum, & 94,5 & $150 \mathrm{u2}^{2}$ & $26+10$ & 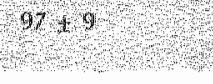 \\
\hline \multirow[t]{2}{*}{ Cosativity } & $\begin{array}{l}\text { Gtriattem } \\
\text { Uhmg protein }\end{array}$ & $0.6 \pm 0.3$ & $8.3+06$ & $0.2 \pm 0.3$ & $8.1 \pm 0.4$ \\
\hline & $\begin{array}{l}\text { Serum } \\
\text { (U/nol: }\end{array}$ & $2.3 \pm 0.4$ & $10=0.3^{\circ}$ & $0.4(n-1)$ & $0.4 \pm 0.1003\}$ \\
\hline
\end{tabular}

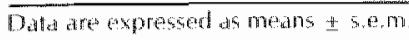

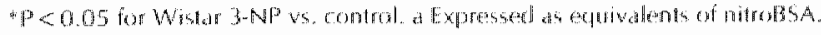


We previously characterised the effect of treatment of Lewis and Wistar rats by immunocylochemical staining for GFAP (Chapter 5), the marker for astrogliosis in this model [711. As shown before, increased GFAP irnmunostaining was observed in the striatum in 3-NP affected Wistar rats (Fig. 5A and B). No increased GFAP staining or lesions were observed in 3NP treated Lewis rats (data not shown).

In the Wistar rats, the nNOS immunostaining was relatively preserved in the 3-NP treated rats (Fig. 5C), while the calbindin immunostaining was severely decreased (Fig. 5D). We characterised the effects of 3-NP treatment in Wistar rats further by immunostaining of markers for microglia, i.e. ED1 and MHCli, which was absent in controls (Fig 6A-D). No effects of treatment with 3-NP on CNPase staining was observed, indicating the preservation of structural integrity of oligodendrocytes in this model (data not shown)
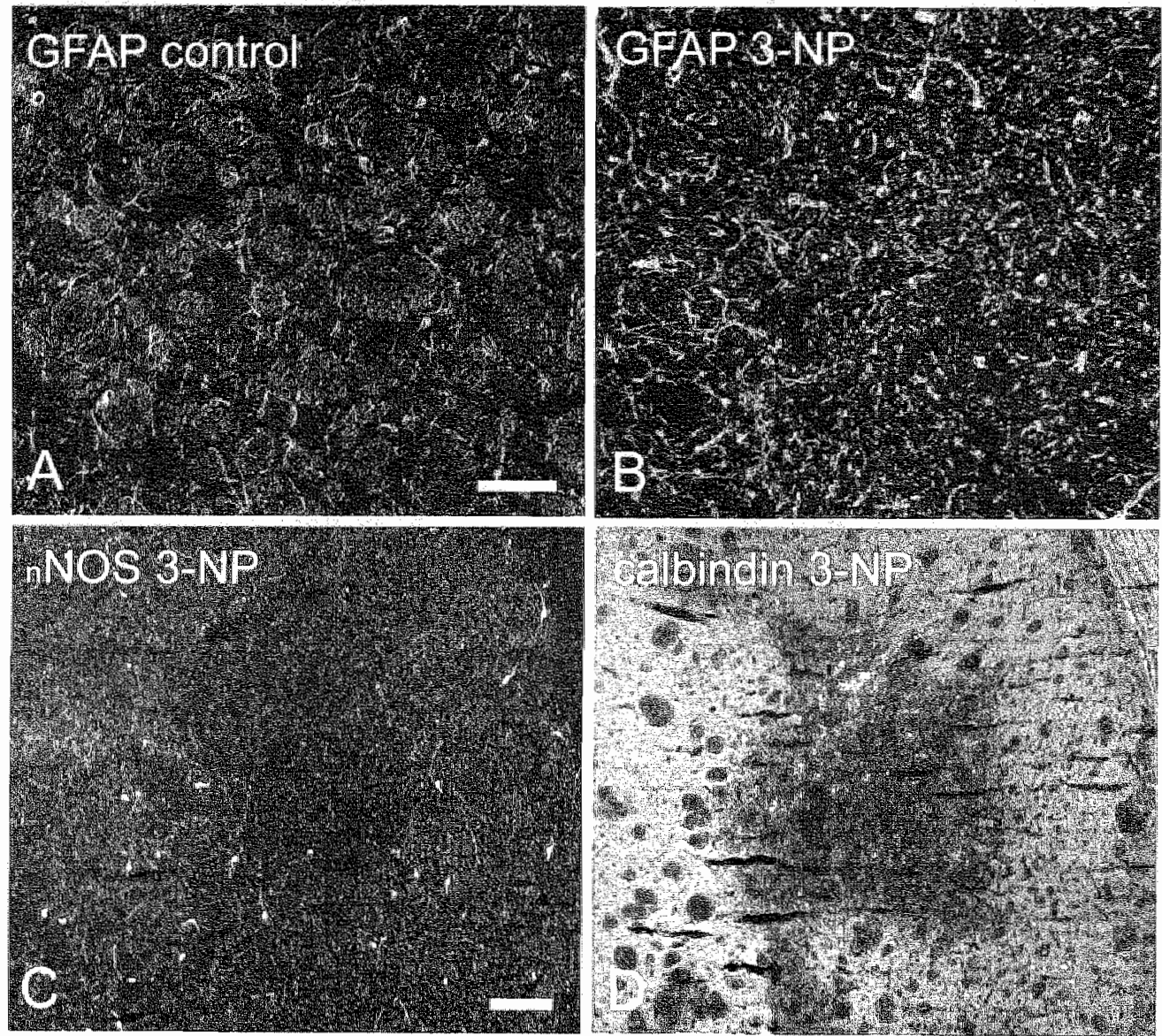

Fig. 5

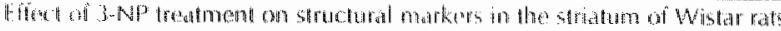

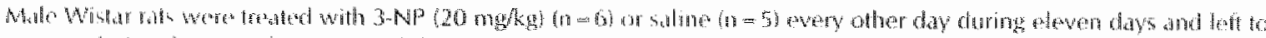

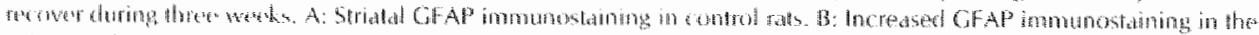

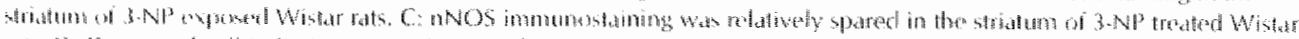

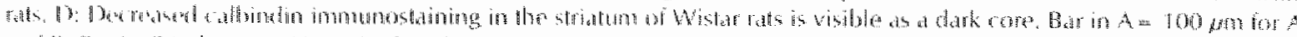

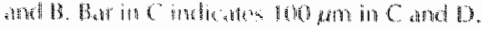




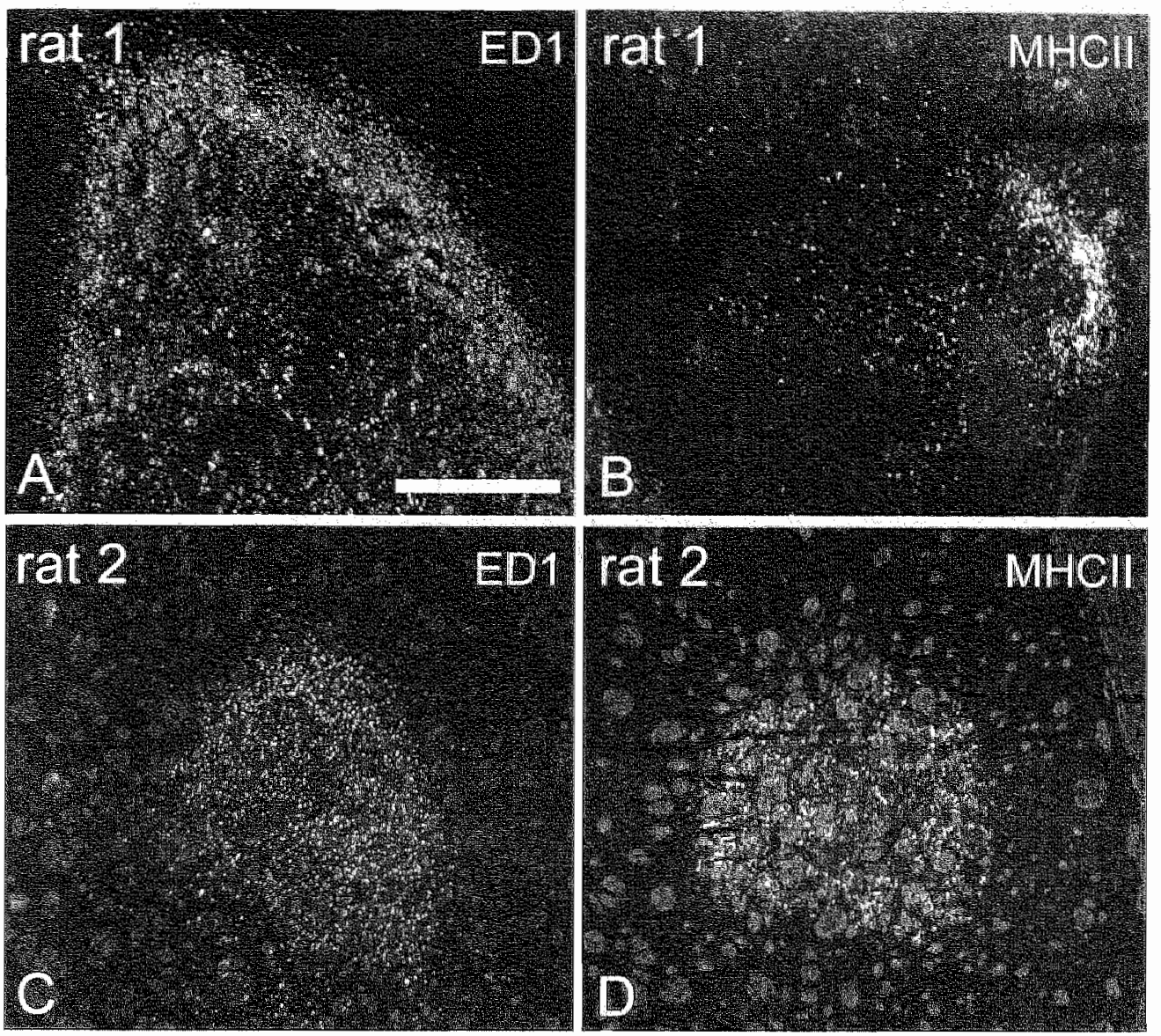

Fig. 6

Efect of 3-NP treament on macrophage/nicroglat markers in the strialum of Wistar mats.

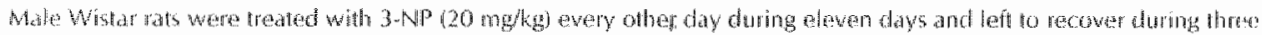

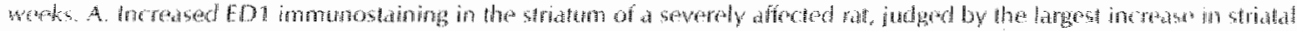

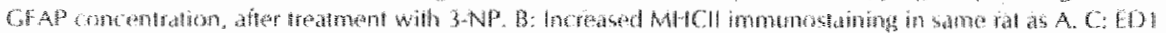

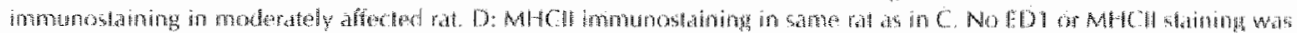

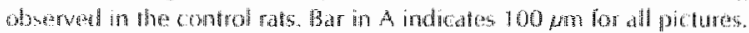

\section{Discussion}

In the present study we inwestigated the hypothesis that 3-nitrotyrosine is a sensitive marker for oxidative stress induced neurodegeneration in the central nervous system. We compared the characteristics of the 3-NP induced 3-nitrotyrosine formation to other markers of oxidative damage, such as C.S activity, GFAP concentration or cell death in both whole brain spheroid cultures and in 3-NP exposed rats. The results of the whole brain cultures from Lewis rats. showed that 3-rittrotyrosine concentration was increased at a concentration of $3-\mathrm{NP}(0.5 \mathrm{mM})$ that thad still no effect on cell death, GFAP concentration or CS activity. The same result was observed regarding the exposure time needed to provoke alterations in the markers studied. Thus, in our experiments using spheroid cell cultures obtained from Lewis rats, 3-nitrotyrosine was a more sensitive marker for oxidative stress induced neurodegeneration than G5, GFAP or cell loss. 


\section{Sensitivity of 3-nitrotyrosine as an indicator for oxidative stress}

As 3-NP is an inhbitor of succinate dehydrogenase, exposure of cells or rats to this toxic compound results in oxidative stress. 3. Nitrotyrosine formation as a marker for oxidative stress has been reponted previously in the 3-NP animal model $[6,22,57]$. Un addition, 3-nitrotyrosine has been associated with several neurodegenerative diseases including Huntington's disease, Alzheimer's disease, and Parkinson's disease $[13,23,28]$. Our experimental results, showing increased 3-nitrotyrosine formation at lower dose and time period compared to alterations in other markers, are in agreement with the suggestion made by 5 et al $\llbracket 63 \rrbracket$ that 3-nitrotyrosine formation might be an early marker in neurodegenerative processes. Their suggestion was based on observations in brains of patients with Alzheimer's disease, where 3-nitrotyrosine formation was observed in cells not exhibiting tangles [63]. Similarly, 3-nitrotyrosine staining was observed in slightly affected cells in Lewy bodies in brains of patients with Parkinson's disease [23].

3-Nitrotyrosine formation in proteins has been observed in the close vicinity of blood vessels after focal cortical lesions [8]. Therefore, we hypothesised that elevated 3-nitrotyrosine levels might be found in serum of 3-NP exposed rats. However, no detectable 3-nitrotyrosine formation was observed in serum after treatment of Wistar or Lewis rats with 3-NP. It might be possible that 3-nitrotyrosine had been formed shortly after exposure and was disappeared thereafter, as was observed in brain tissue after focal ischemia in neonatal rats [15]. Similarly, in humans with celiac disease 3-nitrotyrosine formation was only observed in patients in acute phase of the disease [64]. The metabolism of 3-nitrotyrosine might occur by denitrase activity of yet unidentified enzymes [33].

\section{Sensitivity of GS towards oxidative stress}

GS enzyme activity is known to be sensitive towards oxidalive stress. Putatively, the metalcontaining catalytic site of GS is sensitive to oxidation upon which the activity of the enzyme is lost [37]. Decreased activity and concentration of GS has been observed in Alzheimer's clisease brains $[27,54,60]$. In our results, GS was not as sensitive a marker as 3-nitrotyrosine for 3-NP exposure in the spheroid cultures obtained from Lewis rats. However, some caution is needed in the interpretation of these resulls. The results may depend on the dose of 3-NP or the developmentall stage of the cultures. Up to seven times as high GS activity was observed in onemonthold spheroid cultures as compared to two-weeks-old spheroids, reflecting outnumbering of neurons by astrocytes in old cultures $[14,44]$. Interestingly, we showed decreased GS concentration in serum of 3-NP treated Wistar rats. Thus, GS may also be used as a peripheral biomarker in serum for oxidative stress and excitotoxicity induced by 3-NP, ar least in Wistar rats.

\section{Effect of inhibitors of hee radical formation}

\section{L-NAME}

Previous studies showed attenuation of 3-nitrotyrosine formation in vivo by inhibition of NOS activity with 7nitroindazole $[57,58]$. The present results, in contrast, showed a potentiation of 3-ritrofyrosine formation after coincubation of spheroids with 3-NP and L-NAME. Our results are at least in agreement with several studies. For example, increased 3-nitrotyrosine concentration in foefal rat limbs was observed after intra-amniotic injections of L-NAME [19]. Increased 3-nitrotyrosine formation was also observed after exposure of rats to L-NAME in a model for preechiampsia [55] and another report showed that lipopolysaccharide-induced 3- 
nitrotyrosine fomation could not be inhibited by L-NAME 162,76]. These findings are in contrast with the decreased peroxynitrite formation and 3-nitrolyrosine formation that has been obseved after supplementation of L-NAME during traumatic brain injury in mice [42], and in an rat ischemic lung modiel [31]. It might be that the concentration of L-NAME used in our experiments was not high enough to completely inhibit all NOS (NOS/ENOS/nNOS) activity in our relatively chronic experiments. However, we previously showed that exposure of spheroids to the same concentration for 10 min resulted in almost complete inhibition of NO-mediated cGMP formation after stimulation with Nmethyl Duspartic acid (Chapter 2) [66]. Alternatively, NOS or peroxynitrite may play only a minor tole in the formation of 3-nitrotyrosine [25]. Possibly the formation of superoxide radicals is the rate-limiting event in 3-nitrotyrosine fomation. Superoxide reacts with NO at a rate constant of $6.7 \pm 0.9 \times 10 \% \mathrm{~mol} / \mathrm{s}[30]$ and NO has a presumed half-life of 3-55 [16]. Thus the reaction of superoxide with NO is limited by the diffusion of cytosolic produced NO to superoxide, which is produced mainly in the mitochondria. Increased NO production by the neuronal form of NOS may not be the rate limiting step in 3-nitrotyrosine formation in the aged brain, as supported by the reduced concentrations of arginine and citulline, the substrates for NO formation by NNOS, in aged rat brains. Furthermore, reduced nNOS MRNA and enzyme expression was observed in aged rat cerebellar tissue $[43,75]$, although ageing is accompanied by increased 3-mitrotyrosine concentrations, at least in thuman in cerebrospinal fluid [68\#. In contrast, iNOS expression was shown to be increased in the hypothalamus of the aged rat brain [70].

\section{GLUTATHIONE}

We observed a protective effect on total protein concentration, as a measure of cell loss, by vitamin $E$ and DTT, though not by glutathione, which is a weaker reducing agent than DTT. Glutathione is an important antioxidant in brain tissue and a concentration of $1-2 \mathrm{mM}$ has been measured in human whole brain tissue [59]. The lack of protective effects of glutathione is probably not caused by a too low dose of glutathione as a previous report showed that peroxynitrite-induced GS-inactivation was completely inhibited by $1 \mathrm{mM}$ glutathione, the same concentration as we used [36]. In addition, cell death after exposure to peroxynitrite could be inhibited in rat hippocampal neurons by $0.5 \mathrm{mM}$ g/utathione [36]. 3-NP treatment diminished intracellular glutathione concentrations in cultured astrocytes and neurons [49] and 3nitrotyrosine formation was increased after 3-NP exposure in glutathone peroxidase knock wout mice as compared to will-type control mice [3.5]. Nacetylcysteine injections (i.p.) before exposure of rats to 3-NP protected against oxidative damage induced by $3-\mathrm{NP}[20]$. Nacetylcysteine is a precursor for glutathione and may have a longer half-life than glutathione. Thus, the lack of a protective effect in our experiments is likely due to the short half-life of glutathione in combination with chatacteristics of the culture system.

\section{Extrapolation cell cullure - animal model}

In 3-NP exposed spheroids from Lewis rats, we observed an increased 3-nitrotyrosine concentration, whereas no change in the mean concentration was observed in striatal homogenates of 3-NP exposed Lewis or Wistar rats in vivo. In addition, no increase in CFAP concentration was observed in the 3-NP exposed spheroids from both Lewis and Wistar rals. Nevertheless the 3-NP exposed Wistar animals were characterised by increased GFAP concentration, which is in agreement with the literature $[51,71]$. The differences between the in vitro models and in vivo models may be due to differences in experimentation between the in vivo and in witro model. For example, peripheral metabolism of 3-NP may be important in the in vivo model, since the toxim is injected peripherally in the rats and we showed effects on peripheral molecules in this experiment (Chapter 6). Blood-brain-barrier (BBB) disruption may 
puy an impontant role in the pathogenesis of lesions in the in vivo model since it occurs shortly after exposure to 3-NP $[46,47]$. In the spheroid cultures, the toxin has a more direct access to the neuronal tissue as the BBB is limited to a "gha limitans" in these cultures [73]. Another explanation for the differences observed in the effects of 3-NP between the in vitro and in vivo model mav be the dlifferences between the experimental time period of five days in spheroid cultures and the five weeks experiment duration in the rat model. Nevertheless, several similarities between the in vivo and in vitro models exist. For example, in both models activation of microglia was shown. In addition, in the cell culture model as well as in Wistar rats the CABAEgic neurons were affected after 3-NP exposure, while nNOS containing neurons were relatively spared, which is in accordance with the literature [10].

Similar to what has been reported for the 3-NP rat model, we also observed strain differences in the sensitivity of spheroid cultures towards 3-NP treatment [50]. No increased 3-nitrotyrosine concentration was observed in the spheroids obtained from Wistar rats, whereas there was an increase in the spheroids obtained from Lewis rats. This discrepancy may be due to increased antioxidant capacity of the tissue obtained from Wistar rats compared to tissue of Lewis rats, as it has been reported that Wistar rats have, in comparison to Lewis rats, higher antioxidant activity in plasma 152], lower responsiveness of lung tissue towards ozone [17] and higher glutathione peroxidase activity in retinal tissue [72].

Taking into account the similarities between the 3-NP spheroid model and the 3-NP rat model, we propose that whole brain spheroid cultures might be a suitable model to study certain aspects of 3-NP induced neurodegeneration. The model might be a good alternative for in vivo animal experimentation in this respect

\section{References}

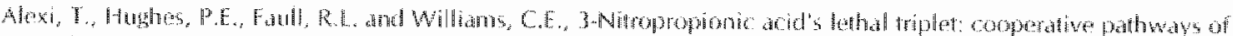

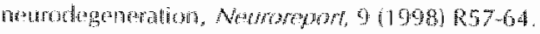

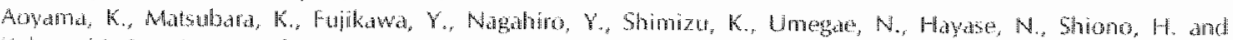

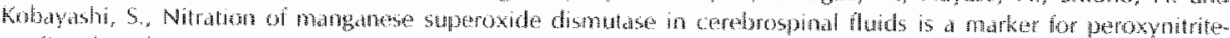

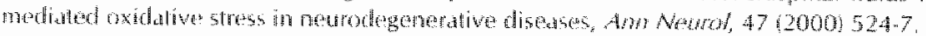

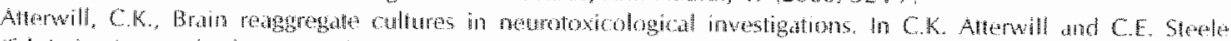

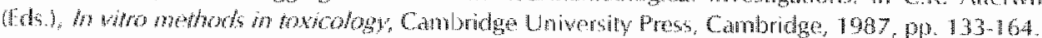

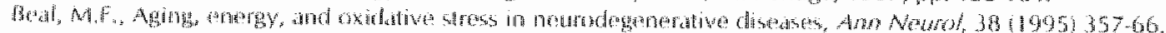

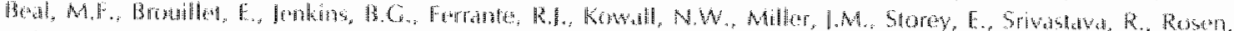

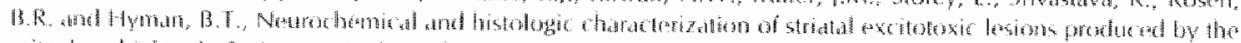

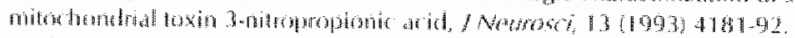

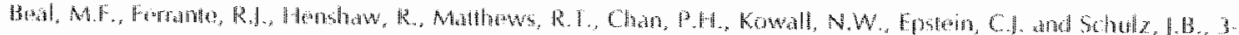

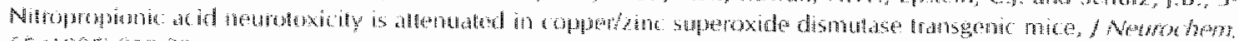
$6(100 \%)+1923$.

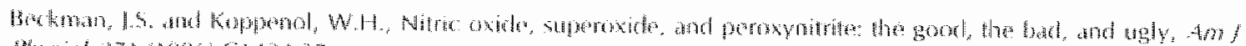

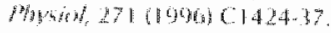

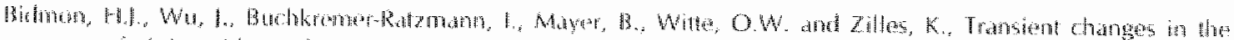

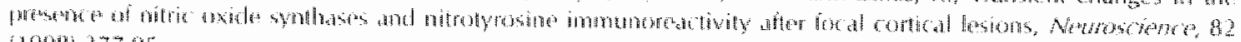
119989789.95

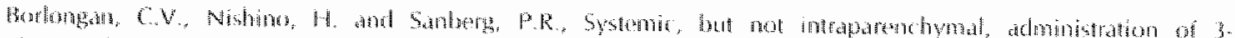

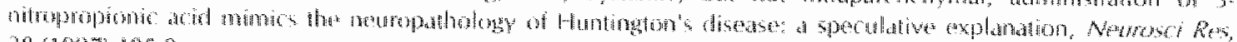
$20.9997 \pi 99$.

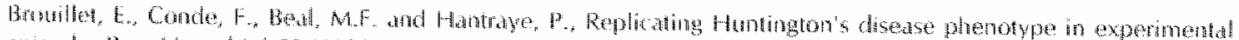

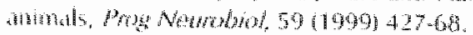

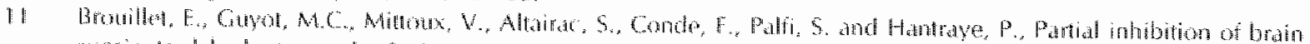

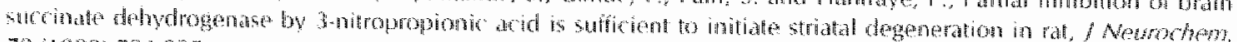
$7001098794-6905$

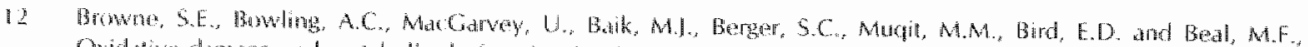

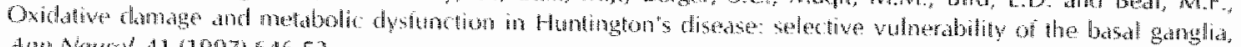

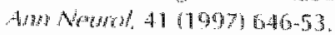

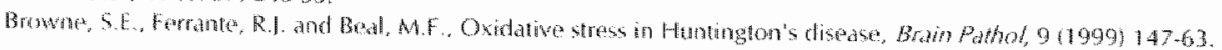




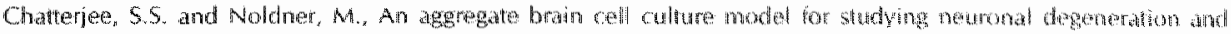

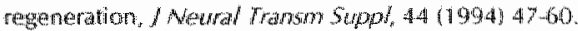

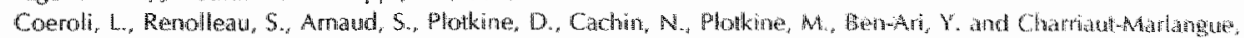

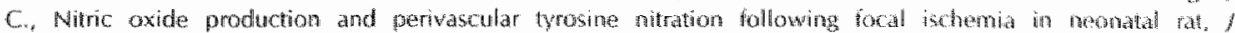
Neurochem, $70(1998) 2516-25$.

Cxapski, $G$, and Goldstein, S., The role of the reactons of NO whth superowide and oxygen in bologicd systems: at kinetic appiroach, Frea Radic Brol Med, 19 (1999) 785-94.

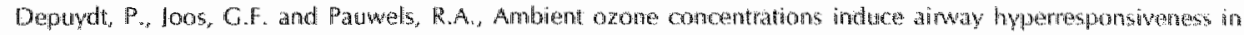

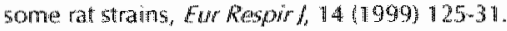

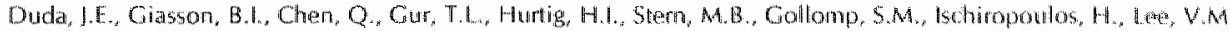
and Trojanowski, I.Q.. Widespread nitration of pathologicat inclusions in newrodegenerative sunucheinopathies, Am fi Pathol, 157 (2000) 143945

Fantel, A.G., Stamps, L.D., Tran, T.T., Mackler, B., Person, R.E. and Nekatai, N., Role of free radicals in the limb

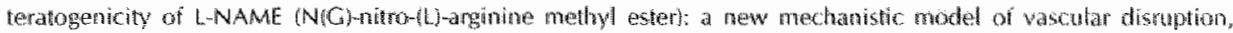
Teratology, $60(1999) 151-60$.

Fontaine, M.A., Geddes, I.W., Banks, A. and Butterfield, D.A., Ffect of exngenteus and endogenous amboxidants an 3-nitropionic acidinduced in wiwo oxidative stress and striatal lesions insights into twintington's distase, / Newrochem, 75 (2000 $1709-15$

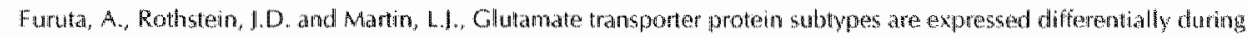
rat CNS development, / Neurosci, 17 (1997) $8363-75$.

Galpen, W.R., Matthews, R.T. Beal, M.F. and Isacson, O., NCF attenuates 3-nimotymosine formation in a 3 -Ne model of Huntington's disease, Neurarepont 7 (10996) 263942 .

Good, P.F., Hsu, A., Wemer, P. Perl, D.P. and Olanow, C.W. Protein mitation in Pakinson's edscease, Meuropanthol Exp Neurol, $57(1998) 338-42$.

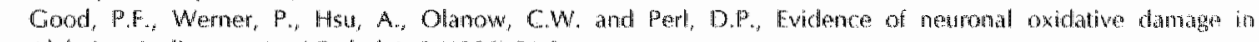
Alzheimer"s dissese. Am / Pand, 149 (1996) $21-8$.

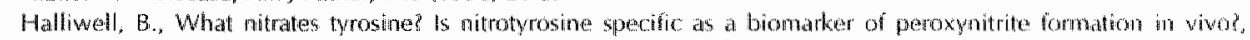
FEBS Let, 411,1997$) 157-60$.

Hassel, B. and Sonnewald, U., Salective inhibition of the tricaboxylic acid cycle of CABAargic neurons whth 3 m nitroptopionic acid in vivo, f Newochem, $65(1995) 118491$.

Henstey, K, Hatl, M., Subramaniam, R., Cote, P., Harris, M. Aksenow, M. Aksenova, M. Gablita, S.P., Wu, I.F. Camey, J.M. and at al, Brain regional correspondence between Alzeimer's disease histopathology and biomakers of protein oxidation, / Newrochem, $65(1995) 2146-56$.

8. Henstey, K, Maid, M.L., Yu, Z., Sang, H. Markesbery, W.R, and Floyd, R. A. Blectiochemical analysis of protein mitwotyrosine and dityrosine in the Alzheimer bran indicates region-specific accumulation, f Nathosci, 183 (1998) $3126-32$

9 Hebison, A.E., Simonian, S.X., Norris, P.l. and Emson, P.C., Relationship of netronal nitric oxter shnthase

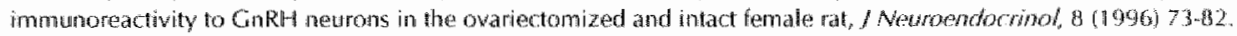
Huie, R.E. and Padmala, $S$, The reaction of NO with superoxide, Free Rafic Res Common, 18 (1993) $195-9$.

Hschiroponlos, H. al-Mehdi. A.B. and Fisher, A.B., Reactwe species in ischemic rat hang injury: contribution of

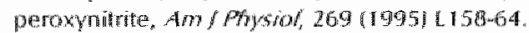

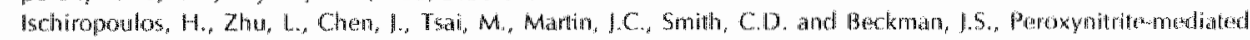
tyrosine aitration catalyzed by superoxide dismutase, Arot Bochem Bhophys; 298 (1992) $431-7$.

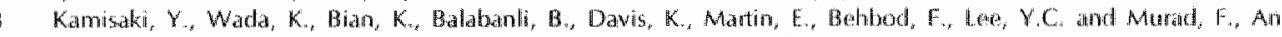

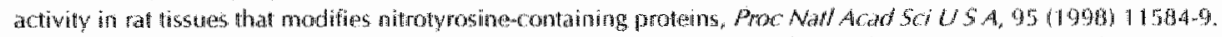

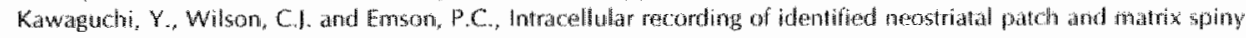
cells in a slice preparation preserving corticat inpuls, / Neurophysiol, 62 (1989) 1052-68.

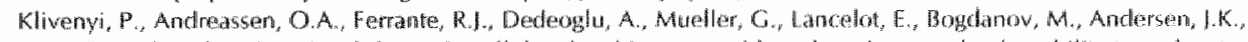

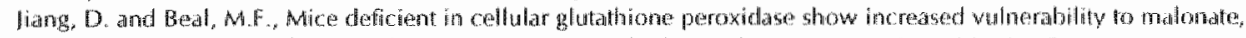

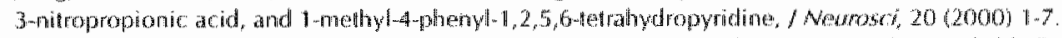

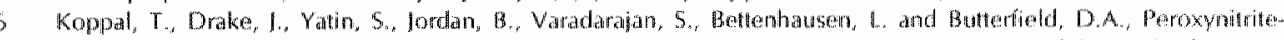

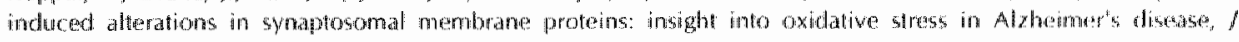
Nevirochen, 72 (1999) 310-7.

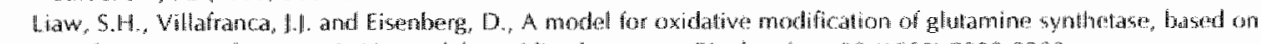

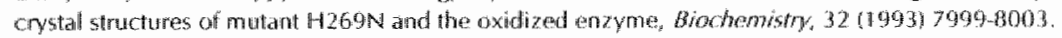

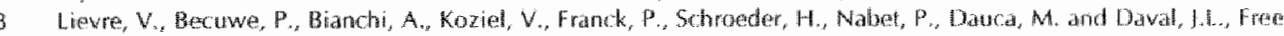
radical production and changes in superoxide dismutases associated with bypoxia/reoxygandicninduced apopiosis of embryonic ral forebrain meurons in culture, Free Radic Brol Aled, 2920001291.301.

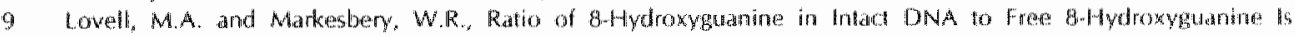
moreased in Althemer Disease Ventricular Cerebrospinal fluid, Awh Newol, 58 (2001) $392-396$.

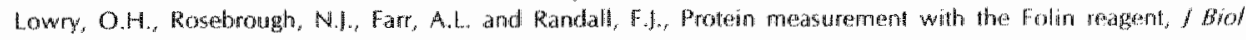
Chem, $193(1951) 265-275$.

Mathews, R., Yuang, L., lenkins, B.G., ferrante, R.,., Rosen, B.R., Kadduah-Darutk, R. and Flint Bewal, M. Neuroprotective effects of creatine and cyclocreatine in animal models of huntinglun "s desease, whe jownd of 


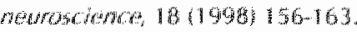

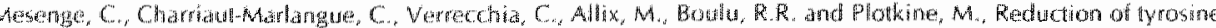

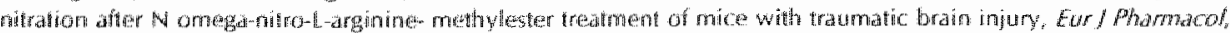
$353(1939) 53 \%$

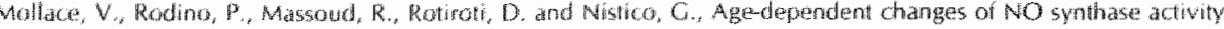

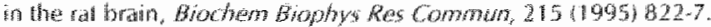

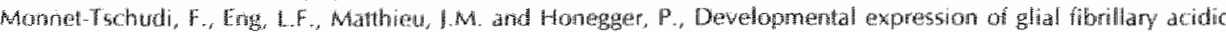

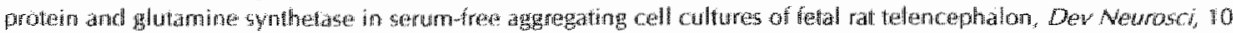
(1) 9899$) 16 \%+172$

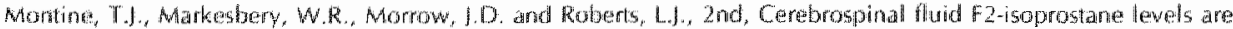

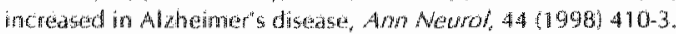

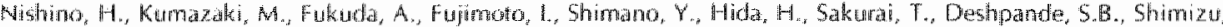

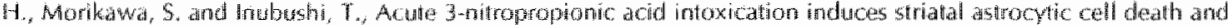

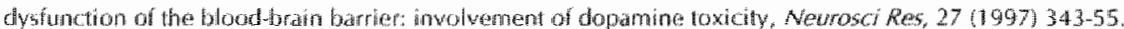

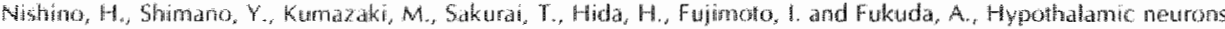

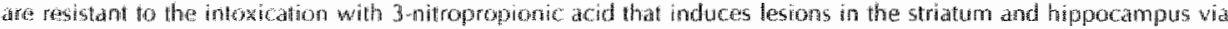

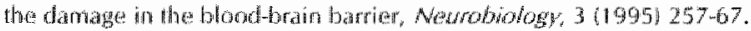

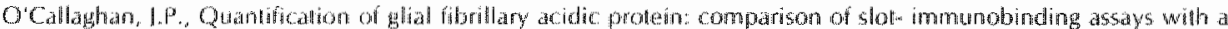

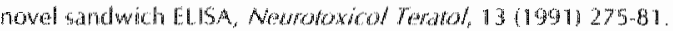

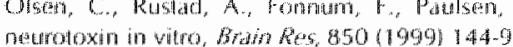

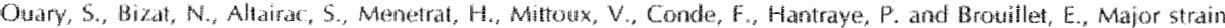

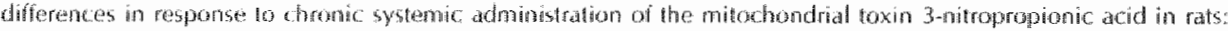

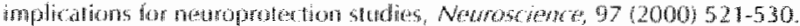

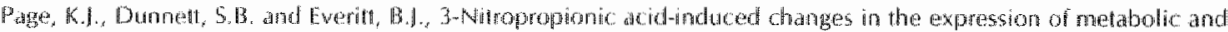

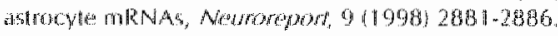

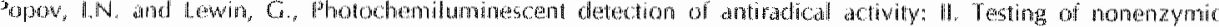

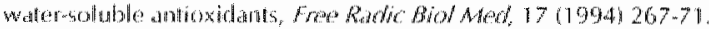

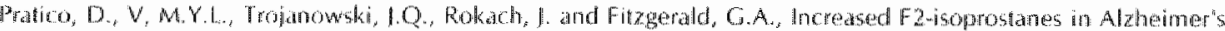

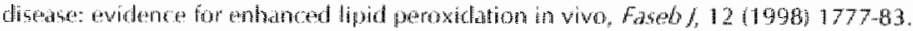

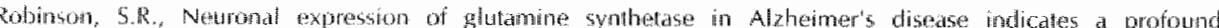

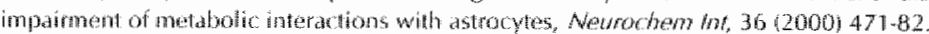

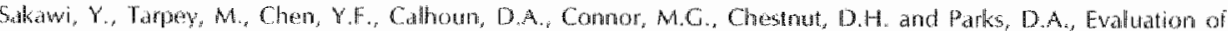

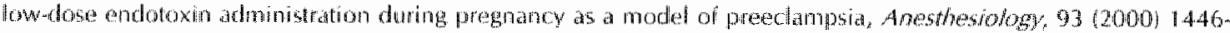
i:

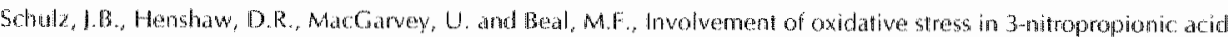
newrotoxicity, Noworhem ho, $29(1996) 167-71$.

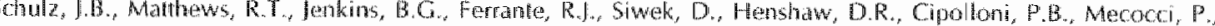

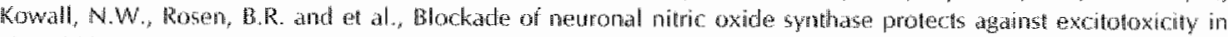
wivo, Nawrost, 1511995$) 841029$

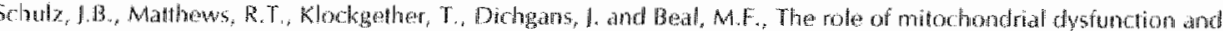

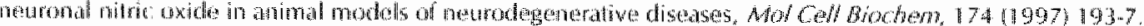

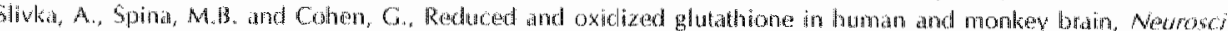
10,$741197 ; 112-8$.

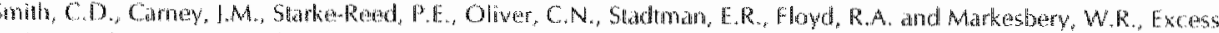

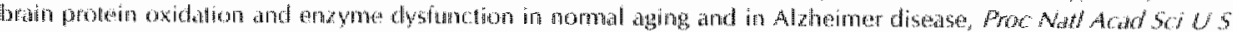
$4.98)(1901) 10540-3$

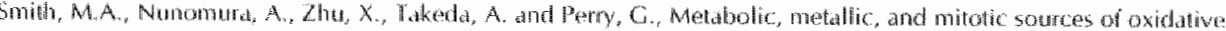

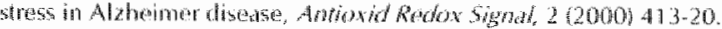

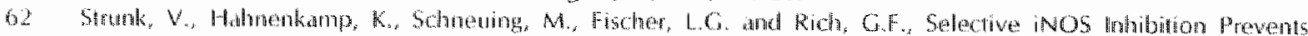

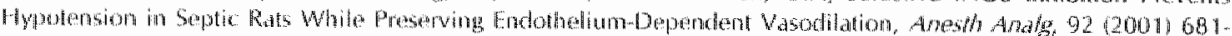
tit. 7.

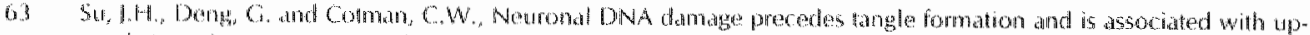

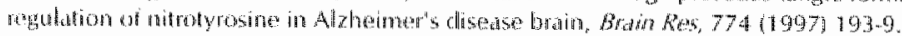

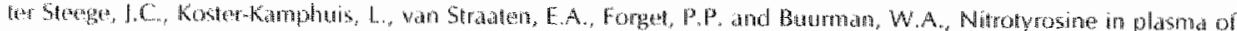

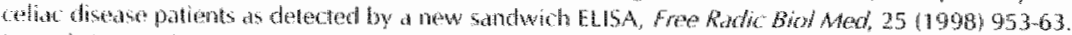

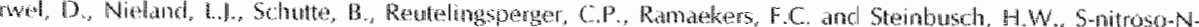

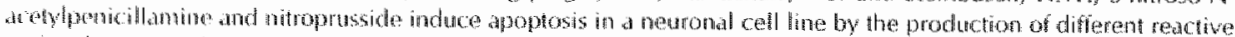

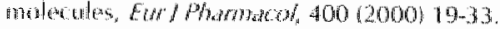

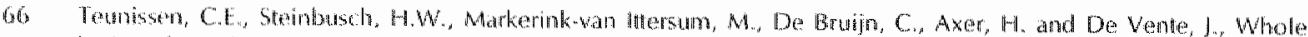

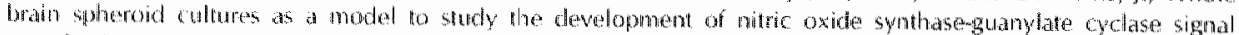

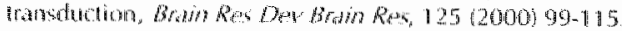

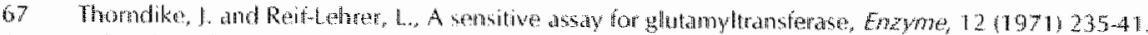

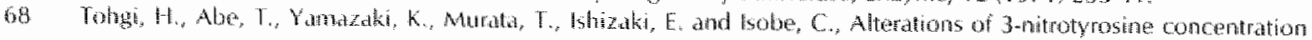




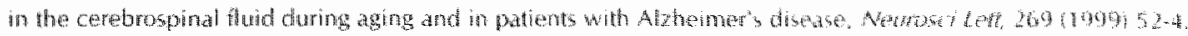

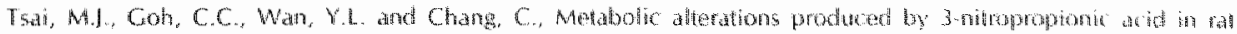

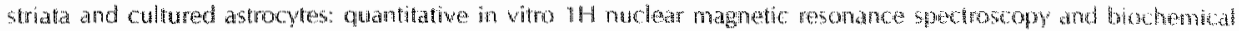

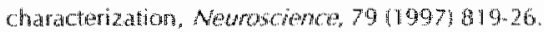

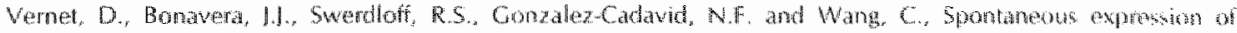

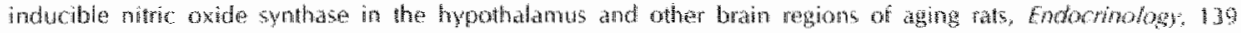
(1) 998$) 3254-61$

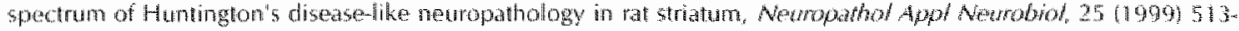
21.

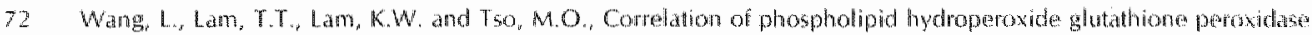

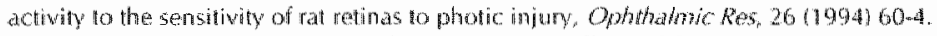

Woodhans, D. A. Atterwil, C.K. and Balazs. R., The eftects of thyoid homone on diferemiation and newrolicment expression in rat brain aggeregating cultures, Neuropathol Appl Nounobiol, $12(1986) 577-92$.

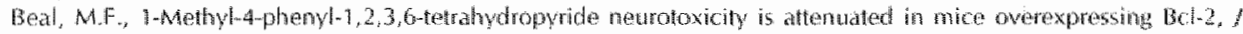
Neurosi 18 11996) 845-52.

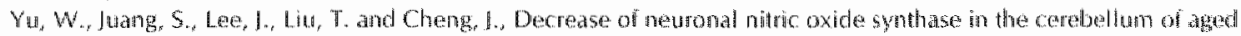
rats, Newrosciteti, 2912000$) 37-40$.

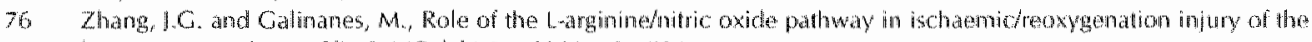
human myocardium. Clin 5 ci Cokft 99 (2000) $497-504$ 



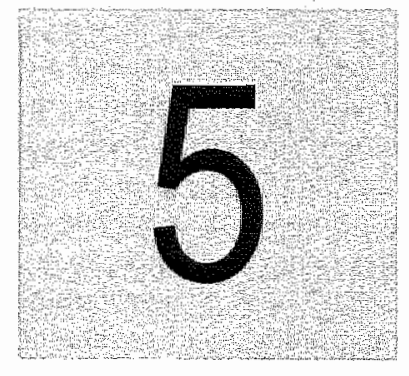

\section{Behavioural correlates of striatal glial} fibrillary acidic protein in the 3nitropropionic acid rat model: disturbed walking pattern and spatial orientation

Charlotte Teunissen, Harry Steinbusch, Maaike Angevaren, Marije Appels, Chris De Bruijn, Jos Prickaerts and Jan de Vente

European Graduate School of Neuroscience (Euron), Universiteit Maastricht, Department of Psychiatry and Neuropsychology, The Netherlands

Neuroscience, $105 / 1$ (2001) 153-167. 


\section{Abstract}

The 3-nitropropionic acid animal model is a model where excitotoxicity, mitochondrial dysfunction and oxidative stress, mechanisms common to various neurodegenerative diseases, are involved. The present study investigated whether behavioural alterations in this model were related to striatal damage.

Wistar and Lewis rats were exposed to 3-nitropropionic acid and their behavioural performance (open field, walking pattern and Morris Water Maze task) was tested after the injections and after a recovery period of three weeks. No changes in activity were found in the open field test. Altered walking pattem was observed in the footprint analysis, although a different response was observed in the Wistar rats compared to the Lewis rats. Initially increased latency times were observed during visual discrimination learning in the Morris Water Maze task in 3-nitropropionic acid treated Wistar rats compared to Wistar controls. During spatial discrimination leaming (invisible platrorm) in the Morris Water Maze task the swirmming welocity was decreased in both rat strains as a result of 3 -nitropropionic acid treatment. Increased striatal glial fibrillary acidic protein concentration in Wistar rats correlated with several parameters of the footprint analysis and with the latency and distance in visual as well as spatial discrimination learning in the Morris Water Maze.

It is concluded that measurement of walking pattern and spatial onientation performance are sensilive indicators to monitor behavioural changes in relation to striatal degeneration in the $3-$ nitropropionic acid animal model. in addition, Lewis rats are less sensitive towards 3nitropropionic acid treatment than Wistar rats.

\section{Introduction}

Neurodegenerative diseases, such as Alzheimer's disease, Parkinson's disease and Huntington's disease (HD) are multifactorial diseases in which mechanisms as excitotoxicity, mitochondrial dysfunction and oxidative stress interact with each other, resulting in complex neurochemical and behavional alterations $[12,22,32,38]$. In the $3-n$ itropropionic acid $(3-N P)$ rat model all these Ilire mechanisms are probably involved (reviewed in: [1]). 3.NP irreversibly inhibits succinate dehydrogenase, an enzyme integral to complex 11 of the mitochondrial electron transport chain and the tricarboxylic acid cycle. This compromises mitochondrial function and results in depletion of ATP, oxidative stress and activation of the N-methyl D-aspartic acid (NMDA) receptors already at normal glutamate concentrations $[3,7,47]$.

The pathology in the 3-NP model is characterised by selective striatal degeneration, concomitant with astrogliosis of which the hallmark is increased expression of glial fibrillary acidic protein (CIFAP) $14,16,35,40,501$. The GABA-ergic spiny neurones are in particular vulnerable for 3 -NP induced toxicity, though the reason for this remains to be clarified [21]. The bethavioural symptoms in rats exposed to a chronic low dose of 3 NP have been characterised as alteration in locomotor activity and recumbence (reviewed in: $[8,9]$. The model may be a good replication of chronic neurodegenerative processes, such as HD. However, HD has an important feature not represented in the 3-NP model, i.e. the expanded polyglutamine repeat of the Huntingtin protein [9].

Between rats treated with 3-NP, there exists a large variability in the behavioural and pathological reactions. Even when rats of the same age and strain are used under the same experimental conditions, a large variation within experimental groups has been observed [9,19]. Various factors contribute to this variation, such as the rat strain and the age of the 
animals. Rats of 4-and 12-months-old seemed to be more wherable than young rats aged 1 month [10]. The difference in behavioural symptoms might also depend on the dose and the way of administration of the toxin as a chronic low dose (i.p. injections) induced a womphase response, hyperactivity followed by hypoactivity, whereas acute doses induced hyperactivity only [6].

The hypoactivity in the chronic model has been studied by monitoring spontaneous activity in open field tests 16,28$]$. Although it is thought that the behavioural deficits are a conseguence of specific striatal degeneration, inhibition of succinate dehydrogenase occurs also in the periphery in this model [1]. It might be that the observed hypoactivity in previous studies was caused by a general suppression of energy availability, rather than by any specific striatal damage. This is supported by results from several studies showing alterations in activity without concomitant altered GFAP concentrations, the hallmark of striatal damage in this model $[19,48]$. We hypothesised that in this model behaviourd changes must correlate with a cellular marker which reflects striatal degeneration. In view of the variation in experimental results isee also discussion) we chose to study an extended number of behavioural tests, incorporating emotional/motivational aspects, learning tasks and motor behaviour, and compared these data with GFAP expression in the striatum. As it has been reported recently that there are important differences between rat strains in this model, we included two related strains in our investigation, i.e. the Wistar rat and Lewis rat (an inbred strain of the Wistar). We wanted to examine if Wistar and Lewis rats were affected similarly under the same experimental conditions. The spontaneous activity of the rats was tested in the open field test. The walking pattern was analysed as a measure for co-ordination of movements, a function of the striatum. Finally, the memory performance was tested in the Morris Water Maze test in both a hippocampus-and striatum-dependent paradigm. To substantiate the relation of the behavioural outcomes with the striatal lesions, the results were correlated with the striatal GifAP concentrations.

\section{Materials and methods}

\section{Animals}

Twelve male Wistar rats were obtained from Janvier, Le Genest-Sant-lske, France. Twelve male Lewis rats were obtained from the local animal breeding facility. The animals were six months old. All animal experiments were performed according to the guidelines of the Universily Comithittee on Animal Welfare, which follow the European Communities Council Directive $(86 / 609 / \mathrm{EEC})$. All efforts were made to minimise animal suffering and to use only the number of animals necessary to produce reliable scientific data, and to utilise alternatives to in vivo technigues, if avallable.

Of each rat strain 7 animals were assigned to the 3-NP treatment group and 5 animals to the control group. All rats were housed individually and had free access to food and water. The rats were kept at normal light/dark cycle (lights on from 6.00 to $18.00 \mathrm{hr}$ ). The excess number of animals in the treatment groups was based on studies reporting large variation, including death, in treatment elfects [8]. The animals in the 3-NP treatment groups received 6 i.p. injections with 3-NP (Sigma, Sigma, St. Louis, USA) in a dose of $20 \mathrm{mg} / \mathrm{kg}$ dissolved in saline (pH adjusted to 7 8) every second day. The use of i.p. injections has been described to provide a suitable model replicating chronic neurodegenerative processes in humans, such as in $\mathrm{HD}[8,9,34]$. The control groups were treated with saline under the same regime. One animall of the 3-NP Wistar rats was severely paralysed after the 3 rd injection and was therefore sacrificed and excluded from further analyses. Another 3-NP treated Wistar rat had lost $35 \%$ of its initial body weight after the $6^{\text {th }}$ 
injection and showed signs of severe hypoactivity. This animal was injected with saline i.p. 15 $\mathrm{mL} / \mathrm{day}$ and fed glucose solution (per os, $5 \mathrm{~mL}$ ) during one week to avoid starvation and dehydration. This animal was excluded only from the second measurement in the footprint anatysis.

The animals were tested in the locomotor tests (open field and footprint analysis) before. directly after the injections, and after a 3 week recovery period (Table 1). In addition, in the two weeks after the injections the rats were tested in the Morris Water Maze test. After completing the behaviourall tests, the rats were decapitated and the right half of the striatum was dissected, homogenised in phosphate buffer $\left(2 \mathrm{mM} \mathrm{NaH} \mathrm{NO}_{4} \mathrm{H}_{2} \mathrm{O}, 0.5 \mathrm{mM}\right.$ ethylenediamineteraacetic acid, $145 \mathrm{mM} \mathrm{NaCl}$, pH 7.4,0.1 g/mL) supplemented with the protease inhibitors soybean

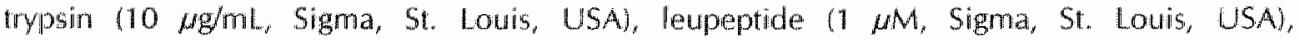
phenylmethylsulfonyl fluoride (1 $\mathrm{mM}$, Sigma, St. Louis, USA and aprotinine $(8.11 \mathrm{U} \sim \mu \mathrm{gg} / \mathrm{mL}$, Fluka, Buchs, Switzerland) and stored at $-80^{\circ} \mathrm{C}$. The left half of the brain was used to prepare slices of $3000 \mu \mathrm{m}$ thickness and these were fixated in $4 \%$ freshly depolymerised paraformaldehyde during 3 hours. After overnight immersion with $10 \%$ sucrose solution, the slices were quickly frozen in Tissue-Tek ${ }^{6}$ and stored at $-20^{\circ} \mathrm{C}$.

Table 1 Tume schedule of the experiments

\begin{tabular}{|c|c|c|c|c|c|}
\hline & & & week & & \\
\hline & 1 & 2 & 3 & 4 & 5 \\
\hline \multirow[t]{4}{*}{ trests: } & open thellit & & open field & & open fifeld \\
\hline & footprint analysis & & tootprint analysis & & footprint analysis \\
\hline & & & $\begin{array}{l}\text { Morris Waler } \\
\text { Maze: }\end{array}$ & $\begin{array}{l}\text { Morris Water } \\
\text { Maze: }\end{array}$ & \\
\hline & & & -visible platiorm & $\begin{array}{l}\text { winvisible platform } \\
\text {-probe trial }\end{array}$ & \\
\hline freatment: & start injections & injertions & finish inpections & & decapitation \\
\hline
\end{tabular}

\section{Open field}

The open field consisted of an area of $51 \times 51 \times 38 \mathrm{~cm}$. Locomotor activity was monitored during 30 min with a video tracking system (EthoVision, Noldus Equipment). Four rats were tested simultaneously. Average velocity and distance moved were calculated.

\section{Footprint analysis}

For evaluating locomotor pattern the footprint analysis was performed as described previously $[13,29]$. The hind feet of the rats were stained with black nutrition ink. The rats were then allowed to walk through a canal $(0.11 \mathrm{~m} \times 0.11 \mathrm{~m} \times 2 \mathrm{~m})$ covered with blank sheets of paper. The prints thus obtained were scanned with a conventional paper scanner and analysed in a computerised footprint analysis program (Footprints, Version 1.22 [27]). The following parameters were measured (Fig. 1): spreading toe 1-5, spreading toe 2-4, step width, step length, paw length, angle of paw axis relative to the canal length and area touched per paw.

\section{Morris Warer Maze Task}

The visual discrimination learning of the rats was assessed in a circular black water tank. (diameter $1.22 \mathrm{~m}$ ) filled with clear tap water $\left(20-22^{\circ} \mathrm{C}\right.$ ). During this experiment with a visible 


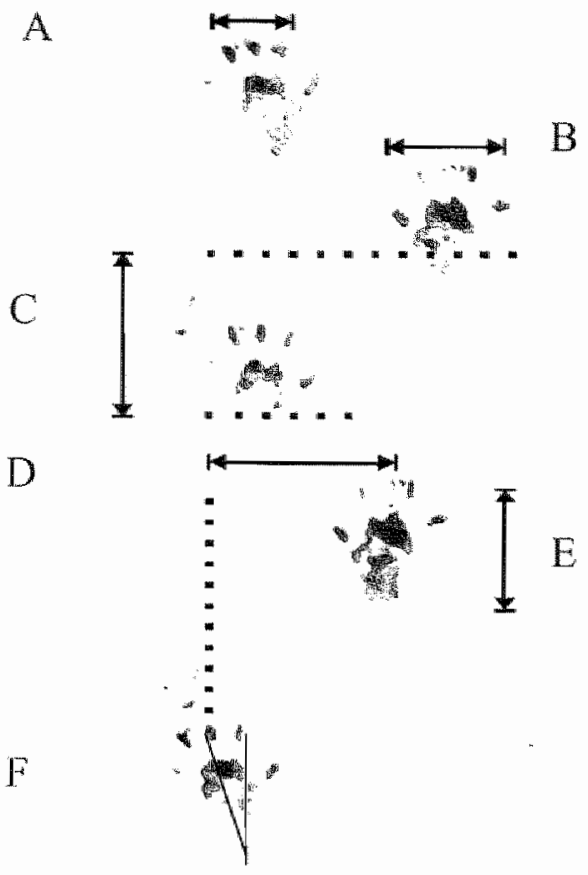

Fig. 1 Parameters inwestigated in the footprint analysis.

Parameters measured are: A: Spleading inner toes (2-4). B: Spreading toe 1-5. C: Step lengih, i.e. distance between wo subsequent feet on the $y$-axis. D: Step widh, i.e. distance between two subsequent feet on the $x$-axis. F: Foot lingth, i.r?

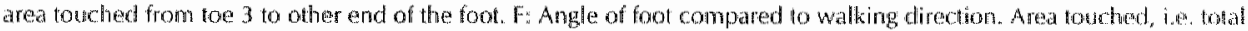
surface of foot in black ink (not indicated in figure).

platform the rats were trained on four daily sessions of four trials to find a white platform (diameter $11 \mathrm{~cm}$ ) that was $1 \mathrm{~cm}$ above the water level. The location of the platform and the starting positions were randomised over the four quadrants of the tank for each trial. A trial lasted until the rat had found the platform or until 60 s had elapsed. If the rats could not find the platform within 60 s they were placed on the platform for $3 \mathrm{~s}$. In the consecutive week, the rals were given a total of four daily sessions, each session consisting of four trials, to find a black platform (diameter $11 \mathrm{~cm}$, which was placed $1 \mathrm{~cm}$ under the water level. During these drials the platform remained at a fixed position, while the slarting posilions were randomised. Trials were ended after a rat had climbed onto the platform or untill 60 s had elapsed. The day after the last triall, a probe trial was given to reveal whether the rals actually learned the position of the platform. For this purpose the platform was removed from the water tank and the belyavour of the rats was monitored during $30 \mathrm{~s}$. Registration of the behaviour of the rats during all trials was done using a video tracking system (Ethovision, Noldus Equipment, Wageningen, The Netherlands) and stored on a computer. The parameters analysed were: distance, i.e. the length of the path swum from the starting position to the platform; latency, i.e. the lime needed to find the platform; swimming velocity, i.e the mean speed of swimming during a trial. In the analysis of the probe trial the time the rats spent in the different quadrants was evaluated. 


\section{GFAP anzyme-linked immunosorbent assay (LLISA)}

GF AP concentrations in striatal homogenates (concentration homogenates $0.2 \mathrm{mg}$ protein/ml was determined using an ELISA as described by O'Callaghan [37]. The primary antibody ufinal concentration $10 \mu \mathrm{g} / \mathrm{mL}$ in PBS was a mouse anti-GFAP monoclonal antibody obtained from Sigma (St. Louis, USA, clone G-A-5) and its specificity has been described by the supplier. The rabbit anti-GFAP polyclonal antibody was a generous gitt trom F.R.C. Ramaeckers (Department of Molecular and Cellular Biology. Universiteit Masstricht, dilution 1:500). Purified bovine GFAP protein (Progen Biotechnik GmbH, Heidelberg, Germany) was used as a standard. The detection system used was anti-rabbit IgG labelled with an alkaline phoshatase conjugate (dilution 1:3,333, Vector, Burlingtiame, USA) and p-nitrophenyiphosphate in diethanolamine solution (Bio-Rad Laboratories, Veenendaal. The Netherlands). Extinction was measured at 405 nm on a Benchmark microplate reader (Bio-Rad Laboratories, Veenendaal, The Netherlands).

\section{Immunohistochemistry}

Cryostat sections (20 $\mu m$ thick) were prepared. Sections were air-dried at room temperature for $20 \mathrm{~min}$. After three times wasthing in Tris-buffered salline containing $0.3 \%$ (v/v) Triton $X-100$ (TBS-T, $p H$ 7.6), sections were incubated overnight at $4^{\circ} \mathrm{C}$ with the primary antibodies. The same mouse GFAP monoclonal antibody as for the GiFAP ELISA was used for the GFAP immunohistochemistry (dilution $1: 1,600$ in TBS-T). The polyclonal antibodies against nNOS and calbindin were kindly provided by P.C. Emson, Cambridge, UK. The antibody against nNOS was raised in sheep (1:750 dilution) [23]. The antibody against calbindin, a protein found in GABAergic structures [41] dilution 1:1,000) was raised in rabbit [26]. After three times washing the sections were incubated with a secondary antibody against mouse or rabit labelled with a CV3 fluorescent Jackson Immunoresearch Laboratories, Inc., Pennsylvania, USA, dilution 1:800) during one hour. After three additional washes the sections were immersed with glycerol (Merck, Darmstadt, Germany) in TBS (3:1) and coverslipped. Sections were processed for standard hematoxylin-eosin staining. Photomicrographs were made with a Olympus microscope, model AX70 TRF, equipped with a narrow band MNIBA-type FITC filter and a MNG filter for CY 3 iluorescence.

\section{Quantification of CFAP immunohuorescence}

CFAP immunofluorescence in the dorsal striatum was recorded with a Sony (Power HAD) CCDcamera. Unages were imponed into a computer with an image analysis program iSoft Imaging System, AnalyS15 3.0, Münster, Germany). Fuorescence intensity was converted into grey values, divided in 64 classes. A histogiam of the division of the pixels over fluorescence intensity classes was calculated. The class with the maximum pixel number was monitored and the amount of pixels in the other classes were expressed as percentage of the pixels found in this class. Two sections every $200 \mathrm{~km}$ throughou the slice were processed in this way. The rotal number of striatal sections per rat included in the measurements is indicated in the legends of the ligures. Sections were stained simultaneously and recorded on the same day to minimise experimental variation.

\section{Statistical analysis}

Data are expressed as means \pm standard error of means $\{ \pm$ s.e.m. Treatment and strain effects in the data of the Morris Water Maze test were evaluated with a three-factorial (Session * Treatment : Strain) analysis of variance (ANOVA) with repeated measures over Session. For differences between the four groups (Wistar control, Wistar 3-NP, Lewis control, Lewis 3-NP) 
on a single session, ANOVA was used with Scheffe's test as Posthoc analysich Theatment and strain effects in the data of open field and footprint analysis were evaluated with a threem lactorial (Time * Treatment * Strain) ANOVA with repeated measures over Time. For the probe trial, preferences for quadranis were evaluated per group by analysing the time spent in each quadrant with t-statistics, to analyse whether the time spent in a quadrant differed from the chance level $(7.5 \mathrm{~s})$. In addition, fime spent in the quadrant where the platform had been was analysed with a two-factorial ANOVA (Strain * Treatment). Differences in GFAP concentrations as measured with the ELISA were evaluated with a one-factorial ANOVA of the ranks. To reveal differences between 3-NP-treated and control rats within a rat strain, a student's t-lest was used. Normality was tested with the Kolmogorov 5 mimov test.

A correlation analysis (Pearson) between GFAP concentrations and pertormance on behavioural tasks was performed. Of the data obtained with the Morris Water Maze test the mean, i.e. height of the curve, and linear components, i.e. slope of the curve, of the learning curves were calculated over session 1-4 (visible plattorm) and session 5m8 (invisible platform) of the Morris Water Maze. Of the datta obtained with the open field and the footprint analysis the mean and linear components over the 3 time points of the measurements were calculated. For the area touched and the angle of the feet a quadratic regression component was calculated as the variance in these parameters was explained better by a quadratic expression than by a linear expression.

The level of significance was $P<0.05$ for all analyses. A P $<0.1$ was estimated as a trend.

\section{Results}

The body weight initially decreased in the 3-NP exposed Wistar rats (Fig. 2). The decrease in body weight was significantly different from the control Wistar rats alter 10 days $\quad 1-3.20$, $P<0.05$ ). In the period after cessation of the injections, these animals recovered to obtain a body weight that was not significanty different from control Wistar rats on day 15 . In contrast, the Lewis rats did not show any difference between control and 3 NP exposed animals.

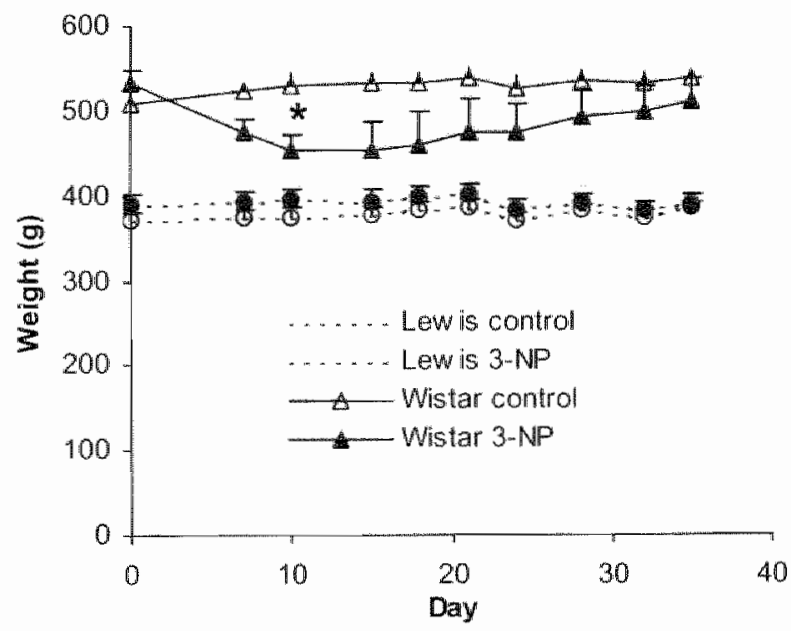

Fig. 2 Weigh of the rats toring the experingent.

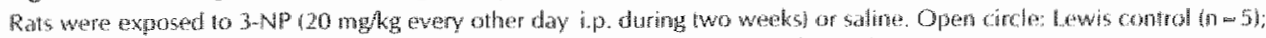

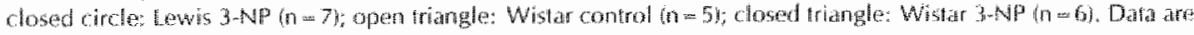

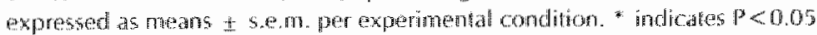




\section{Open Field}

The locomotor activity of all animals was tested in the open field before and directly after cessation of the injections and after a three week recovery period. The mean locomotor activity (1 s.e.m.) in the open field before starting the treatment was $2265 \pm 210 \mathrm{~cm}$ for Lewis $3 \mathrm{NP}$ exposed rats, $1572+306 \mathrm{~cm}$ for Lewis controls, $2135 \pm 252 \mathrm{~cm}$ for Wistar $3-N$ N exposed rats and $2829 \pm 416 \mathrm{~cm}$ for Wistar controls. In view of the high variance, these data represented in the graph were normalised to the activity before the treatment (Fig. 3). No alterations as a consequence of the injections were observed in the repeated measurements and this may be explained by the large variation within the groups. However, more in detail, a difference in distance moved existed between the Wistar 3 NP and Wistar control group at the second measurement $(t=-2.58, P<0.05$ ). Although close inspection of Fig 3 would suggest a treatment effect in the Lewis strain at the third measurement, this was not statisically demonstrated. No dilference in speed of movement of the animals was observed in the repeated measurement analysis or on each of the measurements (data not shown).

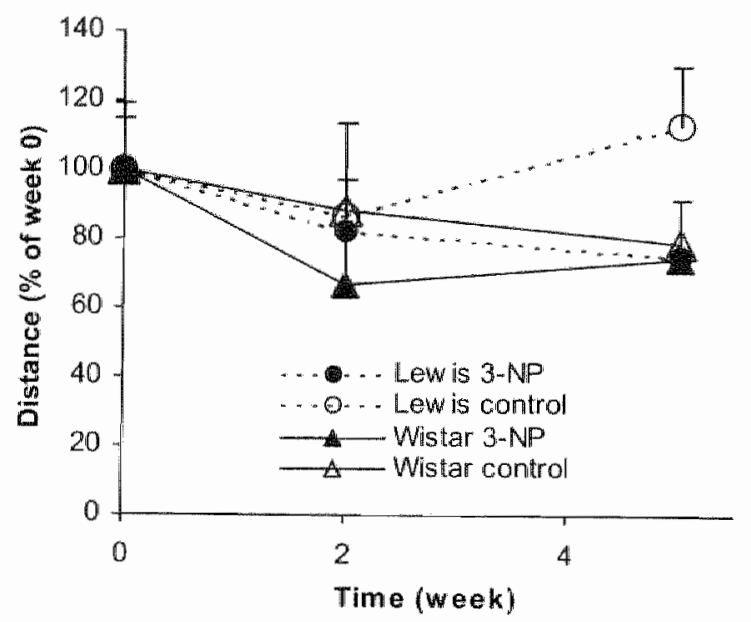

Hig. 3 Totald distance moved in the open field test.

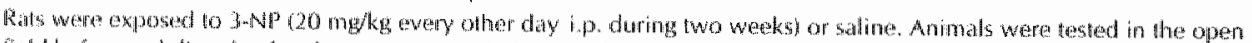

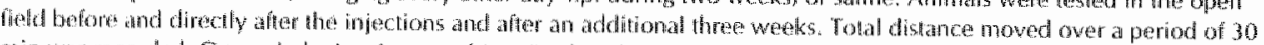

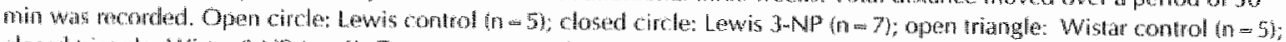

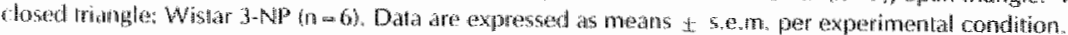

\section{Morris Water Maze}

The results from the Morris Water Maze experiments for all groups of rats on the parameters latency, total distance moved and velocity, are summarised in Fig. 4.

\section{VISUAL DISCRIMINATION}

The escape latencies were reduced in all animals during the visual discrimination learning sessions (visible platform) $(F(3,63)=142.05, P<0.01)(F i g$ 4A). Overall, the 3-NP treated animals had a longer latency time compared to the controt animals over the sessions (general mean Treatment: $F(1,19)=4.52, P<0.05$ ). More in detail, in the Wistar rats, the latency time was longer in the $3 \mathrm{NP}$ exposed rats compared to the control Wistar rats during the first session 


\section{A Visual discrimination learning}

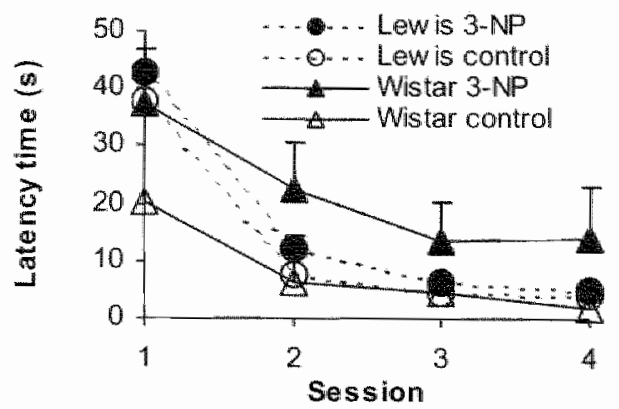

C Visual discrimination learning

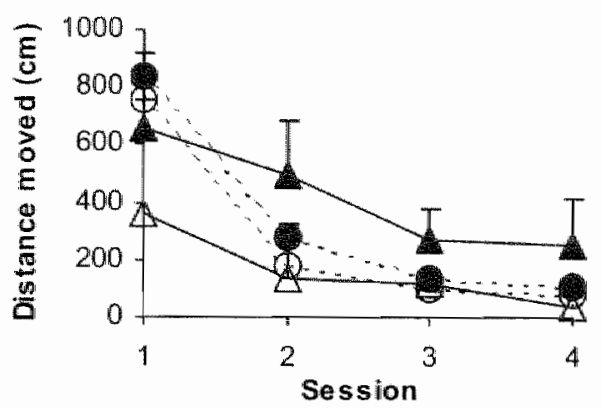

\section{E Visual discrimination learning}

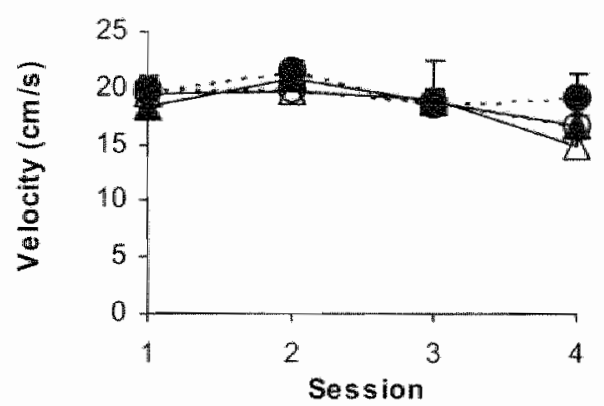

B Spatial discrimination learring

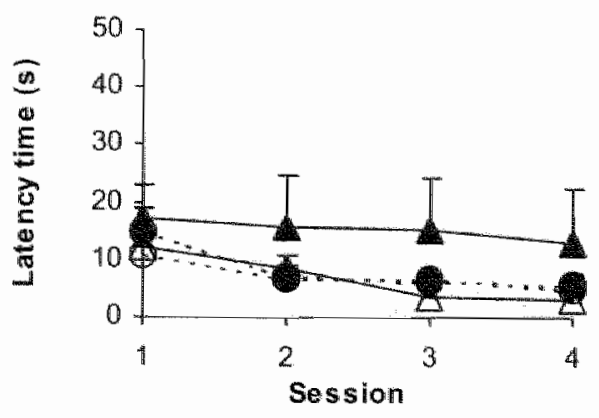

D Spatial discrimination learning

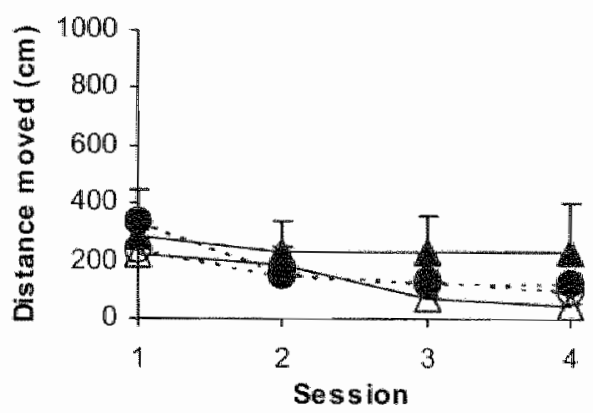

F Spatial discrimination learning

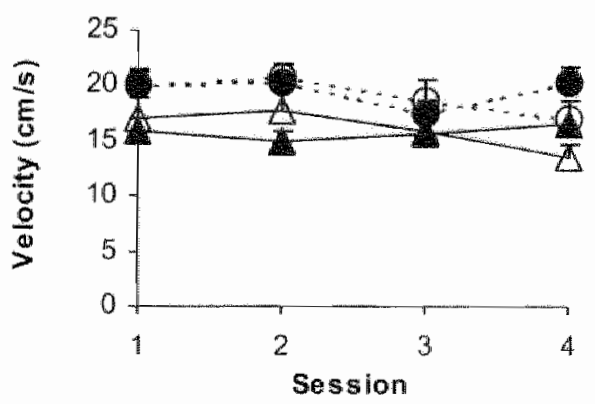

Fig. 4 Menory perfonmance in the Morris Water Maze task.

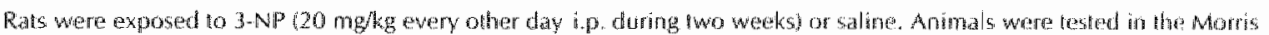

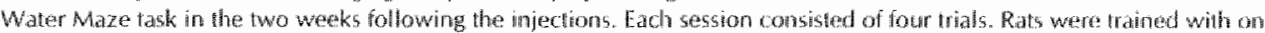

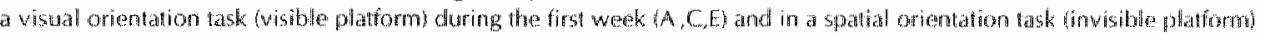

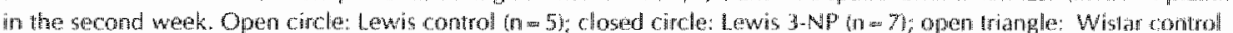

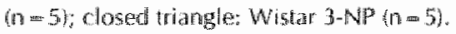

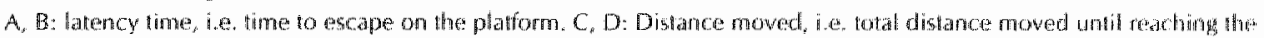

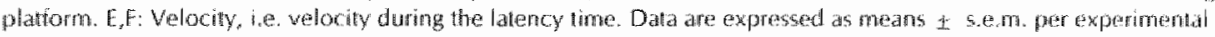
condition. 
with the visual platform $(t=2.86, P<0.05)$. From the second session on, the latency time of these treated Wistar rats was comparable to control Wistars. The 3-NP treated Lewis rats had a similar penformance as the control lewis rats.

The total distance moved during this visual discrimination task reduced in all groups during the sessions $(\mathrm{F}(3,21)-11.27, \mathrm{P}<0.001)(\mathrm{Fig}$. $4 \mathrm{C})$. Overall, the $3-\mathrm{NP}$ treated animals of both strains traversed a longer distance than the control animals during this task (general mean Treatment: $F(1,19)=4.53, P<0.051$

When the velocity was examined in the wisual discrimination task, we round that all groups. showed a decrease in velocity during the training sessions $(F(3,21)=5.31, P<0.01)$ with no significant effect of 3-NP treatment on this parameter (Fig. 4E).

\section{SPATIAL DISCRIMINATION}

Latency times en total distance moved were reduced during the training sessions for spatial discrimination (invisible platiorm) in all groups $(\mathrm{F}(3,21)=7.84, \mathrm{P}<0.001$, Fig. $4 \mathrm{~B}$ and $F(3.21)=5.71, P<0.01$, Fig. $4 D$, respectively). No differences after $3-N P$ treatment for both parameters were observed. However, an effect due to 3-NP treatment was observed in the decrease in swimming velocily during this task (Session theament: $F(3,63)=3.24, P<0.05$ ) (Fig. 4F).

One day after the last trial session a probe trial, in which the platform had been removed, was given. Under normal conditions in such a probe trial, it is expected that animals spend more time than the chance level in the quadrant where the platform had been located $7.5 \mathrm{~s}$ in each quadrant over a total period of 30 si. The results in Fig. 5 demonstrate that all animals spent more time in the quadrant where the platform had been, indicatting preserved spatial memory function. The time spent in the quadrant where the platrom had been was lower in the 3-NP treated animals, although this decrease was only a trend (Treatment: $F(1,19)=4.23, P<0.054$ ).

\section{Footprint analysis}

The effects of $3-N P$ exposure on locomotor function were tested in the footprint analysis before and directly after cessation of the injections and after a three week recovery period (fig. 6). One animal, the one that had lost $35 \%$ of its initial body weight, was excluded from the measurements because of its lack in motor activity all the time of the second measurement. Footprint analysis revealed significant differences in walking pattern between Lewis and Wistar rats on the parameters area (Fig. 6A), distance bewween toe 24 (Fig. 6B), stride width (Fig. $6 \mathrm{C}$, distance between toe 1-5 (Fig. 6F) and foot length (Fig. 6F)general mean Strain: $F>5.8, P<$ 0.05 for all parameters?.

A graph of the mean surface of the area touched per paw of the four groups during the expenments is depicted in Fig. $6 \mathrm{~A}$. Analysis of area touched revealed an alteration over time in both stains (Time: $F(3,63)=55.43, P<0.0011$ and an effect due to 3-NP treatment in both strains (Treatment * Time effect $(F(3,63)=3.67, P<0.05)$ which was however different for both strains $(F(3,63)=6.66, P<0.01)$. Close inspection of the second measurement after wo weeks revealed a signiffcant increased area touched in the Lewis 3-NP compared to Lewis control animals $(t=-2.60, P<0.05)$ and a trend for the decrease in area touched between Wistar $3-N P$ and Wistar control animals $(t=2.24, P<0.055)$.

The spreading of the inner toes, as measured by the distance between toes $2-4$ was not significantly affected Treatment * Time effect $(F(3,63)=3.12, P<0.056)$. Nevertheless, a significant interaction between Stram * Treatment * Time was observed, indicating that the pattern of decreased distance of these inner toes after 3-NP treatment differed between the Lewis and Wistar ratts $(F(3,63)=3.63, P<0.05)$ (Fig. 6 B). 


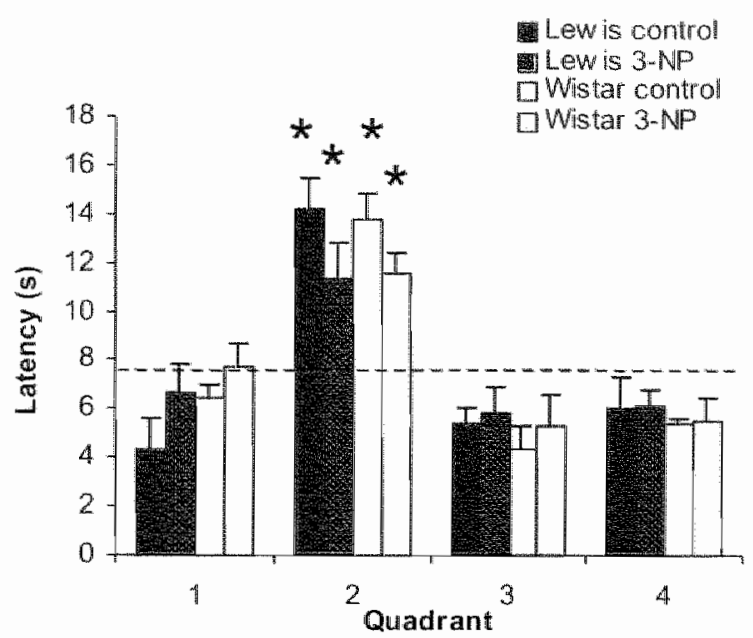

Fig. 5 Performance during the probe trat of the Morris Walce Maze lakt.

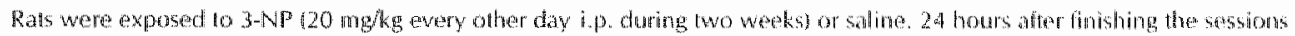

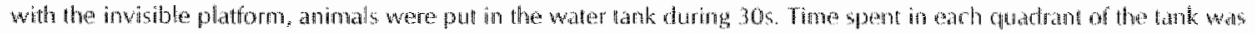

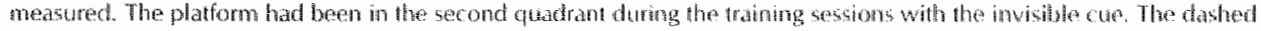

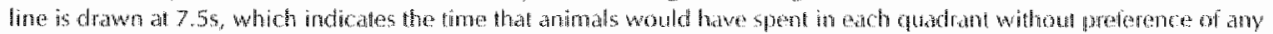

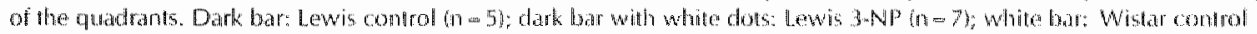

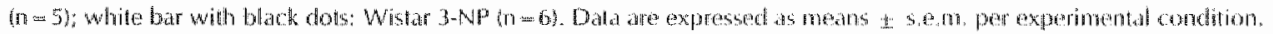

* indicates $p<0.05$.

The stride width (Fig. 6C), the angle of the feet towards the axis of the walking direction (Fig. 6D) and spreading toe 1-5 and foot length (Fig. GE and F) were not aifected by 3-NP treatment. Finally, analysis of the stride length revealed a significant increase in length due to 3 -NP treatment (Time * Treatment $(F(3,63)=3.58, P<0.05)$ idlata not shown).

\section{GFAP determination (immunoassay/immunocytochemistry)}

After completion of the behavioural experiments, the animals were decapitated and GFAP concentration was determined in striatal homogenates. The results in Table 2 show that the striatal GFAP concentration in the Lewis 3-NP group was comparable to that in control Lewis rats. The mean GFAP concentration in striatal homogenates was higher in the 3-NP treated Wistar rats compared to the Wistar control group $(t=2.29, P<0.05)$. Some variation existed within the Wistar 3-NP exposed group as shown by the relatively large s.e.m. as compared to the other groups (22.3 ng/mg protein in Wistar 3-NP group vs. 5.1-9.7 in the other groups).

The increase in striatal GFAP in the 3NP exposed Wistar rats was confirmed using immunohistochemistry (Fig. 7). In the most affected animal a clear lesion was observed with a border of increased GFAP staining (Fig. 7B). In one animal withoul any overt lesion, a core with increased GFAP staining could still be observed (Fig. $7 \mathrm{C}$ and compare Fig. $7 \mathrm{D}$ vs. $7 \mathrm{E}$ ). Im addition, increased GFAP staining was observed in the corlex and corpus callosum in the 3-NP exposed animals.

Image analysis of the GFAP irnmunofluorescence, as an objective and sensitive method to determine diference in immunofluorescences, was performed. The results of the inage analysis in the dorsal stratum further confirmed the increased GFAP concentrations in the 3-NP exposed Wistar rats. The histogram of the grey value distribution was detemined throughout the obtained stratal slice of $3000 \mathrm{~km}$ at intervals of $200 \mathrm{\mu m}$. The immunofluorescence palterm 

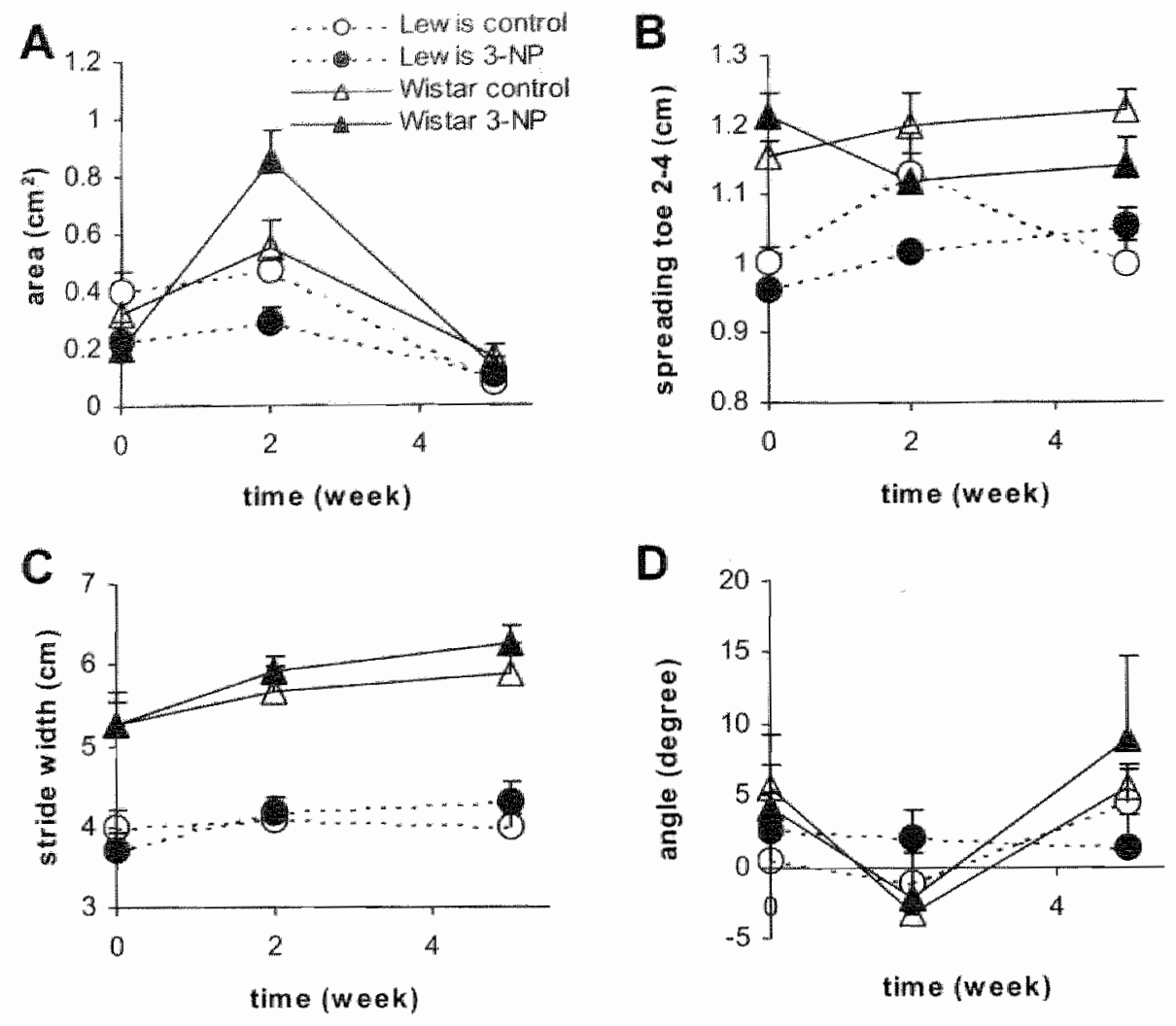

time (week)
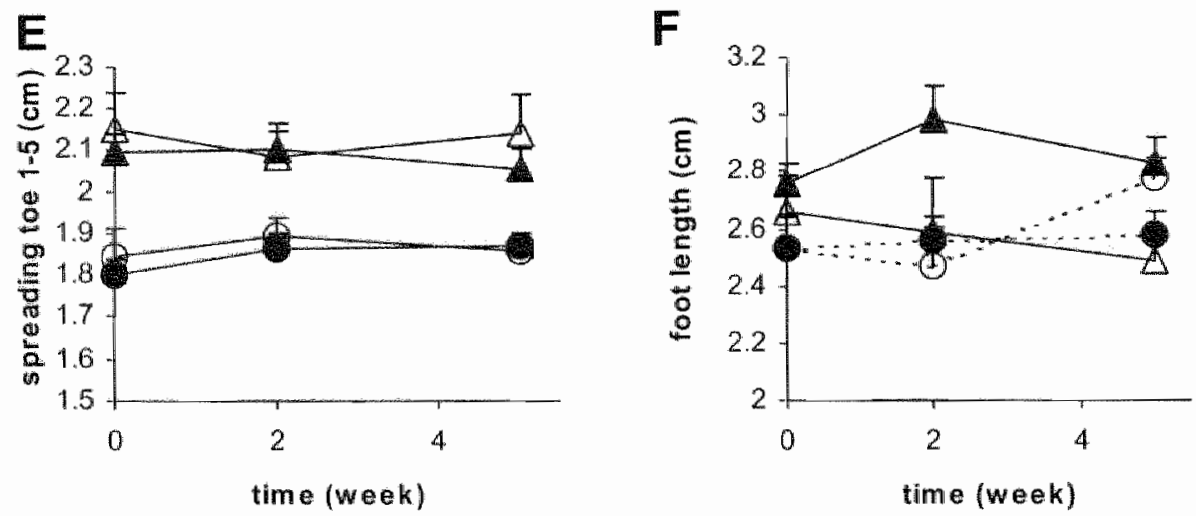

Fig. 6 Walking patten as mastred with the footpring antlysts.

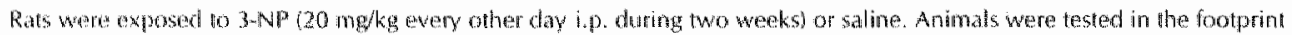

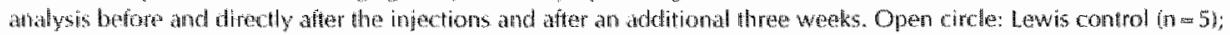

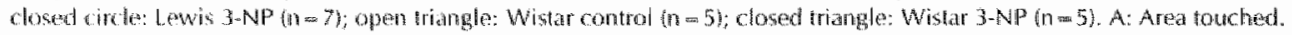

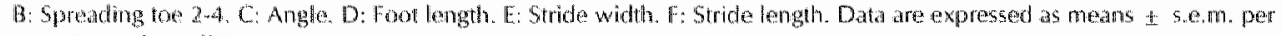
expermental condition. 


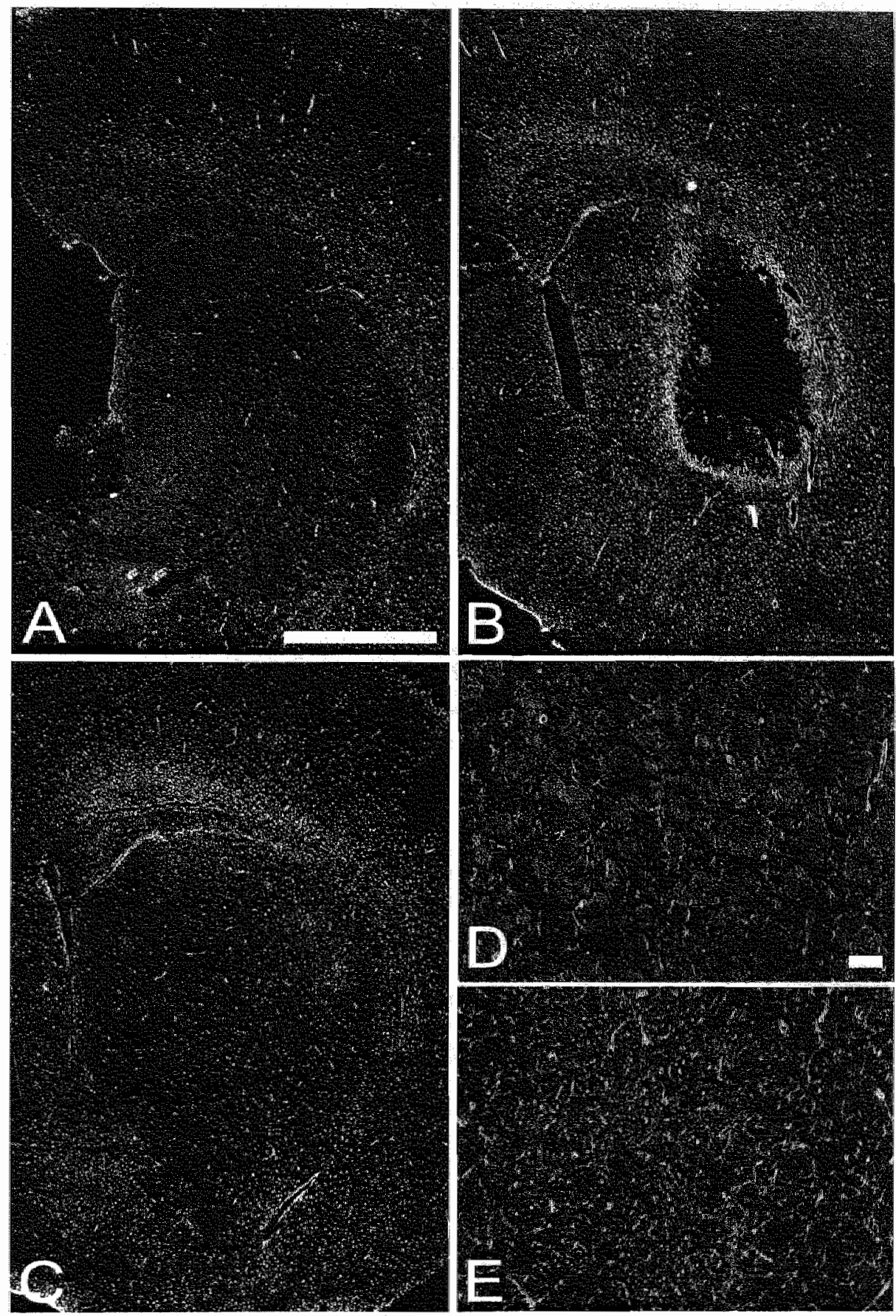

Fig. 7

Striatal GFAP immulinostaining in What rals.

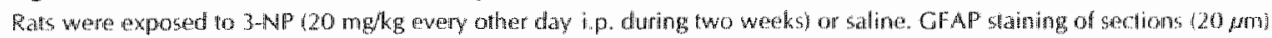
was performed throge weeks after stopping the injections.

A: Control wall B: 3-NP exposed rat whth severe lesion. C: 3-NP exposed rat with mild lesion. Bar for A-C indicates 2 mon.

D. Higher maghification of dorsolateral stratum of confol sat. E: Higher magnification of dobsolateral stratum of rat with mild hasion showing astrogliosis. Bar for D and $\mathrm{E}$ indicates 100 pm. 
appeared to be relatively homogeneous over the whole slice in the Wistar rats as judged by correlation andysis between the section position and the percentage of pixels in each class. Thus, the peak in pixels was found in the same class and the slope of the histogram was similar throughout the whole striatal slice of a ratt. As shown in the histogram of Fig. 8 , the maximum in number of pixels was found in a higher intensity dass in the 3-NP exposed Wistar rats compared to control Wistar rats (class 7 ws. class 6, P<0.05). More immunofluorescence was still present in the 3-NP treated Wistar rats in the following intensity classes as shown by a larger number of pixels in those classes, although the differences between 3-NP and control Wistar rats in each of those classes were not statistically significant nor showed a trend. The GFAP concentration in homogenates, as estimated by ELISA, correlated with the position of the class where the peak in pixels was found $(r=0.602, \mathrm{P}<0.05)$.

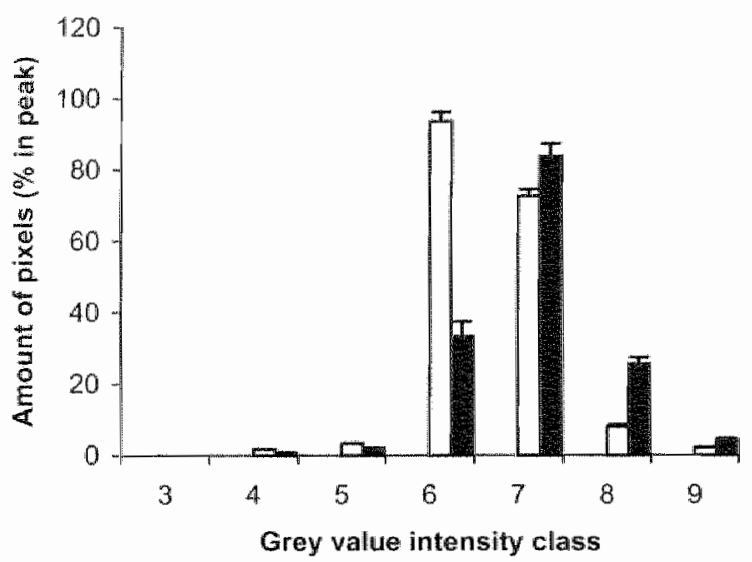

Fig. 8 Histogram of division of pixels over intensity classes of OFAP immunostaining in strata of Wistar fats.

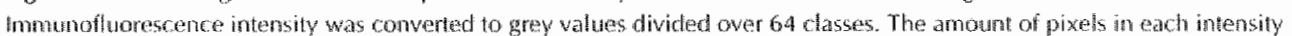
class was axpersed as percentage of the pixels tound in the class where the peak in pixels was found. The rasults of class 3 to 9 atre shown as no pixats were present in all other classes. White bar: Wistar control on 5 ) black bar: Wistar 3 . Pup

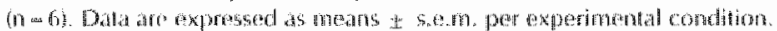

\section{Conelalion of GFAP concentration in striatal homogenates with results of behavioural frests}

We perfomed a correlation analysis between the results of the behavioural tests and the GFAP concentrations. The data in Table 2 and Fig. 9 and 10 show the parameters where a correlation was found.

\section{FOOTPRINT PARAMETERS VS. STRIATAL GFAP}

When examining the footprint analysis a correlation was found between several parameters of the footprint test and striatal GIFA concentration in the 3-NP treated Wistar group only (Fig. 9AD). Note that the most affected rat was excluded from the footprint analysis. The linear component of the fool length correlated negatively with striatal GFAP in the 3-NP treated Wistar group, while no correlation was found in any of the other groups (fig. 9A). A positive correlation existed bewwen striatd GFAP concentration and the quadratic component of the angle of the feet in the Wistar 3-NP treated rats (Fig. 9B). The mean walue over the 3 measurements of distance between tos 1-5 correlated positively with striatal GFAP 


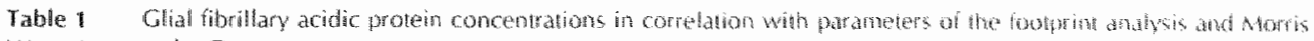

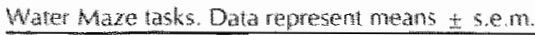

\begin{tabular}{|c|c|c|c|c|c|c|}
\hline & & & \multicolumn{2}{|c|}{ Hewas } & \multicolumn{2}{|c|}{ Wistir } \\
\hline & & & control & $3-\mathrm{NPB}$ & mantmol & Matp \\
\hline & $\begin{array}{l}\text { GFAP } \\
\text { ngimg proteing }\end{array}$ & & $\begin{array}{l}78 \pm 10 \\
n=5\end{array}$ & $\begin{array}{l}100 \text { t } 100 \\
n=7\end{array}$ & $\begin{array}{l}96 \pm 5 \\
7 x=5\end{array}$ & $\begin{array}{l}153 \pm 224 \\
h=6\end{array}$ \\
\hline \multirow[t]{4}{*}{ Footprinti analysis } & $\begin{array}{l}\text { Lemgth } \\
\text { (linear) }\end{array}$ & & $\begin{array}{l}0.17 \\
13=5\end{array}$ & $\begin{array}{l}0.36 \\
11=7\end{array}$ & $\begin{array}{l}0,01 \\
n=5\end{array}$ & $\begin{array}{l}0.90^{\circ} \\
0+2 y\end{array}$ \\
\hline & $\begin{array}{l}\text { Anglo } \\
\text { (chuadratic) }\end{array}$ & & $\begin{array}{l}-0.37 \\
n=5\end{array}$ & $\begin{array}{l}0.32 \\
n=7\end{array}$ & $\begin{array}{l}0.51 \\
13 \times 15\end{array}$ & $\begin{array}{l}0.88^{\circ} \\
n=5\end{array}$ \\
\hline & Step 1 - 5 (mean) & & $\begin{array}{l}-0.17 \\
n=5\end{array}$ & $\begin{array}{l}-0.43 \\
n=7\end{array}$ & 0.08 & $\begin{array}{l}0.93^{\circ} \\
0=5\end{array}$ \\
\hline & Angle tmeany & & $\begin{array}{l}0.11 \\
n=5\end{array}$ & $\begin{array}{l}0.411 \\
11 \mathrm{~m} .7\end{array}$ & $\begin{array}{l}0.54 \\
17=5\end{array}$ & $\begin{array}{l}0.911 \\
n=5\end{array}$ \\
\hline \multirow[t]{4}{*}{ Morris Water Maze } & $\begin{array}{l}\text { Visual } \\
\text { Discrimination }\end{array}$ & $\begin{array}{l}\text { Latency } \\
\text { Whineary }\end{array}$ & $\begin{array}{l}0.02 \\
n=5\end{array}$ & $\begin{array}{l}-0.47 \\
n=7\end{array}$ & $\begin{array}{l}0.34 \\
i t=5\end{array}$ & $\begin{array}{l}0.89^{*} \\
n=6\end{array}$ \\
\hline & & $\begin{array}{l}\text { Distarce } \\
\text { (linear) }\end{array}$ & $\begin{array}{l}-0,20 \\
n=5\end{array}$ & $\begin{array}{l}0.46 \\
n=7\end{array}$ & $\begin{array}{l}0.33 \\
13-5\end{array}$ & $\begin{array}{l}0.60^{4} \\
n-6\end{array}$ \\
\hline & $\begin{array}{l}\text { Spedat } \\
\text { Discrimination }\end{array}$ & $\begin{array}{l}\text { Latency } \\
\text { Wlinear }\end{array}$ & $\begin{array}{l}-0.43 \\
n=5\end{array}$ & $\begin{array}{l}0.57 \\
0.7\end{array}$ & $\begin{array}{l}-0,18 \\
11-5\end{array}$ & $\begin{array}{l}0.94 \\
n=6\end{array}$ \\
\hline & & $\begin{array}{l}\text { Dislance } \\
\text { llinear }\end{array}$ & $\begin{array}{l}-0.35 \\
110=5\end{array}$ & $\begin{array}{l}0.60 \\
n=7\end{array}$ & $\begin{array}{l}-0.25 \\
11=5\end{array}$ & $\begin{array}{l}0.87 * \\
n=6\end{array}$ \\
\hline
\end{tabular}

P $<0.05$.

* not significant from Lewis control

concentration in this group (Fig. 9C), while the mean value over the three measurements of the angle correlated negatively with the striatal GFAP concentration in these Wistar 3-NP rats (Fig. 9D).

\section{VISUAL AND SPATIAL DISCRIMINATION PARAMETERS VS. STRIATAI GFAP}

Significant correlation between striatal GFAP concentration and the parameters from the Morris Water Maze were only obtained for the 3-NP treated Wistar group and are depicted in Fig. 10. A positive correlation between the latency and striatal GFAP concentration was found in the visual discrimination task. This correlation was influenced by the most affected rat with the highest GFAP concentration (Fig. 10A). The positive correlation beween the distance moved in the visual discrimination task was less dependent of the most severely altected rat in the 3w1 treated Wistar rats (Fig. 10B). The latency (Fig. 10C) as well as of the distance moved (Fig. 100) in the spatrial discrimination task correlated positively with the GFAP concentrations in the 3NP treated Wistar animals, though these relations were also influenced by the most severely affected rat showing the highest GFAP concentration. The differences in the mean value of the linear components between the Wistar 3-NP and Wistar control rats can be explaned by the lower initial distance and latency, and therefore a lower slope of the linear change of the Wistar control rats (See Fig. $10 \mathrm{C}$ and Fig. 4A, C). Correlation analysis of the mean value over the four sessions of the latency and distance also revealed a significant correlation with GFAP on both the visual and spatial discrimination lasks. Again, these correlations were largely influenced by the most affected rat (data not shown). 


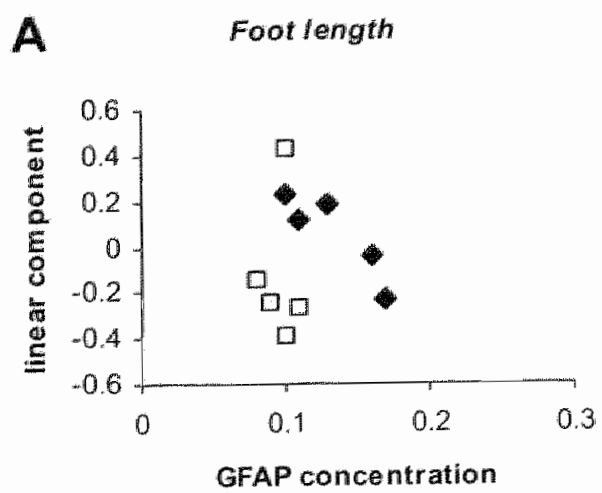

C

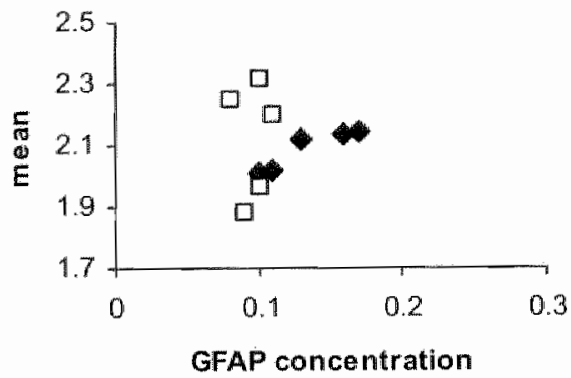

B Angle to axis of walking direction

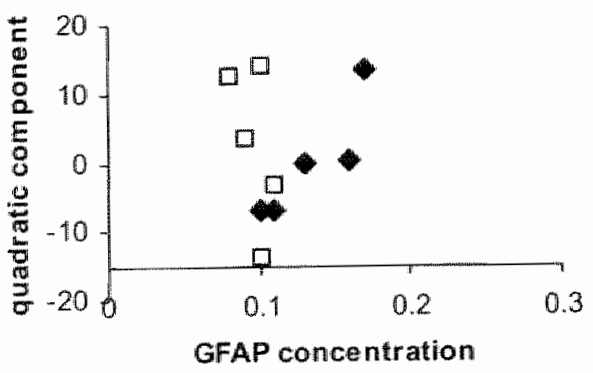

D Angle to axis of walking direction

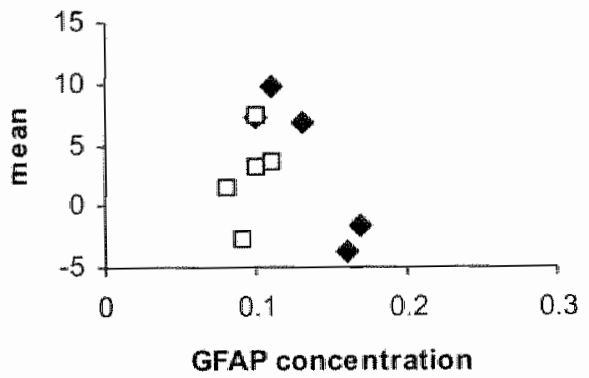

Fig 9 Corretation between GFAP concentrations and patameters of the footprint analysis in Wistear rats. GFAP concentrations were detemined in homogenates with an ELISA. For correlation andysis the linear or quadratic component of the mean over these mealumements was calculated. A: foot length. B: angle (quadratic component). C:

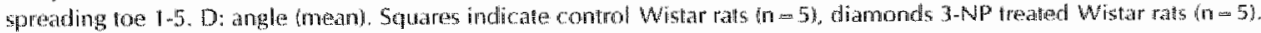

\section{Discussion}

In the present study we investigated whether behavioural alterations are related to striatal degeneration after exposure of two related rat strains to $3-N P$. The 3 NP model involves mechanisms as excitotoxicity, oxidative stress and mitochondrial dysfunction leading to selective striatal degeneration. We presented clear evidence for striatal degeneration in the Wistar rats (see also below). As the striatum is involved in co-ordination of motor movement, we expected to observe changes in motor behaviour of the 3-NP treated animals. We indeed found evidence for motor disturbances when analysing locomotion pattern in the 3-NP treated Wistar rats. In addition, we found small effects of 3-NP treatment during wisual discrimination of Wistar rats in the Morris Water Maze test. Finally, we found important differences between Lewis and Wistar rats on some parameters of these behavioural test.

\section{Strain differences}

As a large variation between rat strains in this amimal model is indicated by several studies [reviewed by [8]), we used two different rat strains, Wistar and Lewis rats. A decrease in body 
weight was observed only in the Wistar rats after treatment with $3+N P$. in a previously performed experiment (C. Teunissen) we continued the injection regime $20 \mathrm{mg} / \mathrm{kg}$ every oher day) for another three weeks in Lewis rats, and even then no alterations in striatal GFAP concentrations and immunostaining were observed.

Although the differential reaction of the two strains towards 3-NP treatment may be surprising, differences in performance in behavioural tasks between theses strains have been reported previously, for example in the open field test $[42,43]$ or in the forced swimming test $[2,30]$. In the present paradigm, the dosage regimen used may have been important. Even at a dose of 36$45 \mathrm{mg} 3-\mathrm{NP} / \mathrm{kg} / \mathrm{day}$ during 5 days, although effective in inducing striatal lesions and behavioural alterations, Lewis rats were less sensitive than Sprague-Dawley or Fischer rats [39]. With a dose of $20 \mathrm{mg} / \mathrm{kg} /$ day of $3-N P$ the reports on behavioural changes in Wistar rats have not been unequivocally $[17,33]$. Therefore, there appears to be a large inter-individual reaction toward 3-NP treatment, which is corroborated by our results. It has been suggested that this variability is caused by differences in 3 -NP biodistribution [39].

\section{A Latency visual discrimination}

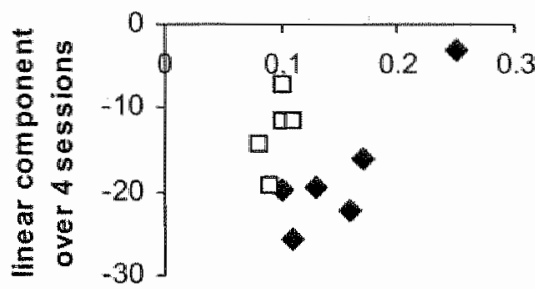

GFAP concentration

\section{Latency spatial discrimination}

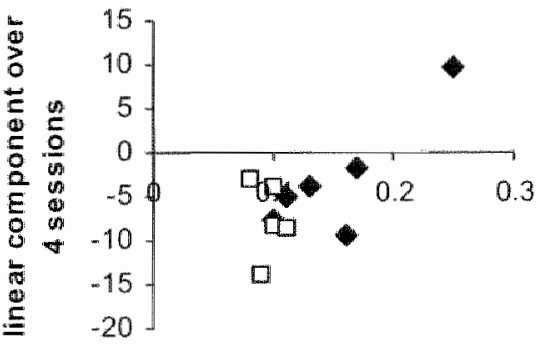

GFAP concentration
B

Distance moved visual discrimination

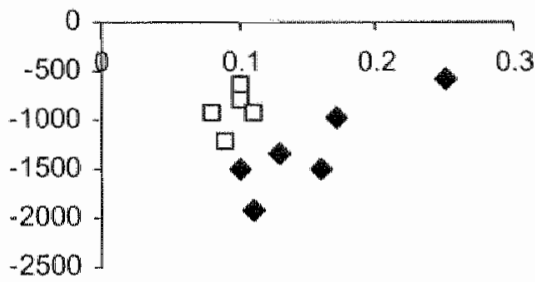

GFAP concentration

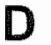

\section{Distance moved spatial discrimination}

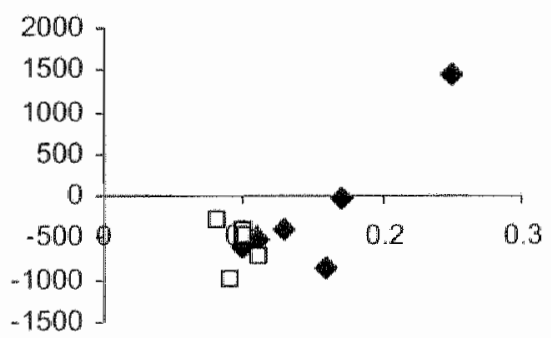

GFAP concentration

Fig. 10 Correlation between GFAP concentrations and paramelers of the Morris Water Mare of Wistal fals.

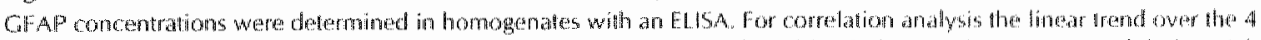

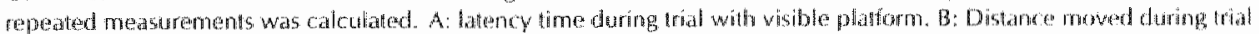

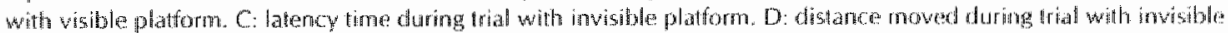

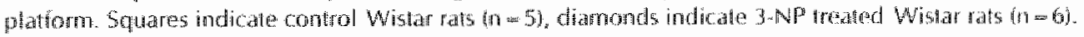




\section{Neurodegeneration in the 3-NP treated Wistar rats}

After staining for calbindin, a marker for CABA-ergic neurons, decreased immunostaining was observed while the nitric oxide syrthase (NOS)-containing neurons were relatively spared in the 3.NP treated animal (Fig. 11), which is in agreement with descriptions of this model in the literature [9]. We characterised the lesions by an increase in GFAP using different approaches. Both methods, quantifying striatal GFAP using an ELISA and the (semi) quantitative analysis of GFAP immunofluorescence, showed an increase of GFAP in the striatum, as is commonly reported in the 3-NP rat model [40] as well as in the non-human primate model [9]. increased GFAP expression likely is an early phenomenon in this model. Ultrastructural changes in astrocyte end-feet have been observed already within hours after a second systemic injection with 3-NP (20 $\mathrm{mg} / \mathrm{kg})$ [35]. The expression of GFAP mRNA was shown to be increased while the striatum and overlying cortex were still intact after systemic injection of $3-N P$ in Lister Hooded rats (20 mg/kg i.p. daily for 14 days) [40].
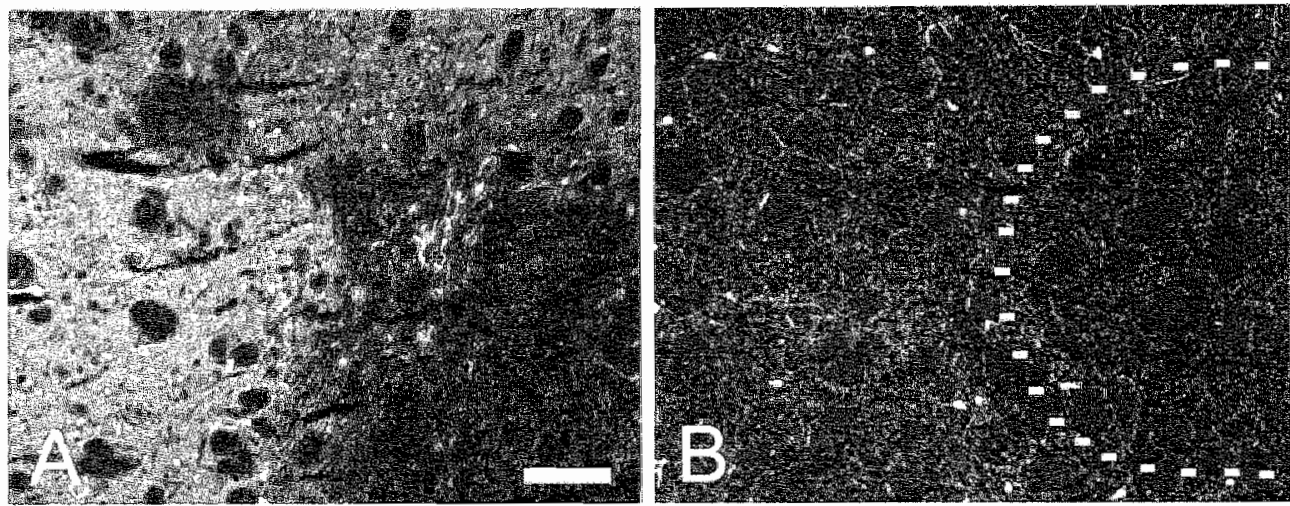

Fig. 11

Striatal cabindin and NOS immunostaning in Wistar rats

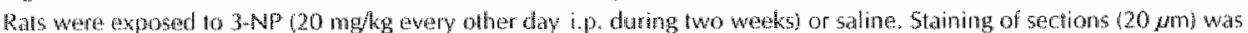
performed lhuese weeks after stopping the injections.

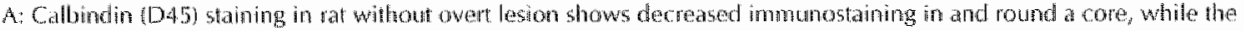

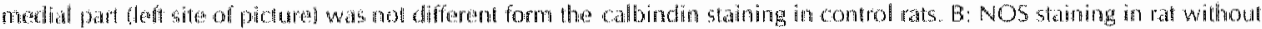

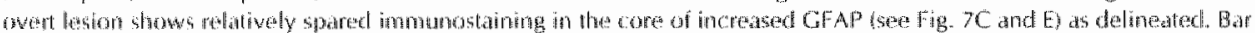
indication $2 \mathrm{~mm}$.

Comelation analysis of GFAP expression and behavioural parameters in the 3-NP treated rats.

\section{OPEN FIELD BEHAVIOR}

We found no correlation between spontaneous artivity in the open field and the increased GFAP concentration in the striatum. This might suggest that there is no correlation between stratal damage and spontaneous activity after treatment with 3-NP. Several studies, however. did monitor altered spontaneous activity in the open field paradigm $[6,28,45]$. One of those studies monitored spontaneous activity during prolonged periods of monitoring time (12 hr compared to $30 \mathrm{~min}$ in our study) and altered activity was observed especially during the dark [28]. Another study showed a transient change in spontaneous activity [45]. Possibly our open field paradigm was not sensitive enough to measure transient alterations in spontaneous activity. On the other hand, other studies reponted alterations in activity without concomitant striatal abnomalities in animals chronically exposed to 3-NP $[19,48]$. A transient hypoactivity 
was observed by others [17] and it is known that 3-NP can induce bradycardia in isolated atrat [31]. Together with the observed early transient loss in body weight (Fig. 2), it might be that the reported early changes in spontaneous behaviour are to some extent the results of the general energy deprivation which is inevitable after systemic application of 3-NP.

\section{LOCOMOTION PATTERN}

The main function of the basal ganglia is co-ordination of movements [49], justifying the analysis of movement patterns in relation to degeneration in this brain structure $[11,25]$. We saw alterations in the spreading of toe $2-4$ and in the surface of the area touched directly after cessation of the injections. We showed a negative correlation between striatal GFAP concentrations and the angle and length of the feet and a positive correlation between GFAP concentration and the spreading of toe $1-5$ in affected Wistar rats, while the means of theser parameters were unchanged. These results substantiate that this test could be a useful tool in monitoring subtle changes related to striatal degeneration. An increase in area touched after striatal degeneration has also been shown by others [44]. Guyot ef al [19] demonstrated a correlate between velocity on traversing a beam and loss of striatal neurons. The striatal damage was quantified by meuron counts by these authors. We did not assess altered concentrations of neurons, but quantified the GFAP concentration. This may be an earlier marker, as astrocytes were shown to be more vulnerable than neurons towards 3-NP treatment in vitro [18] as well all in vivo [35]. Thus, the differences with the results of Guyot ef al. may be due to difference in severity of the effect of 3 NP and the difference between the tests used.

Altered locomotion pattern may be a reflection of the locomotor disturbances observed in HD. The 3-NP treated rat has been reported as the best model available to date for replicating features of HD. However, the model lacks of course the polyglutamine expansion in the Huntingtin protein. For investigating more specifically the disease process of HD, transgenic mice with expansion of the polyglutamine repeat may be exposed to 3-NP [5,24]. The 3-NP rat model involves cognitive alterations, also indicated by our results, locomotor disturbances, blood-brain-barrier damage, excitotoxicity and oxidative stress $[15,20,35]$, features that may also be related to Alzheimer's disease or Parkinson's disease [3]. Therefore, the 3-NP treatment rat: might be used as a model for neurodegenerative processes in general or for studying disorders where the blood-brain-barrier is affected.

\section{VISUAL AND SPATIAL DISCRIMINATION LEARNING}

We observed impared visual discrimination learning in the 3-NP exposed Wistar rats and an effect on velocity during the spatial discrimination task in the Morris Water Maze test. These results also indicate the existence of motor impairment. The distance moved and latency during visual as well as spatial discrimination learning correlated with striatal CFAP, although the mean value of these parameters were not attered due to 3-NP treatment, and a slight decrease in performance in the probe trial was found, indicating a slightly imparred cognitive penformance. Although a function of the striatum in this rest has been reported [14], this suggests also some degree of hippocampal involvement since spatial discrimination leaning in the Morris Water Maze is thought to be related to the hippocampus. Previous studies reporled that the hippocampus of Wistar and Sprague-Dawley rats was affected ahter systemic intoxication with 3-NP [4,34,36]. To our knowledge, only one sludy addressed spatial learning performance in the Morris Water Maze test after treatment with 3-NP. The investigators slarted the treatment with 3-NP after the learning trials, which is a hippocampus independent paradigm, and found impaired performance in the probe trial [15]. Results of another study, using another spatial orientation task, also point to cognitive alterations in the 3-NP animal model [45]. 


\section{Concluding remarks}

3-NP treament of Wistar rats resulted in subtle changes in locomation pattern and slighty impaired performance in the Morris Water Maze test. Some of these changes, i.e. the angle and the length of the feet, the spreading of toe $1-5$ as well as distance moved and latency during visual and spatial discrimination leaming, correlated with the increased striatal GFAP expression in the 3-NP treated Wistar rats.

The 3-NP treatted Wistar rats showed large variations in the performance in the Morris Water maze and in GFAP concentration. These results are consistent with previous reports showing large inter-individual variability 19,19$]$ and, although it has been suggested that this is caused by large differences in bio-distribution among animals, if might as well indicate that there are yet unknown lactors determining the vulneralbility towards 3-NP-induced excitotoxicity and oxidative stress, even if the genetic make-up and the experimental conditions of animals are similar. This variability may refled the variability of severity of symptoms observed in patients with neurodegenerative processes, such as in HD since these patients, with silmilar amount of trinucleotide expansion, considerable variation in symptoms occurs [46].

Finally, the occurrence of neurodegenerative mechanisms as oxidative stress and excitotoxicity and symptoms as cognitive disturbances and altered locomotion may indicate that the model has also features of Alzheimer's disease and Parkinson's disease.

\section{References}

Alexi, T., Hughes, P.E, Fathll, R, L, and Williams, C.E, 3-Nitropropionic acid's lethal triplet: coopelalive pathways of neilladegeneration, Newroreport, 9 (1998) $R 57.64$

Armario, A. Cawalda, A and Marti, 1 , Comparison of the behawiourat and endocrine response to forced swimming stress in five inbred strains of ratss. Pspchoncuroendocrinology, 20 (1995) 879.90.

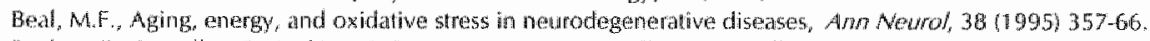

Beal, M.F., Broultet, E., Jenkins, B.C., Ferante, R.l., Kowall, W. W. Miller, I.M., Storey, E., Srivastawa, R., Rosen, B.R. and Hymar, B.T. Neurochemicall and histologic charaterization of striatal excitotoxic lesions produced by the mitochondial toxin s-mitropnopionic acid, J Newroso, $13(1993) 4187-92$.

Bogdanow, M.B., Ferante, R.I., Kuermmete, 5, Kivenyi, P. and Beal, M.F, Increased valneability to 3 mitropropionic acti in an animat model of Hunlington's disease, / Nevrochem, 71 (1998) 2642-4.

Borlongan, C.V., Koutouzis, T.K. Freeman, T.B., Hather, R.A., Cahill, D.W and Sanberg, P.R, Hyperactivin and

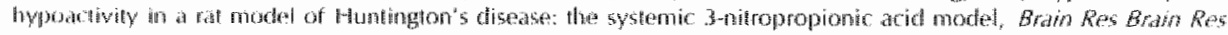
Protor, 111997$) 253 \%$

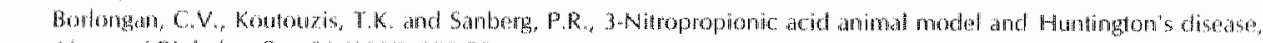

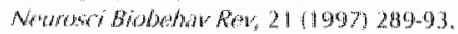

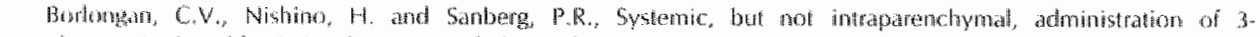

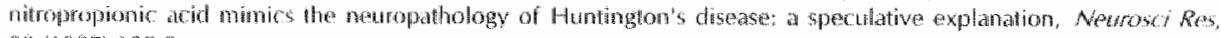
$280(1997) 1859$

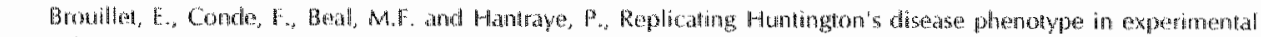

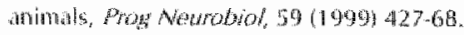

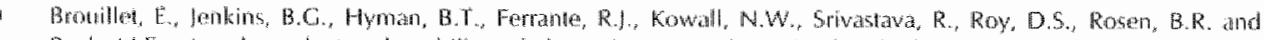

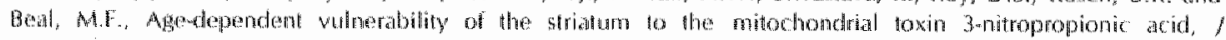
Newmen, 6011993$) 356 \%$.

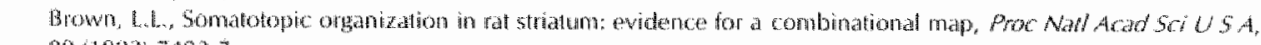
$89(1902) 7403-7$

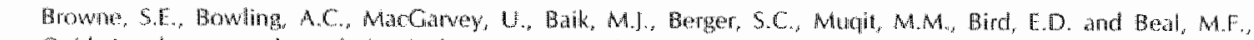
Oxhdive damege and metabolic dyefunchion in Huntington's dinease selective vulnexbitity of the basal ganglia.

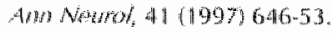

3. de Madinceli, L., Freed, WI and Wyat, R., An index of the functional condition of rat sciatic nerwe based an me astrements made from walking tracks, Exp Nourol, 77 (1982) 63443.

4 Devan, B.D., Gosd, E.H. and Petr. H.L., Dhssociation of hippocanpal and striatal contributhons to spatial navigation in the water mat2s. Newo wol Lam Mem, $66(1996) 305-23$.

Dutkword, E,A., Koutouzis, T.K., Boslongan, C.V., Gordon, M.M., Willing, A.E. Cathill, D.W. and Sanberg, P.R., Rats neceiving systemic 3-nitropropiontic acid demonstrate impaiment of memory in Morris water maze, Psychoblopis $27(1999) 561-566$. 


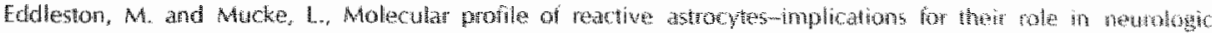
disease, Newrochence, $54(1999)$ 15-36.

17 Eradir, O.l. and Starr, M.S. Stratal clopame dapletion and behavioural semstization indered by

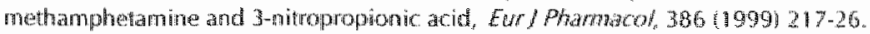

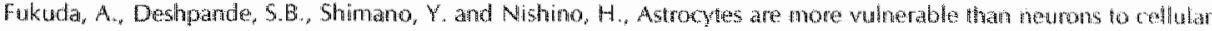

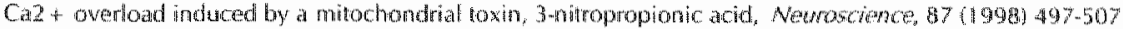

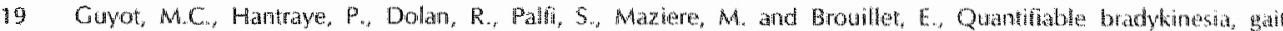
abnomalities and Huntington's disease-tike stratal lesions in rats chronically treated with 3-nitropropionic atcid, verrosichen, $79(1997) 45-56$.

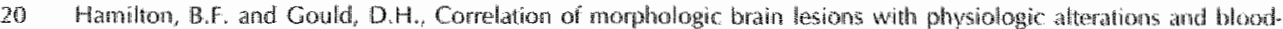

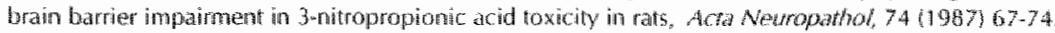

Hassel, B. and Sonnewald, U., selective inhibtion of the tricarboxylic acid cycle of GABAargic neurons with 3 nitropropionic acid in wivo, / Newrochem, 6511995118491.

22 Heales, 5.I., Bolanos, J.P., Sfewart, V.C., Brookes, P.S., Land, I.M. and Cark, I.B., Nitric oxide, mitochondras and neurological disease, Biochin Biophys Acra, $140119991215-28$.

23 Harbison, A.E, Simonian, S.X., Noths, P.J. and Emson, P.C, Relationship of meuronal aitric oxide synthese

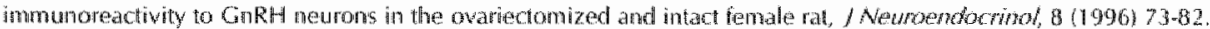
Hickey. M. A and Morton, A. Mice vansgenic for the Huntington's disease mutation are resistant to dronic $3-$ mütropropionic acid-induced strialal toxicity. / Neurochen, 75 (2000) $2163-7 \%$.

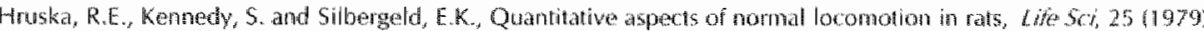
$171-9$.

Kawaguchi, Y., Wilson, C.J. and Emson, P.C., Intracelllular recording of identified neostriatid patch and matrix spiny calls in a shce preparation presening contical inpus, / Nazurophysol, 62 (1989) 1052-65

27 Kandor, K., Dulfer, B.G., Hammann, A. and Van der Stay, F.J., A low-cost method lo andyse finotprim pattanks, Netrosict Methods, 75 (1997) 49-54.

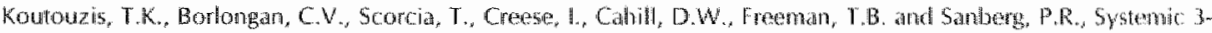
nitroproplonic acid: Iong-term effects on locomotor behavior, Bran Res, 646 (1994) 242-6

29 Kunkel-Bagden, E. and Bregman, B.S., Spinal cord transplants enhance the recowery of locomotor function alter spinal cord injury at birth, Fxp Brain Res, $81(1990) 25-34$.

30 Lahmame, $A_{*}$ and Armario, A, Differential responsweness of inbred strains of rats io antideprescants in the forced swimming test: are Wistar Kyoto nats an animal model of subsensitivity to antidepressents? Psychophasmarology (Berl) $123(1996) 191-8$.

31 Lopez, P.S., Castillo, C.H., Pastelin, G.H., Hemandez, MR., Suarez, M.t, Sanchez, M.L. and Iscalane, B.A,

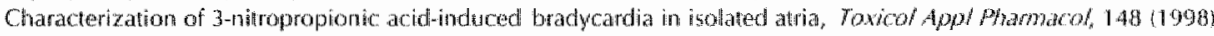
1-6.

32 Markesbery. W.R., The role of oxidative stress in Azheimer disease, Anch Neurol, 56 (1999) 1449.52.

33 Nishino, H., Fujimoto, I., Shimano, $\gamma_{n}$, Hida, H., Kumazaki, $M$. and Fukuda, A., 3-Nitropopionic acid produces striatum selective lesions accompaned by iNOS expression, /Chem Neurowat, 10 (1996) 209212.

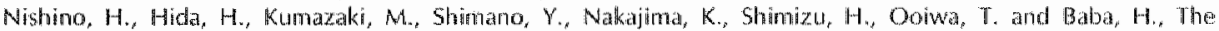
striatum is the most vulnerable region in the brain to mitochondrial energy compromise: a hypothesis to explain its specific walnerability, / Neurothuma, $170000251-60$.

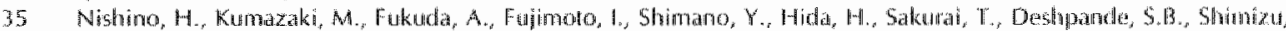

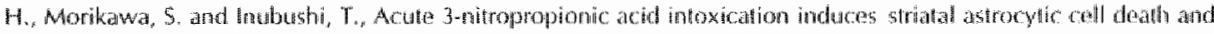

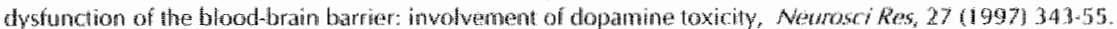

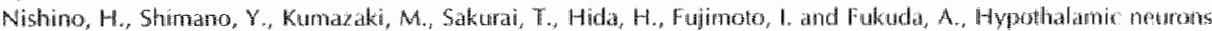

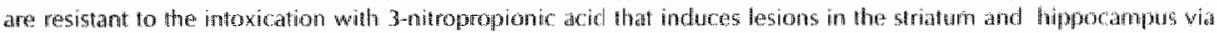
the darmage in the blood brain batrier, Neurobiology, 3 (1995) 257-67.

37 Orallaghan, I.P., Quantification of glial librillay acidic protein: comparison of slich immunobinding assays with a novel sandwich ELISA, Neurowoxicol Teratol, $13119911275-81$.

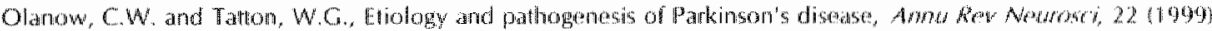
$123-44$.

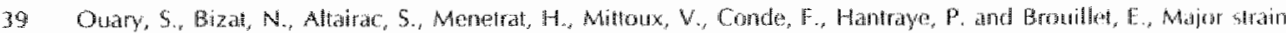

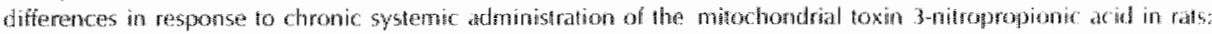
implications for neuroptotection studies, Newrascience, $97120001521-530$

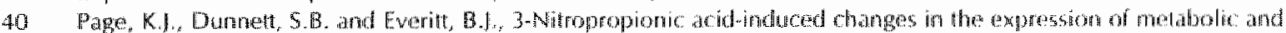

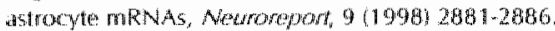

Pickel, W.M. and Heras, A. Utrastruciural Hocalization of calbindin-D28k and GABA in thes matrix compartment the rat caudate putamen nuclei, vetroscience, 71 (1996) $76 \%-70$.

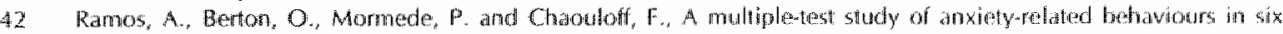
inbred rat strans, Biehaw Brain Res, 85 (1997) $57-69$

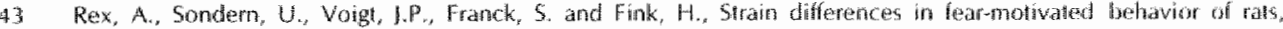
Phambarcol Brachem Behav, $54(1996) 107-11$.

44 Schallert, T, Whishaw, 1.Q., Ramirez, V.D and Teitelbaum, P., Compulsive, abnomil walking catsad by

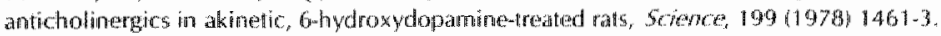




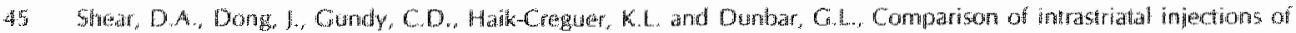
Guinolinic acid and 3-ribropropionic arid for wse in antimal models of Hunimgton's disease. Prog

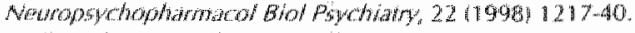

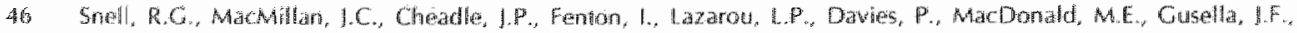

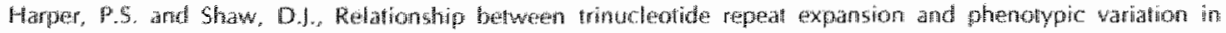
Hunington's disedse, Nat Gene, 4 (1199.51 $193 \%$.

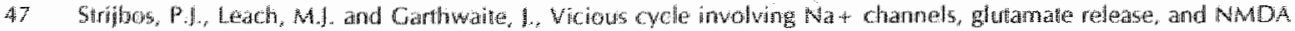

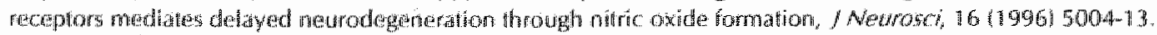

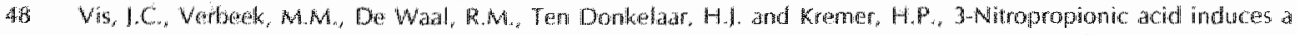

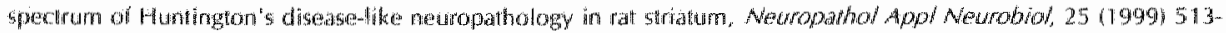
21.

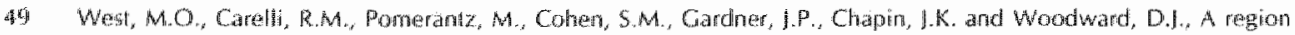

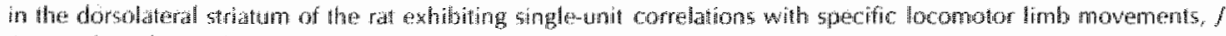
Nexurophysiol, G4 1990123346 .

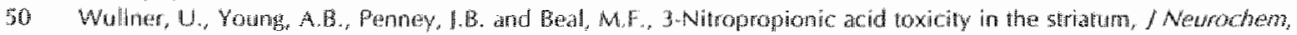
$63(1994) 1772+81$. 


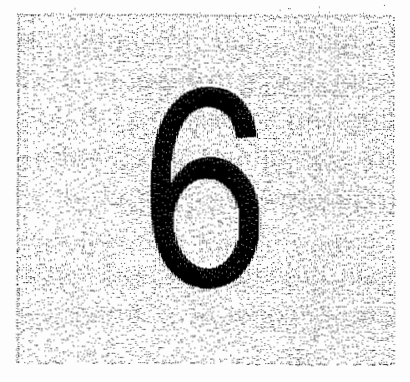

\section{Concentrations of different sterols in the striatum and serum of 3-nitropropionic acid treated Wistar and Lewis rats}

Charlotte Teunissen, Monique Mulder, Jan de Vente, Klaus von Bergmann", Chris De Bruijn, Harry Steinbusch and Dieter Lütjohann ${ }^{2}$

European Graduate School of Neuroscience (Euron), Universiteit Maastricht, Department of Pychiatry and Neuropsychology, The Netherland's a Department of Clinical Pharmacology, University of Bonn, Germany 


\section{Abstract}

In the present study, we examined the long-term effect of oxidative stress induced neurodegeneration on sterol concentrations in striatum and serum of Wistar and Lewis rats.

Three weeks after treatment with 3-nitropropionic acid, no differences in 245hydroxycholesterol concentrations were observed in striatal homogenates and serum. Ratios of striatal campesterol to cholesterol were higher after 3-nitropropionic acid treatment as compared to controls of both rat strains suggesting an increased passage of this exogenous plant sterol across the bloodbrain-barrier. Ratios of lathosterol to cholesterol in serum and striatum were lower in treated rats as compared with controls of both rat strains. Absolute concentrations. of serum and striatal cholesterol precursors and plant sterols differed between the controls of both rat strains.

It was concluded that the changes observed in sterol concentrations in the striatum and serum indicate that cholesterol homeostasis may be affected during neurodegenerative processes. associated with blood-brain-bartier damage.

\section{Introduction}

Cholesterol is one of the main lipid constituents of neuronal cell membranes, subcellular membranes and myelin. Results from previous studies indicate that cholesterol homeostasis is altered during ageing and during neurodegenerative processes in mammals $[6,14,17,30]$. Results from animal studies showed that cholesterol biosynthesis in the white matter of rat brains, as measured by alterations in the concentrations of desmosterol, was already disturbed alter 4 hrs and continued for up to 2 months after acute hypoxia [9]. Results from human studies showed an increase in the concentration of cholesterol precursors in the white matter during aging [30]. Furthermore, the ratio of cholesterol to phospholipids was decreased in the temporal gyrus grey matter of patients with Alzheimer's disease [19]. In an epidemiological study, it was assumed that the prevalence of Alzheimer "s disease could be lowered by treatment with $3 \beta$ hydroxy-3B-methylglutary coenzyme $A$ reductase, an important enzyme in the rate-limiting step of cholesterol biosynthesis [31].

Cholesterol is synthesised in the brain in situ [27]. However, there must be a transport process for the elimination of excess brain cholesterol into the periphery via the blood-brain barrier $(B B B)$, which is nearly impermeable to cholesterol itselti.

Peripheral chollesterol is eliminated and converted into bile acids by the liver through modification and degradation of the cholesterol side chain. In the brain, in contrast, no evidence for such metabolism is found $[8,26]$. There must be a slow but steady exchange of cholesterol or its metabolites with intact side chains between the brain and the periphery, as indicated by the presence of radiolabelled cholesterol in the serum even 2 weeks after injection of ${ }^{14}$ radiolabelled cholesterol in rat brains [8]. The exact mechanism of brain cholesterol tramsport via the BBB is so far unknown. It may be mediated by either apolipoproteins and/or facilitalted transport by enzymatically oxidised cholesterol products including 245 hydroxycholesterol $(245 \mathrm{OH}-\mathrm{Chol})[4,17]$. The oxysterol $245 \mathrm{OH}-\mathrm{Chol}$ seems to be synthesised almost exclusively in the human brain $[4,17,20]$ and a specific cytochrome P-450 species (CYP 46) is involved in its conversion from cholesterol [16]. This enzyme, cholesterol 24Shydroxylase, is mainly localised in neuronal cells, whereas its product, 24S-OH-Chol, is primarily located in myelin [20]. The unique cerebral origin of $245-\mathrm{OH}$-Chol present in the circulation has led to the suggestion that serum or plasma concentrations of this oxysterol may 
reflect nomal or disturbed turnover of cholesterol in the brain. Recently, it was shown that plasma concentrations of $245-\mathrm{OH}-\mathrm{Chol}$ were elevated in early stages of Azheimer's disease and decreased in later stages of this disorder [18,25].

In view of the above observations we wished to investigate the possibility that the concentration of 245-OH-Chol may alter as a consequence of neurodegeneration. In a previous study, we used the 3-nitropropionic acid (3-NP) rat model for neurodegeneration (Chapter 5). Treatment with 3-NP causes selective strialial degeneration in rodents by mechanisms including mitochondrial dysfunction, oxidative stress, excitotoxicity and BBB dysfunction [1, 12.21]. Glial fibrillary acidic protein (CFAP) is the hallmark of severity and astrogliosis in the 3-NP affected striatum $[24,29]$. Thus, we showed increased GFAP concentrations in the striatum of Wistar rats treated with 3-NP, although no increased mean GFAP concentration was observed in Lewis rats. Funthermore, we showed that the GFAP concentrations were related to several behavioral alterations, including altered walking pattern in the Wistar rats as well as in the Lewis rats, and with learning impairment in the Morris Water Maze in the Wistar rats (Chapter 5). In the present study, we examined therefore the concentration of striatal and serum concentrations of 245 OH-Chol in the rat 3-NP model for neurodegeneration. For comparison, we measured the concentrations of the plant sterols campesterol, sitosterol, the cholesterol precursors, desmosterol and lathosterol, and the metabolite cholestanol in striatal homogenates as well as in serum. To examine if levels of the sterols measured are related to the severity of local brain damage, the serum and striatal sterol ratios to cholesterol were correlated with the GFAP concentration in striatal homogenates. Since we and others have reported that important strain differences exist in the sensitivity towards 3-NP treatment $[23,28]$ (Chapter 5), we included rats of two related rat strains, Wistar and Lewis rats.

\section{Experimental procedures}

\section{Materials}

3-NP, soybean trypsin, leupeptide, phenylmethylsulfonyl fluoride and the mouse anti-GFAP monoclonal antibody (clone G-A-5) were purchased from Signa (St. Louis, USA). Aprotinine was purchased from Fluka, Buchs, Switzerlland. The rabbit anti-GFAP polyclonal antibody was a generous gift from F.R.C. Ramaeckers Department of Molecullar and Cellular Biology. University of Maastricht. Purified bovine GFAP protein was purchased from Progen Biotechnik GmbH (Heidelberg, Germany. The alkaline phoshatase conjugated antimabbit IgC was obtained from Vector (Burlinghame, USA). p-Nitrophenylphosphate in diethanolamine solution was obtained from Bio-Rad Laboratories (Veenendaal, The Netherlands). All chemicals and solvents were of analytical grade.

\section{Animals}

Wistar rats were obtained from Janvier (Le Genest-Saint-Isle, France) and Lewis rats from the local animat breeding facility. Rats were treated with $3-N P$ as follows: Six month old male Wistar $(518 \pm 11 \mathrm{~g})$ and Lewis rats $(382 \pm 8 \mathrm{~g})$ were treated with $3-\mathrm{NP}(20 \mathrm{mg} / \mathrm{kg}$; Wistar: $n-6$, Lewis: $n=7)$ or saline $(0.9 \% \mathrm{NaCl}, 1 \mathrm{~mL} / \mathrm{kg} ; \mathrm{n}=5$ for each rat strain) by intraperitoneal injections every other day over $\| 1$ days. All animals had free access to regular rat chow and water. During the three weeks after cessation of the injections, behavioural tests were performed; the results of which have been reported (Chapter 5). After finishing these tests, the rats were sacrificed, blood samples were taken and striatal homogenates were prepared from the left hemisphere. All animal experiments were performed according to the guidelines of the 
University Committee on Animal Welfare, which follow the European Communities Council Directive 186/609/EE. The local Animal Ethics Committee approved the animal experiments.

\section{Serum and siriatum sampling}

After decapitation, blood was immediately collected in serum collection tubes, allowed to clot for 1 hour and serum was separated by centrifuging at $2000 \mathrm{~g}$ and $4^{\circ} \mathrm{C}$ for 10 min. The serum samples were stored at $-20^{\circ} \mathrm{C}$ until analysis. The right half of the striatum was dissected into slices which were weighed and homogenized in phosphate buffer $\left(2 \mathrm{mM} \mathrm{NaH} \mathrm{PO}_{4} \mathrm{H}_{2} \mathrm{O}, 0.5\right.$ mM ethylenediaminetetracetic acid, $145 \mathrm{mM} \mathrm{NaCl}, \mathrm{pH} 7.4,0.1 \mathrm{~g} / \mathrm{mL}$ s supplemented with the protease inhibitors soybean trypsin $(10 \mu \mathrm{g} / \mathrm{mL}$ ), leupepide (T $\mu \mathrm{M})$, pheny/methylsulfonyl fluoride ( $1 \mathrm{mM})$ and aprotinine $(8.1 \mathrm{UU}-1 \mu \mathrm{g} / \mathrm{mL})$ at a total concentration of $10 \%(\mathrm{wt} / \mathrm{vol})$ and stored at $-20^{\circ} \mathrm{C}$ until analysis.

\section{Biochemical assays}

The striatal concentration of GFAP was determined with an ELISA according to the method of O'Callaghan [22]. The primary antibody was the monoclonal anti-GFAP antibody ifinal concentration of $10 \mathrm{\mu g} / \mathrm{mL}$ in PBS). A rabbit anti-GFAP polyclonal antibody was used as the secondary antibody (dilution 1:500). Puritied bovine GFAP protein was used as a standard. The detection system used was anti-rabbit IgG labelled with an alkaline phoshatase conjugate (dilution $1,3,333$ ) and p-nitrophenylphosphate in diethanolamine solution. Extinction was measured at $405 \mathrm{~nm}$ on a Benchmark microplatte reader (Bio-Rad Laboratories, Veenendaal, The Netherlands). Striatal protein concentrations were determined according to the method of Lowry [15].

\section{Sterol analysis by gas-liquid chromatography selective ion monitoring}

Sterols and oxysterols were extracted from serum and brain homogenate by chloroform/methanol (2:1; w/v) after saponification and neutralisation. Fifty $\mu$ g 50 -cholestane (Sigma) $(50 \mu \mathrm{L}$ from a stock solution $5 \alpha$-cholestane in cyclohexane; $\mathrm{mg} / \mathrm{mL}$ ) and $200 \mathrm{ng}$ racemic. $\left[23,23,24,25 \mathrm{~m}^{2} \mathrm{H}\right] 24 \mathrm{OH}-\mathrm{Chol}$ (Medical Isotopes Inc., Pelham) (50 $\mu \mathrm{L}$ from a stock solution $24-$ $\mathrm{OH}-\mathrm{Chol}$ in toluene; $4 \mu \mathrm{g} / \mathrm{mL}$ ) were added as internal standards. The solvents were evaporated and the sterols and oxysterols were trimethylsilylated. The trimethylsilyl (TMSi) ethers of the neutral sterols were separated on an U1tral2 $15 \%$ Phenyl-Methyl-Silicon, crosslinked) fused sillica capillary column (50 $\mathrm{m} \times 0.32 \mathrm{mn}$ i.d. 0.52 mm phase thickness, Hewlet Packard (HP), Böblingen, Germany. Gas-liquid chromatography was performed with a HP5890 gaschromatograph. The oven temperature was initially kept at $150^{\circ} \mathrm{C}$ for 3 min, then increased at $30^{\circ} \mathrm{Cmin}$ to a linal temperature of $290^{\circ} \mathrm{C}$. An aliquot of one $\mu /$ was injected by an automated injector (HP) in a splitless mode all $280^{\circ} \mathrm{C}$. Hydrogen was used as carrier gas with an inlet pressure of $10 \mathrm{psi}$ and flame ionisation detection was performed at $280^{\circ} \mathrm{C}$. The concentrations of cholesterol, cholestanol, lahosterol, desmosterol, campesterol and sitosterol were calculated from the ratio of the peak areas of the corresponding sterol to the area of 5 ch-cholestane multiplied by the amount of internal standarcl $(50 \mu \mathrm{g})$ added to a defined serum or brain homogenate volume. Identity of the sterols were proved by comparison of the mass spectra of authentic compounds. Combined gas chromatography-mass spectrometry-selected ionmonitoring (GC-MS-SIM) analysis for quantification of 245-OH-Chol was pertormed on an HP Ultra 1 colum ( $25 \mathrm{~m} \times 0.2 \mathrm{~mm}$ i.d. $\times 0.33 \mu \mathrm{m}$ film thickness) using an HP5890 Series II plus gas-chromatograph combined with an HP5972 mass selective detector. An aliquot of $1 \mu l$ was injected by automated injection in a splitless mode at an injection temperature of $280^{\circ} \mathrm{C}$. The oven temperature was kept at $180^{\circ} \mathrm{C}$ for $1 \mathrm{~min}$, thereafter increased at a rate of $20^{\circ} \mathrm{C} / \mathrm{min}$ to 
$250^{\circ} \mathrm{C}$ and immediately raised at a rate of $5^{\circ} \mathrm{Cmin}$ to a finat remperature of $300^{\circ} \mathrm{C}$. TMSi-ether of authentic and deuterated 24-OH-Chol were measured at m/2 $413\left(\mathrm{M}^{*} \text {-OTMSi-CH(CH) }\right)^{-}$and $\mathrm{m} / 2416$ (M-OTMSi-CD(CH) $)$, respectively, after electron impact ionization at $70 \mathrm{~V}$. The temperature of the transfer line was kept at $280^{\circ} \mathrm{C}$.

\section{Statistics}

Sterol concentrations are expressed as ratio to cholesterol and indicated as $R$ _ Sterol. Data are expressed as means \pm standard error of means (s.e.m.). Statistics for differences in GFAP and sterol concentrations and sterol to cholesterol ratios between the four treatment groups were pertormed using a two-factorial ANOVA (Treatment * Strain). The ANOVA was pertormed on the ranks if normality was not assumed. Nomality was tested with the Kolmogorov Smirnov test. Differences between the treated and control rats within a rat strain were analysed using a two-tailed Student's t-test. Differences between the control rats of both strains were calculated using a two-tailed Student's t-test. The non-parametric Mann-Whitney U-test was used instead of the Student's t-test if nomality was not assumed. Contelation analysis was performed with a Pearson's correlation coefficient. The level of significance used was $5 \%$.

\section{Results}

\section{Neurodegeneration}

The results in Table IA show that the striatal GFAP concentration in the Wistar 3-NP exposed group was higher than in the Wistar control group $(t=2.29, \mathrm{P}<0.05)$. The mean striatal GFAP concentration in the Lewils 3-NP group was comparable to control Lewis rats.

\section{Strain differences in sterol concentrations and the sterol to cholesterol ratios ( $R$ Sterol) for untreated animals}

In the striatum of untreated animals, significant strain differences were observed in campesterol and sitostenol concentrations (Table $1 \mathrm{~A}$, last column). In serum, broad wariation was observed between the basal sterol concentrations of Wistar and Lewis rats. Significant strain differences were observed between the control rats of both strains in the serum ratio of cholestanol to cholesterol, R Cholestanol, desmosterol, R_Desmosterol, lathosterol, R_Lathosterol, campesterol, R_ Campesterol, sitosterol and R_Sitosterol (Table IB, last columm).

Striatal and serum sterol levels after treatment with 3-NP

\section{STRIATUM}

The concentrations of cholesterol, 245-OH-Chol and R 24S-OH-Chol in striatal homogenates Table (A) were comparable in all groups examined, i.e. Wistar control, Wistar 3-NP, Lewis control, and Lewis 3-NP. Significant decreases were observed for the striatal lathosterol concentration, R Lathosterol and R Cholestanol after treatment with 3-NP. The concentrations of the plant sterol campesterol and $\bar{R}$. Campesterol were higher in striatal homogenates of the 3 NP treatment groups.

\section{SERUM}

The concentrations of cholesterol, 245-OH-Chol and R_245-OH-Chol in sera (Table (B) were comparable in all groups examined, i.e. Wistar control, Wistar 3-NP, Lewis control, and Lewis 
3-NP. Significant decreases in the concentration of lathosterol and $R$ Lathosterol were observed in sera of all 3-NP treated rats compared with controls. Strain specific effects on the absolute concentration of cholestanol in serum were observed (interaction cholestanol concentration: Strain Treatment: $F(1,19)=5.62, P<0.05$. Similarly, strain specific changes were observed for the levels of serum desmosterol (Treatment Strain interaction for desmosterol concentration: $F(1,19)=11,09, P<0.01 ; R$ Desmosterol: $F(1,19)=5.45, P<0.05)$. The concentrations of senum desmosterol and $R$. Desmosterol were higher in Lewis 3-NP animals compared to Lewis control animals as confirmed by the t-test $(t=0.29, P<0.05)$. In Wistar rats no increase in serum desmasterol or $R_{-}$Desmosterol was observed. All other parameters were unaffected after treatment with $3-\mathrm{NP}$.

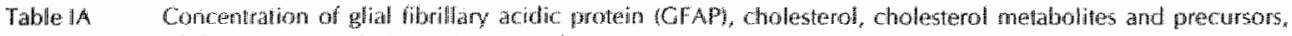

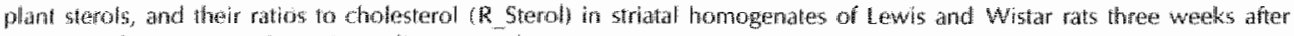
cesschion of remament with 3 wis or saline lcontroll.

\begin{tabular}{|c|c|c|c|c|c|c|c|}
\hline & & \multicolumn{2}{|c|}{ Lewils } & \multicolumn{2}{|c|}{ Wistar } & \multirow{2}{*}{$\begin{array}{l}\text { 3NA US } \\
\text { controt }\end{array}$} & \multirow{2}{*}{$\begin{array}{l}\text { Wistar } \\
\text { control } \\
\text { vs. } \\
\text { Lewhis } \\
\text { control } \\
\text { i }\end{array}$} \\
\hline & & Control & 3un & Contrat & 3-NP & & \\
\hline GFAP & $\mathrm{ng} / \mathrm{mgg}$ & $76 \pm 10$ & $97 \pm 9$ & $94+5$ & $150 \pm 22^{\circ}$ & $a$ & $n .5$ \\
\hline Chalesterol & $\mathrm{mg} / \mathrm{g}$ & $12=1$ & $13 \pm 1$ & $14 \pm 1$ & $15 \pm 1$ & n.s. & n.s. \\
\hline Cholestmol & $\mu g$ & $20 \pm 2$ & $20 \pm 1$ & $23 \pm 1$ & $21+2$ & n.s. & $\mathrm{n} . \mathrm{s}$ \\
\hline R Cholestanol & $\mu g / n g$ & $1.7 \pm 0.0$ & $1.6 \pm 0: 11$ & $1.7 \pm 0.1$ & $1.4 \pm 0.1$ & $C$ & $\mathrm{n} . \mathrm{s}$ \\
\hline Dexsmasterol & $\mu g / g$ & $103 \pm 11$ & $118 \pm 12$ & $107 \pm 8$ & $90 \pm 7.3$ & n.s. & ns.s. \\
\hline R_Desmosterol & $\mu \mathrm{g} / \mathrm{n}$ 月g & $8.4 \pm 0.3$ & $9.2 \pm 1.1$ & $7.7 \pm 0.4$ & $6.2 \pm 0.6$ & n.s. & $\mathrm{n.s.}$ \\
\hline Lathosterol & $\operatorname{mg} g$ & $16 \pm 2$ & $15 \pm 1$ & $20 \pm 2$ & $17 \pm 1$ & a.s. & n.s. \\
\hline R Ithosterol & probigs & $1.3 \pm 0.1$ & $1.1 \pm 0.0$ & $1.4 \pm 0.0$ & $1.2 \pm 0.1^{\circ}$ & $d$ & $n s$ \\
\hline Cumperiteral & $\mu \mathrm{g}$ & $30 \pm 1$ & $35+2$ & $38 \pm 2$ & $50 \pm 6$ & $c$ & $c$ \\
\hline R Campasterot & $\mu \mathrm{g} / \mathrm{mmg}$ & $2.5 \pm 0.1$ & $2.7+0.2$ & $2.7 \pm 0.0$ & $3.4 \pm 0.2^{\circ}$ & $c$ & n.5. \\
\hline Sirosterol & $\mu g / g$ & $19 \pm$ & $27 \pm 6$ & $24 \pm 2$ & $29 \pm 3$ & an.S. & $\therefore$ \\
\hline R Silosterd & Mg/rog & $1.6 \pm 0.2$ & $2.1 \pm 0.4$ & $1.7 \pm 0.0$ & $1.9 \pm 0.1$ & n.s. & $\mathrm{ns}$ \\
\hline 245 OH HChol & $\mu y_{\mathrm{g}}$ & $57 \pm 3.3$ & $65 \pm 3$ & $66 \pm 3$ & $66+3$ & a.s. & ns. \\
\hline R 2450)+Chol & $\mu \mathrm{g} / \mathrm{mg}$ & $4.7 \pm 0.3$ & $5.0 \pm 0.1$ & $4.8 \pm 0.11$ & $4.6 \div 0.3$ & M.s. & n.s. \\
\hline
\end{tabular}

Dala ane expressed as mean \pm s.em.

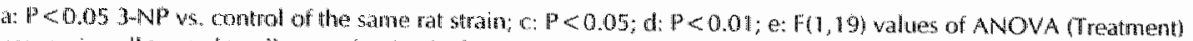
compariag all treatted to all control animals; ff Student 5 tetest; n.s. not significant 


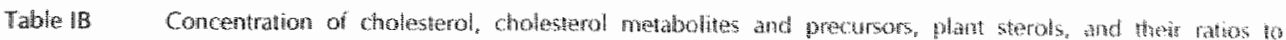

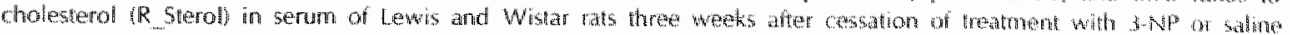
icontroll.

\begin{tabular}{|c|c|c|c|c|c|c|c|}
\hline & & \multicolumn{2}{|c|}{ Lewis } & \multicolumn{2}{|c|}{ Wistar } & \multirow{2}{*}{$\begin{array}{l}3-\mathrm{NP} \\
\text { Wh } \\
\text { control } \\
\end{array}$} & \multirow{2}{*}{$\begin{array}{l}\text { Wistid } \\
\text { control } \\
\text { vis. } \\
\text { bewis } \\
\text { control } \\
\text { 1 }\end{array}$} \\
\hline & & Control & B-NP & Control & HAP & & \\
\hline Cholestanoust & $\mathrm{mg} / \mathrm{dL}$ & $0.7 \pm 0.1$ & $0.0 \pm 0.1$ & $0.6 \pm 0.0$ & $0.5+0.1$ & $\mathrm{As}$ & n.s. \\
\hline R Cholestanol & $\mu g / m g$ & $6.8 \pm 0.3$ & $8.0 \pm 0.5$ & $5.8 \pm 0.2$ & $5.6 \pm 0.3$ & 10.5 & $\mathrm{c}$ \\
\hline Desmosterol & $\mathrm{mg} / \mathrm{dL}$ & $0.6 \pm 0.1$ & $0.8 \pm 0.0^{5}$ & $0.2 \pm 0.0$ & $0.1 \pm 0.0$ & c & d \\
\hline$R$ Desmosterol & $\mu \mathrm{g} / \mathrm{mrg}$ & $5.8+0.7$ & $7.4 \pm 0.3$ & $1.6 \pm 0.1$ & $1.5 \pm 0.1$ & {$[\pi, 3$.} & 4 \\
\hline Lathosteral & $M g i d L$ & $58 \pm 2$ & $46 \pm 4^{1 i}$ & $110=5$ & $73 \pm 10^{5}$ & d & $d^{\text {t? }}$ \\
\hline RE lathosterol & $\mu \mathrm{g} / \mathrm{mg}$ & $0.6 \div 0.1$ & $0.4 \pm 0.0^{b}$ & $1.0 \% 0.1$ & $0.8 \pm 0.1$ & c & $d$ \\
\hline Campenter ol & mg/d! & $2.8 \pm 0.2$ & 3.10 .1 & $4.1 \pm 0.4$ & $3.3 \pm 0.4$ & $\mathrm{~m} . \mathrm{s}$ & 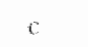 \\
\hline R Campesterol & $\mathrm{Hg} / \mathrm{mg}$ & $28+1$ & $30 \pm 1$ & $38 \pm 2$ & $360 \pm 1$ & n.s. & $\|$ \\
\hline Sitosterol & $\mathrm{mgdL}$ & $2.7 \pm 0.3$ & $29 \pm 0.1$ & $4.4 \pm 0.4$ & $3.5 \pm 0.4$ & Ms. & c: \\
\hline R_sitosterol & $\mu \mathrm{g} / \mathrm{mg}$ & $27 \pm 1$ & $28 \pm 1$ & $40 \pm 2$ & $3.8 \div 2$ & n.s. & 目 \\
\hline 245-OH-HChal & $\mathrm{ng} / \mathrm{m} \mathrm{L}$ & $6.6 \pm 0.9$ & $8.1 \pm 1.6$ & $3.2 \pm 0.4$ & $7.4 \pm 0.5$ & M.s. & 10.5 \\
\hline R_245-OHChot & mig/mg & $6.6 \pm 0.5$ & $7.8 \pm 0.2$ & $7.9 \pm 0.13$ & $8.4 \pm 0.7$ & n.s. & 10.5 \\
\hline
\end{tabular}

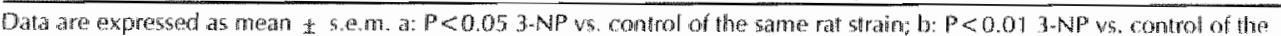

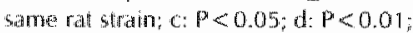

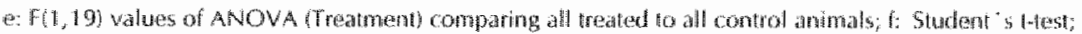

g: Z.score of Manm-Whinn' U lest; $n .5$ : ngt significant

\section{Are serum and striatal sterol levels related to striatal GFAP concentration?}

Although the mean concentrations of some sterols or sterol to cholesterol atios were unchanged, the sterol to cholesterol ratios of some of these parameters correlated with severity of neurodegeneration as expressed in increased striatal GFAP concentrations. The serum R 24S$\mathrm{OH}$-Chol correlated negatively with severity of neturodegeneration in the 3-NP treated Lewis rats $(r=-0.765, P<0.05$ ) (Fig. 1A). In striatal homogenates of Wistar rats treated with 3-NP, a positive correlation between $R$ Cholestanol and severity of neurodegeneration (GFAP) was found $(r=0.809, P<0.05$ ) (Fig. 1B).

The R Sitosterol in striatal homogenates of 3-NP treated Wistar rats correlated positively with GFAP concentration $\{r=0.870, P<0.05$ ) (Fig. $1 \mathrm{C}$. Similar results were obtained in $3-N P$ treated Lewis rats. The R Sitosterol in striatal homogenates of these Lewis rats correlated also positively with GFAP $(r=0.816, P<0.05)$, whereas a negative correlation was observed in the Lewis control rats $(r=-0.935, P<0.05)$ (Fig. 1D). In serum of the Wistar 3-NP treated group, a negative correlation with GFAP concentration was observed for $\mathrm{R}$ Silosterol $(r=-0.936$, 


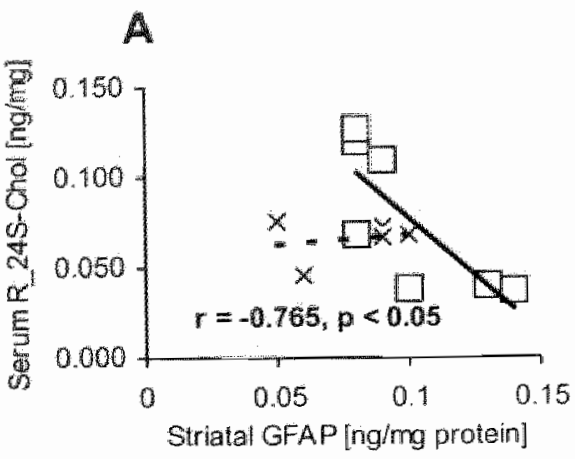

C

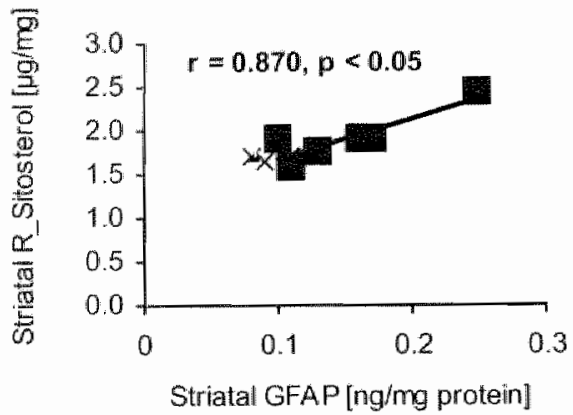

E

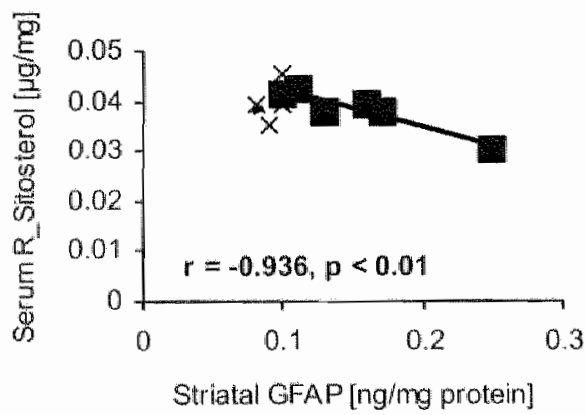

B

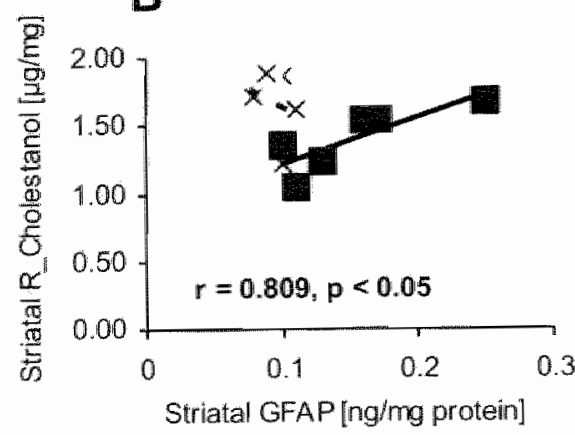

\section{D}

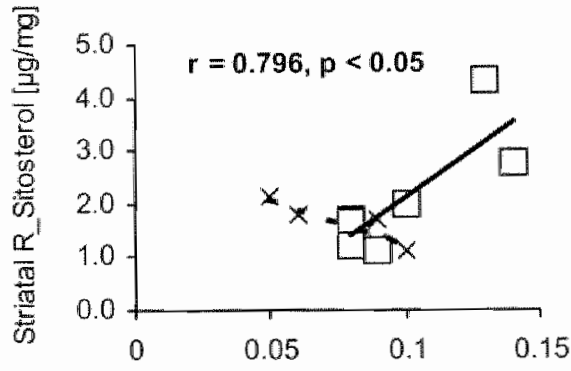

Striatal GFAP [ngrimg protein]

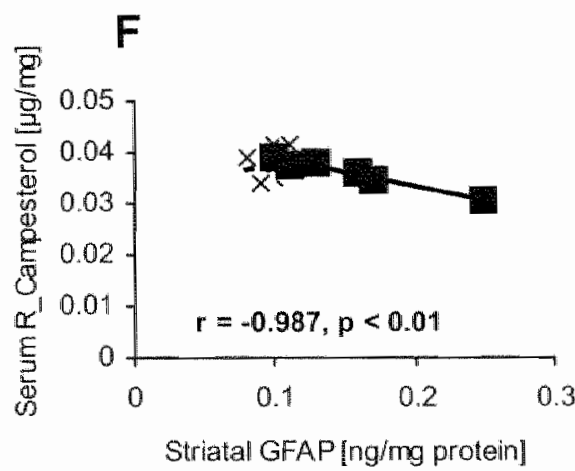

Fig.1 Scatterplots of the correlation between the coneentwations of different sterols and/or whet ratios to

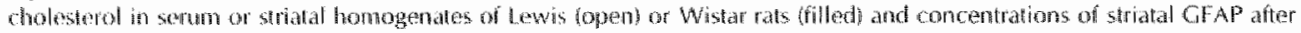

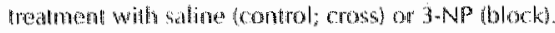

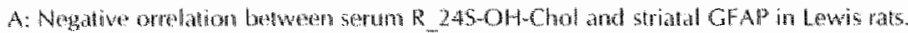

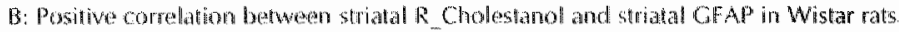

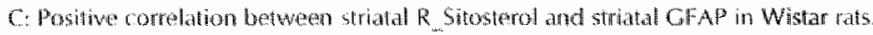

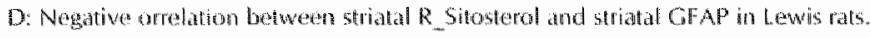

E: Negatiwe orration between serum $R$ Sitosterol and stratal Cl AP in Wistan rats.

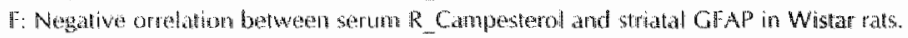


$P<0.01)$ (Fig. 1E) and $R$ Campesterol $(r=-0.987, P<0.01$ (Fig. IF). None of the $R$ Sterols did show a correlation with GFAP in the control rats, except the above-mentioned negative correlation observed for $R$ _Sitosterol in stratal homogenates of Lewis rats. Overall, the most extreme GFAP and sterol concentrations were observed in the most affected rat, that showed a lesion core with degenerated cells and astrogliosis, and severe weight loss (see for more details: Chapter 5).

\section{Discussion}

The aim of the present study was to investigate whether the concentration of 245 OH-Chol may change as a consequence of neurodegenerative processes. We studied therefore the striatal and serum concentrations of $245-\mathrm{OH}-\mathrm{Ch}$ in the 3-NP rat model. We compared these results with striatal and serum concentrations of other sterols including cholesterol precursors (desmosterol and lathosterol), a further metabolite (cholestanol), and two dietary plant sterols (campesterol and sitosterol). Since important strain specific sensitivity towards treatment with 3 -NP has been reported [23](Chapter 5), we included rats from two different strains, Wistar and Lewis rats, in our investigations.

Strain specific differences were observed in the basal concentrations of different sterols, which may indicate strain specific differences in cholesterol homeostasis. Cholesterol homeostasis is a ballance between cholesterol absorption, cholesterol synthesis, transport within the circulation by lipoproteins, and excretion [11]. Strain specific differences may be found at all these levels. First, different pathways may be involved in cholesterol biosynthesis favouning lathosterol formation in Wistar rats and desmosterol formation in Lewis rats. Second, the animals may differ in their absorption or excretion rates for cholesterol and plant sterols as the plant sterol ratios to cholesterol differed between Wistar and Lewis control rats in striatum as well as in serum. In addition, we observed that the Lewis rats were less sensitive towards 3-NP treatment than the Wistar rats, as evidenced by no significant increase in mean striatal GFAP concentration, and no change in body weight during the injection period (Chapter 5). Thuse results are in agreement with a previous study showing very low sensitivity of Lewis rats towards 3-NP treatment [23].

Oxidative stress and excitotoxicity are important mechanisms of the 3-NP model. This model has frequently been described as a model for neurodegeneration as found in Huntington"s disease, and our results may therelore be extrapolated to this human disease [3]. However, our model differs from this neurodegenerative disease on several aspects. We administered 3-NP during 11 days, representing a relatively acute model in contrast to the chronic processes in Huntington's disease. In addition, this disease thas features not represented in the 3 -lvp model, including the expanded polyglutamine tract of the Huntingtin protein in Huntington's disease [7]. The systemic treatment of rats with 3-NP results in a general condition of oxidative stress [1]. This condition is probably also found, although more locally, during plaque and langle formation in Alzheimer's disease [13].

We observed a slightly, but significantly, decreased striatal lathosterol concentration as well as an increased striatal campesterol concentration in 3-NP trealted animals. The presence of the plant sterols in CSF and brain tissue of humans (Lütjohann, unpublished observations) and rals, has been observed previously [9]. Since plant sterols are not endogenously produced in the brain tissue and thus must originate from the circulation, the higher concentrations of the plant sterol campesterol and its ratio to cholesterol in striatal homogenates of 3 -NP treated rats may indicate an increased permeability of the $B B B$. Indeed, it has been reported that $B B B$ damage occurs shortly $(3 \mathrm{hrs}-4$ days) after injection with $3-N P[12,21]$. Since the absolute concentrations of campesterol in stratal homogenates are 1.5 times higher than concentrations of sitosterol in 
both at strans, while serum concentrations are equal, a variable enhanced permeability of the BBB for plant sterols may be induced by $3-N P$ treatment. The half-life of campesterol in the brain is not known, but the hallfife of cholesterol in rat brain slices is estimated to be about 6 months [2]. Thus, it is possible that campesterol, once in the brain, remains present in this tissue for a longer period. Such stable changes have been reported in an unrelated previous study showing increased concentrations of the cholesterol precursors desmosterol and lanosterol in rat brain myelin at 4 hours and 2 months after severe hypoxia [9].

We did not observe significant differences in striatal or serum levels of cholesterol or the oxidised cholesterol metabolite 24S-OH-Chol three weeks after cessation of 3-NP treatment. However, a negative correlation between serum $245-\mathrm{OH}$-Chol concentrations and striatal GFAP concentrations was observed in Lewis rats. The lack of differences in mean serum $245-\mathrm{OH}-\mathrm{Chol}$ concentrations is in contrast to findings of altered mean $245-\mathrm{OH}$-Chol concentrations in plasma of patients with Alzheimer's disease $[18,25]$ and suggests that $245-\mathrm{OH}-\mathrm{Chol}$ is not a marker for neurodegeneration in general.

In addition, it is evident from in vivo and in vitro studies in humans, rats and mice that there is a difference in the site of production of $245-\mathrm{OH}$-Chol between these species $[20]$, indicating the possibility that the data may not be easily extrapolated. Nevertheless, it has recently been reported that different neurological diseases seem to have relatively small effects on the flux of $245 \mathrm{OH}-\mathrm{Chol}$ from the brain except for conditions with very marked destruction of the central nervous system [6], which is in agreement with our findings on the small effects on $245-\mathrm{OH}-$ Chol concentration. Alternatively, since our data are limited to a single measurement three weeks after finishing the injections, the observed changes in 245-OH-Chol concentration will certainly be influenced by the time elapsed between a neurodegenerative insult and the moment of sampling. The importance of sampling time in relation to the neurodegenerative process is stressed by the observation of the increased concentrations of $245-\mathrm{OH}-\mathrm{Chol}$ in plasma of mildlly affected Alzheimer's disease patients [18] and the decrease in 245-OH-Chol serum concentration in more severely affected Alzheimer's disease patients, reported in another study [25]. These resulis suggest that the serum concentration of $245-\mathrm{OH}$-Chol reflects the different stages of (specific) neurodegenerative processes.

We observed significant correlations between striatal GFAP concentration and the concentration of several sterols in serum or striatal homogenates. Increased GFAP concentration has been shown to be a very sensitive marker for 3-NP induced neurodegeneration [29]. in our Wislar rats, intense GIFAP staining of the lesion area was observed in the striatum of rats with obvious cell death (Chapter 5). The expression of GFAP MRNA was shown to be increased while the striatum and overlying cortex were still intact after systemic injection of 3-NP in Lister Hooded rals [24]. GFAP expression has been related to altered sterol composition of the brain. as was shown by previous studies 15,10$]$. Thus, there is clearly a relationship between the altered sterol composition and stratal GFAP expression, although the mechanism remains unknown. It appears from our study that the altered sterol composition of the striatum and serum is a direct consequence of 3-NP induced degenerative processes.

\section{Acknowledgement}

The skilful technical assistance of 5 . Winnen and $M$. Angevaren is gratefully acknowledged. The assistance of $A$. Scheepens is gratefully acknowledged for critically reading the manuscript. This study was supported in part by the Bundesministerium für Bildung, Wissenschaft, Forschung und Technologie (01EC9402) and in part by the Universiteit Maastricht and the Technical University Eindhover. 


\section{References}

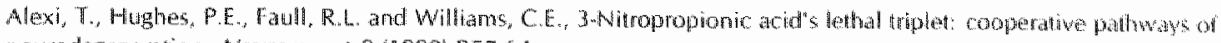
neurodegeneration, Networepon, 9 (1998) R57-64.

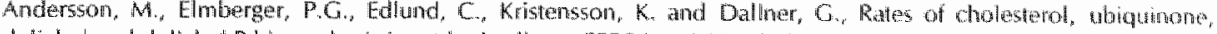

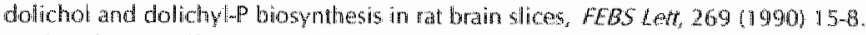

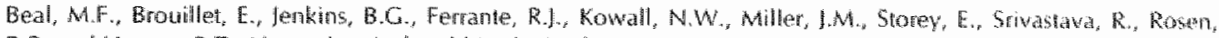
B.R. and Hyman, B.T. Neurochervical and histologic characterization of stratal excilotoxic lesions produced by the mitochondrial toxin 3 -nitropropionic acid, / Nedrosci, 13 (1993) 4181-92.

Bjorkhem, 1. Dictalusy, U. and Lutjoharn, D. Renowal of cholesterol from axtlahepatic sources by axidativen mechanisms, Curr Opin Lipidol, 10 (1999) 161-5.

Bochelen, D., Langley, K, Adamczy, A., Kupterberg. A. Hor, F., Vincendon, G. and Mersel, M., 7bela.

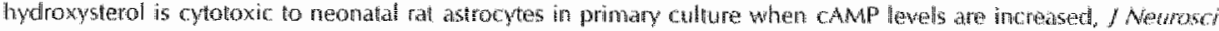
Res, 62 (2000) 99-11:

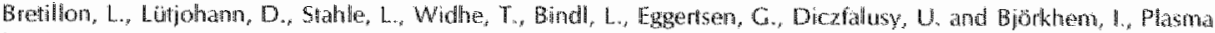
levels of 245 -hydroxycholesterol reflect the balance between cerebay prothetion and hepatic metabolistr and ate inversely related to body surace, I thpid Res, $4112000 \mathrm{D} 840-845$

Bronillet, F., Conde, F., Beal, M.F. and Hantraye, P., Replicating Huntington"s disease phenotwpe in experimentat animals, Prog Neurobio, 5911999$)$ 427-68.

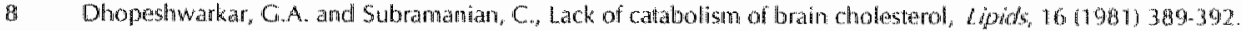

9 Dorsewsk, 1. and Adamczewska-Concerzewicz, $z$, free stenols of the cerebral white matter in expeximenal

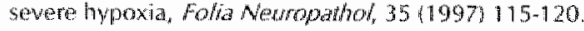

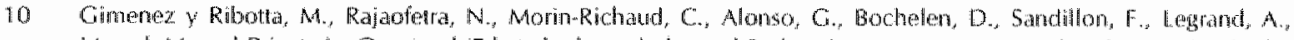
Mersel, M. and Friwat, A. Oxysterol is betathydroxycholesteryl-3-oledtes promotes serotonergic remnervation in the

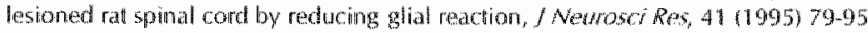

11 Grundy, S.M. George Lyman Duff Memoria Lecture. Mulliactorial etology of lnyperdmolesterotema. Implications for prevention of coromary treant disease, Anterioscles Thromb, 17 (1991) $1619-35$.

12 Hamilon, B.F. and Gould, D.H. Correlation of morphologic buan lesions with physiologic allerations and blond

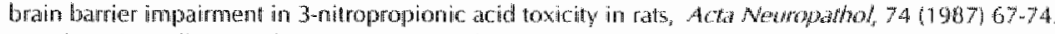

13 Hensley, K, Hail, N, Subramaniam, R., Cofe, P., Haris, M., Aksenow, M. Aksenoy, M., Gablith, S.P., Wu, I.F., Carmey, I.M. and at. Brabn regional correspondence between Alyeimer"s disease histopathalogy and biomarkers of protein oxidation, J Newrochem, $65(1995) 2146.56$.

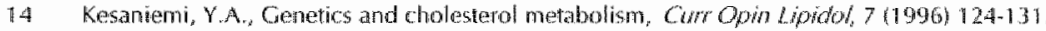

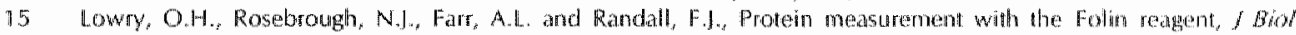
Crem, $193(1951) 265-275$

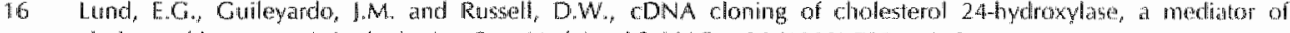
cholemterol homeostasis in the brain, Proc Nath Acad Sri U 5 , 96 (1999! 7236-7243.

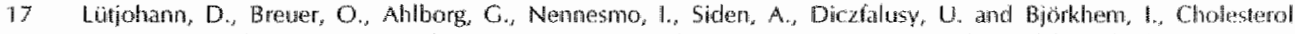
homeostasis in human bain: evidence for an agremdependent flux of 245 -hydroxycholesterol from the brain into the Circulation, Proc Nat Acad Sci U S A, 93 (1996) 9799-9804.

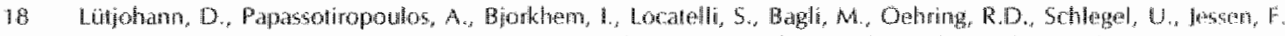

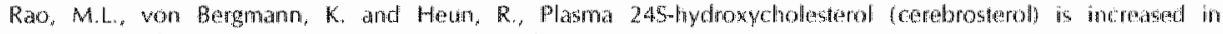
Alzheimer and vascular demented patiants, / Liphid Res, 41 (2000) 195-198.

19. Mason, R.P., Showmaker, W. Shajenko, L., Chambers, T.E. and Herbone, L. G, Evidence for chariges in the

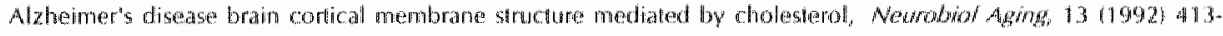
419

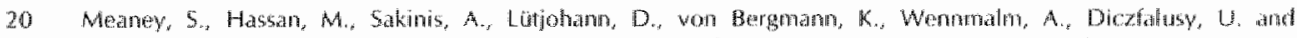
Biorkhem, 1, Studies on the fomation and fiux of oxysterols in viwo with shabe botopes. Evidence that the throge

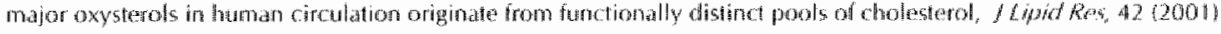
7078 .

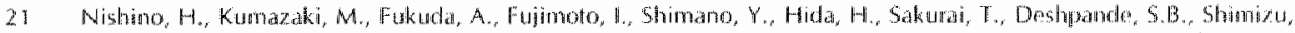

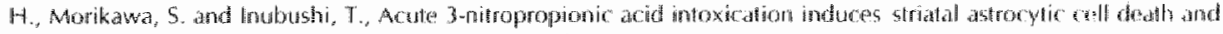

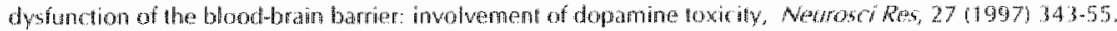

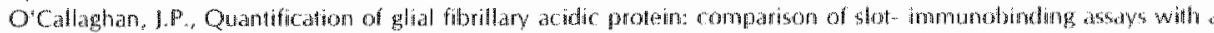
novel sandwich ELSA, Newatoxicol Forato $13 ; 1997) 275$-81.

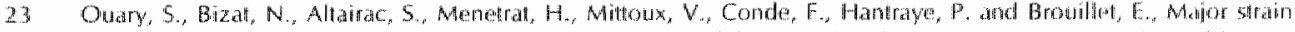

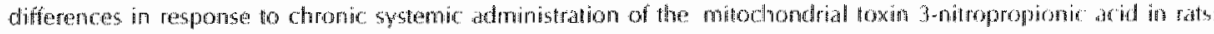

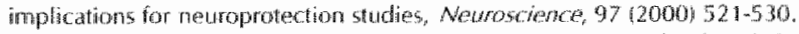

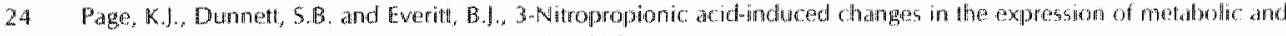
astrocyte mRNAs, Newroreport, 9 (1998) 28812886.

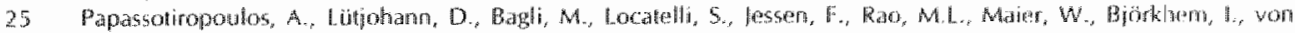

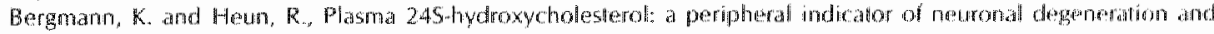

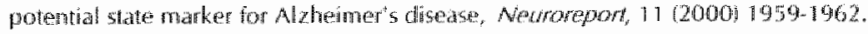




\section{Chapter 6}

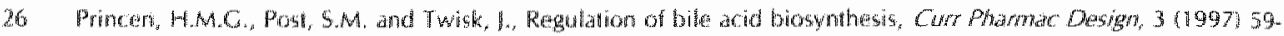

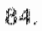

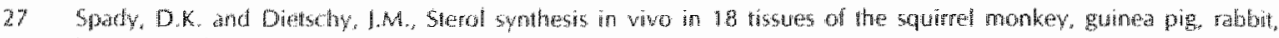
hamster, and rat, fopd Res, $24(1903) 303 \times 315$.

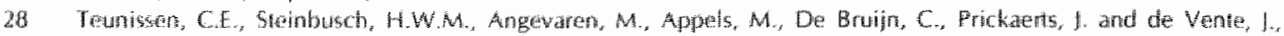

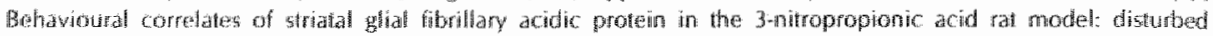

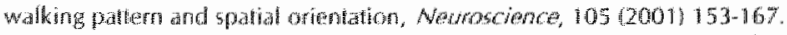

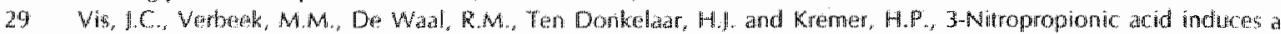

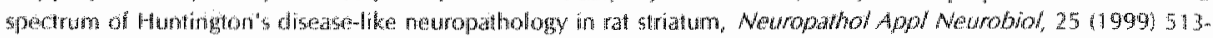
21.

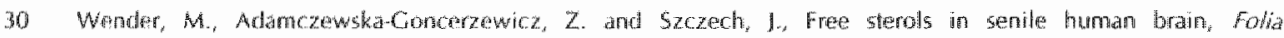

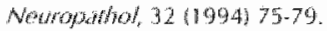

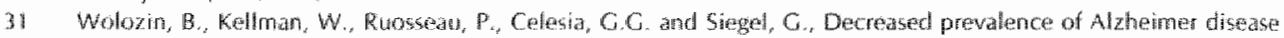

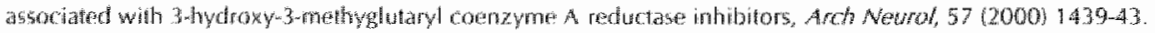




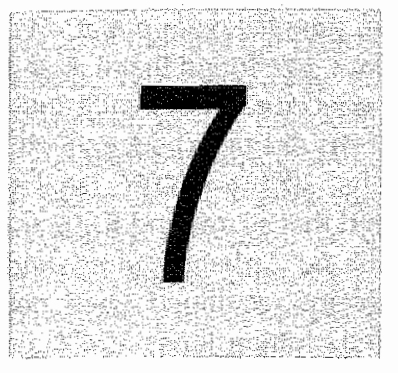

\section{Stability of interleukin 6 , interleukin 6 receptor, interleukin 10 and $\mathrm{CC} 16$ in human serum}

Gunter Kenis, Charlotte Teunissen, Raf De Jongh*, Eugene Bosmans, Harry Steinbusch and Michael Maes.

European Graduate School of Neuroscience (Euron), Universiteit Maastricht, Department of Psychiatry and Neuropsychology, The Netherlands

"Department of Anesthesiology, Ziekenhuis Oost-Limburg, Genk, Belgium. 


\section{Abstract}

We examined the stability of interteukin 6 (IL-6), its soluble receptor (SIL-6R), IL-10 and CC16 or uteroglobin (an endogenous cylokine inhibitor) in human serum, using an accelerated stability resting protocol according to the theory of Arrhenius. Further, the effects of the time delay between blood sampling and sample processing, clotting temperature and the freeze-thaw cycle on the serum llevels of these proteins were determined. Paired serum samples were put at $4^{\circ} \mathrm{C}$, $20^{\circ} \mathrm{C}, 30^{\circ} \mathrm{C}$ and $40^{\circ} \mathrm{C}$ and stored at these temperatures for 11 to 21 days. We found that $1 \mathrm{~L}-6$ and CC16 concentrations did not change at $4{ }^{\circ} \mathrm{C}, 20^{\circ} \mathrm{C}$ and $30^{\circ} \mathrm{C}$. 14.6 concentrations significantly declined after 11 days at $40^{\circ} \mathrm{C}$. The concentrations of sll-6R and IL-10 did not change at $4{ }^{\circ} \mathrm{C}$ but significantly decreased at $20^{\circ} \mathrm{C}$ (after 21 and 14 days respectively), $30^{\circ} \mathrm{C}$ and $40^{\circ} \mathrm{C}$ (after 1 day at both temperatures for both cytokines). Arrhenius plots indicated that $\$ L-6 R$ and $1 L-10$ are stable at $-20^{\circ} \mathrm{C}$ for at least several years. Since their relative stability, no Arrhenius plot could be calculated for $14-6$ and CC16. The concentrations of the proteins examined were not significantly altered by repeated freeze-thaw cycles, nor by extended clotting times at $4^{\circ} \mathrm{C}$ or $20^{\circ} \mathrm{C}$. We conclude that serum siamples for the determination of IL-6, sh-6R and CC16 can be stored at $-20^{\circ} \mathrm{C}$ for longer periods of time, but for IL-10 determinations, storage at $-70^{\circ} \mathrm{C}$ is recommended.

\section{Introduction}

During the past decade, cytokines and other inflammatory mediators have been extensively investigated in various diseases. Their role in infectious diseases and inflammation was thoroughly examined. Accumulating evidence suggests that they play also an important role in autoimmune diseases, psychiatric disorders (major depression, schizophrenia, anxiety and eating disorders) [8,10] and neurodegenerative diseases (e.g. Alzheimer's disease) [2].

To study the role of cytokines in these diseases in humans, samples of biological fluid (serum, plasma, cerebrospinal fluid, symovial fluid, etc. are collected and concentrations of the respective cytokines determined by immunomassays, e.g. radio immuno-assays (RIA's) or enzyme immuno-assays (EIA). However, in these longitudinal studies, one must be aware of two types of bias that can interfere with study results, i.e. the biological and analytical variability.

Biological variability comprises all fluctuations in analyte concentrations due to natural conditions. Seasonal and diumal variability, age, gender, the menstrual cycle and other conditions that change the analyte concentration in wivo are important aspects of biological variability $[6,9]$. Analytical variability can be described as the variation in the analyte concentrations due to preanalytical conditions or the accuracy of the test-system. The present study focuses on the pre-analytical variability as a possible source of error in studies concening cytokines or other serum proteins.

When collecting serum or plasma for cylokine deteminations, care should be given on the processing of the samples, since it is known that anti-coagulants and time before centrifugation may influence cylokine concentration $[4,5,15]$. It is common practice that blood is drawn from the patient and that this sample is transported to the laboratory for processing (centrifugation, separation of plasma or serum and storage) at a later time-point. This delay between sample collection and processing might alter the concentration of cytokines in the sample, since the blood cells are still able to take up or to release cytokines. 
In order to minimise inter-assay variability, it is preferable to collec a cenam number of samples and to analyse them simultaneously. Usually, samples are stored at $-20^{\circ} \mathrm{C}$ or $-70^{\circ} \mathrm{C}$. but it has remained unknowm whether even at these low temperatures cytokines are not degraded by proteolytic enzymes or denatured by the freezing process. Thus, it is of great importance to know how long samples can be stored frozen before activity is lost $\mathrm{reg}$. detection in the immunomassay).

One of the best ways to determine the stability of cytokines or other proteins in samples is to perform a real time stability experiment. This can be dome by storing paired alliquots of a sample and determining the concentration of the protein after certain time points, taking care that the sample is thawed only once. However, this approach requires long experimental periods. Moreover, in real time stability studies, the determination of the protein must be performed at the beginning, during and at the end of the study. These different measurements can often not be done using the same batch of reagents and allso the inter-assay variability is not excluded.

Therefore, estimation of protein stability should rather be performed using an accelerated stability testing protocol $[1,3,7]$. This procedure is based on the Arrhenius equation, which describes a linear relationship between the logarithm of the reaction rate constant (e.g. the degradation rate) and the inverse of the absolute temperature. First, the degradation rate at different temperatures is determined. Aliquots of a sample are stored at different "stress" temperatures that are higher than the storage temperature but denaturing temperatures should be awoided. The recovery of the respective analyte is plotted against the time of storage at each temperature. The slope of the best-fit line gives the degradation rate constant $k$ corresponding to that temperature. Then, the logarithm of $k$ is plotted versus the inverse of the absolute temperature, which gives the so-called Arrhenius-plot. Using the equation of the best fit line. one can calculate the degradation rate constant at every desirable temperature.

The aims of this study were 1) to examine the stability of interleukin 6 (IL-6), soluble IL-6 Receptor (sIL-6R), IL-10 and Clara Cell Protein 16 (CC16, an endogenous cytokine inhibitor) in serum using an accelerated stability testing protocol according to Arrhenus; 2$)$ to examine the influence of clotting time (e.g. the time delay between blood sampling and sample processing on protein concentration measurement by ELISA; and 3) to examine the effects of repeated freeze-thaw cycles on the measured protein concentration.

\section{Materials and Methods}

\section{Samples}

Since serum concentrations of $1 \mathrm{~L}-6$ and $\mathrm{LL}-10$ are very low in healthy humans, the stability of these cytokines was examined in serum of five post-surgical palients admitted to an intensive care unit. The stability of s/L-6R and CC16 was checked in sefum of the same patients and of three healthy wolunteers.

Blood of the patients was drawn from the first stopcock of an arterial line system "Pressure Monitoring Kit, Baxter BV, Utrecht, Holland) after initial elimination of $10 \mathrm{~mL}$ of blood. Then blood was collected in three $10 \mathrm{~mL}$ and eight $5 \mathrm{~mL}$ vacuumed serum tubes $(\$ 5 T$ Vacutainer(i) Hemoguard, Becton Dickinson, Meylan, France). Blood from the healthy volunteers was drawn using Vacutainer Systems (Becton Dickinson, Meylan, Francel. The collected blood was then processed as described below.

\section{Accelerated stability testing protocol}

The blood in the three $10 \mathrm{~mL}$ serum tubes was allowed to clot for 30 minutes at room temperature and subsequently centrifuged for 15 minutes at $1600 \mathrm{~g}$. The serum from each 
subject was pooled in a sterile lube $(50 \mathrm{~mL}$, Falcono, Becton Dickinson) and subsequently fractionated in sterile $1.5 \mathrm{~mL}$ Eppendorf tubes, $0.3 \mathrm{~mL}$ per tube. Then, the ubes were stored at the different stress temperatures. Eleven tubes were put at each stress temperature: $4^{\circ} \mathrm{C}, 20^{\circ} \mathrm{C}$, $30^{\circ} \mathrm{C}$ and $40^{\circ} \mathrm{C}$, and three control tubes were immediately frozen at $-20^{\circ} \mathrm{C}$.

After $1,2,4,7,9,11,14,16,18$ and 21 days respectively, 1 tube from each temperature was frozen at $-20^{\circ} \mathrm{C}$. At the end, all samples were kept at $-20^{\circ} \mathrm{C}$ until cytokine analysis.

\section{Influence of clotting time}

To check the influence of the time delay beween blood sampling and centrifugation, and the influence of clotting temperature, serum iubes were processed as follows. Immediately after blood collection, four tubes were put at $4^{\circ} \mathrm{C}$ and four were kept at room temperature. After 30 minutes, one fube al each temperature was taken and centrifuged for 15 minutes at $1600 \times g$. Serum was immediately collected and frozen at $-20^{\circ} \mathrm{C}$. The same procedure was repeated after 24,48 and 96 hours. Samples were kept frozen until cytokine analysis.

\section{Cytokine analysis}

Cytokines were determined by enzyme linked immunosorbent assays (ELISA) from Eurogenetics (Tessenderlo, Belgium). Analyses were performed according to the instructions of the manufacturer.

\section{Calculations and statistics}

We assumed that the concentration of the proteins in the samples kept frozen during the 21-day stress period remained conslant. The concentration of the proteins in the stressed samples was compared to these control samples and the percentage recovery was calculated. After checking for normality, student t-test was used to compare the recovery after each time point (at each temperature) with the control samples.

Further, the recovery was plotted versus days of heat stress and the slope of the best-fit line was deternined. For $1 L-10$ and $54 L-6 R$, only the first three time points were used to calculate the best fit of the $37^{\circ} \mathrm{C}$ line because the concentration of both cytokines decreased to less than $20 \%$ after 5 days.

Then, the degiadation rate was plotted versus the inverse of the absolute temperature, multiplied by 1000 . The equation of the best-fit line was used to calculate the degradation constant at $-20^{\circ} \mathrm{C}$ and $-70^{\circ} \mathrm{C}$.

The kinetics of protein denaturation are comparable with that of a first order reaction $[3,7]$, what means that the degradation rate is proportional to the concentration of the respective analyte. The equation is:

$$
\begin{aligned}
\cdot d[C] / d t & =k[C] \\
\text { or } \quad \log ([\mathrm{C}] /[\mathrm{C}] \mathrm{o}) & -k t / 2,303
\end{aligned}
$$

where $[\mathrm{C}$ o is the initial concentration and $[\mathrm{C}]$ is the concentrations after timet.

We additionally calculated the time after which $90 \%$ of the respective cytokine can be recovered, by substituting $[\mathrm{CV} / \mathrm{C}] \mathrm{a}=0.90$ in the last equation. 


\section{Results}

\section{Stability}

The stability in serum of slL-6R and CC16 was investigated in fwe patients and three healthy controls, while the stability of IL-6 and IL-10 in serum was investigated in patients only. The concentration of sterile samples of the subjects stored during 21 days at $4{ }^{\circ} \mathrm{C}, 20^{\circ} \mathrm{C}, 30^{\circ} \mathrm{C}$ and $40^{\circ} \mathrm{C}$ were compared to triplicate samples of the same person frozen at $20^{\circ} \mathrm{C}$. The mean concentrations of the serum proteins in patients and controls of the frozen samples are summarised in Table 1. The data depicted in Figure 1 show that the VL-6 concentration in the serum samples stored at $4{ }^{\circ} \mathrm{C}, 20^{\circ} \mathrm{C}$ and $30^{\circ} \mathrm{C}$ remained similar to the concentration in the frozen samples over 21 days. The relative stability of IL-6 in serum at these three temperatures yielded to few data to calculate an Arrhenius-plot. The samples stored at $40^{\circ} \mathrm{C}$ showed a decline down to $88 \%$ starting from day 11 , via the significant lower recovery of $66 \%$ at day 18 $(t=-4.00, P<0.05)$ down to $59 \%$ of the concentration of the control samples at day 21 .

Table 1 Serum concentration of the examined inflammatory markers.

\begin{tabular}{|c|c|c|c|c|c|}
\hline Patlient & $\mathbb{N}$ & $\begin{array}{l}1 \mathrm{H} 6 \\
\text { Mean }( \pm \text { SD) }\end{array}$ & $\begin{array}{l}\text { Sll-6R } \\
\text { Mran (t SD) }\end{array}$ & $\begin{array}{l}11-10 \\
\text { Mean }(+50)\end{array}$ & $\begin{array}{l}\text { CCH } \\
\text { Mean } 1 \text { SD }\end{array}$ \\
\hline$H V+1$ & 2 & $5.9+0.0)$ & $191.6( \pm 6.4)$ & $1.73(100.11$ & $26.4(10.8)$ \\
\hline$H W-2$ & 3 & $0.7+0.6)$ & $207.0(240.4)$ & $1.2\{0.5\}$ & $61.4(1.9 .7)$ \\
\hline$H x^{2}-3$ & 3 & $2.9(+1.2)$ & $204.0( \pm 19.9)$ & $20(1.3)$ & 22361.81 \\
\hline $\mathrm{Pl}$ & 3 & $187.6( \pm 8.4)$ & $184.1(130.3)$ & $26.5(5,1.3)$ & $22.4(1,1,5)$ \\
\hline $\mathrm{P2}$ & 3 & $50.7(12.4)$ & $131.4 \pm 10.4\}$ & $12.1 \pm 3.1 \mathrm{j}$ & $37.2+43.2$ \\
\hline$p 3$ & 3 & $33.3( \pm 2.3)$ & 245.91122 .21 & $13.71+2.1)$ & $73.11 \pm 4.5$ \\
\hline$p 4$ & 3 & $26175( \pm 77.7)$ & $79.9\{ \pm 9.8\}$ & $121.8( \pm 9.2)$ & 16.614 .44 \\
\hline 95 & 2 & $1413: 11.7$ & $212.5+(15.5)$ & $38.8( \pm 5.7)$ & $26.8[ \pm 20,1]$ \\
\hline
\end{tabular}

Concentration of the examined protens in the controt samples (samples that were immediately stored at $-20^{\circ} \mathrm{C}$, and $n 0$ subjected to heat stressi. HV = Heallhy volunteer; $P$.me Pallent.

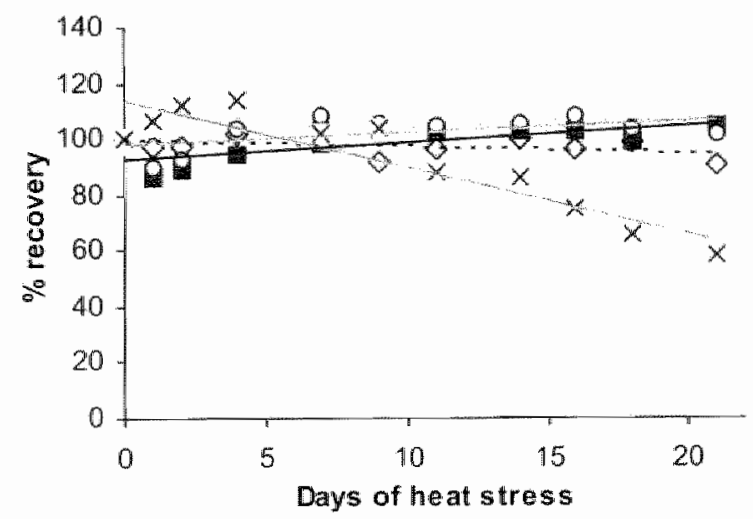

Fig. 1 Recovery of 1 - 6 versus thme of heat stress.

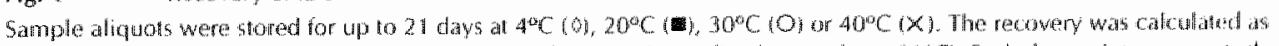

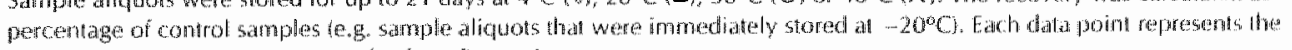
matan $14-6$ recovery in serum samples trom tive patents. 
The dala in Figure $2 \mathrm{~A}$ show the concentration of $5 \mathrm{LL}-6 \mathrm{R}$ in serum at the different temperatures. The $5 / L-6 R$ concentration in serum samples stored at $4^{\circ} \mathrm{C}$ remained similar to that in the frozen samples over the whole duration of the experiment. A significant, apparently linear, decline in the mean concentration of slL-6R in serum stored at $20^{\circ} \mathrm{C}$ was observed down to $87 \%$ of the concentration of the frozen samples affer 21 days $(t=-2.30, P<0.05)$. After one day of storage at $30^{\circ} \mathrm{C}$ the concentration declined down to $91 \%(t=-1.93, P<0.1)$ of the control samples, down to the significant lower recovery of $85 \%$ at day $2(t=-3.08, P<0.01 \%$. The concentration of sllGR in serum stored at this temperature of $30^{\circ} \mathrm{C}$ further declined gradually over time down to $63 \%$ at day $21(t=-7.45, P<0.0001)$. The concentration of sIL-6R in the serum samples stored at $40{ }^{\circ} \mathrm{C}$ showed a $50 \%$ decline after one day $(\mathrm{t}-12.00, \mathrm{P}<0.0001)$ and declined to undetectable concentrations at day 12 . The values of the degradation rate constant at each temperature are shown in Table 2. After calculation of the Arrhenius-plot, the graph and formula shown in Figure $2 \mathrm{~B}$ were obtained. Using this equation, we determined the $k$ value at $-20^{\circ} \mathrm{C}$ and calculated the storage period after which $90 \%$ of s1L-6R can still be recovered at this temperature. The projected $k$-value is $6.398 \times 10^{-6}$ and the predicted storage time is thus approximately 45 years at $-20^{\circ} \mathrm{C}$.

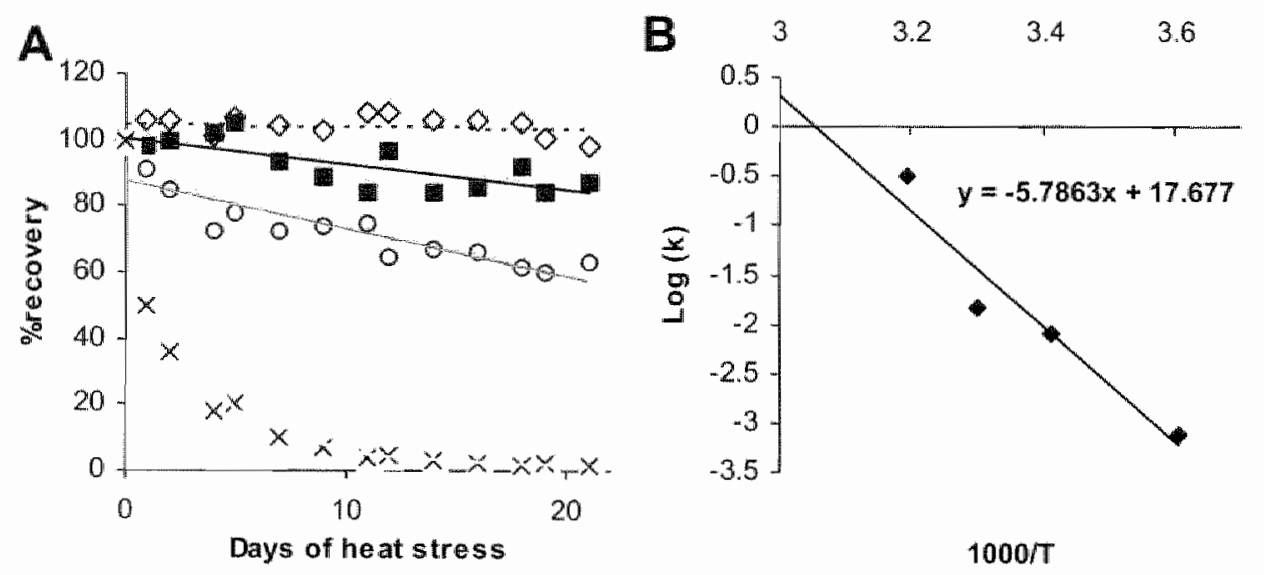

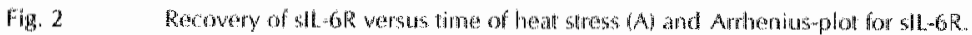

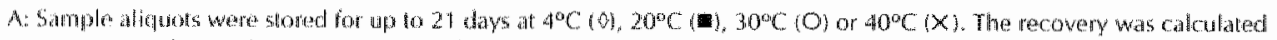

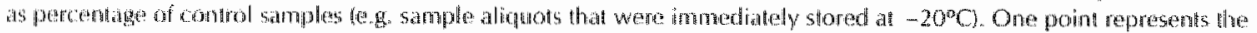

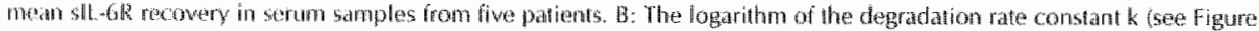

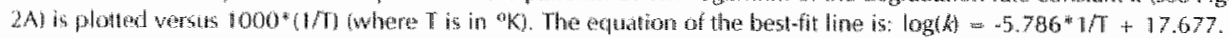

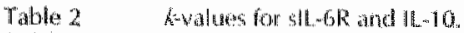

\begin{tabular}{|c|c|c|}
\hline Trmpentatsere $(\mathrm{Cl}$ & $s t L \cdot G R$ & 11.10 \\
\hline 4 & 0.0802 & $-0,0715$ \\
\hline 20 & $0.80 \pi 7$ & 2.9556 \\
\hline 30 & 1.4755 & 4.3875 \\
\hline 40 & 32.191 & 11.901 \\
\hline
\end{tabular}

Pronchage recowery was ploted wersus days of heat stress at each temperature. Values of the degradation rate constant $k$ Wo sll-6R and 10,10 at whese differen stress femperatures were then datermined ats the slope of the best-fin line

The concentrations of $1 \mathrm{~L}-10 \mathrm{in}$ serum samples at the different temperatures during 21 days are shown in Figure 3A. No significant dedine in $1 \mathrm{~L}-10$ concentrations in serum stored at $4^{\circ} \mathrm{C}$ was obsenved during the period of the experiment. The samples stored at $20^{\circ} \mathrm{C}$ showed a decline in 
W-10 concentration to $63 \%$ from day $14(t=-3.54, \mathrm{P}<0.007)$ to $35 \%$ after 21 days. The $11-10$ concentrations in the samples stored at $30^{\circ} \mathrm{C}$ declined from day 7 on $(1=-4.40, p<0.0001)$. The serum concentration of $1 \mathrm{~L}-10$ in the samples at $40^{\circ} \mathrm{C}$ was already decreased to $70 \%$ (t $=$ $2.91, P<0.01)$ after one day of storage. Further decrease during the following days resulted in levels indistinguishable from background at day 14 . The k-values for the degradation at these temperatures are shown in Table 2. Calculation of the Arrhenius-plot yelded the graph and formula demonstrated in Figure 3B. The projected $k$-values at -20 and $-70^{\circ} \mathrm{C}$ are $8.63 \times 10^{-4}$ and $1.776 \times 10^{6}$, respectively. The corresponding storage times at which $90 \%$ of the initial $11 .-10$ concentration can be recowered is 122 days at $-20^{\circ} \mathrm{C}$ and approximately 162 years at $-70^{\circ} \mathrm{C}$.

The concentration of $\mathrm{CC} 16$ in the serum samples remained comparable to the frozen samples at all temperatures as shown in Figure 4. Therefore, no Arrhenius plot could be calculated.

A

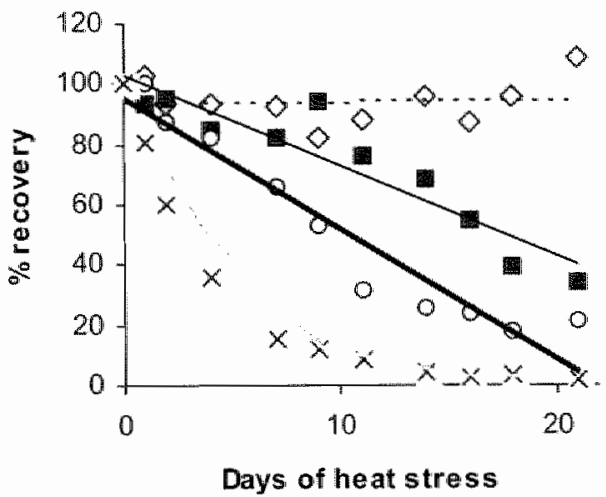

B

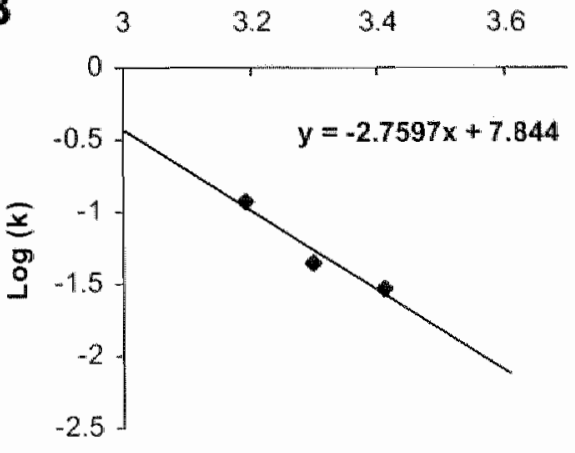

$1000 / T$

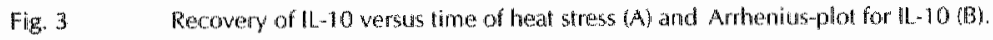

A. Sample atiquots were stored for up to 21 days alt $4^{\circ} \mathrm{C}\left(0,20^{\circ} \mathrm{C}\left(-30^{\circ} \mathrm{C}\right.\right.$ (O) or $40^{\circ} \mathrm{C}$ (X). The recovery was calculated as percentage of control samples (e.g. sample alichots that were inmediately siored at $-20^{\circ} \mathrm{CH}$ One point represents the mean IL-10 recovery in serum samples from five patients. B: The logaritho of the degradation rate constant k (see figure

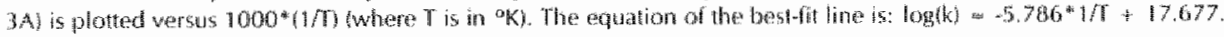

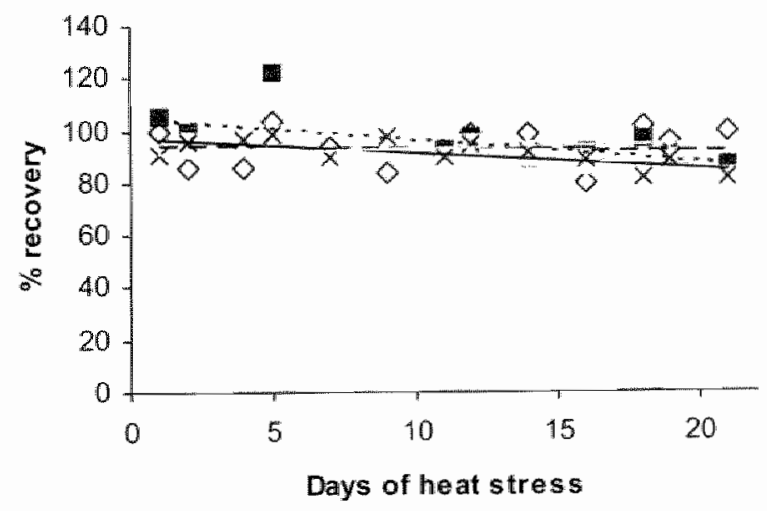

Fig. 4 Recovery of CC 16 versus time of heat stress

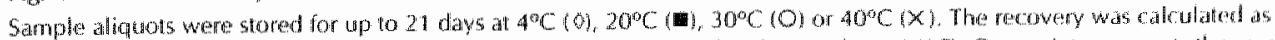

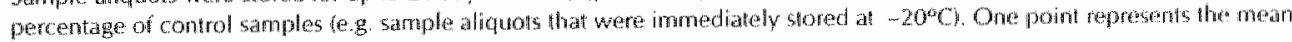
Col6 recovery in serum samples from the palsens. 


\section{Clotring time}

The data depicted in Table 3 show that the serum concentration of IL-6, sIL-6R, IL-10 and CCI6 were not significantly different, at noom temperature, between 30 minutes clotting time and a 4day clotting at room temperature. In addition, no differences in concentration of the proteins were observed when clotting at room lemperature was compared with clotting at $4^{\circ} \mathrm{C}$. More in detail, compared to 15 minutes clotting time, the $511 \mathrm{~L}-6 \mathrm{R}$ and $\mathrm{CC} 16$ concentrations were unchanged up to 10 hours (measurement interval of 11 hour).

Table 3

Infurance of clotting times and temperature on recovery of $1 \mathrm{~L}-6$, sll-6R, $1 \mathrm{~L}-10$ and $\mathrm{CC} 16$.

\begin{tabular}{|c|c|c|}
\hline & $\begin{array}{l}\text { Temperatune } \\
{ }^{\circ} \mathrm{Cl}\end{array}$ & $\begin{array}{l}\text { a recovery } 1 \text { 5.0. } \\
\text { aftur } 4 \text { day clotting }\end{array}$ \\
\hline \multirow[t]{2}{*}{$11.6(n=5)$} & 4 & $97 \geq 19$ \\
\hline & 20 & $84 \pm 11$ \\
\hline \multirow[t]{2}{*}{$54.60^{3}(n=8)$} & 4 & $98+18$ \\
\hline & 20 & $103 \pm 13$ \\
\hline \multirow[t]{2}{*}{$11-10\{0=5\}$} & 4 & $106 \pm 36$ \\
\hline & 20 & $100 \pm 63$ \\
\hline \multirow[t]{2}{*}{$(c 016)=8)$} & 4 & $105 \pm 14$ \\
\hline & 20 & $107 \pm 15$ \\
\hline
\end{tabular}

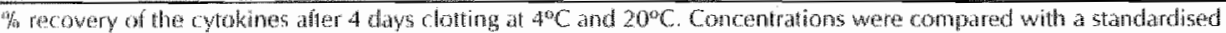

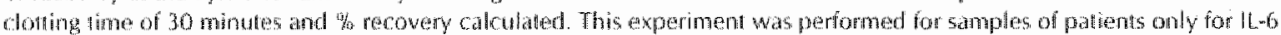
and IL-10, and tor samples of boh patients and controls hor stl $6 \mathrm{R}$ and $\mathrm{CC} 6$.

\section{Influence of freeze-thaw cycles}

The dala summarised in Table 4 show that no significant decline in the concentrations of $1 L-6$, SIL-GR, IL-10 or CC16 was observed after up to four times repeated freeze-thaw cycles.

Table 4

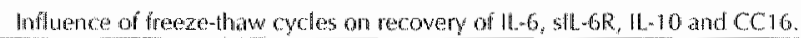

\begin{tabular}{|c|c|}
\hline & 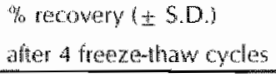 \\
\hline$H L-6(n-3)$ & $101 \pm 10$ \\
\hline sill -6$)$ (n) & $117+3$ \\
\hline $1 L-10(n-3)$ & 77 at 19 \\
\hline$C(6 / 64 n=3)$ & 103 进 2 \\
\hline
\end{tabular}

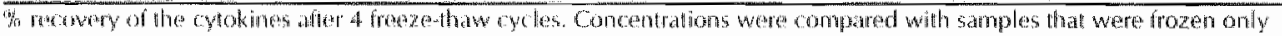
(wimes:

\section{Discussion}

To the best of our knowledge, this study is the first to report on the stability of several inflammatory markers in human serum, examined by means of an accelerated stability testing protocol. The method allows predicting stability for longer storage periods at any temperature. We lound that the recovery of $1 \mathrm{~L}-6$ and $\mathrm{CC} 16$ did not decrease for up to 21 days at $4^{\circ} \mathrm{C}, 20^{\circ} \mathrm{C}$ and $30^{\circ} \mathrm{C}$, and that the $\mathrm{CC} 16$ concentrations even did not change at $40^{\circ} \mathrm{C}$, whereas $1 \mathrm{~L} .6$ concentrations decreased only atter 21 days at this temperature. Soluble $1 \mathrm{~L}-6 \mathrm{R}$ and $1 \mathrm{~L}-10$ were less stable, eg. both proten concentrations did not change at $4^{\circ} \mathrm{C}$ but the recovery decreased gradually at $20^{\circ} \mathrm{C}$ and $30^{\circ} \mathrm{C}$. The predicted storage time after which $90 \%$ of the initial concentration can be recovered was calculated to be 45 years for $5 / L-6 R$ at $-20^{\circ} \mathrm{C}$ and 122 days and 162 years for $11-10$ at $-20^{\circ} \mathrm{C}$ and $-70^{\circ} \mathrm{C}$ respectively. 
The only report to our knowledge that describes an accelerated stablity testing probon with cytokines, is that of Cruz Ruiz et al. [3]. These authors examined the stability of tumour necrosis factor $\alpha$ (TNF-a) in cerebrospinal fluid (CSF) samples, and found it to be stable for at least five years at $-70^{\circ} \mathrm{C}$. They used a pathological CSF-pool (with high TNFa concentration) diluted with a normal CSF-pool to retain any degrading enzymes, and added sodium azide as. preservative. In our study undiluted serum samples were used for the test protocol and we took care that all samples were kept sterile during the stress period.

In our study, the $14-6$ concentrations did not significantly decline at $4{ }^{\circ} \mathrm{C}, 20^{\circ} \mathrm{C}$ and $30^{\circ} \mathrm{C}$. Therefore, it was not possible to calculate the degradation rate constant at those temperatures, and, hence, no Arrhrenius-plot or projected stability times could be calculated. We conclude that $1 L-6$ is very stable and that samples for $1 L-6$ determination can be stored froxen for very long periods before recovery is significantly decreased. These results do not corroborate those from Spong et al., who found that $1 \mathrm{~L}-6$ concentrations in frozen amniotic fluid samples decreased over time [14]. A panel of amniotic fluid samples was measured two times with a one-year interval. The median IL-6 concentration from the second determination was significantly $(20 \%)$ lower as compared to those of 1 year before. However, several factors may explain the discrepancy between these and our findings. First, the high protein concentration in serum may stabilise the IL-6 molecules. Moreover, high concentrations of slL-6R are present in serum as compared to amniotic fluid [11]. These molecules may bind IL-6 and protect it from proteolytic or thermal degradation. Second, detection of proteins in ELISA-systems is dependent on the binding of the antibodies to the respective epitopes on the protein. In some cases, these epitopes are conformationa! structures, which may change due to denaturation or proteolytic cleavage. The antibody then no longer binds its epilope and, hence, recovery in the ELISA decreases. We assume that the antibodies in our ELISA are able to detect partly denatured IL-6. In the study of Spong et al. [14], a decrease in IL-6 concentrations was observed between two independent measurements. The authors do not specify whether the same batch of ELISA was used, thus, interassay variation might be an interfering factor. To overcome this problem, we used an accelerated stability testing, so that all samples could be tested in one run with the same batch of reagents.

Arnother major finding of this study was that the concentrations of IL-6, sIL-6R, IL-10 and CC16 did not change when incubated at $4 \circ \mathrm{C}$ or $20^{\circ} \mathrm{C}$ before centrifugation for up to 4 days. De longh et al. (1997) also reported no change in $14-6$ or $5 / L_{-}-6 R$ concentrations when blood was incubated at $4^{\circ} \mathrm{C}$ for $24 \mathrm{~h}$ before centrifugation [4]. Others showed that II-6 and IL-1 plasma levels were unchanged when stored for up to 3 hours at $37^{\circ} \mathrm{C}$ betore centrifugation [12]. Some studies, however, have shown a decrease in $1 \mathrm{~L}-6$ recovery when blood samples were left unseparated for 24 hours at both $4^{\circ} \mathrm{C}$ and room temperature $115 \rrbracket$, and even after 4 hours at room temperature [5]. Discrepancies between these studies are difficult to explain. In the study of Thavasu et al. [15], IL-6 spiked blood samples were used. White blood cells or thrombocytes may take up the spiked $1 L-6$, whereas in wo generated 11.6 may represent equilibrium between IL-6 production and uptake. Further, comparison between studies might be misteading due to differences in study design and/or immuno-assays le.g. radio- versus, enzyme immunomassays, the antibodies used, etc.).

Finally, we showed that up to four repeated freeze-thaw cycles did not influence the recovery of $1 L-6,51 L-6 R$, IL- 10 and CC16. This confirms the findings of other studies which demonstrated the resistance of $1 L-6, T N F-\alpha, \mathbb{L}-1 \alpha, \| L-1 /$, interferon-y and other serum proteins lleptin, Apolipoprotein Ex to repeated freeze thaw cycles $[5,13,15]$.

It is concluded that serum samples for the determination of $11.6,511-6 R$ and CC. 16 can be stored at $-20^{\circ} \mathrm{C}$ for several years, but for the determination of $\| L-10$, serum samples should be stored at $-70^{\circ} \mathrm{C}$ when longer storage periods are required. Further, neither a time dialay between blood 
sampling and serum separation, nor repeated freeze-thaw cycles have a negative influence on the recovery of these proteins. Thus, we showed that practical aspects of longitudinal and clinical studies (such as long term storage, extended clotting times and repeated freeze-thawing) do not influence the measurement of the proteins examined.

\section{References}

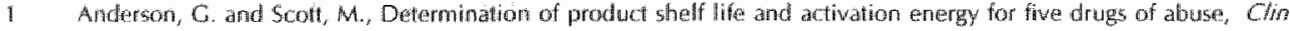
Chem 370991$) 398-402$.

2 Bonaccorso, 5., Lin, A., Song, C. Verkerk, R., Kenis, G., Bosmans, E., Scharpe, S., Vandewoude, M, Dossche, A. and Maes, M. Serotgrin-mmune interactions in elderly volunteers and in patienis wilh Alzheimers ${ }^{\circ}$ disease (DAT):

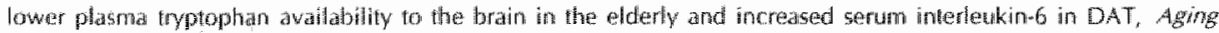
(M/1/6ro) $10(1998) 316-23$.

3 Crun Ruiz, M, Recio Qujano, F, Lopez Contes, 1 . F, Hebes Duvison, M. and Vazquez Rublio, R., Datemination of sheff fife and activation energy for tumor necrosis lactor-alpha in cerebrospinal fuid samples, Chin Chem, 42 11996! $670-4$.

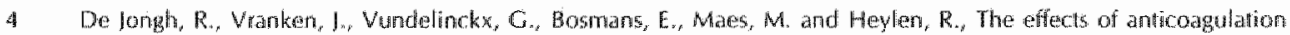
and processing on assays of IL-6, glL-6R, sill-2R and soluble mansferrin receptor, Cytokine, $9(1997) 696-701$. Fibwar, L., Ahaja, R.H., Humphries, S.E. and Mohamed-Ali, V., Effects of sample handing on the sability of interleukin 6, imour aecrosis factor-alpha and lepth, Cyokine, $12 \times 2000117124$.

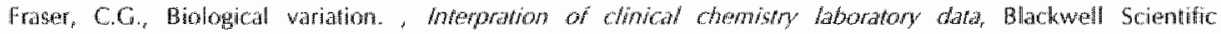
Publications, Oxford, 1986, pp, 111-135.

7 Kirkwood, T.B., Predicting the stability of biological standerds and products, Bionterics, 33 (1977) $736-42$.

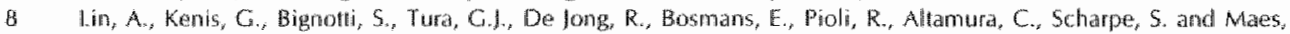
M. The woflammatory response system in treatment-resistant schizophrenia: increased serum moterletkin-6. Schizopher Re, $32(1998) 9-15$.

9 Mars, M. Cooreman, W., Delanghe, 1. Scharpe, 5. Wauters, A., Neels, W. DHondi, P., Pereters, D., Cosyns, P., Ranjan, R. and et al, Components of biological watiation in plasma haptoghobin: relatianstips to plasma fibrinoger

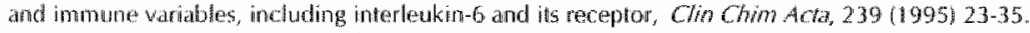

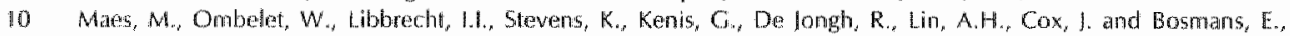
Eifects of pregnancy and delivery on senum concentrations of Clara Cell Protain (CC16), an endogenous anticylokine: lower serum CC16 is related to pastipartum depression, Psychatoy Res, 87 11999) 117-27.

11 Opsjon, 5.L. Wovick, D., Wathen, N.C., Cope. A.P., Wallach, D. and Aderka, D., Soluble tumor necrosis factor receptors and solubis interleukin-6 receptor in fetal and maternal sera, coelomic arid amoliotic fluids in normal and pre-ectamplic pregnancies, /Rephod hmownot, 29 (1995) 119-34.

12 Riches, P., Gooding, R., Mitlar, B.C. and Rowbottom, A.W., Imfluence of collection and separalion of blood samples

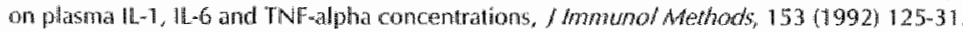

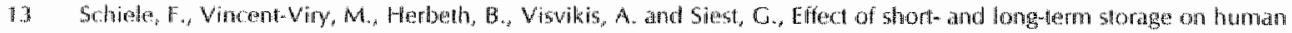

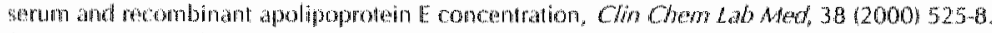

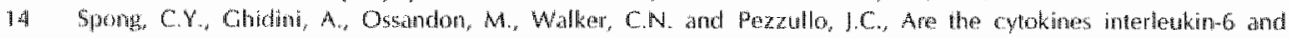

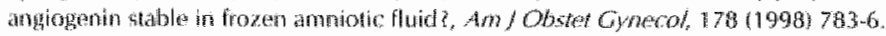

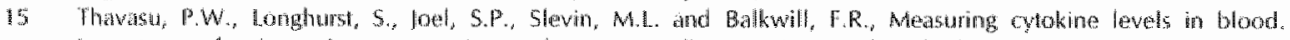

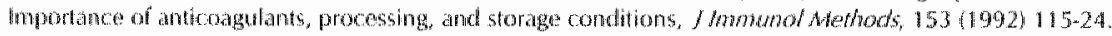




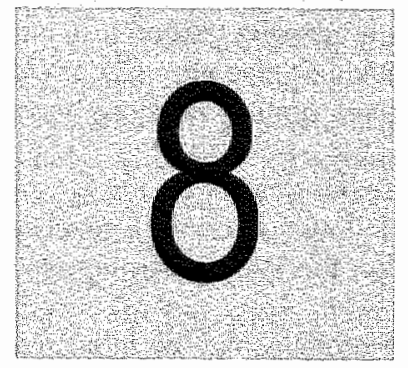

\section{Inflammation markers in relation to cognition in a healthy ageing population}

Charlotte Teunissen, Martin van Boxtel, Hans Bosma, 'Eugene Bosmans, 'Joris Delanghe, Chris De Bruijn, "Annick Wauters, Michael Maes, Jelle Jolles, Harry Steinbusch and Jan de Vente

European Graduate School of Neuroscience (Euron), Universiteit Maastricht, Department of Psychiatry and Neuropsychology, The Netherlands;

"DiaMed EuroGen, Tessenderio, Belgium;

'Laboratorium voor Klinisch Chemie, Universiteit Gent, Belgium;

'AZ Middelheim, Antwerpen, Belgium. 


\section{Abstract}

The aim of this study was to determine whether serum inflammatory protein levels were correlated with cognitive performance in a healthy ageing population.

A cohort of 144 individuals was tested (age 30-80) three times during six years of follow-up in this population based, explaratory study. Domains of cognition that were addressed were cognitive speed (Letter-Digil Coding test), attention and information processing (Stroop test) and memory (Word Learning tests). Serum concentrations of interleukin-6 receptor, Clara cell proten, Cureactive protein, haptoglobin, albumin, total protein and protein fractions were imwestigated.

Haptoglobin levels at baseline correlated negatively with cognitive performance on the Stroop test and on the Word Learning Recall test over the whole 6 years follow-up period. C-reactive protein levels at baseline conrelated negatively with performance on the Word Learning tests over the whole 6 years follow-up period.

In conclusion, relatively high concentrations of these inflammation proteins may be indicative for impared cognitive performance. Further follow-up studies must indicate whether low cognitive performance and high levels of haptoglobin precede cognitive decline.

\section{Introduction}

A decline in cognitive functions is an inevitable feature of normal ageing, but the degree of deterioration varies withim the healthy older population. Some older persons experience a larger decline in certain cognitive functions than others and the patterns of cognitive functions. affected may vary among older people. The processes affecting cognitive deterioration, however, are not understood. Known factors influencing, or possibly conferring a risk for, decline in cognitive performance are among others, age, level of education $[3,13]$, aerobic. fitness [42], gender or hypertension [35]. linsight into biochemical correlates of normal cognitive ageing in individuals may be helpful to understand normal and pathological cognitive ageing. Biochemical correllates may exist for the differentiation between people who age successfully and people who are characterised by a pathological process. While the use of the cerebrospinal fluid (CSF) 10 investigate biochemical correlates in body liquids in nomal healthy individuals is restricted due to thical considerations, the use of peripheral blood samples provides a more readily available tool to assess possible biochemical correlates of cognitive ageing.

Unfortundely, there is no single marker paralleling the cognitive ageing process in body fluids. Several factors may have obstructed the research on such biological makers. First, the brain is separated from the blood by the blood brain barter (BBB), which allows only limited exchange of compounds between the blood and the brain. Nevertheless, the brain is directly linked to peripheral systems, such as the immune system or the endocrine system via the hypothalamuspituitary axis. Secondly, even for the severe forms of cognitive deterioration, dementia, ro conclusive serum marker has been established [45].

Studies on blood constituents in patients with Alzheimer's disease have reported that some serum or plasma compounds may be increased in, at least, a sub-population of patients with dementia of the Alzheimer lype. For example, altered concentrations of pro-inflammatory cytokines, such as interleukin-6 (IL-6), or acute phase reactants, e.g. a-antichymotrypsin have been observed in serum of patients with Alzheimer's disease, though the results on each of these markers varied considerably among the studies $[5,24,30-32,37,43]$. This variation may be due to the low number of patients included, sometimes only 11 [5], or the sensitivities of the 
lests used. Thus, Il-6 concentrations in nomal ageing population as well as in patients with Alzheimer"s disease are often below the detection level of the ELISA-tests used $[2.31$. Recent studies therefore have concentrated on the IL-6 receptor, which shows less variation in blood than IL-6 and is more easily detectable [2].

It is known that the plaques of AD patients are associated with activated microglia and increased expression and protein concentration of inflammatory proteins, e.g. $11.1,11.6, C$ reactive protein (CRP), $\alpha$-antichymotrypsin or tumour necrosis factor- $\alpha[16,17,22,33,34]$. The possibility that mild cognitive deterioration may develop into the pathological state of dementia $[21,25,44]$ led us to hypothesise that inflammation markers as assessed in serum of the ageing population might correlate with cognitive performance.

The aim of the present study was to investigate whether serum inflammation markers could be related to cognitive performance in heathy ageing individuals, or even form a risk factor for cognitive decline. Therefore, in this study we determined serum levels of the inflammation markers IL-6, IL-6 receptor, Clara cell protein 16 (CC16, which is a $15.8 \mathrm{kDa}$ homodimeric antiinflammatory protein and secreted in large amounts in airways by the non-ciliated bronchiolar Clara celts [7]), CRP, albumin, protein fractions, haptoglobin and haptoglobin phenotype. The serum levels of these proteins at baseline were compared with the individual cognitive performance during six years of follow-up in a healthy older population. The study was performed in the course of the MAastricht ageing study (MAAS), a large llongitudinal study involving individuals aged $25 \mathrm{~m} 85$ in the Netherlands.

\section{Methods}

\section{Study population}

Participants in this study were recrutted from a research program investigating determinants of cognitive ageing in the healthy population $[23,41]$. Individuals for this study were randomLy drawn from the Registration Network Family Practices, a research database that contains basic health information of patients in primary care facilities (Metsemakers, Höppener, Knottnerus, Kocken \& Limonard, 1992). Exclusion criteria were clinical evidence of past or present norbidity that can compromise cognifive performance, such as cerebrovascular disease (inclucing stroke), chronic neurological pathology (e.g. dementia, epilepsy or Parkinson's disease), mental retardation or chronic psychotropic drug use. The sample was stratified for age (12 discontinuous groups; $30 \pm 1$ years, $35 \pm 1$ years, ..., $80 \pm 1$ years), gender and wo levels of occupational achievement [23]. In total, 1,823 men and woman were medically and neuropsychologically examined in the baseline examination between 1993 and 1995 . The group was divided into four panels of approximately 450 individuals each.

Individuats aged over 50 years at the beginning of the study (baseline) were re-examined every 3 rd year over a period of 6 years. individuals aged between 30 and 50 years at baseline were re-examined after 6 years. A serum sample was drawn at the beginning and the end of the study and stored at $-80^{\circ} \mathrm{C}$ until analysis. At the end of 1999 , cognitive performance data of three measurements over 6 years of follow-up were avalable from a random group of 144 individuals from the panel that was examined in 1993 for the first time. A complete set of serum and cognitive performance data was available from 93 individuals at baseline. Of 66 individuals a serum sample at both baseline and follow-up was available. An incomplete data set was avallable from the other indivicuals due to technical problems.

The mean age of the 93 individuals at baseline was 57 (SD 11). The gender distribution was 55 man to 37 women. The age of the 66 individuals of whom a complete set of serum data was present was lower (mean 54 years, 5010 . compared to the 27 individuals of whom a serum 
sample was avallable only at baseline (mean 65,5011 , Mann-Whiney $U$ rest: $Z=-4.1$. P $<0.001$. The gender distribution was not statistically different 37 man 1028 women in the 66 individuals, 18 man to 9 woman in the remaining 27 individuals). The performance on cognitive rests was not statistically different between the subgroups (P $>0.05)$.

Five persons used anti-inflammatory drugs on a daily basis. The serum inflammation marker concentrations of these individuals were within the $5 . \mathrm{em}$. of the mean results as shown in Table 1 , though the CRP concentration was elevated in three out of five of these individuals.

The local medical ethics committee approved the study protocol and written informed consent was obtained from all participants.

\section{Cognitive assessment}

Major aspects of cognitive function were tested according to the MAAS-protocol described in detail elsewhere $[23,41]$. The focus was upon processes involving memory, attention and different aspects of cognitive processing speed.

\section{WORD LEARNING TASK (WLT)}

This test is based upon the Auditory Verbal Learning Test [6] and evaluates the ability to acquire and retain new verbal information. Fifteen frequently used monosyllabic words are presented and the subject is instructed to memorise the words. The trial ends with a free recall of the words. This procedure is repeated five times, using the same word set in fixed order. The total number of correctly reproduced words on the five immediate recall trials is recorded (WLTTOT). After 20 minutes the subjed is asked to reproduce the set of words (Delayed Recall). The total number of correctly reproduced words after 20 minutes is recorded [23]. A higher score on this test reflects better cognitive performance.

\section{LETTER-DIGIT CODING TEST (LDCT)}

This paper-and-pencil test is a modified version of the Symbol Digit Modalities Test [39] and measures information processing speed. The subject is recuested to copy numbers in cells that are indexed by a letter. The letter refers to nine letter/number combinations at the top of the form. The total number of correctly copied corresponding numbers in 90 seconds is recorded as test outcome. A relatively better cognitive performance is associated with a higher score on this test.

\section{STROOP COLOUR-WORD TEST (STROOP)}

This perceptual interference test consists of three subtasks. Each subtask consists of a test sheet containing four rows of ten columns of colour names or coloured spots. The test examines the speed at which colour names are read (subtask 1) and the speed at which colour spots are named (subtask II). Subtask III inwolves colour names but the printing ink is different from the colour name. The time needed to name the colour of the printing ink of the words is recorded. Thus, a better cognitive performance is associated with a lower score on the Stroop test, in contrast with the higher scores linked to better cognitive performance on the other three memory tasks in this paper. This test shows robust effects of chronological age [19]. Only the data of subtask $11 \mathrm{l}$ are used in the current study and are referred to as 'Stroop'.

\section{Markers of Inflammation}

For the deternination of $1 L-6,11 L-6$ receptor, CC16 and CRP in serum commercial ELISA kits were used (Eurogenetics S.A., Tessenderlo, Belgium). Detection limit of IL-6 was $10 \mathrm{pg} / \mathrm{mL}$, the detection limit for $1 \mathrm{~L}-6$ receptor was $1 \mathrm{ng} / \mathrm{mL}$, for CC16 the detection limit was $0.1 \mathrm{ng} / \mathrm{mL}$ and 
for CRP the detection limit was $0.25 \mu \mathrm{g} / \mathrm{mL}$. The intra assay coefficients of variance (C.V.) of all assays were below 8\%. Serum haptoglobin concentration was measured by fixed-time immunonephelometry on a BN II analyser (Dade Behring, Marburg, Gemany) and the assay was calibrated against the international CRM 470 reference material [11]. Haptoglobin phenotype was determined using starch gel elechophoresis of haemoglobinusupplemented serum, followed by peroxidase staining [40]. The distribution of the phenotypes $11-1: 12.9 \% ; 2$ T: $50.5 \% ; 2-2: 36.6 \%$ ) was in accordance with the distribution in the north-western European population [28]. Serum protein electrophoresis was pertormed by capillary electrophoresis using the automated Beckman Paragon CZE 2000 (Analis, Namur, Belgivm). The intra-assay CV values for the serum protein fractions are as follows: albumin $1.2 \% ; 0.11 .6 \% ; \alpha 21.6 \%$ and $y$ $4.9 \%$. Considering the long storage period of the samples (up to 6 years), stability of the acute phase proteins IL-6, IL-6 receptor and CC 6 was confirmed under different storage conditions. In addition, stability after repeated freezing-thawing was confirmed (Chapter 7).

\section{Statistical analysis}

Normal distribution was tested using the Kolmogorow-Smimov test. Zero order bivariate correlation analysis was performed using Pearson's correlation coefficient. Because the distribution of CRP was skewed, the ranks of the values of this protein were calculated for the correlation analysis.

The analysis was performed in two phases. First, ordinary least squares multiple regression analysis was performed for the four cognitive tests at baseline with each separate serum protein as potential predictor and age, gender and level of education as covariates as in the following expression:

\section{Cognitive test outcome $=b^{*}$ protein $+\left(b 2^{*}\right.$ age $+b 3^{*}$ gender $+b 4^{*}$ education $)$}

Secondly, the markers showing a significant relation $(\mathrm{P}<0.05)$ with al least two of the cognitive tests, adjusted for age, gender and educational level, multi-level regression analysis was additionally performed. Multi-level repeated measurement analysis was performed to analyse the association between the predictors and cognitive function during the whole follow-up. This method uses all observations, including the observations of persons with only one or two observations [1,15]. Persons who were over 50 years of age were included at all three measurement phases. Person younger than 50 years were included for the first and third phase only. To increase homogeneity regarding age across the different phases, the analyses were redone for persons older than 50 years of age only. The influence of influential cases and oullers was examined using the studentised residuals and Cook's distances in the nomal hierarchical regression analysis. As four cognitive tests were used as outcomes, a Bonferroni adjustment was considered. However, as this study is primarily exploratory in nature, a significance level of $\mathrm{P}<0.05$ was used. Data are expressed as regression coefficient (b) with $95 \%$ conficlence intervals.

All analyses were performed with SPSS statistical software.

\section{Results}

\section{Biochemical measurements}

The mean values of the serum proteins all baseline are expressed in Table 1 . The mean haptoglobin concentration was comparable with reference ranges reported [28]. The mean concentration of total protein, albumin, and protein fractions was in agreement with laboratory 
reference ranges total protein range $60-78$; albumin range $36.0-51.0$; a 1 range $2.7-5.2 ; a 2$ range 3.5.8.2: 7 range $6.2-14.4$ ). The mean and median of the CRP concentrationis were higher compared to proposed reference ranges of $0.98(0.34-2.85) \mathrm{mg} / \mathrm{L}$ for healthy individuals of 27. 75 years old, using a different assay [10]. The mean and range for the $\|$ L-6 receptor concentration as measured in our study were about three times higher then values reported by Angelis for elderly controls $(47.6 \pm 1.3 \mu \mathrm{g} / \mathrm{L}$; range $29.0-61.3 \mathrm{\mu g} / \mathrm{L}[2])$. The mean and range of CC16 concentrations were higher then reported values of $13.3 \mu \mathrm{mg} / \mathrm{L}$ (range $5.2-34.5 \mu \mathrm{g} / \mathrm{L}$ ) for individuals from 18-67 years by Hermans et al. [18].

Tatile 1 Concentiations of serum markets an basel he and after 6 years of hollow-ups.

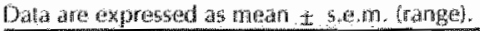

\begin{tabular}{|c|c|c|c|c|}
\hline & \multicolumn{2}{|c|}{ All individuals } & \multicolumn{2}{|c|}{ Indruiduats $>$ m 50 years ofid } \\
\hline & baselinie & Sollow-up & bascline & flollow-np \\
\hline Haptoglobin (a/t) & $\begin{array}{l}1.21 \pm 0.48 \\
(0.08-3.41)\end{array}$ & $\begin{array}{l}1.22 \pm 6.32 E-02 \\
(0.34-2.84)\end{array}$ & $\begin{array}{l}1.26+0.48 \\
0.08-3.41 !\end{array}$ & $\begin{array}{l}1.26 \pm 0.07 \\
(0.43-2.39)\end{array}$ \\
\hline Tolnt protein (g/L) & $\begin{array}{l}66.0 \pm 7.1 \\
443080.09\end{array}$ & $\begin{array}{l}70.7 \pm 1.0 \\
(490 \cdot 910)\end{array}$ & $\begin{array}{l}66.512 .8 \\
(430-80.0)\end{array}$ & $\begin{array}{l}70.9 \pm 1.1 \\
(51.0 \times 83.0\end{array}$ \\
\hline Albumin $(g / \mathrm{L})$ & $\begin{array}{l}36.1 \pm 4.2 \\
126.947 .2 j\end{array}$ & $\begin{array}{l}42.1 \pm 0.6 \\
(29.7 \cdot 53.0\}\end{array}$ & $\begin{array}{l}38.1 \pm 43 \\
426.9 \times 47.2)\end{array}$ & $\begin{array}{l}41.9 \pm 0.7 \\
(30.3-52.0)\end{array}$ \\
\hline a-fractom $(\mathrm{g} / \mathrm{L})$ & $\begin{array}{l}3.67 \pm 0.51 \\
2.204 .800\end{array}$ & $\begin{array}{l}4.52 \pm 0.12 \\
(2.30-7.40)\end{array}$ & $\begin{array}{l}3.72 \pm 0.48 \\
(2.70-4.70)\end{array}$ & $\begin{array}{l}4.57 \pm 0.13 \\
(3.20-7.40)\end{array}$ \\
\hline a24hation $(\mathrm{g} / \mathrm{H})$. & $\begin{array}{l}6.39 \pm 1.55 \\
(3.60-11.305\end{array}$ & $\begin{array}{l}6.01 \pm 0.16 \\
(3.40-8.60)\end{array}$ & $\begin{array}{l}6.56 \pm 1.48 \\
(3.60-11.30)\end{array}$ & $\begin{array}{l}6.15 \pm 0.18 \\
13.50-8.60\end{array}$ \\
\hline Yifration $(\mathrm{g} / \mathrm{h})$ & $\begin{array}{l}9.27+2.33 \\
(4.60 \cdots 10.00)\end{array}$ & $\begin{array}{l}9.80 \pm 0.29 \\
15.50-17.009\end{array}$ & $\begin{array}{l}9.33+2.34 \\
(4.60-18.00)\end{array}$ & $\begin{array}{l}9.75 \pm 0.35 \\
15.50-17.000\end{array}$ \\
\hline IL-6raceptrt (mg/L) & $\begin{array}{l}(58 \pm 42 \\
(62-276)\end{array}$ & $\begin{array}{c}177 \pm 59 \\
(100-290)\end{array}$ & $\begin{array}{l}157 \pm 42 \\
622-276)\end{array}$ & $\begin{array}{r}170 \pm 66 \\
\{100-288\}\end{array}$ \\
\hline CCI6. $\left(\mu \mathrm{B}^{\prime} \mathrm{L}\right)$ & $\begin{array}{l}31.8 \pm 12.7 \\
(3.5 .82 .0)\end{array}$ & $\begin{array}{l}25.6 \mathrm{*}=1.3 \\
0.7-67.11\end{array}$ & $\begin{array}{l}32.6 \pm 12.6 \\
0.5-82.05\end{array}$ & $\begin{array}{l}26.9 \pm 16 \\
(11.0-67.1)\end{array}$ \\
\hline CRP $(n, m y)$ & $\begin{array}{l}5.73+6.74 \\
\text { naediath: } 2.36 \\
60.50 \cdot 27.88\end{array}$ & $\begin{array}{l}7.55+1.57 \\
\text { median: } 2.62 \\
10.50-83.54 \\
\end{array}$ & $\begin{array}{l}6.68 \pm 7.09 \\
\text { median: } 2.19 \\
10.50-27.889\end{array}$ & $\begin{array}{l}6.93 \pm 1.25 \\
\text { median: } 2.51 \\
10.50 \cdot 30.91\end{array}$ \\
\hline
\end{tabular}

\section{Zero onder correlation analysis at baseline}

The results from the correlation analysis of the serum proteins with the cognitive tests for all individuals are shown in Table 2.

Haptoglobin showed a negative correlation with the LDCT $r=-0.208, P<0.05$ ) and a positive relation with outcomes on the stroop test for the whole group. In individuals ollder than 50 years, haptoglobin cotrelated positively with the stroop test $(r-0.278$, $p<0.05)$. These results indicate a relatively decreased cognitive performance when haptoglobin concentrations are higher.

A negative correlation beween albumin concentration and performance on the Stroop test was observed in the whole group $(r-0.238, P<0.05)$ and in the group of older individuals $r=-$ $0.291, p<0.05$ ), indicating better cognitive performance when serum albumin concentrations are higher. Accordingly, a positive correlation between albumin concentration and Delayed Recall was observed in the group of older individuals $(r=0.251, P<0.05)$.

A positive correlation was observed between CRP concentration and the Stroop test $(r=0.248$, $P<0.05)$ and a negative correlation between CRP and the WLTTOT $(r=-0.239, P<0.05)$ in the 
total group. Thus, higher concentration of CRP may indicate a less adequate cognitive periormance. IL-6 was detectable in only twelve of the individuals mean $31.44,5055.04$ (range 10.01-205.05) and therefore those data were not included for analysis. All oher serum proteins did not correlate with cognitive outcome measures.

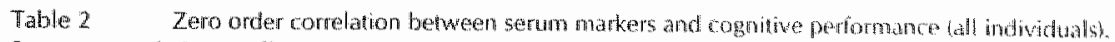

Pearson correlation coeticicients.

\begin{tabular}{|c|c|c|c|c|}
\hline & $51 r 000$ & Q $D C^{2}$ & METCM & Detaygh Real: \\
\hline $\begin{array}{l}\text { Mean }=\text { sem } \\
\text { (rangel }\end{array}$ & $\begin{array}{l}99.1 .27 .01 \\
160.00224 .901 \\
\end{array}$ & $\begin{array}{l}47.25 \div 10.38 \\
2000071.003 \\
\end{array}$ & $\begin{array}{l}42.62+9.3 \\
17.00-61.00 \% \\
\end{array}$ & $\begin{array}{r}9.90: 3.94 \\
60015001 \\
\end{array}$ \\
\hline Maploglobin phenotype & 0.184 & 0,090 & -0.033 & -0.104 \\
\hline Haproglobin (w) & $0,304 * \pi$ & $0.203^{+2}$ & -0.105 & 0.195 \\
\hline Thotal proten (gh) & 0.101 & 0.022 & 0.0 .027 & -10.004 \\
\hline Aloming (gite) & $-0.238^{*}$ & 0.133 & 0.079 & 0.14 \\
\hline o. 自-fractioln $(g / L)$ & 0,010 & 0.030 & 0.012 & -0.112 \\
\hline 2-fraction (g & 0.103 & -0.0095 & -0.039 & 0.055 \\
\hline froftaction $\| \mathrm{g} / \mathrm{t})$ & -0.030 & 0.036 & -0.0137 & -0.100 \\
\hline 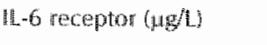 & 0.155 & .0 .121 & 0.117 & -0.090 \\
\hline $\mathrm{CCH}(\mathrm{\mu g} / \mathrm{L}$ & 0.170 & -0.166 & 0.044 & $-0,059$ \\
\hline GRP rong/to & $0.248^{\circ}$ & 0.138 & -0.129 & $-0.239^{\circ}$ \\
\hline
\end{tabular}

$P<0.05 ; * P<0.01$.

\section{Correlation between cognitive performance and serum markers ar baseline adjusted for age, gender and education}

The correlation between the serum markers and the scores on the cognitive tests adjusted for age, gender and education was investigated using multiple regression analysis of all results obtained at baseline. The data obtained for the whole group are presented in Table 3. Relevant for the subgroup of individuals over 50 years old data are placed in the text, due to the similarity of these resulis with the results obtained for the whole group.

A positive association $(b=11.19, P<0.05)$ between haptoglobin concentration and the Stroop test was observed in the total population, indicating decreased cognitive performance in individuals with higher haptoglobin levels. A similar tendency was obtained in the $50+$ subset of individuals $(b=10.80, P<0.1)$. In accordance with these results, a negative correlation between haptoglobin concentration and Delayed Recall was observed for all individuals (b- .. 1.29, $P<0.05)$ and a tendency for the subset of older individuals $(b=-1,27, P<0.1)$. Haptoglobin concentrations are dependent on the phenolype, with lowest concentration in $2-2$ phenotypes $(0.38-1.50 \mathrm{~g} / \mathrm{L})$, then $2-1(0.44-1.83 \mathrm{~g} / \mathrm{L})$ and highest concentration in $1-1$ phenotype (range $0.57 \cdot 2.27 \mathrm{~g} / \mathrm{L}$ ) [28]. Thus, it is important to know if the relation between haptoglobin concentration and cognitive performance is independent of phenotype. When haptoglobin concentration was additionally adjusted for phenotype, the regression coefficients of the correlation will Stroop performance or Delayed Recall increased in magnitude and significance (Table 3 ). 


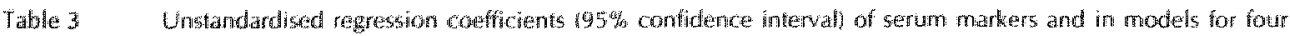

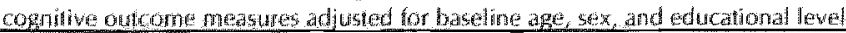

\begin{tabular}{|c|c|c|c|c|c|}
\hline & $n$ & Strops & $\mathrm{LOC}$ & WLTTOT & Delayed Recall \\
\hline \multicolumn{6}{|l|}{$\begin{array}{l}\text { haptoglobin } \\
\text { concentation }\end{array}$} \\
\hline all perople & 92 & $\begin{array}{l}11.9 \\
(0.75,21.64)^{4}\end{array}$ & $\begin{array}{l}-1.73 \\
(-5.53,2.06)\end{array}$ & $\begin{array}{l}-1.51 \\
(-5.28,2.25)\end{array}$ & $\begin{array}{l}-1.29 \\
(-2.52,-0.58)^{*}\end{array}$ \\
\hline$>0050$ yeders & 71 & $\begin{array}{l}10.87 \\
(-1.94,23.60)^{*}\end{array}$ & $\begin{array}{l}0.42 \\
(-4.85,4.01)\end{array}$ & $\begin{array}{l}-0.18 \\
(-5,81,3,45)\end{array}$ & $\begin{array}{l}-11.27 \\
\{-2.78,0.24)^{*}\end{array}$ \\
\hline $\begin{array}{l}\text { haphoglobin } \\
\text { phergotype } \\
\text { all people }\end{array}$ & 92 & $\begin{array}{l}8.16 \\
(0.93,15,39)^{4}=\end{array}$ & $\begin{array}{l}\times 1.14 \\
(-1.72,1.44)\end{array}$ & $\begin{array}{l}0.33 \\
(-2.90,2.24)\end{array}$ & $\begin{array}{l}-0.56 \\
(-1.41,0.29)\end{array}$ \\
\hline$>=50$ years & 71 & $\begin{array}{l}8.42 \\
\left(-0.17,(7.01)^{2}\right.\end{array}$ & $\begin{array}{l}-1.08 \\
(-4.06,1.909\end{array}$ & $\begin{array}{l}0.96 \\
(4.08,2.17)\end{array}$ & $\begin{array}{l}-0.77 \\
(-1.79), 0.26\end{array}$ \\
\hline \multicolumn{6}{|l|}{ haphoglobin } \\
\hline \multicolumn{6}{|l|}{ moncentrations } \\
\hline all peoplles & 92 & $\begin{array}{l}13.97 \\
13.73,24.22\end{array}$ & $\begin{array}{l}-2.08 \\
{[-5.93,1.77]}\end{array}$ & $\begin{array}{l}4.65 \\
(-5.48,2.19)\end{array}$ & $\begin{array}{l}-1.47 \\
(-2.71,-0.24)\end{array}$ \\
\hline$>=50$ years & 71 & $\begin{array}{l}12.99 \\
10.34,25.44 \%\end{array}$ & $\begin{array}{l}-0.66 \\
(-5,15,3.83)\end{array}$ & $\begin{array}{l}-1.41 \\
-6.10,3.29\}\end{array}$ & $\begin{array}{l}-1.46 \\
(-2.97,0.04) *\end{array}$ \\
\hline \multicolumn{6}{|l|}{ lotal promein } \\
\hline all pooplate & 93 & $\begin{array}{l}-0.11 \\
(-0.81,0.58)\end{array}$ & $\begin{array}{l}-0.01 \\
(-0.26,0.23)\end{array}$ & $\begin{array}{l}-0.0 \% \\
0-0.28,0.22)\end{array}$ & $\begin{array}{l}-0.00 \\
(-0.08,0.08)\end{array}$ \\
\hline$y=50$ years & 72 & $\begin{array}{l}-0.12 \\
1-1.03,0.781\end{array}$ & $\begin{array}{l}-0.02 \\
(-0.28,0.34)\end{array}$ & $\begin{array}{l}-0.07 \\
-0.26,0.39)\end{array}$ & $\begin{array}{l}0.06 \\
(-0.04,0.17)\end{array}$ \\
\hline \multicolumn{6}{|l|}{ albuming } \\
\hline all pesople & 93 & $\begin{array}{l}-0.50 \\
(-1.73,0.72)\end{array}$ & $\begin{array}{l}-0.03 \\
(0.46,0.41)\end{array}$ & $\begin{array}{l}0.02 \\
(-0.41,0.46)\end{array}$ & $\begin{array}{l}0.06 \\
-0.09,0.204\end{array}$ \\
\hline$>-50$ years & 72 & $\begin{array}{l}0.60 \\
(-2.12,0.911\end{array}$ & $\begin{array}{l}0.06 \\
(0.45,0.581\end{array}$ & $\begin{array}{l}0.15 \\
(-0.40,0.70)\end{array}$ & $\begin{array}{l}0.15 \\
(-0.03,0.33)\end{array}$ \\
\hline \multicolumn{6}{|l|}{ oldration } \\
\hline all people: & 93 & $\begin{array}{l}2.44 \\
(-7.03,11.92)\end{array}$ & $\begin{array}{l}-1.09 \\
-4.43,2.26 !\end{array}$ & $\begin{array}{l}0.06 \\
(-3.30,3.42)\end{array}$ & $\begin{array}{l}-0.66 \\
(-1.77,0.46)\end{array}$ \\
\hline$y=50$ years & 72 & $\begin{array}{l}-0.07 \\
{[-12.21,12.06]}\end{array}$ & $\begin{array}{l}-0.11 \\
(-4.21,3.99)\end{array}$ & $\begin{array}{l}0.16 \\
(-4.20,4.52)\end{array}$ & $\begin{array}{l}-0.43 \\
-1.88,1.031\end{array}$ \\
\hline \multicolumn{6}{|l|}{ 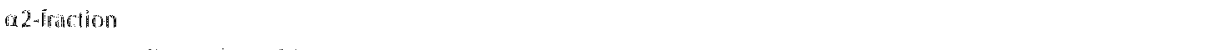 } \\
\hline ה & 9 & $\begin{array}{l}0.90 \\
(-2,3,-4.13)\end{array}$ & $\begin{array}{l}-0.44) \\
(-1,57,0.686)\end{array}$ & $\begin{array}{l}-0.30 \\
(-1,43,0.83)\end{array}$ & $\begin{array}{l}-0.12 \\
-0.50,0.26\end{array}$ \\
\hline$>\quad 50$ years & 72 & $\begin{array}{l}0.76 \\
(-3.21,-4.72)\end{array}$ & $\begin{array}{l}0.37 \\
(-1.71,0.97)\end{array}$ & $\begin{array}{l}0.15 \\
(-1.27,1.50)\end{array}$ & $\begin{array}{l}0.00: \\
(-0.48,0.48)\end{array}$ \\
\hline \multicolumn{6}{|l|}{ pr-fraction } \\
\hline atl people & 93 & $\begin{array}{l}0.21 \\
(-2.70,3.12)\end{array}$ & $\begin{array}{l}0,37 \\
(-0.67,1.41)\end{array}$ & $\begin{array}{l}-0.48 \\
(-1.52,0.55)\end{array}$ & $\begin{array}{l}0.05 \\
(-0.41,0.29)\end{array}$ \\
\hline$>-50$ years & 72 & $\begin{array}{l}0.20 \\
1.3 .20,3.76)\end{array}$ & $\begin{array}{l}0.85 \\
(0,31,200)\end{array}$ & $\begin{array}{l}0.52 \\
(-1.77,0.72)\end{array}$ & $\begin{array}{l}0.07 \\
(0.35,0.49)\end{array}$ \\
\hline \multicolumn{6}{|l|}{ fromation } \\
\hline all propla & 93 & $\begin{array}{l}-0.12 \\
\{-2.25,2.01\}\end{array}$ & $\begin{array}{l}-0.07 \\
1-0.83,0.68 \mathrm{i}\end{array}$ & $\begin{array}{l}0.104 \\
(-0.72,0.809\end{array}$ & $\begin{array}{l}0.05 \\
(-10.30,0.20)\end{array}$ \\
\hline$>-50$ yonts & 72 & $\begin{array}{l}0.34 \\
-2.24,2.91)\end{array}$ & $\begin{array}{l}-0,36 \\
(-1,22,0.51)\end{array}$ & $\begin{array}{l}0.35 \\
\{-0.57,1.28\}\end{array}$ & $\begin{array}{l}0.10 \\
(-0.21,0.41)\end{array}$ \\
\hline \multicolumn{6}{|l|}{ IL 6 megeptor } \\
\hline all people & 90 & $\frac{1.12}{[-0.02,2.26]^{*}}$ & $\begin{array}{l}-0.29 \\
(-0.71,0.12)\end{array}$ & $\begin{array}{l}-0.22 \\
(-0.63,0,195\end{array}$ & $\begin{array}{l}0.06 \\
10.20,0.081\end{array}$ \\
\hline$>=50$ yoars & 71 & $\begin{array}{l}0.82 \\
(-0.5 B, 2,21) \\
\end{array}$ & $\begin{array}{l}0.16 \\
(-0,6,4,0,32)\end{array}$ & $\begin{array}{l}0.31 \\
(-0.80,0.119 \\
\end{array}$ & $\begin{array}{l}-0.08 \\
(0.25,0.08) \\
\end{array}$ \\
\hline
\end{tabular}




\begin{tabular}{|c|c|c|c|c|c|c|}
\hline & & & & & & \\
\hline & & n & subop & tent & MITOT & Detayed Rocall \\
\hline \multicolumn{7}{|l|}{$\mathrm{CCl}$} \\
\hline & all people & 99 & $\begin{array}{l}0.13 \\
(-0.28,0.54)\end{array}$ & $\begin{array}{l}-02 \\
1-0,17,0,12\end{array}$ & $\begin{array}{l}0.13 \\
1001.0 .28)^{*}\end{array}$ & $\begin{array}{l}0.02 \\
-0,02,0.084\end{array}$ \\
\hline & $>=50$ vears & 70 & $\begin{array}{l}0.21 \\
(-0.29,0.701\end{array}$ & $\begin{array}{l}0.04 \\
(-0.21,0,13)\end{array}$ & $\begin{array}{l}0.36 \\
(0.01 .0 .33)^{-2}\end{array}$ & $\begin{array}{l}-0.02 \\
(0.01), 0.104\end{array}$ \\
\hline \multicolumn{7}{|l|}{$\mathrm{CRP}$} \\
\hline & all people & 88 & $\begin{array}{l}0.76 \\
(0.02,1.49)^{* *}\end{array}$ & $\begin{array}{l}-0.06 \\
(-0.33,0.211)\end{array}$ & $\begin{array}{l}-0.11 \\
(-0.38,0.13)\end{array}$ & $\begin{array}{l}0.10 \\
(0.10,-0.01)^{*}\end{array}$ \\
\hline & $>=50$ years & 70 & $\begin{array}{r}0.74 \\
(-0.08,1.56)^{*} \\
\end{array}$ & $\begin{array}{l}0.01 \\
0.0 .28,0.291\end{array}$ & $\begin{array}{l}-0.11 \\
\{0.41,0.19\}\end{array}$ & $\begin{array}{l}0.10 \\
(0.20,-0,003)^{* 6}\end{array}$ \\
\hline
\end{tabular}

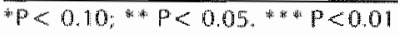

a: additionally adjusted for haptoghobin phenotype

A positive relation between CC16 and the WLTTOT test was observed in the total population $(b=0.13, P<0.01)$ as well as a tendency for the individuals over 50 years only $(b=0.16$, $P<0.1$ ), indicating better cognitive performance in individuals with higher CC16 levels.

A significant positive correlation between CRP concentration and the Stroop test at baseline was observed in the total population $(b=0.76, P<0.05)$ as well as in the subset of 50 +. This effect was a tendency only in the latter subset $(b=0.74, P<0.11$. Thus, high CRP concentrations are observed in persons with a less adequate cognitive performance. In agreement with this, a negative relation between CRP and the Delayed Recall test at baseline was observed in the total population $(b=-0.10 P<0.05)$ as well in the $50+$ subset $(b=-0.10, P<0.05)$.

The correlation between albumin and the Stroop and Delayed Recall as shown in Table 2 disappeared when age, gender and education were accounted for. No correlation between the concentration of total protein, protein fractions or IL-6 receptor and cognitive performance on the current tests was observed.

Correlation between cognitive perfomance and serum markers adjusted for age, gender and education during the whole six years follow-up period

The relation between serum marker concentration at baseline and cognitive performance over the whole six years follow-up was further examined, in order to establish if a causal retation between the parameters existed. This was done for the serum inflammation markers showing choss-sectional correlation with at least two cognitive outcomes in the hienarchical regression analysis. Thus, the correlation of the markers haptoglobin and CRP with cognitive performance during follow-up was investigated using multi-lewel repeated measurement analysis (Table 4). Multilevel repeated measurement analysis allows the simultaneous examination of several observations per person (this increases the power of the study). Furthermore, multi-level analysis allows different numbers of observations between persons.

A tendency towards a negative correlation (positive b) between haptoglobin levals at baseline and performance on the stroop test over 6 years of follow-up was observed for the folal population $(b=8.62, P<0.11)$. A significant negative correlation beween haploglobin concentration at baseline and Delayed Recall during 6 years of follow-up was observed for the total population $(b=-1.17, P<0.05)$ as well as a tendency for the older individuals $(b-1.06$, $P<0$. 11. These relations became even stronger, i.e. $P<0.01$ and larger coefficients, after additional adjustment for haptoglobin phenotype, indicating a relation between haptoglobin concentration and cognitive performance independent of phenotype. 


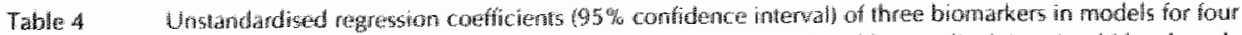

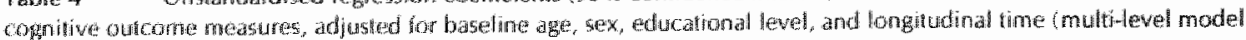
with observations at level 1 and individuats at level 2 . $^{*}$

\begin{tabular}{|c|c|c|c|c|c|}
\hline & $\begin{array}{l}\text { Indits } \\
\text { In }\end{array}$ & Stroop & LDCT & WhTrol & Detayed Recall \\
\hline \multicolumn{6}{|l|}{ Heplonstobin } \\
\hline All peopts & $\begin{array}{l}92 \\
(252)\end{array}$ & $\begin{array}{l}8.62 \\
(0.36,18.13)\end{array}$ & $\begin{array}{l}-2.08 \\
(-5.68,1.52)\end{array}$ & $\begin{array}{l}-2.10 \\
(-5.24,1.04)\end{array}$ & $\begin{array}{l}-1.17 \\
(-2.09,-0.24)^{*}\end{array}$ \\
\hline$>=50$ years & $\begin{array}{l}79 \\
226)\end{array}$ & $\begin{array}{l}8.78 \\
41.70,19.26 !\end{array}$ & $\begin{array}{l}-1.27 \\
(-5.16,2.62)\end{array}$ & $\begin{array}{l}-2.25 \\
(-5.81,1.31)\end{array}$ & $\frac{-1.06}{(-2.18,0.08)}$ \\
\hline \multicolumn{6}{|l|}{ Haptoglobin: } \\
\hline all people & $\begin{array}{l}92 \\
2525\end{array}$ & $\begin{array}{l}10.93 \\
\{1.68,20.18\}\end{array}$ & $\begin{array}{l}-2.43 \\
(-6.96,1.20)\end{array}$ & $\begin{array}{l}-2.29 \\
(-5.47,0.89)\end{array}$ & $\begin{array}{l}-1.10 \\
(-2.09,-0.12)\end{array}$ \\
\hline$>\Rightarrow 50$ yediss & $\begin{array}{l}79 \\
2269\end{array}$ & $\begin{array}{l}11.02 \\
(0.85,21.19)^{*}\end{array}$ & $\begin{array}{l}-1.62 \\
(-5.54,2.29)\end{array}$ & $\begin{array}{l}-2.50 \\
1-6.70, \times .09\end{array}$ & $\begin{array}{l}-1.18 \\
(-2.31 \\
-0.041\end{array}$ \\
\hline \multicolumn{6}{|l|}{$C R P$} \\
\hline All peopll: & $\begin{array}{l}240 \\
1881\end{array}$ & $\begin{array}{l}0.39 \\
(-0.39,1.08)\end{array}$ & $\begin{array}{l}-0.14 \\
(-0.39,0.12)\end{array}$ & $\begin{array}{l}0.18 \\
(-0.40,0.04)\end{array}$ & $\begin{array}{l}-0.09 \\
(-0.15,-0.04)\end{array}$ \\
\hline$y=50$ yoxil's & $\begin{array}{r}217 \\
176)\end{array}$ & $\begin{array}{l}0.33 \\
(-0.35,1.00)\end{array}$ & $\begin{array}{l}-0.10 \\
(-0.35,0.15)\end{array}$ & $\begin{array}{l}-0.27 \\
(-0.45,-0.08)\end{array}$ & $\begin{array}{l}0.00 \\
0.15,-0.00 \%\end{array}$ \\
\hline
\end{tabular}

* $P<0.10 x * p<0.05$.

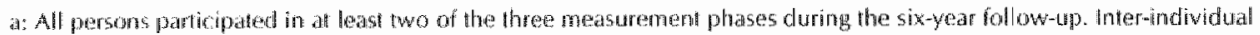
random wation in the intercepts of the assochation between longitudinat the and cogrative function wass signaficant in all analyses. Inter-individual andom variation in the slopes of this association was, however, never statisticalliy significant. This indicates that persons differed in cognitiwe function at basetine first phase), but there were no singnificamb differences baveen pessons in the course of cognitive function tluring follow-up. Interactions between longhtudinat time and the sefum infirmation marthers, i, modeling a diferential longitudinal course of cognive function related to the biomathers, were therefore not investigated further.

b: additionally adjusted for heptoglobin planotype

A serum sample at both baseline and follow-up was available from 66 individuals. When haptoglobin concentration at follow-up was correlated to cognitive pertormance for these subjects at this timepoint, adjusted for age, gender and education, the positive relation failed to reach significance. More in detail, the unadjusted correlation between haptoglobin concentration and cognitive performance was also not significant at follow-up. In fact, when the small number of 66 persons alone were examined at basetine, the relations were similarly not significant datal not shown). When the difference in haptoglobin concentration between baseline and follow-up was correlated to the difference in cognitive performance outcome between baseline and follow-up for the four tests, similarly no correlation was observed (data not shown).

The relation between CRP and cognitive performance was restricted to the word learning tasks. A negative correlation between CRP concentration at baseline and performance on the Delayed Recall lest was observed for the total population $\mathrm{b}=-0.09, \mathrm{P}<0.05$ ) as well as for the subset of $50+$ individuals $(b=-0.08, P<0.05)$. A significant negative conrelation between the CRP concentration at baseline and performance on the WLTTOT during 6 years of follow-up was observed in the subset of $50+$ individuals $(b=-0.27, P<0.05)$. A similar negative correlation was observed for the group as a whole, but this was not significant $(b=-0.18, P>0.1)$.

The influence of the outliers and influential cases on the results was examined. In this way, three indivicuals were excluded from the Stroop test, LDCT and WLTTOT and two individuals from the Delayed Recall test. The relation between haploglobin concentration and the performance on the Stroop test and the Delayed Recall test remained the same in both ordinary least squares multiple regression analysis and the multi-level regression analysis. This was observed for the haptoglobin concentration as such as well as after correction for haptoglobin phenotype. The relation between CRP and the cognitive test as observed by regression analysis 
and multi-level repeated measurement analysis, disappeared after exclusion of the outliers and influential cases.

There were no significant differences in cognitive function between persons over the follow-up period. Therefore, interactions beween longitudinal time and the serum inflammation markers, i.e. modeling a differential longitudinal course of cognitive function related to the biomarkers, were nol investigated further.

\section{Discussion}

The aim of the present study was to investigate whether cognitive performance in the healthy ageing population could be reflected by serum inflammation marker concentrations. The strongest result of the present study was the finding of a correlation of the serum inflammation marker haptoglobin with cognitive performance on the Stroop test and the Delayed Recall test, at baseline as well as over the whole follow-up period of 6 years. In addition, CRP concentration correlated with performance on the Delayed Recall and WLTTOT at baseline as well als over the 6 years follow-up period, at least in the subgroup of older individuals. These effects were independent of age, gender or level of education of the individuals.

There are several indications that the observed correlation might be more specific than just a reflection of a general state of enhanced inflammation as no relation between the concentration of abumin, total protein or $\alpha 1, \alpha 2$ and $\gamma$ protein fractions and cognitive performance was observed. In addition, no detectable increase in IL-6 concentration, a general marker for inflammation, was observed in the majority of serum of individuals in our study. In addition. our findings at least do not show a correlation of the inflammation markers with general cognitive performance but rather a correlation with certain aspects of memory, since no relation with the LDCT test for processing speed was observed.

Haptoglobin may function as an acute phase protein and as an antioxidant. This latter function suggests that high haptoglobin levels may exent a protective effect against oxidative stress, one of the putative mechanisms inwolved in ageing and neurodegeneration $\llbracket 4 \rrbracket$. Haptoglobin plays are role in clearing haemoglobin and its toxin iron group from the circulation by forming a complex with haemoglobin, which interacts with the CD163 receptor for uptake of this complex into macrophages. Haptoglobin and the CD163 receptor ane upregulated by acute phase reactants such as IL-6 [27].

The studies on CRP concentration during ageing and in demented patients are relatively scarce and contradictory [8, 14,29]. CRP is a so-called "first class" acute phase reactant because it is one of the most sensitive plasma proteins indicating inflammatory activity [46]. Haptoglobin has a longer half-life of 5.4 days [36] compared to 6-8 hours for CRP [9] in humans. For CRP, triplicate sampling is recommended to assess an individual serum level due to a relatively high contribution of intra-individual variation to the total variance [12]. Haptoglobin levels give a better reflection of base-line inflammation level than CRP and therefore haptoglobin mal be more valuable.

The correlation between serum CRP and WLTTOT reached significance in the older individuals only, while the corretation between $11-6$ receptor and the performance on the Stroop test was statistically significant only in the whole group. These interesting results are probably due to differences in cognitive performance between the young and old $[20,26]$, as the number of individuals with decreased cognitive functioning decreases more rapidly with age above 60 . The number of individuals of whom a serum sample was avalable was relatively low in our study. The relation between haptoglobin concentration at follow-up and cognitive performance was not significant in cross-sectional analysis at follow-up. This may be explained by several factors including loss of power due to the lower number of individuals of whom a second 
serum sample was available $(n=66)$, variability due to the differences in age or cognitive test oulcomes as described in the methods, or other unknown factors. At least, the results warrant catious interpretation and further research.

Although the results of this study showed a relation between cognition and the makers for inflammation haptoglobin and CRP, the data do not allow drawing conclusions concerning causality or risk estimation. This is probably caused by a lack of changes in cognitive performance over the past six years of follow-up for the individuals in this part of the MAASstudy. Nevertheless, it would be interesting to know if low perfomance on the Delayed Recall, WLTTOT or Stroop test in our study, i.e. the individuals with higher levels of haptoglobin or CRP, is indicative of the development of more severe cognitive deterioration or eventually dementia. As stated in the introduction, it is probable that dementia emerges from gradual cognitive deterioration, although the characteristics of the transition are not clarified [44]. Not all individuals suffering form mild cognitive impaiment do develop dementia [38]. The two outliersfinfluential cases on the Delayed Recall test had extreme bad performance on this test (both had a recall of only two words). Thus, although the results on CRP and cognition may have had less significance for the group as a whole, these wo individuals are highly interesting as they have a very bad cognitive performance and might be at increased risk of deteriorating further in cognitive performance al follow-up. The MAAS-study is still on-going and further follow-up of the individuals will shed more light on the role of inflammation during cognitive decline.

In conclusion, even though the low number, short follow-up time and the lack of correlation on the processing speed task warrant cautious interpretation, our results show a correlation between cognitive performance and some peripheral markers of inflammation in the healthy ageing population. Further follow-up of this population will indicate whether individuals with relatively lower cognitive performance and higher levels of haptoglobin develop dementia in time.

\section{References}

Albandar I.M. and Goldstein, H., Mulli-level statistical models in studies of periodontal diseases, / Periodonto/, 63 (1992) 690.5.

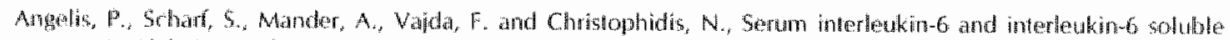

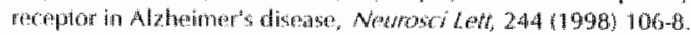

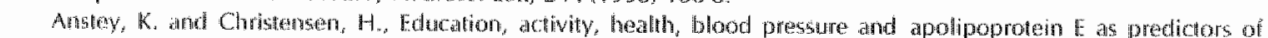

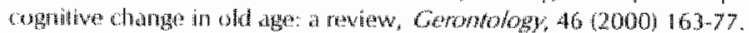

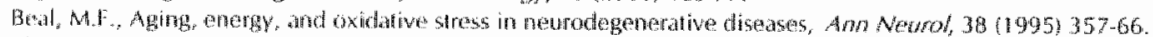

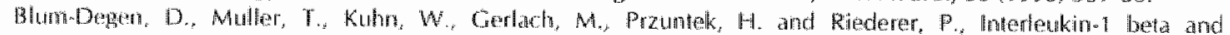

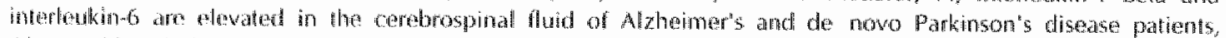

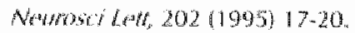

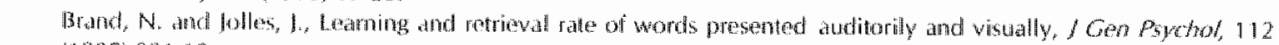
(1905) 201 110 .

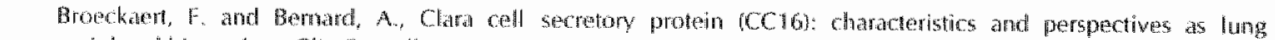

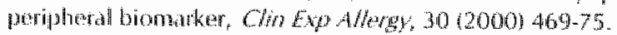

Brtunsgand, H. Andersen Ranberg. K. Jeune, B., Pedesen. A.N., Skinhoi. P. and Pedersen, B.K., A high plasma

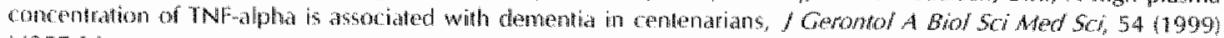
M357.64.

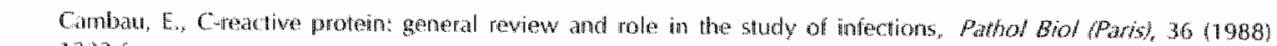
$1232-6$.

Chenilou, O., Henny, J., Steinmet, J., Hertueth, B., Wagnes, C. and Siest, C., High sensitivity Creactive protein:

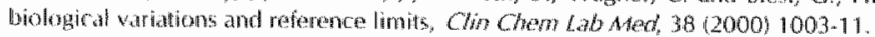

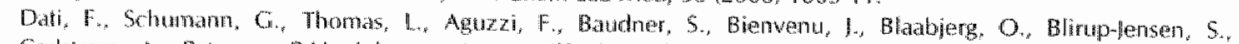

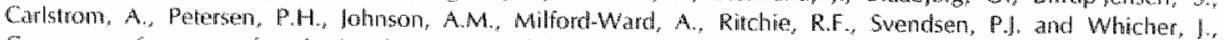
Consensus of a group of professional societies and diagnostic companies on guidelines for interim reference ranges for 1 is proteins in serum based on the standardization against the HFCOBCR/CAP Reference Material ICRM 470$)$. Intemational Federation of Chinical Chemistry. Community Bureat of Reference of the Commission of the European 


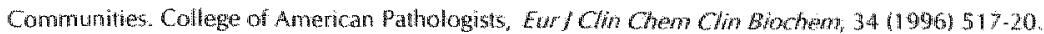

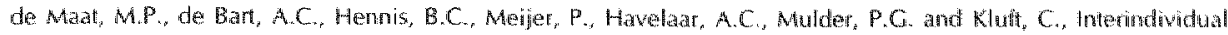
and intrandividua variability in plasma fibrinogen, TPA antigen, PA activity, and CEP in healthy, young voluntears and patients with angina pectoris, Afterioscler Hromb Mac Brol, 16,1996$) 1156-62$.

Elwood, P.C., Gallacher, I.E., Hopkinson, C.A., Pickering, 1., Rabbitt, P., Stollery, B., Brayne, C., Huppert, F.A. and

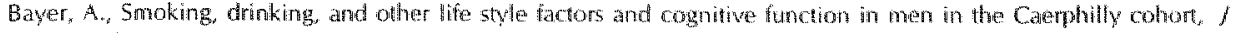
Epideniol Communimy Health, 53 (1999) 9-14t.

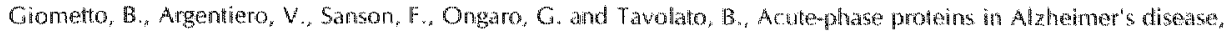
Eur Neurol, 28 (1988) 30-3,

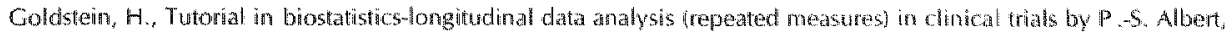
statistics in medirine, 1999, 18, 1707-1732, 5tat Med, 1900001821.

Gonzalez-Scarano, F. and Batuch, G., Microglia as mediatons of unlammatory and degenenatve diseases "Anm Rev" Nermosci, 22 (1999) 21940.

Grifin, W.S. Sheng, I.Ci., Roberts, G.W. and Mrak, R.E., Interteukin-1 expression in different plaque types in

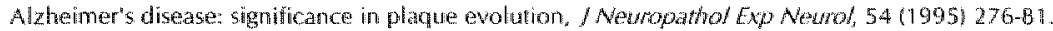

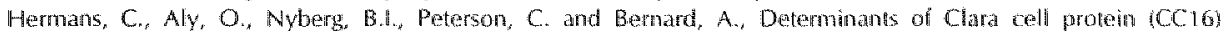
concentration in sefum: a reassessment with two different immumalssys, Chin Chim Acta, 272 (1998) 101-10.

Houx, P.J, Jolles, \}, and Vreeling, F.W., Stoop interferences: aging efferis assessed with the Stroop Color-Word Test, Exo Aging Res, 19 (1993) $209-24$

Houx, P.J., Vreeling, FW. and Jolles, J., Rigorous health screxning reduces age effect on memory scannimg task, Brain Cogn: 15 1991) $246-60$.

Howieson, D.B., Dame, A., Camicioli, R., Sexton, G., Payani, H. and Kaye, I.A., Cognitive markers preceding

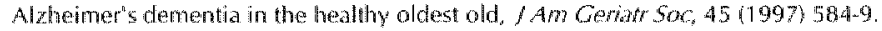

Wwamoto, N. Nishyyma, E., Ohwada, 1. and Arai, H. Demonstration of CRP immunoreactivizy in brains of Alzheimer's disease: immunohistochemical study using formic acid pretreatment of tissue sections, Newrosci Letr, $177(1994) 23.6$.

Jolles, J., Houx, P.J. van Boxtel, M.P.J. and Ponds, R.W.H.M., The Matasirich Aging Sindy Detemninams of cognitive aging., Neuropsych Publishers, Mastistricht, 1995, 192 pp.

Kalman, 1., Juhasz, A, Laird, G., Dickens, P, lardanhazy, T. Rimanoczy, A., Boncz, I., Parryones, W.L. and Janka, $Z$. Serum interleukin-6 levels correllate with the severity of dementia in Down syndrone and in Aluheimer's disease, Acta Nosurol Scand, 9601997$) 236$ uto.

Kawas, C., Gray, S., Brookmeyer, R., Fozard, I, and Zonderman, A., Age-specific incidence rales of Alzheimer"s disease: the Baltimore Longitudimal 5tudy of Aging, Neurology, 54 (2000) 2072-7.

Kaye, I.A., Oken, B.S., Howieson, D.B., Howieson, 1., Holm, L.A. and Dennison, K, Neurologic evaluation of lhe optimally beathy oldest old, Arch Neurol, 51 (11994) $1205-11$.

Kristiansen, M., Graversen, J.H., Jacobsen, C., O., S, Hof Han, H.m, Law, S.K.A. and Moesirup, S., Identification of the hatemoglobin scawenger receptor, Nature, 409 (2001) 198-201.

Langlois, M.R. and Delanghe, J.R., Biological and dimical significance of haptoglobin polymorphism an humans. Cin Chem, 42 (1996) $1589-600$.

Licastro, F., Masliah, E., Pedrini, 5 and That, L., Blood levels of alpha-1-antichymotrpsin and risk factors foo

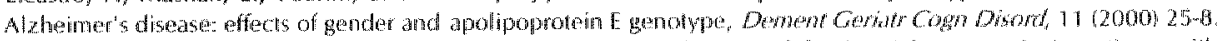

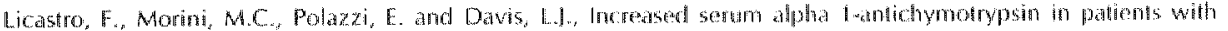

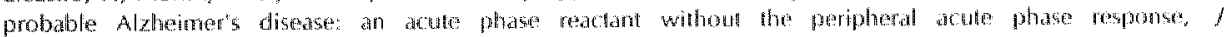
Newroimmunol, 57 (1995) 7 H.5.

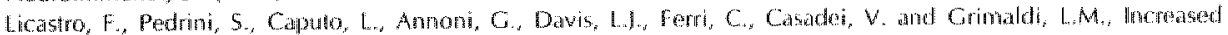

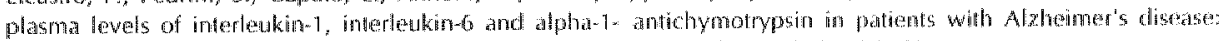

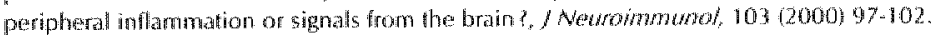

Matsulbara, E, Amari, M., Shoil, M. Harigaya, Y., Yamagatuchi, H. Okalmoto, K. and Hirai, S., Serum concentralon of alpha 1-antichmolrypsin is alevaled in patients with senife dementia of the Alzheimer type, Prog Chin Biof Ress, $317\{1989) 707-14$.

MaGer, P.L. Hagaki, S., Bowes, BE. and MCGeer, EG., Reactive microglia are positive for HLA-DR in the

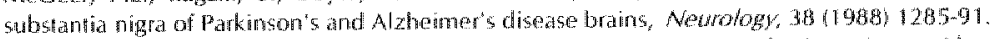

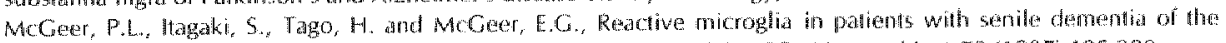

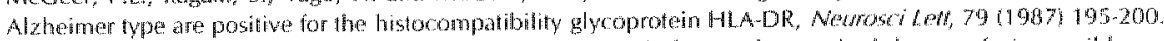

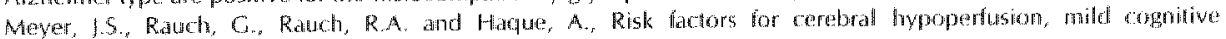
impaiment, and dementia, Nevrobiol Aghg. 21 2000) 161-9.

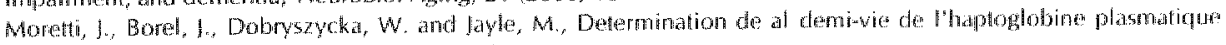
humaine. Biochin Bophys Arta, 69 (1963) 205-2॥1.

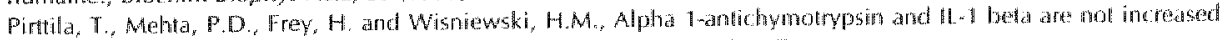
in CSF or serum in Alzheimer's disease, Newobiol Aging 15 (1994) $313 \%$

Shah, 5. Tangalos, E.G. and Petersen, R.C., Mild coggative impairment. When is it a precursor to Aldweimer's disease?, Geriatros, 55,2000$) 62,65-8$.

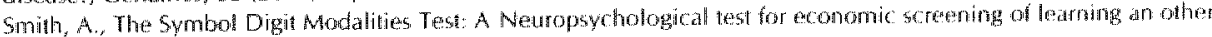
cerebral disonders. Leaming Disonders, 36 (1968) 3491 . 


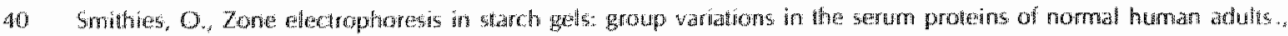
Brochen H, 61019553629641 .

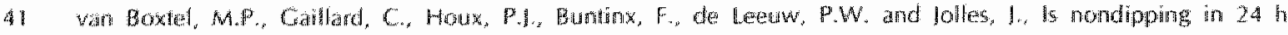

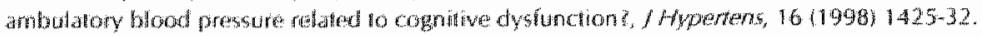

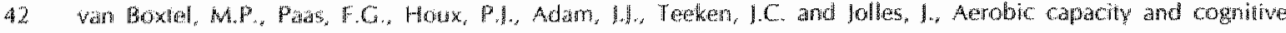

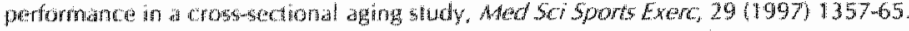

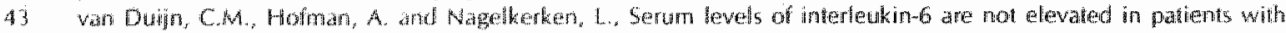

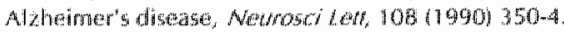

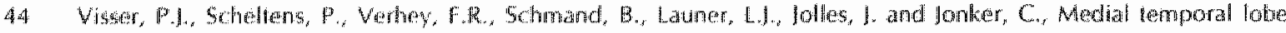

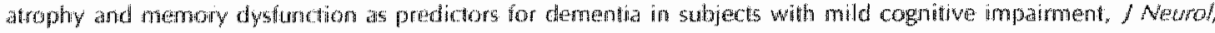
$246(1999) 477+85$.

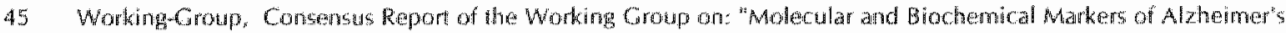

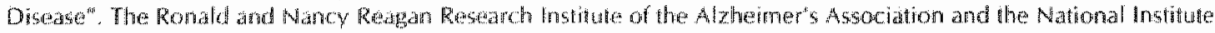

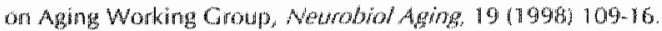

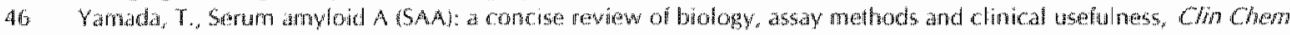
Lab Med, 3719999381.8. 


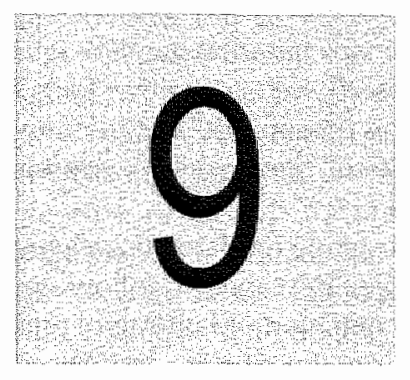

\section{Serum cholesterol precursors and metabolites in relation to cognitive} performance in an ageing population. A longitudinal follow-up study

Charlotte Teunissen, Jan de Vente, "Klaus von Bergmann, Hans Bosma, Martin van Boxtel, Chris De Bruijn, Jelle Jolles, Harry Steinbusch, and "Dieter Lütjohann

European Graduate School of Neuroscience (Euron), Universiteit Maastricht, Department of Psychiatry and Neuropsychology, The Netherlands a Department of Clinical Pharmacology, University of Bonn, Germany

Submitted to Neurobiology of Ageing 


\section{Abstract}

The am of this study was to determine if a causal relation exists between serum concentrations of precursors and metabolites of cholesterol and cognitive performance in a healthy ageing population.

Cognitive function of a cohort of 144 individuals (aged $30-80$ years) was tested at baseline and after six years of follow-up. Domains of cognitive function addressed were cognitive speed (Letter-Digit Coding test, LDCT), attention and information processing (Stroop test) and memory (Word Learning test). Serum concentrations of cholesterol, cholesterol precursors, oxysterols and plant sterols were measured.

The results showed that serum concentrations of lathosterol and lanosterol correlated negatively with cognitive performance on both Word Learning tests at baseline and at follow-up. indlependently of age, sex and education level. In addition, the concentration of lathosterol and lanosterol at baseline of correlated with cognivive performance on the Stroop and Word Learning tests during the whole follow-up period. The concentration of 27 -hydroxycholesterol and 245-hydroxycholesterol showed no consistent correlations with cognitive performance, while cholesterol, desmosterol, sitosterol and campesterol were not related to cognitive performance in this nomal ageing population.

It was concluded that relative high serum ratios of cholesterol precursors indicative for a high rate of endogenous cholesterol synthesis are associated with relatively low memory performance in this normal ageing population. Further follow-up studies must indicate if increased serum concentrations of cholesterol precursors present an increased risk for dementia

\section{Introduction}

Cholesterol is an important lipid constituent of all cellular membranes and myelin. The brain contains about one quarter of the total unesterified cholesterol in human subjects, while this organ constitutes only two percent of the total body mass. Brain cholesterol is mainly synthesised locally and only negligible amounts $(<1 \%)$ of circulatory cholesterol takes part in brain cholesterol metabolism as its transfer is restricted by the blood-brain barrier (BBB) [16]. Within recent years the interest in the description of the chollesterol metabolism and homeostasis in the human brain has increased [19].

Research on serum cholesterol levels in pathological cognitive ageing, such as Alzheimer's disease (AD), did not yield consistent results. In the studies of Merched and Siest, decreased serum high density lipoprotein ( $\mathrm{HDL}$ )-cholesterol concentration in patients with $A D$ were observed compared to controls, whereas Lesser and Bonarek observed increased total and LDLcholesterol in AD patients $[6,20,25,31]$. Very recently Kivipelto ef al. [18] reported that people with high serum cholesterol concentrations had a significant higher risk for development of mild cognitive impaiment in late life. There are several other indications for an involvement of altered cholesterol homeostasis in cognitive deterioration, e.g. Alzheimer's or vascular dementia. 1) Carriers of at least one of the apolipoprotein $E$ (ApoE) 4 allele are at increased risk for the incidence of AD at an early age $[23,36]$. ApoE is involved in intracellular cholesterol transport in the human body and probably also in the brain [9]. The presence of at least one ApoE4 allele is associated with higher circulatory cholesterol concentrations compared to E2 or E3 alleles. Thus, a hypothetical relationship between ApoE-dependent cholesterol homeostasis 
and Alzheimer's disease seems plausible. 2) Several studies showed an assoctation of the ApoE4 allele with lower cognitive performance in the normal ageing population $[2,3,3,35]$. 3) Lower plasma concentration of the oxidised cholesterol 245 fydroxycholesterol $245-\mathrm{OH}-\mathrm{Cho}$ ) has been found in the late stage of Alzheimer's disease patients and patients with severe head trauma [8,28]. Plasma 245-OH-Chol in humans originates exclusively from the brain [5,24]. It has been shown that trydroxylation by a specific 245-hydroxylase may be an impontant mechanism for cholesterol removal out of the brain $[4]$, in addition to ApoE-medialted mechanisms [9]. 4) Recent epidemiological retrospective studies indicate that treatment of individuals with drugs that lower cholesterol synthesis in the whole body, mainly in the liver, $(\beta$-hydroxy- $\beta$-methyl-glutaryl-CoA reductase inhibitors or statins) was associated with decreased prevalence of Alzheimer's disease [42]. Similarly, individuals of 50 years and older who were prescribed statins had a substantially lower risk of developing dementia, independent of the presence or absence of untreated hyperlipedemia [14]. Finally, there is increasing evidence indicating an important link between cholesterol, $\beta$-amyloid $(A \beta)$ and $A D[10,17,19,29,32]$.

These observations lead to the hypothesis that high concentrations of cholesterol are associated with cognitive deterioration. The am of the present study was to investigate if a possible causal relationship exists between serum markers for whole body cholesterol metabolism and cognitive performance in a healthy ageing population. Cholesterol homeostasis is a balance between absorption, de novo synthesis, metabolism, and excretion [13]. Therefore, we investigated the serum levels of cholesterol, the cholesterol precursors lathosterol, lanosterol and desmosterol, the oxidised cholesterol metabolites 245-OH-Chol and 27-OH-Chol (Fig. 1), and the plant sterols campesterol and sitosterol in a healthy ageing population that was pat of a population based study. Cognitive performance was investigated addressing domatins as information processing speed and memory. The individual serum levels were related 10 each individual cognitive performance at baseline, after six years of follow-up, and over the whole six years follow-up period.

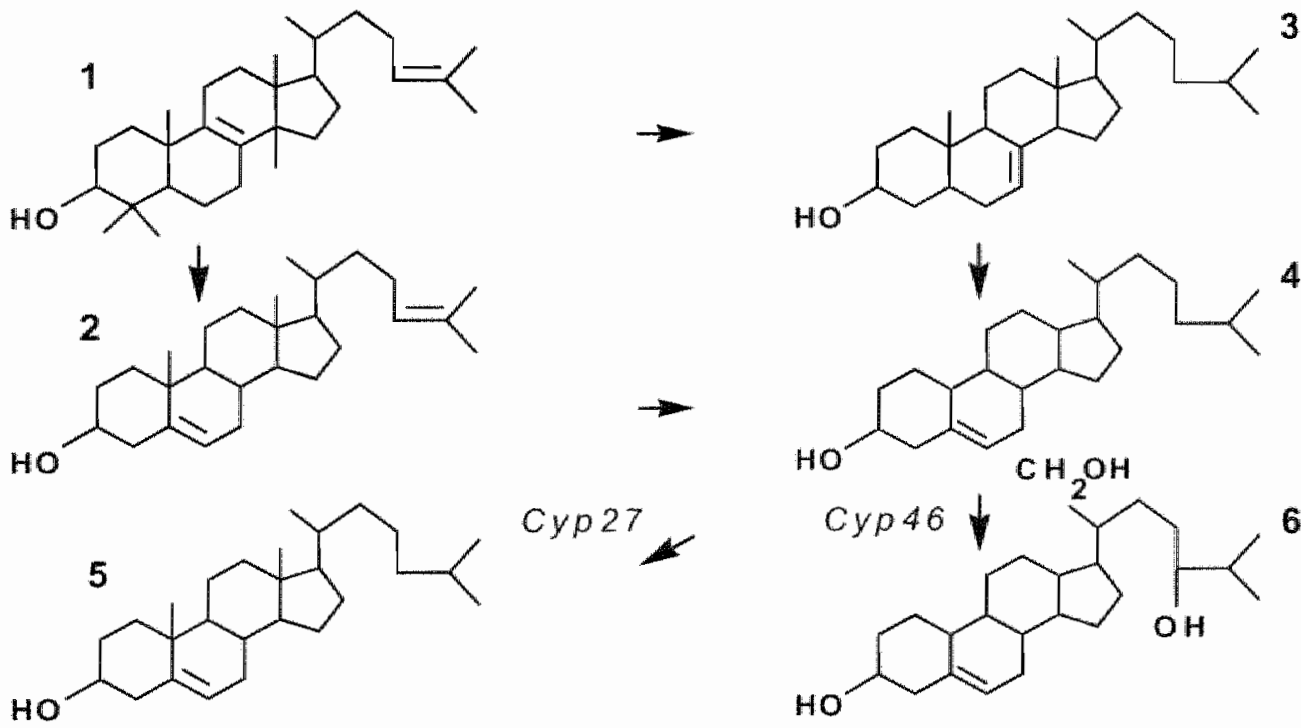

Fig. II Strucures and interelationship benwera the dolestenol precursors and mabolites.

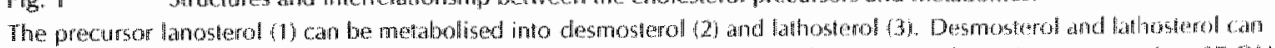

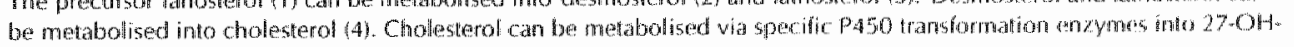
Chalesterol (5) and 245 - bydroxychohesterol $(6)$. 


\section{Methods}

\section{Individuals}

Participants in this study were recruted from a larger research program investigating determinants of cognitive ageing in the bealthy population: The MAastricht Ageing study (MAAS) $[15,38]$. Individuals for this study were randomly drawn from the Registration Network Family Practices, a research database that contains basic health information of patients in primary care facilities (Metsemakers, Hoppener, Knottnerus, Kocken \& Limonard, 1992). Exclusion criteria were clinical evidence of past or present morbidity that can compromise cognitive performance including cerebrovascular disease, chronic neurological pathology, mental retardation or chronic psychotropic drug use. The sample was stratified for age (12 discontinuous groups; $30 \pm 1$ years, $35 \pm 1$ years, .., $80 \pm 1$ years), sex and two levels of occupational achievement [15]. In total, 1,823 men and woman were medically and neuropsychologically examined in the baseline examination beween 1993 and 1995 . The group was divided into four panels of approximately 450 individuals.

Indiwiduals aged $>50$ years at the beginning of the study (baseline) were re-examined every third year over a period of six years. Individuals aged between 30 and 50 years at baseline were re-examined after six years. A serum sample was drawn at the beginning and the end of the study and stored at $-80^{\circ} \mathrm{C}$ until analysis. At the end of 1999 , data of three cognitive performance measurements over six years of follow-up were available from a random group of 144. individuals from the panel that was examined in 1993 for the first time. A complete set of serum samples and cognitive data was available from 92 individuals at baseline and 116 individuals after six years of follow-up, while of a subgroup of 65 individuals these data were available at both time points. An incomplete data set was available from the other individuals due to logistic problems.

The mean age of the 92 individuals at baseline was 57 (SD 11). The sex distribution was 55 man 10.37 women. The age of the 65 individuals of whom a complete set of serum data was present was lower (mean 54 years, 5D 10) compared to the remaining 27 individuals (mean 65 , SD 11, Mann-Whitney $U$ test: $Z=-4.2, P<0.001$ ). The sex distribution was not statistically different $(37$ man to 28 women in the 65 individuals, 18 man to 9 woman in the remaining 27 individuals)(Chi-square, $d f=1: 0.75, P<0.38$. The performance on the cognitive tests was not statistically different between the subgroups $(P>0.05)$.

Eight individuals used cholesterol-lowering drugs and the analyses were also redone without these individuals (see results).

The study was in accordance with the principles of the declaration of Helsinki and the local medical ethics committee approved the study protocol. Written informed consent was obtained from all participants.

\section{Cognirive assessment}

Cognitive function was rested according to the MAAS-protocol described in detail elsewhere [15,38]. We focussed on processes involwing memory, attention and different aspects of information processing speed.

\section{WORD LEARNING TASK (WLT).}

This test is based upon the Auditory Verbal Learning Test [7] and evaluates the ability to acquire and retain new wenbal information. Fifteen frequently used monosyllabic words are presented and the subject is instructed to memorise the words. The trial ends with a free recall of the words. This procedure is repeated live times, using the same word set in fixed order. The total 
number of correctly reproduced words on the five inmediate recall trials is recorded (WLTTOT). After 20 minutes the subject is asked 10 reproduce the set of words (Delayed Recall. The total number of correctly reproduced words after 20 minutes is recorded [15]. A higher score on this test reflects better perfomance.

\section{LETTER DIGIT CODING TASK (LDCT)}

This paperand-pencil test is a madified version of the Symbol Digit Modalities Test [34] and measures information processing speed. The subject is requested to copy mumbers in calls that are indexed by a letter. The letter refers to nine letter/number combinations at the top of the form. The total number of correcty copied corresponding numbers in 60 seconds is recorded as test outcome. Thus, better performance is associated with a higher score on this lest.

\section{STROOP COLOUR-WORD TEST (STROOP)}

This perceptual interference test consists of three subtasks. Each subtask consists of a test sheet containing four rows and ten columns of colour names or coloured spots. The test examines the speed at which colour names are read (subtask 1 ) and the speed at which the colour of spots is named (subtask \|l). Subtask III involves colour names but the printing ink is different from the color name. The time needed to name the colour of the printing ink of the words is recorded. Thus a better cognitive performance is associated with a lower score on the Stroop test. Only the data of subtask III are used in the current study and are referred to as 'Stroop'.

\section{Sierol analysis by gas chromatography flame-ionisation and mass selective detection.}

Sterols and oxysterols were extracted from serum by cyclohexane atter saponification. Fifty $\mu g$ 5 a-cholestane (Sigma), $1 \mu \mathrm{g}$ epicoprostanol (Sigma) and $200 \mathrm{ng}$ racemic $[23,23,24,25 \% \mathrm{H} / 24 \mathrm{~S}-$ $\mathrm{OH}-\mathrm{Chol}$ and $\left[{ }^{2} \mathrm{H}\right.$.] 25R) $27 \% \mathrm{OH}-\mathrm{Chol}$ were added as internal standards. The solvents were evaporated and the hydroxy groups of the sterols and oxysterols were trimethylsilylated. The trimethylsilyt- (TMSi-) ether of cholesterol was separated on an HPI (Methyl Siloxane, crosslinked) fused silica capillary column $(10 \mathrm{~m} \times 0.1 \mathrm{~mm}$ i.d., $0.4 \mathrm{~mm}$ pllase thickness, Hewlett Packard (HP), Böblingen, Germany). Gas-chromatography flame ionisation was performed with a HP6890 gas-chromatograph. The oven temperature was initially kept at $150^{\circ} \mathrm{C}$ for $1.5 \mathrm{~min}$., then incresed at $59^{\circ} \mathrm{Cm}$ m to a final temperature of $290^{\circ} \mathrm{C}$ for $8 \mathrm{~min}$. An alicuot of $1.0 \mathrm{\mu L}$ was injected by an atomated injector (HP) in a pulsed splitess mode a $280^{\circ} \mathrm{C}$. Hydrogen was used as carrier gas with an initial flow of $1.0 \mathrm{~mL} / \mathrm{min}$ and flame ionisation detection was performed at $280^{\circ} \mathrm{C}$ with a constant column and makeup flow mode. The concentration of cholesterol was calculated from the ratio of the peak area of cholesterol to the area of $5 \alpha$ cholestane multiplied by the amount of internal standard $(50 \mu \mathrm{g})$ added to a defined serum volume. Gas chromatography-mass spectrometry-selected ion-monitoring (GC-MS-SIM) analysis for quantification of epicoprostanol, lathosterol, desmosterol, lanosterol, campesterol, sitosterol, $24 \mathrm{~S}-\mathrm{OH}-\mathrm{Chol}$, and $27-\mathrm{OH}-\mathrm{Chol}$ and the deuterated internal oxysterol standards was performed on a DB-XIB column (30 m $\times 0.25 \mathrm{~mm}$ i.d. $\times 0.25$ um film thickness, \& W Scientific (Altech)) using an HP5890 Series II plus gas-chromatograph conbined with an HP5972 mass selective detector. An aliquot of $1.0 \mu \mathrm{L}$ was injected by automated injection in a splitless mode al an injection temperature of $280^{\circ} \mathrm{C}$. Helitum was used as carrier gas with a column gas.flow of 1.0 $\mathrm{mL} / \mathrm{min}$. The initial oven temperature was kept al $150^{\circ} \mathrm{C}$ for $1 \mathrm{~min}$, thereafter increased at a rate of $30^{\circ} \mathrm{C} / \mathrm{min}$ to $290^{\circ} \mathrm{C}$ and kept for $30.33 \mathrm{~min}$. TMSi-ther of epicoprostanol was measured all $m / z 370(M+$-OTMSi), lathosterol at $m / z 458(M+)$, desmosterol at $m / z 456(M+1)$, lanosterol $\mathrm{m} / 2393\left(\mathrm{M}^{*}\right.$. $\mathrm{CH}_{3}$-OTMSi), campesterol at $\mathrm{m} / \mathrm{z} 472\left(\mathrm{M}^{*}\right)$, sitosterol at $\mathrm{m} / 2488\left(\mathrm{M}^{*}\right)$, authentic and deuterated 245-OH-Chol were measured at $\mathrm{m} / \mathrm{z} 413(\mathrm{M}+\mathrm{OTMSi}-\mathrm{CH}(\mathrm{CH}-3) 2)$ and $\mathrm{m} / \mathrm{z} 416$ 
$(\mathrm{M}+-\mathrm{OTMSi}-\mathrm{CH}(\mathrm{CD} 3) 2)$, respectively, and authentic and deuterated $27-\mathrm{OH}-\mathrm{Chol}$ at $\mathrm{m} / 2 \mathrm{z} 56$ $(M+-90)$ and $461(M+-90)$, respectively, after ellectron impact ionisation at $70 e V$. The temperature of the transfer line was kept at $280^{\circ} \mathrm{C}$.

\section{Sitatistical analysis}

Zero-order bivariate correlation analysis was performed using Spearman's correlation coefficient. Normality of the distribution was tested using the Kolmogorov-Smirnov test. Our further analysis was performed in two phases. First, ordinary least squares multiple regression analysis was performed for the four cognitive tests at baseline and each separate serum sterol as potential predictor, with age, sex and level of education as covariates. All variables were treated as continuous variables, except sex (categorical). Second, for the markers showing a significant relation $\left(P<0.05\right.$ ) with at least two of the cognitive tests $s_{x}$ adjusted for age, sex and educational level, an additional multi-level regression analysis was performed. Multi-level repeated measurement analysis was carried out to analyse the association between the predictors and cognitive function during the whole follow-up period, to investigate an eventual risk modulating role of the serum sterol concentrations. This method uses all observations, including the observations of persons with only one observation $[1,1,2]$. To increase homogeneity regarding age across the different phases, the analyses were re-done for persons older than 50 years of age only. As this study evaluates results from four cognitive tests, a Bonferroni adjustment of the significance to $P<0.013$ was considered. However, as this study was primary exploratory in nature, data with a significance level of $P<0.05$ are also shown.

A.ll analyses were performed with SPSS statistical software version 9.0.1. (Chigaco, Illinois U.S.A.).

Tatble 1A. Median (25\% and $75 \%$ percentile, for sterols and sterol to cholesterol concentrations in serum at baselline and at follow-up, all individuats (mean age 57.4 t. 11.6 (29.9-81.3).

\begin{tabular}{|c|c|c|c|c|c|}
\hline & \multicolumn{2}{|c|}{ Absofute values } & \multicolumn{3}{|c|}{ Sterol to chotesterol ratio } \\
\hline & $\begin{array}{l}\text { baseline } \\
(1993) \\
(n-92)\end{array}$ & $\begin{array}{l}\text { follow- } \begin{array}{l}(1999) \\
(n=116)\end{array} \\
\end{array}$ & unit & $\begin{array}{l}\text { baseline } \\
(1993) \\
(m-92)\end{array}$ & $\begin{array}{l}\text { follow-upa } \\
(1999) \\
(07=116)\end{array}$ \\
\hline cholesterol (mg/di) & $\begin{array}{l}155 \\
(122,185)\end{array}$ & $\begin{array}{l}213 \\
(172,237)\end{array}$ & & - & 。 \\
\hline hathosilerol imydy & $\begin{array}{l}0.18 \\
10.14,0.25\rceil\end{array}$ & $\begin{array}{l}0.22 \\
(0.16,0.31)\end{array}$ & $(\mu \mathrm{g} / \mathrm{lng})$ & $\begin{array}{l}1.13 \\
10.87,163)\end{array}$ & $\begin{array}{l}1.07 \\
(0.80,1.49)\end{array}$ \\
\hline desmostarol $(\mathrm{gg} / \mathrm{d}$ ll & $\begin{array}{l}101 \\
(71,128)\end{array}$ & $\begin{array}{l}111 \\
(92,140)\end{array}$ & (ugg/mgl) & $\begin{array}{l}0.61 \\
(0.48,0.78)\end{array}$ & $\begin{array}{l}0.56 \\
(0.47,0.70)\end{array}$ \\
\hline 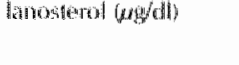 & $\begin{array}{l}11.9 \\
(100,14.4)\end{array}$ & $\begin{array}{l}13.0 \\
(10.6,16.6)\end{array}$ & (ng/ma) & $\begin{array}{l}72.8 \\
(63.1,87.5)\end{array}$ & $\begin{array}{l}66.0 \\
(51.9,78.3)\end{array}$ \\
\hline 245.OH-Chol (noghat & $\begin{array}{l}59.0 \\
40,0,7 \%, 09\end{array}$ & $\begin{array}{l}69.5 \\
652.3,88.08\end{array}$ & (ng/mg) & $\begin{array}{l}38,3 \\
(33,3,46.97\end{array}$ & $\begin{array}{l}34.1 \\
(25.9,40.7)\end{array}$ \\
\hline 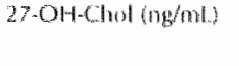 & $\begin{array}{l}161 \\
(1033,203)\end{array}$ & $\begin{array}{l}201 \\
(162,262 \%\end{array}$ & lnging & $\begin{array}{l}110 \\
(81,136)\end{array}$ & $\begin{array}{l}95 \\
(80,125)\end{array}$ \\
\hline Cothesesterol dingenth & $\begin{array}{l}0.32 \\
40.22,0.469\end{array}$ & $\begin{array}{l}0.37 \\
0.26,0.50)\end{array}$ & (wgrmg & $\begin{array}{l}2.04 \\
(1.40,3.19\end{array}$ & $\begin{array}{l}1.79 \\
(1.27,2.66)\end{array}$ \\
\hline sitosterol (ma/dlly & $\begin{array}{l}0.27 \\
10.21,0.401\end{array}$ & $\begin{array}{l}0.311 \\
(0,23,0.42)\end{array}$ & (woghg) & $\begin{array}{l}1.73 \\
1.26,2.70)\end{array}$ & $\begin{array}{l}1.53 \\
(1.11,2.09)\end{array}$ \\
\hline
\end{tabular}




\section{Results}

The absolute sterol concentrations and the sterol to cholesterol ratios ( $R$ _ Sterol) in our ageing population at baseline and after six years of follow-up are summarised in rable $1 \mathrm{~A}$ and $\mathrm{B}$. The values of the cholesterol levels were comparable to levels in the nomal population [37]. The $245-\mathrm{OH} \cdot \mathrm{Ch}$ ol concentration (range $68 \mathrm{ng} / \mathrm{mL}-74 \mathrm{ng} / \mathrm{mL}$ ) in all individuats was lower than reported previously by Bretillon et al. $(77-105 \mathrm{ng} / \mathrm{mL})[8]$. The $27-\mathrm{OH}$-Chol concentration was similar to the concentration observed in previous data obtained in the laboratory in Bonn (mean $146 \pm 43 \mathrm{ng} / \mathrm{ml}$ (range $73-358$ ), for a group of 50 men and 50 females aged $30-79$ yr.).

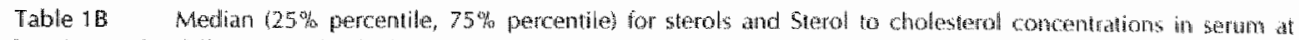
baseline and at follow-up. individuals ower 59 years only imean age $60.7+9.7(49.6-161.3 \%$

\begin{tabular}{|c|c|c|c|c|c|}
\hline & \multicolumn{2}{|c|}{ Absolute values } & \multicolumn{3}{|c|}{ Steral to chatesterol ratio } \\
\hline & $\begin{array}{l}\text { baseline }(1993) \\
(n-77)\end{array}$ & $\begin{array}{l}\text { follow-19p (19,9) } \\
(17 \text { ax } 101)\end{array}$ & unit: & $\begin{array}{l}\text { baseline }(1993) \\
(n-77)\end{array}$ & $\begin{array}{l}\text { follow } \\
\text { (n }=101)\end{array}$ \\
\hline cholesterol (mg/d) & $\begin{array}{l}169 \\
(135,1964)\end{array}$ & $\begin{array}{l}215 \\
(176,239)\end{array}$ & - & - & - \\
\hline lathosterol (mg/d) & $\begin{array}{l}0.18 \\
(0.14,0.253\end{array}$ & $\begin{array}{l}0.22 \\
(0.17,0.31)\end{array}$ & (ug/mg) & $\begin{array}{l}1.09 \\
(0.84,1.54)\end{array}$ & $\begin{array}{l}.04 \\
(0.79,1.21)\end{array}$ \\
\hline desmosterol $(\mu \mathrm{g} / \mathrm{d})$ & $\begin{array}{l}102 \\
(74,135)\end{array}$ & $\begin{array}{l}118 \\
(95,143)\end{array}$ & $(\mu \mathrm{g} / \mathrm{mg})$ & $\begin{array}{l}0.61 \\
(0.48,0.78)\end{array}$ & $\begin{array}{l}0.56 \\
(0.47,0.71)\end{array}$ \\
\hline lanosterol (Mg/d) & $\begin{array}{l}12.2 \\
(10.2,14.4)\end{array}$ & $\begin{array}{l}13.4 \\
(11.1,17.0)\end{array}$ & ing/mg & $\begin{array}{l}72.7 \\
(62.5,87.11)\end{array}$ & $\begin{array}{l}66.4 \\
(52.5,79.1)\end{array}$ \\
\hline 245-OH-Chos (ng/mL) & $\begin{array}{l}60.0 \\
\{51.0,77.5\}\end{array}$ & $\begin{array}{l}72.0 \\
(57.0,90.0)\end{array}$ & lng/mig & $\begin{array}{l}39.3 \\
02.8,47.1)\end{array}$ & $\begin{array}{l}35.0 \\
(27.2,41.5)\end{array}$ \\
\hline 27-OH-Chol (ng/ma) & $\begin{array}{l}169 \\
\{132,202\}\end{array}$ & $\begin{array}{l}208 \\
(166,264)\end{array}$ & $(n g / n g)$ & $\begin{array}{l}108 \\
(73,1340\end{array}$ & $\begin{array}{l}95 \\
(77,124)\end{array}$ \\
\hline campesterol (mg/d) & $\begin{array}{l}0.32 \\
(0.21,0.46)\end{array}$ & $\begin{array}{l}0.35 \\
(0.26,0.49)\end{array}$ & $y \mathrm{gg} / \mathrm{mg})$ & $\begin{array}{l}1.92 \\
(1.30,2.35)\end{array}$ & $\begin{array}{l}1.73 \\
(1.21,2.53)\end{array}$ \\
\hline sitostearol (mg/dli & $\begin{array}{l}0.27 \\
(0.20,0.40)\end{array}$ & $\begin{array}{l}0.31 \\
(0.22,0.42)\end{array}$ & (wghng) & $\begin{array}{l}1.54 \\
(1.19,2.46)\end{array}$ & $\begin{array}{l}1.49 \\
11.06,2.06\end{array}$ \\
\hline
\end{tabular}

\section{Cross-sectional correlation analysis at baseline and atter 6 years of follow-up}

The results from the cross-sectional correlation analysis between the cholesterol precursors; metabolites and plant sterols and cognitive test outcomes both at baseline and at followup are shown in Table 2 and in Figure 2. The sterol concentrations are expressed as ratio to cholesterol and indicated as R_Sterol.

The data in Table 2 show that the cholesterol concentration did not correlate with any of the cognitive tests.

\section{CHOLESTEROL PRECURSORS}

The R Lathosterol showed a negative conrelation with the WLTTOT and Delayed Recall test for the whole group at baseline, while only relation with the Delayed Recall was not obserwed at follow-up (Table 2 and Fig. 2). No significant relation was present between the R L athosterol and the cognitive outcomes on the Stroop of LDCT. The same negative correlation between lathosterol and the WLTTOT and Delayed Recall test was observed when the individuals older than 50 were included only (data not shown). These results indicate a relative better cognifive performance when lathosterol levels are lower, for the unadiusted (to age, sex, education) data. 


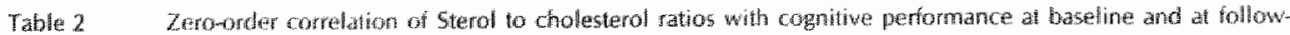

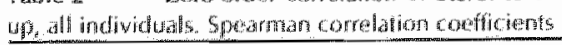

\begin{tabular}{|c|c|c|c|c|c|}
\hline & \multirow[t]{2}{*}{ vime-point } & \multicolumn{4}{|c|}{ Cognitite offcome measurement } \\
\hline & & Stroop & LDCT & WHTTOT & Delayed Recall \\
\hline $\begin{array}{l}\text { Mean } \pm \mathrm{SO} \\
\text { (rringoj }\end{array}$ & Dastelines & $\begin{array}{l}99 \mathrm{~g}, 27 \\
(61,225)\end{array}$ & $\begin{array}{l}47.3=10.4 \\
20.0 .71 .0\end{array}$ & $\begin{array}{l}4.6 \pm 9.1 \\
117.0,61,04\end{array}$ & $\begin{array}{l}8.9 \geq 3.0 \\
(2.0,15.0\end{array}$ \\
\hline cholesteral & bataluines & 0.15 & -0.08 & -0.05 & 0.06 \\
\hline (mug/d) & follow-up & 0.07 & 0.16 & 0.03 & .002 \\
\hline Cathostrent & besculine: & 0.02 & -0.14 & $0.23 *$ & $-0.26 \%$ \\
\hline (Maghos & follow-tup & 0.2 & -0.13 & 0.17 & $-0.19 \%$ \\
\hline desmoshend & bogelding & 0.00 & .0 .09 & -0.14 & $-0.26^{*}$ \\
\hline (uglng) & tollow up & 0.16 & -0.17 & 0.11 & -0.12 \\
\hline bunterol & Dastome & 0.04 & -0.20 & $-0.35 \div$ & $-0.39 *$ \\
\hline (ngarmes) & follow up & 0.16 & -0.2 & $-0.29^{*}$ & $-0.22^{*}$ \\
\hline $245 \mathrm{OH} C \mathrm{Chol}$ & 1.4sertirae & 0.11 & 0.03 & 0.13 & -0.16 \\
\hline (nge/mg) & GAlOW-LP & $0.29^{*}$ & $-0.20^{*}$ & 0.07 & -0.04 \\
\hline 27. On-Chol & baselinace & .0 .01 & $\times 0.01$ & -0.68 & $-0.21^{*}$ \\
\hline (thefong) & followw-zp & 0.09 & -0.15 & 0.13 & 0.13 \\
\hline Cumpasterol & baseline: & -0.02 & 0.05 & $0.27^{*}$ & 0.24 \\
\hline$(4 \mathrm{~g} g \mathrm{mg})$ & follow & -0.114 & 0.09 & 0.16 & 0.14 \\
\hline silosteriol & bastime & 0.01 & 0.05 & $0.28 *$ & 0.21 \\
\hline (wartmg & Collow-up & .0 .13 & -0.01 & 0.18 & 0.15 \\
\hline
\end{tabular}

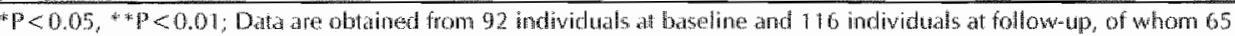
individuals were included in both measurements.

A negative correlation between the R Desmosterol and the Delayed Recall test was observed at baseline (Table 2). Similar results were obtaned for the subgroup of individuals over 50 years old (dala not shown).

The R Lanosterol correlated negatively with the WLTTOT and Dellayed Recall both at baseline and follow-up, while the relation between the R Lanosterol and $L D C T$ was significant only at follow-up (Table 2 and Fig. 2). Similar results were obtained when the individuals older than 50 years were selected only (data not shown). These results indicate a relatively better performance on these aspects of cognition when lanosterol concentrations are lower, unadjusted for age, sex or education.

\section{OXYSTFROLS}

A positive correlation was observed between the R $245-\mathrm{OH}-\mathrm{Chol}$ and performance on the Stroop test at follow up (Table 2). A negative correlation was present between the R $245-\mathrm{OH}$ Chot and pertomance on the LDCI at follow-up. In the group of individuals ofder than 50 years similar relations were observed (data not shown).

The R_27. OH-Chol showed a megative correlation with the Delayed Recall at baseline only, indicating better cognitive performance when serum $27-\mathrm{OH}$-Chol levels are lower. 

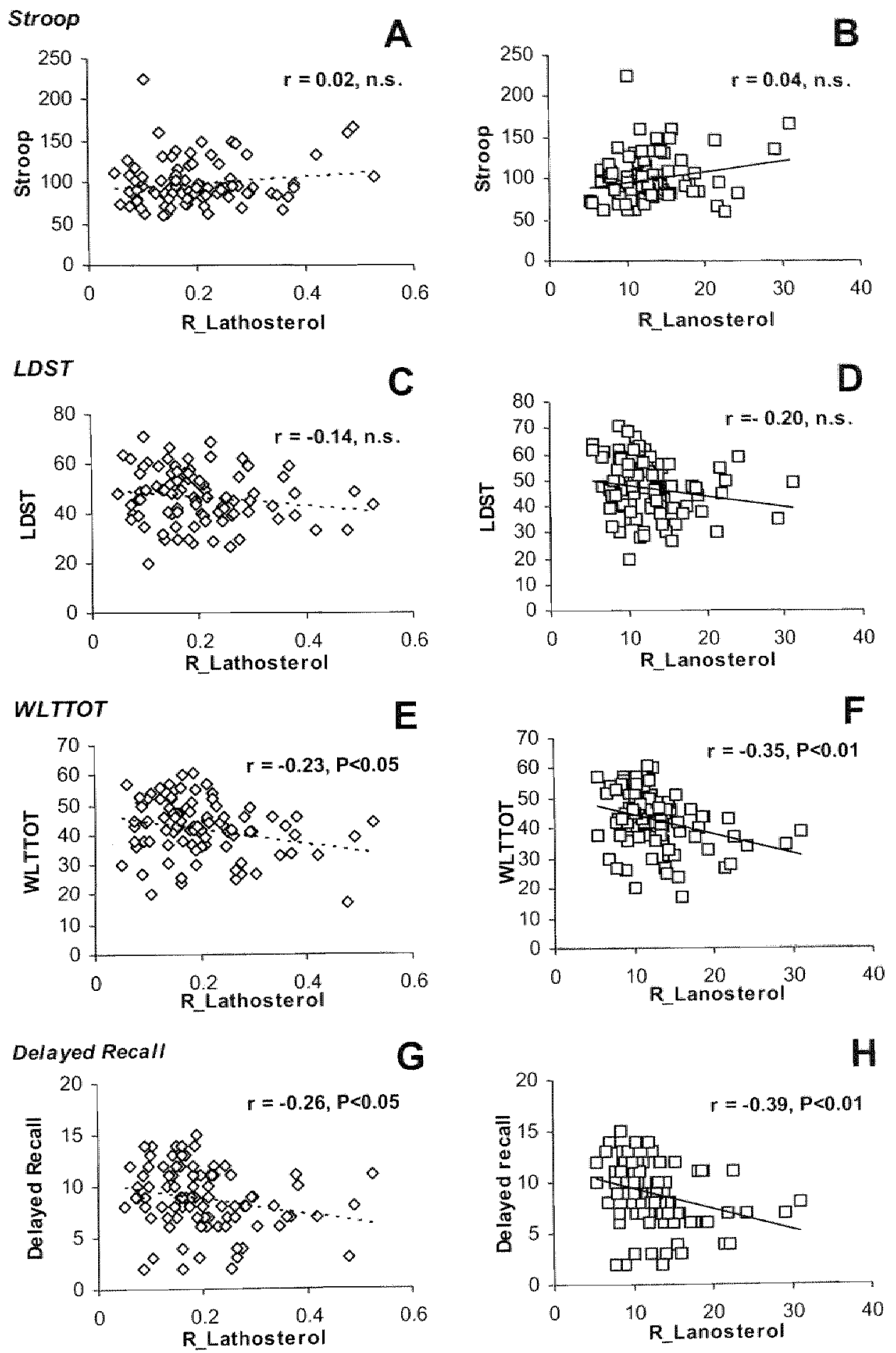

Ingr. 2 baseline.

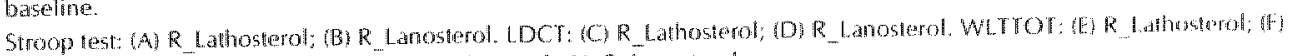

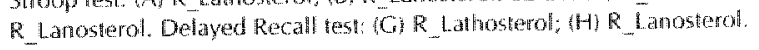




\section{PILANT STEROLS}

A positive correlation was observed between serum R_Campesterol and performance on the WLTTOT and Delayed Recall test at baseline only (Table 2). The R_Sitosierol correlated with the WLTTOT at baseline (Table 2). Similar, though weaker, results were obtained for the group of older individuals for both plant sterols (data not shown).

Cross-sectional correlation between cognitive performance and serum markers at baseline and follow-up adjusted for age, sex and education.

To investigate whether the correlations observed in Table 2 were independent of age, sex and level of education, multiple regression analysis of the data obtained at baseline and at follow-up was performed (Table 3). Due to the similarity of the results for the subgroup of people over 50 years old as compared with the whole population, these results are not discussed in further delail.

The serum cholesterol concentrations did not correlate with any of the cognitive test outcomes, adjusted for age, sex and education.

The R Lathosterol correlated negatively with the WLTTOT and the Delayed Recall test at baseline as well as at follow-up (Table 3). These results indicate a relatively better cognitive performance when lathosterol levels are lower, independent of age, sex and education.

No correlation between the $R$. Desmosterol at baseline with any cognitive test was observed.

A significant positive correlation between the $R$ _Lanosterol and the Stroop test was observed at baseline only. The R_Lanosterol showed a negative correlation with the WLTTOT and Delayed Recall test, adjusted for age, sex and education, at baseline and after six years of follow-up (Table 3). These results indicate a relatively better cognitive performance when lanosteral levels are lower.

The $\mathrm{R} 2245-\mathrm{OH}$-Chol correlated positively with the Stroop test at baseline only (. The R_27-OHChol showed a significant (negative) correlation with the WLTTOT at baseline only. A negative correlation was observed between $\mathrm{R} 27-\mathrm{OH}-\mathrm{Chol}$ and performance on the Delayed Recall test and on the LDCT at follow-up.

No significant relation between the plant sterols R_Campesterol and R_Sitosterol with any of the cognitive outcomes was observed after adjustment to age, sex and education.

The observed relations in this regression analysis remained unaffected by exclusion of the individuals that turned out to be influential cases and outliers, based on the studentized residuals and Cook's distances. Eight other individuals used cholesterol-lowering drugs. The relations remained also largely unaffected after additional exclusion of eight individuals who used cholesterol-lowering drugs at any time during this study.

\section{Longitudinal correlation beween cognitive performance and serum markers adjusted for age, sex and education over the whole six-year follow-up period.}

We next wished to investigate if a causal relation exits between serum sterol concentrations and cognitive decline in time. For this purpose, multi-level repeated measurement analysis was performed [11]. Multi-level repeated measurement analysis accounts for variations in interval of frollow-up and increases the power of the analysis (see second columm of Table 4). This relation 
was investigated for the markers that correlated consistently in time with at least wo cognitive. tests in Table 3.

The R_Lathosterol level at baseline correlated positively with the performance on the stroop test and negatively with performance on the LDCT, WLTrO' and Delayed Recall test over the whole follow-up period. These results indicate a relatively better cognitive performance over a six years period when the R_Lathosterol level is lower at baseline.

The R_Lanosterol levels at baseline correlated positively with the Stroop test and negatively

Table 3 Regression analysis for R Sterols and ourcomes on four cogmine measures, adjusted for age, aducation and sex in all individuals.

\begin{tabular}{|c|c|c|c|c|c|}
\hline & & \multicolumn{4}{|c|}{ Cognitive otitcome medstirement } \\
\hline & & Siroop & LDCT & MLTTOT & Delayed Recall \\
\hline \multirow[t]{2}{*}{ Cholesterol img/de } & baseline & $\begin{array}{l}-0.05 \\
(-0.16,0.06)\end{array}$ & $\begin{array}{l}0.02 \\
10.02,0.061\end{array}$ & $\begin{array}{l}0.03 \\
0.01,0.06\end{array}$ & $\begin{array}{l}0.01 \\
(-0.01,0.02)\end{array}$ \\
\hline & follow-up & $\begin{array}{l}-0.04 \\
(-0.19,0.10)\end{array}$ & $\begin{array}{l}0.00 \\
(-0.04,0.04)\end{array}$ & $\begin{array}{l}0.02 \\
(-0.02,0.05)\end{array}$ & $\begin{array}{l}0.01 \\
10.00,0.020\end{array}$ \\
\hline \multirow[t]{2}{*}{ R_lathosterol (mg/mg) } & baseline & $\begin{array}{l}6715 \\
(-1017,14448)\end{array}$ & $\begin{array}{l}-2439 \\
(-5223,343)\end{array}$ & $\begin{array}{l}-3647^{*} \\
(-6331,-964)\end{array}$ & $\begin{array}{l}-1212^{*} \\
(-2111,-313)\end{array}$ \\
\hline & follow-up & $\begin{array}{l}6721 \\
(-2847,16291)\end{array}$ & $\begin{array}{l}-2325 \\
(-4751,99)\end{array}$ & $\begin{array}{l}-2768^{\circ} \\
(-5125,-412)\end{array}$ & $\begin{array}{l}-894 \\
(-16 ! 6,-171)\end{array}$ \\
\hline \multirow[t]{2}{*}{ Desmosterol (mg/ng) } & baseline & $\begin{array}{l}-5.80 \\
(-27.7,6.1)\end{array}$ & $\begin{array}{l}4.31 \\
(-3.5,12.2)\end{array}$ & $\begin{array}{l}-5.24 \\
(-130,2.5)\end{array}$ & $\begin{array}{l}-0.06 \\
(-2.67,2.56)\end{array}$ \\
\hline & follow-up & $\begin{array}{l}16.63 \\
(-11.0,44.3)\end{array}$ & $\begin{array}{l}-6.360 \\
(-13.4,0.7)\end{array}$ & $\begin{array}{l}-5.30 \\
(-12.2,1.6)\end{array}$ & $\begin{array}{l}-1.77 \\
(-3.80,0.35)\end{array}$ \\
\hline \multirow[t]{2}{*}{ R lanosterol ( $\mathrm{kg} / \mathrm{mg}$ ) } & baseline & $\begin{array}{l}134 \\
(2,266)\end{array}$ & $\begin{array}{l}-36 \\
(-84,12)\end{array}$ & $\begin{array}{l}-66 * \\
(-1) 2,-21)\end{array}$ & $\begin{array}{l}-20 \\
(-36,-5)\end{array}$ \\
\hline & follow-up & $\begin{array}{l}122 \\
\{-134,379\}\end{array}$ & $\begin{array}{l}-62 \\
(-127,2)\end{array}$ & $\begin{array}{l}-72^{*} \\
(-136, \times 9)\end{array}$ & $\begin{array}{l}-32^{*} \\
(-51,-14)\end{array}$ \\
\hline \multirow[t]{2}{*}{$245 \times \mathrm{OH}$-Chol (ng/mg) } & basetine & $\begin{array}{l}3.38 \\
(-0.28,7.04)\end{array}$ & $\begin{array}{l}0.36 \\
(0.98,1.70)\end{array}$ & $\begin{array}{l}-0.93 \\
(-2.24,0.30)\end{array}$ & $\begin{array}{l}-0.20 \\
(-0.65,0.24)\end{array}$ \\
\hline & follow-up & $\begin{array}{l}3.05 \\
(2.67,8.77)\end{array}$ & $\begin{array}{l}-0.46 \\
(-1.92,1.01)\end{array}$ & $\begin{array}{l}0.39 \\
(-1.05,1.83)\end{array}$ & $\begin{array}{l}0.052 \\
(-0.39,0.49)\end{array}$ \\
\hline \multirow[t]{2}{*}{ 27-OH whol (ng/mg) } & baseline & $\begin{array}{l}1.03 \\
(-0.32,2.38)\end{array}$ & $\begin{array}{l}-1,09 \\
(+6,02,3,84)\end{array}$ & $\begin{array}{l}-0.45^{\mathrm{K}} \\
(-0.92,0.01)\end{array}$ & $\begin{array}{l}0.06 \\
(-0.21,0,10)\end{array}$ \\
\hline & follow tip & $\begin{array}{l}0.96 \\
(-0,66,2.58)\end{array}$ & $\begin{array}{l}-0.37 * \\
(-0.78,0.036)\end{array}$ & $\begin{array}{l}-0.21 \\
(-0.61,0.21)\end{array}$ & $\begin{array}{l}-0.1)^{*} \\
(-0.23,0.01)\end{array}$ \\
\hline \multirow[t]{2}{*}{ R Campesterol thg/mg? } & baseline & $\begin{array}{l}650 \\
(-2248,3548)\end{array}$ & $\begin{array}{l}93 \\
(-946,1131)\end{array}$ & $\begin{array}{l}481 \\
(-538,1501)\end{array}$ & $\begin{array}{l}229 \\
-110,569\end{array}$ \\
\hline & foltow-up & $\begin{array}{l}-2116 \\
(-6040,2607)\end{array}$ & $\begin{array}{l}-81 \\
(-1294,1132)\end{array}$ & $\begin{array}{l}858 \\
(-320,2035)\end{array}$ & $\begin{array}{l}133 \\
(-231,498)\end{array}$ \\
\hline \multirow[t]{2}{*}{ R sitasterol (mg/mg) } & baseline & $\begin{array}{l}635 \\
(2597,3867)\end{array}$ & $\begin{array}{l}110 \\
(-1052,1272)\end{array}$ & $\begin{array}{l}762 \\
(374,1897) !\end{array}$ & $\begin{array}{l}273 \\
(-107,65)\end{array}$ \\
\hline & follow-up & $\begin{array}{l}-2849 \\
(-8036,2338)\end{array}$ & $\begin{array}{l}-12 \\
(-1346,1322)\end{array}$ & $\begin{array}{l}1039 \\
6-263,2329\end{array}$ & $\begin{array}{l}169 \\
(231,570)\end{array}$ \\
\hline
\end{tabular}


with pertomance on the WhTT and Delayed Recall test over six years of follow-up. These results indicate at relatively better cognitive performance over six years when lanosterol levels are lower at beseline.

The relations between the levels of the R_Lathosterol and R_Lanosterol and the performance on the WITTOT and Delayed Recall remained unaffected after repeating these analysis with exclusion of the individuals which turned out to be influential cases and outliers. The relation between the precursor levels and the Stroop test observed with the mutti-level analysis was absent after exclusion of the outtiers, thus obtaining results in line with the data of Table 3 . The relations remained also essentially unaffected after exclusion of the eight individuals using cholesterowlowering drugs. The results were similar in the group of all individuals as well as in the group of individuals older than 50 years (data not shown).

Duse to the lack of inter-individual differences in cognitive decline over the follow-up period (see below Table 4), the relation between serum data and differences in cognitive performance, for example the examination of risk modulating effects, could not be investigated further.

Table 4 Ungandardised regression coefficionts (95\% confidence intervall of wo sterol to cholesterol ratios in

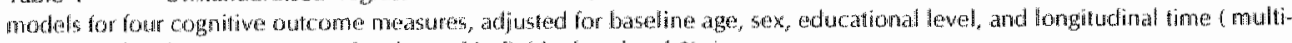
level model with observations at level 1 and individuals at level 2)."

\begin{tabular}{|c|c|c|c|c|c|}
\hline & \multirow[b]{2}{*}{$\begin{array}{l}\text { munber of } \\
\text { individuals } \\
\text { observations }\end{array}$} & \multicolumn{4}{|c|}{ Cognitive outcome meastrement } \\
\hline & & Stroop & LDCT & WLTHOT & Delayed Recall \\
\hline $\begin{array}{l}\text { R Halliosero } \\
\text { wghmg }\end{array}$ & $92 / 252$ & $\begin{array}{l}9134^{\text {क क }} \\
2351,16017)\end{array}$ & $\begin{array}{l}-2781 * \\
-5378,-1840\end{array}$ & $\begin{array}{l}-2855^{-1 k} \\
-5130, .5794\end{array}$ & $\begin{array}{l}-981^{\text {in }} \\
-1710,-252)\end{array}$ \\
\hline $\begin{array}{l}\mathrm{R} \text { Lanostarot } \\
\text { (ng/mg) }\end{array}$ & $92 / 252$ & $\begin{array}{l}169 * \\
52,2867\end{array}$ & $\begin{array}{l}-35 \\
-8046,10)\end{array}$ & $\begin{array}{l}-61^{* *} \\
-99,-22) \\
\end{array}$ & $\begin{array}{l}-19^{\text {ini* }} \\
-31,-61 \\
\end{array}$ \\
\hline
\end{tabular}

* $P<0.05, * P<0,01$

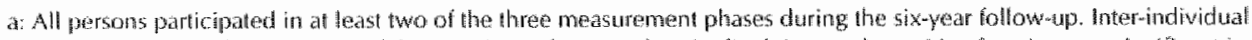
random variaton in the intercepts of the assowation between fongitudinal time and cognitiwe function was significant in all analysas. Intet windwitual random waration in the shopes of this association was, however, never statistically significant.

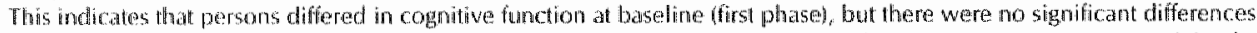
between persons in the course of cognive function during follow-sp. interactions belween longitudinal tithe and the three

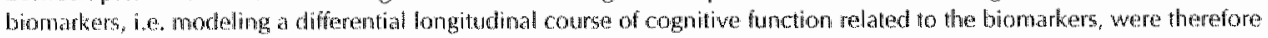

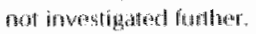

\section{Discussion}

The objective of this study was to determine if a possuble causal relation exists between precursors and oxidised products of cholesterod, reflecting whole body cholesterot homeostasis, and cognitive performance over a six year period in a heallhy ageing population. We investigated the sterol ratios to cholesterol of the cholesterol precursors lathosterol, lanosterol and desmosterol, the cholesterol metabolites $245-\mathrm{OH}-\mathrm{Chol}$ and $27-\mathrm{OH}-\mathrm{Chol}$ and the plant sterols sitosterol and campesterol. First, the sterol ratios to cholesterol at baseline and after six years of follow up were correlated with the individual cognitive performance of those timepoints. As a final step, we studied the relation between the sterol levels at baseline and cognitive performance during the whole follow-up period, using multi-level regression analysis [11]. no investigate whether the concentrations at baseline are indicative for cognitive performance over a longer period, or even cognitive decline.

The results showed a strong negative correlation between the $R$ Lanosterol and $R$ _Lathosterol and cognitive performance as measured with the WLTTOT and Delayed Recall at both time points and over the whole follow-up period. These results indicate a better cognitive 
performance when serum concentrations of cholesterol precursors and especially their ratios to cholesterol are low, which may point to decreased cholesterol synthesis. No correlation between serum cholesterol concentrations and the perfomance of the cognitive tests was observed and one spurious correlation was observed between $245-\mathrm{OH}$ Chol levels and cognitive performance in the zero order correlation analysis after adjustment for age, sex and education. The $27-\mathrm{OH}$-Chol levels showed variable correlations. These results do not support a relation between these oxidised cholesterol metabolites and cognitive perfomance in the normal ageing population. The influence on the analysis of the individuals with the most extreme precursor-concentrations was minimal, as shown by the regression coefficients in the multivariate regression analysis and multi-level regression analysis after exclusion of these cases. Interestingly, exclusion of users of cholesterol synthesis lowering drugs did not alter the relations observed. This study is unique in that it combines biological markers and behavioural data from a population-based longitudinal perspective. The results may therefore have implications on a population level.

To examine if the association of lanosterol and lathosterol ratios to cholesterol with cognition was a reflection of whole body synthesis of cholesterol rather than a reflection of increased absorption [13], we determined if the ratios of campesterol and sitosterol to cholesterol were associated with cognition, too. The serum sitosterol concentration is general considered as a measure of cholesterol absorption [26]. No relation of the ratios of campesterol or citosterol to cholesterol with cognition was observed at baseline or at follow-up, adjusted for age, sex and education, indicating that increased lathosterol levels in relation to cognitive performance may not be due to relatively lower cholesterol absorption. As a final step we examined if the ratio lathosterol to campesterol or to sitosterol, as a new measure of endogenous cholesterol synthesis, was related to cognition. A high lathosterol to campesterol level would then indicate a relatively higher cholesterol synthesis rather than increased absorption. Similar to the results obtained with the lathosterol or lanosterol to cholesterol ratios, significant associations of lathosterol to campesterol or sitosterol ratios and the WLTTOT Or Delayed Recall were observed (data not shown). Thus, relatively high ratio of lathosterol to cholesterol as a reflection of increased cholesterol synthesis was associated with relatively worse performance on the Word Learning tests.

To our knowledge, no research has been done so far on the relation between serum lanosterol or lathosterol and neurodegenerative processes. Nevertheless, a change in lathosterol concentration in relation to neurodegenerative processes is in accordance with our previous findings in vivo, where treatment of rats with the neurotoxin 3 -nitropropionic acid resulted in decreased lathosterol concentration in striatal homogenates and serum (Chapler 6). The striatum is selectively affected after treatment with 3-nitropropionic acid 13. In addition, increased desmosterol concentrations were obsevved in sertum of treated animals. The cholesterol concentrations in the striatum as well as in serum remained unchanged, which is in line with the present results.

It would be interesting to know if low performance on the Delayed Recall, WLTTOT or Stroop test in our study, as found in the individuals with higher levels of cholesterol precursors, is indicative of the development of cognitive deterioration or even dementia. Unfortunately, our data showed little variation in cognitive performance between individuals over the six years of follow-up, and thus we can not yet draw conclusions concerning causality from our data. It is probable that dementia emerges from gradual cognitive deterioration, although the characteristics of the transition are not clarified [39,40]. Not all individuals suffering from mild cognitive impaiment do develop dementia [30]. We may speculate that higher serum levels of cholesterol precursors may be a risk-factor for dementia, as if was shown that inthibilion of 3 hydroxymethyl-3-glutaryl coenzyme $A$ reductase, the rate limiting enzyme of cholesterol synthesis, by statins was associated with decreased prevalence of Alzheimer's dementia 1421 . In 
contrast to better cognitive performance associated with low cholesterol precursor levels, as a possible indication for low cholesterol synthesis, Muldoon ef al [27] observed decreased cognifive performance on tests for attention and psychomotor speed after treatment of hypercholesterolaemic individuals with the statin lovastatin. Similarly, Wardle et al. observed weak decreased performance on a sustained-attention task after cholesterol lowering diet [41]. The contrasts between these results, our results and the results of Wolozin [42] may be explained by differences in study populations, i.e. hypercholesterolemic individuals in the studies of Wardle and Muldoon and AD patients vs. controls in the sudy of Wolozin [42]. while we investigated a nomal ageing population.

Oxysterols are mono-oxygenated derivatives of cholesterol and are formed either by autoxidation or by action of a specific cytochrome $\mathrm{P} 450$ such as cholesterol $7 \alpha$-hydroxylase (Cyp 7A), sterol 27-hydroxylase (Cyp27), and cholesterol 24-hydroxylase (Cyp46) (Fig. 1) [4] [21]. Introduction of an oxygen molecule increases the rate of degradation of cholesterol to more polar compounds, such as bile acids. The concentration of oxysterois in biological fluids is determined by their rate of formation and metabolism, and in general the rate of metabolism of these oxysterols is considerably higher than that of cholesterol [24]. Excess of cholesterol produced in the brain [16] has to be removed into the circulation and enzymatic Cyp450 dependent side-chain hydroxylation to $245-\mathrm{OH}$-Chol, increasing polarity of cholesterol, may be one of the most important export mechanisms [21]. It has been suggested that 245-OH-Choll may be a reflection of total brain mass and possibly a marker for vascular dementia and Alzheimer's dementia $[8,22]$. We found no relation between the oxidised sterols and (nonpathologicall) cognitive performance. This finding is in agreement with previous studies, where decreased 245-OH-Chol concentration was observed only in patients with more severe stages of Alzheimer's dementia or with very severe neurotrauma $[8,28]$. No such decrease was observed in serum of patients with meningitis, brain tumours or multiple sclerosis [8]. These observations indicate a putative monitoring role of this oxysterol in only a few specific neurodegenerative diseases. The lack of correlation with $27-\mathrm{OH}-\mathrm{Chol}$ might have been expected as even in Alzheimer's dementia patients no decreased 27-OH-Chol level have been observed [B].

In conclusion, even though the low number and relatively short follow-up time warrant cautious interpretation, a correlation between the cholesterol precursors lathosterol and lanosterol and cognitive performance was observed in this normal ageing population. The results of this study encourage funther investigations into a causal relation between cholesterol precursors and cognition.

\section{References}

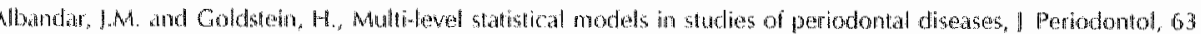
$11902) 6005$.

2 Anstoy. $K$, and Christensen, H. Education, actity, health, blood pressure and apolipoprotein $E$ as predictors of cognilive change in old age: a rewiew, Gerontology, 46 (2000) 163-77.

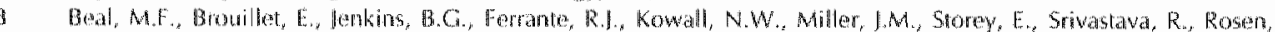

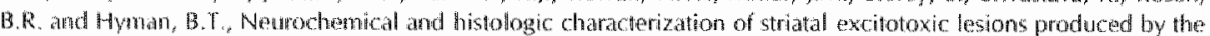

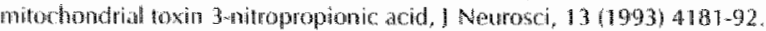

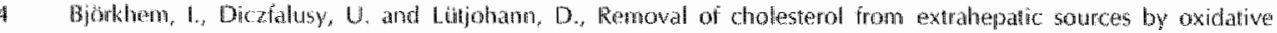
mechatisms, Curr Opin liptol, i0 (19991 161-5.

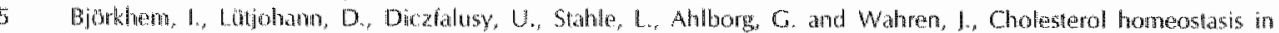
haman brain: tumower of 245 hydroxycholesterol and exidence for a cerebral origin of most of this oxysterol in the circulation, I hipid Res, 39 (19981 1594-1600.

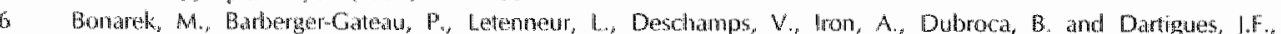
Relatonships between cholesterol, apolipoprotein E polymorphism and dementiar a cross-sectional analysis from the PAQuib study, Newroepidentology, 19 (2000) 1413.

7 Brand, $N$ and lolles, I., Leaming and retrieval tate of words presented auditorily and visualy, Gen Psylhol, 112 
(1985) $201-10$

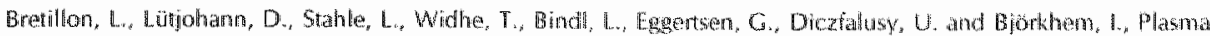

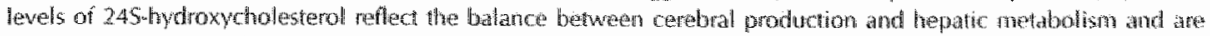
inversely related to body suriace, I Lipid Res, 4 i 12000$)$ 840-845.

9 Dieschy, IM and Tuntey, S.D., Chotesterol metabolism in the brain, Curr Opin Lipidol, 12 (2001) 105.12.

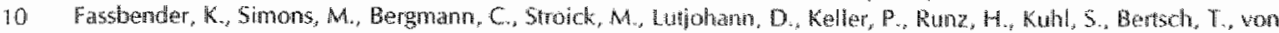

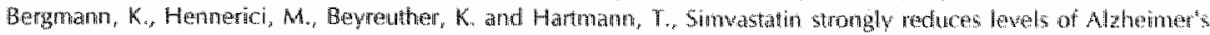
distemse beta armwloid peptides Abeta 42 and Abeta 40 in vitho and in viwo, Proc Nat Acad Sci U 5 A, 101200110. Goldstein, H., Mulilewel Statishical Models, Fdward Anold, London, 1995.

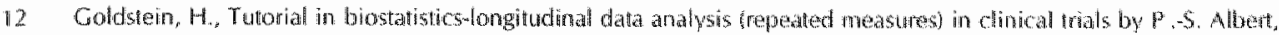
statistics in medicine, 1999, 18, 1707-1732, Stat Med, 19,20001821.

13 Grandy, S.M. George Lyman Duff Memorial Lechere. Aultifatorial etiology of hypercholesterolemia. Implications for prevention of coromany heart disease, Axterioscler Thromb, $11199131619-35$.

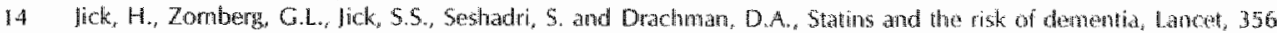
(2000) 1627.31 .

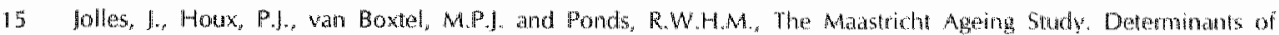
cognitive ageing, Neuropsoch Publishers, Masstricht, $1995,192 \mathrm{pp}$

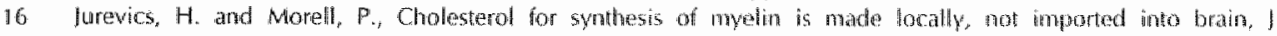
Neurochen, $64(1995) 395-901$.

17 Kakio, A., Nishimoto Si, 5., Yanagisawa, K., Kozutsumi, Y. and Mafsuzaki, K, Cholesterol-dependen famalian of

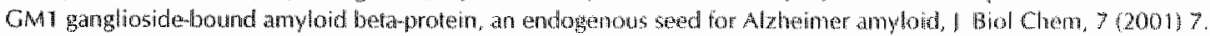

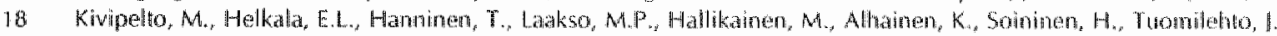

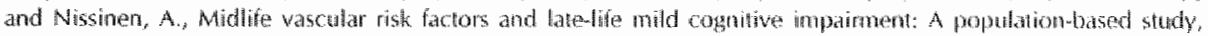
Neurology, 56 (2001) 1683-9.

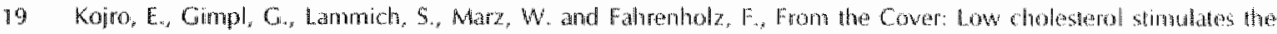

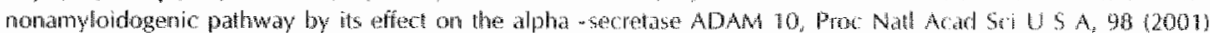
$5815-20$.

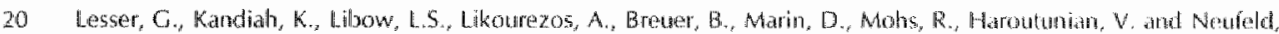

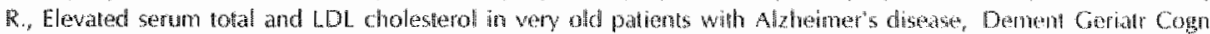
Disord, 12 (2001) $138-45$.

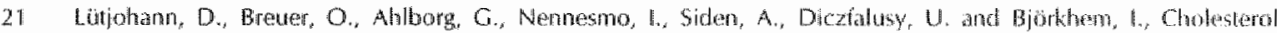
homeostasis in thuman brain: evidence for an age-dependent flux of 24 S-hydraxycholesterol twan the binan into the circulation. Proc Natl Acad Sici U S A, 93 (1996) 9799-9804.

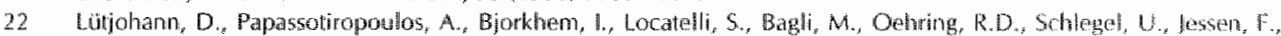
Rao, M.L, wom Bergmann, K. and Hean, R., Plasma 24Smydroxyctolesherol foerebrosterolh is increased in

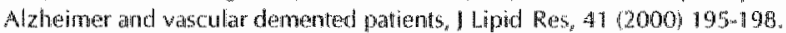

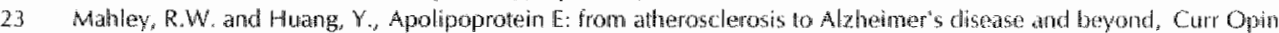
Lipuidol, $10(1999) 207-17$.

24 Meaney, S., Hassan, M., Sakinis, A., fütjoham, D., von Bergmann, K., Wermmatm, A., Diczfallisy, U. and Bjökhem, I. Studies on the formation and flux of oxysterols in wivo with stable isolopes. Evidence that the three

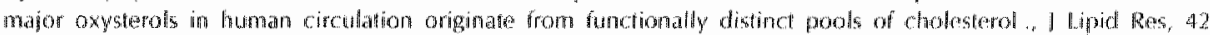
(2001) 70778 .

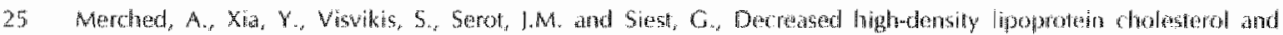

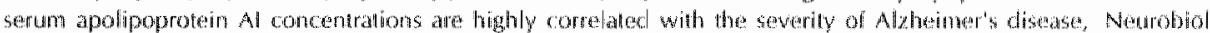
Ageing, $21(2000) 27-30$.

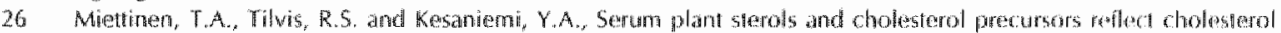

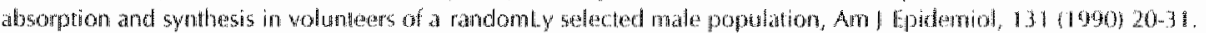

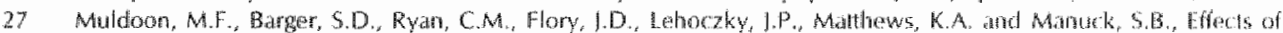

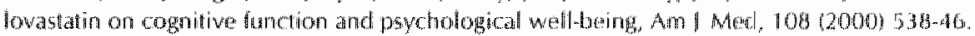

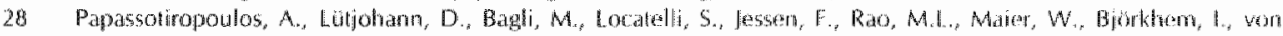

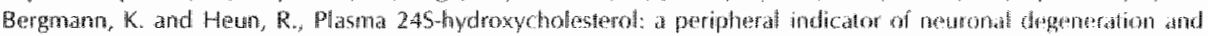
potentiat state marker for Aheiner's disease, Neutoreport. 11 (2000) 1959.1962.

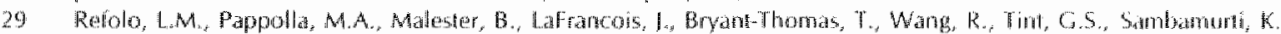

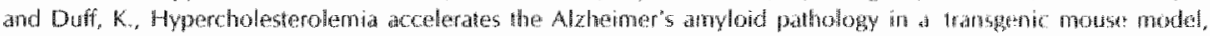
Neurobial Dis, 7 (2000) $321-31$.

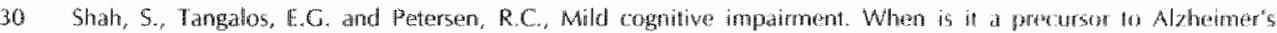
disease?. Gerantics, $55(2000) 62,65-8$.

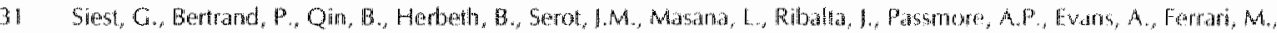

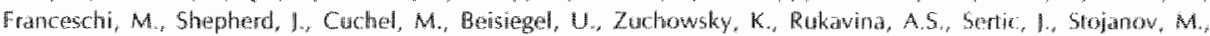

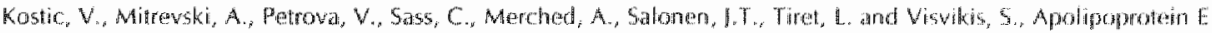

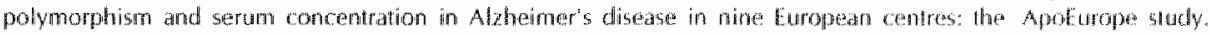
Apoturope group. Olin Chem Lab Med, 36 (2000) 72830 .

32 Simons, M. Keller, P., De Stromper, B., Beygenther, K., Dott, C.G. and Simons, K. Chollegterol dephetum irhibins

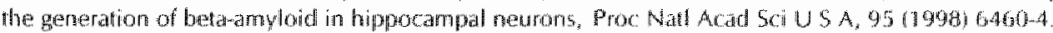




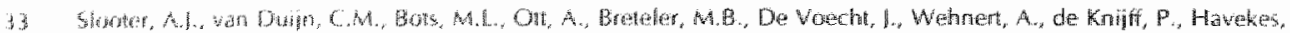

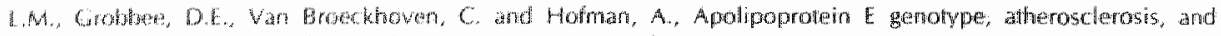

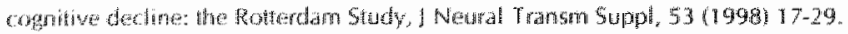

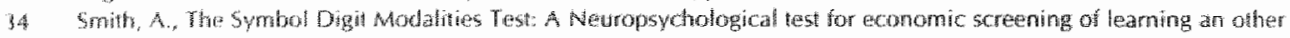
cerebal disorders. Letering Disorders, 360196818391 .

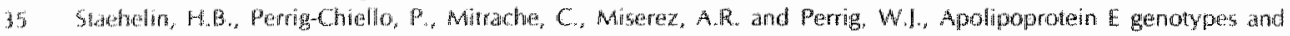

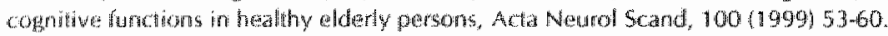

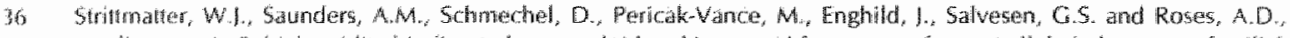

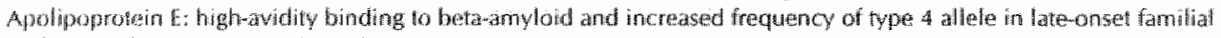

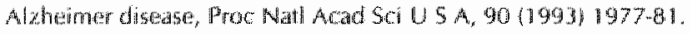

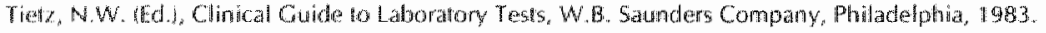

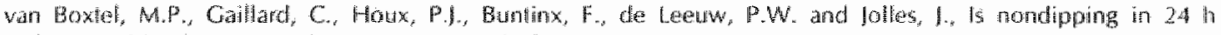

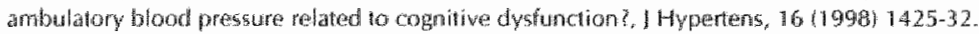

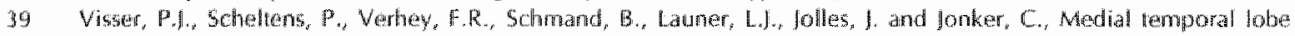

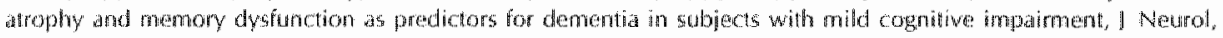
$246119991477-45$.

40 Visser, P., Verhey, F.R. Ponds, R.W., Kester, A, and Joles, 1. Distinction between preclinical Alzheimer's disease

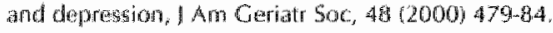

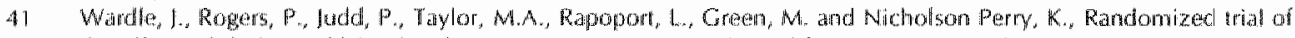

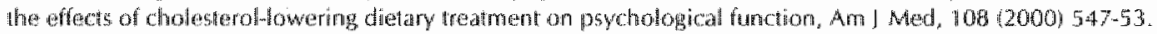

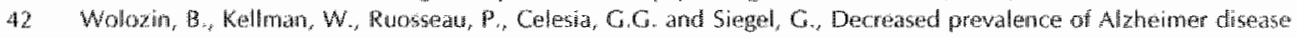
assoclated with 3-hydrosy-3-methyglutury coenzyme A reductase inhibitors, Arch Meurol, 57 12000) $1339-43$. 


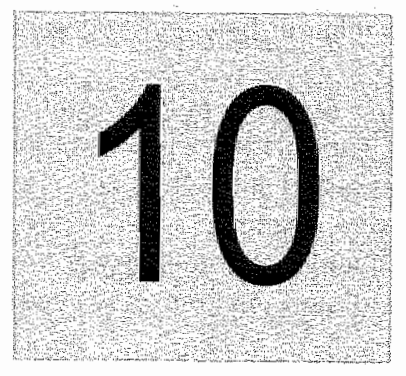

\section{Homocysteine: a marker for cognitive performance? A longitudinal follow-up and patient study}

Charlotte Teunissen, "Henk Blom, Martin van Boxtel, Hans Bosma, Chris De Bruijn, Jelle Jolles," Annick Wauters, "Frans Verhey, 'Fred Vreeling, Harry Steinbusch and lan de Vente

European Graduate School of Neuroscience (Euron), Universiteit Maastricht, Department of Psychiarry and Neuropsychology, The Netherlands;

a Department of Pediatrics, Universiteit Nijmegen, The Nethertands b AZ Middelheim, Antwerpen, Belgium

c Academic Hospital Maastricht, The Netherlands.

Submitted to American Journal of Clinical Nutrition 


\begin{abstract}
Elevated total serum homocysteine concentrations, as a marker for B-vitamin status, are associated with decreased cognitive performance in the ageing population and also with dementia.

The aim of the present study was to investigate whether homocysteine is a risk factor for cognilive decline, and to compare the outcome with the possible relation between cognition and the B-vitamins.

Patients with Alzheimer's disease $(n-34)$, Parkinson's disease $(n-46)$ and other cognitive disturbances $(n-47)$ were compared to a group of normal healthy controls $(n=61)$. Cognition of a cohort of 144 normal healthy individuals (aged 30-80 years) was tested at baseline and after six years of follow-up. Domains of cognitive function addressed were cognitive speed (LetterDigit Coding test), attention and information processing (Stroop test) and verbal memory (Word Learning test). Serum concentrations of homocysteine, folic acid and vitamin B12 were determined.

In all neurological patients together, increased concentrations of homocysteine and decreased vitamin B12 concentrations were observed compared to controls. Serum concentration of homocysteine correlated negatively with cognitive performance on the Word Learning test at baseline in the ageing sample, independent of age, sex, education level or folic acid concentration. Folic acid concentration correlated positively with cognitive performance on the Word Learning test at baseline. Homocysteine concentration at baseline correlated negatively with cognitive performance on the Stroop and Word Learning test during the whole six-year follow-up period.

In conclusion, elevated homocysteine concentrations appeared to be a marker indicative for lower cognitive performance over an extended period in the nomal ageing population.
\end{abstract}

\title{
Introduction
}

Homocysteine concentration in serum is known to be an indicator for the vitamin B status in humans. A deficiency in these vitamins has been frequently described in older individuals, due to an insufficient dietary intake or a reduced intestinal absorption $[2,35]$. Such a decrease in the vitamin $B$ status leads to increased homocysteine concentrations. For some time, elevaled plasma total homocysteine concentration has been known as a risk factor for cardiovascular disease [33]. Results of recent studies hava suggested that elevated tolal homocysteine concentrations may also be a risk factor for both vascular and Alzheimer's dementia (AD) $[9,26]$.

Homocysteine is formed from methionine, an essential amino acid [11]. Methionine is an important methyl donor for several reactions in the mammalian brain, including synthesis and degradation of neurotransmitters and cellular membranes $[3,4]$. Transformation of homocysteine into methione is catalysed by methionine synthase and requires vitamin $B 12$ and folic acid as cofactors. Alternatively, homocysteine may be transformed into cystathionine, catalysed by cystathionine $\beta$-synthase, which requires vitamin $B 6$ as a cofactor, and eventually into cysteine [11]. Increased cellular and peripheral homocysteine concentrations can be caused by, for example the already mentioned dietary influences as well as by genetic mutations in the enzyme cystathionine $\beta$-synthase or methylenetetrahydrofolate reductase $[6,29]$. A causative relation between increased homocysteine concentrations and the brain damage in relation to the reported dementia is mot proven yet 19,22$]$. Excess homocysteine concentrations in the CNS 
may inhibit DNA-repair mechanisms, as was shown in cultured rat hippocampal neurons [20], or inhibit the vasodilating actions of nitric oxide as was shown in humans and mice, by yet unknown mechanisms $[10,36]$.

A relation between elevated total homocysteine concentrations and decreased cognitive performance has also been observed in studies on the normal ageing population $[8,19,28,34]$, and a relation between vitamin B12 and folic acid and cognitive performance has also been shown [34]. However, literature data on the association between these B-vitamins and cognitive performance are not consistent. Thus, an association between folic acid and vitamin $B 12$ and cognitive performance has been confirmed by some epidemiological studies $[14,21]$, whereas this association was absent in other studies $[18,21,23,24]$. In serum of patients with dementia, increased homocysteine concentrations has been reported more consistently compared to the lowered B-vitamins concentrations $[9,26,30]$. In view of these observations, it was suggested that homocysteine may be stronger relatted to cognitive performance than the B-vitamins,

The aim of the present study was to investigate whether homocysteine would be stronger related to cognitive performance than the B-vitamins over an extended period of normal ageing or even be a risk factor for cognitive dedine such as occurs in AD. First, we compared the serum concentration of total homocysteine, vitamin B12 and folic acid between patients with AD, other cognitive disorders (OCD), Parkinson's disease (PD) and a group of normal healthy controls. Secondly, we investigated the serum concentrations of homocysteine, folic acid and vitamin B12 in relation to cognitive function in a longitudinal follow-up study. We related the serum concentration of these molecules with the individual cognitive pertormance on domains of information processing speed, learning, memory and attention. The population was drawn from a longitudinal population based study into the deteminants of cognitive ageing the MAastricht Ageing Study (MAAS: [17,41]).

\section{Subjects and methods}

\section{Patients}

Patients aged between 50-80 years old visiting the outpatient clinic of the departments of Psychiatry ('memory clinic) and Neurology of the Academic Hospital Maastricht were included. Diagnosis of $A D(n=34)$ was based on the diagnosis for probable $A D$ according to NINCDS-ADRA and DSM-IV criteria [27]. Diagnosis of PD $(n-46)$ was based on UK PD Society Brain Bank Clinical Diagnostic criteria. Diagnosis included a clinical examination, i.e. medical history, neurological, somatic and neuropsychiatric status, and routine blood tests. A computed tomographic scan (CT) was pertormed to identily white matter lesions, cortical atrophy and lacunar infarcts. Patients with clinical signs of inflammation were excluded. The patients with OCD $(n=47)$ were diagnosed as having cognitive disturbance (DSM code 294.9)(n 14$)$, mild cognitive impairment $(n=6)$, vascular dementia $(n=7)$, dementia nom-specified $(n=5)$, frontotemporal dementia $(n=4)$, depression induced dementia $(n=4)$, primary aphasia $(n-4)$, brain tumour $(n=1)$, and other $(n=2)$. The term "all neurological patients" is used in this study lo indicate all patients, i.e. AD, PD and OCD.

Serum of healthy controls $(n=61)$, was obtained from a group of participants of the MAAS study (see below). These individuals were also aged $50-80$ years, and the sex distribution was similar to the patient groups. The blood sampling protocol for this group was similar to the patient samples; i.e. serum was collected after $30-60$ min clotting at room temperature. A minimental state examination (MMSE) score [12] was obtained from all controls. A MMSE score was obtained from $33 \mathrm{AD}$ patients, $44 \mathrm{OCD}$ patients and $27 \mathrm{PD}$ patients as part of the diagnostic work-up. Five subjects (fiour OCD patients, one AD patient) had low vitamin B 12 concentraltion 
during the diagnostic wotk-up. At the time of this study, one OCD patient of this group had a high serum concentration of vitamin $B 12$ and three $O C D$ patients had a relatively low serum concentration of vilamin B12, while the $A D$ patient had serum vitamin B12 concentration comparable to the median reported in Table 1.

The patient part of this study was in accordance with the principles of the Helsinki declaration of 1975 as revised in 1983 and was approved by the local ethics committee. Written informed consent was obtained from all individuals or responsible caretakers.

\section{Ageing subjects}

Normal ageing subjects were recruited from the MAAS, which is a larger research program investigating determinants of cognitive ageing in the healthy population [17,41]. Subjects of MAAS were randomly drawn from the Registration Network Family Practices, a research database that contains basic health information of patients in primary care facilities (Metsemakers, Höppener, Knottnerus, Kocken \& Limonard, 1992). Exclusion criteria were evidence of past or present morbidity that may compromise brain function, such as cerebrovascular disease (including stroke), chronic neurological pathology (e.g. dementia, epilepsy or PD), mental retardation or chronic psychotropic drug use. The sample was stratified for age (12 discontinuous groups; $30 \pm 1$ years, $35 \pm 1$ years...80 \pm 1 years), sex and two levels of occupational achievement [17]. In total, 1,823 men and women were medically and neuropsychologically examined in the baseline examination between 1993 and 1995 . The group was divided into four panels of approximately 450 individuals each.

Individuals aged over 50 years at the beginning of the study (baseline) were re-examined after three and six years. Individuals aged between 30 and 50 years at baseline were re-examined after 6 years. A blood sample was drawn, allowed to clot under ice-cold conditions and serum was sampled at the end of each day and stored at $-80^{\circ} \mathrm{C}$ until analysis. At the end of 1999 , cognitive data of two or three measurements over 6 years of follow-up were available from a random group of 144 individuals from the panel that was examined in 1993 for the first tirne. A complete set of serum samples, in addition to the cognitive performance data, was avalable from 93 individuals at baseline and from 116 individuals at follow-up. From 65 individuals a complete set of serum samples was available from both time points. An incomplete data set was available from the other individuals due to technical problems. None of the subjects used Bvitamin supplementation.

The mean age of the 93 individuals at baseline was 57 (SD 11). The sex distribution was 55 man to 38 women. The age of the 65 individuals of whom a complete set of serum data was present was lower (mean 54 years, SD 10) compared to the remaining 28 individuals (mean 65 , SD 11, Mann-Whitney $U$ test: $Z=-4.2, P<0.000$ ). The sex distribution was not statistically different $(37$ man to 28 women im the 65 individuals, 18 man to 10 woman in the remaining 28 individuals) (Chi-square, $\mathrm{df}=1: 0.75, \mathrm{P}<0.38 \%$. The performance on the WLTTOT and Delayed Recall at baseline was slighty better (Mann-Whitney $U$ test: $Z=-1.9, P<0.054$ ) in the subgroup of 65 individuals (WLTTOT mean 44 SD 8) compared to the 28 remaining individuals (WLTTOT: mean 39 SD 11). The performance on the other tests was not statistically different between the subgroups.

This study was in accordance with the principles of the Helsinki declaration of 1975 as revised in 1983 and was approved by the local ethics committee. Written informed consent was obtained from all participants. 


\section{Cogninve assessment}

Cognitive function of the healthy individuals in the follow-up part of this study was fested according to the MAAS-protocol described in detail in Chapter 8 and 9 [17,41]. The focus was upon processes involving memory (Word Learning test total (WLTTOT), Delayed Recall), attention (Letter-digit coding test (LDCT) and different aspects of aftention and cognitive processing speed (Stroop).

\section{Biochemical measurements}

Serum total homocysteine concentration was determined using HPLC with electrochemical detection [40].

VitaminB12 and folic acid were determined using commercial kils (Bayer Immuno 1 . Leverkusen, Germanyl. Some serum sample volumes were insuficient to detemine concentrations of all three markers (see ' $\mathrm{n}$ ' in Table 2).

\section{Statistical analysis}

Normal distribution was tested using the Kolmogorov-Sminnov test. Differences in serum concentrations between patients and controls were analysed with ANOVA on the ranks of the data, with Scheffe's posthoc test, and with logistic regression.

Zero order bivariate correlation analysis between all the serum molecules and the cognitive test outcomes was performed using Spearman correlation.

The further analysis was performed in two phases. First, ordinary least-squares multiple regression analysis was performed for the four cognitive tests at baseline with each separate serum parameter as potential predictors and age, sex and level of education as covariates. All variables were continuous, except for sex (two categories) and education (six categorlies).

Secondly, multi-level regression analysis was additionally performed on parameters showing a conelation $(P<0.05)$ with at least two of the cognitive tests at baseline, adjusted for age, sex and educational level. Thus, multi-level repeated measurement analysis was performed to analyse the eventual causal association between homocysteine at baseline and cognitive function during the whole follow-up. This method uses all observations, including the observations of persons with only one or two observations [1, 13]. Persons who were over 50 years of age were included at all three measurement phases. Persons younger than 50 years were included in the first and third phase only. To increase homogeneity regarding age across the different phases, the analyses were redone for persons older than 50 years of age only. Since four different cognitive tests were used as outcomes, a Bonferroni correction was made of the significance level to $P<0.013$. However, as this study is exploratory in nature, results with significance level of $P<0.05$ are also indicated. Data of the regression analysis are expressed as regression coefficient (b) with $95 \% \mathrm{Cl}$.

All analyses were performed using SPSS statistical software, version 9.0.1. KChigaco, lllinois U.S.A.). 


\section{Results}

Serum concentrations of homocysteine, folic acid and witamin $B 12$ in patients with AD. PD and OCD

Several studies showed increased homocysteine concentrations in serum of patients with vascular dementia and AD $[9,26]$. To validate our study, we investigated the serum concentrations of homocysteine in different patient groups and in controls (Table 1).

The median of the homocysteine concentrations was increased in the total group of neurological patients compared to healthy control individuals Z $Z$-value of Kruskal Wallis test: $2.0, P<0.05$ ), but not in one of the patient groups (ANOVA test: $F(3,190)=2.6$ ). However, when the homocysteine concentrations of the AD patients and controls were compared, homocysteine concentrations in the upper $20 \%$ of values was associated with increased risk for being an AD patient compared to homocysteine concentration in the lowest $20 \%$ of values (Odds Ratio: $4.8 ; 95 \% \mathrm{Cl}: 1.0-22.1$ ). This relation disappeared after adjustment for age (Odds ratio: $3.3,95 \% \mathrm{Cl}: 0.7-16.9)$.

The median of the vittamin $B 12$ concentrations was decreased in the total group of neurological patients compared to healthy control individuals ( $Z$-value of Kruskal Wallis test: $-2.9, \mathbb{P}<0.005$ ). No significant difference between the groups could be found after posthoc comparison with Scheffe's test, although the ANOVA was significant (ANOVA F(3, 190)=3.1, P<0.05), which was probably due to the small differences. The Odds Ratio for the lower $20 \%$ percentile compared to the upper $20 \%$ percentile of the vitamin B12 concentrations was $4.3(95 \% \mathrm{Cl}: 1.1$ 17.2). This significance was lost after including age and sex as covariates in the logistic regression model.

The median of the folic acid concentrations was similar in all groups investigated ( $Z$-value of Kruskal Wallis test: $-0.5, P>0.05$, ANOVA $F(3,190)=0.1, P>0.05)$, and no increased risk for being in the AD group was associated with the $20 \%$ percentiles of values (Odds ratio: $0.9 \% 95 \%$ Cl: 0.2-3.4).

Table - Hornocysteine, vitamin B12 and follic acid concentrations in serum of differen patient grou ps. Data are expressed as median $25 \%$ percentite, $75 \%$ percentilez.

\begin{tabular}{|c|c|c|c|c|}
\hline & $\begin{array}{c}\text { Controls } \\
(n-61)\end{array}$ & $\begin{array}{l}\mathrm{MO} \\
(n=34)\end{array}$ & $\begin{array}{l}P D \\
07: 46:\end{array}$ & $\begin{array}{l}\text { OCD } \\
n=47)\end{array}$ \\
\hline $\begin{array}{l}\text { Homachsteine } \\
\text { (umol/f) }\end{array}$ & $\begin{array}{l}16.5 \\
113,3,19.41\end{array}$ & $\begin{array}{l}17.2 \\
(15.1,21.8)\end{array}$ & $\begin{array}{l}18.9 \\
(16.3,23.2 \mathrm{j}\end{array}$ & $\begin{array}{l}17.1 \\
(15.7,19.1)\end{array}$ \\
\hline 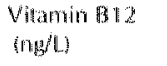 & $\begin{array}{l}405 \\
(340,473)\end{array}$ & $\begin{array}{l}327 \\
(274,406)\end{array}$ & $\begin{array}{l}360 \\
(295,427)\end{array}$ & $\begin{array}{l}347 \\
(288,428)\end{array}$ \\
\hline $\begin{array}{l}\text { Folic alcid } \\
\text { wugh. }\end{array}$ & $\begin{array}{l}4,9 \\
3,5,6,4\end{array}$ & $\begin{array}{l}5.0 \\
3,6,6,4,\end{array}$ & $\begin{array}{l}5.0 \\
(3.7,6.7)\end{array}$ & $\begin{array}{l}4.8 \\
(3.5,5.9)\end{array}$ \\
\hline
\end{tabular}

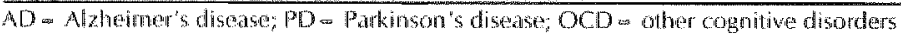

\section{Biochemical measurements in the MAAS}

The results in Table 2 show the serum concentrations of the parameters at baseline and at follow-up measured in the healthy ageing population of the MAAS. The serum homocysteine concentrations were comparable to reference ranges reported by others [33]. The median of the homocysteine concentrations in serum of the healthy controls was lower compared to the walues in the controls presented in Table 1, which were obtained and analysed at a different time point. The vitamin $\mathrm{B} 12$ and folic acid concentration were comparable to the reference ranges of the supplier of the ELISA-kits. 
Table 2 Concentrations of serum markers in the population of the MAdS.

Data are expressed as median $25 \%$ percentile, $75 \%$ percentilel.

\begin{tabular}{|c|c|c|c|c|c|}
\hline & & \multicolumn{2}{|c|}{ baseline } & \multicolumn{2}{|c|}{ follow- } \\
\hline & & $n$ & concentration & $n$ & comcentration \\
\hline \multirow[t]{2}{*}{$\begin{array}{l}\text { Homocysteine } \\
\text { (pmonolfu) }\end{array}$} & all indivicluals & 93 & $\begin{array}{l}130 \\
12,7,15,9\}\end{array}$ & 115 & $\begin{array}{l}14.3 \\
(12.4 .16 .9)\end{array}$ \\
\hline & ower 50 years old & 78 & $\begin{array}{l}13.2 \\
(12.1,86.0)\end{array}$ & 100 & $\begin{array}{l}14.4 \\
112.5,16.91\end{array}$ \\
\hline \multirow[t]{2}{*}{$\begin{array}{l}\text { Visamina } B 12 \\
\text { (mg/L) }\end{array}$} & all indivictuals & 92 & $\begin{array}{l}406 \\
(3 \times 1,470)\end{array}$ & 114 & $\begin{array}{l}396 \\
(327,463)\end{array}$ \\
\hline & over 50 years old & 78 & $\begin{array}{l}400 \\
341,470\end{array}$ & 99 & $\begin{array}{l}384 \\
(327,466)\end{array}$ \\
\hline \multirow[t]{2}{*}{$\begin{array}{l}\text { Folic acid } \\
\text { (rgg/t) }\end{array}$} & all individuals & 92 & $\begin{array}{l}3.9 \\
2.9,49)\end{array}$ & 111 & $\begin{array}{l}5.0 \\
03,0,6.71\end{array}$ \\
\hline & over 50 years old & 78 & $\begin{array}{l}3.8 \\
12.8,4.81 \\
\end{array}$ & 96 & $\begin{array}{l}4.8 \\
0.5,6.7)\end{array}$ \\
\hline
\end{tabular}

\section{Zero order correlation analysis at baseline in the MAAS}

The results of the correlation analysis of serum homocysteine concentration with the cognitive test outcomes are shown in Table 3 and in Fig. 1.

The serum homocysteine concentration was negatively correlated with the outcomes on the immediate recall (WLTTOT)(Fig. 1A) and the Delayed Recall (Fig. 1B) of the Word Learning test at baseline. This result indicates that the performance on these tests was worse when serum homocysteine concentrations were higher. No significant correllation was observed between serum homocysteine concentrations and performance on the Stroop test or the LDCT in all individuals at baseline (Fig. $1 \mathrm{C}$ and D). No correlation between homocysteine concentration and performance on the WLTTOT and Delayed Recall test only was observed at follow-Up. A positive correlation was observed between serum homocysteine concentrations and the outcomes on the Stroop test at follow-up, indicating worse performance when homocysteine concentrations are higher at this time-point.

No correlation was observed between the serum vitamin B12 concentration and the performance on the cognitive tests at baseline, nor at follow-up.

The serum folic acid concentration correlated positively with performance on the Delayed Recall test at baseline, which indicates that relatively high concentrations of folic acid wert associated with better perfomance at baseline only. No correlation of the folic acid concentration with any of the other cognitive test outcomes was observed at baseline; or at follow-up.

The results obtained for the subgroup of subjects aged 50 years or older were of similar magnitude and significance as the results described above and thus not funher described in detaill. 


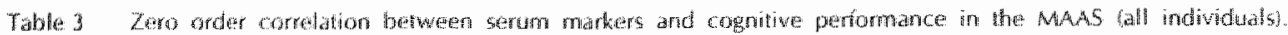

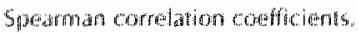

\begin{tabular}{|c|c|c|c|c|c|c|}
\hline & & n & Stroop & $\mathrm{LOCI}$ & WhTrOT & Deldyed lecall \\
\hline $\begin{array}{l}\text { Whan score it So } \\
\text { lronges }\end{array}$ & buseline & 93 & $\begin{array}{l}99 \pm 27 \\
(61,2258\end{array}$ & $\begin{array}{l}47.3 \pm 10.4 \\
20.71 !\end{array}$ & $\begin{array}{l}42.6 \pm 9.1 \\
(17.611\end{array}$ & $\begin{array}{l}8.9 \pm 3.0 \\
(2,35)\end{array}$ \\
\hline \multirow[t]{2}{*}{ Homorysteme } & boseline & 93 & 0.146 & -0.154 & $-0.258^{*}$ & $-0.301 * *$ \\
\hline & follow up & 115 & $0.221 *$ & -0.162 & -0.159 & .0 .029 \\
\hline \multirow[t]{2}{*}{ Wasumin } & busetine & 92 & -0.168 & $0: 031$ & 0.075 & 0.055 \\
\hline & follow-up & 114 & -0.1102 & 0.09 & 0.000 & 0.011 \\
\hline \multirow[t]{2}{*}{ Folic acid } & braseling & 92 & 0.1126 & 0.085 & 0.037 & 0.214 \\
\hline & followups. & 111 & -0.066 & 0.042 & 0.080 & -0.151 \\
\hline
\end{tabular}

$*: P<0.05 ;: P<0.01 .: P<0.013$

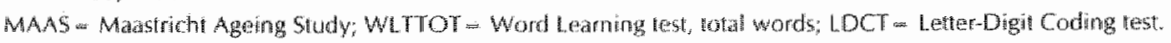

A

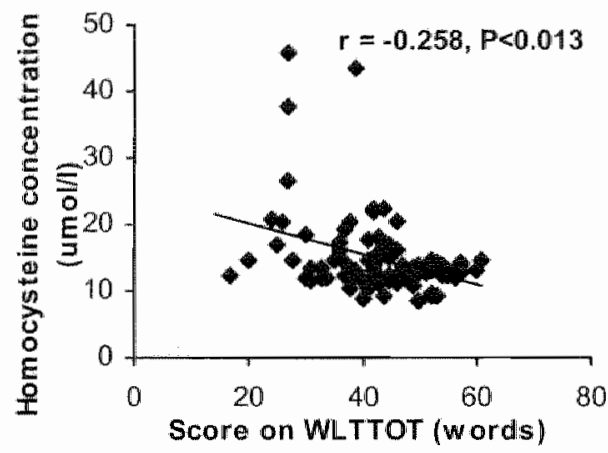

C

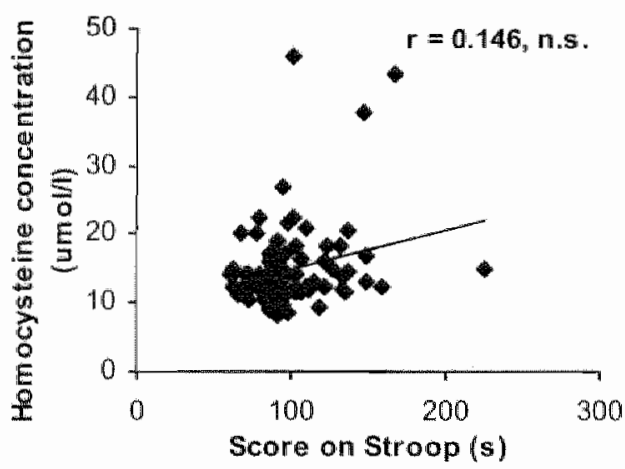

B

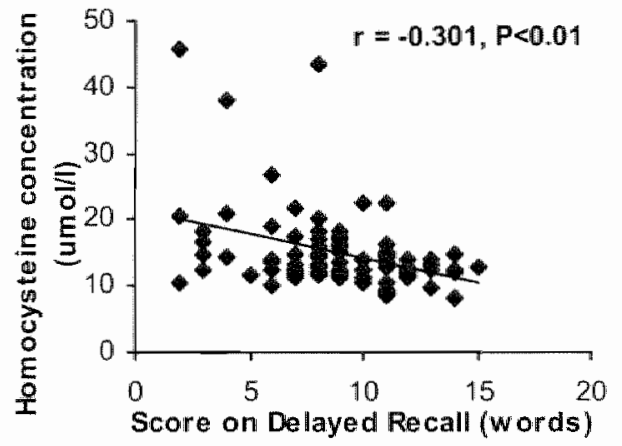

D

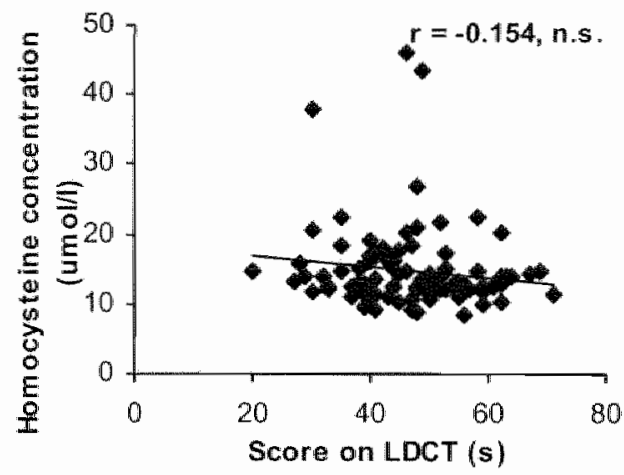

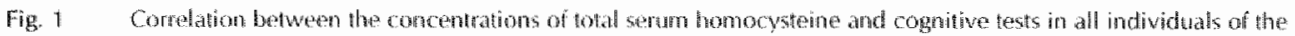
MAAS.

The homocystrine concentration was plotted against the scores on cognitive test at baseline. Spearman correlation anficients.

A. Significant negutive corteltion behween homocystene and pertomance on the WLTTOT.

B: Significan negative corratation beween homogstene and pertormance on the Delayed Recall test.

C: Lack of significant confation betaken homocysteine and performance on the Stropp test.

D: Lack of signifaul correlation betwe thomocysteme and performance on he LDCT. 


\section{Correlation between cognitive performance and serum makkers at baseline and follow-up adjusted for age, sex and education in the MAAS}

The correlation between serum homocysteine concentrations and the scores on the cognitive tests adjusted for age, sex and education was analysed using multiple regression analysis (Table 4).

Serum homocysteine concentrations correlated negatively with performance on the WLTTOT and Delayed Recall after adjustment for age, sex and education at baseline, which is in line with the results of Table 3. However, these correllations were absent at follow-up. No correlation of the serum homocysteine concentrations and performance on the Stroop test or the LDCT was observed at baseline or at follow-up.

No correlation was observed between serum witamin B.12 concentration and any of the cognitive test outcomes.

The serum folic acid concentration correlated positively with performance on the Delayed Recall test at baseline after adjustment for age, sex and educationall level. The correlation of folic acid concentration with the other cognitive test outcomes did not reach significance at baseline or follow-up.

Recently, Morris et al. [28] reported that serum follic acid concentration wats a confounder of the relation between serum homocysteine concentrations and perfomance on a memory test in a cross-sectional study including about 1,200 man and woman aged 60 years and older. Therefore, we investigated if serum folic acid concentration was also a confounder in our study. We were not able to confirm this finding as the regression coefficients between homocysteine and the WLTTOT as well as Delayed Recall were not altered after including serum folic acid concentrations in the regression model (data not shown).

The influence of the outliers and influential cases on the results was examined, using the studentised residuals and Cook's distances in the normal hierarchical regression anallysis. In this way, maximal three individuals were excluded from the analyses. The correlations shown in Table 3 were not modified after exclusion of these observations.

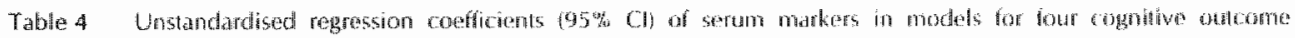

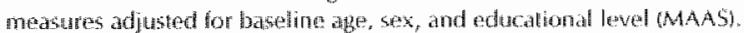

\begin{tabular}{|c|c|c|c|c|c|c|}
\hline & & n & Stroop & $\triangle D C T$ & Whrot & Delangal Rencill \\
\hline \multirow[t]{2}{*}{ Homocysteine } & Disseline & 93 & $\begin{array}{l}0.47 \\
(10.37,1.31)\end{array}$ & $\begin{array}{l}0.11 \\
(\times 0,19,0,41)\end{array}$ & $\begin{array}{l}0.977^{4} \\
\{-0.67,-0.08\}\end{array}$ & $\begin{array}{l}-0.12^{*} \\
(-0.22,-0.02)\end{array}$ \\
\hline & follow-up & 115 & $\begin{array}{l}-0.56 \\
(-2.05,0.94)\end{array}$ & $\begin{array}{l}0.19 \\
(-0.19,0.57\end{array}$ & $\begin{array}{l}(0), 1 \\
(-10,94,0,41)\end{array}$ & $\begin{array}{l}0.10 \\
10.01,0.221\end{array}$ \\
\hline \multirow[t]{2}{*}{ Witanin $B 12^{=}$} & baseline & 92 & $\begin{array}{l}4.24 \\
(-22.92,31.39)\end{array}$ & $\begin{array}{l}-7.66 \\
(-17.19,1.86)\end{array}$ & $\begin{array}{l}0.70 \\
(-8,93,10) 331\end{array}$ & $\begin{array}{l}0.21 \\
-3,46,3,041\}\end{array}$ \\
\hline & follow-up & 114 & $\begin{array}{l}-0.69 \\
(-32.75,31.37)\end{array}$ & $\begin{array}{l}-3.26 \\
-11.47,4.95 \%\end{array}$ & $\begin{array}{l}-17 \\
(-9.20,6.93)\end{array}$ & $\begin{array}{l}0.25 \\
(2.75,2.29)\end{array}$ \\
\hline \multirow[t]{2}{*}{ Folic acid } & baseline & 92 & $\begin{array}{l}-2.31 \\
(-4.98,0,37)\end{array}$ & $\begin{array}{l}0.54 \\
(-0.42,1.51\}\end{array}$ & $\begin{array}{l}0.34 \\
10.63,1.30 \%\end{array}$ & $\begin{array}{l}0.32 \\
(0.01,0.64)\end{array}$ \\
\hline & follow-tup & 111 & $\begin{array}{l}-0.27 \\
(3.03,2.50)\end{array}$ & $\begin{array}{l}0.05 \\
10.74,0.05\end{array}$ & $\begin{array}{l}-0.47 \\
0.4,4.4,0.21\end{array}$ & $\begin{array}{l}0.16 \\
(-0.37,0.05) \\
\end{array}$ \\
\hline
\end{tabular}

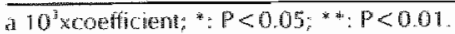

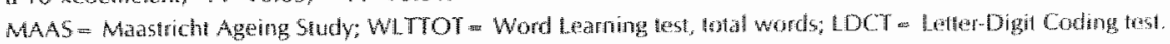


Correlation of serum marker concentration at baseline with cognitive performance during 6 years of follow-up in the MAAS, adiusted for age, sex and education

We investigated the relation between the serum concentrations of homocysteine at baseline and (change in) cognitive performance over the whole follow-up period (Table 5), to investigate an eventual risk modulating role of elevated homocysteine concentrations at baseline. Multi-level repeated measurement analysis allows the simultaneous examination of several observations per person this increases the power of the study. Furthermore, multi-level analysis allows different numbers of observations between persons $[1,13]$.

The results in Table 5 show that the serum homocysteine concentrations at baseline correlated positively with test scores on the stroop test over six years of follow-up. The serum homocysteine concentrations at baseline correlated negatively with test scores on the WLTTOT and Delayed Recall test over the six years follow-up period. These results indicate prolonged relative worse performance when thomocysteine concentrations are high at baseline.

There were no significant differences between persons in the course of cognitive function during follow-up. Interactions between longitudinal time and the three biomarkers, i.e. modeling a risk for decreased cognitive performance related to homocysteine concentration, were therefore not investigated further.

Table 5 Unstandardizad regression coefficient5 $(95 \% \mathrm{Cl}$ ) of serum homocysteine concentration at baseline in models hr four cognitive outcorne measures during six years of follow up, adjusted for baseline age, sex, and educational level MAAS using multiblevel repeated meastrement analysis "

\begin{tabular}{|c|c|c|c|c|c|}
\hline & $\begin{array}{l}\text { obserwations } \\
\text { (n) }\end{array}$ & Stroop & $\mathrm{IMPCT}$ & WLTTOT & Debyed Recall \\
\hline All individuals & $\begin{array}{l}254 \\
(93)\end{array}$ & $\begin{array}{l}0.76 \\
(-0.00,1.52)\end{array}$ & $\begin{array}{l}0.04 \\
(-0.25,0.33)\end{array}$ & $\begin{array}{l}-0.36 \\
(-0.61,-0.11)\end{array}$ & $\begin{array}{l}-0.14 * * \\
(-0.22,-0.06)\end{array}$ \\
\hline
\end{tabular}

\footnotetext{
:P<0.05:*: $P<0.0 \%$
}

a) Al pirsons participated in at least wo of the thee measurenent phases during the six-year follown inter-indivictual random watation in the intercepts of the association between longitudinal time and cognitive function was significant in all analyses. Interindividual random variation in the sloper of this association was, however, never statistically significant. This indicates, that persons direred in cognitive function a baseline (first phase), but there were no significant differences between persons in the course of cognitive function during follow-up. Interactions between longitudinal time and the three biomarkers, i.e. modelling a diferential longitudinal course of cognitue function related to the biomaters, were therefork not investigated lusther.

\section{Discussion}

The aim of the present study was to investigate whether a possiblle causal relation exists between serum concentrations of homocysteine and cognitive performance in a normal healthy group of ageing individuals. In view of the lack of correlation with cognitive performance for witamin $B 12$ and folic acid in some epidemiological studies $[18,21,23,24]$, we hypothesised that serum homocysteine concentrations were more significantly correlated to cognitive performance during extended periods of normal ageing than vitamin B12 or folic acid. First, we investigated the concentrations of these serum parameters of patients with $A D, P D, O C D$ and in an age and sex matched group of nomal healthy controls. Secondly, we investigated serum concentrations of these parameters in a healthy ageing cohort. Individual cognitive performance on tests addressing information processing speed, learning and memory were repeatedly examined in a healthy ageing population over a follow.up period of six years.

Elevated homocysteine concentrations have been observed in several, but not all studies on demented patients $[9,26,32]$. For example, in demented centienarians, no increased 
homocysteine concentration was obserwed [32]. In fact, we also did not observe increase in median serum homocysteine concentrations, although the upper $20 \%$ percentle was associated with a 4.8 times higher 'risk' of being in the AD group compared to the lowest $20 \%$ percentile. The results showed that serum homocysteine concentrations were more strongly related to cognitive performance than vitamin B12 and folic acid concentrations in the normal ageing population of the MAAS, as we observed only one spurious correlation between cognitive performance and the B-vitamins. Results from several previous studies are in line with our findings $[18,23,24,34]$. In some studies a positive correlation between the B-vitamins and cognitive performance in an ageing population was obsened, though less strongly than the negative correlation of homocystene concentrations with cognitive perfomance $[34]$. It is conceivable that such weak relations do not reach significance in a study with a retatively small number of observations as our study. In the study of Riggs et al. [34] only male subjects were included. As there are sex differences in homocysteine concentration 1339 as well as in the performance of our cognitive test [17], it may be possible that the relation between homocysteine and cognitive performance is influenced by sex in the ageing population. We included sex as a covariant in the model and still observed a relation bewween homocysteine and learning and memory as lested with the WLTTOT and Delayed Recall test (Table 4 and 5 . This and the fact that the results in the subgroup of older individuals were similar in magnitude as the results in the total group, suggest that high homocysteine concentrations may be a makter for lower cognitive performance in the general population, as was suggested by previous studies $[8,28]$.

The negative correlation between homocysteine concentration and cognitive performance on the WLTTOT or Delayed Recall test were not present at follow-up. This variation may be due to different population characteristics of the subjects of whom a serum sample was available at follow-up compared to those on baseline. There were two serum samples available from only 65 individuals at both measurements of the total group. This subgroup was similar with respect to cognitive performance but cliffered from the other individuals at baseline on mean age, as described in the method section. Since age may not be a contounder, as suggested above, other unknown parameters may be important.

We tested if folic acid was a confounder of the correlation between homocysteine and cognitive performance. Recently, Morris el al. [28] investigated several possible confounders for the relation between homocysteine and tests for learning and verbal memory in a large population of 1,200 individuals [28]. They also found a negative correlation between homocysteine and both learning and verbal memory, which is in line with our results. From all possible confounders tested, including vitamin $C$ and $E$, total cholesterol, blood pressure and metalls, only folic acid appeared to be a real confounder in their population. The negative correlation between serum homocysteine concentration and the WLTTOT and Delayed Recall at baseline was not contounded by folic acid in our study. Thus, although elevated homocysteine concentrations may be a marker for folic acid or vitamin B12 deficiency, the negative correlation beween homocysteine and cognition was not mediated by folic acid in our study.

Our observations stress the difficulty in drawing conclusions about significance of increased homocysteine concentrations in relation to cognitive performance. Several arguments may argue against a role of increased homocysteine concentration as a risk factor for dementia. First, mildly increased concentrations of homocysteine have been observed in non-pathological cognitive impaiment, as shown by our results, and thus not only in several foms of pathological cognitive impairment [22]. Not all individuals suftering from mild cognitive impairment do develop dementia [37]. Another argument is the fact that the prevalence of dementia was not increased in patients with a mutation in the methylenctetralnydrofolate reductase gene, who have chroniccally mildly increased serum concentration of homocysteine 
[15]. Atthough cognttive problems are common among patients with a mutation in the gene coding for cystathionine $\beta$-synthase, it must be realised that this presents a condition where much higher tomocysteine concentrations occur $[25,29]$. Thus, a causal relationship between mildy elevated homocysteine concentrations and cognitive decline is not yet proven.

Our data cannot elucidate whether elevated homocysteine concentrations are a risk factor for cognitive decline or not. This is probably due to the lack of intra-individual differences in cognitive declime during the follow-up period in our individuals. Similar lack of evidence for homocysteine as a risk factor for cognitive decline was observed in a larger group of individuals investigated in the Rotterdam study $[19]$. However, these authors correlated the plasma homocysteine concentration to the scores on the MMSE, which may not be the most sensitive test to monitor differences in cognitive performance during non-pathological ageing. We observed that individuals with a high homocysteine concentration at baseline were relatively worse performers on the verbal leaming tests (WLTTOT and Delayed Recall), aspects of memory that are affected in early stages of dementia [5]. Furthermore, these individuals remained worse performers over a total period of six years of follow-up. These results may imply that homocysteine concentrations are a state marker for decreased cognitive performance.

In summary, although the small sample size and variable results warrants cautious interpretation of our results, we found that serum homocysteine concentrations correlated negatively with aspects of cognitive performance, independent of serum folic acid or vitamin B 12 concentration. Furthermore, elevated homocysteine concentrations were related to low cognitive performance over an extended period in the normal ageing population. Taken together with the known risk for cardiovascular disease associated with high homocysteine concentrations and the increasing evidence of the involvement of vascular abnormalities in the pathology of AD [31,38], the present results point to increased homocysteine concentrations as a possible risk factor for $A D$.

\section{Acknowledgement}

The authors gratefully acknowledge the financial support by "Van Leersum fonds" of the Dutch Royal Academy of Sciences.

\section{References}

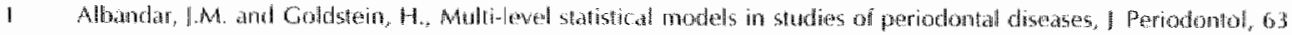
$(1992) 6905$

2 Alkn, R,H. Stabler, S.P. and Lifndenbaum, I., Relevance of vitamins, homochateine and other moabolites in

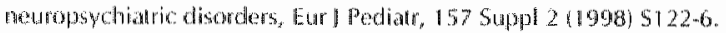

3 Axettrod, I., Methylation reactions in the formalion and metabolism of callecholamenes and other biogenic amines, Phamacol Reve, 18 (1966) 95-113.

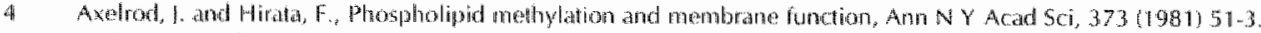

5 Dackman, L. The liak between knowledge and remembering in Alzheimers disease, Scand I Psychol, 39 (1998) $131.9 \%$

6 Blom. H. Genetic determirants of hyperhomocystemaemia: the roles of cystat inonine beta-sunthase and 5,10 -

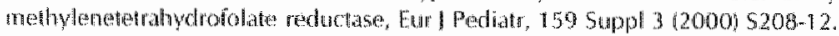

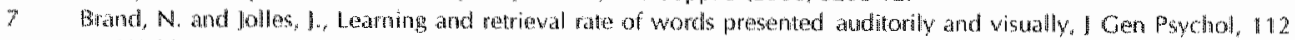
(1985) $201-10$.

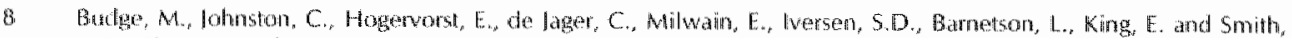

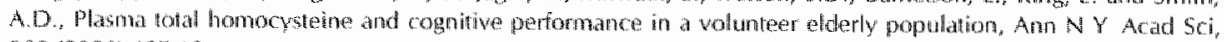
$9032000 \% 407-10$.

9 Clakke, R., Snith, A.D., Jobst, K.A., Refsum, H., Sulton, L. and Weland, P.M., Folate, vitamin B12, and serum total homogsteine levels in confirmed Azheimer disease, Aroh Neurot, 55 (1998) 1449-55. 


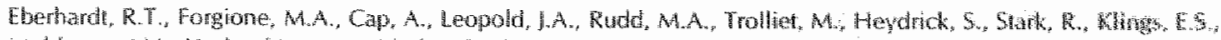

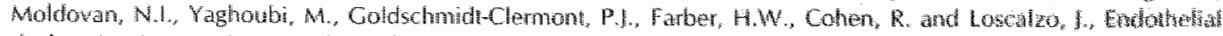

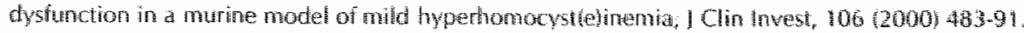

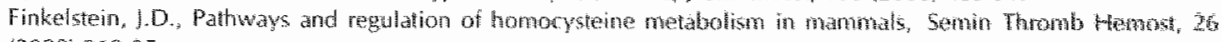
(2000) $219-25$.

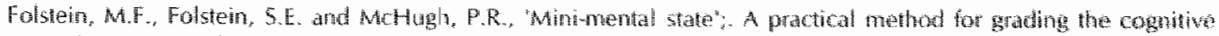
state of patients for the clinician. I Psychiatr Res, $12(1975189.98$.

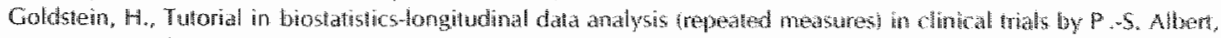
statustics in medicine, 1999, 18, 1707-1732, Shal Med, $19(2000) 1821$

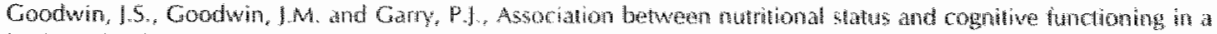

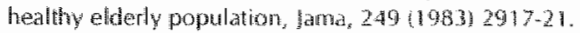

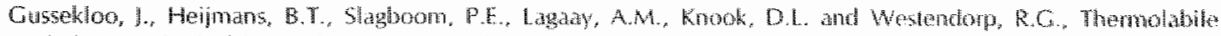

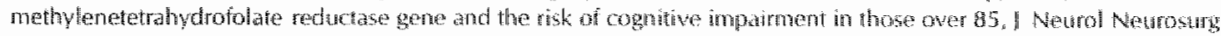
Psychiakry, 67 (1999) 535-8.

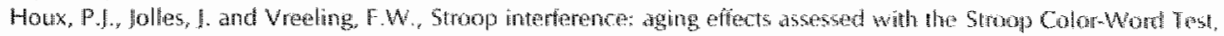
Exp Aging Res, $19: 1993,20924$.

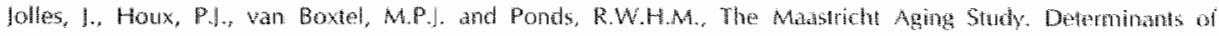
Cognitive aging, Neuropsych Publishers, Maastricht, 1995, $192 \mathrm{ph}$

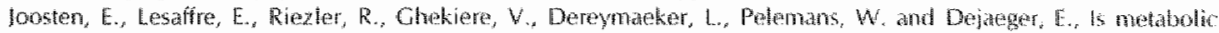

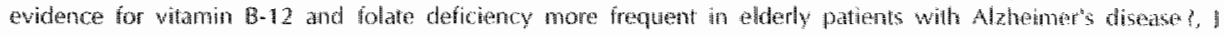
Gerontol A Biol Sci Med Soi, $52(1997)$ M76-\%

19 Kalmür, S, Latner, L.J, Lindemans, J., Bots, M.L., Holman, A. and Brefeler, M.M., fotal homocysteino and cognelive decline in a commonitybased sample of elderly subjects: the Rotterdam Study, Am I Epidemiol, 150 (1999) 2839 .

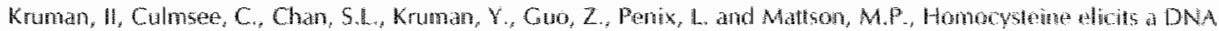

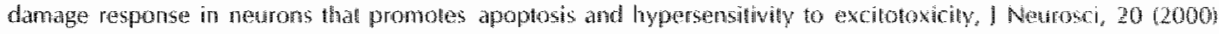
6920-6

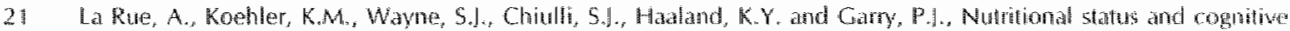
functioning in a nomally agging sample: a 6-y reassessment, Am J Clin Nutr, 65 (1997) $20-9$

Lehmann, A., Giotthites, C.G. and Reghand, B., Identification of cognitive impairment in the elderly: homocystane is an early marker, Dement Geriatr Cogn Disond, 10 (1999) 12-20.

23 Levitt. A.J. and Karlinsky, H., Folate, vitamin B12 and cognitive impationent in patients with Alzheimer's disease, Acta Psychiate 5rand, $86(1992) 301-5$.

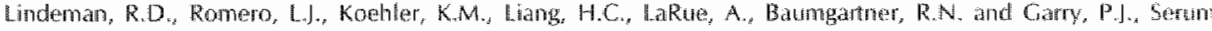

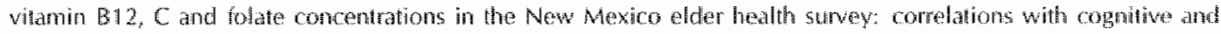
affective functions, I Am Coll Nutr, $19(2000) 68-76$.

Manson, M.A. Lelland, P.M., Aarsland, A. and Svardal, A.M., Redox status and protein bincling of plasma

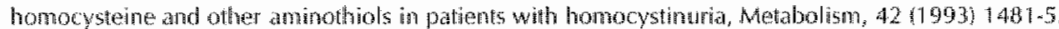

MCCaddon, A, Dawines, G., Hadson, P., Tandy, S and Cathell, H., Total serum homocysteine in senile dementia of Aheimer type, Int J Geriatr Psychiatry, 13 (1998) $235 \%$

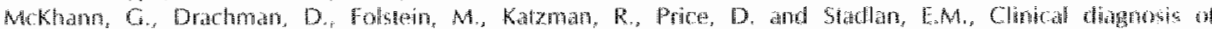

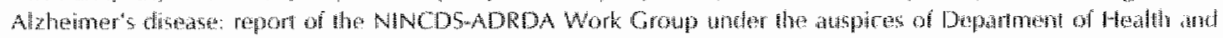

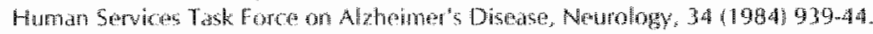

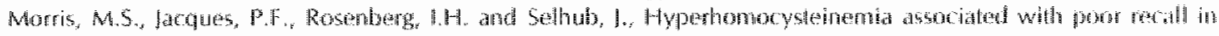
the thard National Health and Nutrision Eamination Survey, Am \& Chin Nutr, 73 2001) 927.33

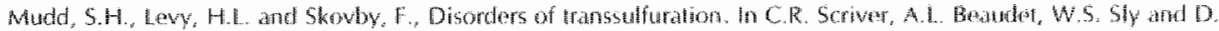

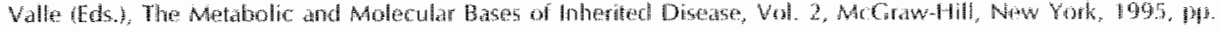
$1279-1327$

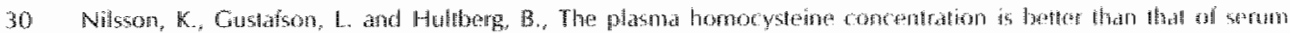

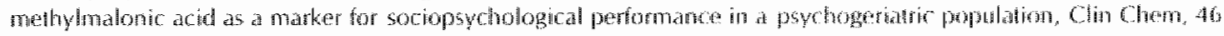
(2000) 691-6

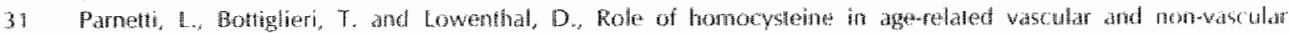
disedses, Agrig (Mileno), 91997241.57

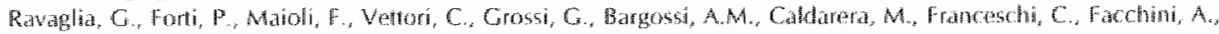

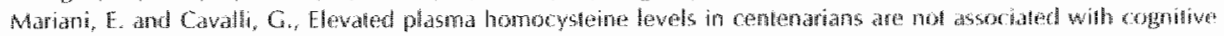
impaiment, Medr Ageng Dev, $12120001251-61$.

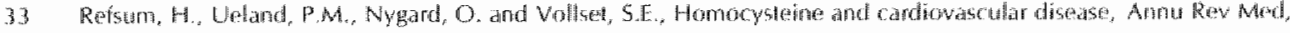
$491998131-62$

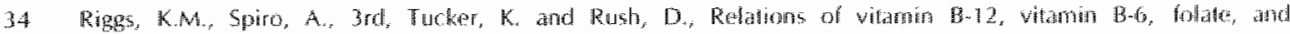

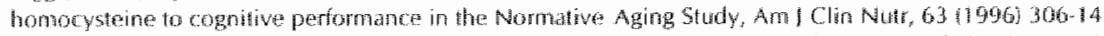

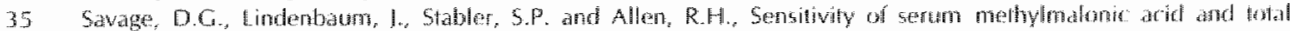

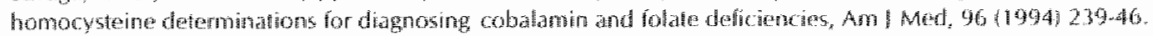

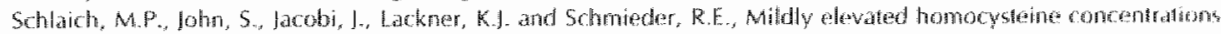

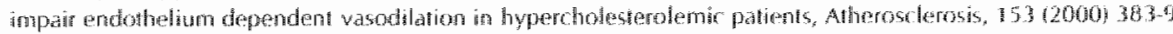




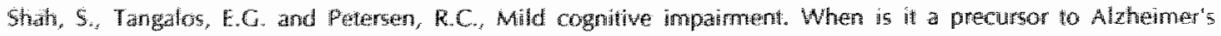
disease?, Ceriatrics, $5512000762,65-8$.

36. Skoog, 1., Kalaria, R, and Breteler, M.M. Vascular factors and Alzheimer disease, Azheimer Dis Assoc Disord, 13 suppl $3(1999) 5106-14$.

39 Smith, A, The Swmbol Digit Madtalthes Test: A. Neuropsychological test for economic screentmg of learning an other cercabral disorders., Learring Disorders, 3607968$)$ 83-91.

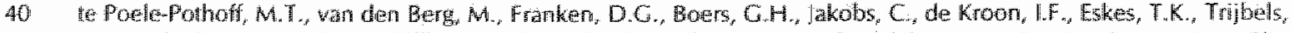
I.M. and Blom, H.J. Three different methods bor the determiration of fotal homocysteine in plasma, Amn Clin Biochem, 3209951820.20 .

41 van Boxtel, M.P., Gatland, C., Houx, P.,. Buntinix, F., de Leeuw, P.W. and Jolles, J., lis nondipping in 24 h ambulatory blood pressure restated lo cognitive dystunction?, J Hypertens, 16 (1998) 1425-32. 


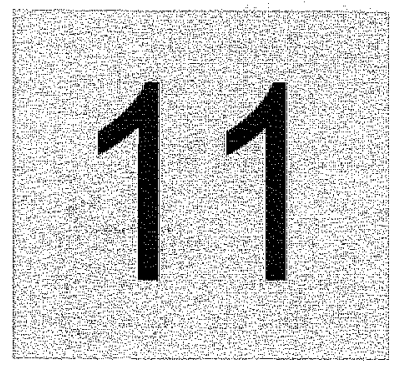

\section{Evaluation of 29 serum markers in patients with $A D, P D$ and other cognitive disorders}

C.E. Teunissen, ${ }^{a} \mathrm{D}$. Lütjohann, ${ }^{\mathrm{a}} \mathrm{K}$. von Bergmann, ${ }^{\mathrm{b}} \mathrm{F}$. Verhey, ${ }^{\mathrm{b}} \mathrm{F}$. Vreeling, 'A. Wauters, 'E. Bosmans, *H. Bosma, *M.P.J. van Boxtel, *M. Maes, "J. Delanghe, 'H.J. Blom, ' ${ }^{M}$ M.M. Verbeek, 'P. Rieckmann, * ${ }^{*}$. De Bruijn, *H.W.M. Steinbusch, *I. de Vente.

European Graduate School of Neuroscience (Euron), Universiteit Maastricht, Department of Psychiatry and Neuropsychology, The Netherlands

"Department of Clinical Pharmacology, University of Bonn, Germany.

${ }^{\circ}$ Academic Hospital Maastricht, The Netherlands.

'AZ Middelheim, Antwerpen, Belgium

"Diamed Eurogen S.A., Tessenderlo, Belgium.

"Laboratory of Clinical Chemistry, University Hospital Gent, Belgium

'Laboratory of Pediatrics and Neurology, UMC Nijmegen, The Netherlands

s Department of Neurology, UMC Nijmegen, The Netherlands

"Department of Neurology, University of Würzburg, Germany.

Submitted to Neurology 


\section{Abstract}

Alzhemer's disease (AD) probably involves several pathobiochemical mechanismo. We investigated whether the different disease mechanisms in $A D$ may be reflected by a pattern of alterations in serum markers and that combination of such markers might lead to the development of an in vitro diagnostic test for $A D$ at an early stage.

Serum of patients with $A D(n=34)$, Parkinson's disease (PD) $(n=46)$, other cognitive impaiments $(n-47)$ and healthy controls $(n-61)$ was analysed for several markers.

Serum concentrations were determined of 1) eleven markers related to inflammation; 2) twelve sterol intermediates and plant sterols; 3) two brain-specific proteins; 4) four metabolites and witamins of the methionine pathway.

The combination of decreased concentrations of lanosterol and IL-6 receptor and increased concentration of cysteine improved the discrimination between $A D$ patients and healthy controls compared to the discrimination by one of these markers alone. The combination of the marker concentrattions in healthy controls did not correlate with cognitive autcomes on tests for learning, memory and infomation processing speed, which may imply that the model is only applicable for palients.

In conclusions, we show that the complex pathology in $A D$ is reflected in serum. In some patients evidence of only one pathobiochemical mechanism may be detected, while in other patients a combination of different disease mechanisms is detectable. The decreased phytosterol concentrations in serum of patients with Parkinson's disease calls for closer attention to the dietary habits of these patients and further investigation of their sterol metabolism.

\section{Introduction}

Neurodegenerative diseases of the elderly including AD and PD pose an increased burden on our ageing society. The definite diagnosis of $\mathrm{AD}$ is based on clinical examination, neuropsychological testing and postmontem histological examination of the brain. No diagnostic test based on body fluids such as CSF, serum or plasma assays is available to diagnose $\mathrm{AD}$ patients. There is evidence that several serum molecules are elevated in at least a subgroup of $A D$ patients compared to controls. For example, serum concentrations of interleukin-6 (IL-6) were increased in AD patients compared to controls $[25,29,32]$, though these findings were not replicaled by other studies $[4,48]$. Several studies have shown that the serum concentration of the oxidised cholesterol metabolite, 24S-hydroxychollesterol (24S-OHChol), correlated negatively with severity of cognitive impairment in AD patients, i.e. the lowest concentrations were observed in the most affected patients $[31,42]$. 245-OH-Chol is probably a brain specific cholesterol metabolite since it is almost exclusively derived from the brain. It is synthesised from cholesterol by an enzyme of the cytochrome P450 superfamily, the NADPHdependent cholesterol 245-hydroxylase (Cyp46) [30]. In addition, increased plasma homocysteine levels have been observed in AD patients compared to normal controls [7,35]. The combined measurement of amyloid $\beta(A \beta)$ and tau protein concentrations in cerebrospinal fluid (CSF), the main constituents of the characteristic plaques and tangles in sporadic AD patients brains, has yielded an improved tool to differentiate AD patients from patients with vascular dementia or PD $[3,14,26]$. However, measuring plasma $A \beta$ ( $A, \beta 40$ and/or $A \beta 42)$ has so far not been successful for diagnostic purposes. Thus, some studies found no difference between plasma $A B(1-42)$ and $A \beta(1-40)$ concentrations of sporadic cases of $A D$, compared to controls $[20,45]$. However, in another study plasma $A \beta(1-42)$ and $A \beta(1-40)$ were measured in 96 normal healthy individuals. Individuals with $A \beta(1-42)$ concentrations in the upper two 
quartiles had a three to four fold increased risk at developing AD companed lo persons in the lowest quartile [34].

in all these reports, a large overlap in marker concentrations between AD patients and nomal volunteers was evident. In addition, no sufficient sensitivity and specificity for discriminating $\mathrm{AD}$ patients from controls or patients with other cognitive disease has been obtained for the above serum or plasma measurements [49]. Most studies focussed on one or more markers related to one specific disease mechanism. However, the pathology of AD probably involves several disease mechanisms, e.g. inflammation [10], altered cholesterol metabolism $[12,33]$, plaque formation $[15,21]$ and antioxidant status [41]. In view of this varieg of disease mechanisms in AD, we hypothesise that the heterogeneous pathophysiology of $\mathrm{AD}$ may be reflected in serum. This implies that a combination of markers related to differen mechanisms might improve the discrimination between AD patients, patients with other neurological and/or neurodegenerative diseases, and controls. In the present study, we aimed at identifying patterns of alterations in several serum marker concentrations in relation to AD. For this purpose, we investigated markers related to several mechanisms in serum of patients with AD and of healthy controls. Patients with PD or other cognitive disorders (OCD) were included to examine whether alterations observed were common for neurodegenerative disorders in general or specific for $\mathrm{AD}$.

The serum markers were selected based on the following mechanismis involved in neurodegeneration: inflanmation, formation of the characteristic plaques in AD, vitamin status and altered cholesterol homeostasis. In addition, serum markers had to be independent of dietary and circadian thythms. We thus analysed the sera for: 1) inflammation markers: interleukin-6 (LL-6), HL-6 receptor (LL-6 recepton), Clara cell protein 16 (CC16, an inflammation modulator [9]), C-reactive protein (CRP), protein fractions (including albumin) and haptoglobin: 2) markers of brain specific damage ICAM-5 (telencephalin) and glial fibrillary acidic protein (GFAP); 3) homocysteine, cysteine, vitamin B12 and follic acid. 4) In addition, we determined the serum concentrations of several markers for cholesterol homeostasis: cholesterol, the cholesterol precursors desmosterol "lathosterol, lanosterol, the cholesterol metabolites $245-\mathrm{OH}$ Chol, 27-hydroxycholesterol (27-OH-Chol) and cholestanol and the diet-derived plant sterols avenasterol, brassicasterol, campesterol, sitosterol , and stigmasterol.

\section{Methods}

\section{Patients}

Patients aged over 50 years visiting the oulpatient departments of Psychialtry (memory clinic) and Neurology of the Academic Hospital Maastricht were included. Diagnosis of $A D(n=34)$ Was based on the criteria for probable AD according to general accepted criteria (NINCDSADRA and DSM-IW) [1,38]. Diagnosis of PD (n-47) was based on UK PD Society Brain Bank Clinical Diagnostic Criteria. Diagnosis included a dinical examination, i.e. medical history. neurological, somatic and neuropsychiatric status, and routine blood tests. A computed tomographic scan was performed to identify white matter lesions, cortical atrophy, infarcts and lacunar infarcts in $A D$ and patients with other cognitive disorders (OCD). Patients with clinical signs and symptoms of an inflammation process were excluded. The OCD patients (n 47 ) were diagnosed as having cognitive disturbance (DSM oode 294.9)( $\mathrm{n}=14 \mathrm{l})$, mild cognitive impaiment $(n=6)$, vascular dementia $(n-7)$, dementia non-specified $(n-5)$, fronto-temporal dementia $(n=4)$, depression induced dementia $(n=4)$, primary aphasia $(n=4)$, brain fumour $(n=1)$ and unknown $(n=2)$. The term "all neurological patients" is used in further text to indicate all patients, i.e. with AD, PD or OCD. A mini mental state examination (MMSE) Score 
[13] was obtained from $33 \mathrm{AD}$ patients, 44 OCD patients and $27 \mathrm{PD}$ patients as pant of the diagnostic work-up.

Serum of sex and aged matched healthy controls $(n=61)$ was obtained from participants of an onugoing population based study [22]. These individuals were also aged $50-80$ years, and the sex distribution was similar to the patient groups. The blood sampling protocol for this group was similar to the patient samples; i.e. serum was collected after $30-60$ min clotting at room temperature. A MMSE score wats obtained from the controls at the time of blood sampling.

The study was in accordance with the principles of the Helsinki declaration and approved by the local ethics committee. Written informed consent was obtained from all individuals or responsible caretakers.

\section{Normal healthy individuals from a follow-up study}

Serum was also obtained from healthy individuals that were participants of a longitudinat follow-up study as part of the MAastricht Ageing study (MAS). The MAAS is a larger research program investigating determinants of cognitive ageing in the healthy population [22,47]. Individuals for this study were randomly drawn from the Registration Network Family Practices, a research database that contains basic health information of patients in primary care facilities (Metsemakers, Höppener, Knotnerus, Kocken \& Limonard, 1992). Exclusion criteria were clinical evidence of past or present morbidity that can compromise cognitive performance, such as cerebrovascular disease, chronic neurological pathology, mental retardation or chronic psychotropic drug use. The sample was stratified for age $(12$ discontinuous groups; $30 \pm 1$ years, $35 \pm 1$ years, ...80 \pm 1 years), sex and two levels of occupational achievement [22]. Cognitive function was tested according to the MAAS-protocol described in detail elsewhere $[22,47]$. We focused on processes involving leaming (Word Learning test, WLTTOT), memory (Delayed Recall test), attention and different aspects of infomation processing speed (Letter Digit Coding Test (LDCT) and Stroop Coloured Word test). These tests have been described in detail in Chapter 9. A complete set of serum and cognitive performance data was available from 92 individuals at baseline and 116 individuals after 6 years of follow-up. The mean age of the 92 individuals was 57 (SD: 12), the man to woman ratio was 55 to 37 . More details about these groups have been described in Chapter 8-10.

\section{Serum markers}

Serum concentrations of inflammatory proteins and acute phase proteins were determined. For IL-6, IL-6 receptor, CC16 and CRP commercial ELISA kits were used (Eurogenetics S.A., Tessenderlo, Belgium). The detection limits were as fol $/ \mathrm{ows}: 10 \mathrm{pg} / \mathrm{mL}$ for $1 \mathrm{~L}-6,1 \mathrm{ng} / \mathrm{mL}$ for $1 \mathrm{~L}-6$ receptor, $0.1 \mathrm{ng} / \mathrm{mL}$ for CC16 and $0.25 \mu \mathrm{g} / \mathrm{mL}$ for CRP. The intra-assay coefficients of variance (C. V.) of all assays were below $8 \%$. Serum haptoglobin concentration was measured by fixedtime immunonephelometry on a BN II analyzer (Dade Behring, Marburg, Germany) and the assay was calibrated against the international CRM 470 reference matterial [8]. Haptoglobin phenotype was determined using starch gel electrophoresis of haemoglobin-supplemented serum, followed by peroxidase staining [44]. Serum protein electrophoresis was performed by capillary electrophoresis using the automated Beckman Paragon CZE 2000 (Analis, Namur, Belgium). The intra-assay $C V$ values for the serum protein fractions are as follows: albumin $1.2 \% ; \alpha, 1.6 \% ; \alpha z 1.6 \%$ and $\gamma 4.9 \%$

Serum total homocysteine and cysteine concentrations were measured by an automated high performance liquid chromatography (HPLC) method with reverse phase separation and fluorescence detection [46]. Vitamin B12 and tolic acid concentrations were determined using commercial kits (Bayer Immuno 1 assays, Bayer, Leverkusen, Germany). 
Serum telencephalin concentration and GFAP concentrations were determined as described $[19.43]$. Sterols and oxysterols were determined as described in Chapter 9.

\section{Statistical analysis}

Differences in demographic variables were detemined with a Students' t-test. Chi-Square contingency test was used for comparison of frequency distributions beween groups.

Serum data were expressed as medians with $25 \%$ and $75 \%$ percentiles. Normality of distributions was examined using a Kolmogorov-Sminov test. Log-transfomation did not improve normality for the serum markers. Therefore, all analyses on serum varables were performed with non-parametric tesis. Differences between the serum concentrations of alt neurological patients together and controls were determined using the Mann Whitney U-test. Differences between groups were examined using analysis of variance (ANOVA) of the ranks, using Scheffe's post-hoc analysis.

Logistic regression was performed for models with AD against healthy controls as dichotomous dependent variable and the marker concentrations as conthuous independent variables. Linearity of the logistic models was determined using quartiles of the marker concentrations as independent, categorical, wariables. The model fit was tested using the Log-fikelihood Ratio. A receiver operating characteristics (ROC) curve was established to examine the specificity of the models in relation to the sensitivity. Multiple regression analysis was performed with the serum markers as independent variable and MMSE or duration of disease as dependent variables. Correlation between the combination of difterent serum markers and cognitive test outcomes in the normal ageing population was performed with hierarchical multiple regression analysis, adjusted for age, sex and education level.

As markers related to four mechanisms were investigated (cholesterol homeostasis, vitamin status, inflammation, brain specific alterations), a Bonferroni adjustment of the significance level was adjusted to $P<0.013$ was made. However, results with significance level of $P<0.05$ are also indicated.

All statistical analyses were performed using SPSS statistical software.

\section{Results}

The demographic data of the individuals are presented in Table I.

The age distribution was not significantly different between the study groups (ANOVA F (3, 187), $P<0.11$ ). The man to woman ratio was statistically even distributed among the groups, though slightly more women were present in the AD patient group compared to the other groups (Chisquare test, $\mathrm{df}=3, \mathrm{P}<0.40$ ). The mean MMSE score was lower in both the AD and OCD group compared to the PD and control group as calculated by Scheffe's post-hoc analysis (ANOVA $F(3,165), P<0.0001)$.

\begin{tabular}{|c|c|c|c|c|}
\hline & $n$ & $\begin{array}{l}\text { A.ge } \\
\text { mean (range) }\end{array}$ & $\begin{array}{l}\text { Maledfenale ratio } \\
\text { (percentage formahe }\end{array}$ & $\begin{array}{l}\text { MASE } \\
\text { MHeany (rongra) }\end{array}$ \\
\hline Controls & 61 & $68 \cdot(55-86)$ & $35 / 26(43 \%)$ & $28.4(20-301 n=61$ \\
\hline$A D$ & 34 & $73(53-95)$ & $14 / 20(59 \%)$ & $20.36-291+7=33$ \\
\hline $\mathrm{PO}$ & 47 & $70(42-89)$ & $30 / 17\left(36^{* 45}\right)$ & $26.6145-30): 11=27$ \\
\hline $\mathrm{OCD}$ & 48 & $70(52-85)$ & $28 / 20\left(42^{6 \%}\right)$ & $22.177 \cdot 300 n=4.4$ \\
\hline
\end{tabular}




\section{Biochemical measurements}

Table 2 summarises the haptoglobin concentrations per phenotype. The median haploglobin concentration per phenotype was lower in the 2-2 phenotype compared to the 2-1 or 1-1 phenotype, which is in accordance with data from previous studies [27]. The median concentration of the protein fractions (Table 3), interleukins (Table 3), homocysteine (Table 4) and sterols (Table 5 ) in serum of the control subjects were similar to previous data from the participating laboratotes.

\begin{tabular}{|c|c|c|}
\hline & frequency & $\begin{array}{l}\text { haptoglobin concentration (mgingl } \\
\text { mean trange) }\end{array}$ \\
\hline $1-1$ & $20 \%$ & $\begin{array}{l}1.68 \\
(1.28,1.86)\end{array}$ \\
\hline $2-1$ & 55 & $\begin{array}{l}1.45 \\
11.21,1.77 !\end{array}$ \\
\hline $2-2$ & 25 w & $\begin{array}{l}1.12 \text { a } \\
(0.79,1.54\end{array}$ \\
\hline
\end{tabular}

an: $P<0.01$ for $2-2$ vs. $2 \times 1$ oir $\mathbb{1}-1$

\section{Serum concentrations of interleukins in the patient groups (Table 3 )}

The serum concentration of the IL-6 receptor was significantly lower in all neurological patients compared to the control group (Table 3, second last column). The IL-6 receptor concentration differed from the control group in the PD group as shown by scheffé's post-hoc analysis.

Serum CC16 concentration was increased in all neurological patient groups together, while this increase also reached significance in the single group of $\mathrm{PD}$ patients only compared to controls $(-125 \%$ of control value). The serum concentrations of the other inflammation markers were not significantly different among the groups (Table 3 ).

\section{Serum concentrations of homocysteine, cysteine and related witamins (Table 4)}

The median serum homocysteine concentration was increased in all neurological patients together compared to healthy control individuals, but not in one of the patient groups separately. The median cysteine concentration was significantly increased in serum of all neurological patients. In addition, serum cysteine concentration was increased in AD patients compared to controls as shown by AMOVA and Scheffe's post-hoc analysis. The median vitamin B12 concentration was decreased in all neurological patients compared to healthy control individuals, but not in ane of the patient groups separately. No differences between the groups were observed for the folic acid concentrations.

\section{Telencephalin and GFAP}

The serum GFAP concentrations were above detection limit of the ELISA in only part of the individuals in each patient group (AD:23\%; PD:29\%; OCD:37\%; controls:38\%). The median of the GFAP concentration of the detectable samples was not significantly different between the groups (data not shown). The serum ICAM-5 (telencephalin) concentrations were also above the detection limit in only a minority of individuals (AD:24\%, PD:22\%, OCD:16\%, controls:21\%) and no difference in the median of the serum concentrations of the detectable values was observed between the groups (data not shown. 


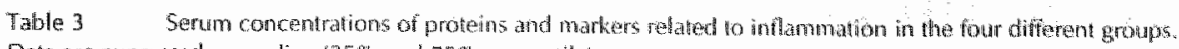

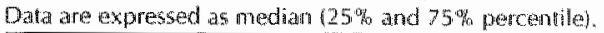

\begin{tabular}{|c|c|c|c|c|c|c|}
\hline & $\begin{array}{l}\text { controls } \\
\text { in w } 61 \mathrm{j}\end{array}$ & $\begin{array}{l}A D \\
n=34)\end{array}$ & $\begin{array}{l}\mathrm{m} D \\
6 \mathrm{in}=46 \mathrm{j}\end{array}$ & $\begin{array}{l}O C D \\
\left.(7)=\frac{45}{6}\right)\end{array}$ & $\begin{array}{l}\text { Patiments } \\
\text { vs. } \\
\text { coturols }\end{array}$ & $(3,1) 01^{3}$ \\
\hline $\begin{array}{l}\text { torat protein } \\
\text { mugimus }\end{array}$ & $\begin{array}{l}72 \\
170,761\end{array}$ & $\begin{array}{l}70 \\
168,731\end{array}$ & $\begin{array}{l}72 \\
(68,75)\end{array}$ & $\begin{array}{l}70 \\
667.751\end{array}$ & n.s. & $\mathrm{n} . \mathrm{s}$. \\
\hline $\begin{array}{l}\text { albumin } \\
\text { (mag/nL) }\end{array}$ & $\begin{array}{l}42.7 \\
140.7,446161\end{array}$ & $\begin{array}{l}41.6 \\
(40.6,43.5)\end{array}$ & $\begin{array}{l}42.5 \\
40.0,43.7)\end{array}$ & $\begin{array}{l}42.7 \\
37.9,446)\end{array}$ & his. & $n, s$ \\
\hline $\begin{array}{l}\text { protein al } \\
\text { (mgimls }\end{array}$ & $\begin{array}{l}4.8 \\
(4.3,5.2)\end{array}$ & $\begin{array}{l}4.6 \\
(4.2,5.0)\end{array}$ & $\begin{array}{l}4.6 \\
44.0,5.3)\end{array}$ & $\begin{array}{l}4.6 \\
44.2,5.11\end{array}$ & $n s$ & IUS. \\
\hline $\begin{array}{l}\text { protein a2 } \\
\text { (molnt) }\end{array}$ & $\begin{array}{l}6.0 \\
(5,2,6.5)\end{array}$ & $\begin{array}{l}6.1 \\
(5,3,6.8)\end{array}$ & $\begin{array}{l}6.2 \\
\{5.5,7.0\}\end{array}$ & $\begin{array}{l}6.2 \\
15.6,6.91\end{array}$ & $n, s$. & $n s$ \\
\hline $\begin{array}{l}\text { protein } \beta \\
\text { (mglmL) }\end{array}$ & $\begin{array}{l}8.2 \\
(7.4 .8 .9\end{array}$ & $\begin{array}{l}8.5 \\
{[7.2,9.11}\end{array}$ & $\begin{array}{l}7.7 \\
(7.0,8.8)\end{array}$ & $\begin{array}{l}7.7 \\
7.11,8.6\end{array}$ & $\mathrm{a} s \mathrm{~s}$ & $\mathrm{nas}$ \\
\hline $\begin{array}{l}\text { proteiny } \\
\text { (mglml }\end{array}$ & $\begin{array}{l}10.3 \\
(8.5 .12 .7)\end{array}$ & $\begin{array}{l}9.5 \\
(9.9,+1,0)\end{array}$ & $\begin{array}{l}10.1 \\
(2.5,1.2 .41\end{array}$ & $\begin{array}{l}9.8 \\
(8.1,11.04\end{array}$ & n.s. & ms.s. \\
\hline $\begin{array}{l}\text { haptogglobin } \\
\text { (mg/mit.) }\end{array}$ & $\begin{array}{l}1.32 \\
(0.91,1.66)\end{array}$ & $\begin{array}{l}1.27 \\
(1.08,1.68)\end{array}$ & $\begin{array}{l}1.46 \\
(1.25,1.80)\end{array}$ & $\begin{array}{l}1.42 \\
\square 1.04,1.800\end{array}$ & $\mathrm{~ns}$ & $n_{1}, s$ \\
\hline $\begin{array}{l}11-6 \\
\text { log }\end{array}$ & $\begin{array}{l}2.51 \\
10.56,4.02)\end{array}$ & $\begin{array}{l}2.54 \\
(1.35,4.23)\end{array}$ & $\begin{array}{l}3.09 \\
\{1.51,5.57\}\end{array}$ & $\begin{array}{l}2.02 \\
0.50,4.75)\end{array}$ & $\mathrm{n}, \mathrm{s}$ & 0.5 \\
\hline $\begin{array}{l}\text { HL-6 receptor } \\
\text { (ng/mL) }\end{array}$ & $\begin{array}{l}106 \\
(95,124)\end{array}$ & $\begin{array}{l}89 \\
(67,113)\end{array}$ & $\begin{array}{l}330 \\
466,107)\end{array}$ & $\begin{array}{l}38 \\
(75,1100\end{array}$ & $d$ & $d$ \\
\hline $\begin{array}{l}\text { CC16 } \\
\text { ing/mat }\end{array}$ & $\begin{array}{l}34.3 \\
22.4,43.31\end{array}$ & $\begin{array}{l}38.5 \\
(25,8,55,5)\end{array}$ & $\begin{array}{l}42.8 \mathrm{~b} \\
31.2,59.11\end{array}$ & $\begin{array}{l}37.5 \\
(27.4,50.49\end{array}$ & $b$ & c \\
\hline $\begin{array}{l}\text { Creactive protein } \\
\text { (Hg/no })\end{array}$ & $\begin{array}{l}2.08 \\
(0.68,4.24)\end{array}$ & $\begin{array}{l}1.47 \\
10.38,3.40)\end{array}$ & $\begin{array}{l}2.01 \\
\{0,70,5,1.8\}\end{array}$ & $\begin{array}{l}2.07 \\
10.5 .5,4.18\}\end{array}$ & $\mathrm{n}, \mathrm{s}$ & n... \\
\hline
\end{tabular}

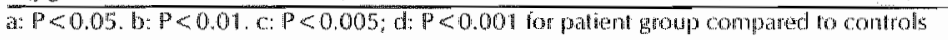

ased on Zwalue from Mann-Whithey U test; 0 based on ANOVA comparing all four groups

n.s.: not significarol

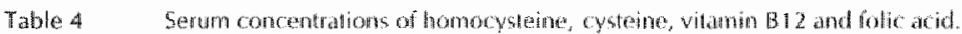

Data are expressed as median ( $25 \%$ and $75 \%$ percentifu).

\begin{tabular}{|c|c|c|c|c|c|c|}
\hline & $\begin{array}{l}\text { controls } \\
4 n-\infty 611\end{array}$ & $\begin{array}{l}M D \\
(n=34)\end{array}$ & $\begin{array}{l}P D \\
m=-469\end{array}$ & $\begin{array}{l}0 \mathrm{DD} \\
(n-47)\end{array}$ & $\begin{array}{l}\text { Panchis } \\
\text { vs. } \\
\text { controls" }\end{array}$ & $(9,190)^{4}$ \\
\hline $\begin{array}{l}\text { homocystene } \\
\text { (fumol/d) }\end{array}$ & $\begin{array}{l}16.5 \\
(13.3,19.45)\end{array}$ & $\begin{array}{l}17.2 \\
(15.1,21.8)\end{array}$ & $\begin{array}{l}18.9 \\
16,3,23.2)\end{array}$ & $\begin{array}{l}17.1 \\
(15.7,19.1)\end{array}$ & ij & $\pi \times$ \\
\hline $\begin{array}{l}\text { Cysteinge } \\
\text { Humolfh! }\end{array}$ & $\begin{array}{l}267 \\
245,295)\end{array}$ & $\begin{array}{l}289 \mathrm{a} \\
259,319\end{array}$ & $\begin{array}{l}285 \\
(262,307)\end{array}$ & $\begin{array}{l}276 \\
259,294\end{array}$ & $\mathrm{~b}$ & $a$ \\
\hline 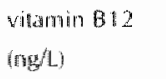 & $\begin{array}{l}405 \\
040,473 j\end{array}$ & $\begin{array}{l}327 \\
2274,406)\end{array}$ & $\begin{array}{l}360 \\
295.4271\end{array}$ & $\begin{array}{l}347 \\
288,4281\end{array}$ & c & a \\
\hline $\begin{array}{l}\text { folic acid } \\
\text { (Hy) }\end{array}$ & $\begin{array}{l}4.9 \\
0.5 .6 .44\end{array}$ & $\begin{array}{l}5.0 \\
(3,6,6.41\end{array}$ & $\begin{array}{l}5.0 \\
(3.7,6.7)\end{array}$ & $\begin{array}{l}4.8 \\
(3.5,5.9)\end{array}$ & Fos.s. & 0.5 \\
\hline
\end{tabular}

a. $\mathrm{P}<0.05, \mathrm{~b}: \mathrm{P}<0.01, \mathrm{P}<0.005 ; \mathrm{d}: \mathrm{P}<0.00 \mathrm{for}$ patien grou compared ta controls

* based on Z-value from Mamm-whiney u tast; (c) based on MOVA comparing all four growps

m.5.: not sigenificant 


\section{Sterols}

Serum total cholesterol concentration was not significantly different from controls in any of the patient groups (Table 5). The ratio of sterols to cholesterol provides a more reliable estimation of the sterol balance in an individual than the absolute concentrations. Therefore, data are expressed ais sterol to cholesterol ratios $(\mathbb{R}$ sterols) (rable 5).

The cholesterol precursor R. lathosterol was increased in all neurological patients together companed to controls. The $R$ lanosterol in serum was significantly decreased in all neurological patients. The decrease in $R$ Tanosterol was significant in the group of AD patients compared to controls as shown by Scheffés post-hoc analysis. No significant alterations were observed for the cholesterol precursor $R$ _desmosterol between the patient groups.

The cholesterol metabolite $R$ cholestanol was comparable in all groups. No difference in $R$ 24S-OH-Chol was observed in all neurologicall patients compared to nomal healtiny control individuals or in any of the single patient groups. The oxysterol R 27-OH-Chol was decreased in all neurological patients compared to controls, down to $86 \%$ of controls in OCD patients.

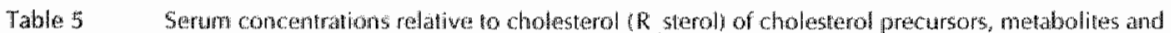
phylosterols th the four different groups Data ane expressed ats median (25\% and 75 \% percentile).

\begin{tabular}{|c|c|c|c|c|c|c|}
\hline & $\begin{array}{l}\text { controls } \\
(n=61)\end{array}$ & $\begin{array}{l}\mathrm{AD} \\
(n+2=34)\end{array}$ & $\begin{array}{l}\text { PD } \\
\text { (I) }=46 \%\end{array}$ & $\begin{array}{l}\mathrm{OCD} \\
n=47)\end{array}$ & $\begin{array}{l}\text { Patients } \\
\text { vis. } \\
\text { controls }\end{array}$ & 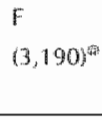 \\
\hline $\begin{array}{l}\text { chollesterol } \\
\text { (mgholl) }\end{array}$ & $\begin{array}{l}248 \\
(205,294)\end{array}$ & $\begin{array}{l}259 \\
228,2888\end{array}$ & $\begin{array}{l}216 \\
(1186,264)\end{array}$ & $\begin{array}{l}243 \\
(200,334)\end{array}$ & n.s. & a \\
\hline $\begin{array}{l}\mathbb{R} \text { lathosterot } \\
\text { He/mg }\end{array}$ & $\begin{array}{l}0.70 \\
(0.59,0.85)\end{array}$ & $\begin{array}{l}0.79 \\
10.53,1.051\end{array}$ & $\begin{array}{l}0.80 \\
\{0.63,1.08\}\end{array}$ & $\begin{array}{l}0.83 \\
(0 \times 56,1.08)\end{array}$ & $a$ & n.s. \\
\hline $\begin{array}{l}\text { 鹤 lanosterol } \\
\text { (ng/mg) }\end{array}$ & $\begin{array}{l}71.6 \\
(59.3,97.7)\end{array}$ & $\begin{array}{l}58.3 \mathrm{c} \\
449.3,71.7)\end{array}$ & $\begin{array}{l}67.2 \\
(59.9,83.6)\end{array}$ & $\begin{array}{l}65.8 \\
(52.4,74.5)\end{array}$ & $c$ & $c$ \\
\hline $\begin{array}{l}\text { R_desmosterol } \\
\text { hug/mg! }\end{array}$ & $\begin{array}{l}0.46 \\
10.36,0.55)\end{array}$ & $\begin{array}{l}0.42 \\
10.31,0.47)\end{array}$ & $\begin{array}{l}0.44 \\
(0.33,0.58)\end{array}$ & $\begin{array}{l}0.44 \\
0.33,0.51)\end{array}$ & $\mathrm{n}, 5$ & n.s. \\
\hline $\begin{array}{l}\text { R_chotestanol } \\
\text { (ug/ng) }\end{array}$ & $\begin{array}{l}1.17 \\
(0.94,1.41)\end{array}$ & $\begin{array}{l}1.09 \\
10.95,1.311\end{array}$ & $\begin{array}{l}1.05 \\
(0.89,1.30)\end{array}$ & $\begin{array}{l}1.06 \\
(0.90,1.28)\end{array}$ & m.s. & n.s. \\
\hline $\begin{array}{l}R_{2} 2 \text { ans.OH-Chal } \\
\text { (ng/mg }\end{array}$ & $\begin{array}{l}35 \\
129,401\end{array}$ & $\begin{array}{l}32 \\
126,381\end{array}$ & $\begin{array}{l}344 \\
(29,43)\end{array}$ & $\begin{array}{l}31 \\
(26,36)\end{array}$ & $\mathrm{m}, \mathrm{s}$ & $c$ \\
\hline $\begin{array}{l}\mathrm{R} 27 . \mathrm{OH}-\mathrm{Chol} \\
\text { (ng/ngl) }\end{array}$ & $\begin{array}{l}111 \\
189,133)\end{array}$ & $\begin{array}{l}99 \\
(83,112)\end{array}$ & $\begin{array}{l}100 \\
187.121 !\end{array}$ & $\begin{array}{l}96 \\
(76,113)\end{array}$ & $\mathrm{b}$ & $n, 5$ \\
\hline $\begin{array}{l}\text { R campested } \\
\text { hyg/mg! }\end{array}$ & $\begin{array}{l}1.89 \\
\{1.25,2.49\}\end{array}$ & $\begin{array}{l}1.54 \\
11.18,2.00 \%\end{array}$ & $\begin{array}{l}1.32 \mathrm{c} \\
(0.92,1.80)\end{array}$ & $\begin{array}{l}1.48 \\
1.16,2.06\end{array}$ & $d$ & b \\
\hline 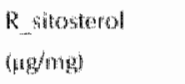 & $\begin{array}{l}1.61 \\
(1.18,2.20)\end{array}$ & $\begin{array}{l}1.37 \\
11.08,1.71 \%\end{array}$ & $\begin{array}{l}1.216 \\
10.8 \%, 1.71\}\end{array}$ & $\begin{array}{l}1.28 \\
11.00,1.689\end{array}$ & $d$ & $b$ \\
\hline $\begin{array}{l}\text { R stigmasterol } \\
\text { (nighmg) }\end{array}$ & $\begin{array}{l}40.1 \\
(30.6,55.3)\end{array}$ & $\begin{array}{l}36.0 \\
(28.7,46.0)\end{array}$ & $\begin{array}{l}34.6 \\
\{22.7,42.8\}\end{array}$ & $\begin{array}{l}31.6 \mathrm{a} \\
24.0 .42 .31\end{array}$ & $\mathrm{~d}$ & $b$ \\
\hline $\begin{array}{l}\text { R_avenusherol } \\
\text { mag/mgl }\end{array}$ & $\begin{array}{l}50.6 \\
(39.3,63.1)\end{array}$ & $\begin{array}{l}38.9 \mathrm{a} \\
130.9,45.95\end{array}$ & $\begin{array}{l}31.0 \mathrm{c} \\
(23,6,55.2)\end{array}$ & $\begin{array}{l}39.8 \mathrm{a} \\
(29.8,50.9)\end{array}$ & $\mathrm{d}$ & $c$ \\
\hline $\begin{array}{l}\text { R_brassicasterol } \\
\text { lng/mgl }\end{array}$ & $\begin{array}{l}84.7 \\
(52.6,135.2)\end{array}$ & $\begin{array}{l}64.1 \\
(45.7,123.6)\end{array}$ & $\begin{array}{l}49.9 \mathrm{c} \\
(35.4,80.2)\end{array}$ & $\begin{array}{l}80.2 \\
(40.6,98.2)\end{array}$ & b & $b$ \\
\hline
\end{tabular}

a: $P<0.1$, b: $P<0.05, c: P<0.01, d: P<0,001$ for patient grou 0 compared to controls

\# based on Z-walte hom Mam-Whitney U fest; b based on ANOWA comparing all tour groups 10.s.: not significant 
All plant sterols las $R$ sterol) were decreased in all neurological patients compared to control individuals. This was partly due to the prominent decreased ratios in the PD group. The $R$ _campesterol, $R$ _sitosterol as well as $R$ brassicasterol were significantly lower in PD patients Compared to the control group as shown by Scheffés posthoc analysis, down to $59-75 \%$. The R_stigmasterol was significantly decreased in the $O C D$ group only. The median $\mathbb{R}$ avenasterol was lower in ewery single patient group compared to the control group (AD: $77 \%, \mathrm{PD}: 61 \%$, OCD: $79 \%$.

\section{Combination of different markers in a logistic regression model}

Our hypothesis was that a combination of several markers that are related to different mechanisms would improve discrimination of patients with $\mathrm{AD}$ from controls than a single marker alone, in terms of sensitivity and specificity. To test this hypothesis, we used a logistic regression model in which we combined several markers. First, significance of markers was calculated in logistic regression models including the single marker as independent variable (AD ws. control as dependent variable). The markers that showed the highest significant relation with AD compared to controls were stepwise included in the model. The models were adjusted to age and sex. The significance of the markers within the combined model was examined, and the improvement in log likelihood ratio of the model after adding a marker was calculated. Due to the limited number of individuals (34 AD patients and 61 controls) maximal three markers were added in the model, besides sex an age.

Table 6 Odds Ratios and Waid's p-values and $95 \%$ confidence indervils for models io discriminete between AD patients and controls adjusted tor age and gender

\begin{tabular}{|c|c|c|c|c|}
\hline Model & Coefficient & p-vallue & Odds Ratio & $\begin{array}{l}95 \% \text { ronficlinese } \\
\text { isviterwal }\end{array}$ \\
\hline Mondel 1: & -0.050 & 0.001 & 0.952 & $0.9244-0.981$ \\
\hline \multicolumn{5}{|l|}{$\mathbb{R}$ lanosterol } \\
\hline \multicolumn{5}{|l|}{ Modiel 2: } \\
\hline R I inosterol & -0.050 & 0.003 & 0.952 & $0.921-01984$ \\
\hline 11.-6 receptor & -0.033 & 0.003 & 0.968 & $0.947-7-0.989$ \\
\hline \multicolumn{5}{|l|}{ Modte: 3} \\
\hline R_lanosterol & .0 .056 & 0.002 & 0.945 & $0.911 \cdots 0.980$ \\
\hline Le receptor & -0.034 & 0.003 & 0.967 & 0.5460 .9189 \\
\hline cysteme & 0.024 & 0.025 & 1.024 & $1.009 \times 1.045$ \\
\hline
\end{tabular}

First, R lanosterol was included (model 1 in Table 6), next the inflammation marker IL-6 receptor (model 2) and finally cysteine (model 3, Chi-Square of the log-likelihood between model 2 and model $3: 5.7, \mathrm{df}=1, \mathrm{P}<0.05$ ). The results in Table 6 show that model 3 vielded significant Odds Ratios for all three markers included. In Fig. 1, the ROC-Curves for comparing specificity and sensitivity of the models are depicted. The graphs illustrate the improvement of the models to discriminate between AD patients and controls after inclusion of additional markers, shown by a shift of the curve to the upper left comer of the graph.

The correlation of the combination of the three serum markers with MASE score or duration was investigated in the AD patient group using multiple regression models. MMSE or duration of disease were dependent wariables and the serum concentrations of markers from model 3 were independent variables, adjusted to age and sex. No significant correlation was observed for these nodels (MMSE: $F(3,28)=1.13, P>0.05$; duration of the disease: $F(3,29)=1.19$, $p>0.05$;

Next, the model validity for discriminating $A D$ patients from the other patient groups, PD and OCD patients, was examined. The data in Table 7 list the sensitivity and specificily at a 
probability level of 0.5 for the three differen models, as an example. These results indicate that the speacificity of the AD models (model 2 and model 3) was less for PD or OCD than for control individuals at this probability level.

\section{Correlation of combination of serum markers with cognitive outcomes in a normal ageing population}

Next, we investigated if the combination of the serum markers in the AD models described above would be related to cognitive performance in the normal ageing population. Therefore, data of normal ageing, healthy individuals participating in a longitudinal ageing study were investigated. A complete set of serum and cognitive outcome data was present form 92 individuals at baseline and 116 at follow-up. Serum concentrations had been determined in this study for $R$ lanosterol and IL-6 receptor. The cognitive outcome variables addressed fields as speed (LDCT), werbal learning and memory (WLTTOT and Delayed Recall), attention and information processing (Stroop). The significant relation between $R$ lanosterol and cognitive outcomes on the Word Learning tests has been described in detail elsewhere (Chapter 9!.

Table 7 Specificity and sensitivily of combined models in other patient giroups at a probability level of 0.5 .

\begin{tabular}{|c|c|c|c|c|}
\hline & cortiful & $\mathrm{AD}$ & $\mathrm{PDD}$ & $O C D$ \\
\hline AD modal & $87 \%$ & 48 or & $70 \%$ & $62 \%$ \\
\hline A.D hodoel 2 & $830 \%$ & $610 \%$ & $62^{m} t_{4}$ & $48 \%$ \\
\hline AD modis 3 & $02 \%$ & 64 of & $53 \%$ & $52 \%$ \\
\hline
\end{tabular}

a) sensitivily

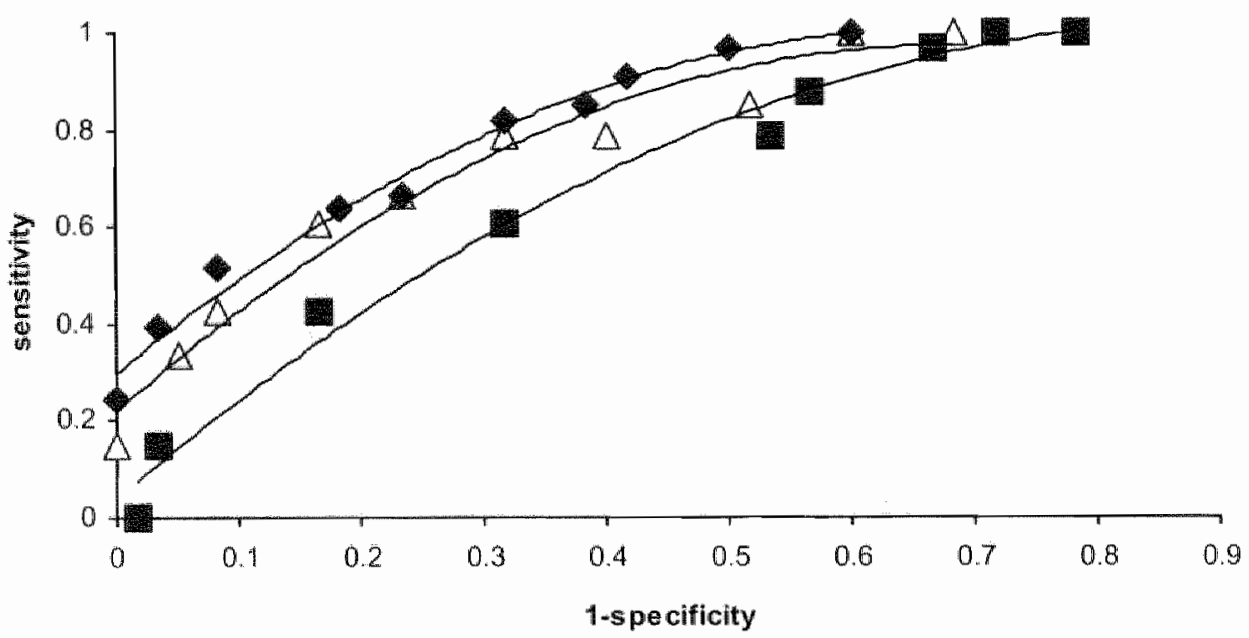

Fig. i Rechite operating chanacteristics (ROC)-cunve comparing models including a combination of markers with mad als ind lading al single maker.

ROCS for models comparing AD patients with controls are depicted. Alodels with higher sensitivity and specificity have a steeper cuves to left of the graph. Squares: data ponts of the model including serum $\mathrm{R}$ lanosterol. Triangles; data points of

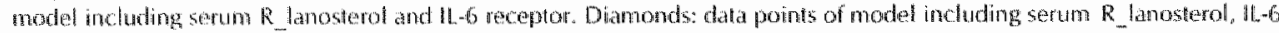
aroptor and cysteina. 
Thus, multiple regression analysis was performed with cognitive outcomes as dependent variables and the serum marker concentrations as independent variables, adjusted for age, sex and education.

The results showed that no significance was obtained for the regression model correlating cognitive outcome data at baseline to $\mathrm{R}$ lanosterol and $\| \mathrm{L}-6$ receptor $(S t r o o p: F(6,80)=1.04$; LDCT: $F(6,81)=1.14 ;$ WLTTOT: F $(6,81)=1.72 ;$ Delayed Recall: F $(6,81)=1.35)$. Due to this lack of correlation at baseline, no further analysis of the follow-up data was performed.

\section{Discussion}

In the present study, we hypothesised that the complex pathology of AD is reflected in concentrations of serum markers specific for different pathological mechanisms. This hypothesis implies that a combination of serum markers related to severall disease mechanisms will improve the discrimination befween patients with AD, PD or OCD patients and controls. To test this idea, we investigated 29 serum markers in patients, of which eleven were related to inflammation processes, welve to cholesterol metabolism, four to vitamin stafus and of which two were brain specific marker proteins.

The markers that were significant in logistic regression models including single markers were combined in a logistic regression model to investigate whether significant models were obtained after inclusion of additional markers related to different mechanisms. We showed that the ROC curve for the sensitivity and specificity to discriminate AD patients from controls was improved compared with the model including $\mathbb{R}$ _lanosterol alone. The combination of $R$ lanosterol with serum concentrations of $I L-6$ receptor and cysteine yielded a logistic regression model with a sensitivity of $64 \%$ and a specificity of $82 \%$ assuming a probability of 0.5 for discrimination $\mathrm{AD}$ patients from healthy controls. Thus, the combination of markers related to cholesterol homeastasis, inflammation and vitamin status, mechanisms that are all involved in the neurodegenerative process, yielded an improved sensitivity for the discrimination of $\mathrm{AD}$ patients from healthy controls. Moreover, these results indicate that the complex pathology of $A D$ is reflected in serum marker patterns.

The specificity of the model for discriminating AD patients from PD or OCD patients was lower than the specificity for controls (Table 7). This may be due to the lack of specificity of decreased $1 \mathrm{~L} 6$ receptor concentration for $\mathrm{AD}$ patients. The combination of the three serum markers did not correlate with the outcomes of the MMSE in the AD patients, or with the duration of the disease. The combination of $R$ lanosterol and $\| L-6$ receptor did not correlate with cognitive pertormance in the normal ageing population of the MAAS. These results together maly imply that the present combination of serum markers may not be used for an estmaltion of further disease progression and may be valid only in already diseased persons. Nevertheless, the logistic regression models obtaned from our data may be a first step in the direcion of a diagnostic test based on serum analyses. In any case, our model will need replication and extension belore the results can be used in clinical practice. Although a blood sample is easy to obtain compared to CSF, several specialised techniques are needed for examination of our markers, such as CCIMS, ELISA and HPLC, which increases the costs of the diagnosis.

The changes in plant sterol concentrations observed in $P D$ patients and, although fo a lesser extent, in the $\mathrm{AD}$ and $\mathrm{OCD}$ patients is a new and highly interesting finding 50 far, limited research has been done on the relation between cholesterol precursors, metabolites or plant sterols in relation to neurodegenerative processes. As mentioned in the introduction, decreased concentration of $245-O H$-Chol has been reponted in severely affected AD patients $\llbracket 6,31,42 \rrbracket$ The lack of decreased median $245 \mathrm{OH}$-Chol concentration in our patient groups maly be due 10 the low sample size or the relatively mild severity of dementia in the AD and OCD group. 
We observed decteased concentrations of the $\mathrm{L}-6$ receptor and increased concentration of CC16 in PD patients. The latter is known as an immunosuppressor [9]. Serum inflammatory proteins in PD patients have not been widely investigated. Only a few previous studies investigated serum $\mathbb{L}-6$ receptor concentration and CC16 concentrations in AD patients and reported decreased concentration of these inflammatory proteins. Stability of $1 L-6$ receptor levels within patients is an advantage compared to the rather rapid variation in time for IL-6 levels. Our results support the idea that $\mathbf{L}-6$ receptor may be a more suitable marker in studies comparing serum of AD patients to serum of controls. The findings of a changed pattern of inflammation markers in the neurological patients are in line with the inflammatory hypothesis in neurodegenerative disease $[10,36]$. In addition, these results may encourage investigations on the use of non-steroid anti-inflammatory drugs in PD patients too, similar to AD patients $[5,37]$. We did observe increased homocysteine and decreased vitamin B12 concentrations in all neurological patients, which is in agreement with results showing increased homocysteine concentrations in patients with Alzheimer's or vascular dementia [7,24]. The lack of significantly increased median homocysteine concentration and decreased vitamin B12 concentration in the $A D$ patients as a single group may be explained by the low number of patients in this group $(n=34)$. In the study of Clarke et al. [7] the mean concentration in $A D$ patients was only slightly increased compared to controls, and the number of patients $(n=164)$ and controls $(n=108)$ included in their study was much larger than in our study. The increased cysteine concentration in serum of AD patients was a remarkable finding. The only report also addressing blood concentrations of this amino acid showed increased plasma cysteine concentrations in patients with $A D$ and $P D$ [17]. Interestingly, elevated cysteine concentrations have been related to increased risk for cardiovascular disease $[11,40]$.

Observations of increased telencephalin concentrations in some $\mathrm{AD}$ patients prompted us to include this marker in our analysis [43]. We observed high concentrations in some AD, PD and $O C D$ patients, while the telencephalin concentrations in the majority of individuals remained below detection levels. We did not observe significant differences in detectable serum telencephalin concentrations between the groups. Similarly, our results did not show differences in median measurable GFAP concentrations between the four different groups. In fact, the majority of the samples were below the detection limit of the ELISA. Increased GFAP serum concentrations have been shown in stroke patients [19]. In addition, increased GFAP auto-antibody titers have been observed in serum or CSF of AD patients compared to controls [39]. Thus, the sensitivity of the current assays for both GFAP and telencephalin are limited for delection of small differences in serum concentrations between patients with chronic neurodegenerative diseases and controls.

A striking observation was the strongly decreased serun concentration of plant-derived sterols, which is indicative of changes in sterol homeostasis. Cholesterol homeostasis is a balance between excretion, cholesterol synthesis and sterol absorption after intake [16]. A difference in serum levels of PD patients due to decreased plant sterol intake seems unlikely, since il was reported in epidemiological research that PD patients consume a diet with vegetable content comparable to healthy controls. In contrast, increased animal fat intake was associated with PD $[2,18]$. Our data do not indicate that the general cholesterol synthesis might be disturbed, as the lanosterol or desmosterol levels were similar to controls in PD patients, though the lathosterol concentration was increased in the group of neurological patients as a whole. At present, no mechanism is known for the relation between altered plant sterol concentrations in PD or in $A D$ and the results of the present correlation study do not allow drawing conclusions concerning causality. Plant sterol supplements have been shown to be effective in lowering cholesterol concentrations $[23,28]$. Given the interrelationship between plant sterol concentrations and cholesterol metabolism and the finding of low plant sterol concentrations in 
neurological patients, especially in PD patients, it would be interesting to investigate if use of plant sterols supplements may be beneficial in lowering prevalence of $\mathrm{PD}$ or $\mathrm{AD}$.

In conclusion, we have shown that the complex pathology in AD is reflected in a pattern of altered serum concentrations of markers. In some patients evidence of only one mechanism may be detected, while in other patients a combination of difference disease mechanisms is detectable. The strikingly decreased plant sterol concentrations in all patients with neurodegenerative diseases draws immediate attention to dietary habits, absorption, metabolism and excretion of these compounds in relation to the CNS.

\section{Acknowledgement}

This study was supported by the "Van Leersum fonds" of the Royal academy of Sciences, The Netherlands.

\section{References}

American, A.P. Dhagnostic and Statistical Marmat of Wental Dronders, 4 edn. APA, washington DC, 1987, 21.29 pp.

Anderson, C, Checkoway, H., Franklin, G.M, Beresford, S.; Smith-Weller, T. and Swanson, P.D., Dietwin factors in Parkinson's disease: the role of food groups and specific foods, Mov Disord, 14 (1999) 21-7.

Andreasen, N., Minthon, L., Vanmechelen, E, Vanderstichele, H., Davidsson, P., Winblad, B. and H3tennonw, K., Cerebrospinal fluid tat and Abeta 42 as predictors of dewelopment of Alzheirner's disease in patients with mild cognitive impaiment, Neurosci Lett, 273 (1999) 5n

Angelis, P., Scharf, S., Mander, A., Vajda, F. and Christophidis, N., Serum interleukin-6 and interteukin-te soluble receptor in Alzhe iner's disease, Neurose Latr, 244 ("11998) $106-8$.

Breitner, I.C., Welsh, K.A., Helms, M.I., Caskell, P.C., Gau, B.A., Roses, A.D., Pericak-Vance, M.A. and Saundeys, A.M., Delayed onset of Alzheimer's disease with nonsteroidal anti-intiammatory and histamine Hz blocking drugs. Neurobiol Aging 16 (1995) 523-30.

Bretillon, L., Lüthohanm, D. Stahle, L, Widhe, T., Bindl, L., Eggentsen, G., Diczfalusy, U. and Bjorkhem, I., Pasma levels of 24S-hydroxycholesterol reflect the balance between cerebral production and hepatic metabolism and are inversely related to body surface, / Lipid Res, 41 (2000) 840-845.

Charke, R., Smith, A D. Jobst, K.A. Refsum, H., Sulton, L, and lieland, P.M., Folate, vatamin BI2, and servim lotal homocysteine hevels in confirmed Alzheimer disease, Arch Nourol, 55 (1996) 1449-55.

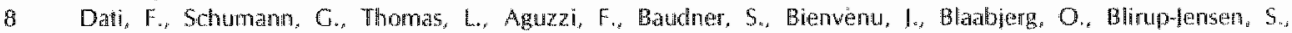

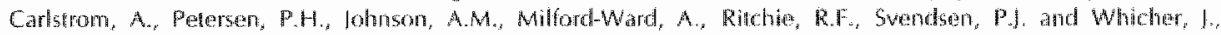

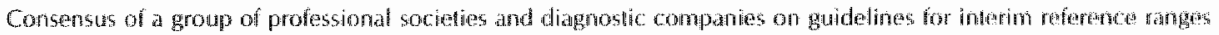
for 14 proteins in serum based on the standardization against the IFCC/BCR/CAP Reforence Material (CEM 470 . Internationd Fedenation of Clinical Chemistry. Community Bureat of Reterence of lwe Commission of the Eutopean Communities, College of American Pahologists, Eu/ Clin Chem Clin Bochem, $34(1996) 51720$.

9) Dierynck, 1, Bermard, A., Roels, H. and De Ley, M, The thuman Chara coll proten: bionemical and biological

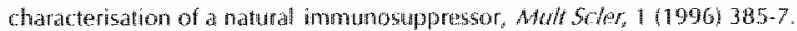

10 Elkelerboom, P., Rozemuller, A.l., Hoozemans, J1, Veerhus, R, and van Cool, W.A., Noumintammathm and

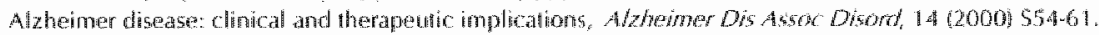

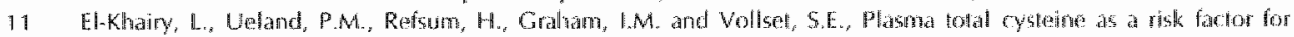

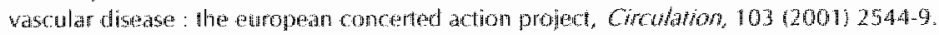

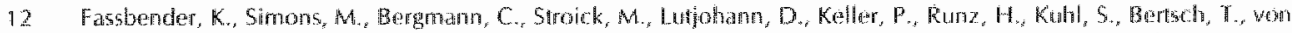

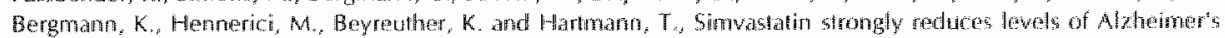

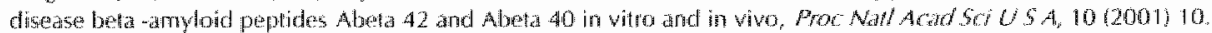

13 Folstein, M.F. Folstein, 5.E. and MCHagh, P.R. "Mani-mental state", A practical method for grading the cogeritive

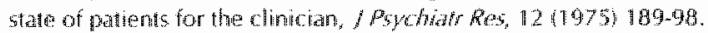

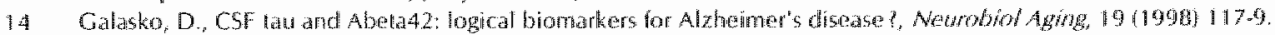

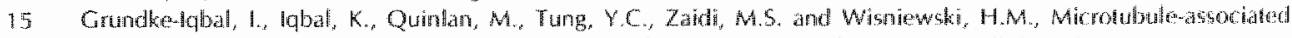

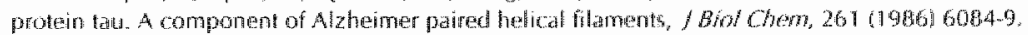

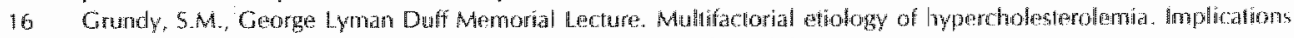
for prevention of cononary heart disease. Arteriosctex Thromb. $11109111619-35$.

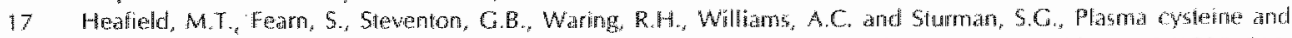

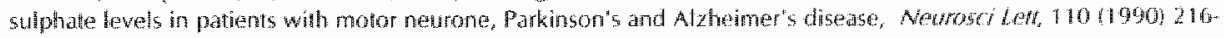


20.

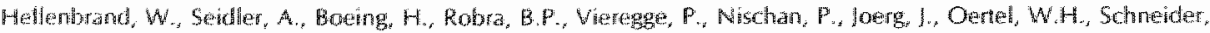

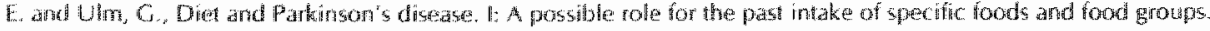

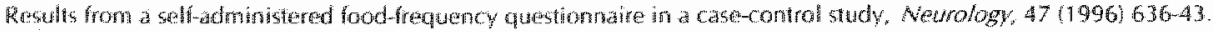

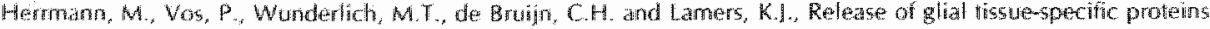

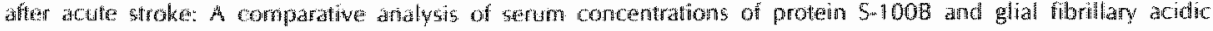
protein, strake, $3120002670-7$.

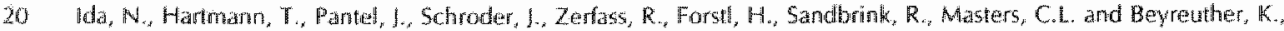

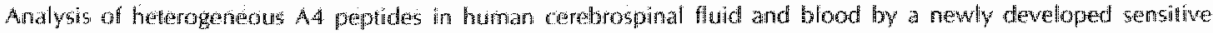

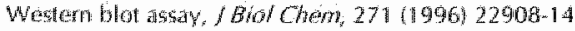

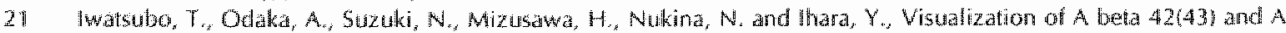

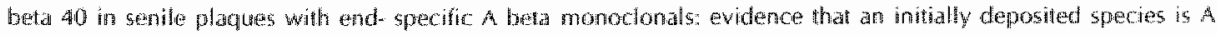
betar $42(43)$. Nestron, $13(1994) 45-53$.

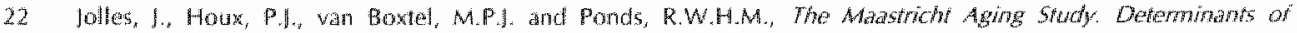
cogrithe aghing, Neverosych Pubishers, Madricht, $1995,192 \mathrm{pp}$.

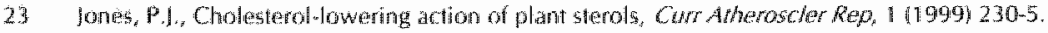

24 poosten, E., Lesafre, E. Riezler, R., Chekere, V., Deramaker, L, Pelemans, W. and Dejager, E., Hs netabolic. evidence for whanin B-12 and folate deficiency more frequent in elderly patients with Alzheimer's disease?, Goronol A Bol Sci Med Sci, 52 (11997) M76-9.

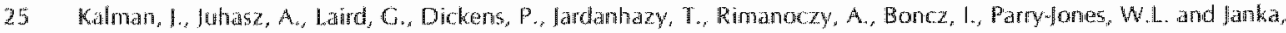
Z. Serum interteutch-6 lewels corretate with the semerity of dementia in Down syndrome and in Alzheimet's disease. Aeda Neurol Seand, $96(1997) 236-40$

26 Kanail, M. Matsubara, E, lsoe, K., Urakami, K, Nakastrinal, KK, Arail, H., Sasaki, H., Mbe, K., Iwalsubo, T, Kosaka,

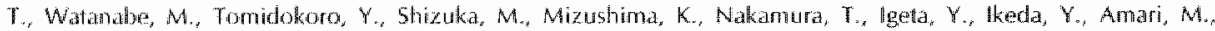
Kawarabayashi, T, shiguro, Ko, Hargaya, Y., Wakabayashi, K., Okamolo, K, Hirai, 5. and Shoji, M., Longitudual

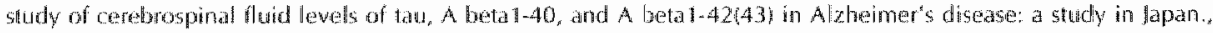

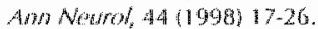

27 Langlois, MR. and Delanghe, IR., Biological and thicen significance of haptoghobin polymorohism in humans, Cin Chom, 4211996$) 1599-600$.

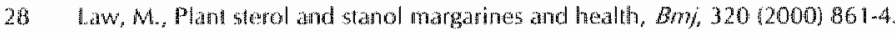

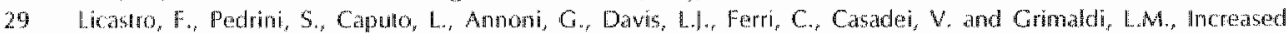

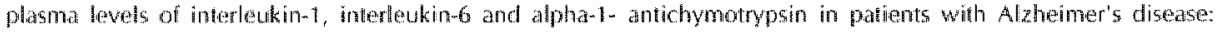
peripheral inflammation or signals from the brain? / Neurownowot, 103 (2000) $97-102$.

30 Lund, E.G., Guilevardo, I.M. and Russell, D.W., c..WA cloning of cholesterol 24-hydroxylase, a mediator ot cholesterol homeostasis in the brain, Proc Nal Acad So U SA, 96 (1999) $7238-7243$.

31 Euljohann, D., Paphssotiropoulos, A., Bjorkhem, 1. Locattelti, S., Bagli, M., Oehring, R.D., Schlegel, U., Jessen, F., Rao, M,L., von Bergmann, $K$. and Heun, R., Plasma 245-hydroxycholesterol fcerebrosterob is increased in Alzhemer and vascular demented patients, / hipid Res, $4 \|$ i2000) 195-198.

32 Maes, M. DeVos, N., Wauters, A., Demedis, P., Maurits, W.W., Neels, H., Bosmans, E., Altamufat; C., Liñ , A., Song, C. Vandenbroucke, $M$. and 5 charpe, $S_{n}$, inflammatory markers in younger vs elderly mormal volunteens and in

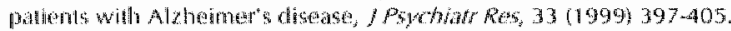

33 Manon, R.P., Sthomather, W.., Shajenko, L., Chambers, TE, and Herbette, L. G., Ewidence for changes in the

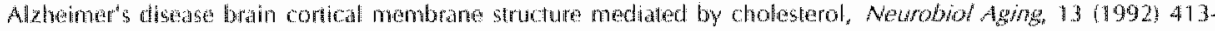
$41^{2}$.

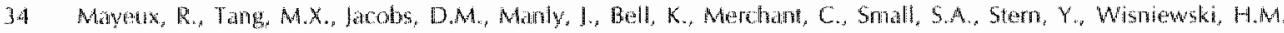

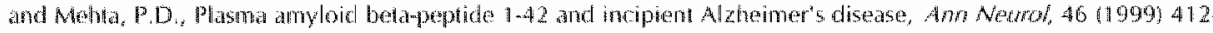
6.

35 McCadelon, A., Davies, G.. Hudson. P., Tandy, S. and Caltell, H., Total sertam homocysteme in senile dementia of

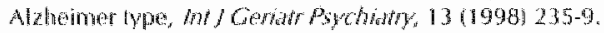

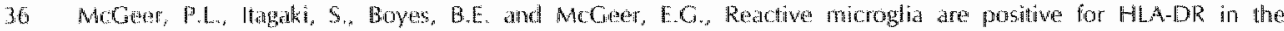
substantian migra of Parkinson's and Azheimer's disease brains, Netrology, 38 (1988) $1285.5 \%$.

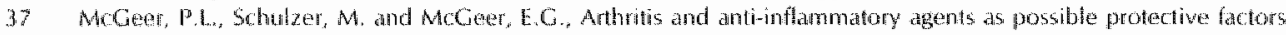

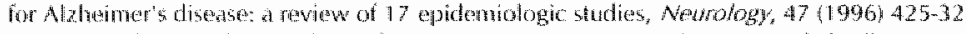

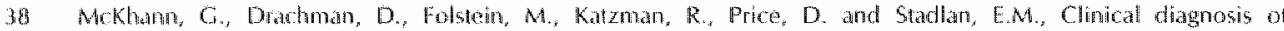
Aluhemer's distase: report of the NINCDS-ADRDA Work Croup under the auspices of Department of Heath and

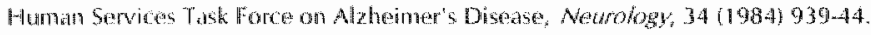

39 Mecoco, P., Parweti, L., Romano, G., Scarelli, A., Chionne, F., Cechetti, R., Polidoni, M.C., Palumbo, B., Cherubin, A. and Senin, U., Serum anti-GFAP and ant 5100 attoantbodies in brain aging, Alzhemer's disease and

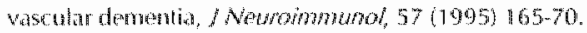

40 Mils, B.J., Weiss. M.M. Ling, C.A. Liu, M.C. and Ziegler, C., Blood glutatione and oysteme changes in cardiovasculall dicease, / Lab Clin Med 135 t2000) 396401

41 Nourhashemi, F, Gillette-Guyonne, S., Andrieu, S., Ghisolí, A., Ousset, P., ., Grandjean, H., Grand, A. Pous, 1 ,

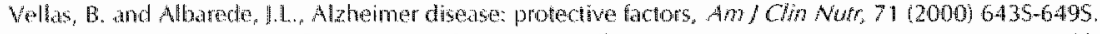

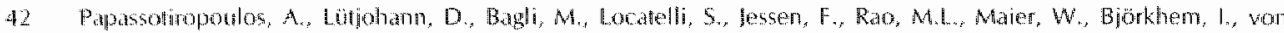




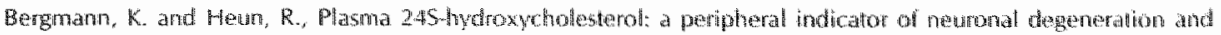

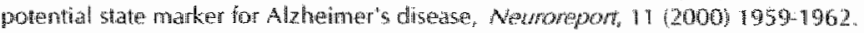

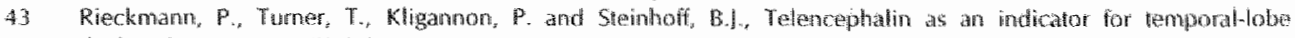
dysunction. Lancer, 352 (1998) 370 1 .

44 Smithes, 0 ., Zone ectrophoresis in starch gels: group variations in the senm protens of nomal human adults.. Biochem /, 61 (1955) 629-64).

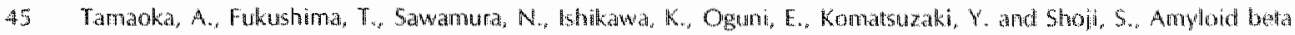

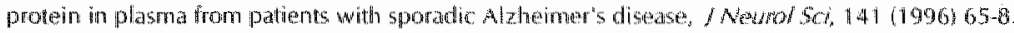

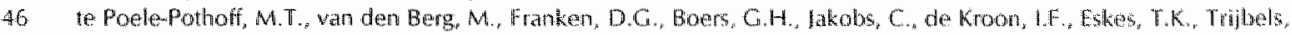

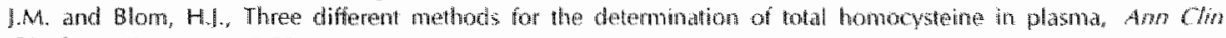
Biachem, 3211995,21820 .

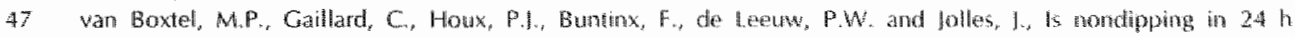

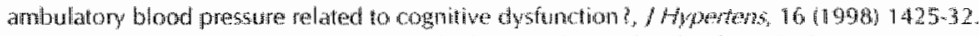

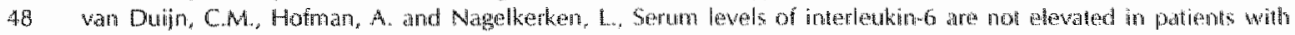
Altheimer's disease, Neurosci Leti, 108 (1900) 350 -4.

49. Working-Group, Consensus Report of the Working Group on: "Molecular allud Biochemical Marthers of Alaheinner's

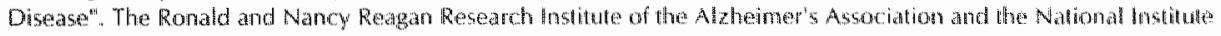
on Aging Working Group, Newobiof Aging, $19119981109-116$. 
$-$ 


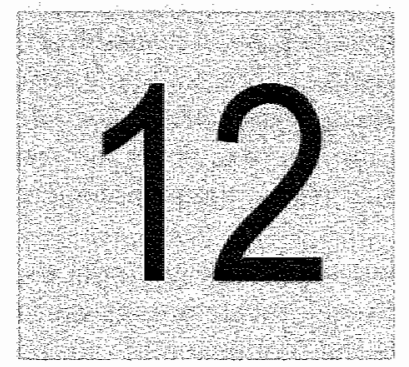

General discussion 


\section{Design of the thesis}

Neurodegenerative diseases such as Alzheimer's disease (AD) are posing an increasing impact on the ageing society, where life expectancy increases. The diagnosis of AD is currently made based on clinical examination, using neuropsychological test and exclusion of e.g. vascular dementia [78]. The definite diagnosis is based on the presence of amyloid plaques and neurofibrillary tangles in post-mortem tissue [5]. No in vitro diagnostic test is yet avallable for AD or cognitive deterioration during normal ageing. This may be due to, among other reasons, the limited insight in the etiology of $\mathrm{AD}$. $\mathrm{AD}$ is a multifactorial disease with several possible causes and mechanisms leading to a common endpoint of cell death. There appears to be a consensus opinion in the literature that a condition of oxidative stress is part of the pathology of AD $[29,51]$.

Oxidative stress is defined as a misbalance between increased production of oxygen radicals, e.g. superoxide or nitric oxide, and the anti-oxidative defences and repair mechanisms against oxygen radical-induced protein-, lipid-, RNA- or DNA-damage [26]. The production of oxygen radicals is a nomal phenomenon in tissue, for example in the immune defence or during mitochondial energy production [28]. The organism has a number of defence mechanisms against these aggressive chemical species and oxidative stress may result from the slow decline of efficiency of these defence mechanisms during normal ageing [36]. Increased oxidative stress can occur during conditions of excitoxicity [3], inflammatory processes [18] and vascular abnormalities [59]. These conditions may in turn be influenced by a large number of environmental and life-style influences such as dietary intake of vitamins [63], smoking [87], (acute) physical exercise [14,68], and exposure to pesticides [72]. Changes in levels of markers for oxidative stress have been observed in the demented brain. e.g. increased presence of nitrated proteins, the lipid peroxidation products isoprostanes, the oxidised DNA product 8hydroxy-2'-deoxyguanosine or decreased activity of the oxidative stress sensitive enzymes glutamine synthetase and creatine kinase $[32,49,71,83]$. In addition, increased cerebrospinal fluid (CSF) concentration of 8-deoxyguanidine, isoprostanes or 3-nitrotyrosine residues in proteins have been observed $[50,90]$. Finally, lower concentrations of the antioxidants vitamin $A, C$ and $E$ have been reported in serum or CSF of AD patients $[20,38,39,74,81,94]$.

The presence of an inflammation process in $A D$ is evidenced by increased transcription of interleukins in activated microglia and astroglia in association with the plaques $[53,56]$. Furthermore, epidemiological and retrospective clinical data suggest a positive effect of nonsteroid-anti-inflammatory drugs on the prevalence of AD $[7,57,76]$. Moreover, several studies observed increased concentrations of inflammatory proteins as a-antichymotrypsin or interleukin-6 (1L-6) in serum of AD patients $[22,42,47,48,64,88]$.

Recent studies showed that vascular risk factors may be involved in AD $[13,45]$. Increased serum homocysteine level, a risk fiactor for cardiovascular disease, has been observed in AD patients compared to controls $[10,46]$. Carriers of the Apolipoprotein $E 4$ allele have an increased risk for incidence of AD at an early age [85] and sightly decreased apolipoprotein $\mathrm{E}$ Concentrations in serum of AD patients have been observed, independent of ApoE isoform [80]. So far, no single serum marker has been found that could discriminate AD patients from controts with high specificity and sensitivity. Most of the studies focussed on one serum marker in rellation to one specific mechanism that might be part of the AD pathophysiology. One formidable obstacle to overcome in order to discriminate between $\mathrm{AD}$ and healthy individuals is the fact that the concentration ranges for markers in serum showed a large overlap between patients and controls. In view of the heterogeneity of disease mechanisms in $A D$, it was hypothesised in the current thesis that the combination of different markers related to different 
pathological mechanisms might improve the discrimination between AD patients and healthy individuals. The purpose of this thesis was to propose a biomarker-index, i.e. a risk indication of pathological cognitive decline, from the combination of markers relatted to the different pathological mechanisms. This biomarker-index should be applicable on a routine basis and, when fully developed, even be suitable for screening large populations. This implied the investigation of a llarge number of parameters, rationalised on the basis of the above mentioned mechanistically defined categories: oxidative stress markers, inflammation markers, vascular risk factors, i.e cysteine and homocysteine and related compounds, markers for cholesterol homeostasis and brain specific structural markers.

To provide the rationalisation of the hypothesis with a solid basis, the relation between these markers and neurodegenerative processes was investigated on four levels: first in cell culture studies, secondly in an in vivo animal model. Next, the relation between cognitive performance and a set of serum parameters was investigated in nomal, non-pathologically ageing humans, that were followed at three time-points over a period of six years (MAastricht Ageing study (MAAS) $[40,91])$. Lastly, the set of markers investigated in this longitudinal study was analysed in serum of patients diagnosed for $\mathrm{AD}$.

No experimental evidence was present for 3-nitrotyrosine as an early marker for AD and it was not known if it could be measured in human serum. Thus, the purpose was to investigate the sensitivity of this marker in experimental in vitro and in vivo models for neurodegeneration. As an in vitro model, we exposed whole brain spheroid cultures, a three-dimensional culture system, to 3-nitropropionic acid, which is an oxidative stress-inducing toxin (Chapter 2 and 4 ).

Furthermore, no in vivo experimental evidence was avallable for a relation between changes for the markers glial fibrillary acidic protein (GFAP) and 245 -hydroxycholesterol, and early slages of neurodegenerative processes. 24S-hydroxycholesterol is a brain specific cholesterol metabolite that was decreased in severely affected AD patients $[8,66]$. Therefore, siudies were undertaken to examine these two brain specific markers in relation to neurodegeneration in the 3-nitropropionic acid rat model (Chapter $4-6$ ).

For the studies examining marker concentrations during non-pathological cognitive ageing in humans, serum of participants of the MAAS was manestigated (Chapter 8-10). In this follow-up study, serum samples were stored at three time-points over six years. Some serum molecules may have been subject to negative effects due to prolonged storage. Therefore, the stability of proteins was estabished for which the literature did not provide enough evidence for stability (Chapter 7). For the studies on attered serum composition in more severe and defined neurodegeneration, serum marker patterns in patients with $\mathrm{AD}$ were examined. In order io obtain insight in the specificity of the changes for $A D$, the concentrations in these patients were compared with those observed in patients with other cognitive disorders, patients with Parkinson's disease and in controls (Chapter 11 ).

\section{3-Nitrotyrosine}

A good candidate as a serum marker for oxidative stress in neurodegeneration was 3 nitrotyrosine. Increased concentrations of 3 -nitrotyrosine have been observed in CSF of AD patients $[1,33,90]$, as well as in several animal models for oxidative 5 tress $[44,79,92]$. The presence of 3-nitrotyrosine in intact and degenerating neurons in the AD brain lead to the suggestion that 3-nitrotyrosine might be an early marker for neurodegenenation 186. .

We found that 3-nitrotyrosine could be a sensitive marker for increased oxidative stress in the spheroid cell culture tissue (Chapter 4). Such increase was clearly observed in spheroids obtained from Lewis rats, though much weaker in spheroids obtained from Wistar bats after exposure to 3-nitropropionic acid. Since 3-nitrotyrosine formation was not inhibited by the 
usual antioxidants present in brain fissue (witamin $E$, glutathione (Chapter 4 ) or superoxide dismulase (37)), it was expected that 3-nitrofyrosine formation could also be present in the in vivo rat striatum. Nevertheless, no significantly increased 3-nitrotyrosine formation was detectable in serum or striatum after chronic treatment with 3-nitropropionic acid in our vivo model. This may point to a limited extrapolation possibility of the in vitro model to the in vivo situation. A possible explanation might be that 3-nitrotyrosine may have disappeared due to denitrating enzyme activity [43], which may not be operative in the spheroid cultures. Furthemore, nitration may only occur during acute phases of degeneration. It is known from human studies that 3-nitrotyrosylation of serum proteins may occur only during acute phases of e.g. multiples sclerosis, a condition which is accompanied with increased peripheral inflammation [65]. No acute inflammation reaction was observed in the treated rats at the lime when serum was collected, evidenced by a lack of increased serum IL-6 levels (data not shownil).

These results, taken together with the detection limit of the ELISA used, argued against a role for 3-nitrotyrosine as a serum marker in chronic neurodegeneration. Therefore, the 3-nitrotyrosine levels were not determined in serum of the patients, let alone of the healthy ageing individuals of the MAAS.

It has been proposed that nitrotyrosine formation occurs via peroxynitrite formation under conditions of oxidative stress [37]. In wiew of the widespread occurrence of NO synthase in the brain, the toxic propenties of NO have attracted a lot of attention $[11,12]$. However, NO has several functions in normal physiological processes: e.g. vasodilation, influence on neurotransmission via cGMP formation or in immune reactions [6,21]. Superoxide is a byproduct of the respiratory chain, and is released in several enzyme reactions [54]. So far, there is no evidence that protein nitration is an exclusive feature of oxidative stress as compared to normal oxidation reactions. Our observation of 3-nitrotyrosine formation in control cultures, being almost detectable at the start of the experiments and further increasing during exposure lime (Chapter 4), argues against such a point of view. However it cannot be excluded that our control cultures were subject to some background condition of oxidative stress. More convincing evidence comes from the in vivo experiments, where a background level of 3nitrotyrosine was observed in animals treated with saline (Chapter 4). The reaction of nitric oxide with superoxide is limited by diffusion, which indicates that a reaction occurs immediately when these molecules meet or collide [35]. No protection, but rather increased production of 3-nitrotyrosine residues is provided by superoxide dismutase, the superoxidescavenging enzyme [37]. However, recent views state that nitration of proteins may also occur by other routes than peroxynitrite, via direct nitration by NO or other unknown mechanisms, arguing against a role of superoxide or oxidative stress in 3-nitrotyrosine formation during noimal physiological processes [19,27].

\section{Sterol concentrations}

The decreased concentration of 24S-hydroxycholesterol in patients with AD led to the question if changes in the concentration of these and other sterols could also be related to neurodegenerative processes (Chapter 6). The occurrence of altered serum concentrations of sterols in the several models used in this thesis (animals, normal ageing people and neurological patients may indicate the involvement of a common process occurring in several disease entilies, such as oxidative stress. A possible relation between cholesterol homeostasis and oxidative stress was evidenced by altered sterol concentrations in serum and in striatal tissue in our vivo model for oxidative stress. In addition, altered desmosterol concentration was obseved after experimental hypoxia [15]. But, unfortunately, the data from this thesis are 
insufficient, as is the yet avallable literature to fully explain the relationship bewween altered sterol levels and oxidative stress.

In general, the cholesterol concentration is determined by dietan intake, de novo synthesis, absorption rates and excretion [25]. Decreased serum plant sterol to cholesterol ratio, such as in our patients, is indicative for decreased cholesterol absotption. To balance the decreased cholesterol absorption, several mechanisms are avalable, e.g. decreased excretion of cholesterol or increased cholesterol synhesis. There are indications for this latter mechanism in our patients, since increased median concentration of the cholesterol precursor lathosterol was observed in serum of the group of neurological patients as a whole.

A contrasting direction of results between the animal and humans studies was observed, i.e. decreased serum lathosterol to cholesterol ratio in 3-NP treated rats opposed to increased lathosterol concentration in all neurological patients and in worse performing individuats in the MAAS. This may have implications for the extrapolation between the current animal model and human studies addressing this issue. The serum samples in this model were laken only at one time-point, i.e. three weeks after finshing the treatment. For further reseurch, it would be interesting to measure serum parameters in earlier stages, for example during treatment and directly after finishing the injections. In such an experimental setting more insight may be obtained in a possible role of sterols as early markers, as may be suggested by the relation between the sterols and cognitive perfomance in the MAAS (Chapter 9). lincreased concentrations of cholesterol precursors lathosterol and lanosterol were associated with decreased cognitive perfomance in the MAAS. This is in line with the results of decrealsed lanosterol to cholesterol ratio in the patient groups together (Chapter 11). The cholesterol excretion was not measured, and thus, no final conclusions can be drawn about the mechanisms from our observations.

New observations in our experiments were the findings on the diet derived plant sterols. No other studies ever investigated the plant sterol concentrations in relation to neurodegenerative diseases and especially Parkinson "s disease, where the most striking decreases in median concentration were found. A decrease in the concentration of the plant sterol avenasteroll was also observed in AD and patients with cognitive disorders (Chapter 11). In the rat 3nitropropionic acid model, increased concentration of campesterol was observed in striatal homogenates of treated Wistar rats (Chapter 5). These results suggest that allered plant sterol concentrations are a common finding associated with several forms of neurodegeneralion; in in vivo models and in human neurodegeneration

\section{Homocysteine}

Increased serum homocysteine concentration is a known risk factor for cardiovascular disease [73]. In view of the possible vascular involvement in AD, it has been suggested that the increased homocysteine concentrations present an increased risk for dementia $[10,16,41,46,611$. In agreement with those previous studies, increased levelts of homocysteine concentrations were observed in all neurological patients together in our patient study (Chapler 11) In the animal model, including 3mitropropionic acid treated and control animatls of two rat strains, no altered serum homocysteine concentrations were observed (data not shown). A negative correlation was observed between cognitive performance and homocysteine concentrations in the MAAS (Chapter 10). Since the relation with cognition was based on linear regression analysils using data of 93 subjects, determining a concentration of honwocysteine above which abnormal cognition might be expected for a single human being may be difficull. Only small concentration differences and thus a large overlap with controls were observed in previous studies $[10,62]$. Significant differences between groups may only be observed in liarge 
populations and this limits the applicability of homocysteine concentration as a risk factor for an individual.

\section{Inflammation markers}

In the spheroid cultures as well as in the in vivo animal experiment, we showed that the neurodegenerative processes were associated with an neuro-inflammation reaction (Chapter 4 ). From the data presented in the overview of the literature on narker concentrations in serum or CSF of demented patients, it became clear that the results with respect to inflammation markers are very variable. The concentrations of a number of markers in serum of $\mathrm{AD}$ patients showed a large overlap with controls. Furthermore, concentrations of IL-1 and IL-6 were frequently below detection limits of the current tests, and results from severall studies were contradictory. The most often investigated inflammation markers were IL-1, IL-6, TNF- $\alpha$, and $\alpha-A C T$ (Chapter 1 ). In the present thesis also one of these markers (IL-G) was investigated, and some less wellinvestigated markers were included like $14-6$ receptor, Clara cell 16 (CC16), C-reactive protein and haptoglobin. In addition, protein fractions $(\alpha 1, \alpha 2, \beta, \gamma)$, total protein, and albumin concentrations were measured.

The IL-6 receptor concentration was decreased in the AD patient group and also in the PD and the OCD group (Chapter 11). These results are in line with the wo studies so far known on decreased IL-6 receptor concentrations in CSF and one in serum of AD patients [30,31]. This marker correlated negatively with cognition in the Stroop test at baseline in the MAAS (Chapter 8), which indicates that better cognitive performance was associated with lower IL-6 receptor concentrations at that lime-point. These results may imply that inflammation processes are involved in cognitive decline, though the mechanism of the interaction between the inflammalion marker and cognition is not clear. The IL-6 concentration was not different in our patient groups compared to controls and no relation with cognition in the healthy ageing popullation of the MAAS was observed (Chapter 8 and 11 ). In fact, the $1 L-6$ concentrations were below detection limit in most of the samples of the MAAS, as was also observed by others $[48,67]$. Unless the sensitivity of the $L_{-}-6$ assay is improved, these results support the idea that it would be better to measure IL-6 receptor concentrations in further experiments.

A highly significant result was observed for the increased concentration of CC16 in PD patients. CC16 is an inflanmation suppressor [9] and has not been investigated before in this context. These results may imply that the CC16 concentration is increased as a consequence of an ongoing inflammation. The decreased IL-6 receptor concentrations observed in these PD patients fits well in this line of reasoning. In the normal ageing population of the $M A A S$, however, high concentrations of CC16 were associated with better performance on the WLTTOT. Since signs of inflammation are very clear in post mortem AD brains [23,24], CC16 would be expected to be changed in the AD patients group. Howewer, no difference in CC16 concentration was observed in serum of these patients. Thus, the $\mathrm{CC} 16$ concentration may be altered in relation with several aspects of neurodegeneration, in non-pathological as well as in pathological conditions, though the specificity and the mechanism are yet unclear.

Haptoglobin concentration seemed to be a stable marker that correlated negatively with Cognitive performance on the WLTTOT and Delayed Recall test at baseline, in cross-sectional analysis, as well as with cognitive performance over the whole six years follow-up period in the normal ageing population of the MAAS (Chapter 8). However, when the haptoglobin data were analysed at follow-up (cross-sectional), no correlation between haptoglobin concentration and cognitive performance was observed. This may be due to loss of discriminative power, as a complete data set was available only from a subset of the baseline sample at follow-up. The haptoglobin concentrations were similar in the neurological patient groups and controls. As an 
explanation for these results, it may be hypothesised that haptoglobin concentrations are altered in non-pathological cognitive perfomance, though nomalised during further pathological cognitive decline.

\section{Glial fibrillary acidic protein (GFAP)}

Increased GFAP expression is the hallmark of astrogliosis, the vigorous response of astrocytes to brain injury [17] (Chapter 1). From the data in Chapter 4 it was suggested that increased GF AP was a less sensitive marker than 3-nitrotyrosine formation, at least in spheroids oblained from Lewis rats. In the study presented in Chapter 5 , it was shown that increased GFAP concentration in the striatum correlated with several aspects of behavioural alterations in the 3 -nitropropionic acid animal model. Similarly, the results from Chapter 6 showed that increased GFAP concentrations correlated with changes in sterol metabolism. Untortunately, no test was available to measure serum CFAP concentrations in serum of affected animals. In the human sera of the patient groups, GFAP was detectable in only part of the patients IAD: 23\%: PD: $29 \%$; OCD: $37 \%$; controls $38 \% 1$. Probably increased serum levels of GFAP can only be observed in acute severe neurodegeneration, since increased serum GFAP concentrations have been reported in stroke patients [34]. Thus, GFAP is a sensitive manker for neurodegeneration in brain tissue in vivo. However, it is not a wery sensitive marker for neurodegeneration associated with oxidative stress in vitro or in human serum.

\section{Biomarker-index}

It would be ideal, and in line with the aims of this study, if a biomarker-index could be proposed, i.e. a risk indication of pathological cognitive decline. This index would be composed from the pattern of a combination of different marker concentrations in an individual.

The selection of markers investigated in Chapter 8-11 was based on the several mechanisms, oxidative stress, cholesterol metabolism, vitamin status and inflammation. Other selection criteria were: the stability of a marker in serum during variable sampling conditions, independence of circadian rlythms and dielary influences and stability during prolonged storage. The individuals in the MAAS are being lested cluring more than two hours on different times of the day. If sampling would have to be performed on an exact time point, insurmounfable logistical problems would have arisen. Thus, the samples were laken on different times of the day and under different dietary conditions. This situation has set an important dimit to our choice of marker molecules. The blood samples were processed once per day, and in this way, the time elapsed during blood sampling and serum processing was also variable. The samples from the MAS were stored for a long period, sometimes even more than $5 \mathrm{x}$ years elapsed between sampling and determination. Some samples were stored at $-80^{\circ} \mathrm{C}$ and others af $-20^{\circ} \mathrm{C}$. The stability of several inflammation markers in serum under our conditions was not clear from the literature. The control experiments described in Chapter 7 showed that $1 \mathrm{~L}-6, \mathrm{CC} 16$ and $1 \mathrm{~L}-6$ receptor were indeed stable in serum during prolonged storage. Interleukin-10, in contrast, was less stable in serum and therefore not inchuded in our analysis.

An attempt was made to construct a biomarker-index from the serum markers described in the pathological states, i.e. in patients with AD, PD and OCD (Chapter 11). The data showed that a model including cysteine, lanosterol to cholesterol ratios and $1 \mathrm{~L}-6$ receptor improved the ciliscrimination between the AD patients and heathy controls in terms of specificity and 
senstivity. These resuls were independen of age and gender. The model was relatively specific for differentiating $A D$ patients from controls $182 \%$ specificity at a sensitiwity of $64 \%$ based on the marker concentrations used. However, the specificity of the model for discriminating $A D$ patients from other cognitive disorders or PD patients was lower $152-53 \%$. This may also be due to the fact that the decreased $11-6$ receptor concentration was not specific for AD, as in Serum of the PD or OCD patients also an decrease in median IL-6 receptor concentration was observed. In addition, even in comparison with control individuals, a large overlap was observed.

As a final sllep, the combination of lanosterol and IL-6 receptor as a biomarker index was investigated in the normal healthy ageing individuals of the MAAS. No improvement in the correlation was observed after inclusion of $14-6$ receptor to the model containing only the lanosterol level (adjusted for age, gender and education). The biomarker model for AD did also not correlate with the MMSE in AD patients or the controls in the patient study in Chapter 11. These observations may indicate that the biomarker model based on the data of the present study may not reflect differences in normal ageing, but may be valid only for discriminating $A D$ patients from controls.

\section{Future perspectives}

No conclusions addressing causality from our correlation studies can be drawn. This was partly due to the lack of inter-individual differences in cognitive decline between the persons in the MAAS. Another limitation of this study was the incompleteness of the set of individual serum samples of each person in the MAAS and thus the small power of the study. Further prospective research should be performed to establish whether the biomarker index for AD indeed describes an increased risk and to determine at what cut-of point a person may be at increased risk for $\mathrm{AD}$. The marker pattern should be compared with the progression of the disease, in order to understand if the individual marker pattern is stable in the diseased state or correlates with disease progression. Other studies should be undertaken that include patients with mild cognitive impairment and these subjects should be followed to determine if a marker pattern could reflect an increased risk for dementia. Untortunately, it was not possible to replicate our combination approach in the animals, since not all markers measured in human serum could be measured in animals with the current method isterols and homocysteine could be measured in animals, several interleukins and GFAP cotild not.

The imposed setection criteria for markers investigated in this thesis may have several implications for our findings with respect to the marker pattem. In the receiver operating Charactenistics (ROC)-Curve presented in Chapter 11, still a considerable area is present to the lett of the curve and thus there is still yet unexplained variation in the patient data. Other markers may as well or fit even better into the model. For example, the data on isoprostane concentration in AD patients seemed very promising at least in CSF [75]. Only two conflicting reports about serum measurements are presently available $[58,70]$. The sampling of isoprostanes should be perfomed under very strict conditions, and it was thus not possibte to measure these compounds in our study $[2,69]$. Finally, it has to be realised that refinement of the present model will require a major investment in terms of personnel and especially in the costs of running all necessarily analyses. 


\section{Conclusions}

Thus, prospects for development of an in vitro diagnosis based on serum marker concentrations for neurodegenerative disorders in the near future are not wery good. Nevertheless, recent development of experimental therapeutics for $A D$, such as vaccination against $A B$ [93] or implantation of stem cells [82], inevitably lead to the question at which time poim prevention has to be started. It is difficult to predict if imaging techniques will be developed that fall that early diagnosis or a risk indication can be given. However, in view of the human genome project and the emergence of a plethora of studies on genetic risk factors for familial as well as for sporadic AD $[4,52,55,60,77,84,89]$, an interesting option might be the combination of a biomaker-index with genetic risk factors.

\section{References}

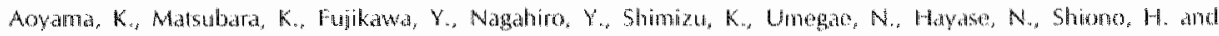

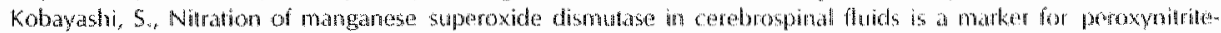
mediated oxidaliwe stress in neurodegenerative diseases, Am Neurol 47 (2000 5247 .

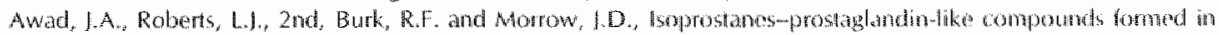

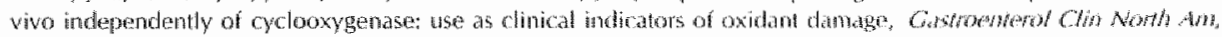
$25(1996) 409-2 \pi$

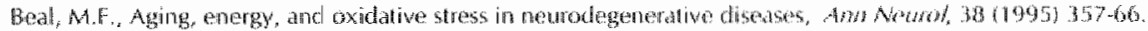

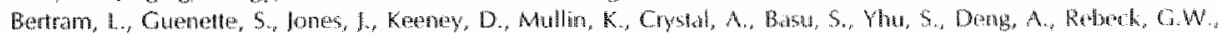

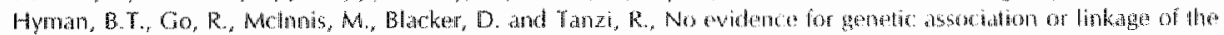

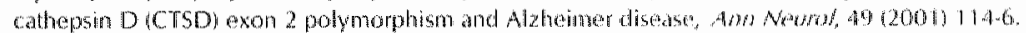

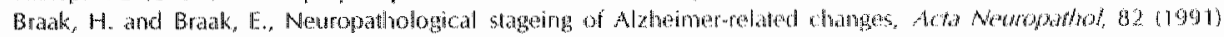
239.59 .

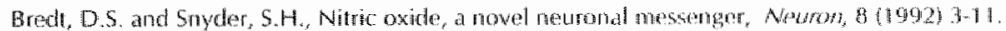

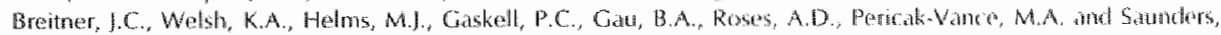

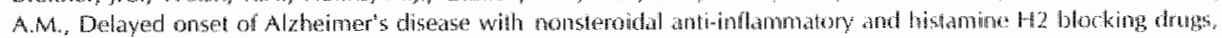
Newobiol Aging $1611995 \% 52330$

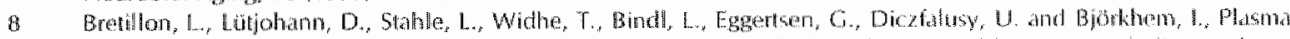
fenels of 245 -hydroxychosterol reflect the balance between cerebral production and hepatic matabolism and are inversely related to body surace, flapd Res, 41 (2000) 840 -845.

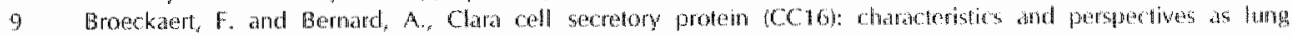
peripheral biomarker, Clin Exp Alkergy, $30120000469-75$.

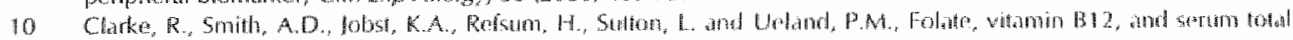

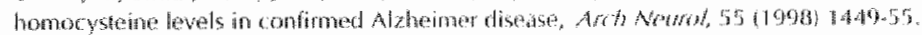

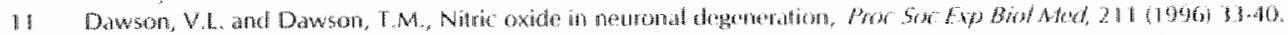

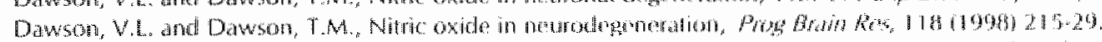

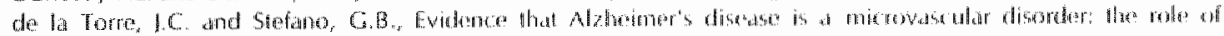

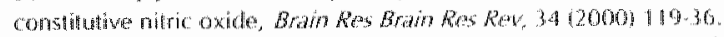

\section{0 .}

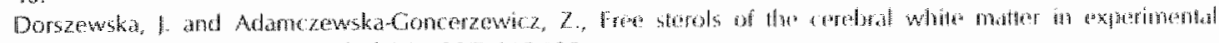
severe hyposia, folia Nesropinhol, 3501997$) 115-120$.

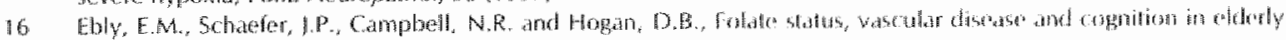
Cunatlitas, Age, Agetrge, $27(1998) 4135-91$.

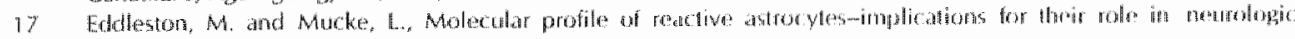
disedse, Neurosioncas, 54 (1993) 15 -36.

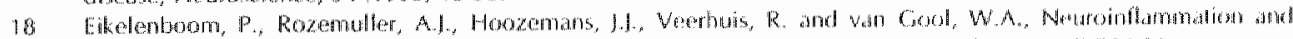

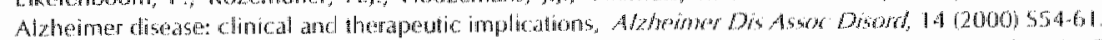

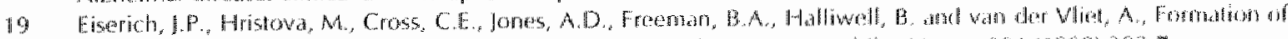

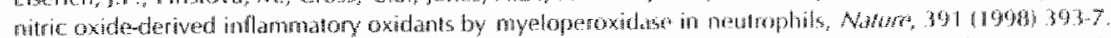

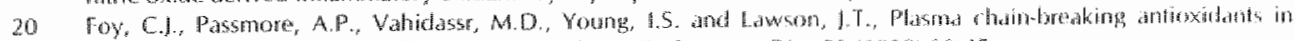

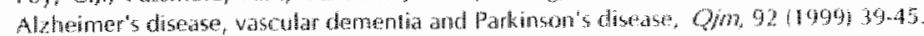

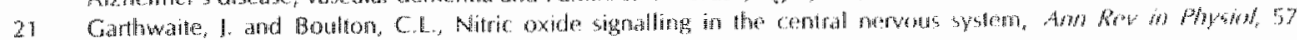
(1995) $683-706$.

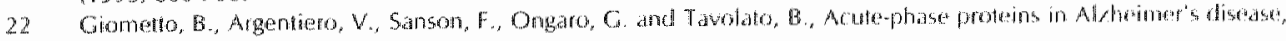
Eu"Newo/, 28 11988j 30-3. 


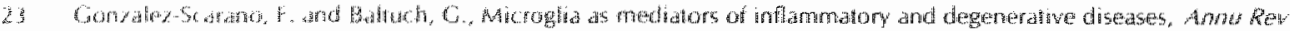
Noitris $22(1959) 219-40$

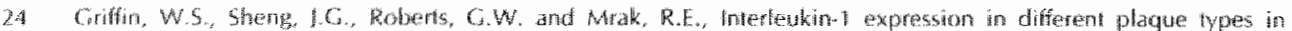

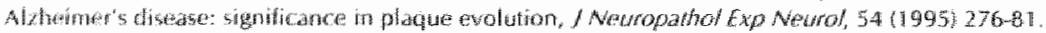

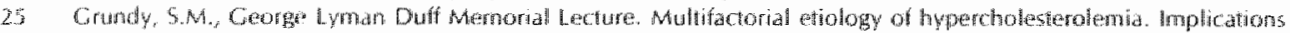

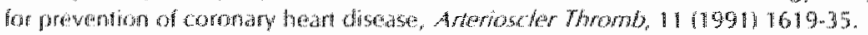

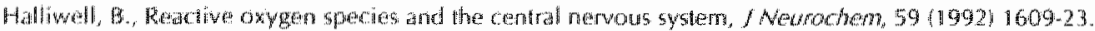

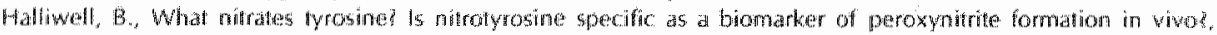
FEBS Lah, $411(199 \pi) \div 5,60$

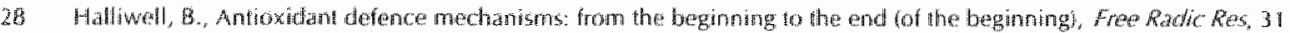
$(1999) 261,72$.

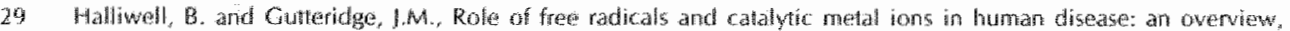
Mathods Enymol, 186 (1900) I-85.

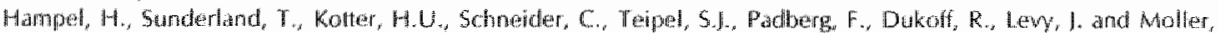
H.1. Decreased soluble inferleukinw6 seceplor in cerebrospinal fluid of patients wh Azheimers clisease, Bran Res, $700(1998) 356-9$.

Mampel, H. Teigel, S.I. Padberg. F, Haslinger, A., Riemenschneider, M., Schwarz, M.J., Koher, H.U., Scheloke, M., Buch, K. Stubner, S., Dukoft, R., Lasser, R, Muller, N., Sunderland, T., Rapoport, S.I. and Moller, H.l.

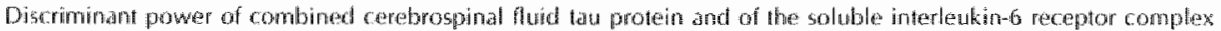
in the diagnosis of Alaheimut"s disedse. Bran Ras, 823 r19991 104.12.

Henstey, K., Mall, N., Subramanim, R., Cole, P., Harris, M. Aksenow, M. Aksenowa, M., Gabbila, S.P., Wu, I.F.,

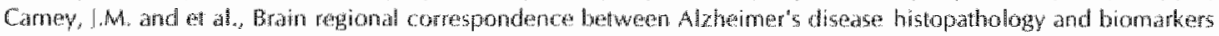
of protain oxidation, / Neturochern, 6511995 ) 2146-56.

Henstey, Ko, Maid, MA., Wu, Z., Sang, Harkestory, WR. alld Floyd, R.A., Electrochemixal analysis of protein

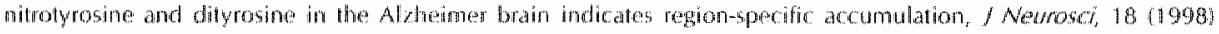
$0126-32$

Hermenn, M. Vos, P., Wundertich, M.T. de Bruin, C.H. and Lamers, K.J., Release of glial tissuespecific protens after acuse stroke: A comparative analysis of serum concentrations of protein S-ioos and glial fibrillary acidic protein, srose, 31 12000) $2670 \mathrm{~m}$.

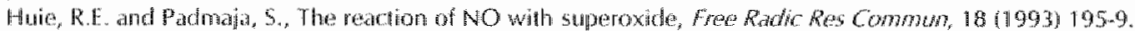

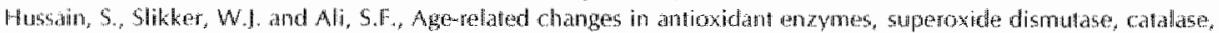

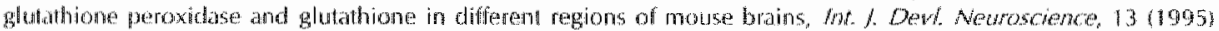
a $11-817$.

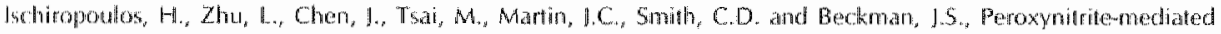
Iynosine nitration catalyzed by superoxide dismutase. Arch Biochem Bwopys, 290 (1992) $431-7$. Heandel, C. Nicolas, M.B., Dubois, F., Nabet-Bellewille, F. Penin, F, and Cuny, G, Lipid peroxidatum and free radical scatenger in Alzheimes co disease, Cerontologk, 35 (11989) 275482 .

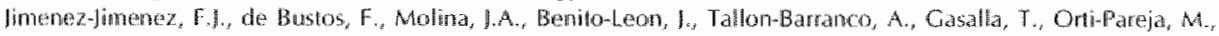
Cuiliamon, F, Rubio, 1.C., Arenas, I, and Enriquez-de-Salamanca, R. Cenebrospinal fluid levels of alphatocopherol

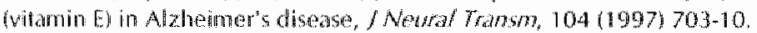

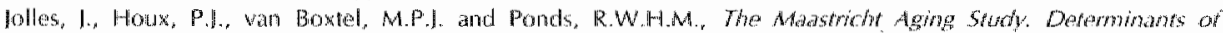

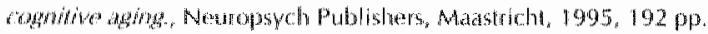

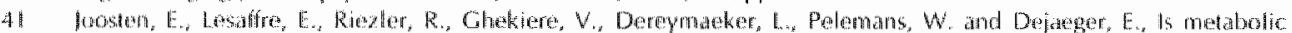

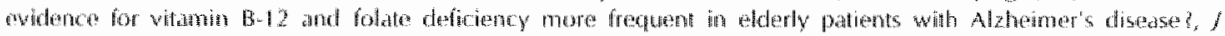

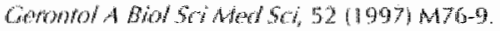

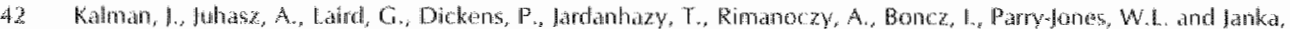

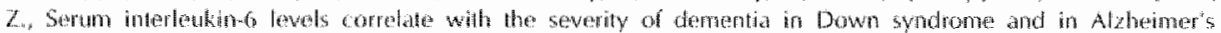

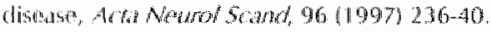

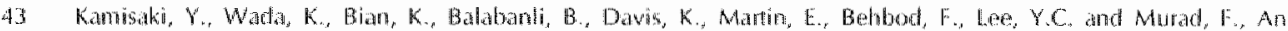

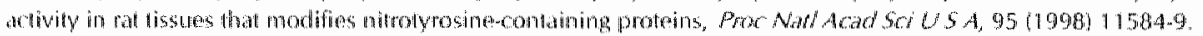

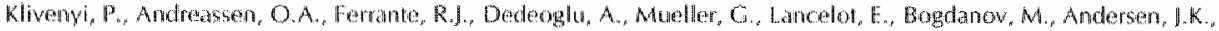

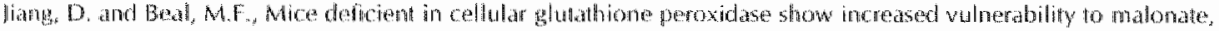

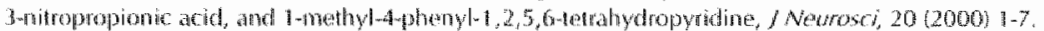

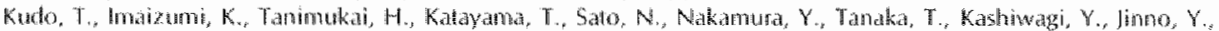
Tohyana. M. and Takeda, M. Aro cerebrowacular factors involved in Alzhemer"s disease?, Neyrobior Aging. 21 (2000) $215-24$

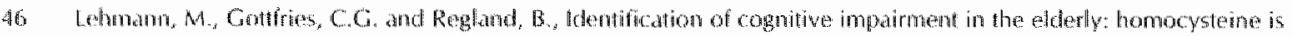

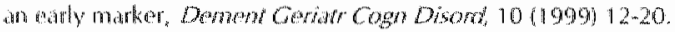

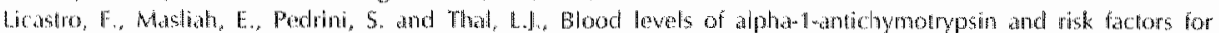

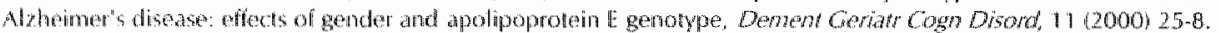

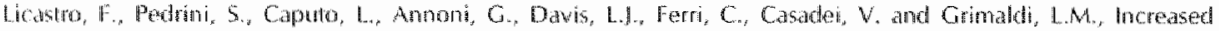

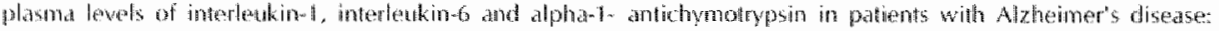

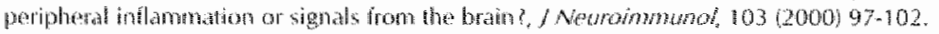


in Azheimer's disease ventricular CSF, Nearochom. $72199991771-6$.

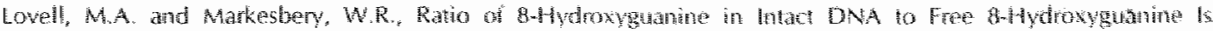

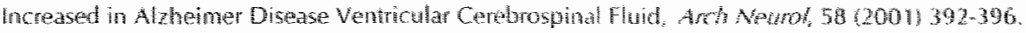

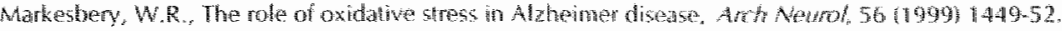

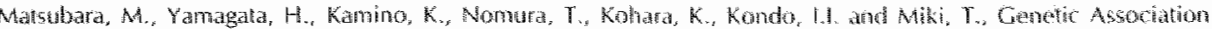

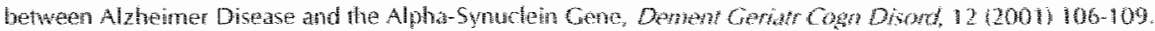

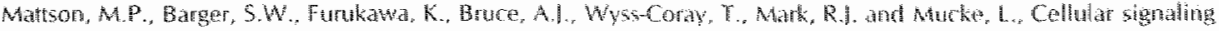

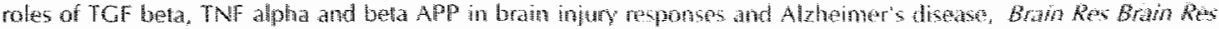
Rey, $23(11997) 4761$.

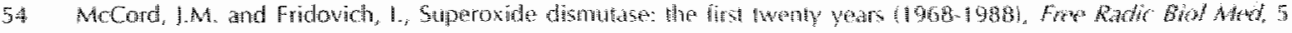
(1988) 363-9.

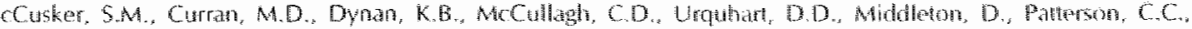

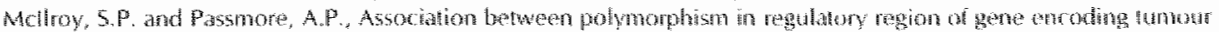

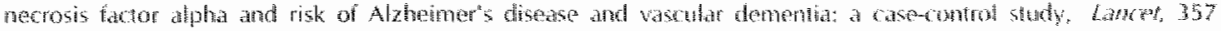
120011436 .

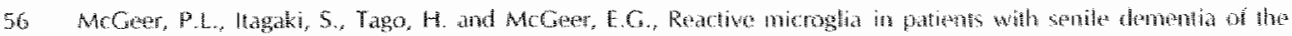

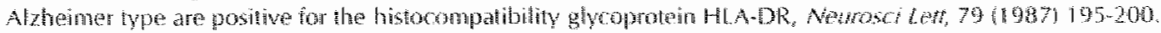

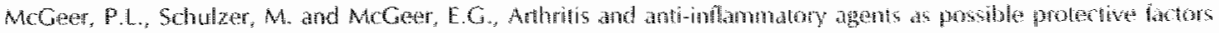

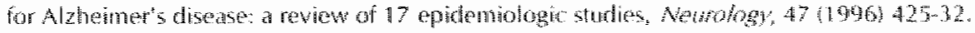

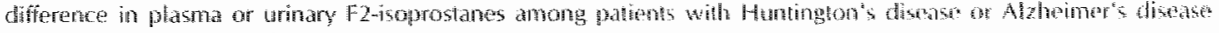
and controls. An Nedrol, 480000 9 950 .

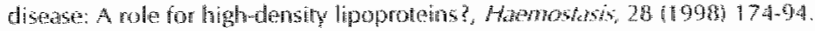

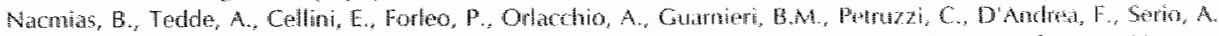

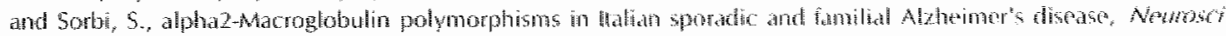
Lat, $299(200119-12$.

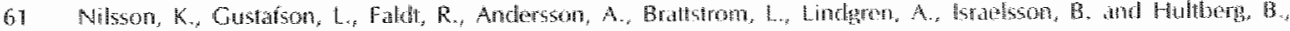

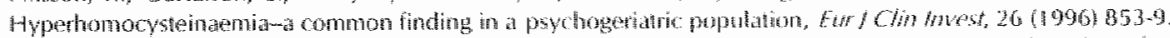

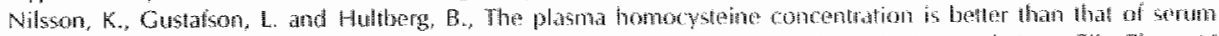
methylmalonic acid as at marker for sociopsychological pertormance in an psychogeriatro population, Chin chen, 46 $(2000) 691.6$.

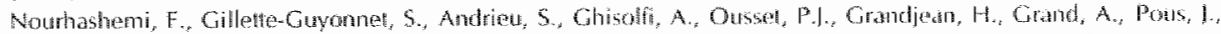

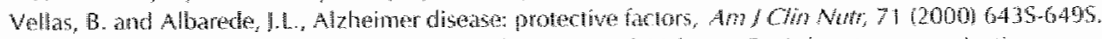

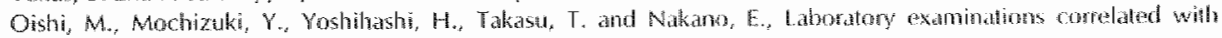
severily of dementia, Anr Clin Lab Sci, 26 a $1996 \% 340-5$.

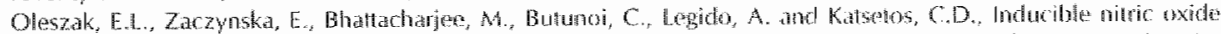

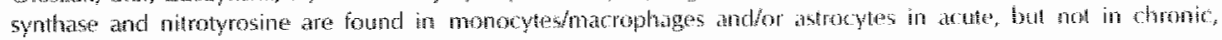

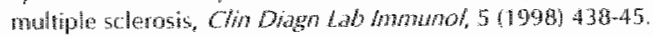

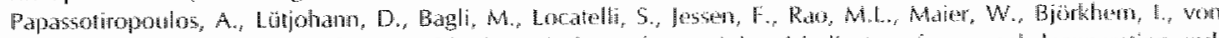

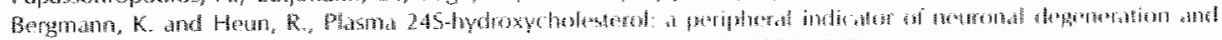

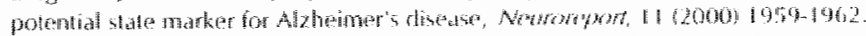

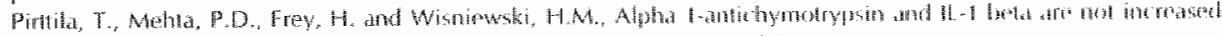

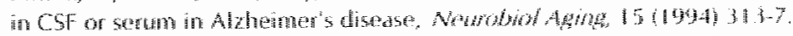

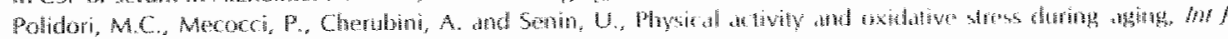

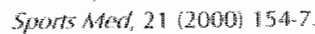

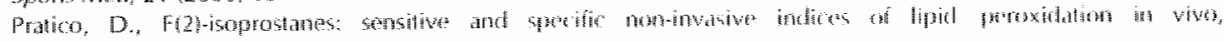
Atherowdrosis 147 (1999) 1-10.

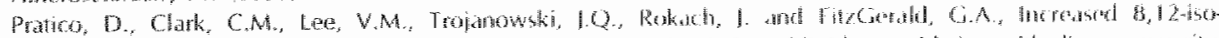

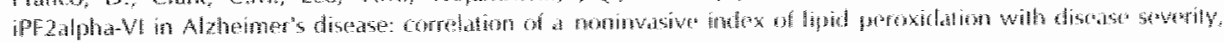
Am Netsol, 48 8000) 809-12

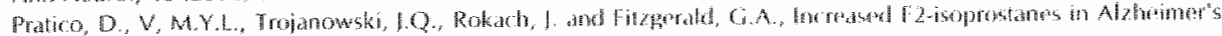

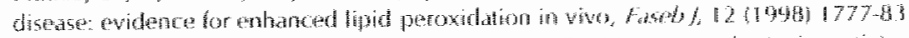

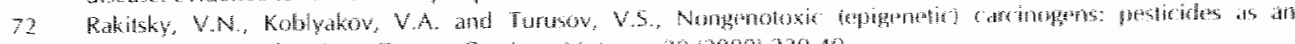

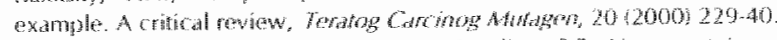

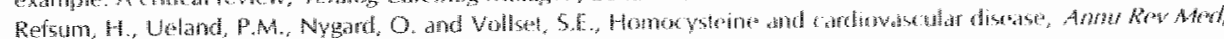
$49(1998) 31-62$

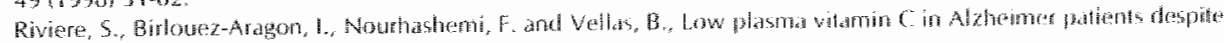
an adequate diet, hil Getiat Pychidr, 13 (1999) 749.54 .

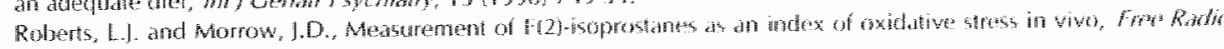
Biol Med 28 (2000) 505-513.

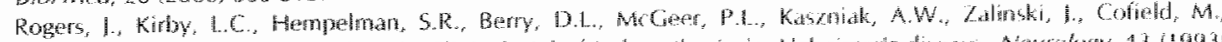

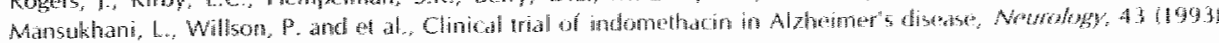
1609 an 1 


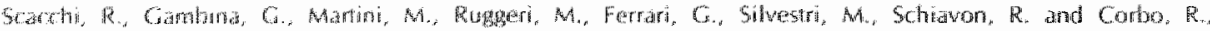

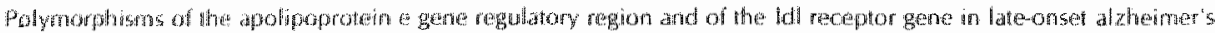

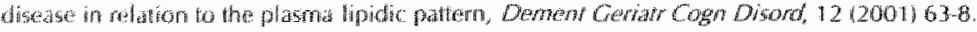

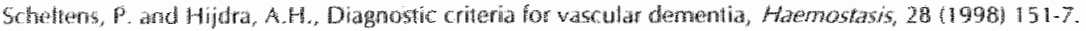

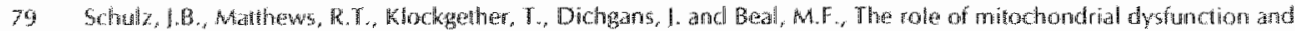

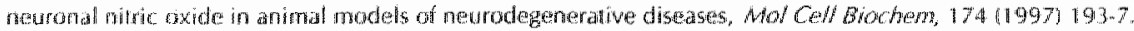

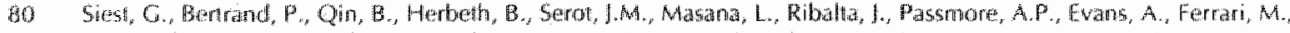

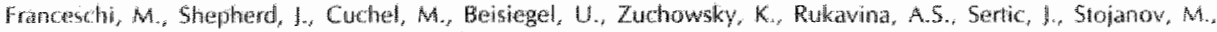

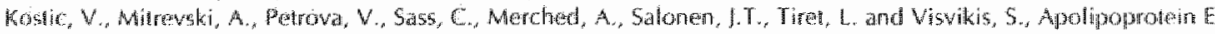

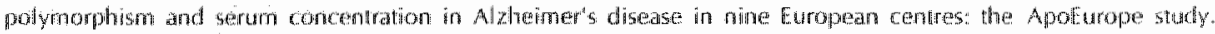

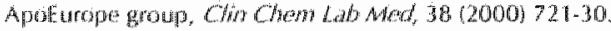

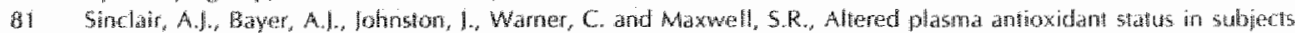

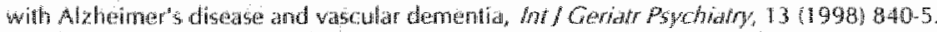

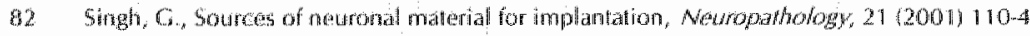

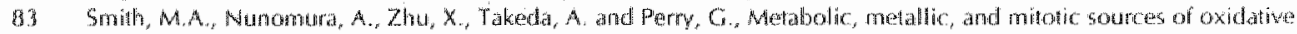

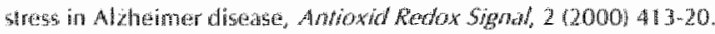

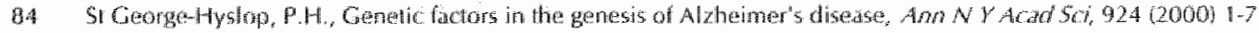

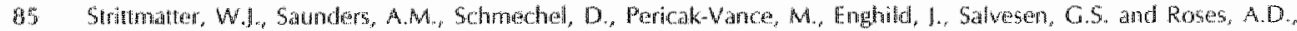

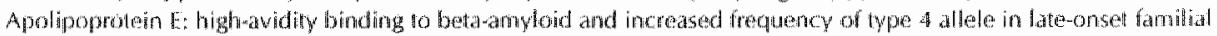

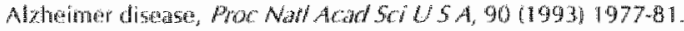

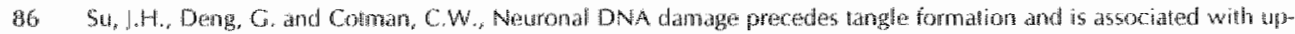

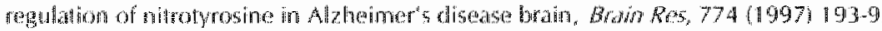

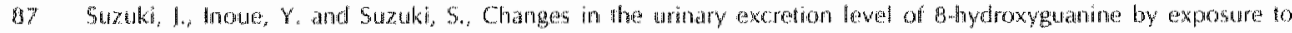

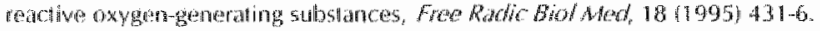

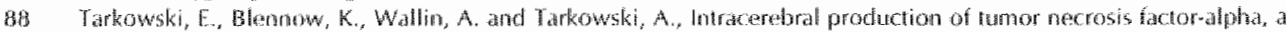

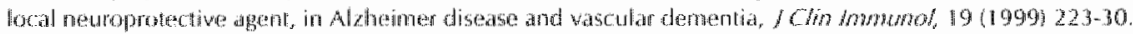

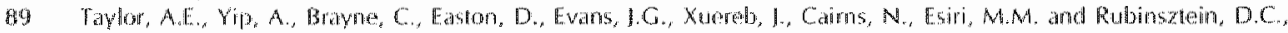

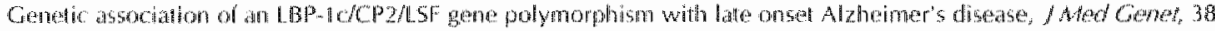
(2001) $232-233$

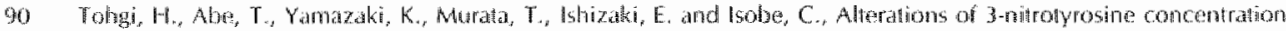

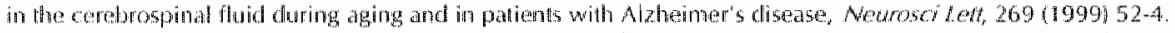

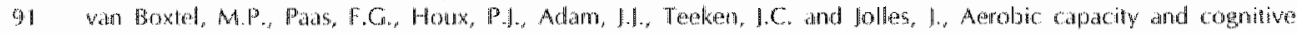

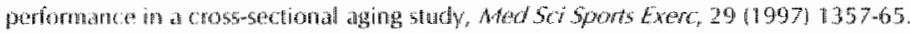

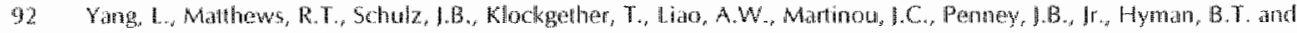

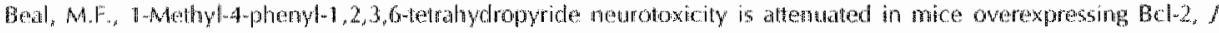

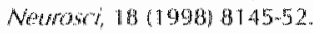

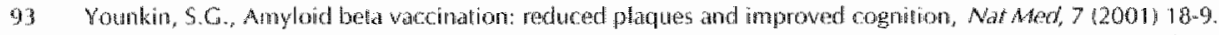

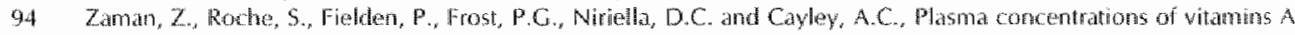

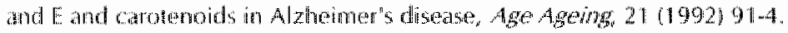




\section{Summary}

The present thesis addressed the question whether it might be possible to develop an in vitro diagnosis for neurodegenerative diseases using serum marker molecules. Taking into account the complex pathophysiology of these diseases, the investigations were based on lhe assumption that a combination of marker molecules related to different pathophysiological mechanisms would enlarge the chances for success. The relation between these markers and neurodegenerative processes was investigated on four levels: first in cell culture studies, secondly in an in vivo animal model. Next, the relation between cognition and serum parameters in serum of normal ageing humans was examined and lastly, serum of paltents with defined neurodegenerative diseases was analysed.

In Chapter 1, an overview of the current state of knowledge on biological markers in serum or plasma and cerebrospinal fluid (CSF) in relation to dementia was given. The objective was to identify markers that could be included in a model combining markers from different pathophysiological backgrounds.

Increased tau protein and decreased concentrations of amyloid $\beta$ in CSF have been reponted consistently in Alzheimer's disease (AD) patients compared to controls.

The CSF levels of all markers for inflammation give a variable picture. So far, only consistent changes have been reported for the increase in haptoglobin fragments. The results on the inflammation markers interleukin-6 (LL-6) and tumour necrosis factor-a (TNF-a) in serum were pointing to increased IL-6 concentration and decreased TNF-a concentration in AD patients, though several studies showed no difference. Increased all-antichymotrypsin concentration in serum might be the most convincing, most imwestigated serum inflammation marker to date.

Studies on the oxidative stress markers 3-nitrotyrosine, the DNA oxidation product 8 hydroxyguanine, and isoprostanes have shown increased concentrations of these markers in CSF when patients with AD were compared to controls. Increased mean concentrations of autoantibodies against glial fibrillary acidic protein (GFAP) in serum and CSF of AD patients was observed. The altered serum concentration of $245-\mathrm{OH}$-Cholesterol (increased in mild dementia, decreased in severe dementia), telencephalin and CFAP autoantibody increased in serum and (SF) might present as brain specific markers. However, there are relative few data awailable on these possible marker molecules.

The decreased serum and CSF concentration of vitamins $(A, B, C$ and E) may give an indication of the oxidative status in general. Increased homocysteme concentration has generally been observed in serum of AD patients, though some overlap with controls exists. The slighlly decreased concentrations of Apolipoprotein $E$ in serum of $A D$ patients indicate what this is a wery insensitive marker.

In summary, so far, differences in serum or CSF concentrations have been obseved for sovera! marker molecules related to each pathophysiological mechanism. However, a large overlap in mean concentrations and variable results have been observed in studies comparing AD patients with controls for the single markers. These results support the idea that investigating serum concentrations of markers reflecting only one of the pathophysiological meachanisms may not be sufficient to diagnose AD patients.

The aim for this thesis was to investigate a large number of possible marker molecules which are related to oxidative processes, inflammation, of fipid metaloolism. In addition, two brain specific structural markers that are present an low level in serum of healthy individuats were investigated. Firstly, some of these markers were investigated in animal modrats for neurodegeneration 10 provide evidence for the relevance of these substances as edrly marker for neurodegeneration. For these animal studies a model was chosen that had the possibilities 
fo study oxidative stress in relation to excitotoxicity, inflammation, and brain specific structura! changes. Next, the marker molecules were to be analysed in sera of a group of nomal ageing individuals at three different time-points over a lime span of six years. These serum values were to be correlated with the outcomes on four differen neurocognitive assessments done at the same timepoints as the serum sampling. Finally, these outcomes would be compared with concentrations of these markers in serum of a diagnosed group of $A D$ patients. The results obtained in AD patients were to be compared to serum concentrations in patients with Parkinson's disease or other cognitive disorders. The purpose of this study was to search for a combination of markers which would held some prognostic value for diagnosing $A D$ with a calculated specificity and sensitivity.

In Chapter 2, an in witro cell culture model was used to investigate whether functional nitric oxide (NO)-cyclic guanosine $3^{\prime}, 5^{\circ}$ - monophosphate (CGMP) pathways were present in differentiated spheroids. In the literature a role for the NO-CGMP signalling pathway during development and neurodegeneration has frequently been suggested. Whole brain spheroids provide a suitable model to study these phenomena. In three-week-old spheroids, soluble guanylate cyclase was stimulated with $\mathrm{N}$-methyl D-aspartic acid or sodium nitroprusside (NO donor. The results showed that the NO synthase-cGMP pathway is present in the culture system. Soluble guanylate cyclase-dependent CGMP formation was found in NO synthase containing neurons, in neurons of the GABAergic, glutamatergic and chollinergic system, and in astroglia and oligodendroglia. Activation of particulate guanylate cyclase by atrial natriuretic peptide also triggered an increase in CGMP production. Particulate guanylate cyclase was found in astroglia and in microglia, as well as in glutamic acid decarboxylase and calbindin containing structures, and in neuronal NO synthase containing neurons. Chronic imhibition of NO synthase during culture development had no effect on soluble or particulate guanylate cyclase functioning. Similarly, inhibition of soluble guanylate cyclase during culture development did not have any effect on NO synthase and particulate guanylate cyclase functioning.

It was concluded that NO synthase and both soluble and particulate guanylate cyclase are present in whole brain spheroid cultures and that their activity can be influenced by several stimuli. The spheroid culture system constitutes a suitable model to study the NO-cGMP pathway during brain development and degeneration in mammals.

The presence of soluble and particulate cyclase in astroglia, as observed in Chapter 2 , led to the question if these two enzymes could also be simultaneously present in one astrocyte. Therefore, in Chapter 3, the localisation of particulate and soluble guanylate cyclase was studied in hippocampal astrocytes.

Counting the colocalisation of CGMP immunoreactivity with the astrocytic marker GFAP after stimulation of brain slices with sodium nitroprusside $(0.1 \mathrm{mM})$ or atrial natriuretic peptide $(100$ nM) il was shown that at least $67 \%$ of the hippocampal astrocytes contained both guanylyl cyclase isolorms. In addition, it was shown that a large number of atrial natriuretic peptide, brain-derived natrituretic peptide or sodium nitroprusside responsive cells contain the $\beta \pi$ subunit of the soluble guanylyl cyclase.

The results show that, in at least a subset of hippocampal astrocytes, soluble and particulate guanylyl cyclases are simultaneously present in the same cells.

The aim of the study presented in Chapter 4 was 10 investigate whether 3-nitrotyrosine formation can be used as an early marker for neurodegenerative processes. The dose response relation of 3-nitrotyrosine was investigated in a model for 3-nitropropionic acid exposure. This was performed in whole brain spheroid cultures as well as in animals, using Lewis and Wistar rats. 
Increased 3-nitrotyrosine concentration in spheroid cultures from Lewis rats was observed at lower dose and shorter exposure time as compared to alterations in GFAP concentration, decrease in glutamine synthetase activity or cell loss. In addition, staining of GABAergic neurons was affected, while neuronal NO synthase staning was preserved. Dithiothreitol, but not glutathione, inhibited the formation of 3-nitrotyrosine. Interestingly, Ninitro-1.arginine methyl ester potentiated the 3-nitrotyrosine formation after five days of exposure to 3 nitropropionic acid. Spheroid cultures obtained from Wistar rats did not show increased 3 nitrolyrosine concentration after exposure to 3-nitropropionic acid during five days. In the striatum of 3-nitropropionic acid exposed Lewis and Wistal rats, no change in 3-nitrotyrosine concentration was observed. The concentration of GFAP was increased and activation of microglial cells was observed in treated Wistar rats.

It was concluded that 3-nitrotyrosine was a more sensitive marker than GFAP and glutamine synthase in spheroid cell cultures of Lewis rats. The strain-dependent formation of 3 nitrotyrosine residues indicates an influence of genetic background on the sensitivity of this biological marker for oxidative stress. Finally, the similanites between the 3 -nitropropionic acid spheroid model with in the vivo model indicate that the spheroid cultures provide a good alternative for chronic exposure of animals to neurotoxins.

The study presented in Chapter 5 investigated whether behavioural alterations in the 3mitropropionic acid animal model were related to striatal damage. The 3-nitropropionic acid animal model is a model where oxidative stress, excitotoxicity and mitochondrial dystunction, phenomena common to various neurodegenerative diseases, are irvolved.

Wistar and Lewis rats were exposed to 3-nitropropionic acid and their behavioural performance (open fteld, walking pattern and Morris Water Maze task) was tested after the injections and after a recovery period of three weeks. No changes in activity were found in the open field test. Altered walking pattern was observed in the footprint analysis, although a different response was observed in the Wistar rats compared to the Lewis rats. Initially increased latency limes were observed during visual discrimination learning in the Morris Water Maze lask in 3nilropropionic acid treated Wistar rats compared to Wistar controls. During spatial discrimination learning (invisible platiorm) in the Morris Water Maze task the swimming velocity was decreased in both rat strains as a result of 3 -nitropropionic acid treatment. Increased striatal GFAP concentration in Wistar rats correlated with several parameters of the footprint analysis and with the latency and distance in visual as well as spatial discrimimation learming in the Morris Water Maze.

It was concluded that measurement of walking pattem and spatial orientation perfomance atre sensitive indicators to monitor behavioural changes in relation to stratal degeneration in the 3 nitropropionic acid animal model. In addition, Lewis rals are less sensitive towards 3 nitropropionic acid treatment than Wistar rats.

In Chapter 6 , the long-term effect on strialal and serum 245-hydroxycholesierol concentrations was examined in this rat model for oxidative stress induced neurodegeneration.

Concentration of 245 -hydroxycholesterol, a brain specific cholesterol metabolite, was determined in striatal homagenates as well as in serum samples from Wistar and Lewis rats treated with 3-nitropropionic acid. In addition, concentrations of cholesterol, cholesterol precursors and metabolites, and the diet derived plant sterols were determined.

Three weeks atter cessation of treatment, no difference in 245 -hydroxycholesterol concentrations was observed in striatal homogenates or serum of treated rals. However, ratios of campesterol to cholesterol in the striatum were higher after 3 -nitropropionic acid treatment as compared with controls of both rat strains suggesting an increased passage across the bloodbraimbarrier (BBB) for this plant sterol. Ratios of lathosterol to cholesterol in serum and striatum were lower in treated rats as compared with controls of both rat strains. Absolute concentrations 
of serum and stratal cholesterol precursors and plant sterols differed between the controls of both rat strains.

It was conduded that the changes obserwed in sterol concentrations in the striatum as well as in serum in this model indicate that chalesterol homeostasis is affected during neurodegenerative processes associated with BBB damage.

In Chapter 7, important technical sources of variability in serum concentrations of some proteins related 10 inflammation reactions were investigated. Especially the stability of $I L-6$, its receptor (IL-6 receptor), $\mathrm{L}-10$ and Clara cell 16 (CC16, an endogenous cytokine inhibitor) in human serum was studied, using an accelerated stability testing protocol. Further, the effects of the time delay between blood sampling and sample processing, clotting temperature and repeated freeze-thaw cycles on the serum levels of these proteins were determined.

Series of serum samples were incubated at $4^{\circ} \mathrm{C}, 20^{\circ} \mathrm{C}, 30^{\circ} \mathrm{C}$ and $40^{\circ} \mathrm{C}$ during 1 to 21 days. It was shown that IL-6 and CC16 concentrations did not change upon incubation at $4{ }^{\circ} \mathrm{C}, 20^{\circ} \mathrm{C}$ and $30^{\circ} \mathrm{C}$, while $1 \mathrm{~L}-6$ concentrations significanly declined after 11 days at $40^{\circ} \mathrm{C}$. The concentrations of $1 \mathrm{~L}-6$ receptor and $\| \mathrm{L}-10$ did not change at $4^{\circ} \mathrm{C}$, but were significantly decreased at $20^{\circ} \mathrm{C}$ after 21 and 14 days respectively. The concentrations of these two inflammation markers were decreased after 1 day at $30^{\circ} \mathrm{C}$ or $40^{\circ} \mathrm{C}$. Arthenius plots indicated that $\mid L-6$ receptor and $I L-10$ are $\$$ table at $-20^{\circ} \mathrm{C}$ for at least several years. Since their relative stability in serum, no Arrhenius plof could be calculated for $11-6$ and CC16. The concentrations of the proteins examined were not sigmificantly altered by repeated freeze-thaw cyctes, nor by extended clotting times at $4^{\circ} \mathrm{C}$ or $20^{\circ} \mathrm{C}$.

It was concluded that serum samples for the correct determination of $1 L-6,11-6$ receptor and $\mathrm{CC} 16 \mathrm{can}$ be stored at $-20^{\circ} \mathrm{C}$ for longer periods of time. However, for IL-10 determinations, storage at $-70^{\circ} \mathrm{C}$ is recommended. These findings are of special importance for large screening programs and longitudinal studies.

The aim of Chapter 8 was to investigate whether a causal relation exists between serum inflammatory protein levels and cognitive performance in a healthy ageing population.

A cohort of 144 individuals was tested lage 30-80) three times during six years of follow-up, as part of the MAastricht Ageing Study (MAAS). Domains of cognition that were addressed were cognitive speed (Letter Digit Coding test), attention and information processing (Stroop rest) and memory (Word Learning test). Serum concentrations of the inflammatory proteins IL 6 receptor, CC16, Creactive protein, haptoglobin, albumin, total protein and protein fractions $(\alpha 1, \alpha 2, \beta$. 7) ware investigated.

The results showed that levels at baseline of haptoglobin correlated negatively with cognitive performance on the Stroop test ( $P<0.05$ ) and on the Word Learning Recall test $(P<0.05)$ during six years of lollow-up. Levels of Creactive protein at baseline correllated negatively with perfomance on the Word Learning fest during six years of follow-up $(P<0.05)$.

In conclusion, relatively high concentrations of these two proteins that reflect inflammation reactions may be indicative for impaired cognitive performance. Further follow-up studies should indicate whether individuals with relatively low cognitive performance and high levels of haploglobin are more prone to progressive cognitive decline in time. These results support the hypothesis of the involvement of inflammation reactions in nomal cognitive ageng.

The study presented in Chapter 9, examined if precursors and metabolites of cholesterol in serum, reflecting whole body cholesterol homeostasis, are, eventually causal, related to cognitive performance in the healthy ageing population of the MAAS.

Serum of a cohort of individuals (aged 30-80 years, total $n=144$ ) from the MAAS was analysed at two time-points over a follow-up period of six years. Domains of cognitive function andressed were cognitive speed (Letter-Digit Coding test), attention and information processing (Stroop test) and memory (Word Learning test). Serum concentrations of cholesterol and the 
cholesterol precursors lathosterol, lanosterol and desmostarol as well as the dietary phant sterols campesterol and sitosterol were investigated. In addition, levels of the oxysterol $245-$ hydroxycholesterol and, for comparison, of 275 -cholesterol were determined.

The results showed that serum concentration at baseline and at follow-up of lathosterol and lanosterol, absolute and as ratio to cholesterol, correlated negatively with cognitive performance on the Word Leaming test. These effects were independent of age, gender and level of education. Multi-level repeated measurement analysis showed that the concentration ass ratio to cholesterol at baseline of lathosterol and lanosterol correlated with cognitive performance during the whole follow-up period. The concentration of 275 -cholesterol, a bile acid precursor ubiquitously produced in the whole body, and 245-hydroxycholesterol, a brain specific cholesterol metabolite, showed only spurious correlations wirh outcomes on the cognitive tests in this normal ageing population. The serum concentrations of the plant sterols campesterol and sitosterol were not related to any of the cognitive outcomes.

It was concluded that relative high serum concentrations of cholesterol precursors and their ratios to cholesterol are associated with relatively low cognitive performance in this nomal ageing population. Further follow-up studies must give the answer to the question whether increased serum concentrations of cholesterol precursors over prolonged periocls of time $>6$ yearsl are a risk factor for increased worsening of the cognilive performance.

The aim of the study in Chapter 10 was to determine if serum homocystene is independently related to, or even a risk factor for, cognitive performance during follow-up in the healthy ageing population. Total homocysteine is a marker for whamin B12 or folic acid deficiency. In the literature, elevated total homacysteine concentrations are associated with a decrease in cognitive performance in the ageing population and with dementia.

A cohort of 144 individuals (aged 30-80 years) from the MAS was tested at baseline and after six years of follow-up. In addition, patient groups diagnosed for Alzheimer's disease $(n=34)$, Parkinson's disease $(n=46)$ or other cognitive disorders $(n=47)$ were compared to a second group of controls $(n=61)$. The same domains of cognitive function as in Chapter 8 and 9 were addressed; i.e. cognitive speed (t.etter-Digit Coding test), attention and information processing (Stroop test) and mentory (Wond Learning test). In addition lo homocysteine, serum concentrations of folic acid and vitamin $B 12$ were investigated.

In all three patient groups together, stightly increased levels of homocysteine, in line with the literature, and decreased vitamin $B 12$ concentrations were obserwed. The results showed that serum concentrations at baseline of homocysteine correlated negatively with cogmitive performance on the Word Learning test in the cohon of the MAAS. These affects were independent of age, gender, education level or folic acid concentration. Folic acid concentration showed a negative correlation with cognitive performance on the word leaming test at baseline only, while the vitamin B12 concentration was not correlated to the oulcomes of the cognitive tests in this population. Muti-level repreated measurement analysis showed that the concentration at baseline of homocysteine correlated with cognitive perfornance durng vhe whole follow-up period.

It was concluded that lotal homocysteine may be related to non-pathological cognitive performance as well as with degenerative processes related to cognitive impaiment. Elevathed homocysteine concentrations appeared to be a marker indicative for low cognitive performance over an extended period in the nomal ageing population.

The aim of the study presented in Chapter 11 was to devise a model to diagnose cognitive impaiment based on serum marker concentrations. This model was formed by relating all the concentration data of 29 serum markers to a diagnosed group of AD patients. The combined model was finally applied to a large group of normal ageing people from the MAAS.

Serum of patients with Alzheimer"s disease $(A D, n=34)$, Parkinson's disease $(P D, n=46)$, other cognitive disorders $i n=47)$ and healthy controls $(n=61)$ was analysed for 29 marker molecules. 
Thus, senm concentrations were determined for: 1) inflammation markers such as C-reactive protein, $1 L-6,146$ receptor, CC16, protein fractions (total, $\alpha 1, \alpha 2, \beta, \gamma$, haptoglobin and albumin; 2) the cholesterol precursors lathosterol, lanosterol and desmosterol; cholesterol; the cholesterol metabolites cholestanol, 245-hydroxycholesterol and 275-cholesterol; the dietary plant sterots campesterol, sitosterol, stigmasterol, brassicasterol and avenasterol; 3) the brainspecific proteins telencephalin and GFAP; 4) metabolites of the methionine pathway. homocysteine, cysteine, witamin B12 and folic acid.

The results showed that a combination of the decreased serum lanosterol concentrations with the decreased LL-6 receptor and the increased cysteine concentration improved the discrimination between AD patients and controls as compared to the serum concentration of these markers alone. No correlation was observed between the combination of these three markers and the severity or duration of the disease, or with age in the AD patients. The combination of the serum lanosterol and $\mathrm{L}-6$ receptor concentration in cognitively healthy controls did not correlate with outcomes on the Word Learning test, the test for attention and information processing (Stroop) nor a test for speed (Lettef-Digit Coding lest). These results imply that the model may not reflect differences in normal cognitive ageing.

It was concluded that the combination of markers related to different pathophysiological mechanisms improved the discrimination of patients with Alzheimer's disease from controls. The decreased sterol concentrations in serum of all neurological patients warrants ciloser attention to the sterol homeostasis in relation to the central nervous system.

Finally, it was concluded (Chapter 12) that: 3-nitrolyrosine and GFAP may be sensitive and early markers in specific in vitro or in vivo animal models for neurodegeneration, and possibly also in human CSF. However, the levels of these markers are too low in relation to the cognitive disorders under investigation to be measured in human serum with the avallable methods. Changes in inflammation markers have been observed in the cell cultures as well as in the in wivo rat 3-nitropropionic acid model. In addition, increased concentrations of haptoglobin, $C$ reactive protein and $\mathrm{CC} 16$ were associated with worse cognitive functioning in our normal ageing population. Finally, decreased concentrations of $\| \mathrm{L}-6$ receptor and increased concentrations of $\mathrm{CC} 16$ were observed in serum of patients with neurodegenerative diseases. These four lines of evidence strongly support the role of inflammation in neurodegenerative processes. Untiottunately, these inflanmation markers are not specific for neurodegeneration. Alterations of cholesterol homeostasis during neurodegeneration, i.e. changes in cholesterol precursor and plant sterol concentrations, was supported by the results obtained in the experiment with the 3enitropropionic acid animal model as well as by the results obtained in the longiludinal reseanch of the MAAS and the remarkable results obtained from the patient study.

The combination of makers related to different pathophysiological mechanisms improved the discrimination of patients with Alzheimer's cisease. Future research will demongtrate if additional markers will enhance the discrimination between patient groups and if the proposed combination model may also be used to make an early risk estimation or even an in vitro diagnosis for AD. 


\section{Samenvatting}

In dit proefschrift stat de vaag centraal of het mogelijk is on een diagnose voor neurodegeneratieve ziekten te onwikkelen op basis van in vitro analyse van serum bestanddelen. Aangezien de neurodegeneratieve ziekten gekenmerk worden door een complexe pathofysiologie, waren de studies gebaseerd op hel uitgangspunt dat de kansen op succes vergroot kunnen worden als een combinatie wordt gemakd van verschillende marker moleculen die gerelateerd zijn aan verschillende pathofysiologische mechanismen. Voorbeelden van deze mechanismen zijn; oxidatieve stress, verandenggem in het cholesterol metabolisme, ontstekingsreacties en vitamine status. De relatie tussen deze markers is op vier verschillende niveaus onderzocht: allereerst in celkweek studies, datma in een in viro diermodel. Vervolgens is de relatie tussen cognitie en marker moleculen onderzoch in serum vam normaal verouderende mensen en tenslotte in serum van patienten met een gediagnostiseerde neurodegeneratieve ziekte.

In Hoofdstuk 1 is een overzich gegeven van de huidige kennis ontrent biologische markers in serum (of plasma) en hersenvloeistof in relatie tot dementie. Het doel was om markers te identificeren die interessant zouden kunnen zijn om een model te vomen warin verschillende markers zouden worden gecombineerd.

Verhoogde concentraties van het tau eiwit en verlagde concentraties van het amyloid p eiwit zijn herhaaldelijk gevonden in hersenvloeistof wan patianten met de ziekte wan Alzheimer.

In de meerderheid van de studies word een verhoogde concentratie van de ontstekingsmarkers al-antichymotrypsine en van haptoglobine fragmenten gevonden in hersenvloeistof van patiënten met de ziekte van Alzheimer. Verlaagde concentraties van de interleukine 6 (IL-6) receptor, glycoproteine $130,1 \mathrm{~L}-1 \beta$ receptor en tumor necrosis factor $\alpha$ (TNF- $\alpha$ ) waren gevonden in hersenvloeistof van gedementeerde patiënten. De resultaten die gerapporteerd zijn over de serum concentraties van $\| \mathrm{L}-6$ en TNF- $\alpha$ suggereren een verlaging, maar verscheidene andere stadies konden geen verschil vinden tussen AD patienten en controles. Verhoogde a antichymotrypsine concentraties in serum is warschijnlijk de meest eenduidige en best onderzochte bevinding wat betreft ontstekingsmarkers in serum.

De resultaten van studies naar de markers vooi oxidatieve stress 3-nitrotyrosine en isoprostanen lieten zien dat deze stoften in verhoogde concentrates vookomen in hat hersenvloeistof van patiënten mel dementie. Een aantal andere sudies liet zien dat verhoogde concentraties van autoantilichamen tegen glial fibrillary acidic protein (GFAP) vookomen in hersmovosisof en serum van patienten met de ziekte wan Alzheimer.

Veranderingen in serum concentraties van 245 -hydroxycholesterol (wat verhougd was in milde dementie en verladgd in ernstige dementie, telencephaline en GFAP autoantilichamen (alle wee verhoogd zouden markers woor hersenspecifieke veranderingen in Alzheimer kunnen zijn. Echter, er zijn nog maar enkele studies naar deze moleculen gewersi tor nu toe. Een indicatie voor een verslechterde algehele oxidatieve balans kan afgeleid worden uil een verlaging in de serum concentraties van vitamines $(A, B, C$ en $E$. Over het atgemeen worden er verhoogde serum concentraties van homocystene gevonden in dementie patienten, hoewel er een grote overlap met controles bestaat. Det apolipoprotein E concentraties waren slechrs licht verlaagd in serum van patienten met Alzheimer, wat erop duid dat dit cen onbruikbare marker is.

Samengeval, tot wu toe zijn er wel veranderingen gevonden in concentratties van verschillende markers gerelateerd atan de verschillende patholysiologische mechanismen in hersenvloe istof of serum. Echter, er bestaall een grote overlap tussen de gemiddelde concentraties en er werden variabele resultaten gevonden in studies die serum of hersenzloeistof van Alzheimer putionten 
en controles vergeteken hebben. Deze bevindingen ondersteunen de visie dat het niet woldoende is om serum concentraties van markers behorend bij slechts éen pathophysiologisch mechanisme le onderzoken.

De opzet van dit proetschrift was om een groot aantal mogelijke markermoleculen te onderzoeken. Deze markers moesten gerelateerd zijn aan oxidatieve processen, ontstekingen of het cholesterol metabolisme. Daarnaast zijn wee hersenspecifieke structuurmarkers onderzocht, die nomal in zeer lage concentraties in serum van gezonde personen voorkomen. Allereerst werden deze markers onderzocht in diemodellen woor neunodegeneratie. Dit was om inzicht te werkrigen in de relevantie van deze markers in neurodegeneratieve processen. In deze studies was voor een diermodel gekozen dall de mogelitkheid gaf om oxidatieve stress te bestuderen in relatie tot excitotoxiciteit, ontsteking, en hersenspecificke structurele veranderingen. Daana werden de markers onderzoch in serum van een groep hormaal verouderende mensen, wan wie een serummonster afgenomen was op drie tijdstippen over een periode van zes jaar. De serum waarden zouden worden gecorreleerd aan prestalies op vier verschillende cognitieve tests die op dezelfde momenten over zes jaar afgenomen waren. Tenslotte werden de uitkomsten van deze studies vergeleken met de serum concentraties in gediagnostiseerde groepen patiënten, te weten patiënten met de ziekte van Alzheimer, Parkinson of andere cognitieve aandoeningen. Deze studie had als doel een model te vormen op basis van een combinatie van markers, met bij voorkeur een prognostische waarde en een redelijke specificiteit en sensiliviteit.

In Hoofdstuk 2 is een celkweek model gebruilkt, het hersensferoüden model. Het doel was te onderzoeken of een functioneel stikstof monoxide (NO) cyclisch guanosine 3',5'-monofosfat (CGMP) signaaliransduclie route aanwezig was in gedifferentieerde sferoiden. In de literatuur is herhaaldelijk gesuggereerd dat de NO-CGMP signaaltransductie een rol kan spelen tijdens de ontwikkeling of degeneratie van het centraal zenuwstelsel. De hersensferoiden vormen een model warin deze fenomenen goed bestudeerd kunnen worden.

In sferoiden van drie weken oud kon de activiteit van het oplosbaar guanylaat cyclase gestimuleerd worden met $\mathrm{N}$-methyl D-aspartaat en met matrium mitroprusside (een NO donor). De resultaten lieten zien dat de NO-CGMP signaaltransductie route aanwezig was in het celkweek systeem. Oplosbaar guanylaat cyclase gemedieerde cGMP vorming werd waargenomen in NO synthase bevattende neuronen, in neuronen van de GABAerge. glutamaterge en cholinerge systemen en ook in astroglia en oligodendroglia. Activatie van hei membrangebonden guanylaat cyclase door atrial natruretisch peptide induceende eveneens een verhoging in CGMP productie. Het membrangebonden cyclase werd wadrgenomen in astroglia, microglia, glutamaat decarboxylase en calbindin bevattende structuren, en in NO synthase bevattende neuronen. Chronische inhibitie van NO synthase gedurende de celkweekontwikkeling had geen effed op het functioneren van de oplosbare en membraangebonden guanylat cyclases. Tevens had de remming van het oplosbare cyclase gecturende de celkweekontwikkeling geen effect of het functioneren van het wO synthase of membraangebonden guanylat cyclase.

De conclusie was dat NO synthase en zowel het oplosbaar als het membrangebonden guanylat cyclase anwezig zijn in de hersensferoiden en dat de activiteit van deze enzymen gestimuleerd kon worden door verschillende stofien. De hersensferoiden vomen een goed model on de NO-CGMP signaaltransductie te onderzoeken tijdens on wikkeling of degeneratie van het centrat zenuwstelsel in zoogdieren.

De anwezigheid van zowel het oplosbare alsook het membraangebonden cyclase in astrocytem, zoals beschreven in Hoofdstuk 2, leidde tot de vraag of deze twee enzymen ook tegelijkertijd in dezelfde astrocy kunnen woorkomen. Daarom is in Hoofdstuk 3 de lokalisatie van het membrangebonden en oplosbaar guanylat cyclase onderzocht in astrocyten in de lippocampus wan de rat. 
Het aantal cellen waarin CGMP gevormd was na blootstelling aan natrum nitroprusside of het atrial natriuretisch peptide, en warin tevens de marker voor astrocyten GFAP anwezig was werd geteld. Hierdoor kon worden laten zien dat in tenminste $67 \%$ van de astrocyten in de hippocampus beide guanylat cyclase isovomen aanwezig zujn. Daamaast kon wordan aangetoond dat de 31 -subunit van hel oplosbare cyclase aamwezig was in een groof aantal van de cellen die reageerden op het atriaal natriuretisch peptide, hersen natrituretisch peptide of natrium nitroprusside.

Samengeval lieten de resultaten zien dat de oplosbaar en membrangebonden guanylat cyclases tegelijkertijd kunnen voorkomen in tenminste een subpopulatie van die astrocyten in de hippocampus.

Het doel van de studie beschreven in Hootdstuk 4 was om le onderzoken of de voming van 3mitrotyrosine een vroege marker is voor neurodegeneratieve ziekten. De dosis-iespons relatie tussen blootstelling aan 3-mitropropionzutu en vorming wan 3-nitrotyrosine was onderzocht. Dit was gedaan in de hersensferoiden en in het diemodel, met behulp van zowel Wistar als Lewis ratten.

De 3-nitrotyrosine concentratie was verhoogd bij een lagere dosis en kortere blootstelling dan veranderingen in GFAP concentraties, glutamine synthase activiteit of celdood in sferoiden afkomstig van Lewis ratten. Daatnaast was de kleuring van GABAerge neuronen aangetast terwijl de kleuring van neuromaal NO synthase intact was gebleven. De 3-nitrotyrosine vorming was geremd door dithiothreitol, matar niet door glutathion, na vijf dagen blootstelling arn 3 nitropropionzuur. Het was verrassend dat N-nitro-larginine methyl ester de vorming van 3 nitroryrosine potentiëerde. In de steroide celkweek afkomstig wan Wistar ratten werd geen vorming van 3-nitrolyrosine waargenomen na vijf dagen blootstelling aan 3-nitropropionzuur. In het striatum van Lewis en Wistar ralten was geen verandering in 3-nitrolyrosine concentratie gemeten. Een verhoogde GFAP concentratie en activering van microglia werd waargenomen in Wistar ralten.

De conclusie was dat 3-nitrotyrosine een gevoeliger marker was dan GFAP en glutamine synthetase in sferö̈de kweken afkomstig uit Lewis ratten. De stamathankelijke vorming van 3nitrotyrosine residuen is een indicatie dat de genetische achtergrond van invloed kan ziin op de gevoeligheid van een marker voor oxidatieve stress. Tenstotte, de overeenkomsten tussen 3 nitropropionzum behandelde steroiden en het diermodel suggereren dat het sleroide celkweekmodel een goed alternatief kan vormen voor chronische blootstelling van diefen aan een hersentoxine.

De studie gepresenteerd in Hoofdstuk 5 onderzoch of gedragsveranderingen gerelateend waren aan striatale schade in het 3-nitropropionzummodel in ratten. Het 3-nitropropionzummodel is een model waar oxidatieve stress, excitotoxiciteit en mitochondride schade optreden, fenomenen die woorkomen bij verschil lende neurodegeneratieve ziekten.

Ratten van de Wistar en Lewis stam werden bloolgesteld aan 3-nilropropionzuur. Hel gedrag van de dieren (open veld, looppatroon en Morris zwemtadk) werd direct ha de injeclies getest en na een herstelperiode van drie weken. Er werden geen gedragsvelanderingen watrgenomen in de open veld test. Een veranderd looppatroon werd wargenomen met bellulp wan de voetsporen test, hoewel een verschillende patroon aanwezig was tussen de Wistar en Lewis ratten. Aanwankelik werd een toegenomen latentietijd wargenomen tijdons de visuele discriminatie leertak in de Morris Water trest in Wistar ratten die behandeld waren met $3-$ nitropropionzuur, vergeteken met niet behandelde Wistars. Gedurende de ruimtelike discriminatie leentak (onzichtbaar platworm in de Morris zwembak was de zwemsnolneid verlaged in beide rattenstammen na behandeling met 3-nitropropionzun. Venhoogde concentraties van het GFAP in het stratum van Wistar ratten correleerde met verscheidene 
parameters van de voetsporen test. Tevens correleerde GFAP concentratie met de tatentierijd en afgelegde afstand in de visuele als ook rumtelijke leerlak in de Morris zwemtaak.

De conclusie was dat de analyse van het looppatroon en rumtelije orientatie gevoelige indicatoren waren on gedragsweranderingen war te nemen in relatie tol degeneratie van het striatum in het 3 fitropropionzuurmodel. Daamaast bleek dat Lewis matten minder gevoelig waren dan Wistar ratten woor de behandeling met 3-nitropropionzuur.

In Hoofdstuk 6 is het langetemineffect onderzocht wan de behandeling met 3 nitropropionzutur op de 245 -hydroxycholesterol concentraties in het striatum en serum.

De concentratie van 24S-hydroxycholesterol, een hersenspecifieke cholesterol metaboliet, werd bepaald in homogenaten van het striatum en ook in serum monsters wan Wistar en Lewis ratten na behandeling met 3-nitropropionzuur. Daarnaast zijn de concentraties bepaald van cholesterol, cholesterol precursors en metabolieten, en de plantesterolen. Deze laatst genoemde sterolen zin van plantaardige oorsprong.

Drie weken nel beẻindiging van de behandeling werd er geen verschil gemeten in de 245hydroxycholesterol concentratie in het striatum of serum tussen behandelde en onbehandelde ratten. Echter, de ratio van campesterol tot cholesterol in het striatum was hoger na behandeling met 3-nitropropionzuur, vergeleken mel de controles. Dit is een indicatie woor een verhoogde passage van deze plantesterol over de bloed-hersenbariere. De ratio van lathosterol ten opzichte van cholesterol in serum en ook striatum was lager in de behandelde ratten wergeleken met de controle ratten. De concentraties van de cholesterol precursors in serum of striatum verschilden tussen de onbehandelde dieren van beide stammen.

De conclusie van deze studie was dat de veranderingen in sterol concentraties in het striatum en senum in dit model een indicatie zijn dat de cholesterol homeostase aangedan kan zijn mijens neurodegeneratieve processen die gepaard gan met aantasting wan de bloed-hersenbarriëre.

In Hoofdstuk 7 zijn mogelijke technische oorzaken voor variatie in serum concentraties onderzocht voor een aantal ontstekingseiwittem. De stabiliteit van IL-6, de IL-6 receptor, IL-10 en Clara cel eiwit (CC16, een endogene onderdrukker wan cytokines) in serum werd onderzocht met behulp van een versnelde stabiliteitstest volgens de theorie van Arthenius. Dalarnaast is het effect van variatie in tijdsduur tussen bloedafname en arwerking van het monster bepaald. Tenslotte zijn de effecten van variatie in de temperatuur tijdens het stollen en van herhaddelijk inviezen en ontooien op de serum concentraties van deze eiwitten bepald. Serum monsters werden geincubeerd bij $4^{\circ} \mathrm{C}, 20^{\circ} \mathrm{C}, 30^{\circ} \mathrm{C}$ en $40^{\circ} \mathrm{C}$ gedurende 1 ton 21 dagen. Het bleek dat de 11.6 en $\mathrm{CC} 16$ concentraties niet veranderden gedurende de incubatie bij $4^{\circ} \mathrm{C}$, $20^{\circ} \mathrm{C}$ en $30^{\circ} \mathrm{C}$. De $1 \mathrm{~L}-6$ concentratie dadde na 11 dagen incubatie bij $40^{\circ} \mathrm{C}$. De concentraties van de $11-6$ receptor en $11-10$ bleven stabiel biij $4^{\circ} \mathrm{C}$, maar namen significant af bij $20^{\circ} \mathrm{C}$ na respectievelijk 21 en 14 dagem. Bif $30^{\circ} \mathrm{C}$ en $40^{\circ} \mathrm{C}$ namen de concentraties van deze twee cylokinos al na I dag incubatie af. Uit extrapolatie van de Arrhenius plots bleek dat IL-6 receptor en $11-10$ stabiel zijn bij $-20^{\circ} \mathrm{C}$ geclurende een groot aantal jaren. Er kon geen Arrheniusplot gemaakt worden voor IL 6 en CC16, omdat deze moleculen relatief zeer stabiel waren in serum. De concentraties van de onderzochte eiwitten waren niet significant veranderd door herhaddelijk inviezen en ontooven of door langdurige stoltijden bij $4^{\circ} \mathrm{C}$ of $20^{\circ} \mathrm{C}$.

De conclusie van deze studie was dat de IL-6, IL-6 receptor en CC16 concentraties in serum met voldoende nauwkeurig bepaald kuninen worden in monsters die gedurende lange tijd bij $20^{\circ} \mathrm{C}$ of kouder zijn bewaard. Fchter, voor de bepaling van IL-10 moeten monsters bij voorkeur bij $-70^{\circ} \mathrm{C}$ worden beward. Deze bevindingen zijn wooral van belang bij grote. screeningsprogramma's of longitudinale situdies.

De studie in Hoofdstuk 8 onderzocht of ontstekingsmarkers in serum gecorreleerd zijn aan het cognitief functioneren in een gezonde verouderende populatie. Tevens was de vraag of deze markers en risicofactor kunnen vormen voor cognitieve achteruitgang. 
Een cohort van 144 personen (leeftijd 30-80 jaan is drie keer getest gedurende zes jaar followup, als onderdeel van de MAastricht Ageing Study (MAAS). De Cognitie is getest op domeinen van snelheid (Letter Digit Coding test), concentratie and informatieverwerking (Stroop test), en verbaal geheugen (Woorden Leer taak). De serum comcentraties van de volgende ontstekingsmarkers in serum werd gemeten: $11-6, C \mathrm{C} 16$, C-reatiet eiwil, haptoglobine, albumine, totaal eiwit en eiwitracties $(\alpha 1, \alpha 2, \beta, y)$

De resultaten lieten zien dat haptoglobine concentraties gemeten aan het begin wan de studie negatief correfeerden met het cognitief functioneren op de stroop test en op de verbale geheugentaak gedurende zes jaar follow-up. De concentraties van het C-reactief eiwit ann het begin van de stuclie correleerde negatief met de cognitieve prestaties op de herinmeringstest wan de Woorden leer taak gedurende zes jaar follow-up.

De conclusie was dat relatief hoge concentraties van de ontstekingseiwitten haptoglobine en Creactief eiwit een indicatie kunnen zijn voor slechte cognitieve prestaties. verdere follow-up studies zouden moeten uitwijzen of personen die relatief slechte cognitie en verhoogde serum haptoglobine concentraties hebben een vehoogd risico hebben voor cognitieve verslechtering op de lange temin. Deze resultaten zijn een ondersteuning woor hel idee dat ontstekingsreacties een rol kunnen spelen in nomale cognitieve veroudering.

In Hoofdstuk 9, is onderzocht of er een correlatie is lussen de concentraties van precursors en metabolieten van cholesterol in serum en cognitieve prestaties in de gezonde, verouderende populatie wan de MAAS.

Het serum van een cohort personen (30-80 jaar, totale $n=144)$ uit de MAAS werd geanalyseerd. De cognitie werd getest op domeinen van sneheid (Letter Digit Coding last), concentratie en informatieverwerking (Stroop test) en verbad geheugen (Woorden Leer taak). De serum concentraties van cholesterol, de cholesterol precursors lathosterol, lanosterol en desmosterol, en de concentraties van de uit het dieef afkomstige plantesterolen campesterol en sitosterol waren gemeten. Daarnaast zijn de serum concentraties van de oxysterol $24 \mathrm{~S}$ hydroxycholesterol en, ter vergelijking, 275 -cholesterol bepald.

De resultaten lieten zien dat de serum concentraties var lathosterol en lanosterol, absoluut en als ratio tot cholesterol, negatief correleenden met cognitief functioneren op de beide onderdelen van de verbale geheugentaak. Dit was te zien zowel aan het begin van de siudie en na zes jaar follow-up. Deze effecten waren onahankelijk van de leefijd, geslacht en opleidingsniveau. Analyse met Multi-level Herhalde Metingen loonde aan dat de ratio van lanosterol en lathosterol tol cholesterol an hel begin wan de studie negatiex correleerde me het cognitief functioneren over de gehele periode. De retaties tussen de serum con monties van 275-cholesterol en 245-hydroxycholesterol en cognitieve preskaties op de vier fosts waten niel consistent. De serum concentraties van de plantsterolen sitosterol er campesterol waren niet gerelateerd an cogntieve prestaties in deze populatie.

De conclusie was dat relatief hoge serum concentralles van cholesterol precursors, zowel de absolute warden als in ratio tot cholesterol, geassocieed waren met relatiei stechte cognitieve prestaties in deze nomatle verouderende populatie. Verder ondierzok met behulp wan followup studies zou een antwoord moeten geven op de vraag of langdurig verhoogde precursor concentraties een risicofactor vormen voor loenemende verslectuterng in hel cognitief functioneren.

In Hoofdstuk 10 is onderzoch of serum concentraties van homocysteine onahlankelijk gerelateerd zinn aan cognitief functioneren. Dit is onderzocht tijdens follow-up in de populatie van de MAAS, en in patienten mel de ziekte van Alzheimer. De tolale serum homocysteine concentratie is een marker voor vitamine $B 12$ en foliumzun deficièntie. Er zijn aanwijzingen in de literatur dat verhoogde homocysteine concentraties geasocieerd zijn met een verslechtering in cognitief functioneren in de gezonde populatie, en met dementis. 
Het cohort wan 144 personen (leeftijd 30-80 jaar) wit de MAAS is onderzocht aan het begin wan de studie en na zes jaar follow-up. Daarnaast zinn patiëntengroepen met de diagnose Alzheimer, Parkinson of andere cognitieve veranderingen vergeleken met een tweede controlegroep. De cognitie was getest met dezelfde tests als in Hoofdstuk 8 en 9 , te weten domeinen wan snetheid (Letter Digit Coding test), concentratie and informatie verwerking (Stroop test) en verbaal geheugen (Woorden Leer laak). Daarnaast zin de serum concentraties wan homocysteine, foliumzur en witamine B12 onderzocht.

Wanneer de patientengroepen tezamen vergeleken werden met de controles, was een lichte verhoging te zien in de totale homocysteine concentraties in serum van de patienten. De serum homocysteine concentraties correleerden megatief met het functioneren op de Woorden Leer laak aan het begin wan het onderzoek in de populatie van de MAAS. Deze effecten waren onathankelijk van leefijd, geslacht, opleiding of foliumzuur concentraties. De foliumzuur concentratie correleerde negatief mel de prestaties op de Woorden Leer taak, maar alleen aan het begin van de studie. De vitamine $B 12$ concentratie correleerde met geen van de cognitieve tests in deze populatie. Analyse met behulp van Multi-Level Herhaalde Metingen liet zien dat de totale homocysteine concentratie arn het begin van de studie correleerde met de cognitieve prestaties op de drie tests over de gehele follow-up periode.

De conclusie was dat verhoogde homocysteine concentraties een indicatie zijn voor relatief slechte cognitieve prestaties over een lange termijn in de normale verouderende populatie.

Het doell van de studie beschreven in Hoofdstuk 11 was om een model te vormen waarmee cognitieve achteruitgang gediagnostiseerd kan worden. Het model werd gevomd door de serum concentraties van 29 serum markermoleculen te evalueren aan gediagnostiseerde patiënten groepen. Tenslotte werd hel gevormde model toegepast op de serum warden van een grote controlegroep van nomal verouderende personen uit de MAAS.

De 29 serum markermolecullen hadden betrekking op de vitamine status, cholesterol homeostase en ontstekingsreacties. Daamaast was de serum concentratie van twee hersenspecifieke structurele eiwitten geanalyseerd. De volgende moleculen waren bepaald: 1) ontstekingsmarkers: C-reactief eiwit, IL-6, IL-6 receptor, CC16, eiwit fracties $(\alpha 1, \alpha 2, \beta$, $y)$ en albumine; 2) cholesterol presursors: lanosterol, lathosterol en desmosterol; cholesterol; de cholesterol metabolieten 245-trycroxycholesterol, 275-cholesterol en cholestanol; de vanuit het dieet afkomstige phytosterolen campesterol, sitosterol, brassicasterol, stigmasterol en avenasterol: 3) hersenspecifieke structurele eiwitten: telencephalline en GFAP; 4) stoffen betrokken bij het methonine metabolisme: homocysteine, cysteine, vitamine B.12 en follumzuur. Het serum was akomstig van patiënten met de ziekte van Alzheimer $(n=34)$, de zielke van Parkinson $(n-46)$, andere cognitieve aandoeningen $(n=47)$ en gezonde controles $(n=61)$. De patiẻnten waren bezoekers van het Academisch ziekenhuis Maastricht en de controles waren deelnemers ann de MAAS.

De resultaten lieten zien dat een combinatie van de lanosterol concentratie met de LL-6 receptor en de cysteine concentratie de onderscheiding van Alzheimer ten opzichte van controles vergrookte vergeleken met de serum concentratie van een enkele marker op aspecten als sensitiviteit en specificiteit. Er was geen correlatie tussen de uilkomsten van het model en de dutur of de mate van dementie wan de Alzheimer patienten. Wanneer dit model toegepast werd op de dala van cognitief gezonde personen, bleek de combinatie van serum lanosterol en IL-6 receptor niet te correleren met de cognitieve prestaties op de tests voot snelheid (Letter Digit Coding test), leren en verbaal geheugen (Woorden Leer takk) of concentratie en informatie verwerking (Stroop) in deze gezonde populatie. Dit duid enop dat de combinatie van markers. in dit model niet correleert met cognitief functioneren tijdens nomale veroudering.

De senum concentratie van alle vijf plantesterolen was verlaagd in patiënten met de ziekte van Parkinson ten opzichte van controles. De verlaggde plantesterol concentraties in serum van 
patiènten met de ziekte van Parkinson geven aan dat verder onderzoek nodig is naar hel dieet van deza patienten in relatie tot het cholesterolmetabolisme.

In het algemeen kon worden geconcludeerd (Hoofdstuk 12) dat 3-nitrotyrosine en GFAP gevoelige en vroege markers kunnen zijn voor neurodegenerative in in wiro en in vivo diermodellen, en mogelijk ook in humaan hersenvloeistof. Echer de concentraties van deze markers in serum zijn te laag om in dit opzicht bruikbad te zijn. Veranderingen in concentraties van ontstekingsmarkers zijn waargenomen in de sleroide celkweek en in het diermodel. Daamaast waren verhoogde concentraties van haploglobine, C-reactiel eiwit en CC16 geassocieerd met relatief slechte cognitieve prestaties in ons cohon wan nomale werouderende personen. Tenslote waren verlaagde concentraties van $1 \mathrm{~L}-6$ receptor, fen verhoogde concentraties van CC16 wargenomen in serum van palienten met neurodegeneratieve ziekten. Dit ondersteunt de hypothese dat ontstekingsprocessen een rof spelen in neurodegeneratieve processen. Een verandering in cholesterol homeostase, h.e. veranderingen in cholesterol precursor en plantesterol concentraties, tijdens neurodegeneratie werd onderbouwd door onze resultaten verkegen in het experiment met hei 3 nitropropionzuur diermodel, de longitudinale resultaten verkregen witt de MAAS en ook door de opmerkelijke resultaten uit het patiëntenonderzoek.

Het model dat gevormd was door de combinatie van serum markers die gerelateerd waren aan de verschillende pathofysiologische mechanismen vergroolte het onderscheidend vermogen ussen patiënten met de ziekte van Alzheimer en controles. Verdere follow-up studies zouden moeten uitwijzen of de toevoeging van andere markers aan het model hel onderscheidend vermogen tussen patientengroepen zou kunnen vergroten en of het gevormile model ook gebruik kan worden om een risicoschatting te doen of om zelfs een in witro diagnose te stellen voor de ziekte van Alzheimer. 



\section{Publications}

C.E. Teunissen, J. de Vente, H.W.M. Steinbusch and C. de Bruin. Biochemical markers related to Alzheimer's dementia in serum and cerebrospinal fluid. Neurobiology of Aging in press.

C.E. Teunissen, M. Markerink-van Ittersum, C. De Bruijn, H.W.M. Steinbusch, and 1. De Vente. Evaluation of 3-nitrotyrosine as a marker for 3-nitropropionic acid induced oxidative stress in Lewis and Wistar rats and strain-specific whole brain spheroid cultures. Brain Resarch In press.

C.E. Teunissen, M. Mulder, I. de Vente, K. von Bergmann, C. De Bruijn, H.W.M. Steinbusch and D. Lutjohanm. Concentrations of different sterols in the striatum and serum of 3mitropropionic acid treated Wistar and Lewis rats. Neurochemica/ Research 26/10 (2001).

C.E. Teunissen, H.W.M. Steinbusch, M. Angevaren, M. Appels, C. De Bruijn, 1. Prickaerts and J. de Vente. Behavioural correlates of striatal glial fibrillary acidic protein in the 3-nitropropionic acid rat model: disturbed walking pattern and spatiat orientation. Neuroscience 105/1 (2001) $153-167$.

C.E. Teunissen, H.W.M. Steinbusch, M. Markerink-van Ittersum, D. Koesling and J, de Vente. Presence of soluble and particulate guanylyl cyctase in the same hippocampal astrocytes. Brain Research 891/1-2(2001) 206-212.

C.E. Teunissen, H.W.M. Steinbusch, M. Markerink-van Ittersum, C. de Bruijn, H. Axer and J. de Vente. Whole brain spheroid cultures as a model to study the development of nitric oxide synthase - guanylate cyclase signal transduction. Developmental Bram Research 125 (2000) 99115

C.E. Teunissen. International Conference on Volatile Organic Compounds in the Enviromment. Congres report. Indoor \& Built Environment 7 (1998) 4-17.

C.E. Teunissen, S.T. Kingsion, D.C. Morse, W.M. Purcell, and C.K. Alterwill. Volatile Organic: Compounds in the Enviromment. Risk Assessment and Neurotoxicity, Vot. 3. Applicalion of rat and chick whole brain spheroids to polychlorinated biphenyl (PCB) neurotoxicity testing. Le Collane della Fondazione Salvatore Maugeri, PI-ME Press, Pavia 1998$) 263272$.

H.E.M.G Haenen., A. Spenkelink, C.E. Teunissen, J.H.M. Temmink, I.H. Koeman, P.J. vat? Bladeren. Transport and metabolism of glutathione conjugates of menadione and ethacrynic: acid in confuent monolayers of rat renal proximal tubular cells. Toxicology 110 (1996) 1-14.

\section{Publications in process}

C.E. Teumissen, D. Lütjohann, K. von Bergmann, F. Verhey, F. Vreeling, A. Wauters, E. Bosmans, M.P.l. varn Boxtel. M. Maes, H. Bosma, J. Delanghe, H. Blom, M. Verbetk P. Rieckmann, C. De Brujn, H.W.M. Steinbusch and I. de Vente. Evaluation study of 29 markers in patients with Alzheimer"s and Parkinson's disease and other cognitive disorders. Nourology Submitted. 
C.F. Teunissen, M.P. van Boxiel, H. Bosma, E. Bosmans, J. Delanghe, C. De Bruijn, A. Waulers, M. Maes, J. Jolles, H.W.M. Stentbusch and J. de Vente. Inflammation markers in relation to cognition in a healthy aging population. Alzheimer Disease and Associated Disorders Submitted.

C.E. Teunissen, J. De Vente, K. von Bergmann, H. Bosma, M. van Boxtel, C. De Bruijn, J. Jolles, H.W.M. Steinbusch, and D. Lütjohann. Serum cholesterol precursors and metabolites in relation 10 cognitive performance in an aging population. A longitudinal follow-up study. Neurobiology of Aging Submitted.

G. Kenis, C.E. Teunissen, R. de Jongh, E. Bosmans, H.W.M. Steinbusch and M. Maes. Stability of interleukin-6, interleukin-6 receptor, interleukin-10 and CC16 in human serum. Cytokine Submitted.

C.E. Teunissen, H. Blom, M. van Boxtel, H. Bosma, C. De Bruijn, I. Jolles, A. Wauters, F. Verhey, F. Vreeling, H.W.M. Steinbuschand ). de Vente. Homocysteine: a marker for cognitive performance? A longitudinal follow-up and patient study. American Joumal of Clinical Nutrition Submitted.

\section{Abstracts}

C.E. Teunissen, M. van Boxtel, F. Verhey, F. Vreeling, D. Lütjohann, A. Wauters, E. Bosmans, M. Maes, J. Delanghe, H. Blom, M. Verbeek, J. Jolles, C. De Bruijn, H.W.M. Steinbusch and J.

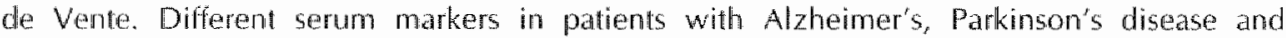
patrents with non-Alzheimer dementia. $5^{\text {th }}$ Dutch Endo-Neuro meeting. Doorwerth (2001).

C.E. Teunissen, M. Mulcler, I. de Vente, K. von Bergmann, C. De Bruijn, H.W.M. Steinbusch and D. Lüfohann. Altered brain and whole-body sterol metabolism in the 3-nitropropionic acid rat model of neurodegeneration. Gordon Research Conference "Oxidative stress and disease" Ventura, U.S.A. (2001).

C.E. Teunissen, M. van Boxtel, C. De Bruin, H.W.M. Steinbusch, H. Dieplinger, L. Jerkovic, A. Wauters, E. Bosmans, M. Maes, I. Delanghe, H. Blom, M. Verbeek, D. Lütiohann, I. Jolles andl. de Vente. Cognitive pertomance in combination with serum markers in an aging population: a six year follow-up study as part of the MAastricht Aging Study (MAAS). Society for Neuroscience Abstracts 25 (2000) $575-573$.

C.E. Teunissen, I. de Vente, $M$, van Duinen, M. Angevaren, C. De Bruijn and H.W.M. steinbusch. Biomarkers for neurodegeneration in the 3-nitropropionic acid animal model. $21^{\text {* }}$ Summer School for Brain Research. Netherlands Institute for Brain Research Amsterdam (1999).

C.E. Teunissen, 1. de Vente and H.W.M. Steinbusch. Spheroid cultures as a model system for studying the NOCGMP Cascade. European fournal of Newroscience 10/suppl.10 (1998) 28.

C.E. Teumissen, 1. de Vente and H.W.M. Steinbusch. Spheroid cultures as a model system for NO-neurotiansmitter interactions. Autumn School MRC Cambridge Centre for Brain Repair $(1997)$ 


\section{Curriculum vitae}

\section{English}

Charlotte Teunissen was borm in Deventer, the Netherlands on August the 15 1971 . She completed her secondary education at the Carolus Clusius College in Zwolle in 1989. She then started her studies Environmental Sciences at the Wageningen University with as specialisation epidemiology and toxicology. She performed two internships to increase her skills in these disciplines. First, she performed an intemship at the department of Human Epidemiology, which took place in wory Coast. West-Africa. Her second internship was performed at the department of Toxicology of the Wageningen University. Since her special interest in the neuroscience was raised, she chose to perform an additional intemship focussed on this subject, which she perfomed at the department of Medical Chenistry at the Veterinary Medical University in Vienna, Austria. After finishing her studies in august 1995, she continued her research at this latter department. She subsequently obtained a grant from the European Science foundation to pertom research using whole brain spheroid cultures at the Universty of Herdfordshire, UK. She started in March 1997 as a PhD student at the department of Psychiatry and Neuropsychology of the University of Maastricht. This department is part of the European Graduate School of Neurosciences (EURON). The experimental work carnied out until September 2001 is presented in this thesis.

\section{Nederlands}

Charlotte Teunissen werd op 15 augustus 1971 geboren te Deventer en groeide op in Wiihe. In 1989 behaalde zij haar diploma aan het Gymnasium van het Carolus Clusius College te Zwolle. Vervolgens is zij begonnem aan haar studie Milleullygiene aan de Wageningen Universiteit waar zij zich gespecialiseerd heeft in de epidemiologie en toxicologie. On zich in deze vakgebieden te bekwamen heeft zij een afstudeervak uitgevoerd bij de vakgroep Humane Ephdemiolagie, welke plaatsvond in Woorkust. West-Arilsa. Vervolgens theefli zii een afstudcervak bif de vakgroep Toxicologie wan de Wageningen Universiteil uitgevoerd. Aangezion inmiddals hat bijzondere interesse voor het central zenuwstelsel gewekt was, heef zij besloten een extra afstudeervak op het onderwerp van de neurotoxicologie wit te voeren. Dit wond plats op de vakgroep Medizinische Chemie van de Veferinair Medizinische Universitát Wien, te Oostenrijk. Na haar aistuderen in augustus 1995 heeft zij bij deze universiteit haar onderzoek voortgezet en heeft vervolgens met een beurs van de European Science foundation evaring opgedaan in het onderzoek met de hersensferoïde celkweek techniek op de University of Herdfordishire, Groot-Brittannie. In Maart 1997 is zij gestart als assistent in opleiding ande vakgroep Psychiatrie en Neuropsychologie aan de Universiteit Masstricht. Deze vakgroep is onderdeell van de European Graduate School of Neurosciences (EURON). Het onderzoek dall aldaar tot september $200 \%$ is uitgevoend stat beschreven in dit proefschrift. 


\section{Dankwoord}

Het einde wan mijn aio-tijd en van dil proefschrift is gekomen en dit betekent ook een afshuiting wan m'n Maastrichtse periode. Een mooi moment om terug te blikken en on een aantal mensen te bedanken. Dit is een heel dik proetschrift geworden en dat kom zeker doordat zoweel mensen, allen expert op een bepaald gebied, in de afgelopen vier jaar enthousiast met mij samen hebben gewerkt.

Als eerste will ik lan de Vente bedanken. Jan, in de afgelopen jaren zijn we een goed team geweest. Het is heel prettig en motiverend om met jou iets to stand te brengen, van pianostukken tot een proefschrift en als finishing touch een heus schildenij! Dan wil ik mijn paranimf Monique Mulder bedanken. Monique je bent in vele opzichten mijn positieve voorbeeld. We hebben weel gediscussieerd en gelachen en ik hoop dat we daar nog lang mee door zullen gaan. Vervolgens wil ik Marjanne Markerink bedanken. Marjanne, jouw positieve levensinstelling en jouw aandacht voor anderen maken je heel bijzonder, Je hebt me bijzonder veell werk uit handen genomen en bent een echte steroiden-expert geworden.

Ik wil mijn promotor Harry Steinbusch bedanken voor de wiiheid, die hij me al die jaren gegeven heeft om al mijn ideeën uit te voeren. Dan mijn Iweede promotor, Chris de Bruijn, ik wil je bedanken voor je ondersteuning in het leggen van externe contacten, naast je kritische suggesties voor de manuscripten.

In de beginperiode ben ik vooral bezig geweest met het opzetlen van de cellkweek, biochemische analysemethoden, en het dierexperiment. Daabij heb ik veel hulp gehad van studenten: Maaike Angevaren, Marije Appels, Fenke Verlinden, Mariska van den Broek, Marlies van Duinen, Joris Verheugten en Emiel Verstraelen. Jullie hebben allemaal mijn onderzoek een stap verder geholpen. Bovendien vond ik het heel gezellig on jullie in de buurt te hebben, ook om mijn vocabulaire weer aan te vullen, zoals bijvoorbeeld met hombuffer, $3-$ D-kweek, kiekje schieten, chips en Charly goes Nature. Dan wil ik ook Patula lansen bedanken voor je brabantse humor en nuchterheid, die hebben de lunches in de laatste maanden extra leuk gemakt. Gunter Kenis, ik will jou bedanken voor de succesvolle experimenten in Genk met wurdoop op de intensive care, en het mooie artikel dat we samen gemaakt hebben. Ilk wens je nog vele geslaagde (sferoüde-jexperimenten en een mooi proefschrift toe Tevens dank ik alle collega's van beide secties van de vakgroep Psychiatrie en Neurospychologite. ik wond het een gezellige en interessante groep. Ook bedankı woor alle hulp als donor. In hel bijzonder wil ik Wiel Honig bedanken voor de rustgevende ondersteuning bij de dierexperimenten en Hellen Steinbusch voor de hulp op het lab en bij het inwerken van studenten. Hans vies will ik bedanken voor zijn onathankelijke waardewolle visie op de discussie.

Toen alle dierexperimenten zo'n beetje liepen ben ik begonnen met de analyses van de serum monsters uit de MAAS, de studie die aan de basis van $m$ 'n proefschrift heen gestaan. In deze context wil ik Jelle Jolles en Martir van Boxtel noemen. Bedankt voor jullie bijdrage wantit de MAAS, de grote interesse en jullie wardevolle adviezen. Vervolgens will ik Hans Bosma bedanken voor de multi-level analyses, en al je kritische, stimulerende adviezen voor de manuscripten. Ik wil ook alle medewerkers en testassistenten van de MAAS bedanken, in het bijzonder Astrid Quist, Carlijn Karimoen, Nico Rozendad en Esmeralda wan Pol. Jullie hebben heel veel werk werzet binnen de MAAS en hebben je allijd bijzonder behulpaam opgestald, dat heb ik altigl heel erg gewardeerd.

Een groot aantal mensen heeft me geholpen hebben bij de laboratoriumanalyse van mijn serummonsters, wat zeer nauwkeurig gedaan was en verrassend snell af was. Ik ben ook heel erg tevreden over de manier waarop jullie meegewerkt hebben aan $m$ in mamuscripten. 
Daamaast was bet voor mij leuk om een kijkje in andere labs kunnen nemen en de nodige (inter-) mationale rijervaring opdoen. Ik wil iedereen heel hartelijk daarwoor bedanken: loris Delanghe Henk Blom, Stefanie Vloet. Dinny Oppenrali, Eugene Bosma, Michael Maes, Belinda Egyed, Karel Lamers, Marcel Verbeek, Herman de Reus, Wieneke wan Geel en Annick Wauters. I also want lo express my gratitude towards Dieter Lutjohamn, Bernard Lindenthal, Silvia Winnen, Anja Kerksiek and Prof. won Bergmamn from the Unwersity of Bonn, for wheir collaboration and for accurately andysing the senum samples. It was a/ways a surprise what kind of and how many sterols you could determine. I hope that we 'll be able to continue ou" good and inspiring cooperation. Peter Rieckmann from the University of Wirzburg thank you for your spontaneous collaboration on lelenceptraline. I hope that we can meet one day. I also warst to thank Lidija Jerkovic, Barbara Schantl, Linda, and Hans Dieplinger for their hospilality and rull suppont for my experiments in lnnsbruck. I enjoyed my stay in the beaukitu mountains of rirol, where we walked (Lidijal and tried some skying and sightsceing in the rain (Barbara). I hope that we will be able to further strengthen our collaboration.

Een volgend belangrijk onderdeel van mijn onderzoek was het verzamelen van patiënten serum, wat heel wat geduld en creatiwiteit vergt. Uiteindelijk hebben we een zeer waardevolle clataset kunnen werkriggen. Daarvoor was de inzet van Fred Vreeling en Frans Verhey, maar ook van alle assistenten en medewerkers van de poli Neurologie en de geheugenpoli, onmisbaar. Daarom wil ik hierbij iedereen bedanken voor je inzet. Ik vond het ook heel inspirerend on op de polis patiènten te ontmoeten, zij zijn tenslothe de doelgroep van dit onderzoek. Tevens wil ik de medewerkers van de prikdienst wan het AZM bedanken voor hun medewerking en alle moeite on de monsters zo snel mogelijk bij mij te laten belanden.

Natuurlijk wil ik $m$ 'n lieve ouders, Bep en Eibert bedanken. Jullie vertrouwen heeft me een basis gegeven om in $m$ ' n leven onbevangen nieuwe uitdagingen aan te gaan. Daabbij will ik ook Martleen en Klaas, Gerrit-jan en Mariët, André's familie en alle kinderen betrekken. Ik heb m'n nomadenbestaan nu zo goed als opgegeven, dus in de toekomst ben ik zeer warschijnlijk op elk feest te vinden!

Alle vrienden en vriendinner, Sandra, Anoesika, 15A en Olories: heel erg leuk dat de meeste wan jullie in de laatste jaren samen met mij Maastricht en omgeving werkend hebben en bij de promotie zijn.

Tot slot: André, liefstelief, je was er altijd met je humor en nuchtere opmerkingen, dichtbij of op middelange abitand. Het is een feest on na 5 jaar echt samen mer jou door het leven te gandin! 